\title{
Development and Resultant Implementation of Custom Software for Analyses of Galactic Neighborhoods
}

\author{
Michael Dane Moore \\ West Virginia University
}

Follow this and additional works at: https://researchrepository.wvu.edu/etd

\section{Recommended Citation}

Moore, Michael Dane, "Development and Resultant Implementation of Custom Software for Analyses of Galactic Neighborhoods" (2014). Graduate Theses, Dissertations, and Problem Reports. 433.

https://researchrepository.wvu.edu/etd/433

This Thesis is protected by copyright and/or related rights. It has been brought to you by the The Research Repository @ WVU with permission from the rights-holder(s). You are free to use this Thesis in any way that is permitted by the copyright and related rights legislation that applies to your use. For other uses you must obtain permission from the rights-holder(s) directly, unless additional rights are indicated by a Creative Commons license in the record and/ or on the work itself. This Thesis has been accepted for inclusion in WVU Graduate Theses, Dissertations, and Problem Reports collection by an authorized administrator of The Research Repository @ WVU. For more information, please contact researchrepository@mail.wvu.edu. 


\title{
Development and Resultant Implementation of Custom Software for Analyses of Galactic Neighborhoods
}

\author{
by \\ Michael Dane Moore \\ Thesis submitted to the \\ College of Engineering and Mineral Resources \\ at West Virginia University \\ in partial fulfillment of the requirements \\ for the degree of
}

Master of Science

in

Software Engineering

James D. Mooney, Ph.D., Committee Chair

Katerina Goseva-Popstojanova, Ph.D.

Jason Best, Ph.D.

Lane Department of Computer Science and Electrical Engineering

Morgantown, West Virginia

2014

Keywords: Database, SQL, HTML5, CSS3, JSON, jQuery, JavaScript, performance, galaxies, morphology, clustering, pointwise dimension 


\begin{abstract}
Development and Resultant Implementation of Custom Software for Analyses of Galactic Neighborhoods
\end{abstract}

\author{
By \\ Michael Dane Moore \\ Master of Science in Software Engineering \\ West Virginia University \\ James D. Mooney, Ph.D., Chair
}

The structure and organization of galaxies and their environments within the universe is a critical and fundamental question in the field of astrophysics. The amount of data available necessitates computer software to aid analyses. Previous research in the field utilized software built in FORTRAN for these analyses. The amount of data now available to astrophysicists is astounding and a new generation of analysis software is needed. In this thesis I present an overview of the questions posed in the field of astrophysics. I also discuss the pointwise dimension, which is the methodology used to conduct analyses of galactic environments. I discuss the development of custom software, Galactic, and the requirements which are obtained from an expert in the field. The development process including initial technology decisions as well as issues encountered and the solutions devised are presented. Particular attention is paid to improving performance relative to previous software. The Galactic software is then used to conduct scientific analysis of a newer data catalog, the 2MASS Redshift Survey (2MRS). Conclusions of this scientific research find that the environments of early-type galaxies are statistically different from late-type galaxies. The environments of barred spiral galaxies are found to be similar to the environments of unbarred spiral galaxies and to spirals for which the presence of a bar cannot be confirmed or rejected. I find that the environments of early-type galaxies are similar to each other; also all types of spirals also share similar environments. This suggests secular evolution is a larger factor than environment for evolution of elliptical vs. lenticular and barred vs .unbarred spirals. I also find that the clustering of disk-type galaxies around disk-type galaxies evolves over time. Finally, conclusions are presented regarding the benefit of collaboration between software engineers and scientists. Also discussed is the importance of domain knowledge and understanding the user's experience. 


\section{Acknowledgements}

I first want to thank my late parents, Marion and Judith Moore, who instilled in me a curiosity and desire to learn and to understand. Whether it was dinosaurs, history, or science they patiently answered my questions and allowed me to discover my abilities.

I want to thank my research advisor, Dr. Jason Best of Shepherd University, for agreeing to take part in this stage of my education. His patience, feedback, and guidance during the development of Galactic, the science, and the writing of this thesis has been invaluable.

I want to thank my committee chair, Dr. James Mooney, who agreed to this atypical cross-institution committee structure. This is especially true since I am not an on-campus student in Morgantown. Also, out of all the courses I took within the program I particularly enjoyed his course on portable software.

I would like to thank Dr. Katerina Goseva-Popstojanova for agreeing to serve on my committee based on the proposed topic alone without having had me as a student in any of her courses.

I want to thank Sara Maene for her feedback and suggestions for my defense presentation.

Finally, I want to thank those friends who encouraged and supported me during this process: Ashley, Dugald, Ginger, Jessica, and Jon. You guys and gals cannot imagine how helpful that has been. 


\section{Contents}

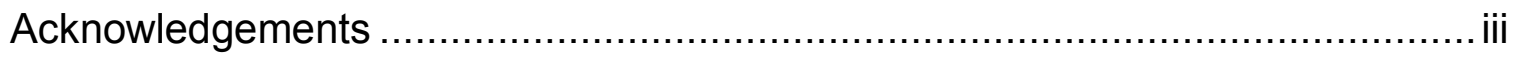

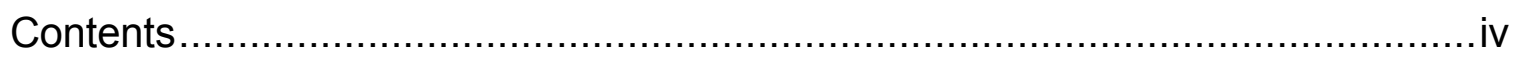

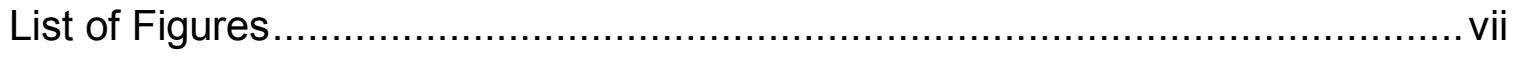

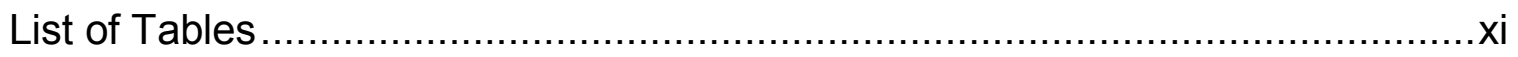

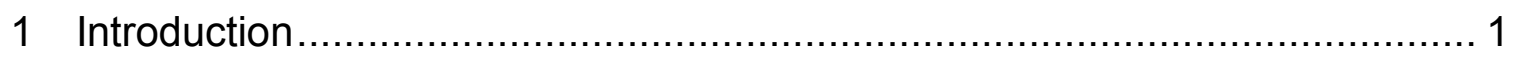

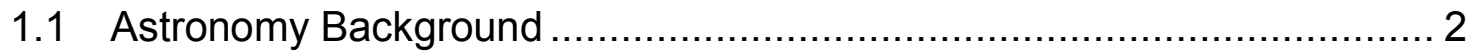

1.1.1 Galaxies and Galactic Morphology …........................................... 2

1.1.2 Redshift and Hubble's Law........................................................... 7

1.1.3 Theories of Galactic Evolution .................................................. 9

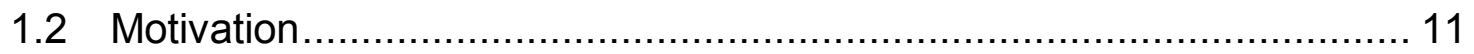

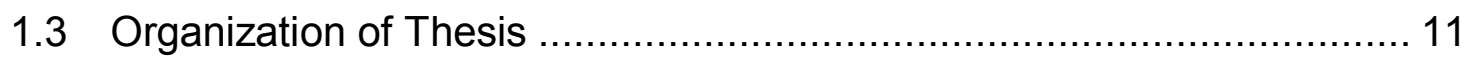

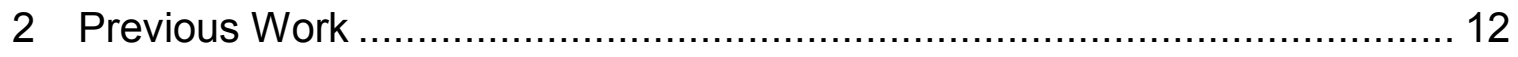

2.1 The Contribution of This Thesis ........................................................... 13

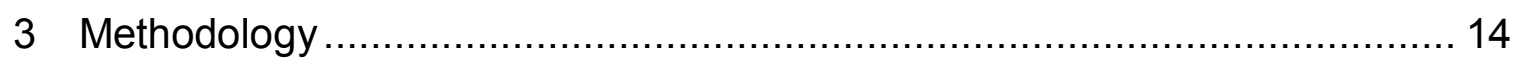

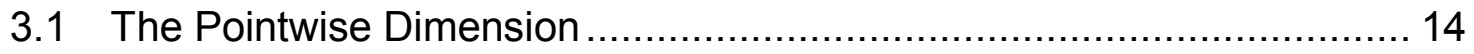

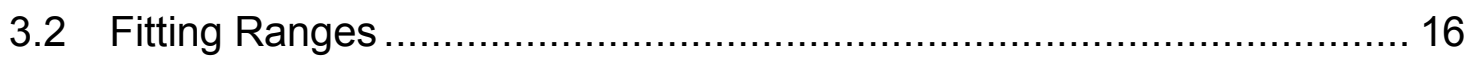

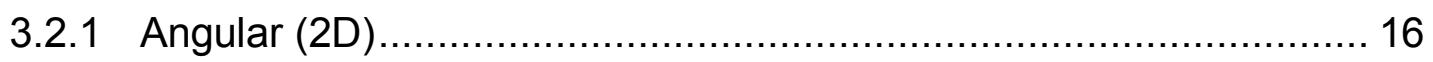

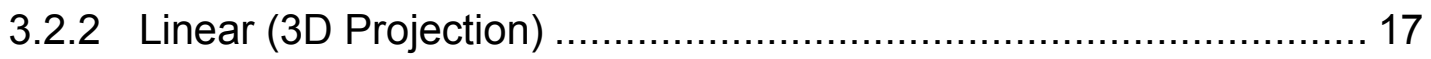

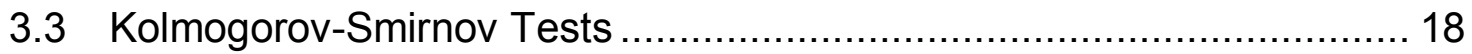

3.4 Data Catalog Selected for Analysis ..................................................... 18

3.4.1 Plots of 2MRS Data as Analyzed ............................................ 21

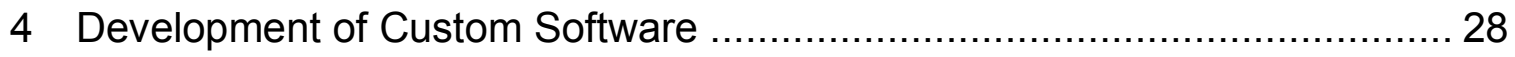

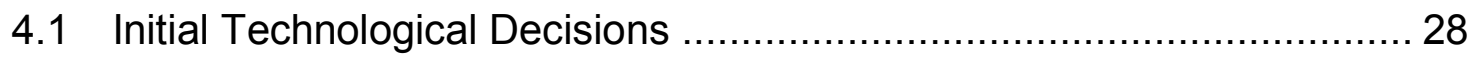

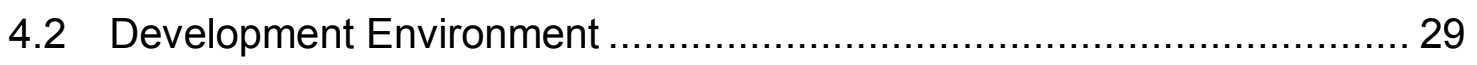

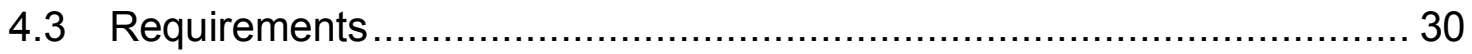

4.3.1 The system shall (mandatory): .................................................. 30 


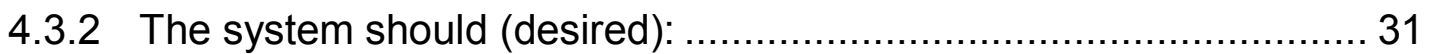

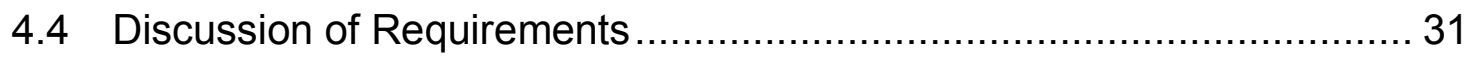

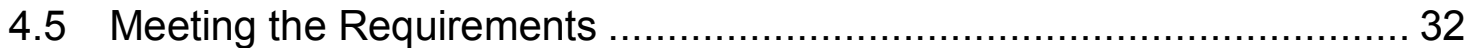

4.5.1 Dynamic Configuration of Data Set Definitions ............................. 32

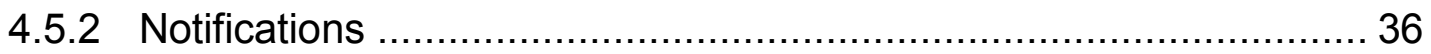

4.5.3 Benefits of Relational Database for Performance and Usability....... 36

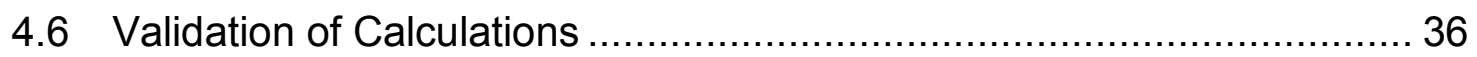

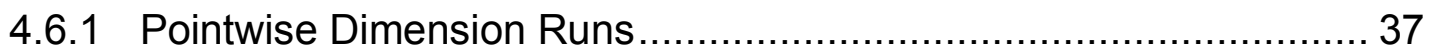

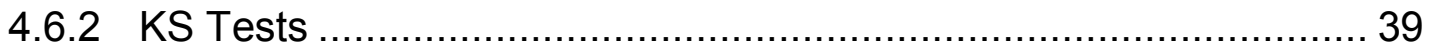

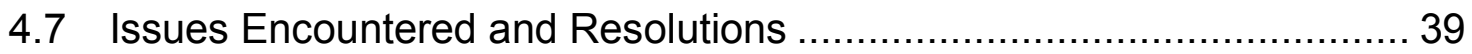

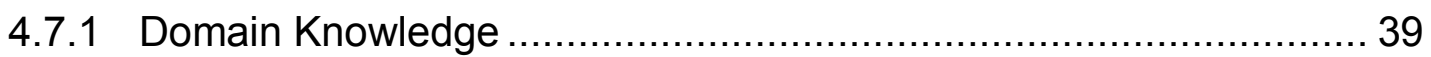

4.7.2 Right Ascension/Declination vs. Galactic Longitude/Latitude .......... 40

4.7.3 SQL Server Spatial Features..................................................... 40

4.7.4 Regular Expressions in SQL Server ........................................... 41

4.7.5 Dynamic Generation of KS Test Result Tables ........................... 42

4.7.6 Considerations for Color-Blind Users ........................................ 43

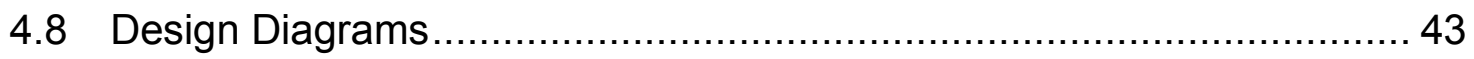

5 Use of Software for Analyses of Galactic Neighborhoods ......................... 47

5.1 Explanation of Data Run Nomenclature........................................... 47

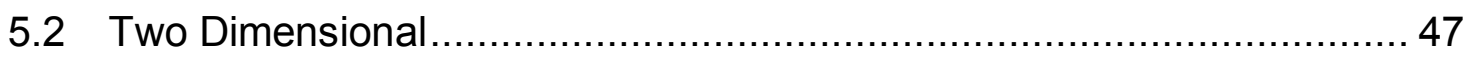

5.2.1 Averages and Standard Deviations .......................................... 47

5.2.2 Impact of Fitting Range on the Pointwise Dimension ...................... 62

5.2.3 Impact of Secondary Morphology on the Pointwise Dimension ....... 74

5.2.4 Impact of Primary Morphology on the Pointwise Dimension ........... 84

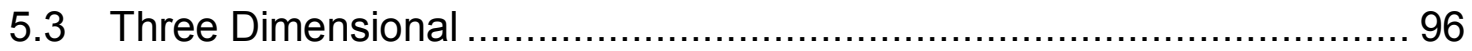

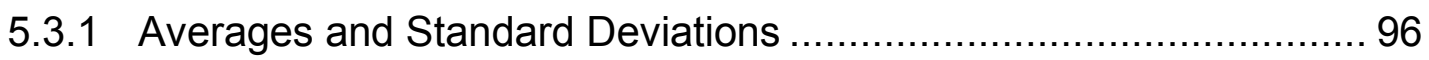

5.3.2 Impact of Fitting Range on the Pointwise Dimension ..................... 110

5.3.3 Impact of Secondary Morphology on the Pointwise Dimension ..... 122 
5.3.4 Impact of Primary Morphology on the Pointwise Dimension .......... 132

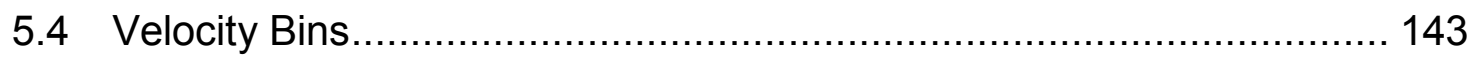

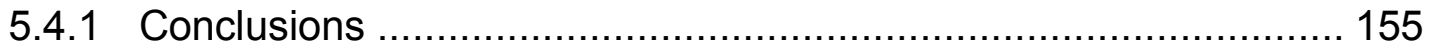

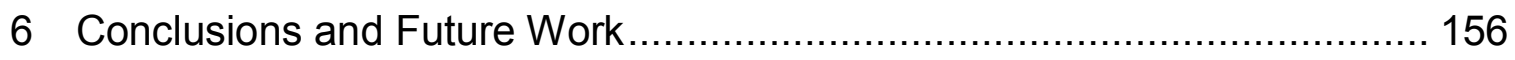

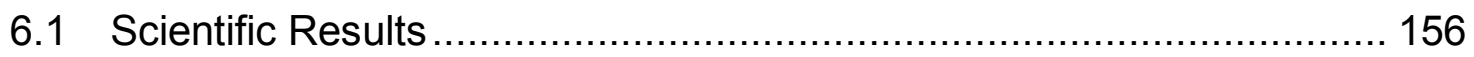

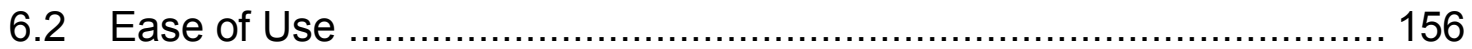

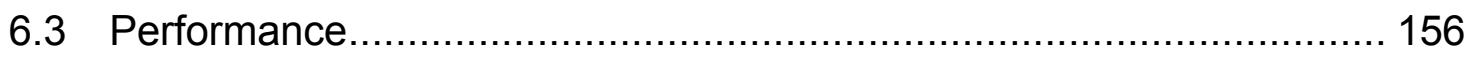

6.4 Importance of Domain Knowledge and Understanding the User Experience 157

6.5 Benefits of Cross-Field Collaboration ............................................. 157

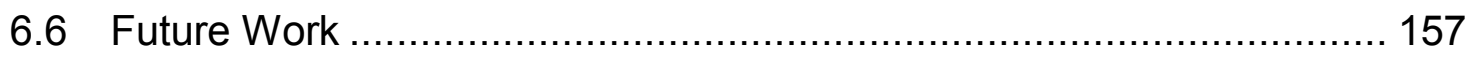

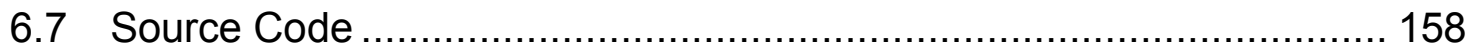

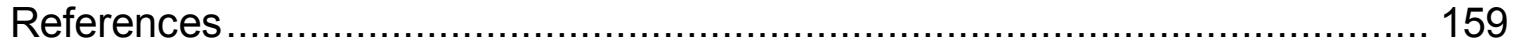




\section{List of Figures}

Figure 1-1 Transistors on processors over time illustrating Moore's Law [2] ........ 1

Figure 1-2 The Hubble Sequence, Courtesy NASA/hubblesite.org [7] ................ 3

Figure 1-3 NGC 1132, Elliptical Galaxy (credit NASA/Space Telescope Science

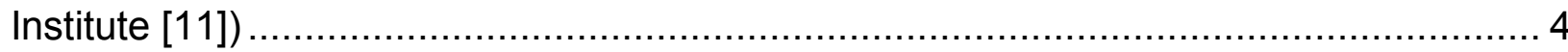

Figure 1-4 NGC 524, Lenticular Galaxy (credit NASA/ESA [12]) ….................. 5

Figure 1-5 M74, Spiral Galaxy (credit NASA/ESA/Hubble Heritage [13]) ............ 5

Figure 1-6 NGC 1300, Barred Spiral Galaxy (credit NASA/ESA/Hubble Heritage [14]) .

Figure 1-7 NGC 1441, Unbarred Spiral Galaxy (credit NASA/ESA Hubble

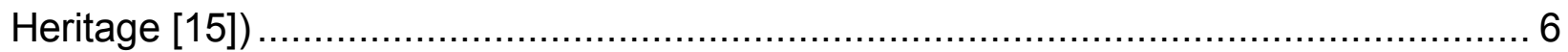

Figure 1-8 Visible Light Spectrum (Wikipedia/public domain [16]) …............... 7

Figure 1-9 Velocity-Distance relationship ............................................... 8

Figure 3-1 Positions of M58 and Surrounding Galaxies at $1^{\circ}$ distance from M58

Figure 3-2 Representative curve for M58 generated using the pointwise dimension (PD) analysis with a fitting range of $1^{\circ}$ and no secondary morphology filter 16 Figure 3-3 2MRS North and South after Discarding Objects Where $-8<b<8$.. 19 Figure 3-4 2MRS North All Galaxies ....................................................... 21

Figure 3-5 2MRS North Ellipticals............................................................. 22

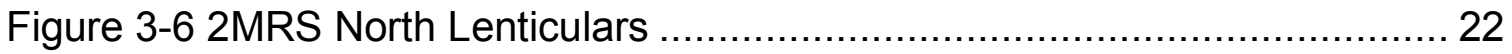

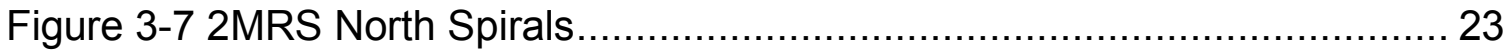

Figure 3-8 2MRS North Barred Spirals ................................................... 23

Figure 3-9 2MRS North Unbarred Spirals ................................................... 24

Figure 3-10 2MRS South All Galaxies .................................................... 24

Figure 3-11 2MRS South Elliptical Galaxies ............................................... 25

Figure 3-12 2MRS South Lenticular Galaxies ........................................... 25

Figure 3-13 2MRS South Spiral Galaxies ............................................... 26

Figure 3-14 2MRS South Barred Spiral Galaxies ....................................... 26

Figure 3-15 2MRS South Unbarred Spiral Galaxies .................................... 27

Figure 4-1 Model View Presenter Pattern - (via Wikipedia Creative Commons

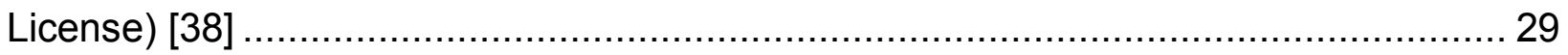

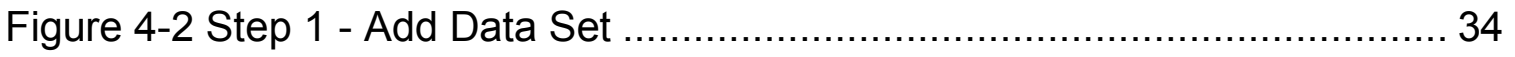

Figure 4-3 Step 2a - Add Data Set .......................................................... 34 


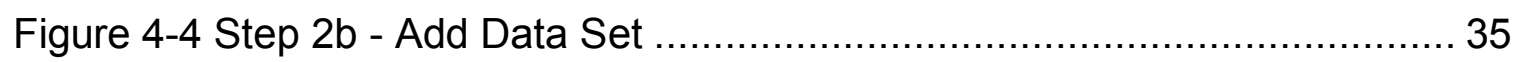

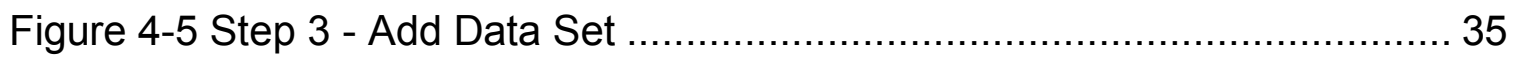

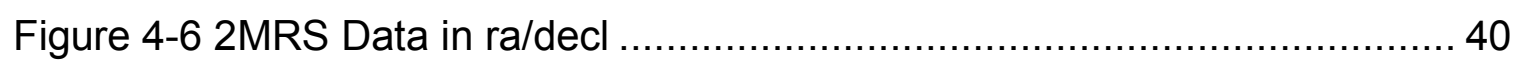

Figure 4-7 Screenshot Depicting KS Test Results Table ................................ 43

Figure 4-8 Database Table Relationship Diagram ........................................ 44

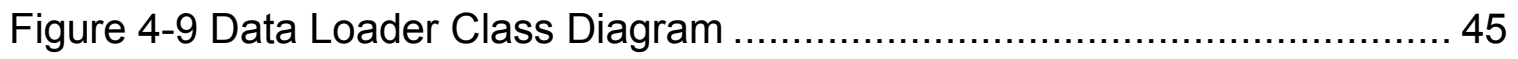

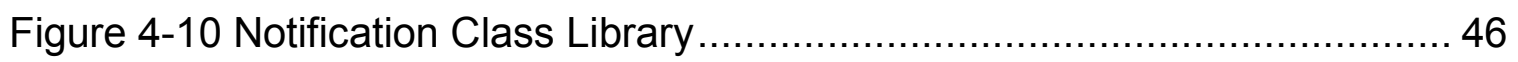

Figure 5-1 2MRS North Small Scale 2D Average PD and $\sigma$ values for All

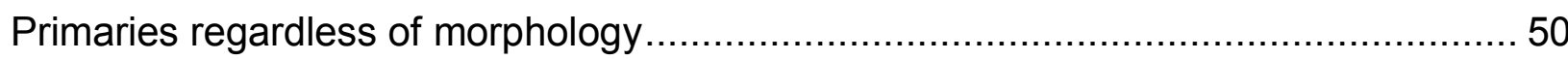

Figure 5-2 2MRS North Small Scale 2D Average PD and $\sigma$ values for Elliptical Primaries 50

Figure 5-3 2MRS North Small Scale 2D Average PD and $\sigma$ values for Lenticular

Primaries

Figure 5-4 2MRS North Small Scale 2D Average PD and $\sigma$ values for Spiral Primaries

Figure 5-5 2MRS North Small Scale 2D Average PD and $\sigma$ values for Barred

Spiral Primaries 52

Figure 5-6 2MRS North Small Scale 2D Average PD and $\sigma$ values for Unbarred Spiral Primaries 52

Figure 5-72 MRS South Small Scale 2D Average PD and $\sigma$ values for All

Primaries regardless of morphology.... 53

Figure 5-8 2MRS South Small Scale 2D Average PD and $\sigma$ values for Elliptical Primaries 53

Figure 5-9 2MRS South Small Scale 2D Average PD and $\sigma$ values for Lenticular

Primaries 54

Figure 5-10 2MRS South Small Scale 2D Average PD and $\sigma$ values for Spiral Primaries 54

Figure 5-11 2MRS South Small Scale 2D Average PD and $\sigma$ values for Barred Spiral Primaries 55

Figure 5-12 2MRS South Small Scale 2D Average PD and $\sigma$ values for Unbarred Spiral Primaries 55

Figure 5-13 2MRS North Large Scale 2D Average PD and $\sigma$ values for All Primaries regardless of morphology. 56

Figure 5-14 2MRS North Large Scale 2D Average PD and $\sigma$ values for Elliptical Primaries 56 
Figure 5-15 2MRS North Large Scale 2D Average PD and $\sigma$ values for Lenticular Primaries 57

Figure 5-16 2MRS North Large Scale 2D Average PD and $\sigma$ values for Spiral Primaries 57

Figure 5-17 2MRS North Large Scale 2D Average PD and $\sigma$ values for Barred Spirals. 58

Figure 5-18 2MRS North Large Scale 2D Average PD and $\sigma$ values for Unbarred Spirals. 58

Figure 5-19 2MRS South Large Scale 2D Average PD and $\sigma$ values for All

Primaries regardless of morphology.... 59

Figure 5-20 2MRS South Large Scale 2D Average PD and $\sigma$ values for Elliptical

Primaries 59

Figure 5-21 2MRS South Large Scale 2D Average PD and $\sigma$ values for Lenticular Primaries 60

Figure 5-22 2MRS South Large Scale 2D Average PD and $\sigma$ values for Spiral Primaries 60

Figure 5-23 2MRS South Large Scale 2D Average PD and $\sigma$ values for Barred Spiral Primaries 61

Figure 5-24 2MRS South Large Scale 2D Average PD and $\sigma$ values for Unbarred Spiral Primaries 61

Figure 5-25 2MRS North Small Scale 3D Average PD and $\sigma$ values for All Primaries regardless of morphology. 98

Figure 5-26 2MRS North Small Scale 3D Average PD and $\sigma$ values for Elliptical Primaries 98

Figure 5-27 2MRS North Small Scale 3D Average PD and $\sigma$ values for Lenticular Primaries 99

Figure 5-28 2MRS North Small Scale 3D Average PD and $\sigma$ values for Spiral Primaries 99

Figure 5-29 2MRS North Small Scale 3D Average PD and $\sigma$ values for Barred Spiral Primaries 100

Figure 5-30 2MRS North Small Scale 3D Average PD and $\sigma$ values for Unbarred Spiral Primaries 100

Figure 5-31 2MRS South Small Scale 3D Average PD and $\sigma$ values for All Primaries regardless of morphology. 101

Figure 5-32 2MRS South Small Scale 3D Average PD and $\sigma$ values for Elliptical Primaries 101 
Figure 5-33 2MRS South Small Scale 3D Average PD and $\sigma$ values for Lenticular Primaries 102

Figure 5-34 2MRS South Small Scale 3D Average PD and $\sigma$ values for Spiral Primaries 102

Figure 5-35 2MRS South Small Scale 3D Average PD and $\sigma$ values for Barred Spiral Primaries 103

Figure 5-36 2MRS South Small Scale 3D Average PD and $\sigma$ values for Unbarred Spiral Primaries 103

Figure 5-37 2MRS North Large Scale 3D Average PD and $\sigma$ values for All

Primaries regardless of morphology.... 104

Figure 5-38 2MRS North Large Scale 3D Average PD and $\sigma$ values for Elliptical

Primaries 104

Figure 5-39 2MRS North Large Scale 3D Average PD and $\sigma$ values for Lenticular Primaries 105

Figure 5-40 2MRS North Large Scale 3D Average PD and $\sigma$ values for Spiral Primaries 105

Figure 5-41 2MRS North Large Scale 3D Average PD and $\sigma$ values for Barred Spirals. 106

Figure 5-42 2MRS North Large Scale 3D Average PD and $\sigma$ values for Unbarred Spirals. 106

Figure 5-43 2MRS South Large Scale 3D Average PD and $\sigma$ values for All Primaries regardless of morphology.... 107

Figure 5-44 2MRS South Large Scale 3D Average PD and $\sigma$ values for Elliptical Primaries 107

Figure 5-45 2MRS South Large Scale 3D Average PD and $\sigma$ values for Lenticular Primaries 108

Figure 5-46 2MRS South Large Scale 3D Average PD and $\sigma$ values for Spiral Primaries 108

Figure 5-47 2MRS South Large Scale 3D Average PD and $\sigma$ values for Barred Spiral Primaries 109

Figure 5-48 2MRS South Large Scale 3D Average PD and $\sigma$ values for Unbarred Spiral Primaries 109 


\section{List of Tables}

Table 3-1 PD data for M58 2D at $1^{\circ}$ with no Secondary Morphology Filter ........ 14

Table 3-2 2MRS Galaxies for Analysis ........................................................ 20

Table 3-3 2MRS North Galaxies for Analysis................................................ 20

Table 3-4 2MRS South Galaxies for Analysis ............................................. 20

Table 3-5 Regular Expression Morphology Filters ........................................ 20

Table 4-1 Frameworks and Libraries Utilized............................................... 29

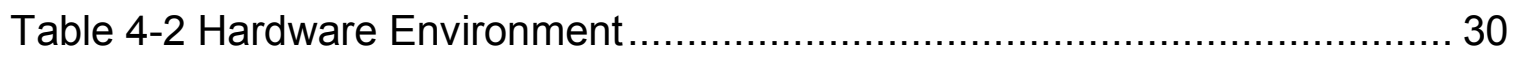

Table 4-3 Software Environment (Workstation) …........................................ 30

Table 4-4 Virtual Environment (Server) ….................................................. 30

Table 4-5 Example Data from 2dFGRS Catalog .......................................... 33

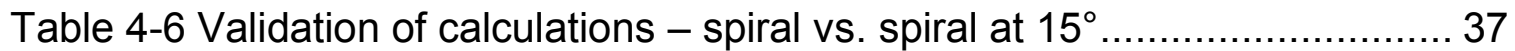

Table 4-7 Validation of calculations - spiral vs. spiral at $15 \mathrm{Mpc}$.................... 38

Table 4-8 Validation of calculations - spiral vs. spiral at $15 \mathrm{Mpc}$ with velocity bin

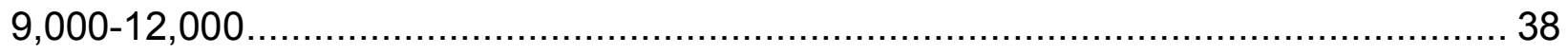

Table 4-9 Validation of Calculations - KS Tests compared to Best ................. 39

Table 4-10 Example KS results from Galactic database ................................. 42

Table 4-11 Example of desired KS test result table using columns.................. 42

Table 5-1 Morphological Filters .............................................................. 47

Table 5-2 2D North Elliptical vs. Spiral Avg. PD ……................................. 48

Table 5-3 2D South Elliptical vs. Spiral Avg. PD ........................................ 49

Table 5-4 2D KS Test Results - comparing two runs having the same primary/secondary filters and varying fitting range filters - limited to primaries with no

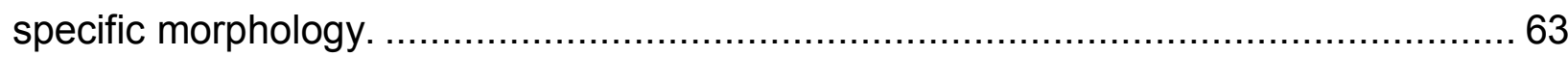

Table 5-5 2D KS Test Results - comparing two runs having the same primary/secondary filters and varying fitting range filters - limited to elliptical primaries.

Table 5-6 2D KS Test Results - comparing two runs having the same primary/secondary filters and varying fitting range filters - limited to lenticular primaries.

Table 5-7 2D KS Test Results - comparing two runs having the same primary/secondary filters and varying fitting range filters - limited to spiral primaries... 68 
Table 5-8 2D KS Test Results - comparing two runs having the same primary/secondary filters and varying fitting range filters - limited to barred spiral primaries.

Table 5-9 2D KS Test Results - comparing two runs having the same primary/secondary filters and varying fitting range filters - limited to unbarred spiral primaries.

Table 5-10 2D KS Test Results - comparing two runs having the same primary filter and same fitting range filter with different secondary filters - limited to elliptical primaries.

Table 5-11 2D KS Test Results - comparing two runs having the same primary filter and same fitting range filter with different secondary filters - limited to lenticular primaries.

Table 5-12 2D KS Test Results - comparing two runs having the same primary filter and same fitting range filter with different secondary filters - limited to spiral primaries.

Table 5-13 2D KS Test Results - comparing two runs having the same primary filter and same fitting range filter with different secondary filters - limited to barred spiral primaries.

Table 5-14 2D KS Test Results - comparing two runs having the same primary filter and same fitting range filter with different secondary filters - limited to unbarred spiral primaries.

Table 5-15 2D KS Test Results - comparing two runs having the same secondary filter and same fitting range filter with different primary filters - limited to primaries with no particular morphology in Run1. $\mathrm{Y}=$ dissimilar to the $99 \%$ level. $\mathrm{N}=$ not dissimilar to the $99 \%$ level. 85

Table 5-16 2D KS Test Results - comparing two runs having the same secondary filter and same fitting range filter with different primary filters - limited to elliptical primaries in Run1.

Table 5-17 2D KS Test Results - comparing two runs having the same secondary filter and same fitting range filter with different primary filters - limited to lenticular primaries in Run1.

Table 5-18 2D KS Test Results - comparing two runs having the same secondary filter and same fitting range filter with different primary filters - limited to spiral primaries in Run1. 
Table 5-19 2D KS Test Results - comparing two runs having the same secondary filter and same fitting range filter with different primary filters - limited to unbarred spiral primaries in Run1.

Table 5-20 2D KS Test Results - comparing two runs having the same secondary filter and same fitting range filter with different primary filters - limited to unbarred spiral primaries in Run1.

Table 5-21 3D North Elliptical vs. Spiral Avg. PD ........................................... 97

Table 5-22 3D South Elliptical vs. Spiral Avg. PD ....................................... 97

Table 5-23 3D KS Test Results - comparing two runs having the same primary/secondary filters and varying fitting range filters - limited to primaries with no specific morphology.

Table 5-24 3D KS Test Results - comparing two runs having the same primary/secondary filters and varying fitting range filters - limited to elliptical primaries.

Table 5-25 3D KS Test Results - comparing two runs having the same primary/secondary filters and varying fitting range filters - limited to lenticular primaries.

Table 5-26 3D KS Test Results - comparing two runs having the same primary/secondary filters and varying fitting range filters - limited to spiral primaries. 116

Table 5-27 3D KS Test Results - comparing two runs having the same primary/secondary filters and varying fitting range filters - limited to barred spiral primaries.

Table 5-28 3D KS Test Results - comparing two runs having the same primary/secondary filters and varying fitting range filters - limited to unbarred spiral primaries.

Table 5-29 3D KS Test Results - comparing two runs having the same primary filter and same fitting range filter with different secondary filters - limited to elliptical primaries.

Table 5-30 3D KS Test Results - comparing two runs having the same primary filter and same fitting range filter with different secondary filters - limited to lenticular primaries.

Table 5-31 3D KS Test Results - comparing two runs having the same primary filter and same fitting range filter with different secondary filters - limited to spiral primaries. 
Table 5-32 3D KS Test Results - comparing two runs having the same primary filter and same fitting range filter with different secondary filters - limited to barred spiral primaries.

Table 5-33 3D KS Test Results - comparing two runs having the same primary filter and same fitting range filter with different secondary filters - limited to unbarred spiral primaries.

Table 5-34 3D KS Test Results - comparing two runs having the same secondary filter and same fitting range filter with different primary filters - limited to primaries with no particular morphology in Run1.

Table 5-35 3D KS Test Results - comparing two runs having the same secondary filter and same fitting range filter with different primary filters - limited to elliptical primaries in Run1.

Table 5-36 3D KS Test Results - comparing two runs having the same secondary filter and same fitting range filter with different primary filters - limited to lenticular primaries in Run1.

Table 5-37 3D KS Test Results - comparing two runs having the same secondary filter and same fitting range filter with different primary filters - limited to spiral primaries in Run1.

Table 5-38 3D KS Test Results - comparing two runs having the same secondary filter and same fitting range filter with different primary filters - limited to unbarred spiral primaries in Run1.

Table 5-39 3D KS Test Results - comparing two runs having the same secondary filter and same fitting range filter with different primary filters - limited to unbarred spiral primaries in Run1.

Table 5-40 KS Tests For Velocity Bins of $3000-6000 \mathrm{~km} / \mathrm{s}$ and $9000-12000 \mathrm{~km} / \mathrm{s}$ limited to all primaries 144

Table 5-41 KS Tests For Velocity Bins of $3000-6000 \mathrm{~km} / \mathrm{s}$ and $9000-12000 \mathrm{~km} / \mathrm{s}$ limited to elliptical primaries

Table 5-42 KS Tests For Velocity Bins of $3000-6000 \mathrm{~km} / \mathrm{s}$ and $9000-12000 \mathrm{~km} / \mathrm{s}$ limited to lenticular primaries.

Table 5-43 KS Tests For Velocity Bins of $3000-6000 \mathrm{~km} / \mathrm{s}$ and $9000-12000 \mathrm{~km} / \mathrm{s}$ limited to spiral primaries

Table 5-44 KS Tests For Velocity Bins of $3000-6000 \mathrm{~km} / \mathrm{s}$ and $9000-12000 \mathrm{~km} / \mathrm{s}$ limited to barred spiral primaries 151

Table 5-45 KS Tests For Velocity Bins of $3000-6000 \mathrm{~km} / \mathrm{s}$ and $9000-12000 \mathrm{~km} / \mathrm{s}$ limited to unbarred spiral primaries 


\section{Introduction}

Advances in hardware and software open doors to new possibilities. This is the case with computers, as seen by the industry keeping up with Moore's Law. This idea, put forth by Intel co-founder Gordon Moore states "The number of transistors incorporated in a chip will approximately double every 24 months" [1]. Figure 1-1 demonstrates this by plotting transistor counts on processors $(y)$ against time $(x)$.

Figure 1-1 Transistors on processors over time illustrating Moore's Law [2]

\section{Microprocessor Transistor Counts 1971-2011 \& Moore's Law}

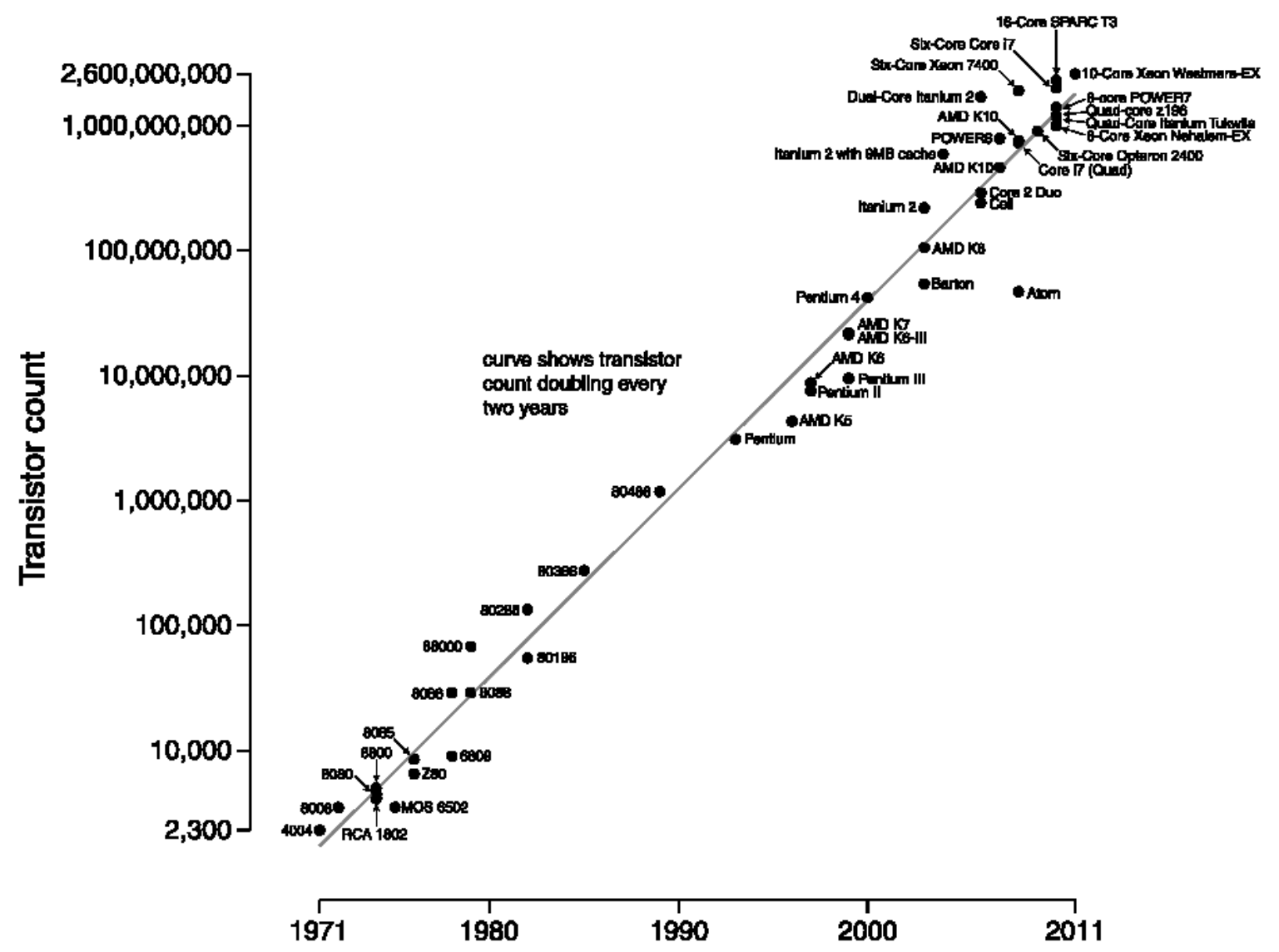

Date of introduction 
By extension this is the case in the field of Astronomy as well. In 1990 NASA launched the Hubble Space Telescope (HST) which is able to make much clearer observations due to its location in orbit above Earth. This mitigates atmospheric issues such as rain or light pollution [3]. Hubble has been serviced five times since then (1993, 1997, 1999, 2002, 2009) [4] adding new scientific instruments. In 1999 NASA launched the Chandra X-Ray Observatory, which like the HST is a telescope in orbit around Earth. [5]. Chandra has a unique orbit that takes it a third of the way to the moon at its highest point. This allows Chandra to escape terrestrial interference for up to 55 hours at a time. [5]

More advanced instruments such as the HST and Chandra permit newer, more complete data collection. The scientific community can create larger data catalogs and use higher quality images to classify celestial objects. These advancements provide room to improve by expanding upon previous work and research that has been conducted on older data catalogs.

\subsection{Astronomy Background}

As the discussion of this thesis will centralize around the software built to conduct data analysis in a field other than computer software, some astronomical background is essential to fully understand the benefits and importance of this work. This analysis studies the large-scale of the universe by comparing the distribution of galaxies.

\subsubsection{Galaxies and Galactic Morphology}

Stars, such as our sun, group together into galaxies, such as our Milky Way. The way these galaxies are shaped is termed galactic morphology. There are four general classifications of galactic morphology: elliptical, lenticular, spiral, and irregular. For the most part galaxies are of the first three classifications.

There are various properties that are related to galactic morphology. For example elliptical galaxies contain little non-stellar gas and the population of stars consists of older, smaller stars. Spirals have more gas and as a result have more active star formation and younger, hotter, more massive stars. Lenticulars are in-between for these properties. Ellipticals exhibit very slow rotation while spirals and lenticulars rotate 
quickly enough to form into disc shapes and also feature a "bulge" at the center of the disk. Finally, spirals feature supermassive black holes at the very center.

Galaxies of the elliptical, lenticular, and spiral classification make up the Hubble Sequence, named for astronomer Edwin Hubble who described the different types of nebulae (now called galaxies) in 1926 [6]. Spiral galaxies are also separated into barred and unbarred variants. Barred spirals have a thick bar-like structure across the diameter of the galaxy.

The elliptical and lenticular classifications are referred to as "early-type" and spirals are referred to as "late-type." This does not indicate an early-type to late-type evolution relationship this simply is the order in which they appear on the Hubble Sequence.

Below is Figure 1-2 which depicts the Hubble Sequence and the various morphological classifications. Note that the S0 classification at the start of the fork are lenticular galaxies.

Figure 1-2 The Hubble Sequence, Courtesy NASA/hubblesite.org [7]

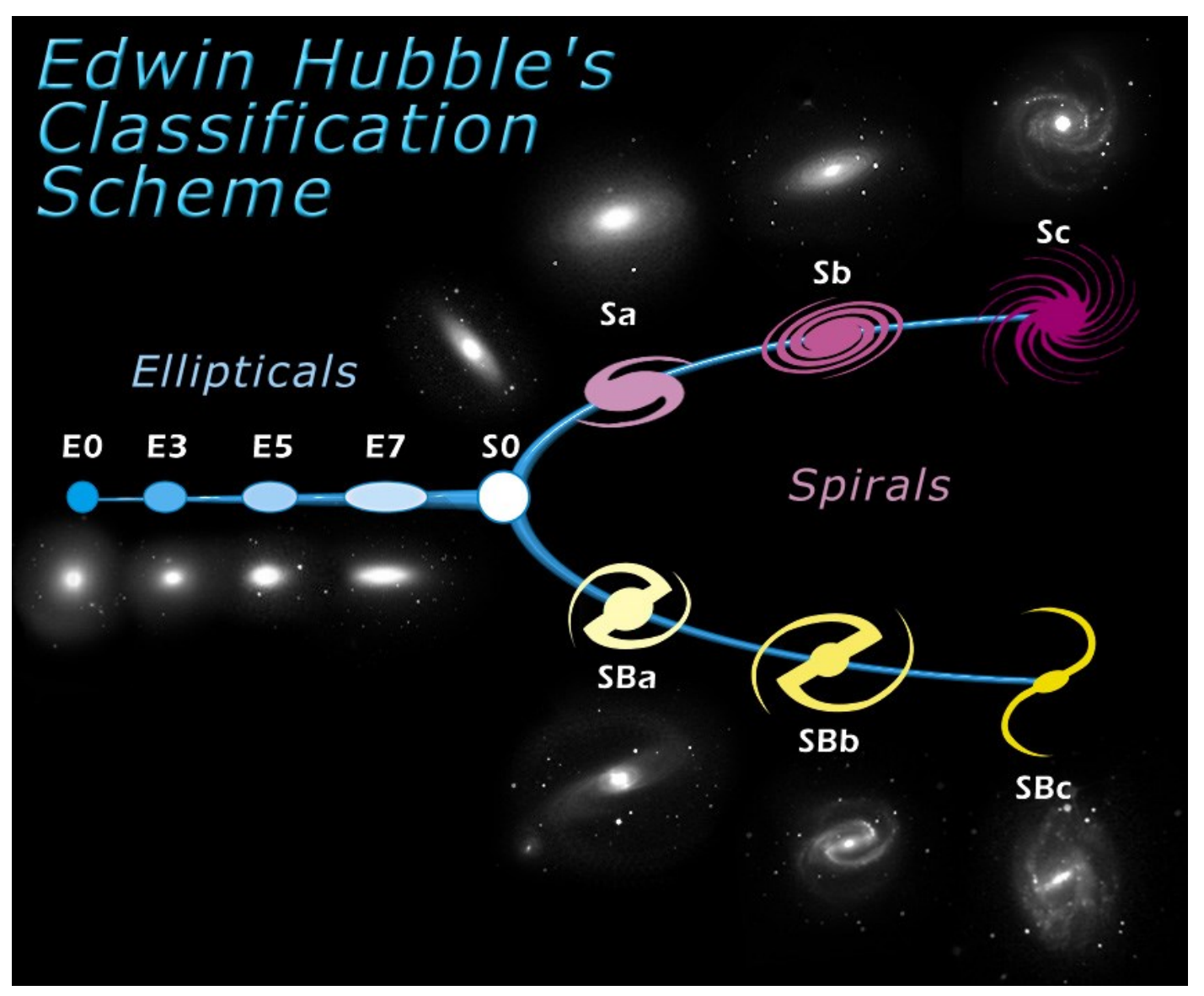


The nature of the clustering (grouping) and evolution of galaxies is a critical piece of the puzzle when investigating the origins of the universe. Galaxies have a number of properties that impact clustering and morphology. An obvious one is the mass of each galaxy, as this dictates the gravitational influence that galaxy has on its neighbors and on the interaction of individual components of the galaxy. Mass within a galaxy is not just the visible stars but objects such as planets, gasses, and dark matter. Visible matter, such as stars or planets make up only about $5 \%$ of the known universe. Dark matter makes up about $27 \%$ of the known universe [8].

Luminosity is another property of galaxies. Luminosity is the measurement of energy emitted by an object within a period of time. Galactic luminosity is related to the amount of normal matter that is organized into stars. Therefore using luminosity and a measure of the rotation of the galaxy compared to the core (velocity dispersion) and the Tulley-Fisher [9] and Faber-Jackson [10] relationships we can estimate the mass of spiral and elliptical galaxies, respectively.

\subsubsection{Example Galaxies}

Following are examples of the various galactic morphologies discussed herein.

Figure 1-3 Shows NGC 1132, an elliptical galaxy. Elliptical galaxies feature rounded edges and range from spherical to football (elongated) shapes.

Figure 1-3 NGC 1132, Elliptical Galaxy (credit NASA/Space Telescope Science Institute [11])

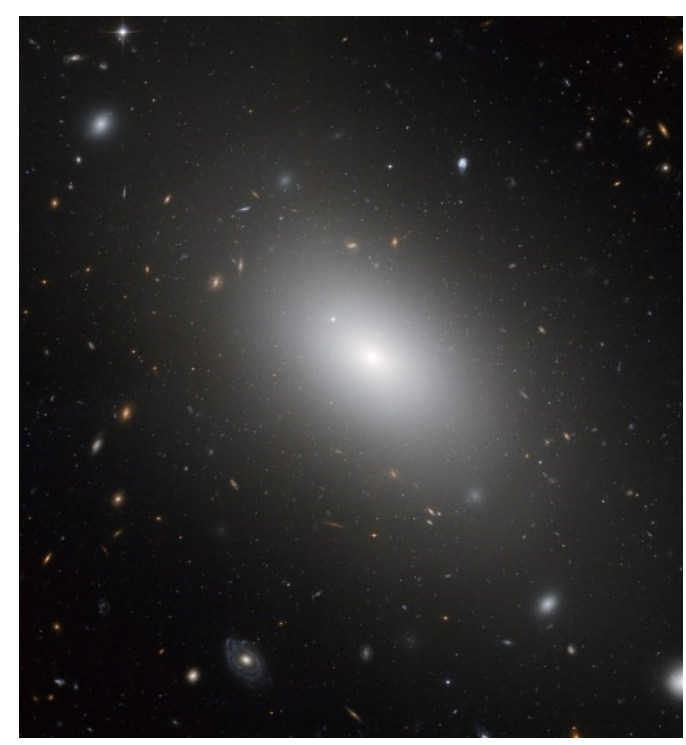


Figure 1-4 shows NGC 524, a lenticular galaxy. Lenticular galaxies are disc-shaped due to increased rotational velocity.

Figure 1-4 NGC 524, Lenticular Galaxy (credit NASA/ESA [12])

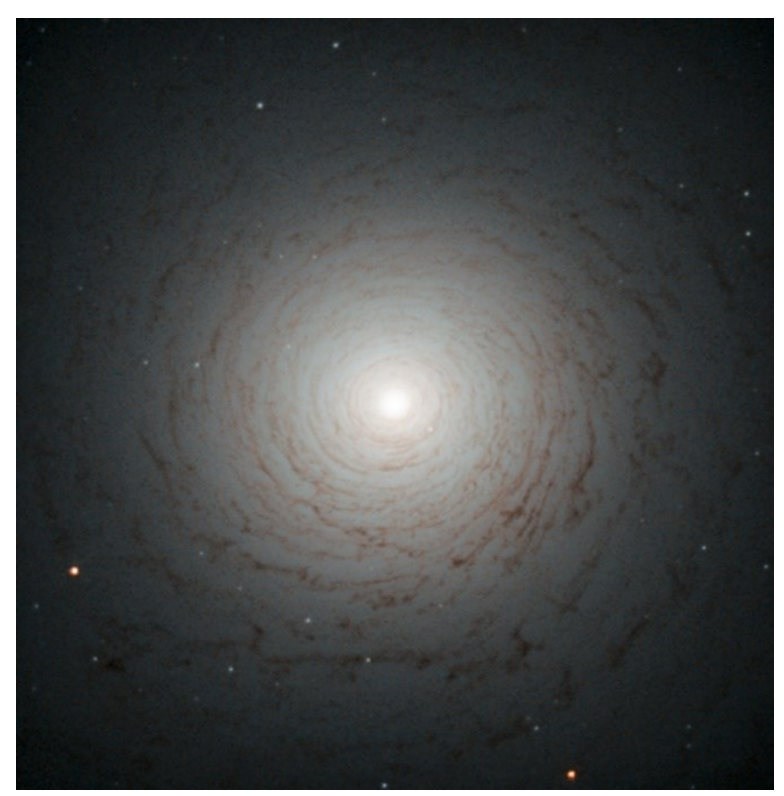

Figure 1-5 shows M74, a spiral galaxy viewed nearly face-on. Spiral galaxies are disc-shaped as well but feature the spiral arms containing stars and dust clouds.

Figure 1-5 M74, Spiral Galaxy (credit NASA/ESA/Hubble Heritage [13])

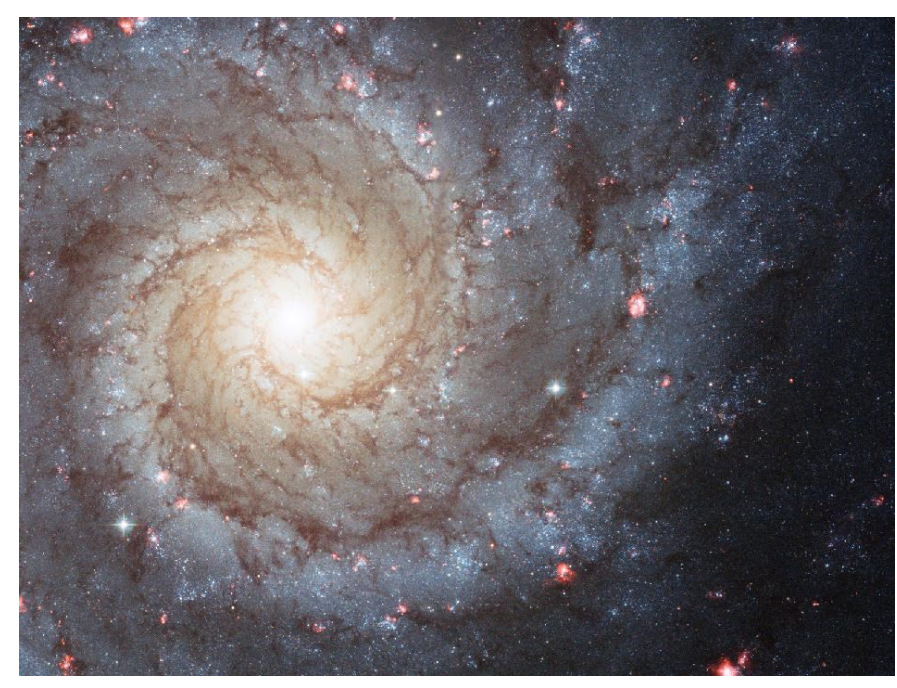


As seen in the Hubble tuning-fork diagram spirals come in barred and unbarred variants. Figure 1-6 shows NGC 1300, a barred spiral galaxy. The bar structure is highlighted in white.

Figure 1-6 NGC 1300, Barred Spiral Galaxy (credit NASA/ESA/Hubble Heritage [14])

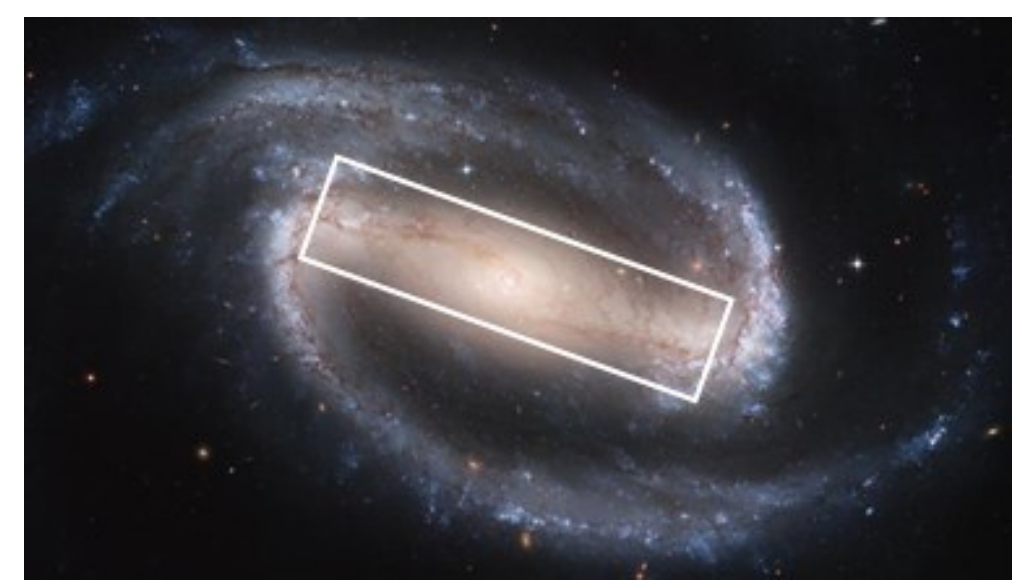

Figure 1-7 shows NGC 1441, which like M74 is also an unbarred spiral galaxy. Viewed closer to edge-on than M74 this galaxy exhibits counter-clockwise rotation (relative to observer) and a denser dust cloud.

Figure 1-7 NGC 1441, Unbarred Spiral Galaxy (credit NASA/ESA Hubble Heritage [15])

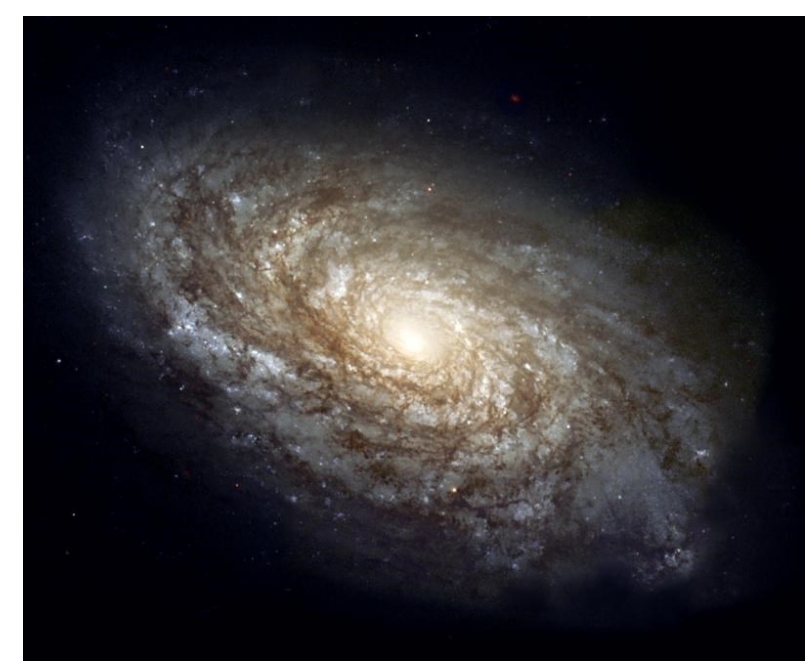




\subsubsection{Redshift and Hubble's Law}

Readers of this paper may be familiar with the concept of Doppler shift as it applies to sound. This is the principle that explains why the pitch of a sound, for example a train's horn, appears to increase as the train approaches and decrease as the train recedes. Consider Figure 1-8 below, which depicts the familiar rainbow of the visible light spectrum.

Figure 1-8 Visible Light Spectrum (Wikipedia/public domain [16])

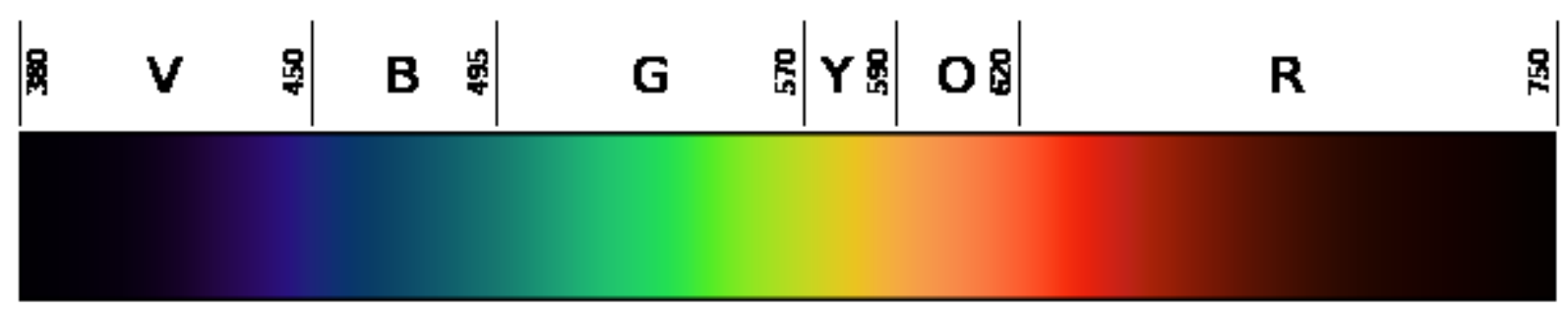

The wavelength of light from stars is shifted toward either the blue $(B)$ or red $(R)$ spectrum if it is coming towards or moving away from the observer. These phenomena are termed blueshift and redshift. The amount of shift is directly related to the velocity of the galaxy toward or away from the observer. That velocity is the radial velocity.

Due to the expansion of the universe, the vast majority of galaxies are travelling away from Earth. For those galaxies we can use the value of redshift with Hubble's Law [17] to calculate the distance of the galaxy in Megaparsecs (Mpc). One Mpc is $3.08567758 \times 10^{22}$ meters.

Hubble's law is an empirical observation [17] that for nearby galaxies a relationship exists between redshift, $z$, recessional velocity, $v$, and the speed of light, $\mathrm{c}$ such that:

$$
v=c Z
$$

The distance, $D$, to such a galaxy can be calculated as:

$$
D=\frac{v}{H_{0}}
$$

Where $\mathrm{H}_{\mathrm{o}}$ is an observed value, termed the Hubble constant, which estimates the rate of expansion of the universe. 
Consider Figure 1-9 below which illustrates the relationship of distance to radial velocity found by Hubble and Hubble and Humason in [17] and [18]. This plot is created using modern data for 40 galaxies from the 2MASS Redshift Survey (2MRS) [19].

Figure 1-9 Velocity-Distance relationship

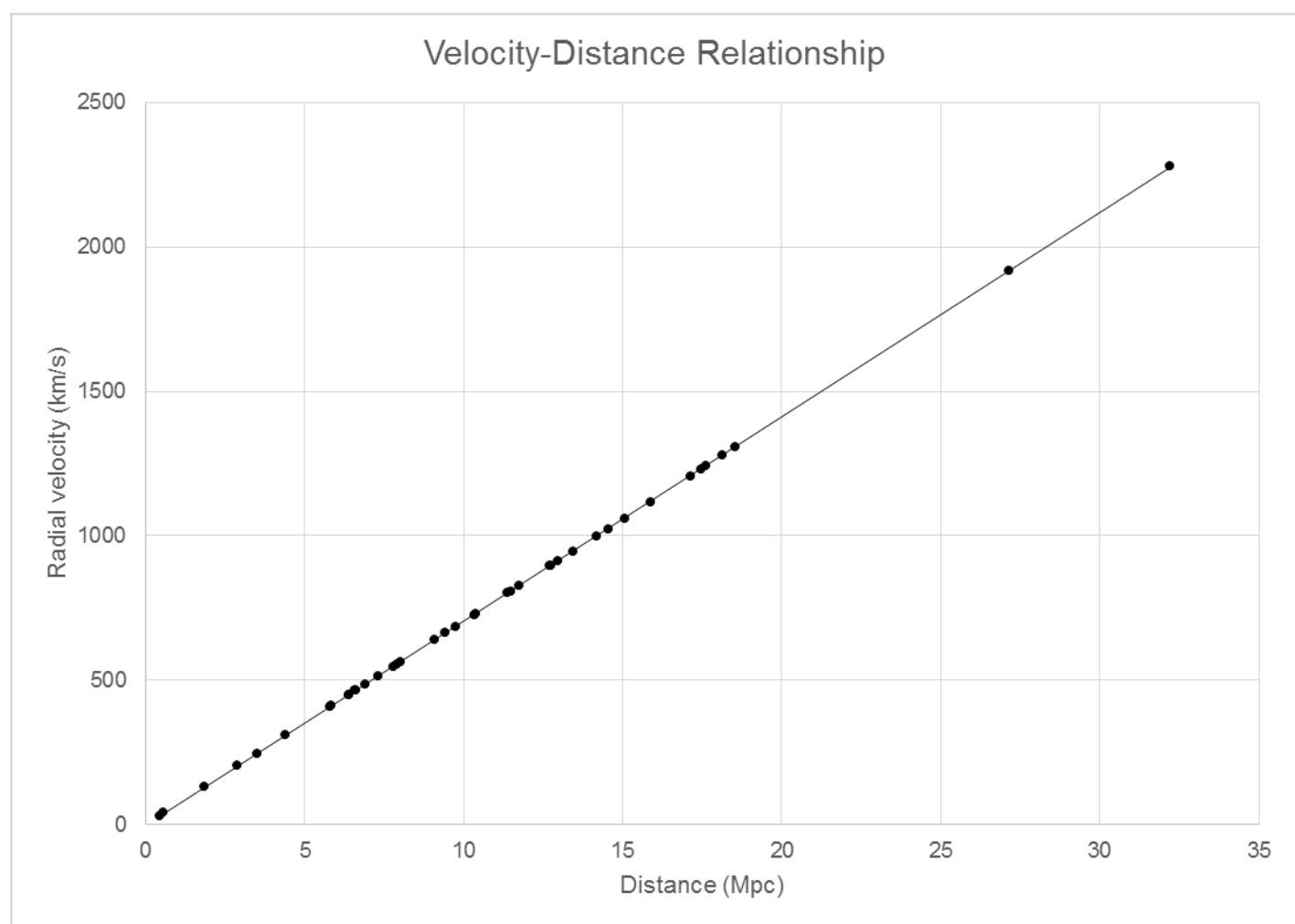

For galaxies that are farther the expansion of the universe must be accounted for. To understand implications of expansion of the universe, consider a rubber band cut so that it is now a rubber strip. Points are marked in ink on the band. As the band is stretched the increase in distance between points is proportional to the initial distance between those points.

Because of this expansion, the true distance to a galaxy by the time the light reaches the observer is greater than when the light was first emitted. The following formula accounts for this:

$$
D=\frac{c z}{H_{o}}\left[\frac{1+\frac{z}{2}}{(1+z)^{2}}\right]
$$




\subsubsection{Theories of Galactic Evolution}

There are several theories of galactic evolution which are discussed in this subsection. Thus far the consensus is that many factors play into galactic evolution.

\subsubsection{Secular Evolution}

One potential form of evolution is secular, where the internal structure of the galaxy itself is the impetus for evolution. Features in a galaxy such as bars or halos interact against/with each other and change the structure of the galaxy over time. Buta [20] provides an in-depth discussion of secular evolution. For the purpose of this paper, which seeks to measure the environmental relationship to galaxy morphology, the salient detail to keep in mind is that secular evolution can be impacted or even erased by non-secular interaction with the environment.

\subsubsection{Morphology-Density}

Research suggests that the density of a galaxy's environment has an influence on the galaxy's evolution. Oemler found in 1974 [21] that clusters of mostly spiral galaxies are similar to the field (sections of the universe outside of dense galaxy clusters) with irregular mass distribution, low density, and no central concentration in the cluster. Elliptical rich clusters show smooth spherical mass distribution with high density and a central concentration. Clusters that are spiral poor and consist mostly of lenticular galaxies have properties that are in-between the spiral rich and elliptical rich properties described above.

In 1976 Davis and Geller [22] find that the environment of elliptical galaxies is approximately twice as dense as those for spiral galaxies. They find that lenticulars reside in between spirals and ellipticals density-wise; in concurrence with Oemler. They also performed comparisons to the total catalog by removing dense clusters (Virgo, Coma) and analyzing that subset. They found that in the cluster-light subset environmental density is still higher for ellipticals than spirals, but the difference is less substantial. In 1980 Dressler [23] offered a theory that the density of environment for elliptical galaxies is responsible for slowing or stopping the formation of disks which are seen with lenticular and spirals. 
More recent research (2010) by Peng et al [24] shows that the environment has a more distinct impact on morphology once large-scale structure has formed $(z \sim 1)$. For higher redshift (immature) galaxies the mass within the galaxy and the related rate of star formation has the more distinct impact.

\subsubsection{Galactic Mergers}

A galactic merger occurs when a galaxy collides and merges with another galaxy. The frequency of galactic mergers depends on the environment and speed of the galaxies in question. Lotz et al [25] find that the major merger rate is more closely tied to the redshift of galaxies than to mass or luminosity. That is, galaxies that are moving faster experience more mergers than slower galaxies of comparable mass and luminosity.

Hibbard and van Gorken [26] studied five in-progress galactic mergers. For four of the five the remnant galaxy appears on-track to become an elliptical galaxy as the gas disks are consumed into the central core. However; for one galaxy merger there was sufficient rotation to retain much of the gas disk, suggesting that a lenticular or even spiral galaxy will result.

Galactic mergers are the most dramatic evolutionary mechanism. However, galaxies at lower velocity experience fewer mergers [25] and more minor mergers.

\subsubsection{Minor Mergers}

Evidence presented by Naab et al [27] derived from cosmological simulations on a supercomputer shows that for high redshift (distant, thus immature) spheroid galaxies accretion of sub-galactic objects evolves said spheroids into galaxies that resemble local (mature) ellipticals. This finding indicates that immature irregular galaxies can interact within their local environment to evolve into elliptical galaxies. This corresponds with the finding of the higher-density environments of ellipticals found by Davis and Geller [22] and Oemler [21] as formation in a denser environment (as other galaxies are forming) provide more opportunity for minor mergers. Lotz et al [25] find that the minor merger rate is approximately three times the major merger rate at $z \sim 0.7$ and does not change much as redshift increases. 


\subsection{Motivation}

Previous research into galactic morphology and distribution by Best [28] [29] [30] was conducted using the software tools available at the time. Dr. Best created FORTRAN software to process the data for his analyses. In some cases analysis of a data catalog can take several days. As the data catalogs continue to grow delays in waiting for a data run grow even longer. Updated software created by engineers with formal training in the software field could greatly improve on this performance.

\subsection{Organization of Thesis}

The remainder of this thesis is organized as follows. Chapter 2 discusses previous work in the field of astrophysics which serves as the foundation for this research. Chapter 3 discusses the scientific methodology of the pointwise dimension as applied to analysis of galactic distribution. Chapter 4 discusses the development of custom software, Galactic [31], for calculation and analysis of the pointwise dimension. Chapter 5 discusses the use of Galactic for analysis of the 2MRS data catalog. Chapter 6 presents conclusions and discusses future work related to this research. 


\section{Previous Work}

In the mid-1990s, Best, Charlton, and Mayer-Kress pioneered the use of a statistic known as the pointwise dimension (PD: discussed in detail in section 3 ) in the study of astronomical clustering [30]. As an example Best (2000) [28] analyzes data from the Las Campanas Redshift Survey (LCRS) [32]. The LCRS PD values are compared to a homogeneous mock catalog used by Shamarin and Yess for a different analysis of LCRS [33]. The PD values of LCRS and the mock catalog are found to be statistically dissimilar to the $99 \%$ level. Best concludes in this research that at scales out to $200 \mathrm{Mpc}$ there is no evidence to suggest that homogeneity surfaces at large scales. This finding is significant because, at a minimum, it establishes that even larger scale is required to meet the Cosmological Principle. The Cosmological Principle states that that at large enough scale the distribution of matter (galaxies and clusters of galaxies) throughout the universe is homogenous. [34]

Another use of the PD is in the analysis of possible relationships between morphology and environment. In morphological uses of the PD Best (1999) [29] analyzes data from the Dressler catalog [35]. This research "finds that for the combined Dressler data, Komolgorov-Smirnov tests show that the environments of the early-type galaxies are statistically different to the $99 \%$ level from the environments of the late-type galaxies. This result is in agreement with the general concept of a morphology-density relationship." [29] However, an analysis of 14 clusters, selected due to the availability of velocity data for most galaxies therein, found a contradictory result in that within those 14 clusters few of the statistical differences noted in the combined catalog are present. This "suggests that the morphology-density relationship is not applicable to at least onequarter of the clusters from which the result was credited in the first place (and in which the relationship should therefore be most evident), and it is therefore not as fundamental a relationship as has been previously assumed." [29] Essentially this research determines that the morphology-density relationship alone does not explain all of the observed results and that models for galactic morphology and evolution should not rely solely on the morphology-density relationship. 


\subsection{The Contribution of This Thesis}

This thesis seeks to examine the environments around different types of galaxies in order to explore possible relationships between the galactic morphologies and their environments. This is achieved by utilizing the method described in previous work by Best [30] [29] [28], the pointwise dimension (PD), to quantify the neighborhood and conducting Kolmogorov-Smirnov (KS) tests to determine if the quantified PDs are statistically dissimilar to PDs representing a different combination of primary and secondary galactic morphologies and fitting range.

This thesis discusses the development of Galactic, which is a software package created to conduct the PD and KS tests in a more user-friendly way than existing software offers. Galactic provides greater performance and usability for analyses than previously available tools. This makes analysis of the data more accessible to students and scientists who do not have development experience or training.

Finally, Galactic is used to conduct new research on the environments of galaxies and relationship to galactic morphology. This includes the first research I am aware of regarding to environments of barred and unbarred spirals. The research conducted with Galactic results in findings that constrain future theories of galactic evolution. 


\section{Methodology}

In this section I introduce the methodology used in this research.

\subsection{The Pointwise Dimension}

The pointwise dimension (PD) originated in the field of fractal geometry [36] and was applied to astrophysics in previous work by Best, Charlton, and Mayer-Kress [30], with additional work by Best [29] [28].

The pointwise dimension is applied in this thesis following Best [29]:

The cumulative count of secondary galaxies within a given radius $r$ from a primary galaxy ( $\mathrm{pg})$ is expressed as $N_{p g}(r)$. In a log-log representation of $N_{p g}(r)$ vs. $r$, there is a scaling region over which a slope can be defined. Within that scaling region, bounded by $r_{\min }$ and $r_{\min }$, the slope, $d_{p g}$, is the PD and is defined as:

$$
d_{p g}=\frac{\log \left[N_{p g}\left(r_{\max }\right)\right]-\log \left[N_{p g}\left(r_{\min }\right)\right]}{\log r_{\text {max }}-\log r_{\text {min }}}
$$

The pointwise dimension is a quantification of the environment (secondary galaxies) around a galaxy (the primary) within a particular fitting range (radius).

Below in Table 3-1 is a 2D example of the data for M58 for all secondaries (regardless of morphology) within a fitting range of $1^{\circ}$. Below in Figure 3-1 is a plot of the positions of M58 and the surrounding galaxies at a fitting range of $1^{\circ}$ which illustrates the galaxies given in Table 3-1. Finally, Figure 3-2 is a plot of an example PD.

Table 3-1 PD data for M58 2D at $1^{\circ}$ with no Secondary Morphology Filter

\begin{tabular}{rrrlrr}
\multicolumn{1}{c}{ Count } & \multicolumn{1}{c}{$\log \mathbf{N}_{\mathbf{p g}}$} & \multicolumn{1}{c}{$\log \mathbf{r}$} & \multicolumn{1}{c}{ Galaxy Name } & \multicolumn{1}{c}{ glon } & \multicolumn{1}{c}{ glat } \\
\hline 1 & 0 & -0.308785504 & NGC_4564 & 289.56033 & 73.92059 \\
2 & 0.301029996 & -0.200268285 & $12363270+1115287$ & 289.77798 & 73.748 \\
3 & 0.477121255 & -0.190537859 & $12363426+1114199$ & 289.81485 & 73.73051 \\
4 & 0.602059991 & -0.178252407 & IC_3574 & 288.79614 & 74.86443 \\
5 & 0.698970004 & -0.170502646 & NGC_4550 & 288.09363 & 74.63187 \\
6 & 0.77815125 & -0.168205376 & NGC_4551 & 288.16608 & 74.68074 \\
7 & 0.84509804 & -0.117161383 & 12353724+1222552 & 288.05057 & 74.79482 \\
8 & 0.903089987 & -0.098823086 & NGC_4606 & 293.24512 & 74.58636
\end{tabular}




\begin{tabular}{rrrlrr}
\multicolumn{1}{c}{ Count } & \multicolumn{1}{c}{ Log $\mathbf{N}_{\mathbf{p g}}$} & \multicolumn{1}{c}{$\log \mathbf{r}$} & \multicolumn{1}{c}{ Galaxy Name } & \multicolumn{1}{c}{ glon } & \multicolumn{1}{c}{ glat } \\
\hline 9 & 0.954242509 & -0.068423075 & NGC_4607 & 293.48456 & 74.57033 \\
10 & 1 & -0.048878902 & MESSIER_089 & 287.93384 & 74.9665 \\
11 & 1.041392685 & -0.01728186 & $12393316+1058036$ & 292.54865 & 73.59927 \\
12 & 1.079181246 & -0.014533898 & $12411573+1123140$ & 293.80518 & 74.07825 \\
13 & 1.113943352 & -0.013624176 & A1238+1227 & 293.50714 & 74.86367
\end{tabular}

Figure 3-1 Positions of M58 and Surrounding Galaxies at $1^{\circ}$ distance from M58

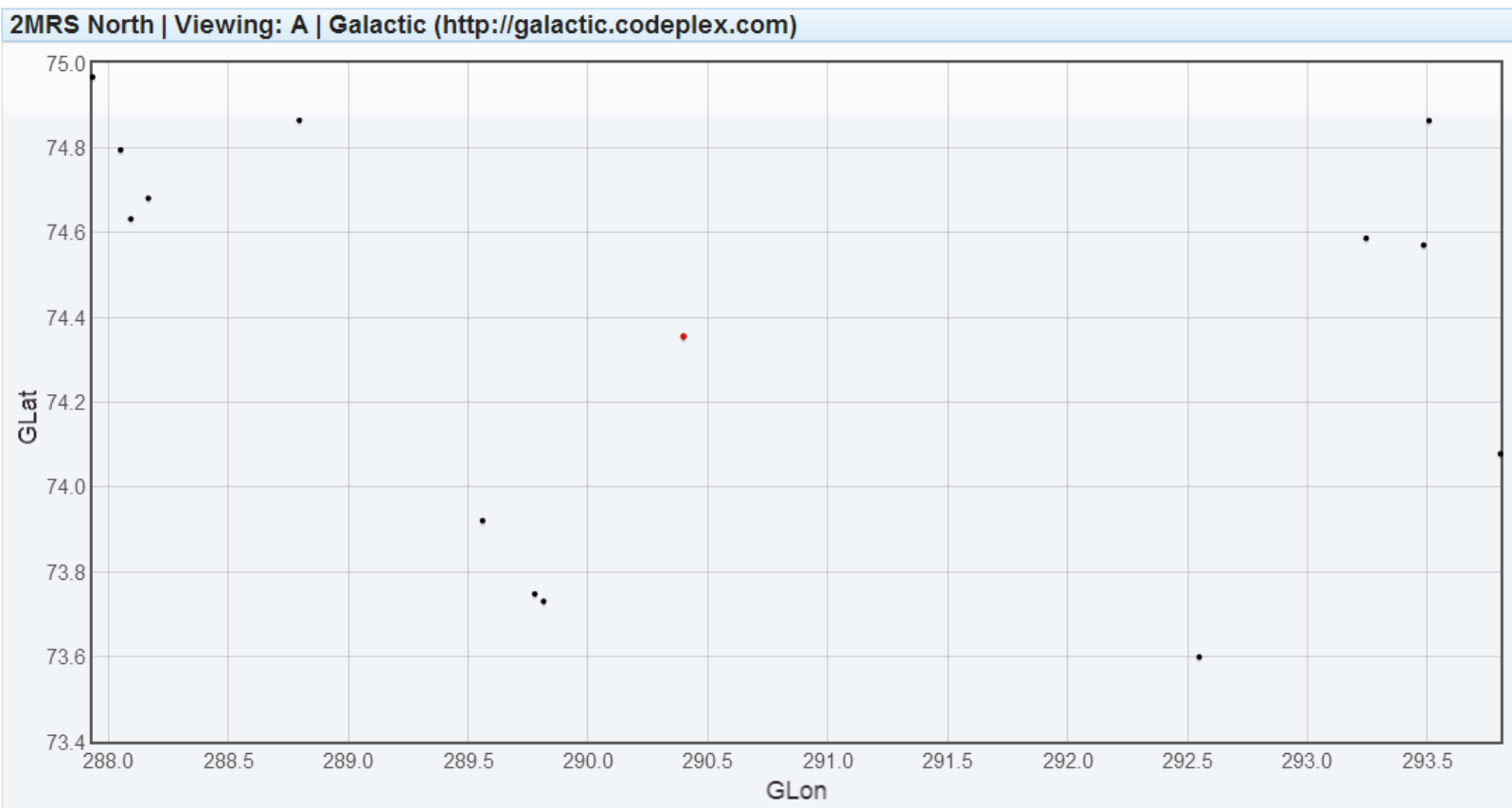


Figure 3-2 Representative curve for M58 generated using the pointwise dimension (PD) analysis with a fitting range of $1^{\circ}$ and no secondary morphology filter

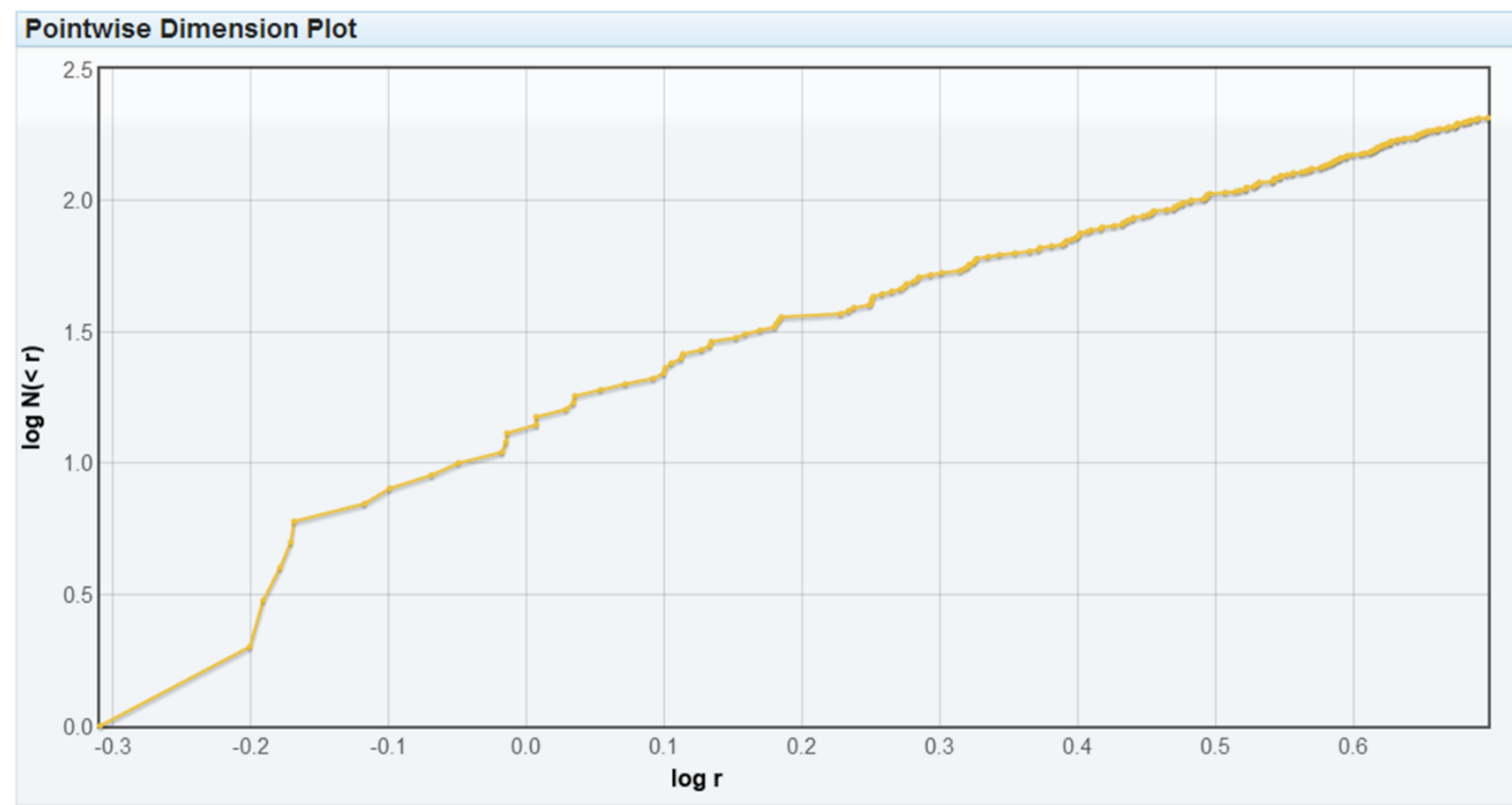

\subsection{Fitting Ranges}

Fitting ranges for this analysis can be calculated as angular separation from the primary galaxy (two-dimension). A linear distance can also be calculated using a threedimensional projection.

\subsubsection{Angular (2D)}

To understand how galactic separations are measured, let me use the analogy of a golf ball. The observer would be at the center of the ball, while the positions of the galaxies would be represented by the dimples on the surface of the ball. As we are measuring on a curved surface the shortest distance between the two points will not be a straight line, but a curve. 
The distance, as a curve, between two points is calculated using the Great Circle formula:

$$
\begin{gathered}
\cos (\theta)=\cos \left(l_{1}-l_{2}\right) \cos \left(b_{1}\right) \cos \left(b_{2}\right)+\sin \left(b_{1}\right) \sin \left(b_{2}\right) \\
\theta=\operatorname{acos}(\theta)
\end{gathered}
$$

Where $I_{1}$ and $l_{2}$ are the galactic longitudes for the two points and $b_{1}$ and $b_{2}$ are the galactic latitudes. Secondary galaxies within a radius corresponding to the angle, $\theta$, in degrees are included for analysis.

\subsubsection{Linear (3D Projection)}

One can use the actual distance to the galaxy in order to convert the angular calculation into a linear projection.

First the actual distance to the galaxy is needed. This is found through the use of the redshift/velocity data and Hubble's Law [17] to calculate the distance to objects which are traveling away from Earth (recessional velocity).

The data catalogs contain the redshift, $z$, and/or the radial velocity, $v$, for that object. If only one value is given the other can be calculated using the following relationship:

$$
z=\frac{v}{c}
$$

Where $c=300,000 \mathrm{~km} / \mathrm{s}$ is the speed of light.

Having a value for $z$; the distance in Mpc can be calculated using Hubble's Law:

$$
D=\frac{c z}{H_{o}}\left[\frac{1+\frac{z}{2}}{(1+z)^{2}}\right]
$$

Where $v$ is a positive value (recessional velocity) and $H_{o}$ is the Hubble Constant. In this paper I use a value of $70(\mathrm{~km} / \mathrm{s}) / \mathrm{Mpc}$ for $H_{o}$. 
The angular separation, $\theta$, converted to radians is scaled to cover an appropriate portion of the sky for the known distance of the primary galaxy. This is expressed as: $r=\theta D$ which yields a radius in Mpc. Secondary galaxies within this radius are included for analysis.

\subsubsection{Velocity Bins}

Because the linear projection cannot account for two objects that are very distant from one another yet share a small angular separation, another method employed with $3 \mathrm{D}$ is the velocity bin. This is a simple concept in that galaxies must have velocity within a specified range to be included for analysis. The application of the velocity bin can be expressed as:

$$
v_{\min } \leq v \leq v_{\max }
$$

Where $v$ is the velocity value of a galaxy, $v_{\min }$ is the minimum velocity to include in the analysis and, $v_{\max }$ is the maximum velocity to include in the analysis.

\subsection{Kolmogorov-Smirnov Tests}

Once the PD has been calculated for the subsets to be investigated KolmogorovSmirnov (KS) two sample tests are used to determine the probability that the two sets of PD data are from the same distribution. When the $p$ value of a two sample KS test is < 0.01 there is less than a $1 \%$ chance that the two subsets are from the same distribution. That is, the environments are statistically dissimilar to the $99 \%$ level.

\subsection{Data Catalog Selected for Analysis}

A new galactic data catalog is analyzed for this thesis. The 2MASS Redshift Survey (2MRS) [19] contains data for 44,599 galaxies representing $91 \%$ of the currently observable sky. The $9 \%$ not covered is obscured by the Milky Way. [19] The 2MRS data catalog is analyzed to exclude galaxies with a negative or zero velocity, so that distance can be calculated using Hubble's Law (which relates positive recessional velocity to distance). 
Also, galaxies with a galactic latitude between 8 and -8 degrees are excluded to obtain a flat border around the Milky Way [19]. Of the 44,599 galaxies in 2MRS, 41,976 are retained for analysis. Galaxies having galactic latitude $>8$ degrees are designated as 2MRS North, and galaxies having galactic latitude $<-8$ degrees are designated as 2MRS South. A plot of both North and South, Figure 3-3, follows to illustrate the resultant combined data to be analyzed in this paper.

Figure 3-3 2MRS North and South after Discarding Objects Where $-8<b<8$

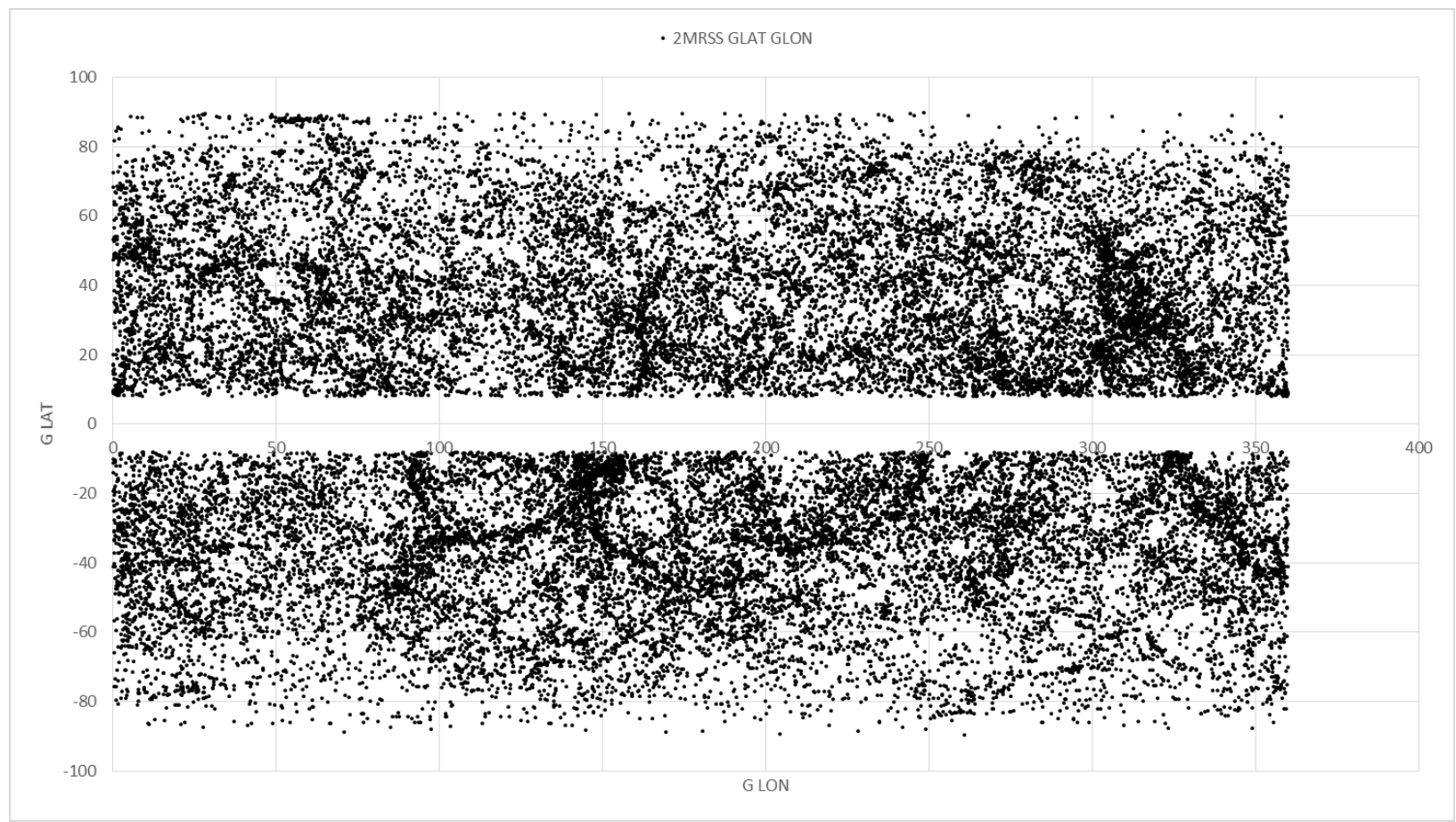

In the subset of 2MRS data catalog analyzed in this paper ellipticals represent $12 \%$, lenticulars are $13 \%$, and spirals are $30 \%$ of the catalog (see Table 3-2, Table 3-3, and Table 3-4 for detail). In addition, since the 2MRS catalog provides sufficient morphological depth where catalogs analyzed in previous work did not; barred spirals and unbarred spirals are compared in addition to the larger category of all spirals. The 2MRS data catalog does not include bar-type for the vast majority of spirals. Those galaxies are treated as spirals. Also barred and unbarred spirals are treated as spirals when looking at the simple spiral morphology. Galaxies that do not fall into the elliptical, lenticular, or spiral categories are either irregular galaxies or galaxies that were classified with uncertainty. The specific filters used are given in Table 3-5 below. 
Table 3-2 2MRS Galaxies for Analysis

\begin{tabular}{rrrrrr} 
& Lenticular & Elliptical & Spiral & Barred Spiral & Unbarred Spiral \\
\hline Count & 5,435 & 5,146 & 12,734 & 1,841 & 1,709 \\
$\%$ & $12.95 \%$ & $12.26 \%$ & $30.34 \%$ & $4.39 \%$ & $4.07 \%$
\end{tabular}

Table 3-3 2MRS North Galaxies for Analysis

\begin{tabular}{r|rrrrr} 
& Lenticular & Elliptical & Spiral & Barred Spiral & Unbarred Spiral \\
\hline Count & 2,980 & 2,488 & 6,778 & 1,135 & 1,115 \\
$\%$ & $13.83 \%$ & $11.55 \%$ & $31.45 \%$ & $5.27 \%$ & $5.17 \%$
\end{tabular}

Table 3-4 2MRS South Galaxies for Analysis

\begin{tabular}{r|rrrrr} 
& Lenticular & Elliptical & Spiral & Barred Spiral & Unbarred Spiral \\
\hline Count & 2,455 & 2,658 & 5,956 & 706 & 594 \\
$\%$ & $12.02 \%$ & $13.01 \%$ & $29.16 \%$ & $3.46 \%$ & $2.91 \%$
\end{tabular}

For the purpose of this paper I will refer to spirals with a given bar type (unbarred or barred) explicitly as barred or unbarred spirals. Where I refer to spirals without reference to a bar type this always indicates a spiral with no given bar type within the catalog.

The morphology classification in the 2MRS data release is in column 23 and described in Table A8 of the paper [19]. I generate regular expressions to use when searching the database. These regular expressions are detailed below.

Table 3-5 Regular Expression Morphology Filters

\begin{tabular}{cll} 
Morphology & \multicolumn{1}{c}{ Regex Filter } & \multicolumn{1}{c}{ Explanation } \\
\hline $\mathrm{A}$ & & All galaxies \\
$\mathrm{E}$ & ${ }^{\wedge}-[4-7]\{1\}$ & Classifications starting -4 to -7 \\
$\mathrm{~L}$ & ${ }^{\wedge}-[1-3]\{1\}$ & Classifications starting -3 to -1 \\
& & Classifications starting 1-7 not followed by another number. \\
$\mathrm{S}$ & ${ }^{\wedge}\left([1-7]\left(\left[^{\wedge} \mid \mathrm{d}\right] \mid \$\right)\right) \mid 20$ & Also classifications starting with 20 \\
$\mathrm{S}_{b}$ & $\wedge([1-7] \mathrm{B}) \mid 20 \mathrm{~B}$ & Classifications starting 1-7B. Also classifications starting with 20B \\
$\mathrm{S}_{u}$ & $\wedge([1-7] \mathrm{A}) \mid 20 \mathrm{~A}$ & Classifications starting 1-7A. Also classifications starting with 20A
\end{tabular}




\subsubsection{Plots of 2MRS Data as Analyzed}

The following figures in this section depict the data as analyzed with breakdowns for each morphological category.

Figure 3-4 2MRS North All Galaxies

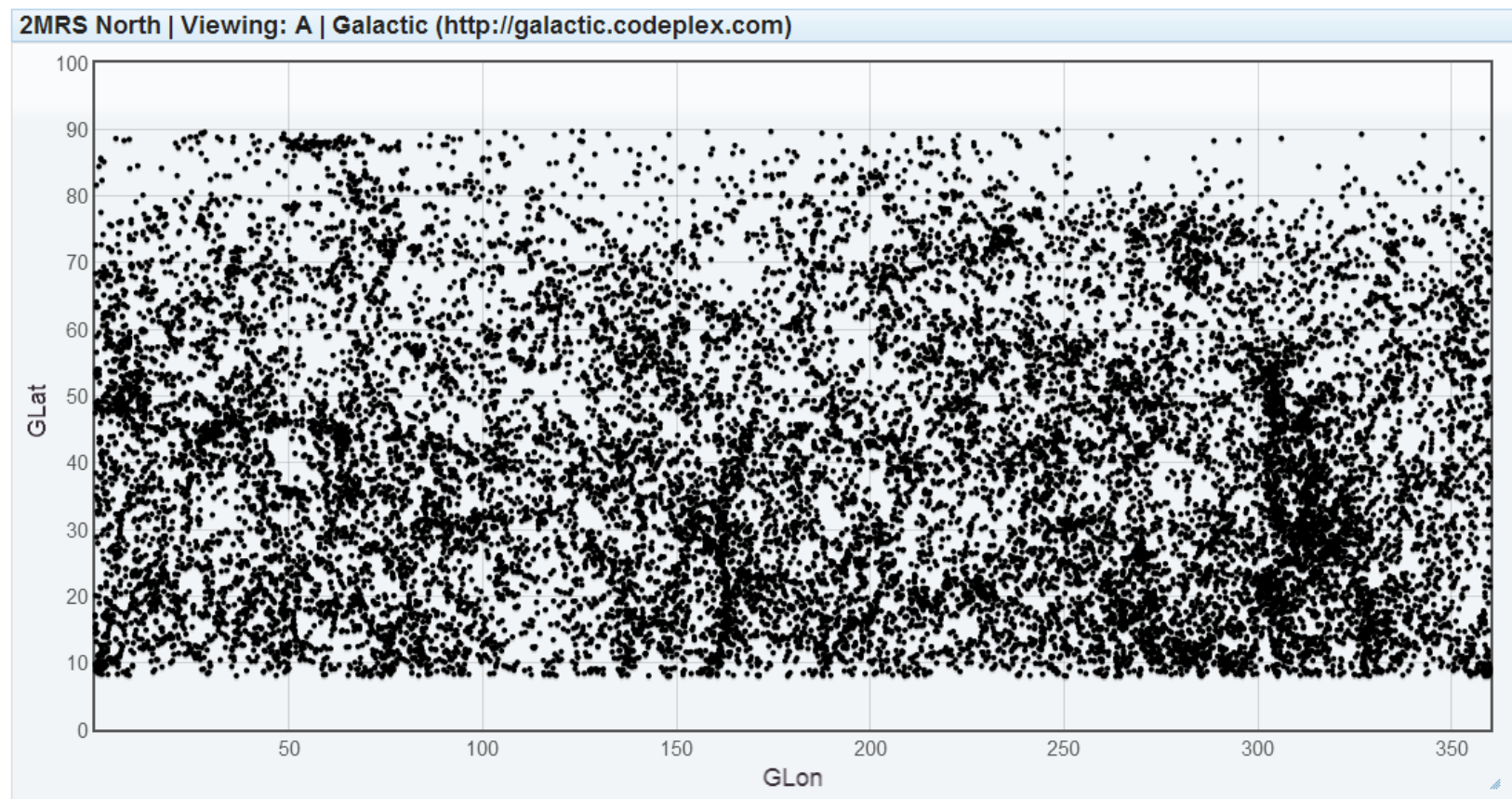


Figure 3-5 2MRS North Ellipticals

2MRS North | Viewing: E | Galactic (http://galactic.codeplex.com)

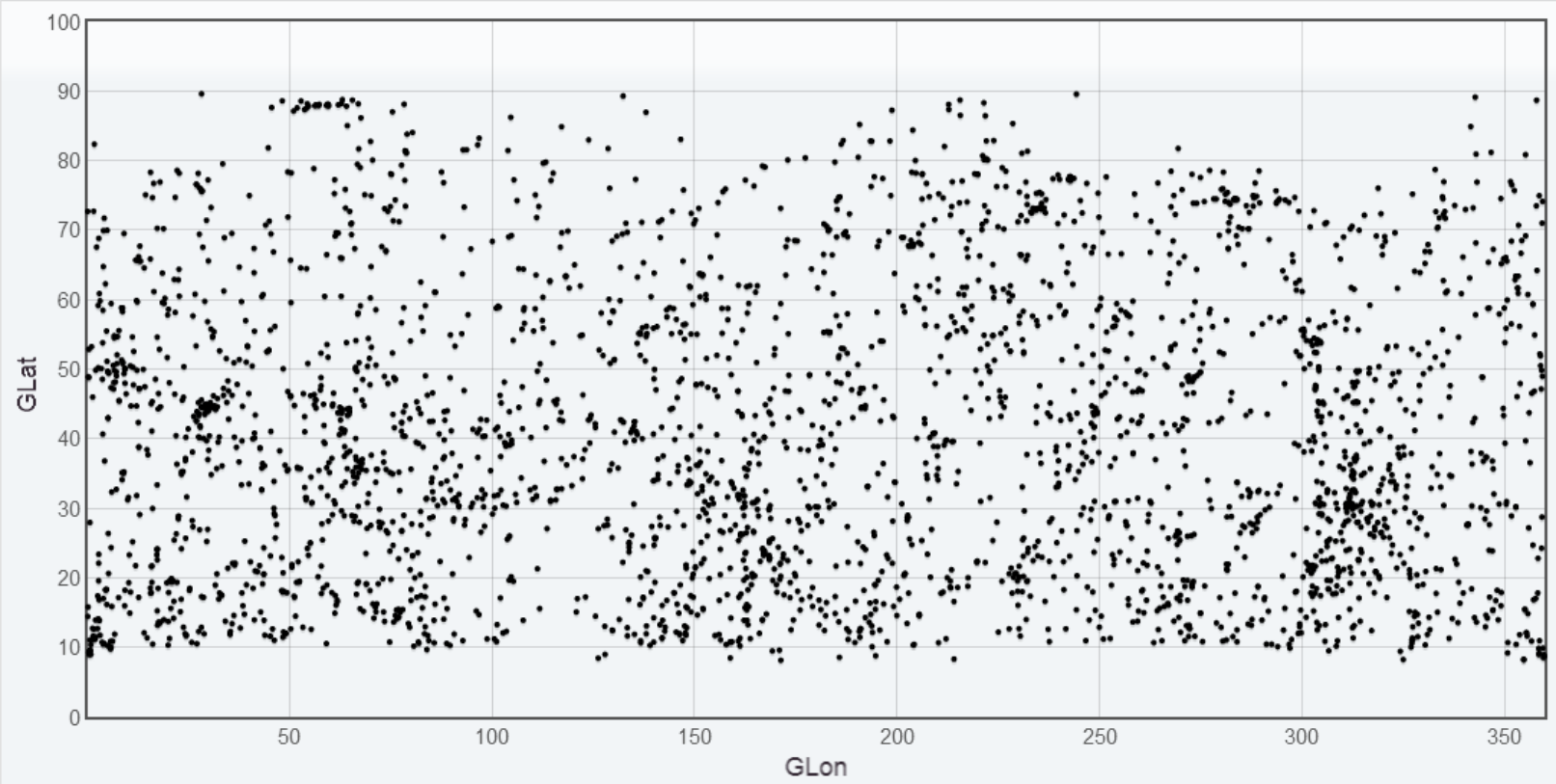

Figure 3-6 2MRS North Lenticulars

2MRS North | Viewing: L | Galactic (http://galactic.codeplex.com)

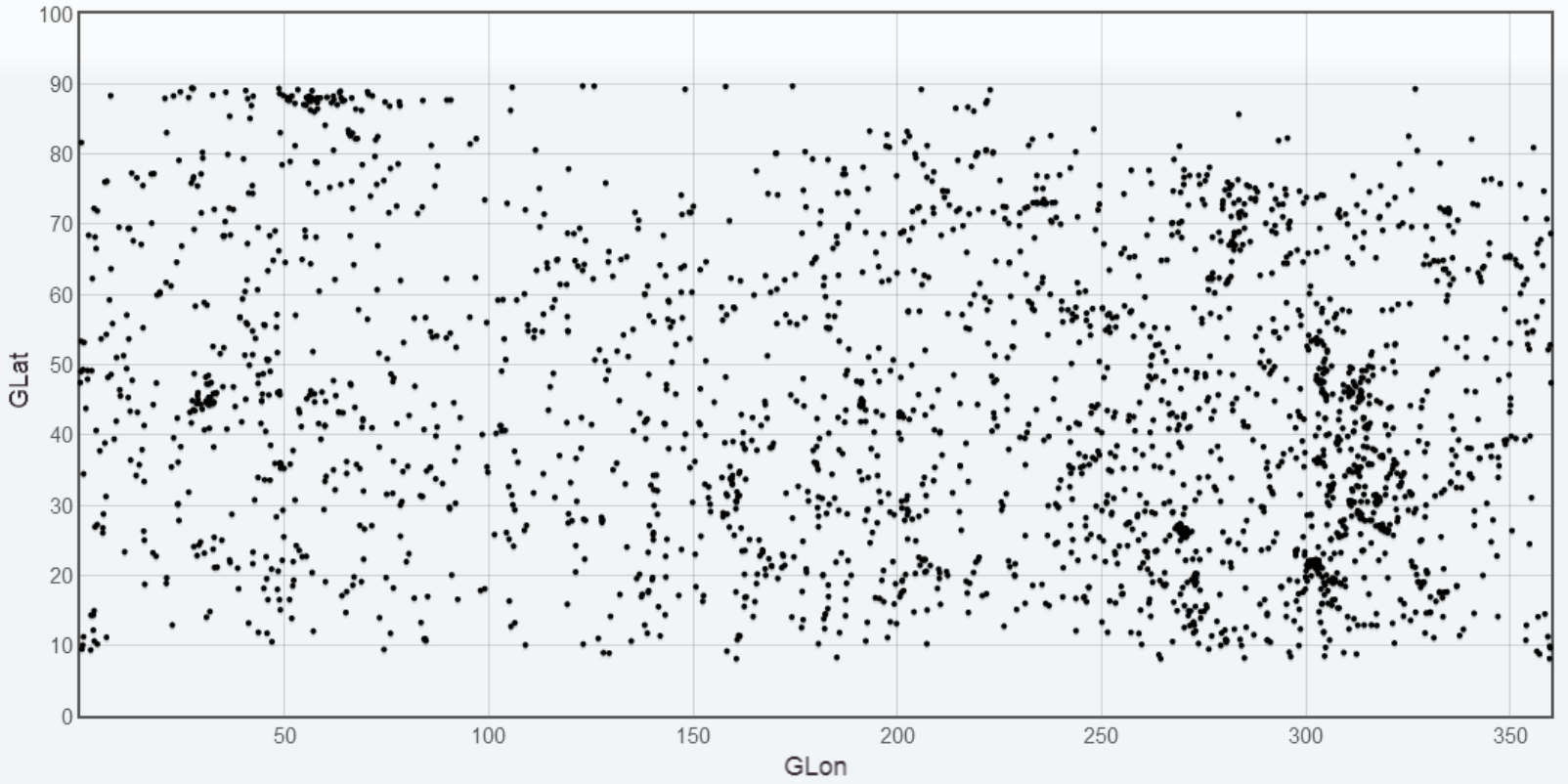


Figure 3-7 2MRS North Spirals

2MRS North | Viewing: S | Galactic (http://galactic.codeplex.com)

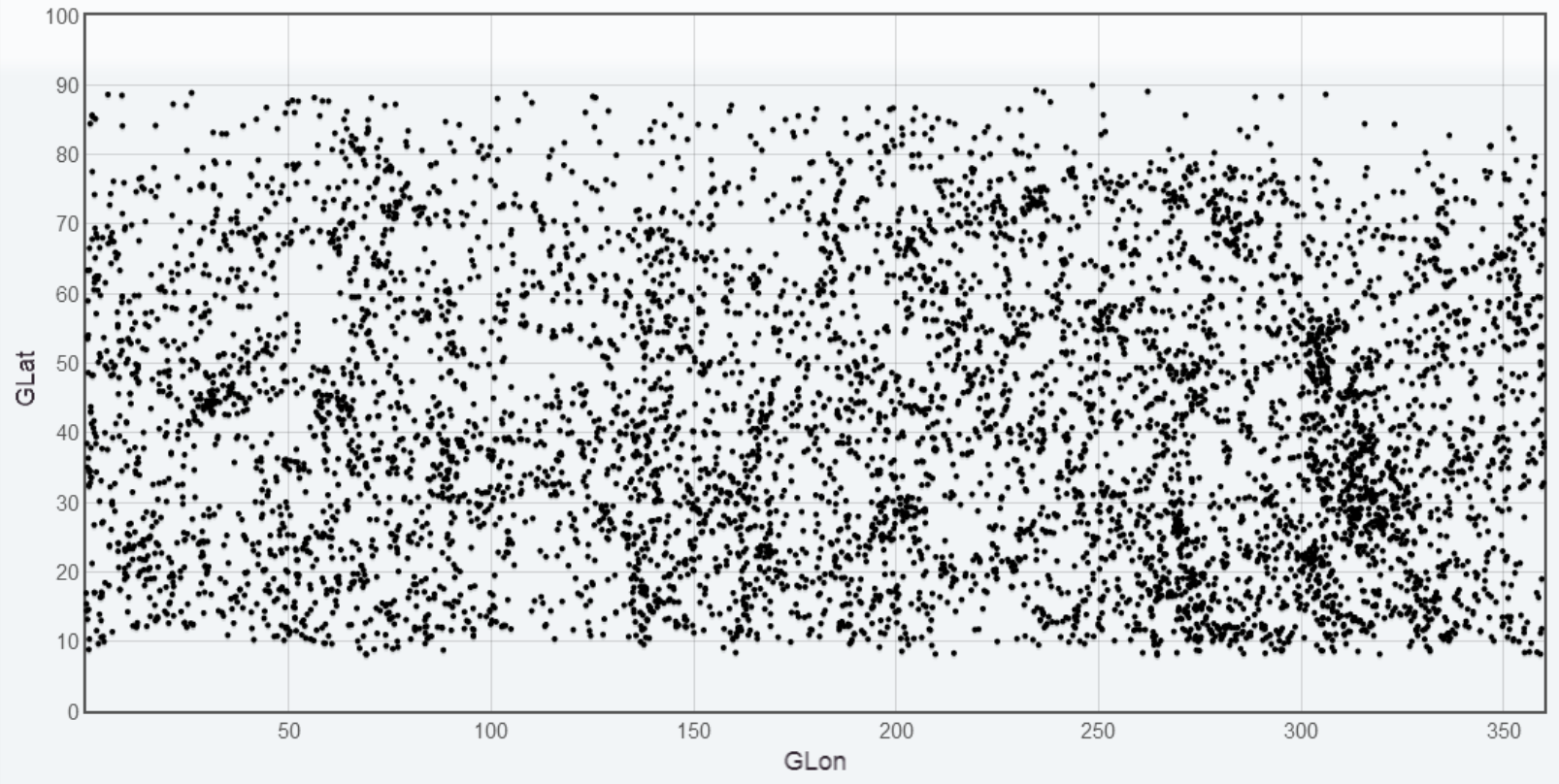

Figure 3-8 2MRS North Barred Spirals

2MRS North | Viewing: S_b | Galactic (http://galactic.codeplex.com)

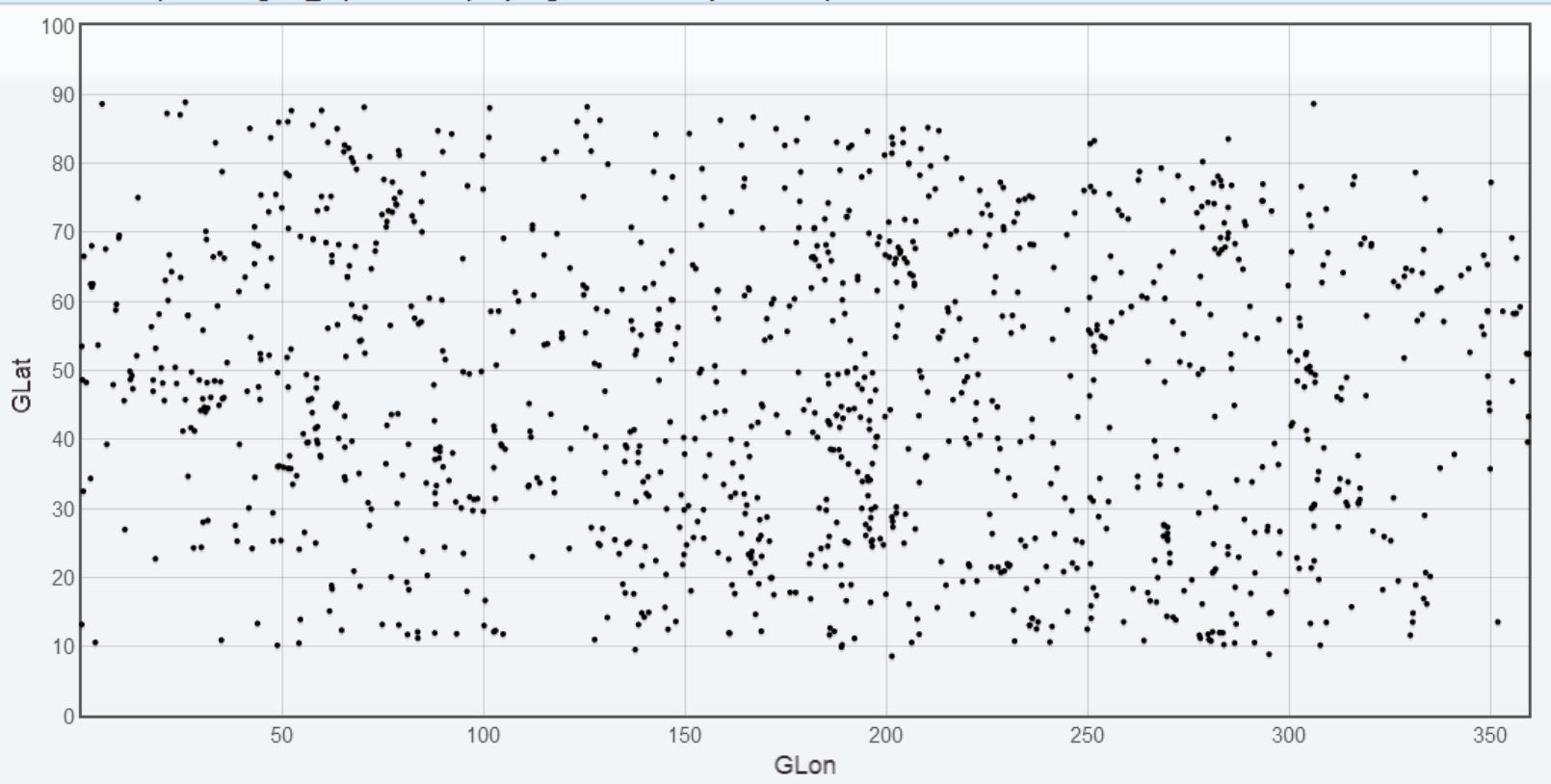


Figure 3-9 2MRS North Unbarred Spirals

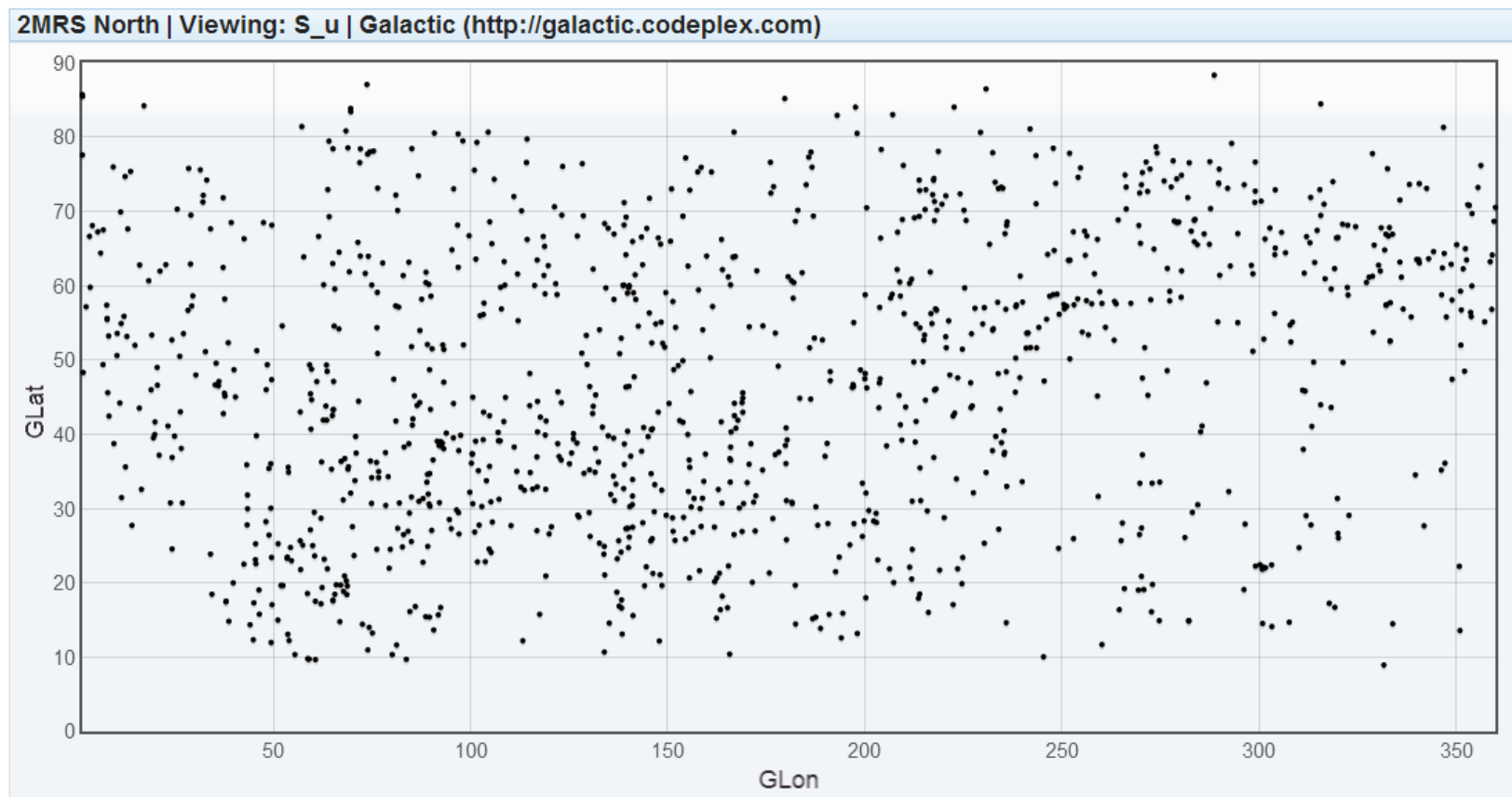

Figure 3-10 2MRS South All Galaxies

2MRS South | Viewing: A | Galactic (http://galactic.codeplex.com)

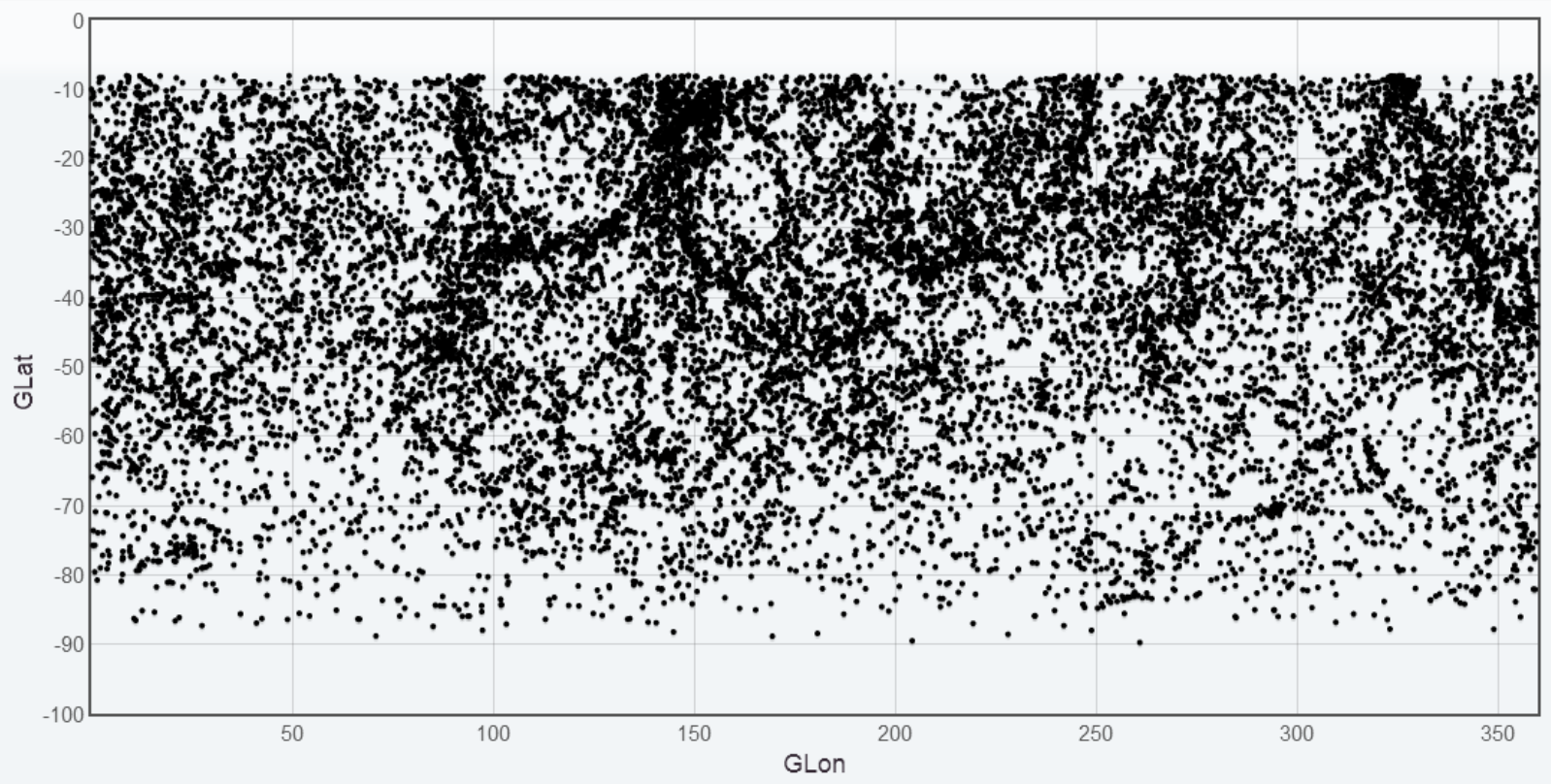


Figure 3-11 2MRS South Elliptical Galaxies

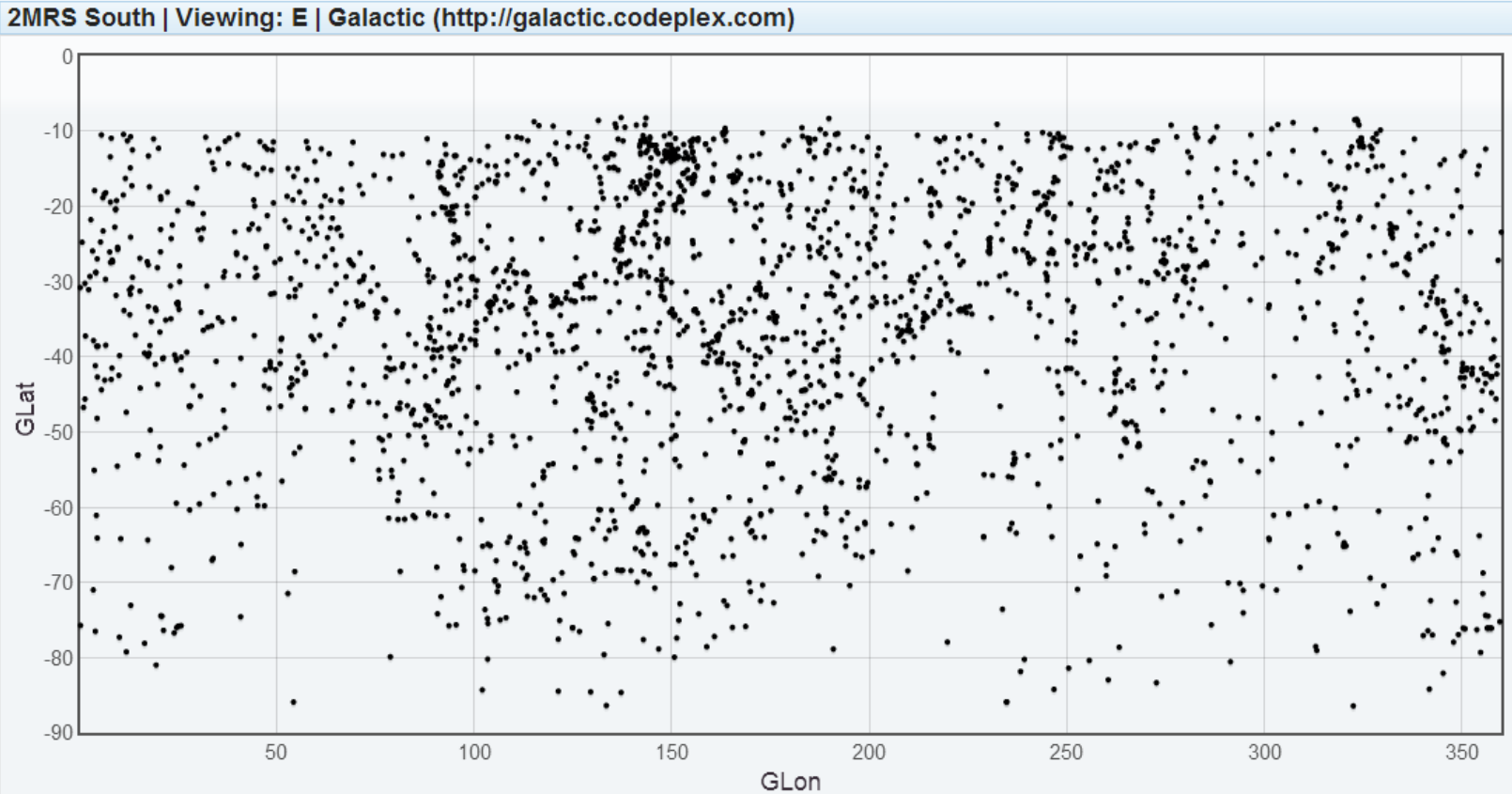

Figure 3-12 2MRS South Lenticular Galaxies

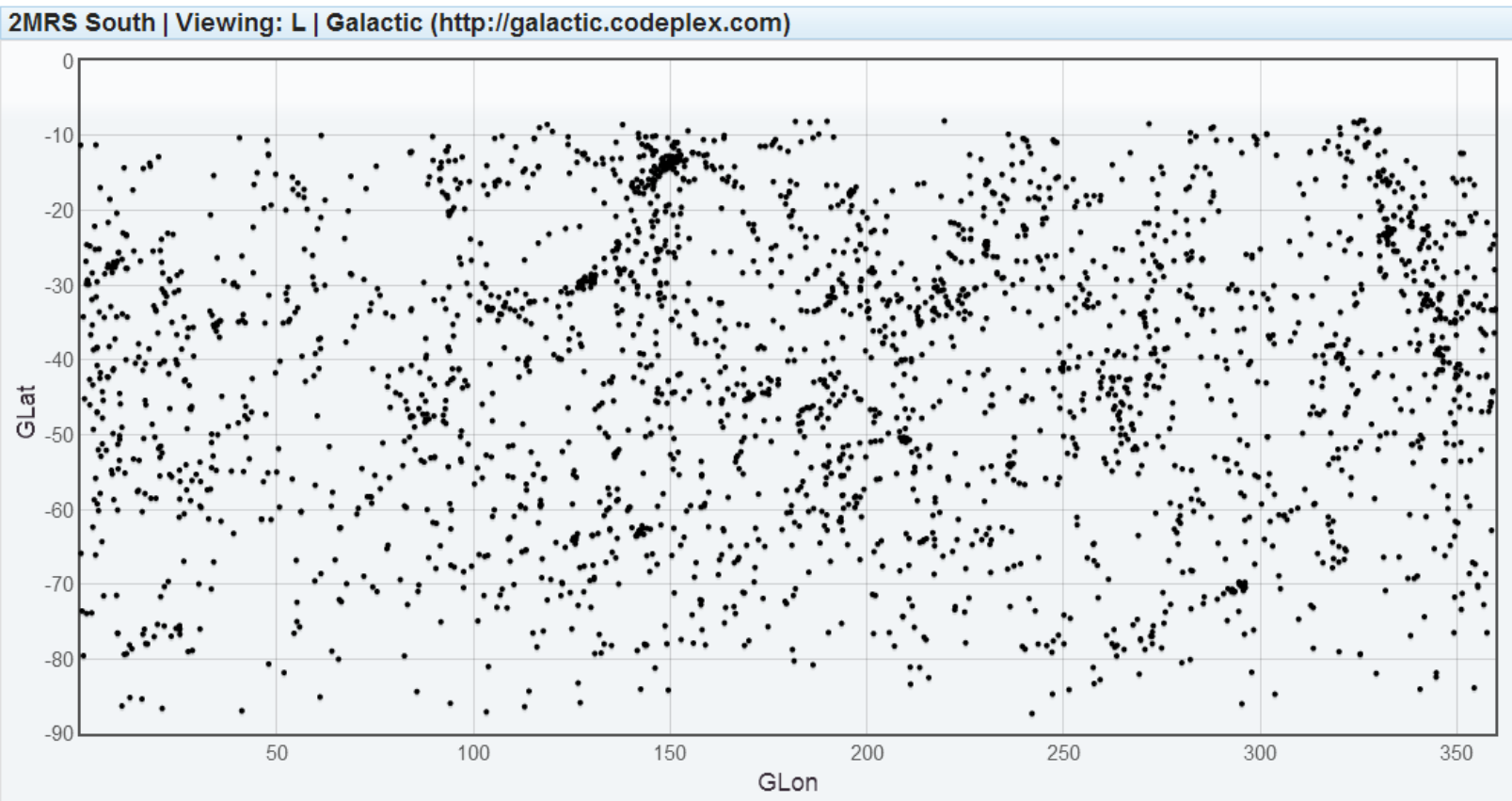


Figure 3-13 2MRS South Spiral Galaxies

2MRS South | Viewing: S | Galactic (http://galactic.codeplex.com)

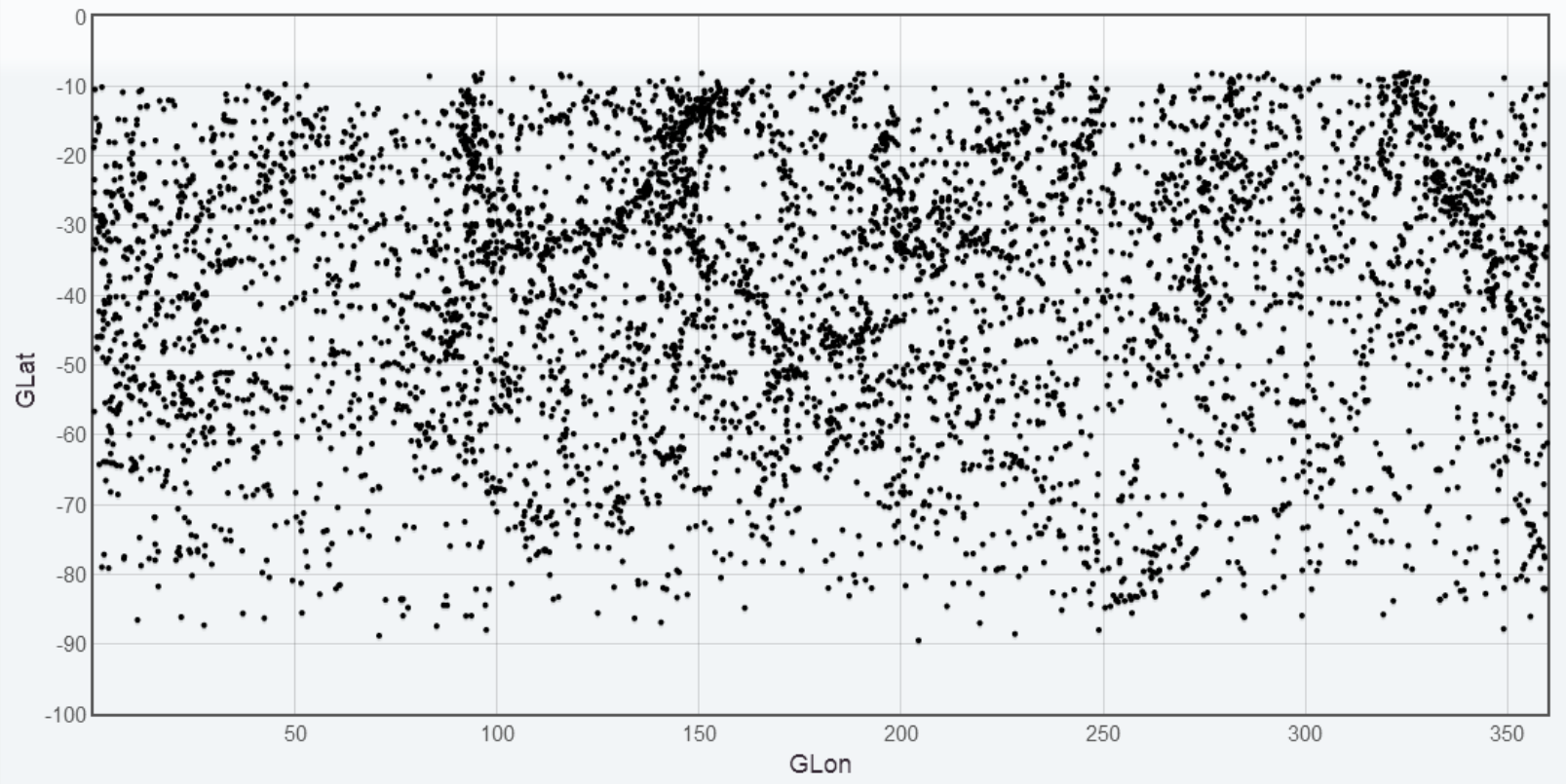

Figure 3-14 2MRS South Barred Spiral Galaxies

2MRS South | Viewing: S_b | Galactic (http://galactic.codeplex.com)

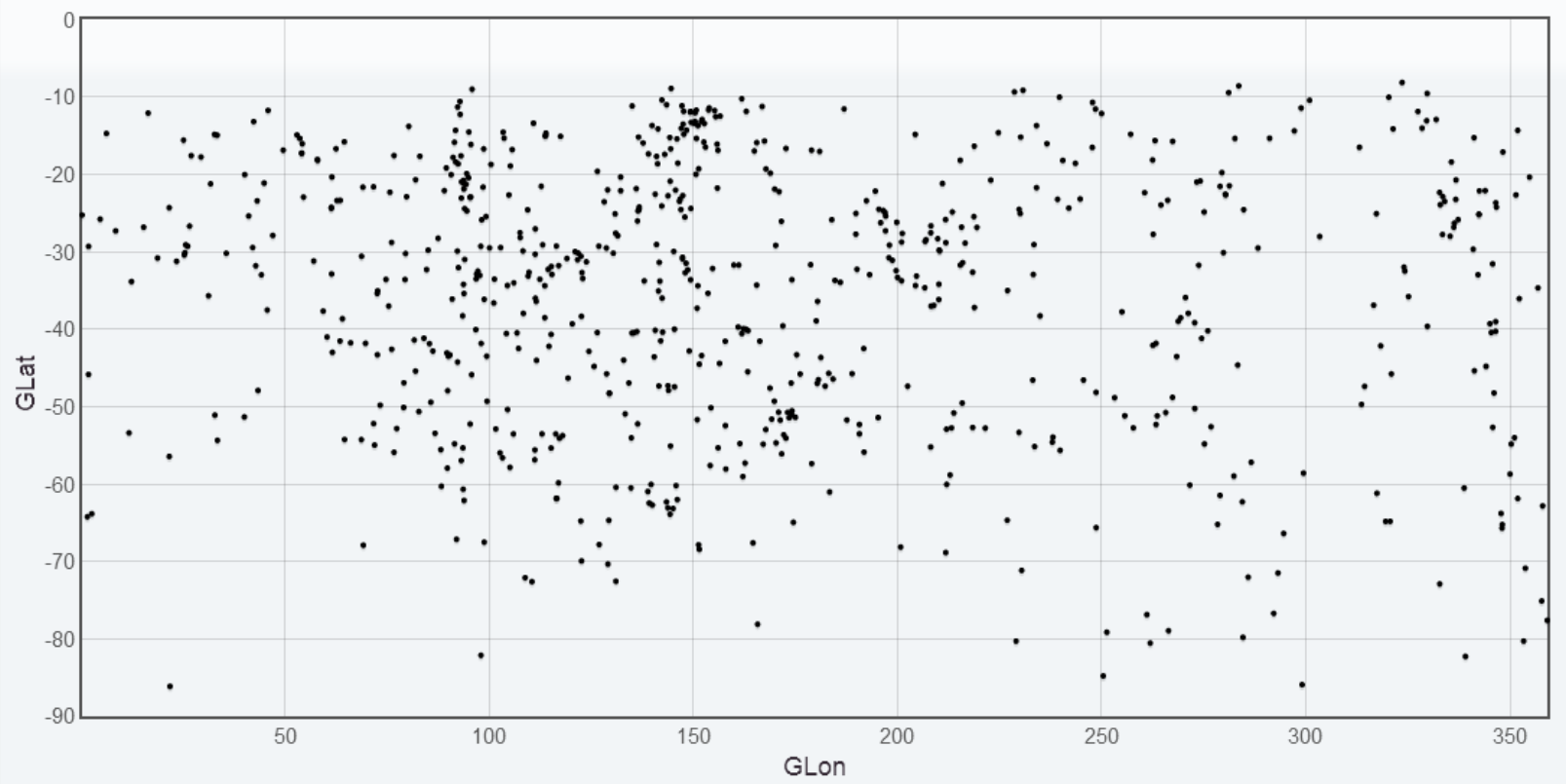


Figure 3-15 2MRS South Unbarred Spiral Galaxies

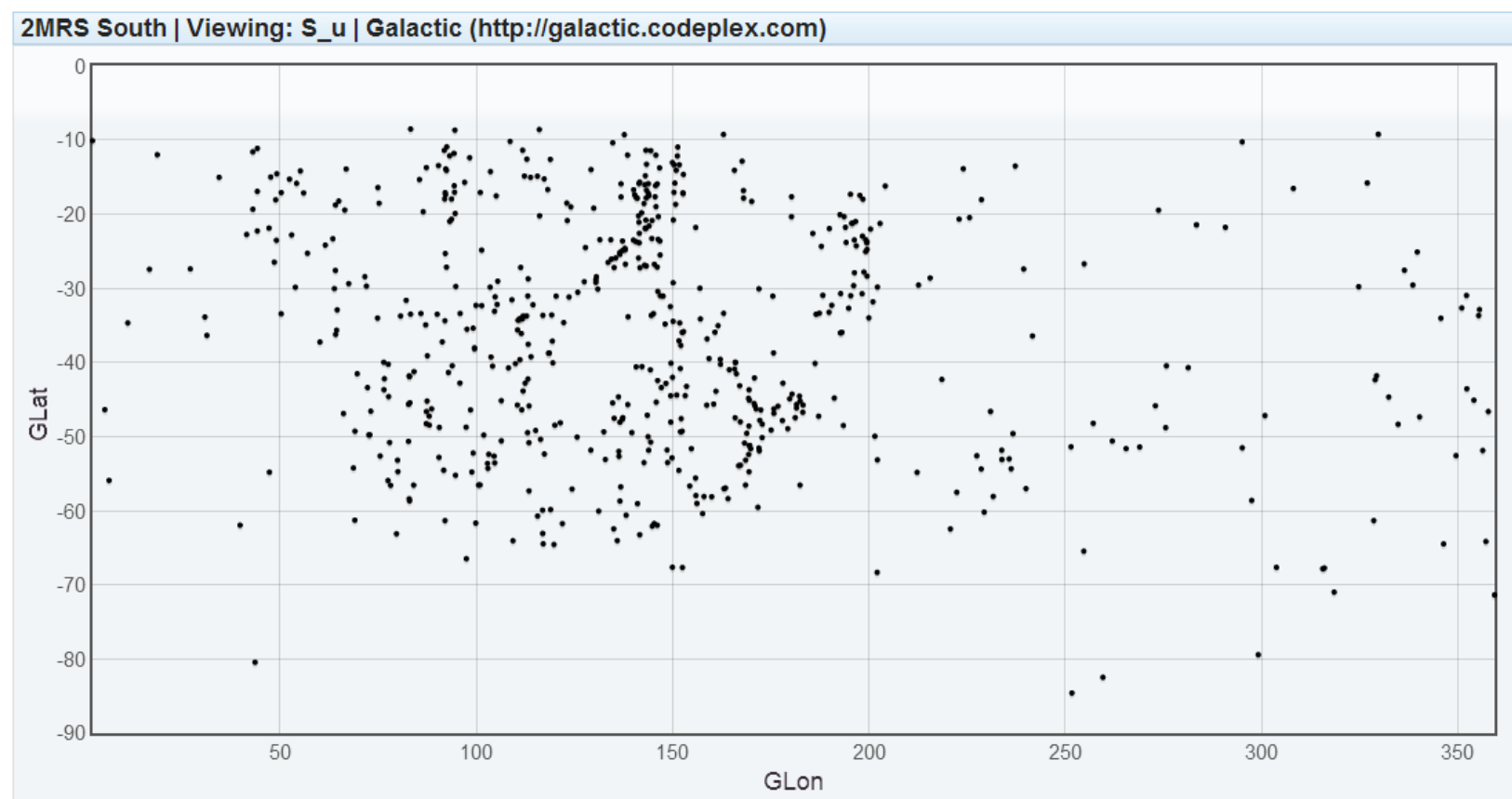




\section{Development of Custom Software}

\subsection{Initial Technological Decisions}

Early in the development phase several decisions have to be made regarding the technologies to utilize for the software. Given there is exactly one resource, myself, for this project it is easy to select technology with developer familiarity. Developer familiarity lowers the learning curve for the tools utilized, which is important because the domain of astronomy is rather unfamiliar and requires a lot of familiarization. While there is no financial cost involved, a lack of developer familiarity represents a cost in time [37].

Based on the skillset of the developer, it is decided to create the system as a web application. The application will use Microsoft SQL Server as the database to store the data sets as well as the results of data runs. The selection of SQL Server for the database mandates the use of Microsoft Windows Server for the operating system. Following that logic, Microsoft's Internet Information Server (IIS) will be the web server, and Microsoft's ASP.NET will be the web-server-side technology. Development for ASP.NET and the HTML user interface is accomplished using Microsoft Visual Studio.

The architecture of the user application is Model-View-Presenter (MVP), a derivative of the Model-View-Controller (MVC) pattern. The MVP pattern is described in Figure 4-1. For this application, the Model is the database structure. The View is an ASP.Net Representational State Transfer (REST) web service which translates tabular data to JavaScript Object Notation (JSON). The Presentation layer is the HTML5 and JavaScript web user interface (UI).

De-coupling the UI (view) from the backend of the system (model) and having the presenter act as a translation interface provides easier portability should that be necessary later in the system's lifecycle. This also permits development of additional Presentation layer Uls if desired, for example a mobile app to track progress of data runs. 
Figure 4-1 Model View Presenter Pattern - (via Wikipedia Creative Commons License) [38]

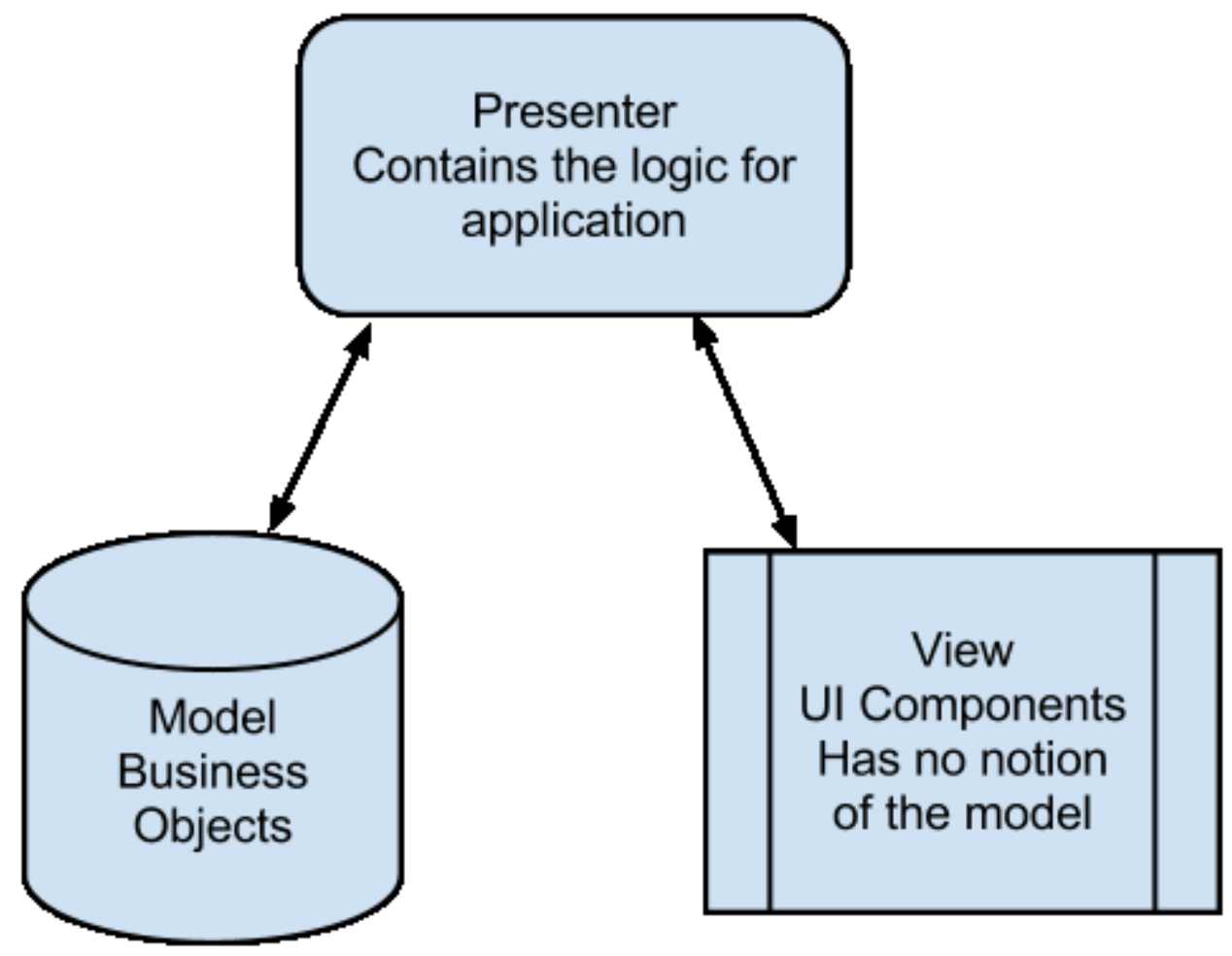

Following onto the decision to create the view (user interface) as a web application, popular open source web frameworks are leveraged due to developer familiarity and the benefits of code reuse [37] [39]. Those frameworks and additional libraries utilized are listed in Table 4-1.

Table 4-1 Frameworks and Libraries Utilized

\begin{tabular}{|l|l|}
\hline \multicolumn{1}{|c|}{ Library } & \multicolumn{1}{c|}{ Description } \\
\hline jQuery [40] & Popular open-source framework for interactive web applications \\
\hline jQuery UI [41] & Popular open-source framework for interactive web applications \\
\hline Font-Awesome [42] & Open-source library of icons \\
\hline jQuery Flot [43] & Open-source library used to create plots in web applications \\
\hline Json.NET [44] & Open-source library used to serialize objects to JSON \\
\hline Accord.NET Framework [45] & Open-source library used for Kolmogorov-Smirnov tests \\
\hline SQL\# [46] & Library used to add regular expression support to SQL Server \\
\hline
\end{tabular}

\subsection{Development Environment}

The software is developed on common consumer grade hardware utilizing a virtual machine for the server software. The workstation was built using parts totaling 
$\$ 1,108.42$ purchased from Newegg [47]. Microsoft's open-source project hosting repository, Codeplex [48], is used for code versioning and issue tracking.

Table 4-2 Hardware Environment

\begin{tabular}{r|l} 
Processor & Intel Core i7-3770K Quad Core @ \\
& 3.5Ghz (3.9Ghz Turbo) with Hyper \\
Threading \\
Memory (RAM) & 32GB DDR3 1600 \\
Network & Dual 1000 Mbit Ethernet \\
Storage & 256GB Solid State, 2TB Platter
\end{tabular}

Table 4-3 Software Environment (Workstation)

Operating System $\mid$ Windows $8.1 \times 64^{1}$

Integrated Development Environment Visual Studio $2013^{2}$

Virtual Machine Oracle VM VirtualBox [49]

\author{
Table 4-4 Virtual Environment (Server) \\ Processor 4 virtual cores @ 3.5GHz \\ Memory (RAM) 16GB \\ Operating System Windows Server 2012 R2 Standard $x 64^{3}$ \\ Database Software SQL Server 2014 Standard x64 \\ Network Dedicated pass-through of one 1000Mbit \\ Ethernet adapter.
}

\title{
4.3 Requirements
}

\subsubsection{The system shall (mandatory):}

1. Support processing of multiple galactic data sets with minimal (or optimally no) development to add support for new data formats.

2. Use the fractal (PD) method to calculate the distribution of galaxies in a neighborhood and search for correlations in the below data values:

3. Utilize and store the following data elements from data sets

a. Galaxy name

b. Position (in galactic longitude and latitude)

c. Galaxy morphology

d. Galaxy distance/redshift

4. Run responsively on common hardware available in an academic setting and/or hosting such as Amazon Web Services or Microsoft's Azure

\footnotetext{
${ }^{1}$ Upgraded from Windows 8 x64 during development

2 Upgraded from Visual Studio 2012 during development

3 Upgraded from Windows Server 2012 Standard x64 during development

${ }^{4}$ Upgraded from SQL Server 2012 Standard during development
} 
5. Function properly with modern browsers (IE10, Firefox, Chrome)

6. Support multiple user accounts for tracking data runs.

7. Perform calculations faster than previous software does

8. Provide visualization of the galactic neighborhood, charts of the Pointwise Dimension, and the ability to save both as image files.

9. Watermark charts and graphs with the application name, source data set, and parameters such as the center galaxy and the maximum distance.

\subsubsection{The system should (desired):}

1. Provide notification to user when a data run is complete

2. Perform calculations in the most efficient manner possible

\subsection{Discussion of Requirements}

Each data catalog has its own format. Typically these catalogs are flat text files using a tab or space delimited scheme to separate the data into columns. Newlines or line breaks are used to represent rows in the data. Comments in the catalog vary, as do the position of the columns of importance for our analysis.

For example, the columns of interest in 2MRS are 4 and 5 (galactic longitude and latitude), 23 (morphology), 25 (velocity), and 29 (galaxy name) [19]. Another catalog considered for evaluation, the $2 \mathrm{dF}$ Galaxy Redshift Survey (2dFGRS) as downloaded [50] has the columns of interest at 1 and 2 (ra/decl), 5 (galaxy name), and 9 (redshift). Editing a parser to handle this is fairly easy, however it is greatly desired to be extremely easy to add support for new catalogs.

The system will conduct analyses using the Pointwise Dimension as used in previous work [30] [29] [28]. This also requires the system to handle galactic positions as well as morphology, redshift/velocity, and galaxy name (for plotting).

The software system should also perform well without extravagant hardware requirements, using modern operating systems and web browsers. Since the system is developed as a web application, it is necessary to have user accounts.

Given that previous software can take a long time to run, performance is an important consideration. It also is necessary to generate plots of the PD and the galactic data set, similar to charts and plots in [30] [29] [28], which can be saved as an image for inclusion in papers, such as this thesis. Watermarking of these charts with relevant information is also necessary. 
Desired features include notification to the user upon completion of a data run, and the fastest performance, beyond a simple improvement over previous software possible.

\subsection{Meeting the Requirements}

\subsubsection{Dynamic Configuration of Data Set Definitions}

Data catalogs provided in the literature follow no particular format. The information provided varies, as well as the units for values. As an example, following is a small sample from the 2MRS data release [19] with rows word-wrapped. The 2MRS data contains a lot of columns. The columns of interest (morphology, radial velocity, galactic latitude and longitude, and galaxy name) in the scope of this paper are underlined.

\footnotetext{
\#ID RAdeg DECdeg $\stackrel{b}{\perp}$ k_c h_c j_c k_tc h_tc j_tc e_k e_h ej e_kt e_ht e_jt e_bv r_iso r_ext b/a flgs type ts $\underline{v}$ e_vc vsrc $\underline{\text { CAT ID }}$

$00424433+4116074 \quad 10.6847141 .26875121 .17430-21.573190 .797 \quad 0.9291 .5520 .7430 .8811 .4970 .0160 .016$ 0.0150 .0170 .0170 .0160 .6833 .2083 .4910 .473 Z111 $\underline{3 A 2 s}$ ZC $-\underline{300} \quad 4$ N 1991RC3.9.C...0000d MESSIER 031

00473313-2517196 11.88806 -25.28880 $97.36301-87.96452 \quad 3.815 \quad 4.1324 .858 \quad 3.765 \quad 4.0774 .7980 .0160 .015$

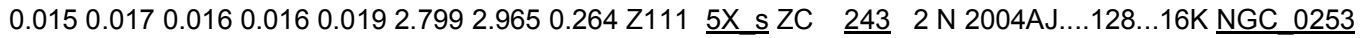

$09553318+6903549148.88826 \quad 69.06526142 .0919040 .90022 \quad 3.8984 .1314 .7843 .8034 .0434 .6900 .0160 .016$ 0.0150 .0180 .0180 .0160 .0802 .6882 .8780 .517 Z111 2A2s ZC $\quad-\underline{34} \quad 4$ N 1991RC3.9.C...0000d MESSIER 081

$13252775-4301073201.36565-43.01871309 .5163919 .417613 .9484 .2444 .9313 .9014 .2034 .8760 .0150 .016$ 0.0150 .0160 .0170 .0160 .1152 .4452 .6130 .957 Z111 -2_P ZC $\underline{547} 5 \mathrm{~N}$ 1978PASP...90..237G NGC 5128

$13052727-4928044196.36366-49.46790305 .2715113 .340174 .4714 .7905 .5084 .4214 .7355 .4440 .0160 .016$

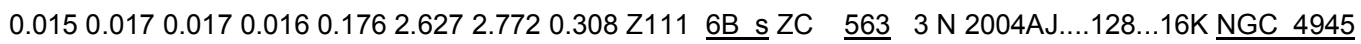

As another example, below in Table 4-5 are a few entries from the 2df Galaxy Redshift Survey (2dFGRS) catalog [50] Note that in this catalog right ascension and declination (RAJ2000, DEJ2000 columns) are used for position instead of galactic latitude and longitude. Also redshift is given as $z$ (actual redshift) and not $v$ (velocity) as in 2MRS. Finally, this catalog does not include a column for morphological classification. 
Table 4-5 Example Data from 2dFGRS Catalog

\begin{tabular}{|c|c|c|c|c|c|c|c|c|}
\hline $\begin{array}{l}{ }^{\text {RAJ22000 }} \\
\text { deg }\end{array}$ & $\begin{array}{l}\text { _DEJ2000 } \\
\text { deg }\end{array}$ & recno & SeqNum & Name & $\begin{array}{l}\text { RAJ2000 } \\
\mathrm{h}: \mathrm{m}: \mathrm{s}\end{array}$ & $\begin{array}{l}\text { DEJ2000 } \\
\mathrm{d}: \mathrm{m}: \mathrm{s}\end{array}$ & z.obs & $\mathrm{z}$ \\
\hline 3.61271 & -32.2707 & 1 & 1 & TGS436Z001 & 001427.05 & -321614.6 & 0.2981 & 0.2981 \\
\hline 3.62729 & -32.9669 & 2 & 2 & TGS496Z001 & 001430.55 & -325800.7 & 0.1229 & 0.1228 \\
\hline 3.58629 & -32.388 & 3 & 3 & TGS435Z001 & 001420.71 & -322316.8 & 0.1038 & 0.1038 \\
\hline 3.60196 & -32.4966 & 4 & 4 & TGS436Z002 & 001424.47 & -322947.6 & 0.2066 & 0.2065 \\
\hline 3.608 & -32.7115 & 5 & 5 & TGS435Z002 & 001425.92 & -324241.5 & 0.1036 & 0.1036 \\
\hline
\end{tabular}

The requirement to support easy addition of new data catalogs with minimal (or no) development work is an interesting one. Students of astronomy who will eventually use this software with Dr. Best may not have formal training in software development. While these students do not have the development training, they nonetheless will be able to interpret the scientific data generated. A paper was prepared by an undergraduate student of Dr. Best in 2013 utilizing the pointwise dimension to analyze the large-scale distribution of quasars [51]. This shows that having software development skills isn't necessarily a prerequisite to perform data analysis.

At first glance a trained software developer might follow an Object Oriented approach to create a base Parser class and extend that class for each new data catalog, for example $2 \mathrm{mrsParser}$ and 2 dgrfsParser. One then defines the column positions, unit conversions, and delimiters in the sub-class.

This approach would obviously require additional development. The literature shows that maintenance activities on software degrade the quality of the software and contribute to software aging [52] [53]. As a result of that degradation a legacy system eventually must be reengineered [52], which poses a problem because, the resources available to perform the maintenance on the system (astronomy undergraduates) may not have any formal software engineering background. In addition any future maintenance performed increases the software complexity and the cost of future maintenance. Therefore a goal is to future-proof the software as much as possible, and not consider just the first release [54]. It is important to note that no software can be fully future proofed, especially given that most software will be in use for a long time [53].

The above proposed OOP approach is therefore rejected. 
A solution is devised to meet this requirement while considering the above concerns for skillset of future maintainers and amount of maintenance. That solution is to guide the user through a three-step (two and confirmation) process to name the catalog, upload the data file, define the format of the file using a drag and drop GUI, and save the catalog definition in the database to be processed in the background.

In step1, Figure 4-2, the user uploads the data file, names the data set, and provides the format for comments appropriate for the data set.

Figure 4-2 Step 1 - Add Data Set

\section{Add Data Set (1/3)}

Data Set File: Choose File 2mrs_first_50_or_so.txt

Data Set Name: 2MRS Demo

Comments Start with (separate multiples with comma like "|,I"): \# Upload

In step 2a, Figure 4-3, the user previews the valid data (non-comments) and can edit the comment format until correct. The user also uses the buttons to add regular expression pieces to define the format of the catalog. The regular expression is shown below the buttons. The user can drag and drop the pieces to re-order them, or click the close icon on the piece to remove it.

Figure 4-3 Step 2a - Add Data Set

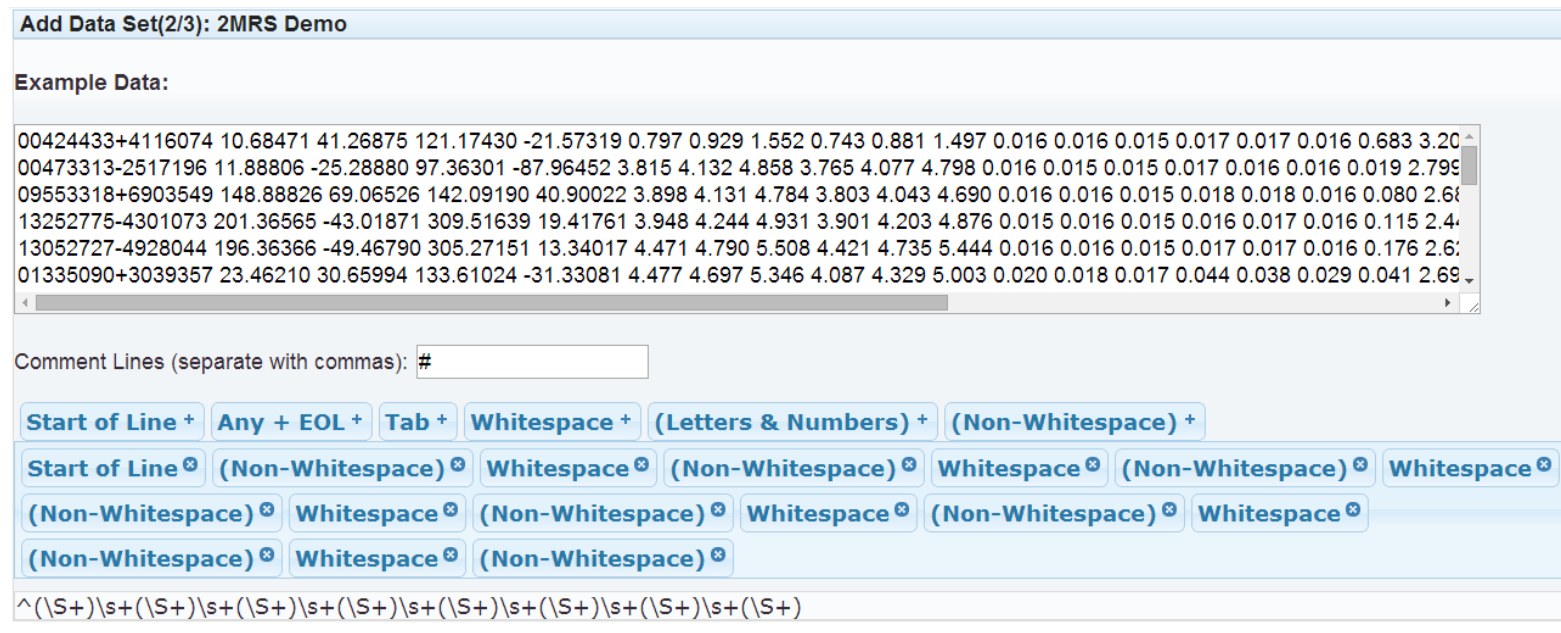


In step 2b, Figure 4-4, the user then drags and drops the required fields onto the correct columns to map the required field to the column in the data set.

Figure 4-4 Step 2b - Add Data Set

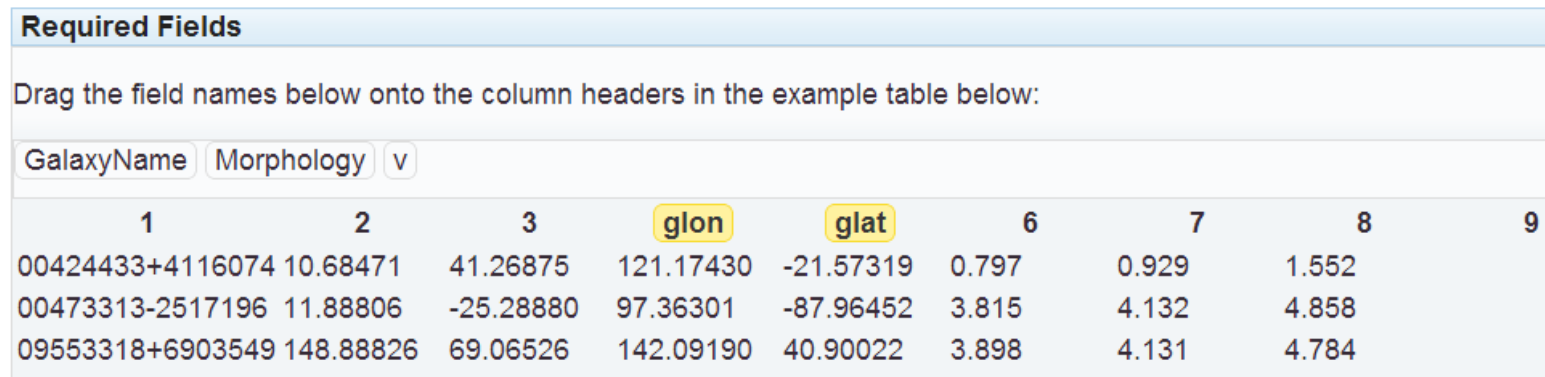

Finally in step 3, Figure 4-5, the user is presented a summary of the settings to be used and can choose to be notified when the data set is done loading into the database.

Figure 4-5 Step 3 - Add Data Set ${ }^{5}$

\begin{tabular}{|c|c|}
\hline \multicolumn{2}{|c|}{$\begin{array}{l}\text { Add Data Set (3/3) } \\
\text { Summary }\end{array}$} \\
\hline Id & 205 \\
\hline DataSetName & 2MRS Demo \\
\hline DataSetFileName & C:Ifakepath $12 \mathrm{mrs}$ first_50_or_so.txt \\
\hline Regex & $\wedge(\mid S+)|s+(\mid S+)| s+(\mid S+)|s+(\mid S+)| s+(\mid S+)|s+(\mid S+)| s+(\mid S+) \mid l s+(\mid S+)$ \\
\hline CommentChars & \# \\
\hline glon & 4 \\
\hline glat & 5 \\
\hline GalaxyName & 7 \\
\hline Morphology & 8 \\
\hline$v$ & 9 \\
\hline
\end{tabular}

Email me when data is loaded:

Pushover notification when data is loaded:

Save Properties \& Queue Data Load

${ }^{5}$ For illustrative purposes only. The actual 2MRS definition has much higher column positions. 
The data set information is then saved to the database and picked up by the next execution of the data set loader SQL Server Agent job. This is a C\# console application that runs on the database server. It connects to the database, collects the pending data set load information, parses the data file using the information collected, and saves the catalog into the database. Then if the user requested notifications it sends the notification.

\subsubsection{Notifications}

Notifications are provided via Email or Pushover [55]. Pushover is a service with apps for Apple iOS and Google's Android mobile. The Pushover API is an HTTP based REST API that is used by Galactic to send notifications to mobile devices.

\subsubsection{Benefits of Relational Database for Performance and Usability}

Use of SQL Server to store the data catalogs, data runs, and results offers benefits for performance. In past work [30] [29] [28] the software parsed the data catalog into memory, and then iterated over the data for data runs outputting files containing the primary galaxy's name, location, and the PD based on the filters. A separate step then processed the output files for KS tests.

SQL is optimized for set-based filtering and processing, so it is not necessary to iterate over the data during a data run. Filtering galaxies by fitting range or morphology is a simple Boolean comparison within the WHERE clause of the SQL statement. This represents fewer computation cycles and therefore improved performance.

Another benefit of using SQL Server is that concurrent access to the data is possible, where it was not in previous software. Once a data set is loaded it is possible for the system to simultaneously process multiple data runs while also providing data for plots of galactic positions or the PD values of a particular galaxy.

Finally, the use of a database permits user accounts which allows multiple users to conduct research on different or the same data sets at once.

\subsection{Validation of Calculations}

This section discusses the steps taken to validate the data calculations of the software, compared to the software used in previous work by Best [30] [29] [28]. 


\subsubsection{Pointwise Dimension Runs}

Pointwise dimension runs are made both in Dr. Best's original software and in Galactic using spirals as the morphological filter for primary and secondary galaxies. The $2 \mathrm{D}$ run is for $15^{\circ}$ and $3 \mathrm{D}$ is at $15 \mathrm{Mpc}$. A third run is conducted in 3D at $15 \mathrm{Mpc}$ with a velocity bin of $9,000<=v<=12,000$.

The outputs from Galactic and the software from Best are placed into Excel spreadsheets. The output is then sorted by galactic latitude and longitude so that each primary galaxy resides on the same row in both outputs. A column is created to find the difference in values using an Excel formula, expressed as $\Delta d=d_{b}-d_{m}$ where $d_{m}$ is my value for PD and $d_{b}$ is Best's value. The final step is to create an Excel formula to find the maximum absolute delta PD. The formula is expressed as $\max (a b s(\Delta d))$. The results of these validations follow including subset (for brevity) tables of the output.

First, the 2D run spirals vs. spirals at 15 degrees, the results of which are presented in Table 4-6. The maximum absolute $\Delta d$ is zero.

$\begin{array}{crccc}\text { Table 4-6 Validation of calculations - spiral vs. spiral } \\ \text { glon } & \text { glat } & \boldsymbol{d}_{\boldsymbol{m}} & \boldsymbol{d}_{\boldsymbol{b}} & \boldsymbol{\Delta} \boldsymbol{d} \\ 0.23412 & 38.45351 & 1.47 & 1.47 & 0.00 \\ 0.26156 & 53.81665 & 1.72 & 1.72 & 0.00 \\ 0.32161 & 35.0564 & 1.47 & 1.47 & 0.00 \\ 0.32594 & 63.35838 & 1.73 & 1.73 & 0.00 \\ 0.35577 & 53.50912 & 1.75 & 1.75 & 0.00 \\ 0.45156 & 58.97009 & 1.96 & 1.96 & 0.00 \\ 0.51596 & 48.63602 & 1.84 & 1.84 & 0.00 \\ 0.5405 & 54.04232 & 1.79 & 1.79 & 0.00 \\ 0.54199 & 36.36618 & 1.58 & 1.58 & 0.00 \\ 0.70827 & 32.49183 & 1.37 & 1.37 & 0.00 \\ 0.73542 & 32.33817 & 1.41 & 1.41 & 0.00 \\ 0.77376 & 34.67246 & 1.34 & 1.34 & 0.00 \\ 0.79108 & 34.36919 & 1.32 & 1.32 & 0.00 \\ 0.84191 & 66.54572 & 1.77 & 1.77 & 0.00\end{array}$


Next, the 3D run spirals vs. spirals at $15 \mathrm{Mpc}$, the results of which are presented in Table 4-7. The maximum absolute $\Delta d$ is zero.

\begin{tabular}{ccrrr}
\multicolumn{5}{c}{ Table 4-7 Validation of calculations - spiral vs. spiral at $15 \mathrm{Mpc}$} \\
glon & glat & $\boldsymbol{d}_{\boldsymbol{m}}$ & \multicolumn{1}{c}{$\boldsymbol{d}_{\boldsymbol{b}}$} & \multicolumn{1}{c}{$\boldsymbol{\Delta} \boldsymbol{d}$} \\
0.01263 & 16.96581 & 2.02 & 2.02 & 0.00 \\
0.09469 & 15.41123 & 1.60 & 1.6 & 0.00 \\
0.23412 & 38.45351 & 1.33 & 1.33 & 0.00 \\
0.26156 & 53.81665 & 1.57 & 1.57 & 0.00 \\
0.32161 & 35.0564 & 1.43 & 1.43 & 0.00 \\
0.32594 & 63.35838 & 1.75 & 1.75 & 0.00 \\
0.35577 & 53.50912 & 1.26 & 1.26 & 0.00 \\
0.45156 & 58.97009 & 2.13 & 2.13 & 0.00 \\
0.51596 & 48.63602 & 1.95 & 1.95 & 0.00 \\
0.5405 & 54.04232 & 1.70 & 1.7 & 0.00 \\
0.54199 & 36.36618 & 1.73 & 1.73 & 0.00 \\
0.70827 & 32.49183 & 1.08 & 1.08 & 0.00 \\
0.73542 & 32.33817 & 1.11 & 1.11 & 0.00 \\
0.77376 & 34.67246 & 1.17 & 1.17 & 0.00
\end{tabular}

Finally, the $3 \mathrm{D}$ run at $15 \mathrm{Mpc}$ with velocity bin 9,000-12,000, the results of which are presented in Table 4-8. The maximum absolute $\Delta d$ is zero.

Table 4-8 Validation of calculations - spiral vs. spiral at $15 \mathrm{Mpc}$ with velocity bin 9,000-12,000

\begin{tabular}{rcrrr}
\multicolumn{1}{c}{ glon } & glat & \multicolumn{1}{c}{$\boldsymbol{d}_{\boldsymbol{m}}$} & \multicolumn{1}{c}{$\boldsymbol{d}_{\boldsymbol{b}}$} & \multicolumn{1}{c}{$\boldsymbol{\Delta} \boldsymbol{d}$} \\
1.20411 & 37.84016 & 1.52 & 1.52 & 0.00 \\
1.80189 & 40.67618 & 1.70 & 1.7 & 0.00 \\
2.05379 & 61.58296 & 0.96 & 0.96 & 0.00 \\
2.89738 & 60.47928 & 0.79 & 0.79 & 0.00 \\
3.03604 & 50.14746 & 1.37 & 1.37 & 0.00 \\
3.3305 & 51.36156 & 1.17 & 1.17 & 0.00 \\
3.51719 & 59.75629 & 0.91 & 0.91 & 0.00 \\
3.54629 & 50.87012 & 1.14 & 1.14 & 0.00 \\
3.69532 & 33.13222 & 2.20 & 2.2 & 0.00 \\
3.9305 & 37.74599 & 1.96 & 1.96 & 0.00 \\
4.64688 & 40.01719 & 1.61 & 1.61 & 0.00 \\
5.11931 & 51.74884 & 1.70 & 1.7 & 0.00 \\
5.21268 & 49.20739 & 1.45 & 1.45 & 0.00 \\
6.11798 & 48.2239 & 1.31 & 1.31 & 0.00
\end{tabular}




\subsubsection{KS Tests}

After the PD runs in 2D are validated, data runs are conducted in 2D and KS tests ran to obtain the $p$ values of the new, $p_{m}$ to the old $p_{b}$. The difference in $p$ values is expressed as $\Delta p=p_{b}-p_{m}$. The results of that comparison are presented below in Table 4-9.

Table 4-9 Validation of Calculations - KS Tests compared to Best

$\begin{array}{cccc}\text { Runs Compared } & \boldsymbol{p}_{\boldsymbol{m}} & \boldsymbol{p}_{\boldsymbol{b}} & \boldsymbol{\Delta} \boldsymbol{p} \\ \text { SS30 vs. AA30 } & 2.78 \mathrm{E}-28 & 2.61 \mathrm{E}-28 & -1.70 \mathrm{E}-29 \\ \text { AA15 vs. AA30 } & 1.09 \mathrm{E}-43 & 1.01 \mathrm{E}-43 & -8.00 \mathrm{E}-45 \\ \text { AA15 vs. EA15 } & 1.38 \mathrm{E}-52 & 1.42 \mathrm{E}-52 & 4.00 \mathrm{E}-54 \\ \text { AA15 vs. EE15 } & 4.70 \mathrm{E}-70 & 5.23 \mathrm{E}-70 & 5.30 \mathrm{E}-71\end{array}$

\subsection{Issues Encountered and Resolutions}

In this section I discuss issues encountered and the resolutions devised.

\subsubsection{Domain Knowledge}

As this software is intended to improve research capabilities within the field of astrophysics, it clearly was necessary for me to become familiar with this domain. As specific domain knowledge is required, many scientists develop their own custom software, though many do not have formal software engineering training [56]. Acquiring needed domain knowledge is an important part in the early phases of any software development project, be it new development or maintenance. The familiarity of an engineer with the related domain has a significant impact on program understanding. Essentially, an engineer with expert level domain knowledge will more quickly understand the system and will make fewer mistakes in design. [37]

The importance of domain knowledge is readily apparent from projects I have worked on in the industry. For example, the system I currently support is used by the U.S. Coast Guard to gather data that is used to plan search and rescue (SAR) missions. The head trainer at SAR school provided me with the same hands-on training that the enlisted and officers in the field receive. This has positively influenced design decisions, especially within the user interface, because I am familiar with the needs of the field users. 
Since my formal education in astronomy and astrophysics consisted of two undergraduate courses with Dr. Best it was necessary to get up to speed. Dr. Best provided resources (papers, notes, and discussion support) to assist in this endeavor. Also my subsequent use of the system to conduct scientific research placed me in the role of user and forced me to notice and acknowledge issues and to conceive of improvements that benefit the user's experience.

\subsubsection{Right Ascension/Declination vs. Galactic Longitude/Latitude}

Initial development utilizes right ascension and declination for the position of galaxies. This results in a curve in the plotted data (see Figure 4-6 below) which complicates calculation of the primary galaxy's angular separation to the edge of data coverage.

Figure 4-6 2MRS Data in ra/decl

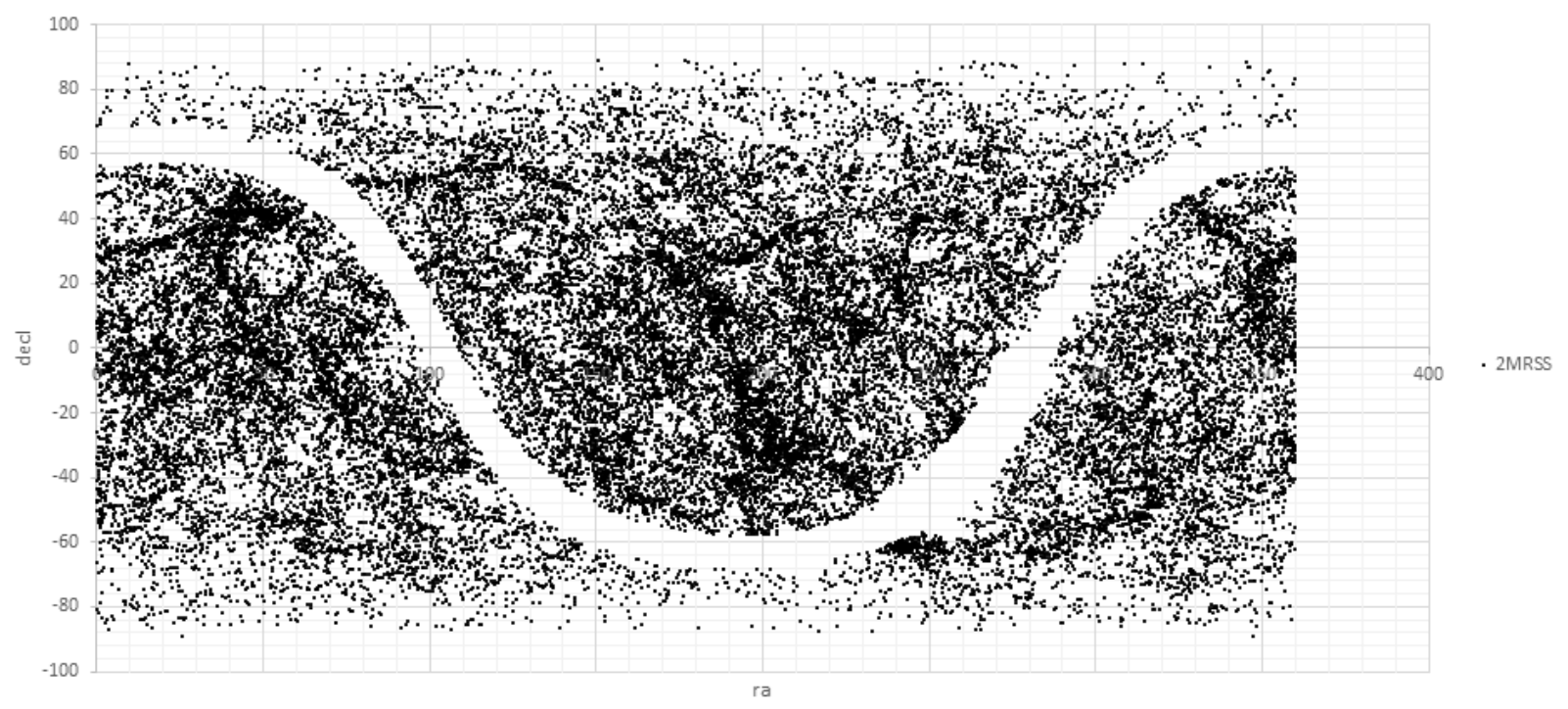

The decision was made to switch to galactic longitude and latitude which provides a clean northern/southern border for the data, as evidenced earlier in Figure 3-3.

\subsubsection{SQL Server Spatial Features}

The initial development uses SQL Server Spatial Geometry::Point [57] to store the ra/decl values for galactic position. Generation of spatial indexes yields improved 
computation speed for the STDistance [58] function used to calculate angular separation between galaxies.

Switching to galactic longitude and latitude breaks the usefulness of this because SQL Spatial Geometry types do not wrap across the x-axis. It is not feasible to use SQL Spatial Geography types because all of the common Spatial Reference Identifiers (SRIDs) [59] expect an ellipsoidal Earth with longitude ranging from -180 to 180 . There is no SRID found that can accommodate the 0-360 galactic longitude scale.

The angular separation calculation is refactored to utilize the Great-Circle distance formula as in previous work by Best [30] [29] [28] and discussed in section 3.2.1 of this paper.

\subsubsection{Regular Expressions in SQL Server}

The initial morphological filtering in SQL Server is conducted using the SQL WHERE LIKE <pattern> [60]. This method is not powerful enough to handle the scenario where Spiral galaxies in $2 \mathrm{MRS}$ are identified as starting with a single digit from 1-7 and no following digit OR starting as the digits "20". This was discovered during the validation of calculations discussed above. A free library, SQL Sharp [46], is used to add regular expression comparison features to SQL server. The morphological filters are defined as regular expressions which work very well with acceptable performance. 


\subsubsection{Dynamic Generation of KS Test Result Tables}

A particular challenge was the generation of the KS test result tables. Because the fitting ranges are dynamically specified by the user and therefore exist as rows in the database and not columns, a traditional SELECT statement will not suffice.

Consider Table 4-10 which illustrates (after appropriate JOIN statements) the nature of $\mathrm{KS}$ test results as rows in the database.

Table 4-10 Example KS results from Galactic database

\begin{tabular}{|l|r|l|r|c|}
\hline Run1 & Fit1 & Run2 & Fit2 & P \\
\hline SA & 1 & SA & 2 & 0.00 \\
\hline SA & 1 & SA & 3 & 0.00 \\
\hline SA & 1 & SA & 4 & 0.00 \\
\hline SA & 1 & SA & 5 & 0.00 \\
\hline SA & 2 & SA & 3 & 0.00 \\
\hline SA & 2 & SA & 4 & 0.00 \\
\hline SA & 2 & SA & 5 & 0.00 \\
\hline SA & 3 & SA & 4 & 0.00 \\
\hline SA & 3 & SA & 5 & 0.00 \\
\hline SA & 4 & SA & 5 & 0.00 \\
\hline
\end{tabular}

I used SQL Server's PIVOT feature [61] along with a list of the requested fitting ranges (from the user) to pivot the rows into columns to create the desired result. An example of the desired format corresponding to the data in Table 4-10 follows in Table 4-11.

Table 4-11 Example of desired KS test result table using columns

\begin{tabular}{|lccccccc|}
\hline & \multicolumn{1}{c|}{ Fitting Range 2 } \\
Run & FR1 & $\mathbf{1}$ & $\mathbf{2}$ & $\mathbf{3}$ & $\mathbf{4}$ & $\mathbf{5}$ \\
\hline $\mathrm{SA}$ & 1 & $\mathrm{Y}$ & $\mathrm{Y}$ & $\mathrm{Y}$ & $\mathrm{Y}$ \\
\hline $\mathrm{SA}$ & 2 & & $\mathrm{Y}$ & $\mathrm{Y}$ & $\mathrm{Y}$ \\
\hline $\mathrm{SA}$ & 3 & & & $\mathrm{Y}$ & $\mathrm{Y}$ \\
\hline $\mathrm{SA}$ & 4 & & & & $\mathrm{Y}$ \\
\hline
\end{tabular}




\subsubsection{Considerations for Color-Blind Users}

It is important to consider color blind users as this is a common affliction (10\% of men have some form of color blindness [62]). In the KS test result tables and confidence tables CSS classes are used to style the $\mathrm{Y}$ and $\mathrm{N}$ values green and red. While the lack of color difference does not impair understanding $Y$ vs. $N$ it does not result in the at-a-glance differentiation desired. To achieve at-a-glance differentiation for color-blind users the CSS classes are modified as such: green added bold text, and red added underlining. A screenshot from the application, Figure 4-7, illustrates this.

Figure 4-7 Screenshot Depicting KS Test Results Table

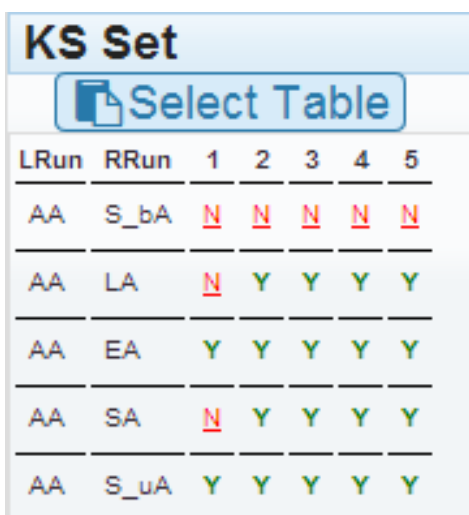

\subsection{Design Diagrams}

Following are diagrams to present the design of the system. 
In Figure 4-8 is the relationship of database tables in Galactic.

Figure 4-8 Database Table Relationship Diagram

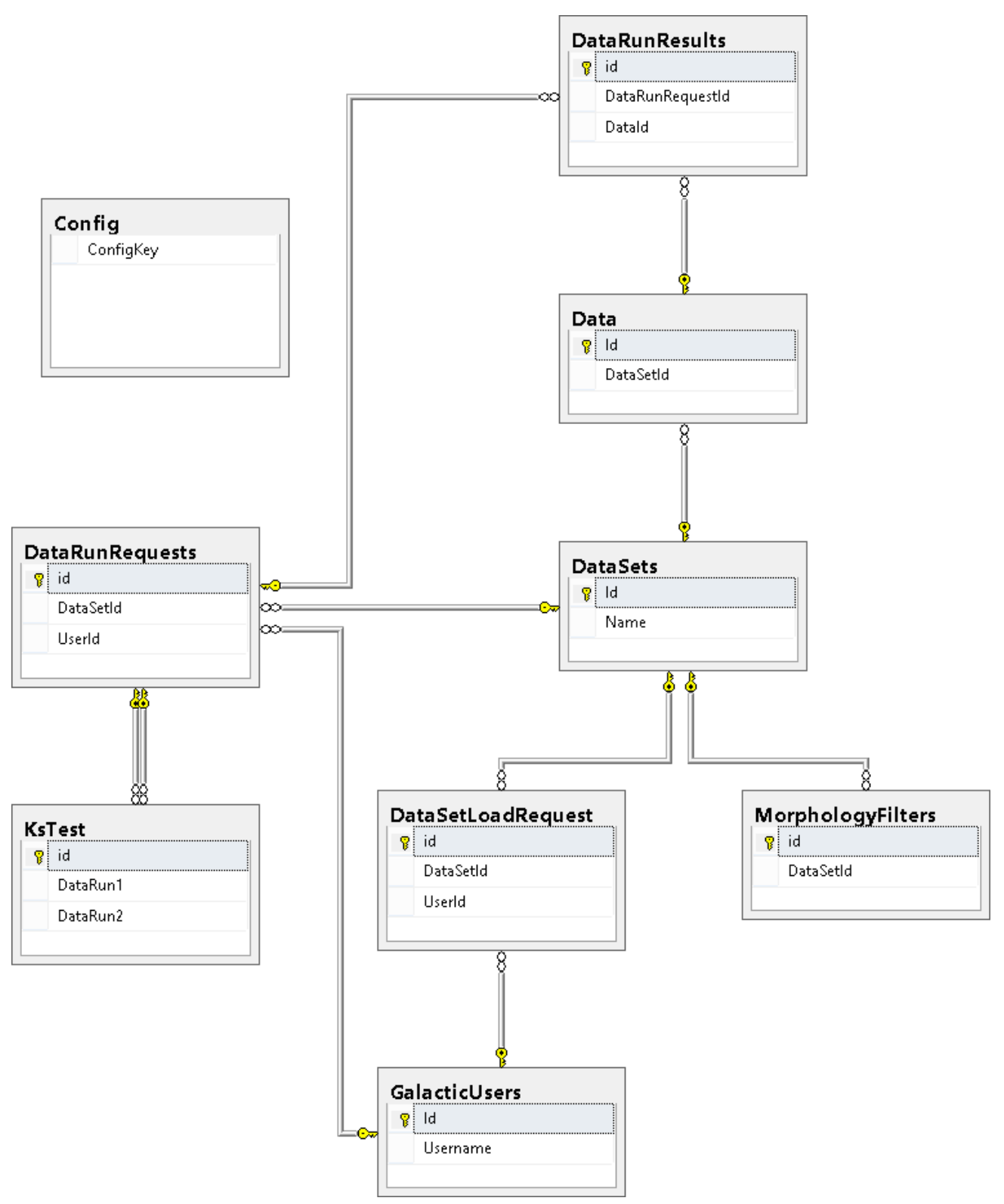


Figure 4-9 depicts the class diagram for the data set loader.

Figure 4-9 Data Loader Class Diagram

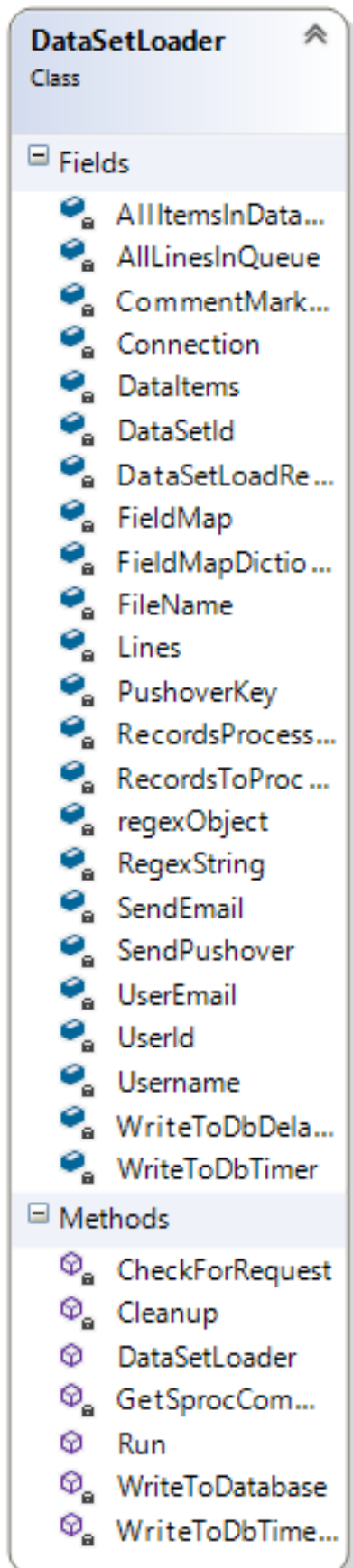

\begin{tabular}{|c|c|c|}
\hline $\begin{array}{l}\text { Datas } \\
\text { Class }\end{array}$ & etData & ล \\
\hline \multicolumn{2}{|c|}{$\square$ Fields } & \\
\hline$\theta$ & C & \\
\hline 0 & Distance & \\
\hline$\theta$ & $\mathrm{H}$ & \\
\hline$\theta_{a}$ & RedshiftUnit & \\
\hline \multicolumn{3}{|c|}{$\Xi$ Properties } \\
\hline * & GalaxyName & \\
\hline 8 & glat & \\
\hline 8 & glon & \\
\hline \& & Morphology & \\
\hline 8 & $v$ & \\
\hline 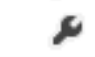 & $z$ & \\
\hline \multicolumn{2}{|c|}{- Methods } & \\
\hline$\varphi$ & ToString & \\
\hline$\varphi$ & TryParse & \\
\hline
\end{tabular}

\begin{tabular}{|l|}
$\begin{array}{l}\text { Config } \\
\text { Class }\end{array}$ \\
$\square$ Fields \\
$\theta$ connection \\
$\theta$ EmailSettings \\
- PushoverSettings \\
$\square$ Properties \\
$\approx$ Values \\
$\square$ Methods \\
$\ominus$ Config \\
$\ominus$ GetConfigValue... \\
\hline
\end{tabular}


Figure 4-10 depicts the class diagram for the notification subsystem.

Figure 4-10 Notification Class Library

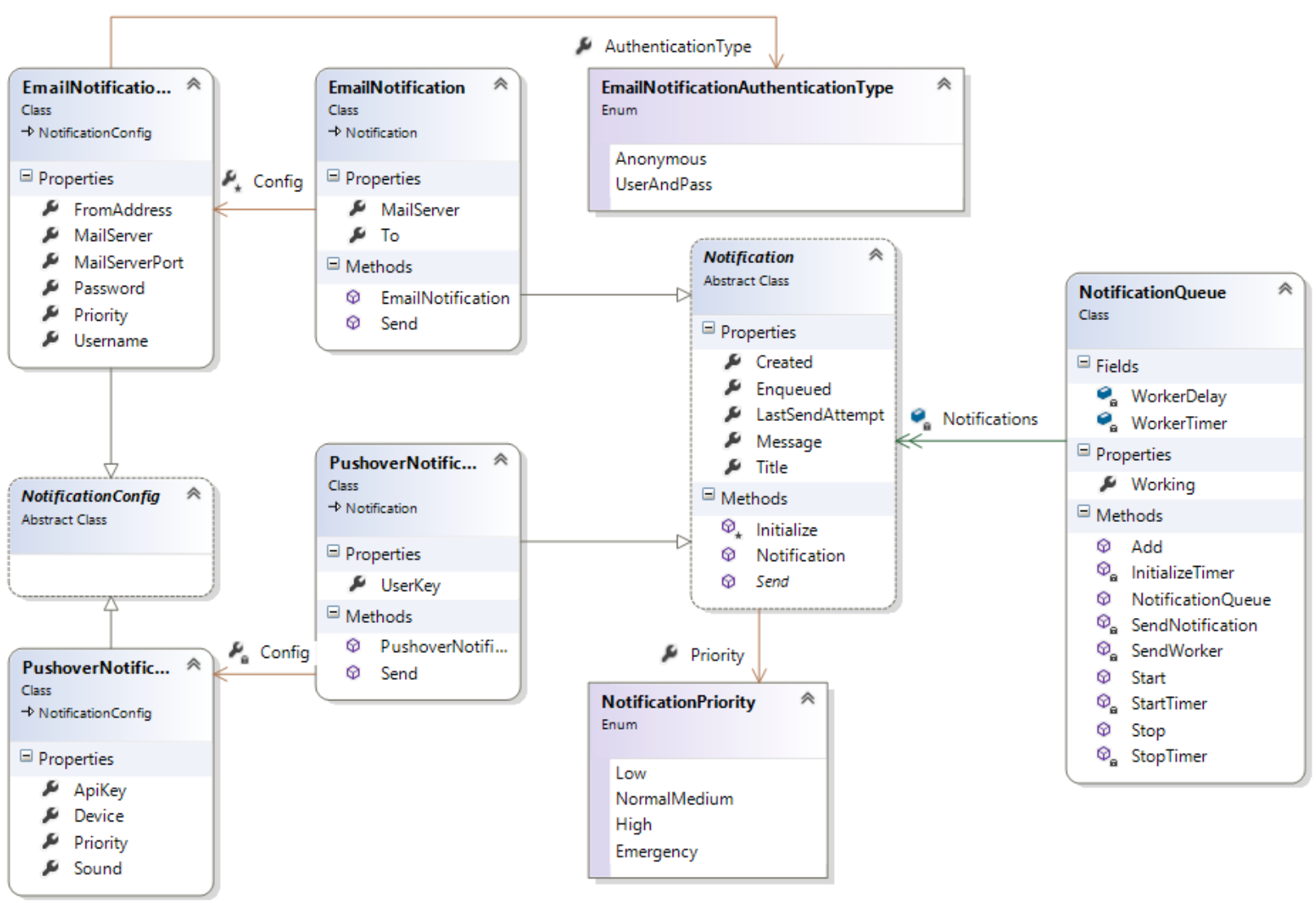




\section{Use of Software for Analyses of Galactic Neighborhoods}

\subsection{Explanation of Data Run Nomenclature}

Data runs within this paper are given titles that reflect the primary and secondary morphological filters as well as the fitting range size, the fitting range unit (Deg or $\mathrm{Mpc}$ ), and the velocity bin if appropriate. The format describing a data run is as follows: one or two characters describing the primary morphological filter followed by one or two characters representing the secondary morphological filter. This is followed by two to three digits representing the fitting range filter. A space and the fitting range unit Deg (degrees) or Mpc (Megaparsecs) follows. In the case of Mpc a velocity bin may be listed as $v_{\min }-v_{\max }$ which represents the minimum and maximum velocity values for a galaxy to be included in the run. Table 5-1 describes the morphological abbreviations used.

Table 5-1 Morphological Filters

\begin{tabular}{ll} 
Filter & \multicolumn{1}{c}{ Meaning } \\
\hline $\mathbf{A}$ & All galaxies regardless of morphology \\
$\mathbf{E}$ & Elliptical galaxies \\
$\mathbf{L}$ & Lenticular galaxies \\
$\mathbf{S}$ & Spiral galaxies \\
$\mathbf{S}_{\mathrm{b}}$ & Spiral galaxies with bar features \\
$\mathbf{S}_{\mathrm{u}}$ & Spiral galaxies with no bar features
\end{tabular}

For example: AA05 Deg indicates a run with primary of all galaxies, secondary of all galaxies, at a fitting range of 5 degrees. As another example, SbE01 Mpc 3000-6000 indicates a run for primary of barred spirals, secondary of elliptical at a fitting range of one Mpc using a velocity bin of $3000 \leq v \leq 6000$.

\subsection{Two Dimensional}

\subsubsection{Averages and Standard Deviations}

Below are the average PD values with error bars for the population standard deviation, $\sigma$. The average is calculated via SQL Server's avg [63] function, and $\sigma$ is calculated using stddevp [64]. The run names describe the parameters of the run as described in section 5.1 of this paper. 
For the purposes of this paper I define $2 \mathrm{D}$ small scale as fitting ranges from $1^{\circ}$ to $5^{\circ}$ in $1^{\circ}$ increments. $2 \mathrm{D}$ large scale is defined as fitting ranges from $5^{\circ}$ to $30^{\circ}$ in $5^{\circ}$ increments.

In both small and large scales there is a consistent trend for smaller $\sigma$ values as the fitting ranges are increased; however, this trend is not universal. For example, EE02 Deg has a larger $\sigma$ value than EE01 Deg or EE03 Deg in 2MRS North.

Another trend observed for small scale is that the averages tend to fit within the $\sigma$ value of the runs matching the same primary and secondary morphology filters. This trend is also not universal. The run $\mathrm{S}_{b} \mathrm{~S}_{b} 01$ Deg in both hemispheres has a much higher average PD value than the other runs in that hemisphere. It is so high that it exceeds the $\sigma$ value for all other $S_{b} S_{b}$ runs. Interestingly the equivalent run for unbarred spirals, SuSb01 Deg has a very small $\sigma$ value by comparison. This suggests that at a fitting range of $1^{\circ}$ that unbarred spirals have a consistent environment while barred spirals have an inconsistent environment.

At the small scale ellipticals show a less dense environment than spirals. Refer to Table 5-2 and Table 5-3 which are sorted by average PD in ascending order. For both hemispheres the average PD for any EA run with a fitting range of $5^{\circ}$ or less is smaller than the average for any SA run with a fitting range of $5^{\circ}$ or less.

Table 5-2 2D North Elliptical vs. Spiral Avg. PD

Sorted by Avg. PD Ascending

\begin{tabular}{|c|c|c|}
\hline \multicolumn{3}{|c|}{ North } \\
\hline Run & Avg. PD & $\boldsymbol{\sigma}$ value \\
\hline EA02 Deg & 1.510155 & 0.945335 \\
\hline EA03 Deg & 1.542704 & 0.573129 \\
\hline EA04 Deg & 1.589278 & 0.490025 \\
\hline EA05 Deg & 1.628452 & 0.434908 \\
\hline EA01 Deg & 1.678289 & 2.604508 \\
\hline SA03 Deg & 1.690279 & 0.575642 \\
\hline SA02 Deg & 1.700727 & 1.446155 \\
\hline SA04 Deg & 1.727625 & 0.481278 \\
\hline SA05 Deg & 1.761395 & 0.426753 \\
\hline SA01 Deg & 1.902609 & 2.876529 \\
\hline
\end{tabular}


Table 5-3 2D South Elliptical vs. Spiral Avg. PD

Sorted by Avg. PD Ascending

\begin{tabular}{|c|c|c|}
\hline \multicolumn{3}{|c|}{ South } \\
\hline Run & Avg. PD & $\boldsymbol{\sigma}$ value \\
\hline EA02 Deg & 1.507207 & 0.920086 \\
\hline EA03 Deg & 1.523494 & 0.627817 \\
\hline EA04 Deg & 1.56572 & 0.503726 \\
\hline EA05 Deg & 1.603922 & 0.444636 \\
\hline EA01 Deg & 1.64061 & 1.805112 \\
\hline SA02 Deg & 1.654338 & 0.87333 \\
\hline SA03 Deg & 1.663094 & 0.588874 \\
\hline SA04 Deg & 1.700353 & 0.493356 \\
\hline SA05 Deg & 1.729073 & 0.433941 \\
\hline SA01 Deg & 1.825007 & 2.233109 \\
\hline
\end{tabular}

The complete plots of average PD and $\sigma$ values for 2D follow. 


\subsubsection{2MRS North Small Scale}

Figure 5-1 2MRS North Small Scale 2D Average PD and $\sigma$ values for All Primaries regardless of morphology

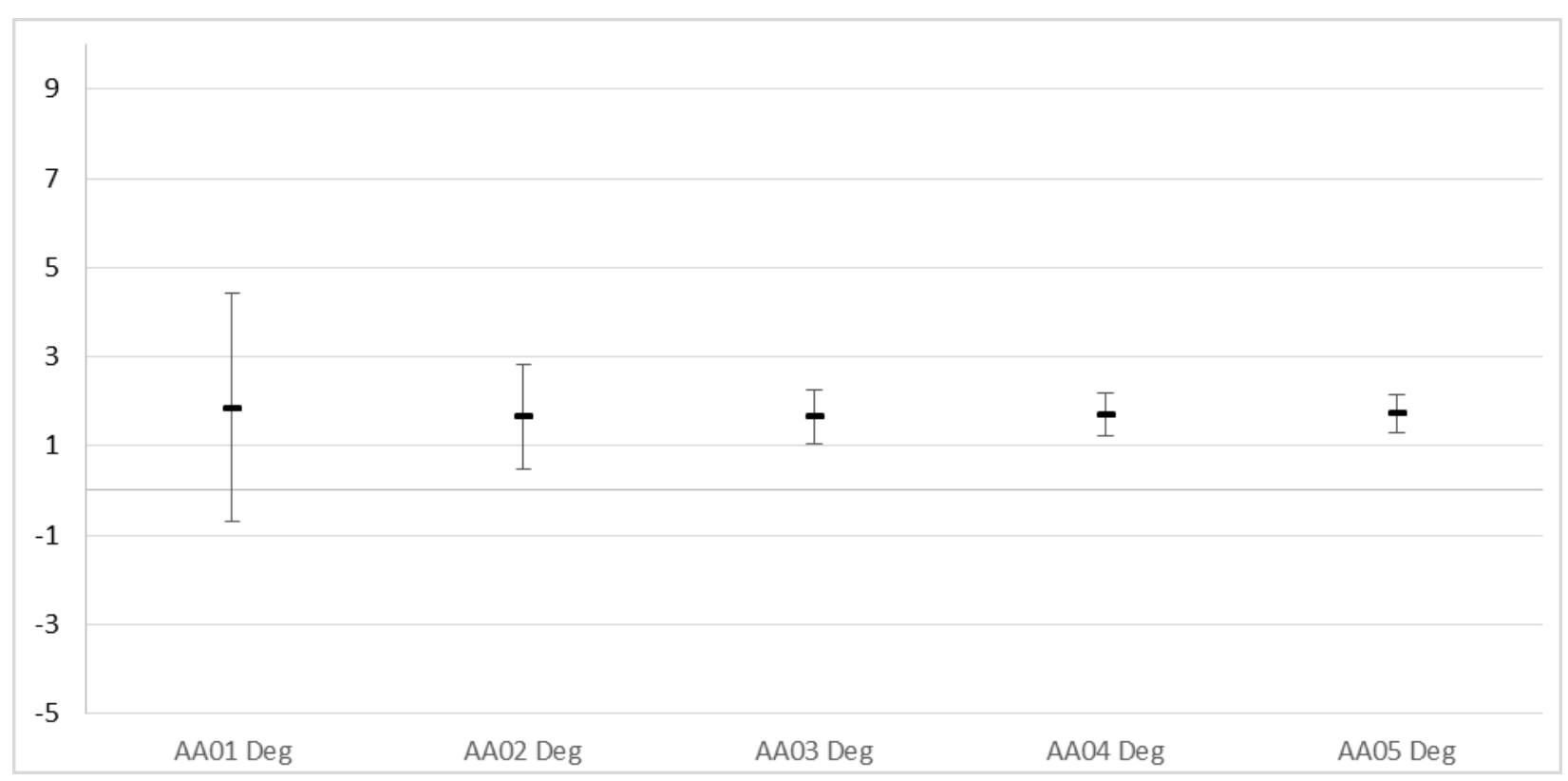

Figure 5-2 2MRS North Small Scale 2D Average PD and $\sigma$ values for Elliptical Primaries

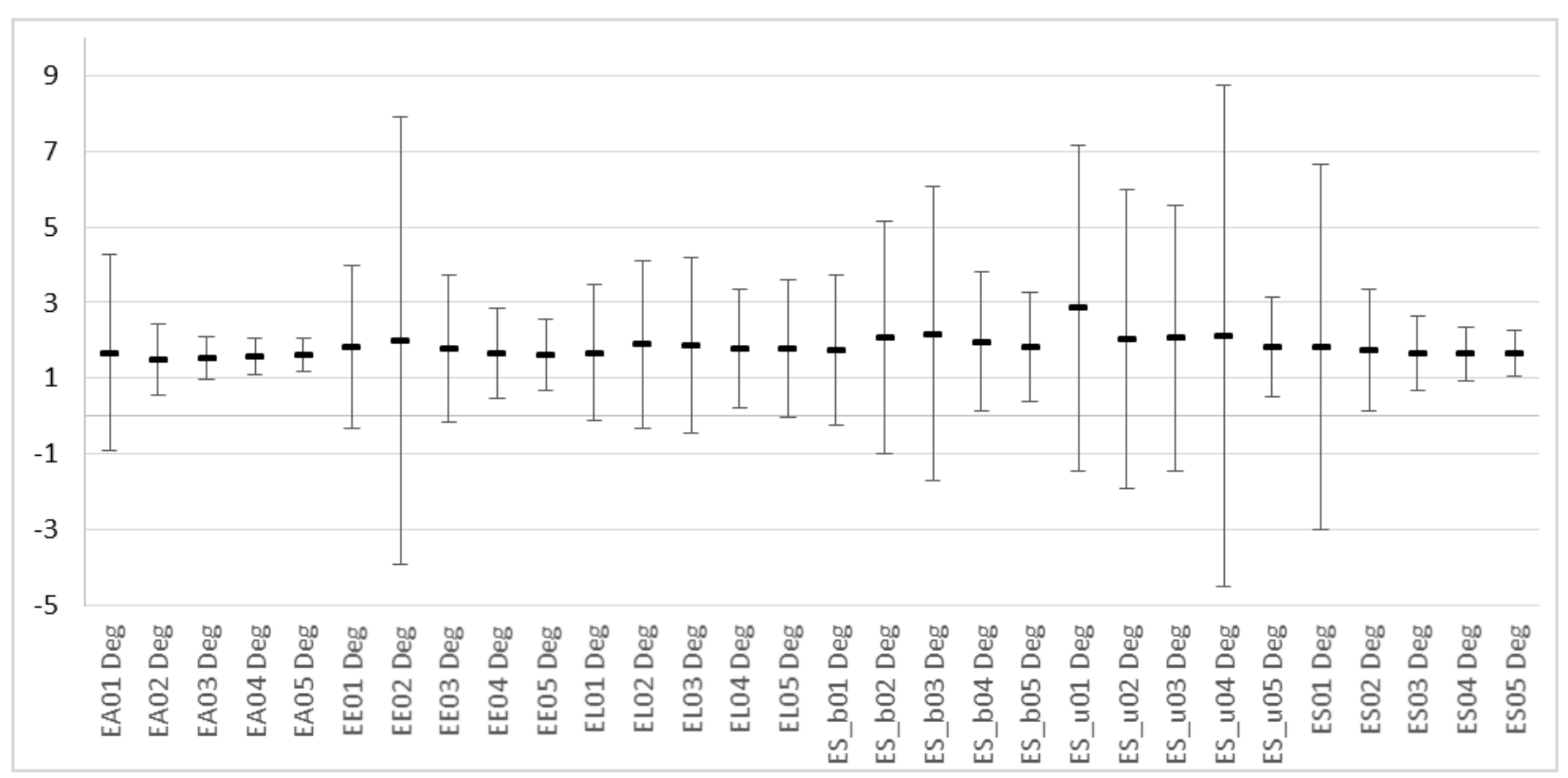


Figure 5-3 2MRS North Small Scale 2D Average PD and $\sigma$ values for Lenticular Primaries

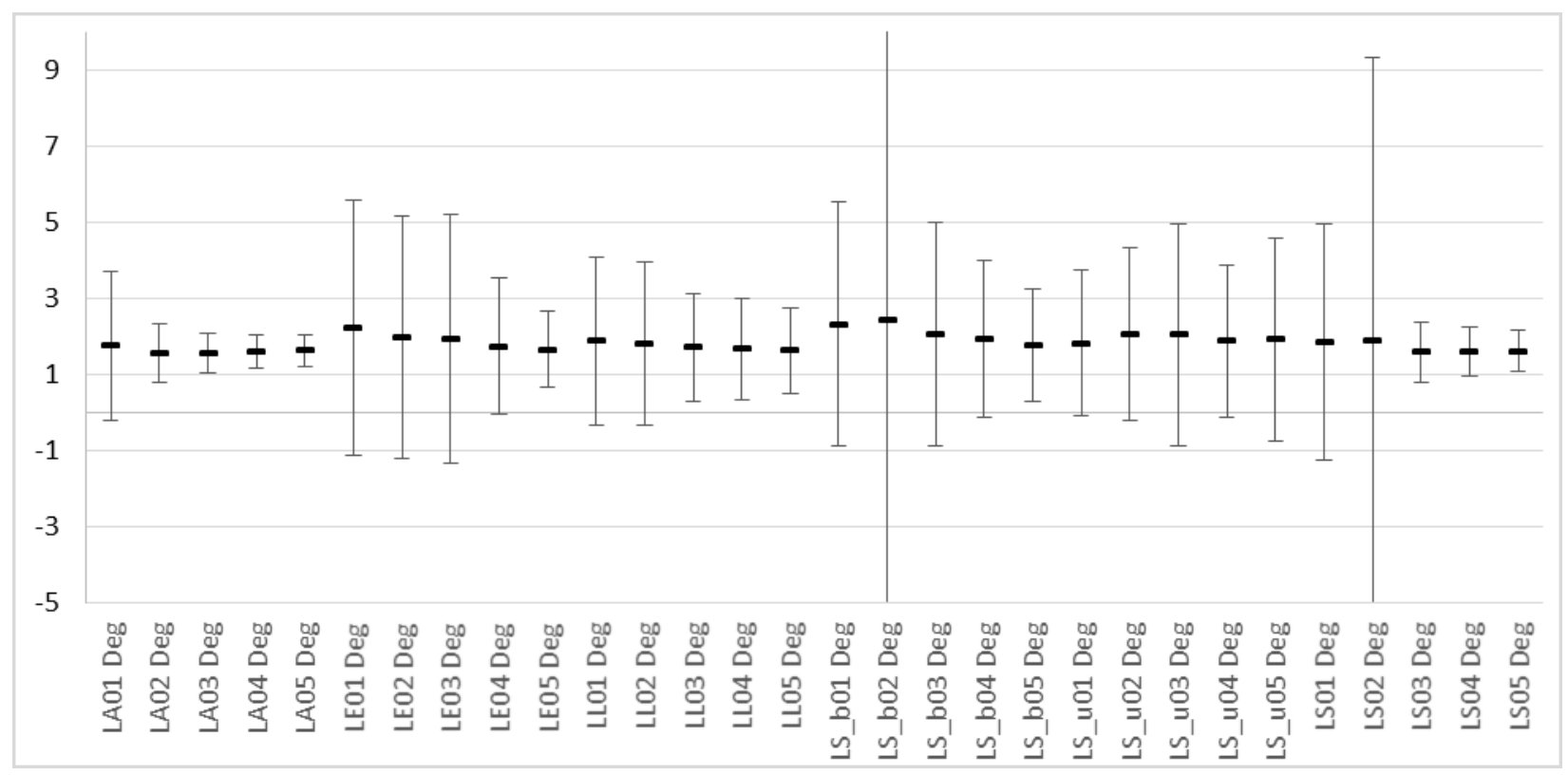

Figure 5-4 2MRS North Small Scale 2D Average PD and $\sigma$ values for Spiral Primaries

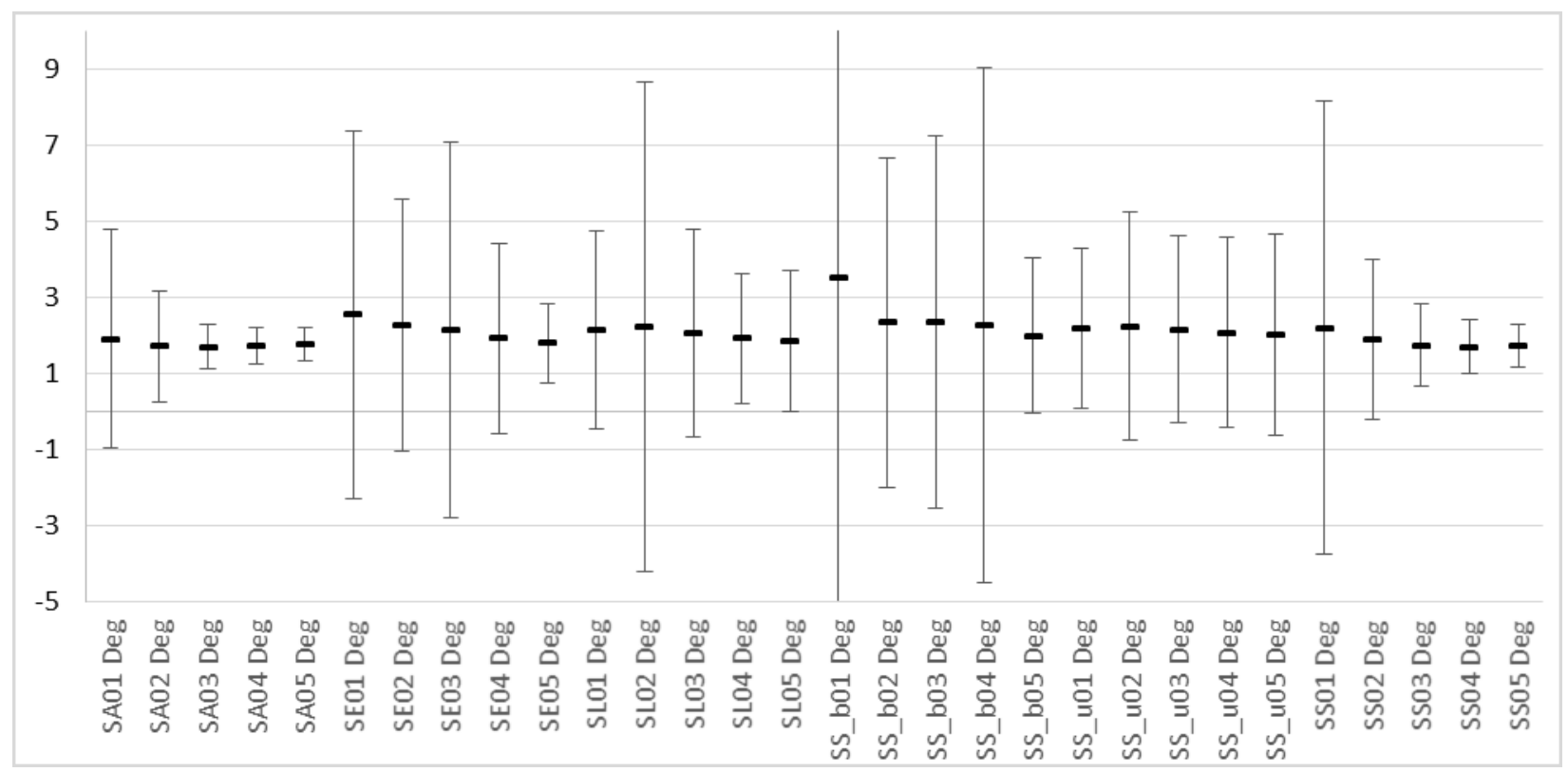


Figure 5-5 2MRS North Small Scale 2D Average PD and $\sigma$ values for Barred Spiral Primaries

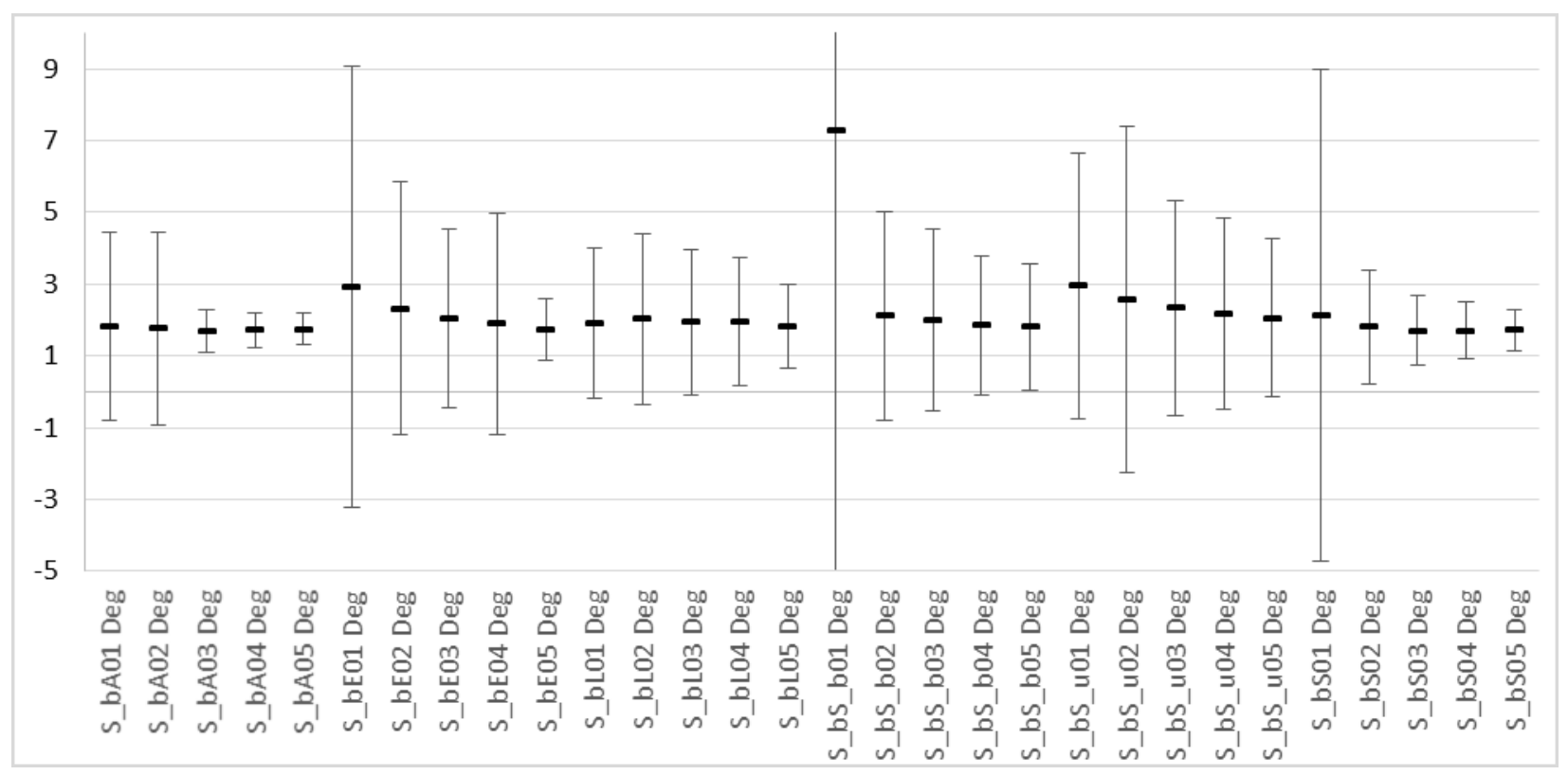

Figure 5-6 2MRS North Small Scale 2D Average PD and $\sigma$ values for Unbarred Spiral Primaries

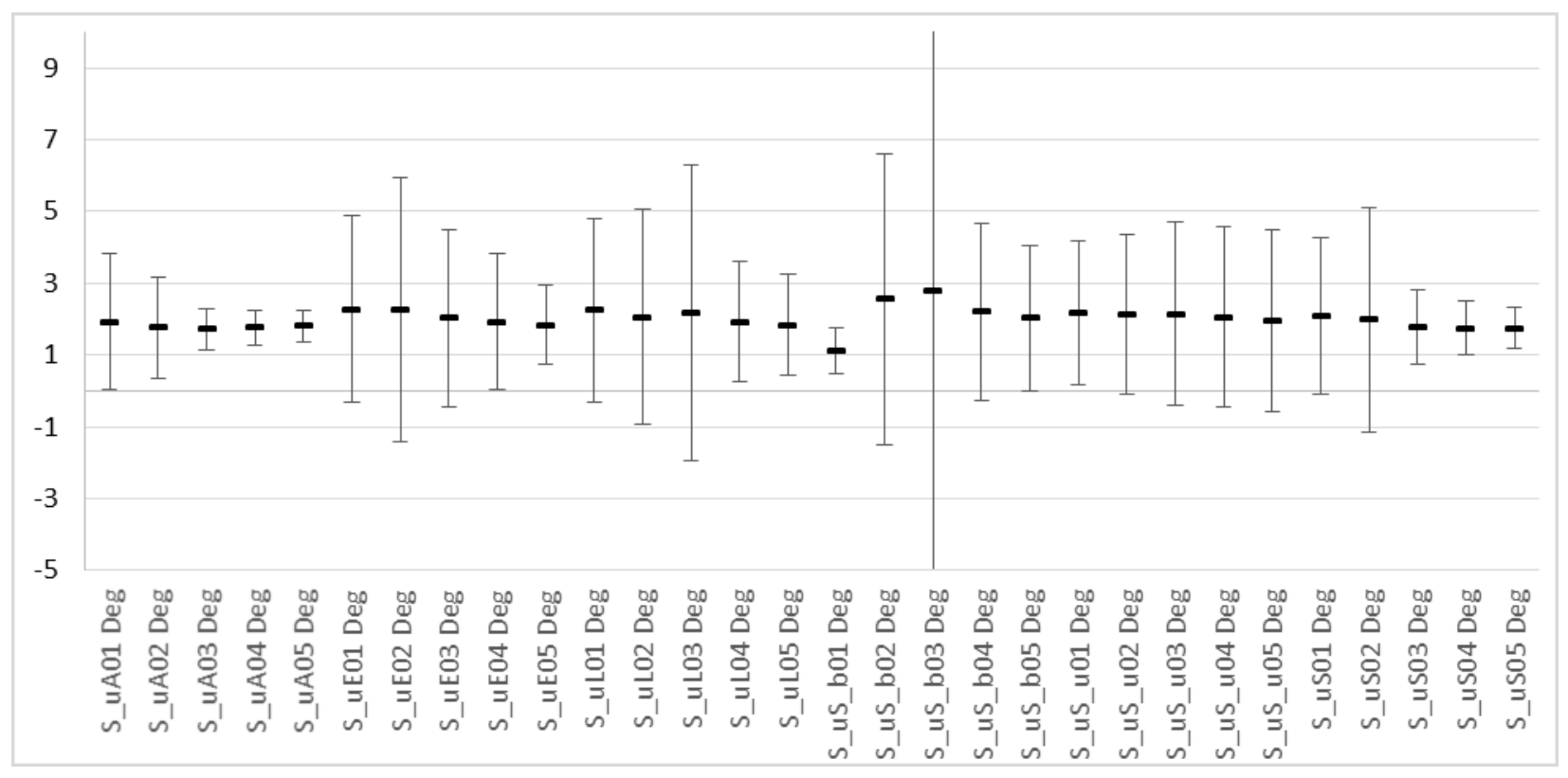




\subsubsection{2MRS South Small Scale}

Figure 5-72 MRS South Small Scale 2D Average PD and $\sigma$ values for All Primaries regardless of morphology

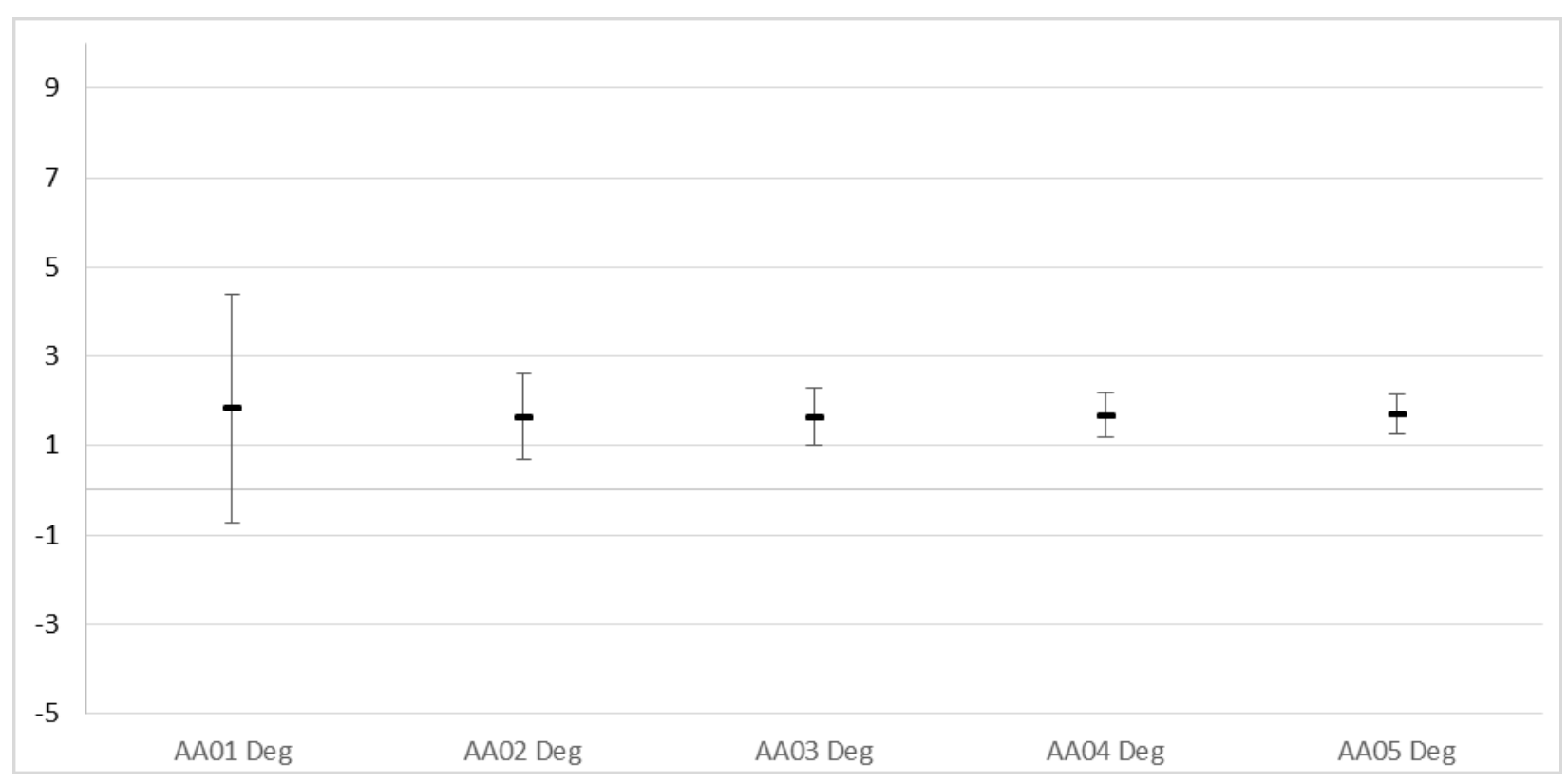

Figure 5-8 2MRS South Small Scale 2D Average PD and $\sigma$ values for Elliptical Primaries

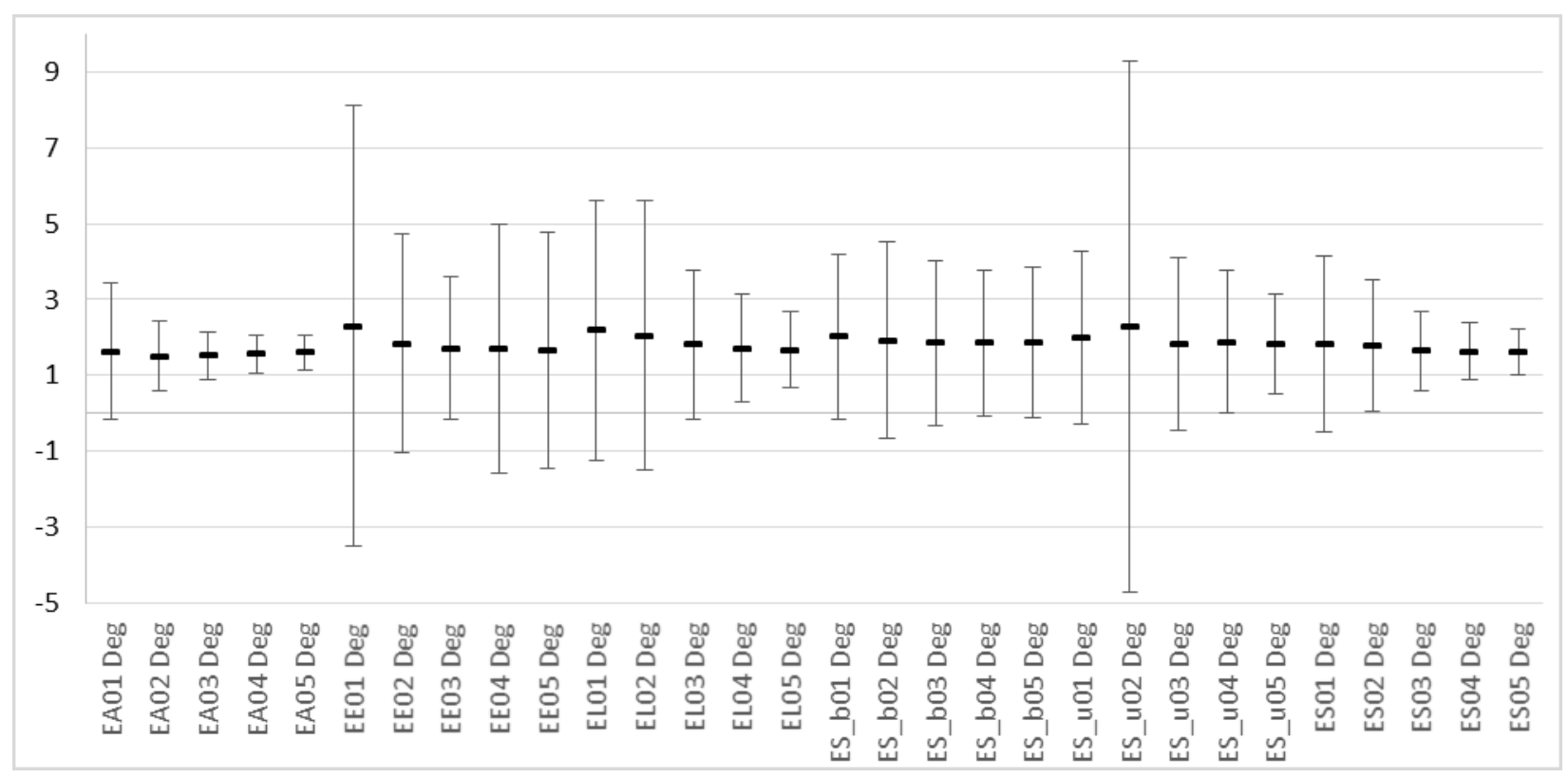


Figure 5-9 2MRS South Small Scale 2D Average PD and $\sigma$ values for Lenticular Primaries

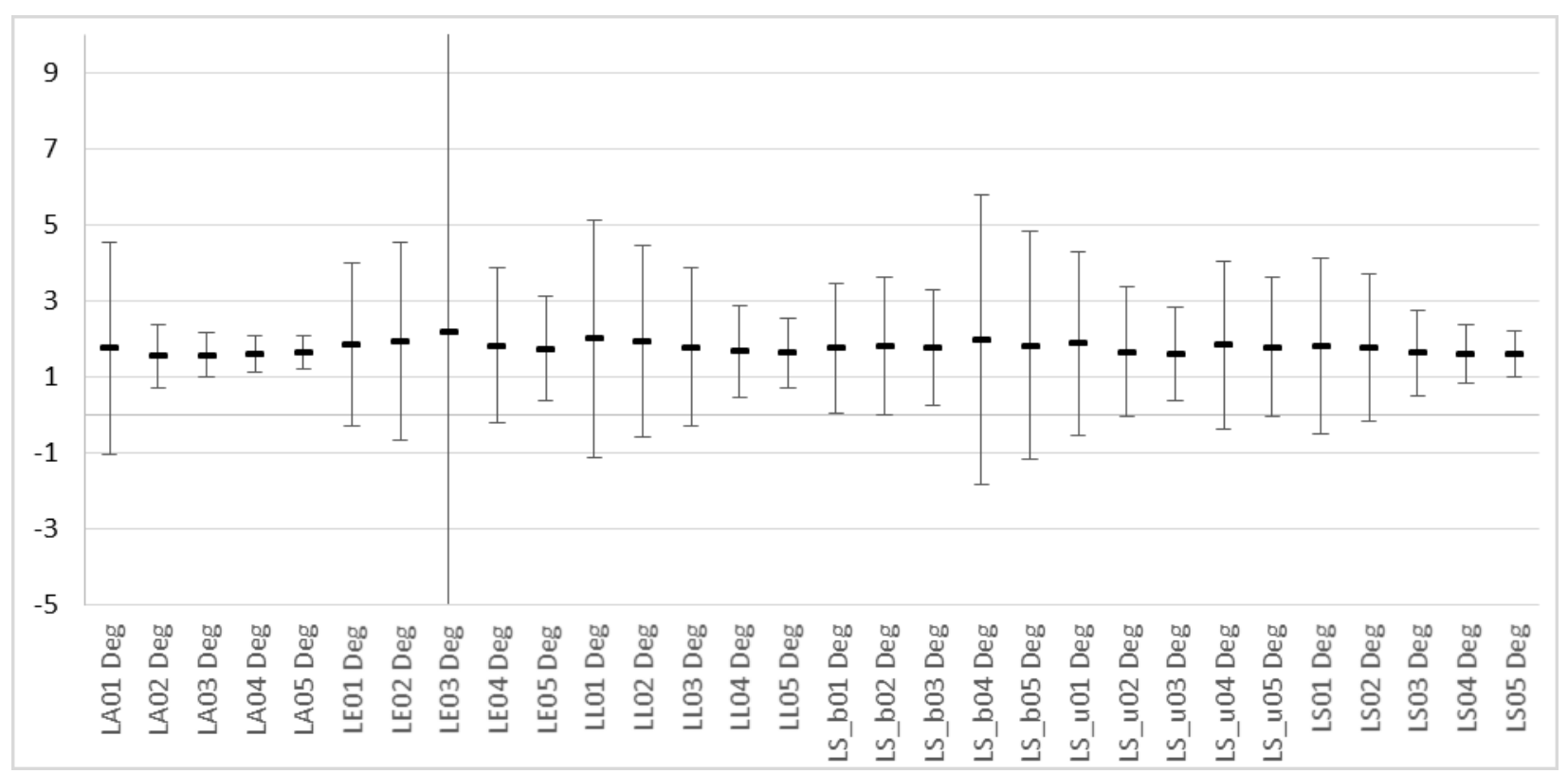

Figure 5-10 2MRS South Small Scale 2D Average PD and $\sigma$ values for Spiral Primaries

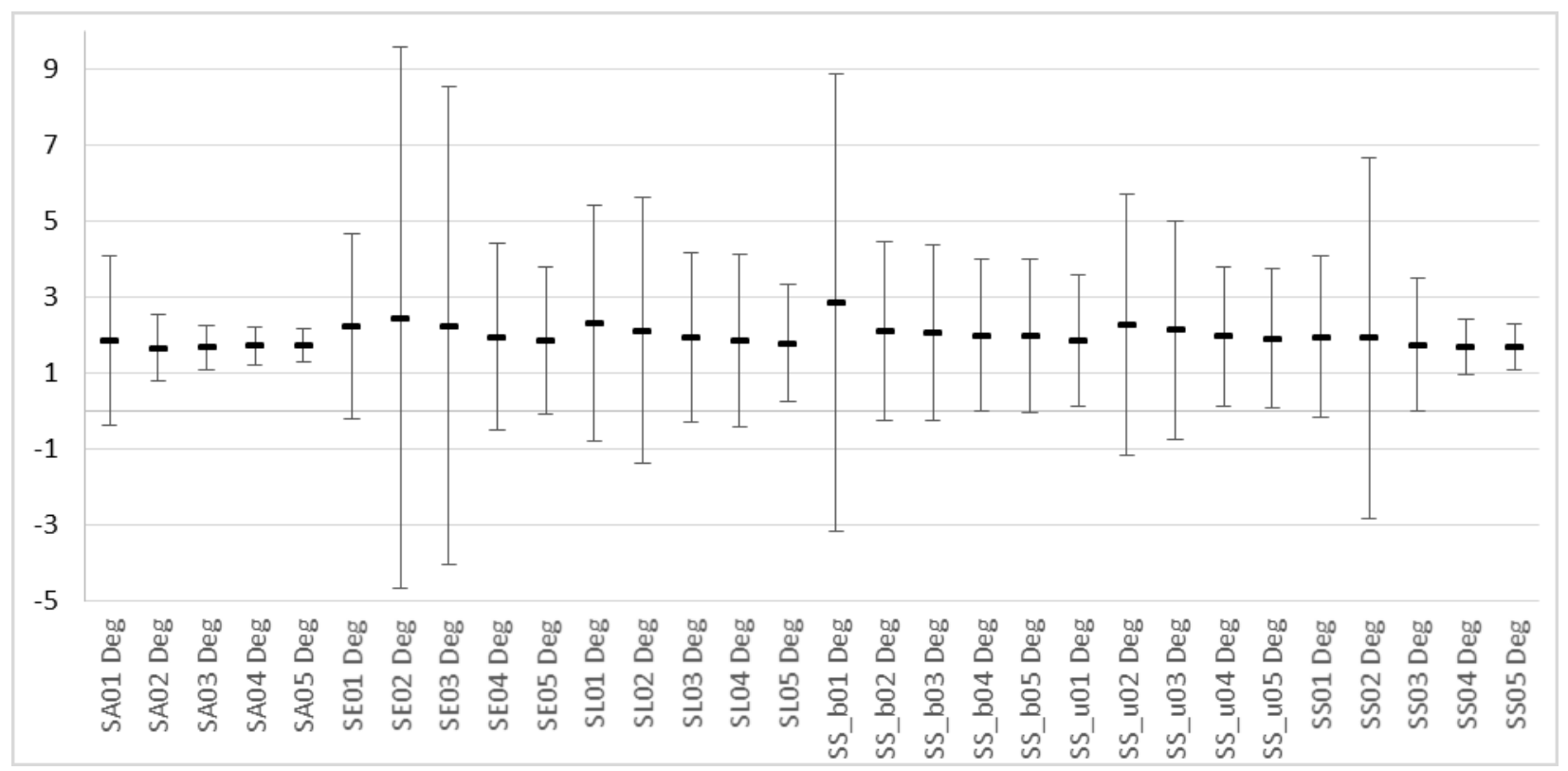


Figure 5-11 2MRS South Small Scale 2D Average PD and $\sigma$ values for Barred Spiral Primaries

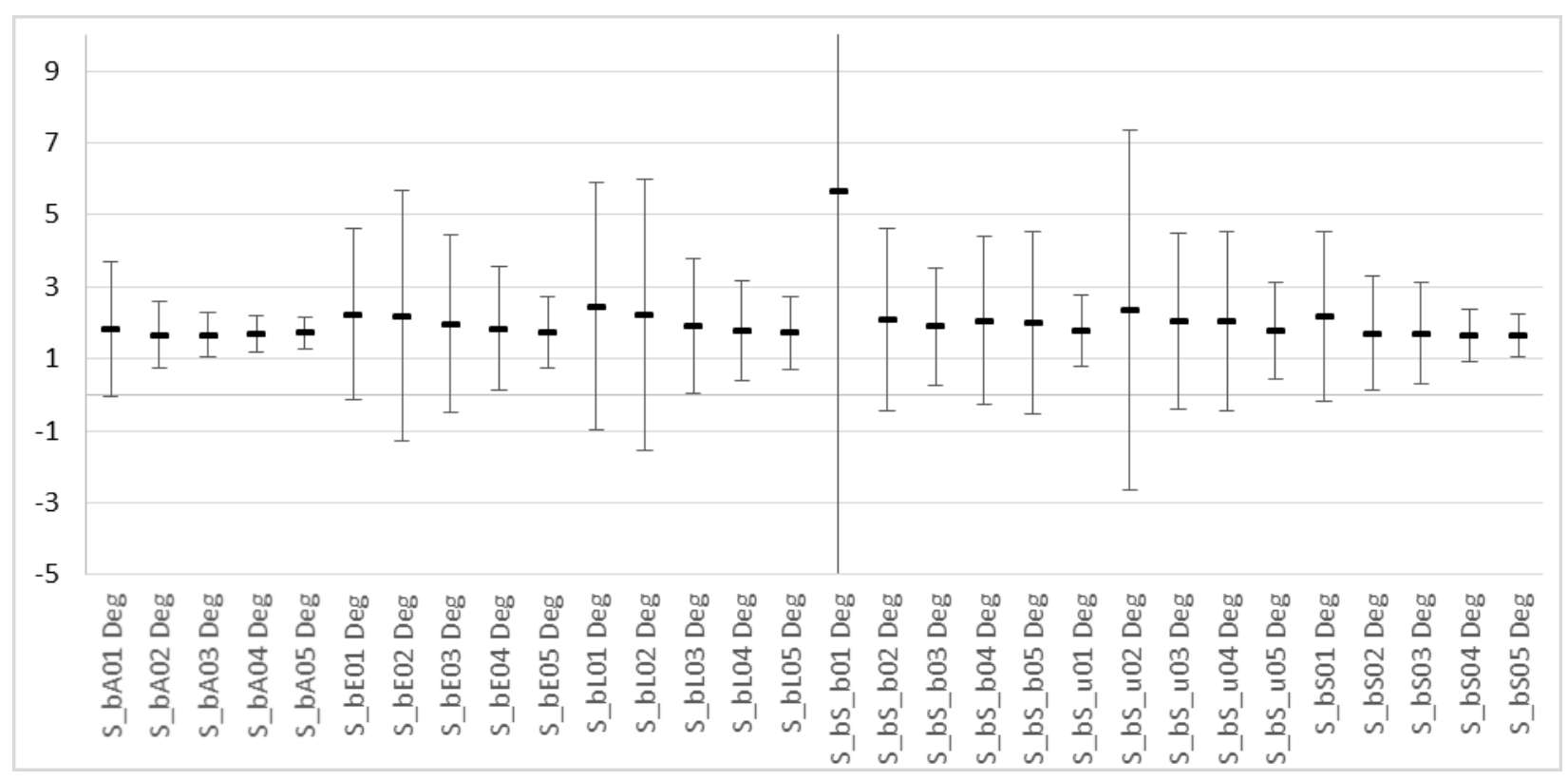

Figure 5-12 2MRS South Small Scale 2D Average PD and $\sigma$ values for Unbarred Spiral Primaries

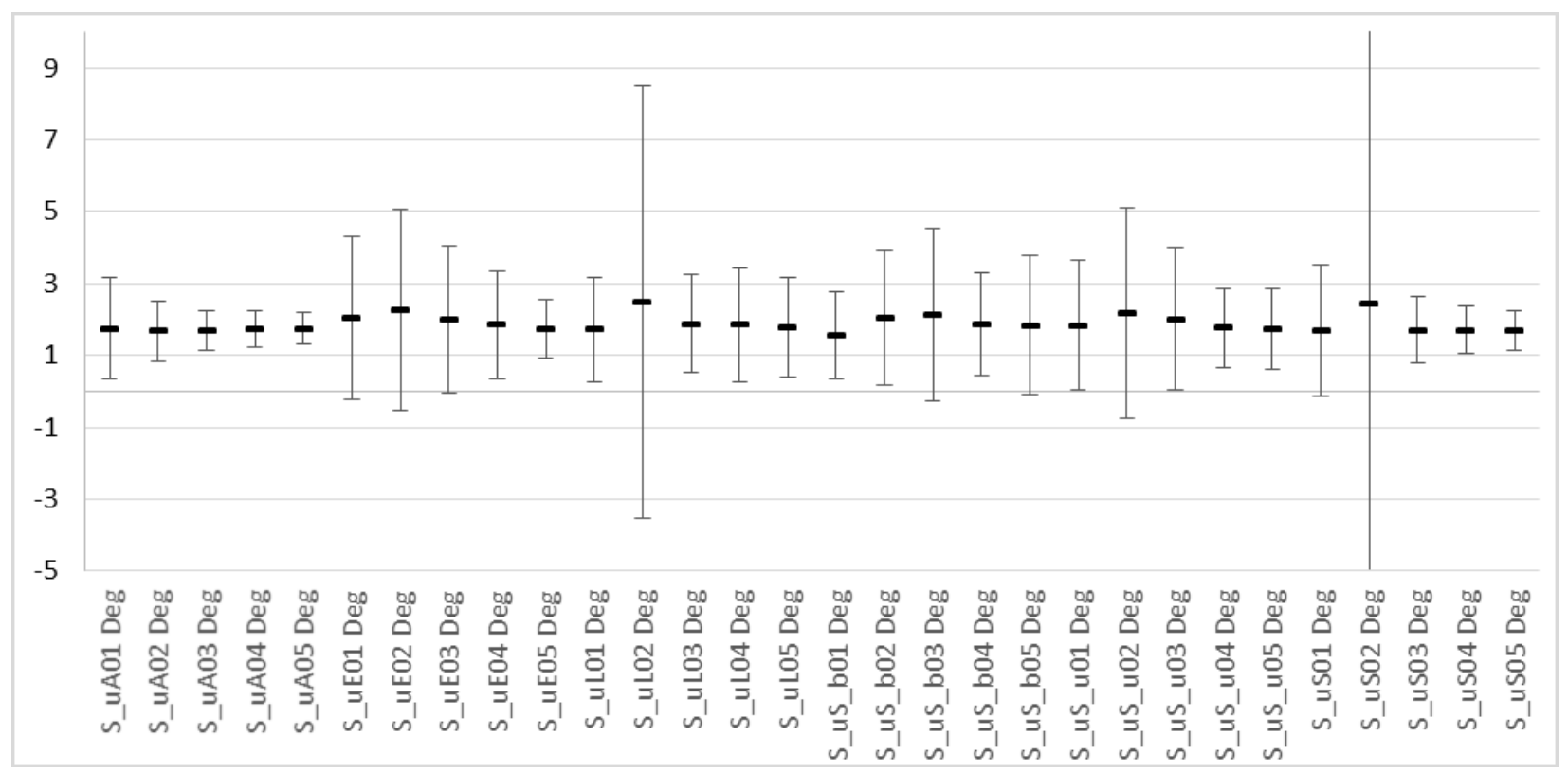




\subsubsection{2MRS North Large Scale}

Figure 5-13 2MRS North Large Scale 2D Average PD and $\sigma$ values for All Primaries regardless of morphology

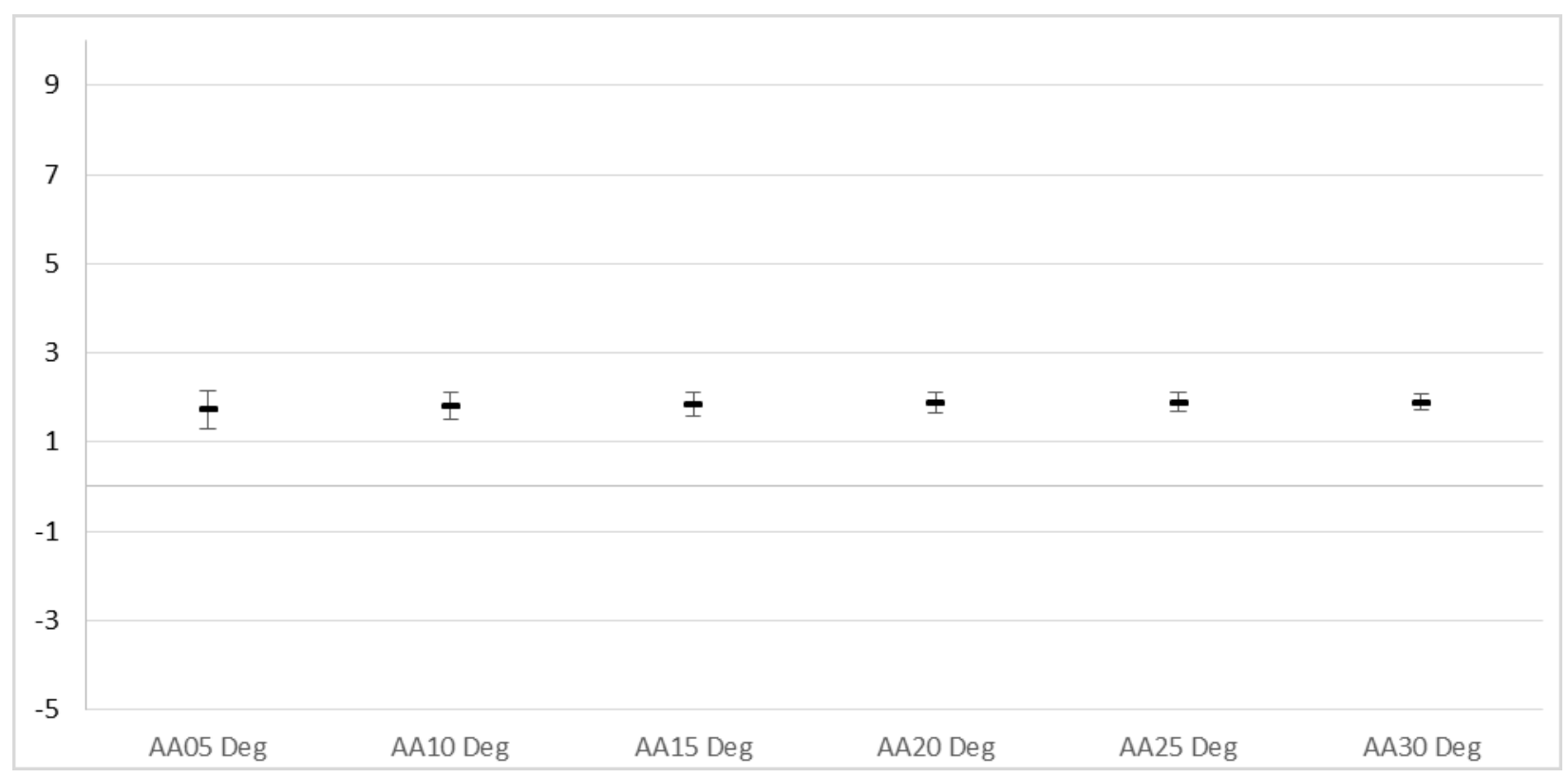

Figure 5-14 2MRS North Large Scale 2D Average PD and $\sigma$ values for Elliptical Primaries

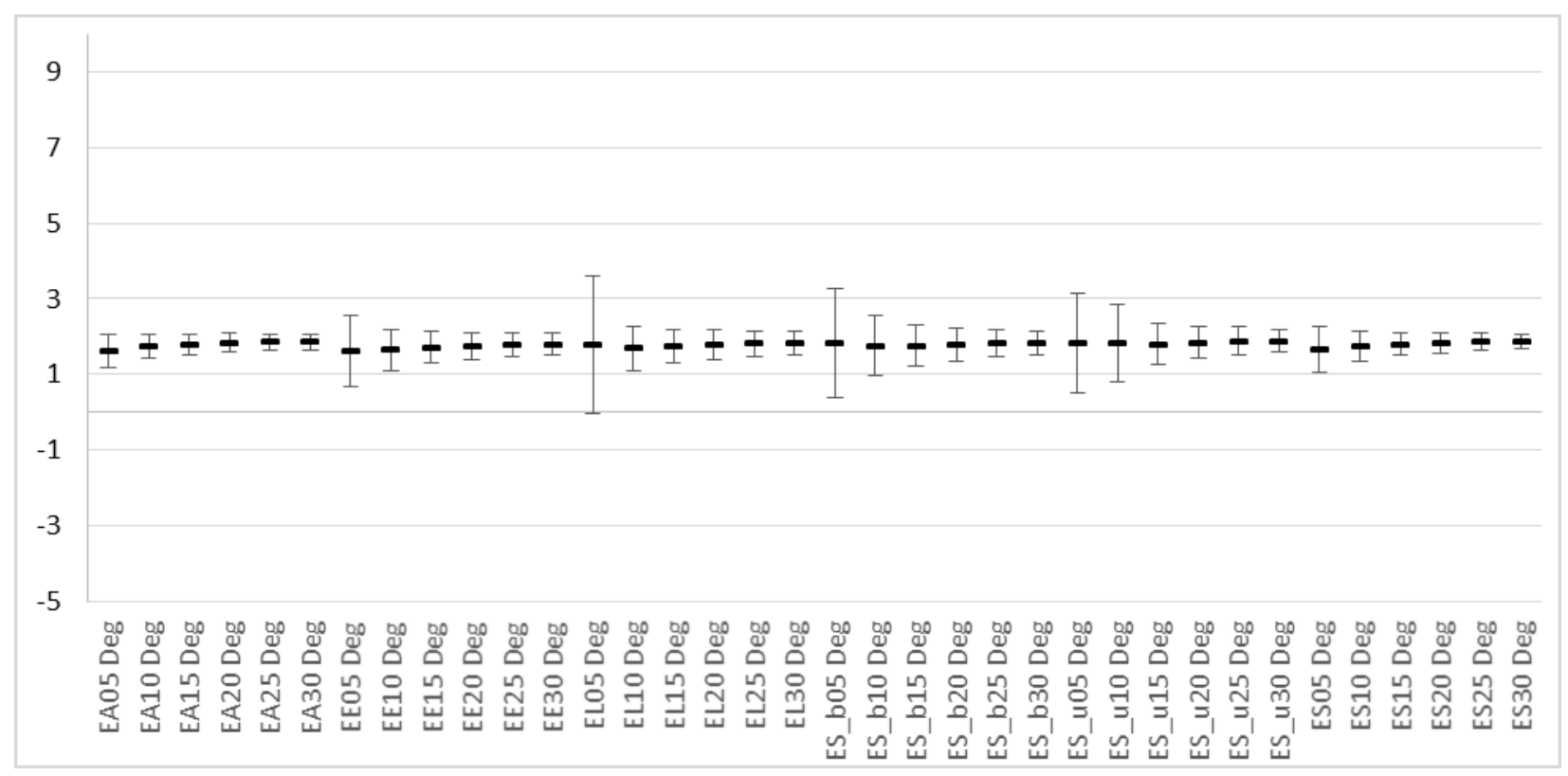


Figure 5-15 2MRS North Large Scale 2D Average PD and $\sigma$ values for Lenticular Primaries

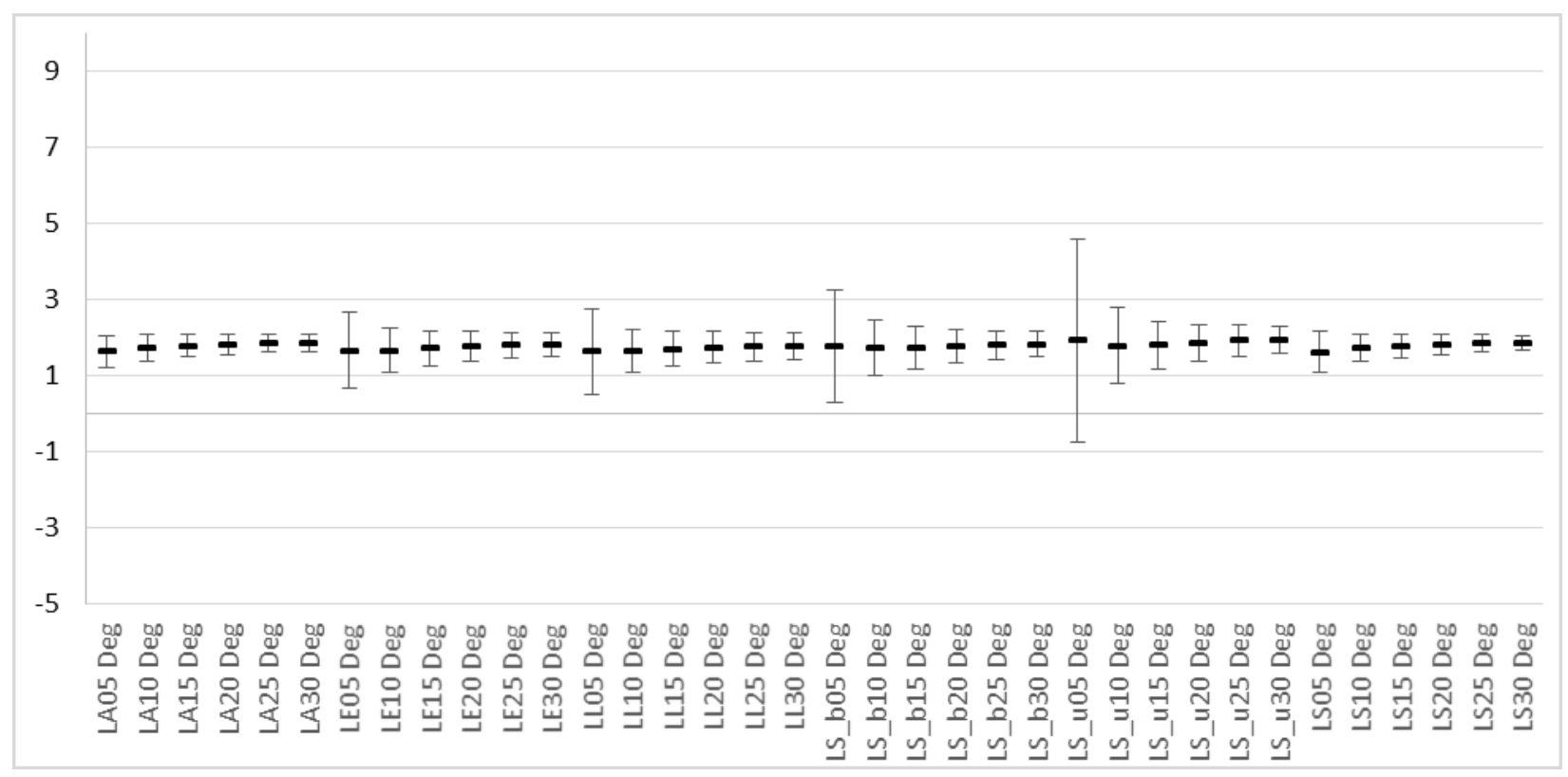

Figure 5-16 2MRS North Large Scale 2D Average PD and $\sigma$ values for Spiral Primaries

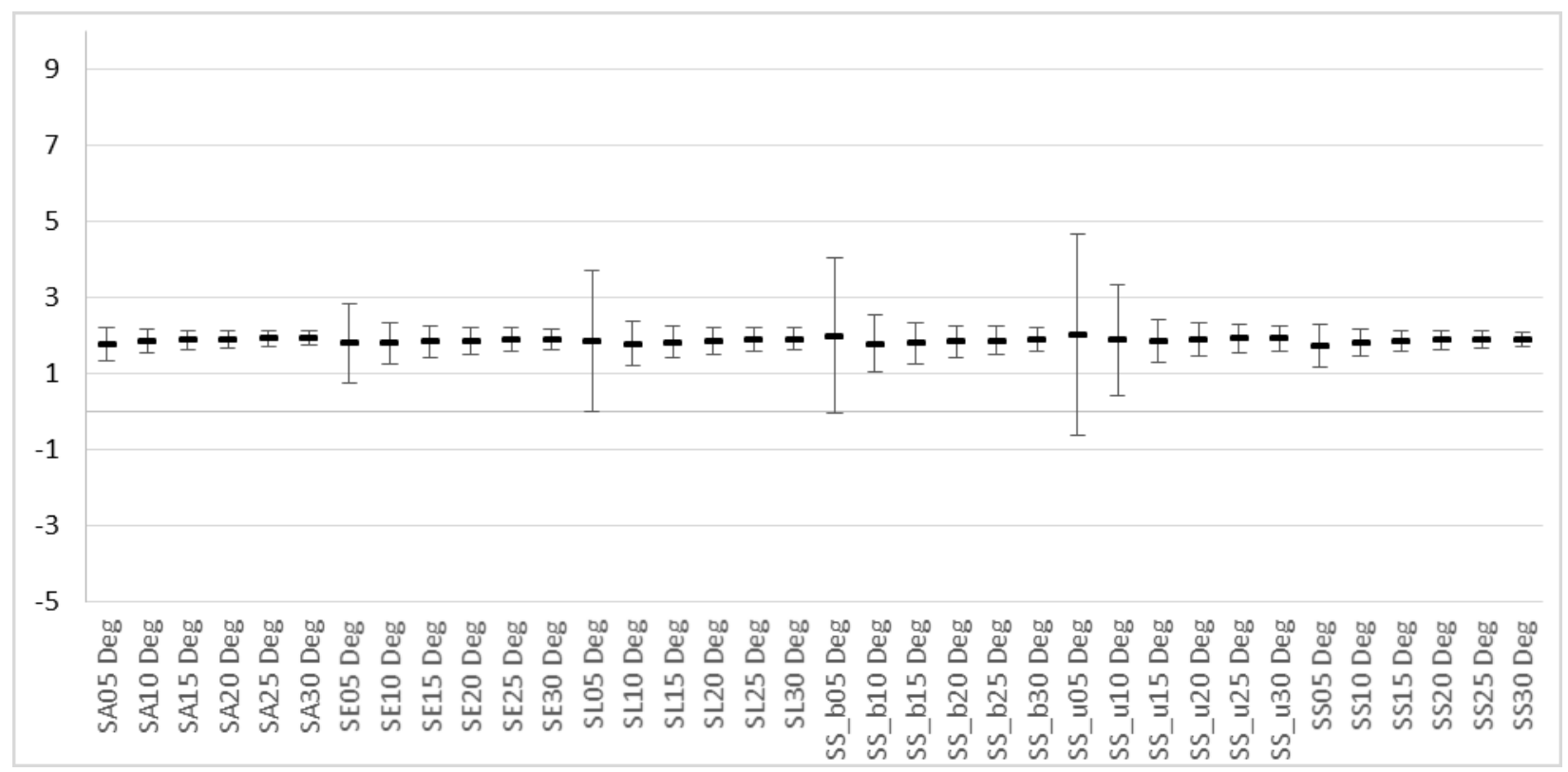


Figure 5-17 2MRS North Large Scale 2D Average PD and $\sigma$ values for Barred Spirals

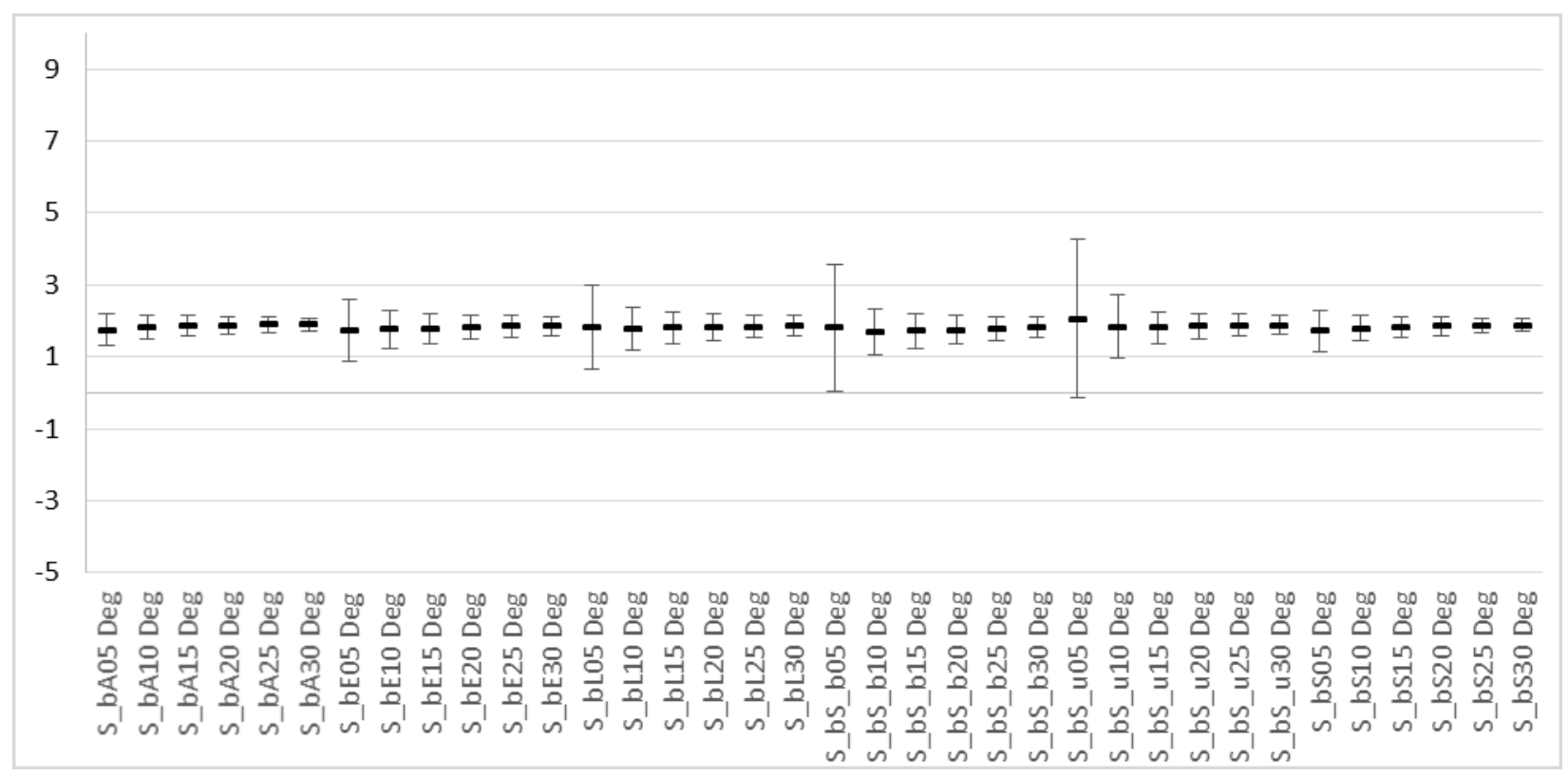

Figure 5-18 2MRS North Large Scale 2D Average PD and $\sigma$ values for Unbarred Spirals

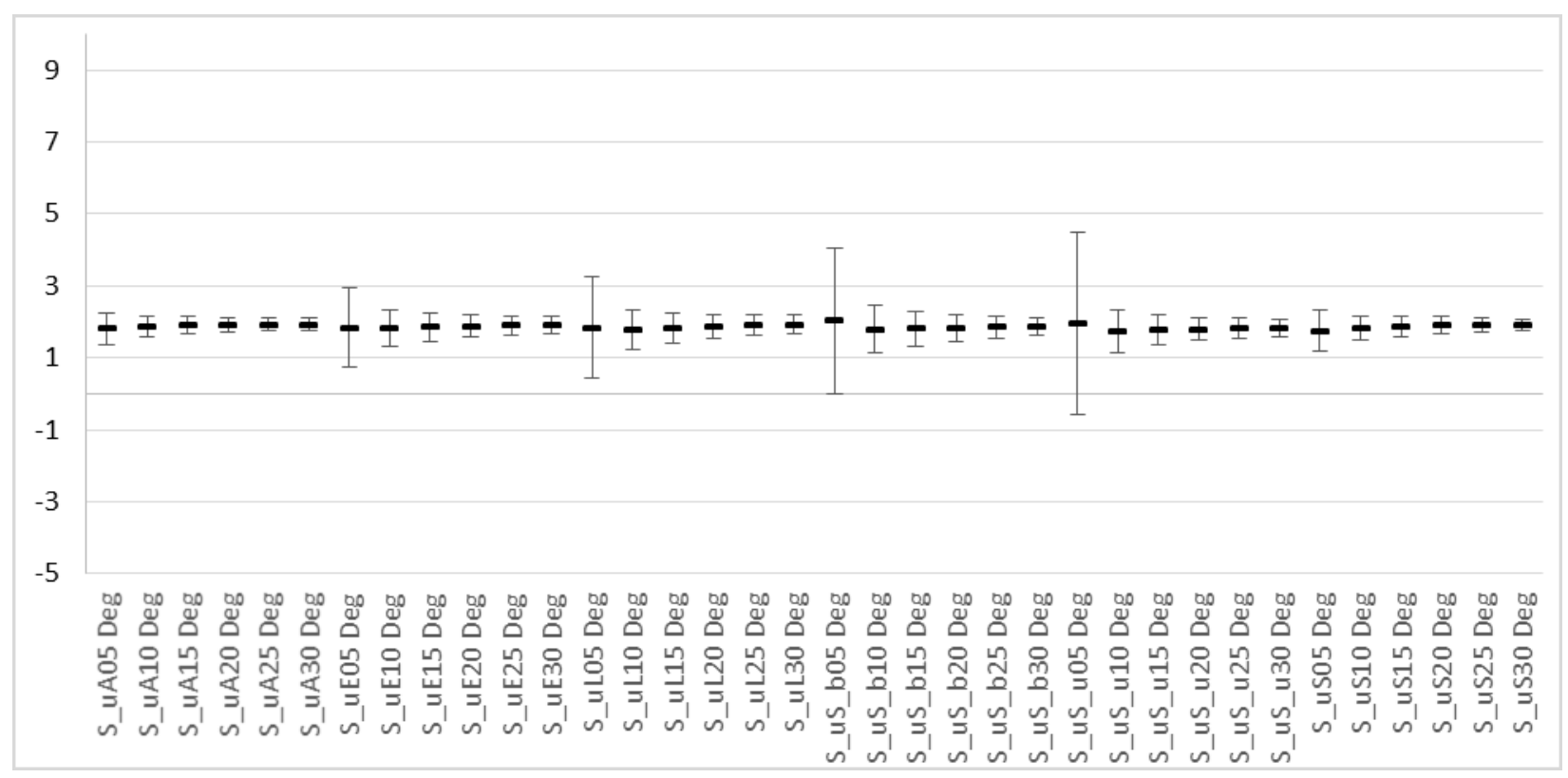




\subsubsection{2MRS South Large Scale}

Figure 5-19 2MRS South Large Scale 2D Average PD and $\sigma$ values for All Primaries regardless of morphology

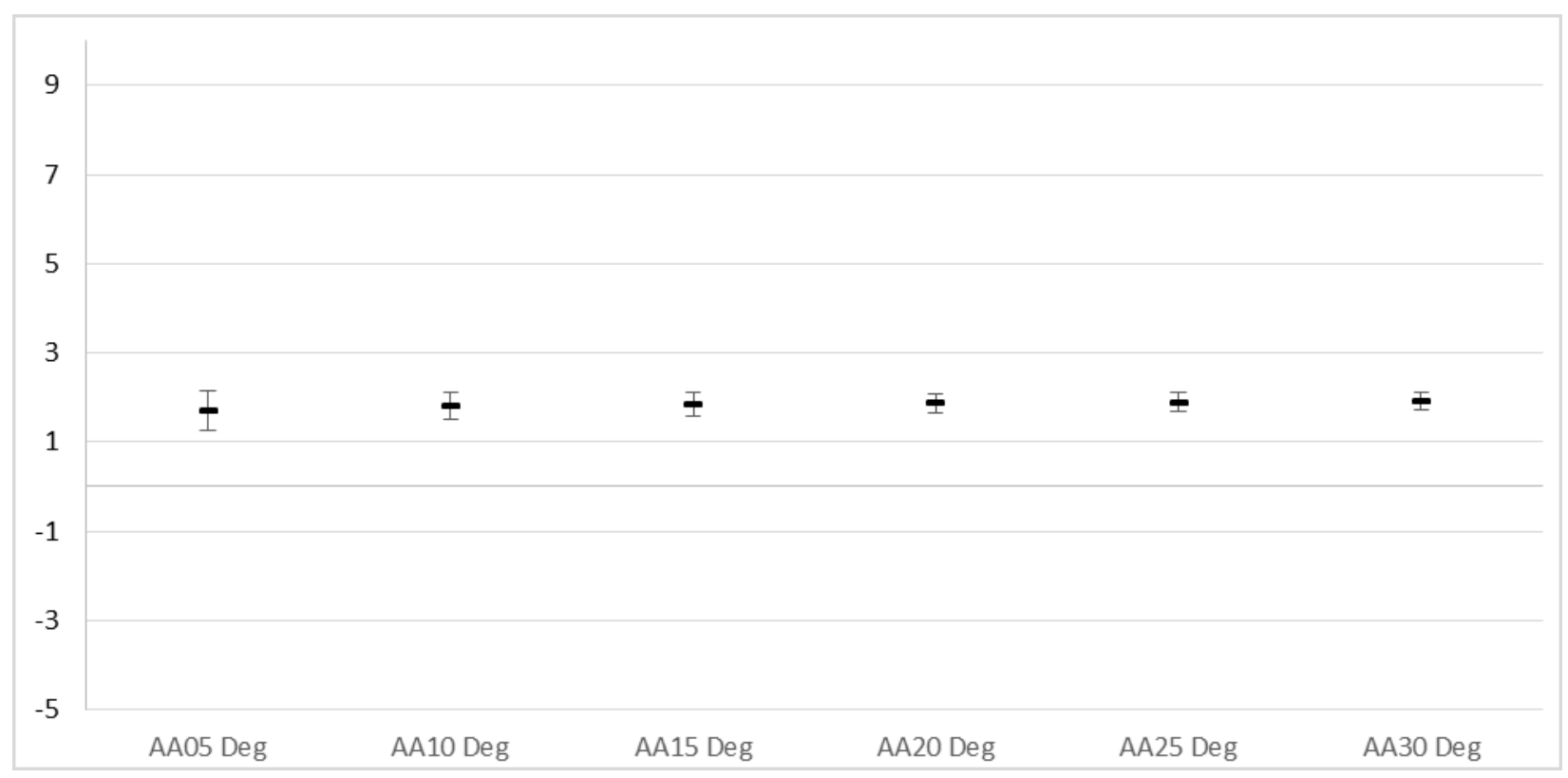

Figure 5-20 2MRS South Large Scale 2D Average PD and $\sigma$ values for Elliptical Primaries

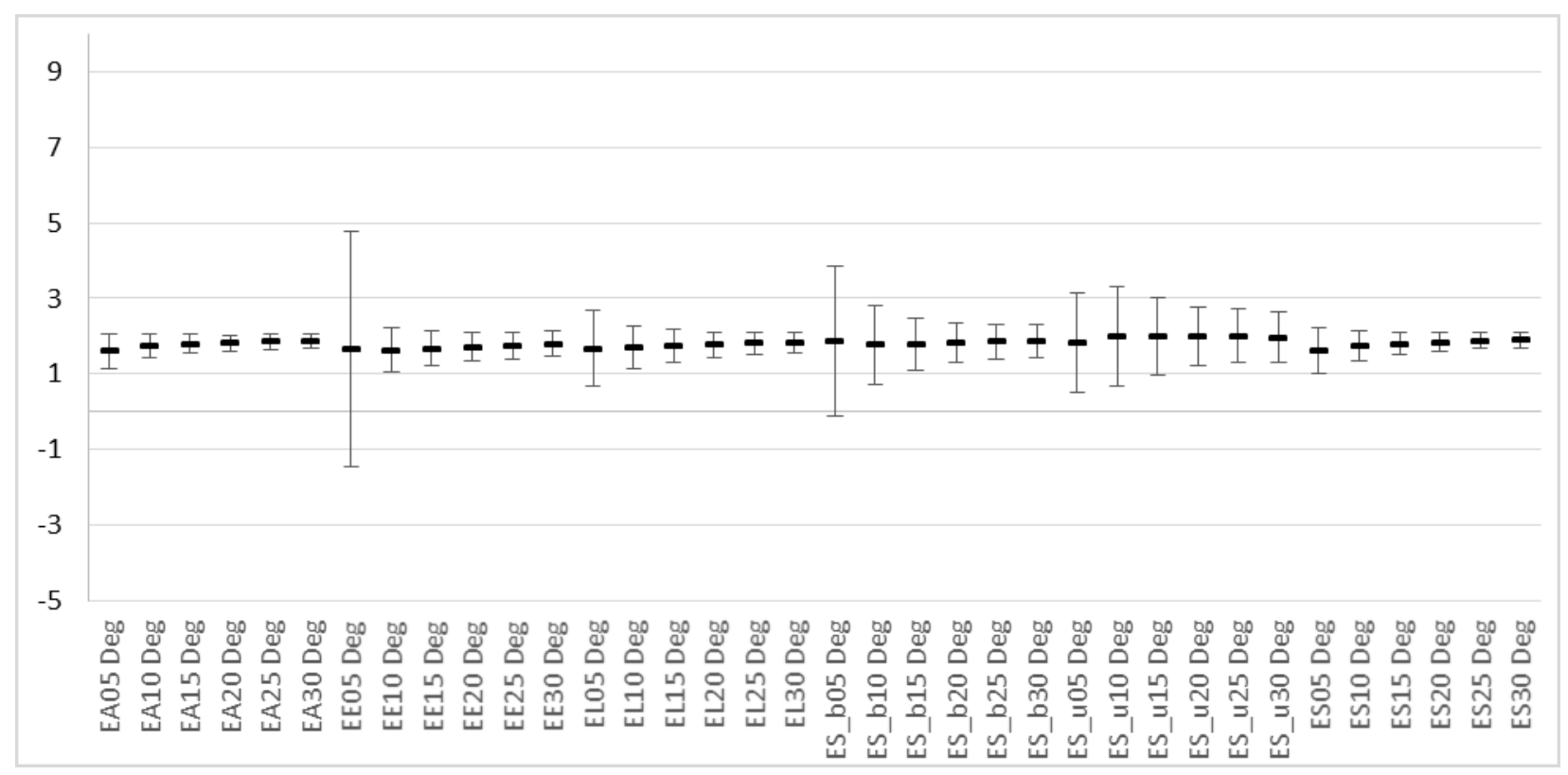


Figure 5-21 2MRS South Large Scale 2D Average PD and $\sigma$ values for Lenticular Primaries

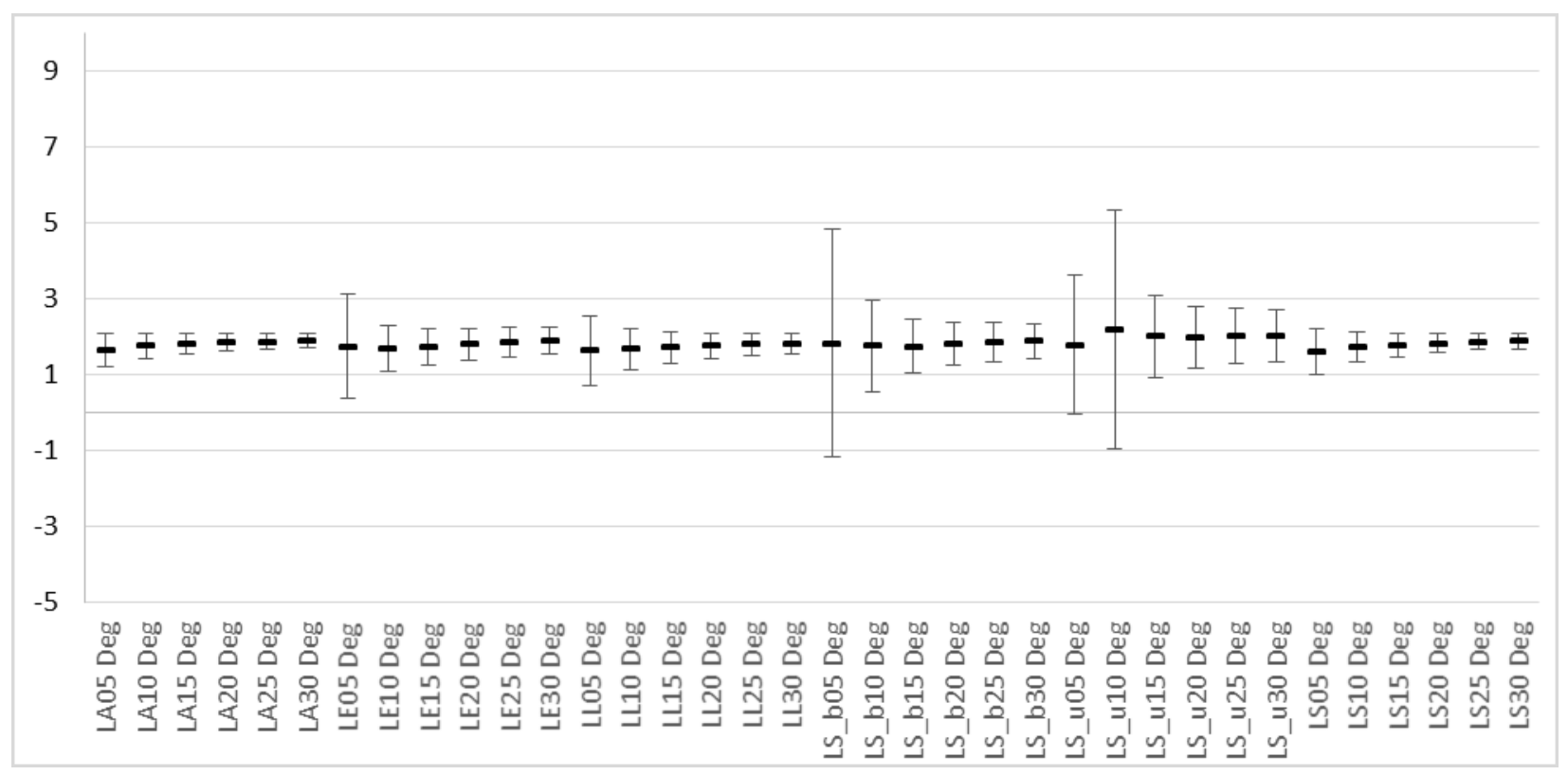

Figure 5-22 2MRS South Large Scale 2D Average PD and $\sigma$ values for Spiral Primaries

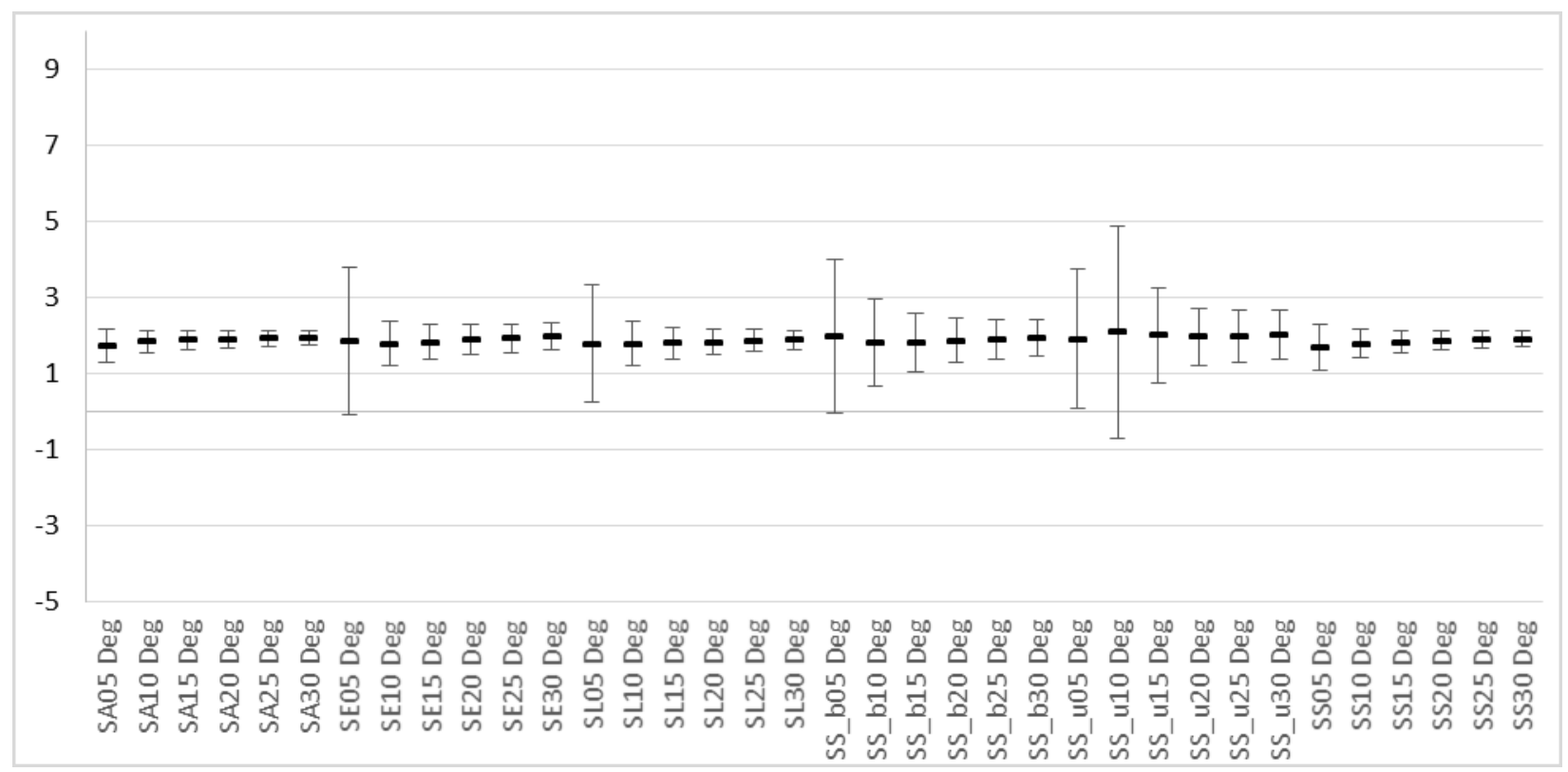


Figure 5-23 2MRS South Large Scale 2D Average PD and $\sigma$ values for Barred Spiral Primaries

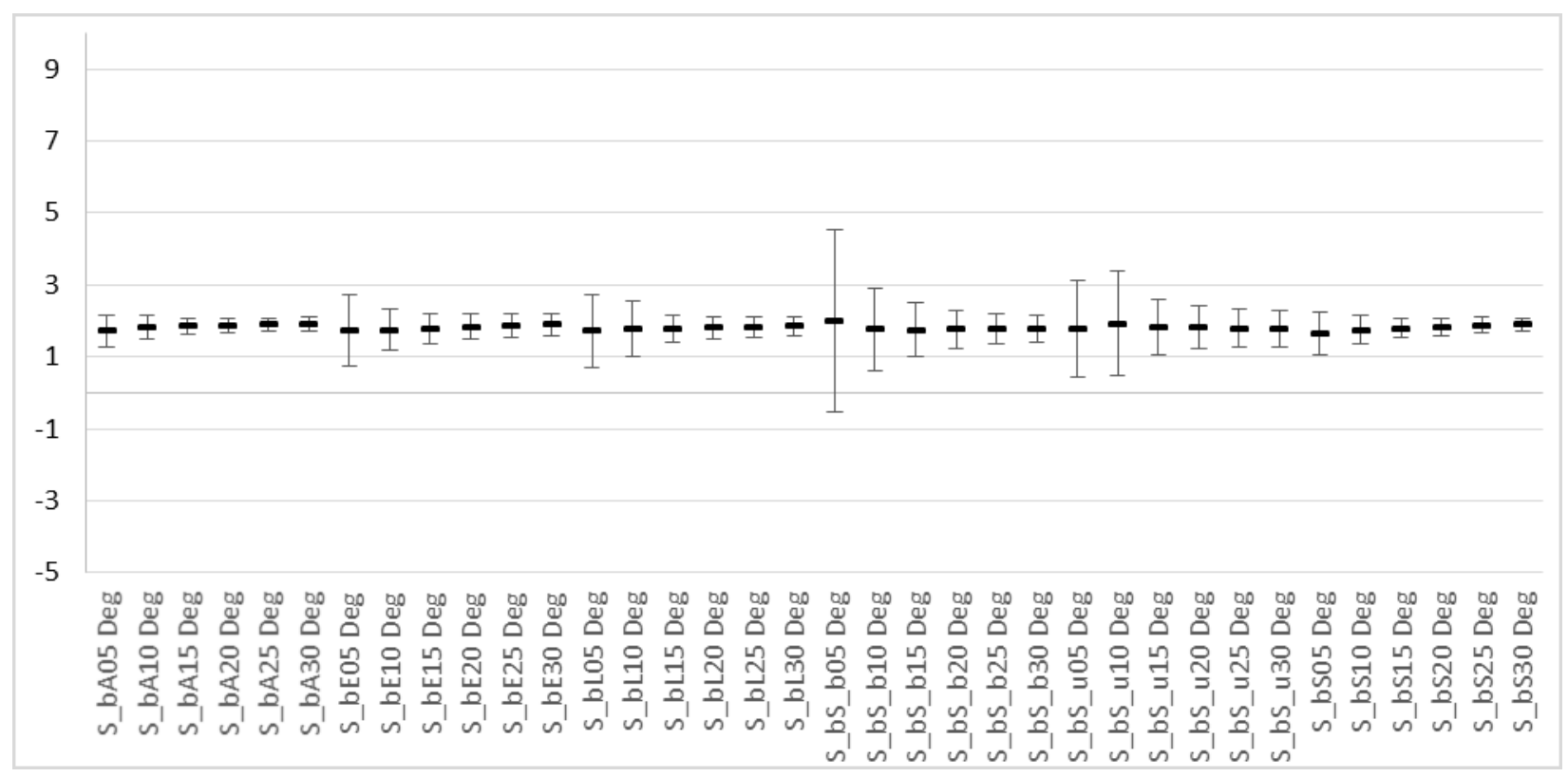

Figure 5-24 2MRS South Large Scale 2D Average PD and $\sigma$ values for Unbarred Spiral Primaries

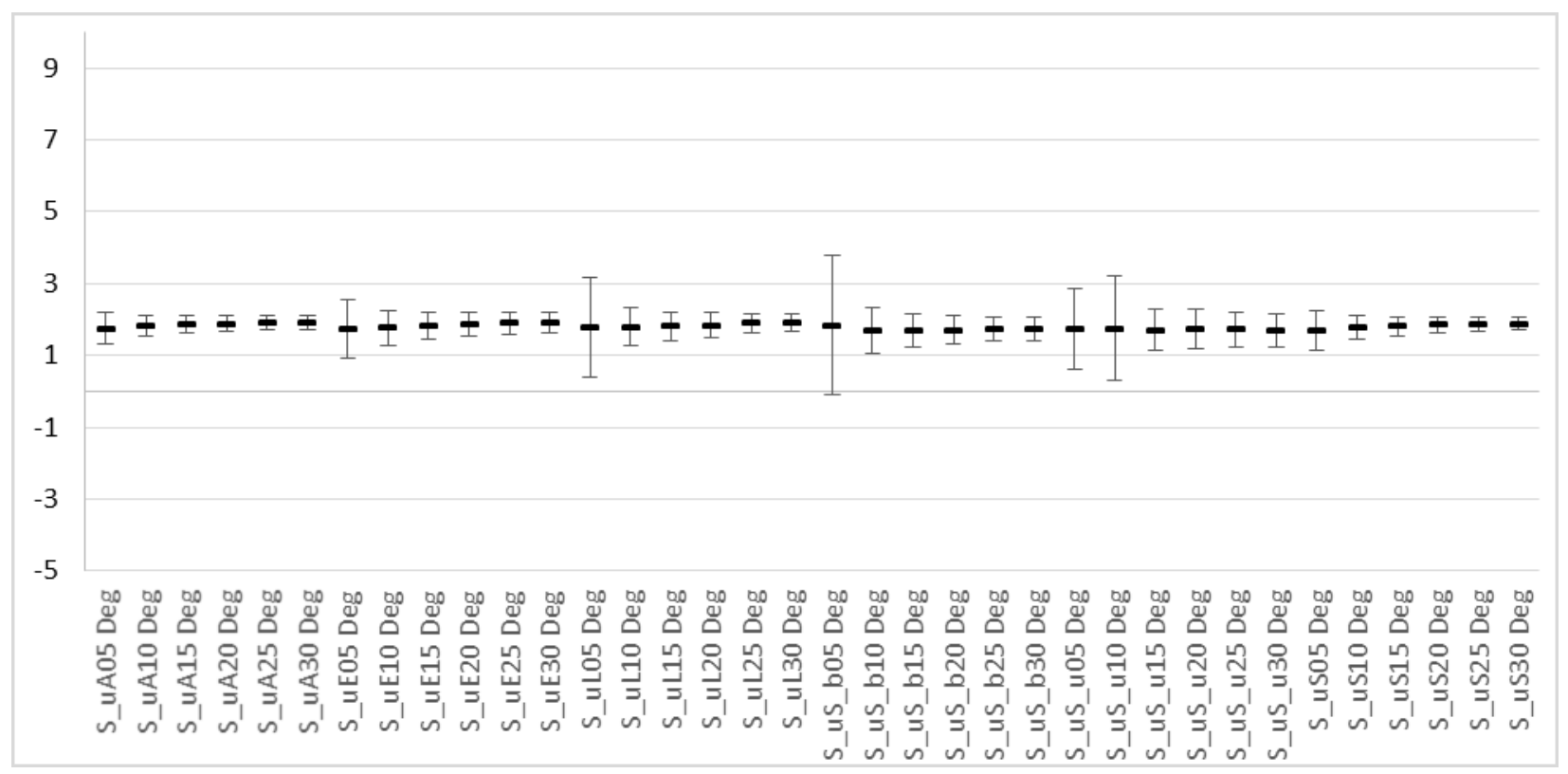




\subsubsection{Impact of Fitting Range on the Pointwise Dimension}

Data runs are created using the same primary and secondary morphological filters at varying fitting ranges. These runs are then analyzed in KS tests.

In all of the following tables $\mathrm{Y}$ indicates that the $p$ value from the $\mathrm{KS}$ test is < 0.01 . This means that there is less than a $1 \%$ chance that the two compared data sets are drawn from the same distribution. That is, the compared data sets are statistically dissimilar to the $99 \%$ level. A value of $\mathrm{N}$ indicates the inverse. That is, the datasets are not statistically dissimilar to the $99 \%$ level. 
When considering all galaxies in Table 5-4 without morphological filtering, fitting range clearly is a factor as all comparisons are statistically dissimilar.

Table 5-4 2D KS Test Results - comparing two runs having the same primary/secondary filters and varying fitting range filters - limited to primaries with no specific morphology.

$\mathrm{Y}=$ dissimilar to the $99 \%$ level. $\mathrm{N}=$ not dissimilar to the $99 \%$ level. FR1 = Fitting Range 1

\begin{tabular}{|c|c|c|c|c|c|c|c|c|c|c|c|c|c|c|c|c|c|c|c|c|c|c|c|}
\hline \multirow[b]{3}{*}{$\begin{array}{c}\text { Run } \\
1\end{array}$} & \multirow[b]{3}{*}{$\begin{array}{c}\mathrm{FR} \\
1\end{array}$} & \multicolumn{10}{|c|}{ North } & \multicolumn{12}{|c|}{ South } \\
\hline & & \multicolumn{10}{|c|}{ Fitting Range 2 (degree) } & \multicolumn{12}{|c|}{ Fitting Range 2 (degree) } \\
\hline & & 1 & 2 & 3 & 4 & 5 & $\begin{array}{l}1 \\
0\end{array}$ & $\begin{array}{l}1 \\
5\end{array}$ & $\begin{array}{l}2 \\
0\end{array}$ & $\begin{array}{l}2 \\
5\end{array}$ & $\begin{array}{l}3 \\
0\end{array}$ & $\begin{array}{c}\text { Run } \\
1\end{array}$ & $\begin{array}{c}\text { FR } \\
1\end{array}$ & 1 & 2 & 3 & 4 & 5 & $\begin{array}{l}1 \\
0\end{array}$ & $\begin{array}{l}1 \\
5\end{array}$ & $\begin{array}{l}2 \\
0\end{array}$ & $\begin{array}{l}2 \\
5\end{array}$ & $\begin{array}{l}3 \\
0\end{array}$ \\
\hline AA & 1 & & $\mathbf{Y}$ & $\mathbf{Y}$ & $\mathbf{Y}$ & $\mathbf{Y}$ & & & & & & $\mathrm{AA}$ & 1 & & $\mathbf{Y}$ & $\mathbf{Y}$ & $\mathbf{Y}$ & $\mathbf{Y}$ & & & & & \\
\hline AA & 2 & & & $\mathbf{Y}$ & $\mathbf{Y}$ & $\mathbf{Y}$ & & & & & & $\mathrm{AA}$ & 2 & & & $\mathbf{Y}$ & $\mathbf{Y}$ & $\mathbf{Y}$ & & & & & \\
\hline AA & 3 & & & & $\mathbf{Y}$ & $\mathbf{Y}$ & & & & & & $A A$ & 3 & & & & $\mathbf{Y}$ & $\mathbf{Y}$ & & & & & \\
\hline AA & 4 & & & & & $\mathbf{Y}$ & & & & & & $\mathrm{AA}$ & 4 & & & & & $\mathbf{Y}$ & & & & & \\
\hline AA & 5 & & & & & & $\mathrm{Y}$ & $Y$ & $\mathrm{Y}$ & $\mathrm{Y}$ & $Y$ & $\mathrm{AA}$ & 5 & & & & & & $Y$ & $Y$ & $Y$ & $Y$ & $\mathrm{Y}$ \\
\hline AA & 10 & & & & & & & $Y$ & $\mathrm{Y}$ & $\mathrm{Y}$ & $\mathrm{Y}$ & $\mathrm{AA}$ & 10 & & & & & & & $Y$ & $Y$ & $Y$ & $Y$ \\
\hline $\mathrm{AA}$ & 15 & & & & & & & & $Y$ & $Y$ & $Y$ & $\mathrm{AA}$ & 15 & & & & & & & & $Y$ & $Y$ & $Y$ \\
\hline $\mathrm{AA}$ & 20 & & & & & & & & & $Y$ & $Y$ & $\mathrm{AA}$ & 20 & & & & & & & & & $Y$ & $Y$ \\
\hline AA & 25 & & & & & & & & & & $Y$ & AA & 25 & & & & & & & & & & $Y$ \\
\hline
\end{tabular}


When ellipticals are considered as primary galaxies in Table 5-5, statistical dissimilarity is found for the vast majority of secondaries and fitting ranges. The major exception is for secondaries of barred and unbarred spiral which are not consistently dissimilar below $5^{\circ}$. This suggests fitting range is less of a factor for secondaries of barred and unbarred spiral around elliptical primaries.

Table 5-5 2D KS Test Results - comparing two runs having the same primary/secondary filters and varying fitting range filters - limited to elliptical primaries.

$\mathrm{Y}=$ dissimilar to the $99 \%$ level. $\mathrm{N}=$ not dissimilar to the $99 \%$ level. FR1 = Fitting Range 1

\begin{tabular}{|c|c|c|c|c|c|c|c|c|c|c|c|c|c|c|c|c|c|c|c|c|c|c|c|}
\hline \multicolumn{12}{|c|}{ North } & \multicolumn{12}{|c|}{ South } \\
\hline \multicolumn{12}{|c|}{ Fitting Range 2 (degree) } & \multicolumn{12}{|c|}{ Fitting Range 2 (degree) } \\
\hline $\begin{array}{c}\text { Run } \\
1\end{array}$ & $\begin{array}{c}\mathrm{FR} \\
1 \\
\end{array}$ & 1 & 2 & 3 & 4 & 5 & $\begin{array}{l}1 \\
0 \\
\end{array}$ & $\begin{array}{l}1 \\
5 \\
\end{array}$ & $\begin{array}{l}2 \\
0 \\
\end{array}$ & $\begin{array}{l}2 \\
5 \\
\end{array}$ & $\begin{array}{l}3 \\
0 \\
\end{array}$ & $\begin{array}{c}\text { Run } \\
1 \\
\end{array}$ & $\begin{array}{c}\text { FR } \\
1 \\
\end{array}$ & 1 & 2 & 3 & 4 & 5 & $\begin{array}{l}1 \\
0 \\
\end{array}$ & $\begin{array}{l}1 \\
5 \\
\end{array}$ & $\begin{array}{l}2 \\
0 \\
\end{array}$ & $\begin{array}{l}2 \\
5 \\
\end{array}$ & $\begin{array}{l}3 \\
0 \\
\end{array}$ \\
\hline EA & 1 & & $\mathrm{Y}$ & $\mathrm{Y}$ & $\mathrm{Y}$ & $\mathrm{Y}$ & & & & & & $\mathrm{EA}$ & 1 & & $Y$ & $Y$ & $\mathrm{Y}$ & $\mathrm{Y}$ & & & & & \\
\hline EA & 2 & & & $\mathbf{Y}$ & $\mathbf{Y}$ & $\mathbf{Y}$ & & & & & & EA & 2 & & & $\mathbf{Y}$ & $\mathbf{Y}$ & $\mathbf{Y}$ & & & & & \\
\hline EA & 3 & & & & $\mathbf{Y}$ & $\mathbf{Y}$ & & & & & & EA & 3 & & & & $\mathbf{Y}$ & $\mathbf{Y}$ & & & & & \\
\hline EA & 4 & & & & & $\mathrm{Y}$ & & & & & & $\mathrm{EA}$ & 4 & & & & & $\mathrm{Y}$ & & & & & \\
\hline EA & 5 & & & & & & $\mathrm{Y}$ & $\mathrm{Y}$ & $\mathrm{Y}$ & $\mathrm{Y}$ & $\mathrm{Y}$ & EA & 5 & & & & & & $\mathrm{Y}$ & $\mathrm{Y}$ & $\mathrm{Y}$ & Y & $\mathrm{Y}$ \\
\hline EA & 10 & & & & & & & $\mathrm{Y}$ & $\mathrm{Y}$ & $\mathrm{Y}$ & $\mathrm{Y}$ & EA & 10 & & & & & & & $\mathrm{Y}$ & $\mathrm{Y}$ & Y & $Y$ \\
\hline EA & 15 & & & & & & & & $\mathrm{Y}$ & $\mathrm{Y}$ & $\mathrm{Y}$ & $\mathrm{EA}$ & 15 & & & & & & & & $\mathrm{Y}$ & $\mathrm{Y}$ & $\bar{Y}$ \\
\hline EA & 20 & & & & & & & & & $\mathrm{Y}$ & $\mathrm{Y}$ & EA & 20 & & & & & & & & & $\mathrm{Y}$ & $\mathrm{Y}$ \\
\hline EA & 25 & & & & & & & & & & $\underline{N}$ & EA & 25 & & & & & & & & & & $Y$ \\
\hline $\mathrm{EE}$ & 1 & & $\underline{N}$ & $Y$ & $Y$ & $\mathrm{Y}$ & & & & & & $\mathrm{EE}$ & 1 & & $\underline{N}$ & $Y$ & Y & $Y$ & & & & & \\
\hline EE & 2 & & & $Y$ & $\mathrm{Y}$ & $Y$ & & & & & & $\mathrm{EE}$ & 2 & & & $Y$ & $\mathrm{Y}$ & $Y$ & & & & & \\
\hline $\mathrm{EE}$ & 3 & & & & $Y$ & $\mathrm{Y}$ & & & & & & $\mathrm{EE}$ & 3 & & & & $Y$ & $\mathrm{Y}$ & & & & & \\
\hline$\overline{E E}$ & 4 & & & & & $\bar{Y}$ & & & & & & $\mathrm{EE}$ & 4 & & & & & $\bar{Y}$ & & & & & \\
\hline $\mathrm{EE}$ & 5 & & & & & & $Y$ & $Y$ & $Y$ & $Y$ & $Y$ & $\mathrm{EE}$ & 5 & & & & & & $Y$ & $Y$ & $Y$ & $Y$ & $Y$ \\
\hline $\mathrm{EE}$ & 10 & & & & & & & $Y$ & $\mathrm{Y}$ & $Y$ & $Y$ & $\mathrm{EE}$ & 10 & & & & & & & $\mathrm{Y}$ & $\mathrm{Y}$ & $Y$ & $\bar{Y}$ \\
\hline $\mathrm{EE}$ & 15 & & & & & & & & $Y$ & $Y$ & $Y$ & $\mathrm{EE}$ & 15 & & & & & & & & $Y$ & $Y$ & $Y$ \\
\hline $\mathrm{EE}$ & 20 & & & & & & & & & $Y$ & $Y$ & $\mathrm{EE}$ & 20 & & & & & & & & & $\underline{N}$ & $Y$ \\
\hline $\mathrm{EE}$ & 25 & & & & & & & & & & $\underline{N}$ & $\mathrm{EE}$ & 25 & & & & & & & & & & $\bar{Y}$ \\
\hline EL & 1 & & $\underline{\mathrm{N}}$ & $\mathrm{Y}$ & $\mathrm{Y}$ & $\mathrm{Y}$ & & & & & & $\mathrm{EL}$ & 1 & & $\underline{\mathrm{N}}$ & $\underline{N}$ & $Y$ & $\mathrm{Y}$ & & & & & \\
\hline EL & 2 & & & $\underline{N}$ & $Y$ & $\bar{Y}$ & & & & & & $\mathrm{EL}$ & 2 & & & $Y$ & $Y$ & $\bar{Y}$ & & & & & \\
\hline$\overline{E L}$ & 3 & & & & $\mathrm{Y}$ & $\bar{Y}$ & & & & & & $\overline{E L}$ & 3 & & & & $\underline{N}$ & $\bar{Y}$ & & & & & \\
\hline $\mathrm{EL}$ & 4 & & & & & $\underline{N}$ & & & & & & $\mathrm{EL}$ & 4 & & & & & $\mathrm{Y}$ & & & & & \\
\hline$\overline{E L}$ & 5 & & & & & & $Y$ & $\mathrm{Y}$ & $Y$ & $Y$ & $Y$ & $\mathrm{EL}$ & 5 & & & & & & $\mathrm{Y}$ & $\mathrm{Y}$ & $\mathrm{Y}$ & $Y$ & $\mathrm{Y}$ \\
\hline$\overline{E L}$ & 10 & & & & & & & $Y$ & $\mathrm{Y}$ & $\mathrm{Y}$ & $\mathrm{Y}$ & $\overline{E L}$ & 10 & & & & & & & $\mathrm{Y}$ & $\mathrm{Y}$ & $\bar{Y}$ & $\bar{Y}$ \\
\hline $\mathrm{EL}$ & 15 & & & & & & & & $Y$ & $Y$ & $\mathrm{Y}$ & $\mathrm{EL}$ & 15 & & & & & & & & $\mathrm{Y}$ & $\mathrm{Y}$ & $Y$ \\
\hline EL & 20 & & & & & & & & & $Y$ & $Y$ & $\mathrm{EL}$ & 20 & & & & & & & & & $\mathrm{Y}$ & $Y$ \\
\hline EL & 25 & & & & & & & & & & $Y$ & $\mathrm{EL}$ & 25 & & & & & & & & & & $\underline{N}$ \\
\hline
\end{tabular}




\begin{tabular}{|c|c|c|c|c|c|c|c|c|c|c|c|c|c|c|c|c|c|c|c|c|c|}
\hline ES & 1 & $\mathbf{Y}$ & $\mathbf{Y}$ & $\mathbf{Y}$ & $\mathbf{Y}$ & & & & & & ES & 1 & $\mathbf{Y}$ & $\mathbf{Y}$ & $\mathbf{Y}$ & $\mathbf{Y}$ & & & & & \\
\hline ES & 2 & & $\mathbf{Y}$ & $\mathbf{Y}$ & $\mathbf{Y}$ & & & & & & ES & 2 & & $\mathbf{Y}$ & $\mathbf{Y}$ & $\mathbf{Y}$ & & & & & \\
\hline ES & 3 & & & $Y$ & $\mathbf{Y}$ & & & & & & ES & 3 & & & $\mathbf{Y}$ & $\mathbf{Y}$ & & & & & \\
\hline ES & 4 & & & & $Y$ & & & & & & ES & 4 & & & & $\mathrm{Y}$ & & & & & \\
\hline ES & 5 & & & & & $\mathbf{Y}$ & $\mathbf{Y}$ & $\mathbf{Y}$ & $\mathbf{Y}$ & $\mathbf{Y}$ & ES & 5 & & & & & $\mathbf{Y}$ & $\mathbf{Y}$ & $\mathbf{Y}$ & $\mathbf{Y}$ & $Y$ \\
\hline ES & 10 & & & & & & $\mathbf{Y}$ & $Y$ & $\mathbf{Y}$ & $\mathbf{Y}$ & ES & 10 & & & & & & $\mathbf{Y}$ & $\mathbf{Y}$ & $Y$ & $Y$ \\
\hline ES & 15 & & & & & & & $\mathrm{Y}$ & $\mathrm{Y}$ & $\mathrm{Y}$ & ES & 15 & & & & & & & $\mathrm{Y}$ & $\mathrm{Y}$ & $\mathrm{Y}$ \\
\hline ES & 20 & & & & & & & & $\mathbf{Y}$ & $\mathbf{Y}$ & ES & 20 & & & & & & & & $\mathbf{Y}$ & $Y$ \\
\hline ES & 25 & & & & & & & & & $\underline{N}$ & ES & 25 & & & & & & & & & $\underline{N}$ \\
\hline $\mathrm{ES}_{\mathrm{b}}$ & 1 & $\underline{N}$ & $\underline{N}$ & $Y$ & $Y$ & & & & & & $\mathrm{ES}_{\mathrm{b}}$ & 1 & $\underline{N}$ & $\underline{N}$ & $\underline{N}$ & $\underline{N}$ & & & & & \\
\hline $\mathrm{ES}_{\mathrm{b}}$ & 2 & & $\underline{N}$ & $\mathrm{Y}$ & $\mathrm{Y}$ & & & & & & $\mathrm{ES}_{\mathrm{b}}$ & 2 & & $\underline{N}$ & $\underline{N}$ & $Y$ & & & & & \\
\hline $\mathrm{ES}_{\mathrm{b}}$ & 3 & & & $\underline{N}$ & $\mathrm{Y}$ & & & & & & $E S_{b}$ & 3 & & & $\underline{N}$ & $\underline{N}$ & & & & & \\
\hline $\mathrm{ES}_{\mathrm{b}}$ & 4 & & & & $\underline{N}$ & & & & & & $\mathrm{ES}_{\mathrm{b}}$ & 4 & & & & $\underline{N}$ & & & & & \\
\hline $\mathrm{ES}_{\mathrm{b}}$ & 5 & & & & & $\mathrm{Y}$ & $\mathrm{Y}$ & $\mathrm{Y}$ & $\mathrm{Y}$ & $\mathrm{Y}$ & $\mathrm{ES}_{\mathrm{b}}$ & 5 & & & & & $\mathrm{Y}$ & $\mathrm{Y}$ & $\mathrm{Y}$ & $\mathrm{Y}$ & $\mathrm{Y}$ \\
\hline $\mathrm{ES}_{\mathrm{b}}$ & 10 & & & & & & $\mathrm{Y}$ & $\mathrm{Y}$ & $\mathrm{Y}$ & $\mathrm{Y}$ & $\mathrm{ES}_{\mathrm{b}}$ & 10 & & & & & & $\mathrm{Y}$ & $\mathrm{Y}$ & $Y$ & $\mathrm{Y}$ \\
\hline $\mathrm{ES}_{\mathrm{b}}$ & 15 & & & & & & & $\mathrm{Y}$ & $\mathrm{Y}$ & $\mathrm{Y}$ & $E S_{b}$ & 15 & & & & & & & $\mathrm{Y}$ & $Y$ & $\mathrm{Y}$ \\
\hline $\mathrm{ES}_{\mathrm{b}}$ & 20 & & & & & & & & $\mathrm{Y}$ & $\mathrm{Y}$ & $E S_{b}$ & 20 & & & & & & & & $\mathrm{Y}$ & $\mathrm{Y}$ \\
\hline $\mathrm{ES}_{\mathrm{b}}$ & 25 & & & & & & & & & $\underline{\mathrm{N}}$ & $\mathrm{ES}_{\mathrm{b}}$ & 25 & & & & & & & & & $\underline{N}$ \\
\hline $\mathrm{ES}_{\mathrm{u}}$ & 1 & $\underline{N}$ & $\underline{\mathrm{N}}$ & $\underline{\mathrm{N}}$ & $\underline{\mathrm{N}}$ & & & & & & $\mathrm{ES}_{\mathrm{u}}$ & 1 & $\underline{\mathrm{N}}$ & $\underline{\mathrm{N}}$ & $\underline{\mathrm{N}}$ & $\underline{\mathrm{N}}$ & & & & & \\
\hline $\mathrm{ES}_{\mathrm{u}}$ & 2 & & $\underline{N}$ & $\mathrm{Y}$ & $\mathrm{Y}$ & & & & & & $\mathrm{ES}_{\mathrm{u}}$ & 2 & & $\underline{N}$ & $\mathrm{Y}$ & $\mathrm{Y}$ & & & & & \\
\hline $\mathrm{ES}_{\mathrm{u}}$ & 3 & & & $\underline{N}$ & $\mathrm{Y}$ & & & & & & $\mathrm{ES}_{\mathrm{u}}$ & 3 & & & $\mathrm{Y}$ & $\mathrm{Y}$ & & & & & \\
\hline $\mathrm{ES}_{\mathrm{u}}$ & 4 & & & & $\underline{\mathrm{N}}$ & & & & & & $\mathrm{ES}_{\mathrm{u}}$ & 4 & & & & $\underline{\mathrm{N}}$ & & & & & \\
\hline $\mathrm{ES}_{\mathrm{u}}$ & 5 & & & & & $Y$ & $\mathrm{Y}$ & $Y$ & $Y$ & $Y$ & $\mathrm{ES}_{\mathrm{u}}$ & 5 & & & & & $\mathrm{Y}$ & $\mathrm{Y}$ & $Y$ & $Y$ & $\mathrm{Y}$ \\
\hline $\mathrm{ES}_{\mathrm{u}}$ & 10 & & & & & & $\mathrm{Y}$ & $Y$ & $\mathrm{Y}$ & $\mathrm{Y}$ & $\mathrm{ES}_{\mathrm{u}}$ & 10 & & & & & & $\mathrm{Y}$ & $\mathrm{Y}$ & $\mathrm{Y}$ & $Y$ \\
\hline $\mathrm{ES}_{\mathrm{u}}$ & 15 & & & & & & & $\mathrm{Y}$ & $\mathrm{Y}$ & $\mathrm{Y}$ & $\mathrm{ES}_{\mathrm{u}}$ & 15 & & & & & & & $\mathrm{Y}$ & $\mathrm{Y}$ & $\mathrm{Y}$ \\
\hline $\mathrm{ES}_{\mathrm{u}}$ & 20 & & & & & & & & $\mathrm{Y}$ & $\mathrm{Y}$ & $\mathrm{ES}_{\mathrm{u}}$ & 20 & & & & & & & & $\underline{N}$ & $\underline{N}$ \\
\hline $\mathrm{ES}_{\mathrm{u}}$ & 25 & & & & & & & & & $\underline{\mathrm{N}}$ & $\mathrm{ES}_{\mathrm{u}}$ & 25 & & & & & & & & & $\underline{N}$ \\
\hline
\end{tabular}


When considering lenticulars as primary galaxies in Table 5-6, and secondaries without morphological filtering, dissimilarity is found for almost all comparisons. When considering $L$ primaries with $S$ as secondary nearly all comparisons are dissimilar. When considering $L$ primaries and secondaries other than $S$ there are some instances of similarity at $5^{\circ}$ but very few above $5^{\circ}$. As seen above for $E$ primaries, secondaries of $\mathrm{S}_{\mathrm{b}}$ and $\mathrm{S}_{\mathrm{u}}$ show very little dissimilarity below $5^{\circ}$ but are consistently dissimilar above $5^{\circ}$. This suggests that the clustering of $S_{b}$ and $S_{u}$ around $L$ galaxies is impacted less by the fitting range. Also interesting, the clustering of late-type galaxies around $L$ show more instances of similarity below $5^{\circ}$ than was noted for $\mathrm{E}$ primaries.

Table 5-6 2D KS Test Results - comparing two runs having the same primary/secondary filters and varying fitting range filters - limited to lenticular primaries.

$\mathrm{Y}=$ dissimilar to the $99 \%$ level. $\mathrm{N}=$ not dissimilar to the $99 \%$ level. FR1 = Fitting Range 1

\begin{tabular}{|c|c|c|c|c|c|c|c|c|c|c|c|c|c|c|c|c|c|c|c|c|c|c|c|}
\hline \multicolumn{12}{|c|}{ North } & \multicolumn{12}{|c|}{ South } \\
\hline \multirow[b]{2}{*}{$\begin{array}{c}\text { Run } \\
1 \\
\end{array}$} & \multirow[b]{2}{*}{$\begin{array}{c}\mathrm{FR} \\
1 \\
\end{array}$} & \multicolumn{10}{|c|}{ Fitting Range 2 (degree) } & \multirow[b]{2}{*}{$\begin{array}{c}\text { Run } \\
1 \\
\end{array}$} & \multirow[b]{2}{*}{$\begin{array}{c}\text { FR } \\
1 \\
\end{array}$} & \multicolumn{10}{|c|}{ Fitting Range 2 (degree) } \\
\hline & & 1 & 2 & 3 & 4 & 5 & $\begin{array}{l}1 \\
0 \\
\end{array}$ & $\begin{array}{l}1 \\
5 \\
\end{array}$ & $\begin{array}{l}2 \\
0 \\
\end{array}$ & $\begin{array}{l}2 \\
5 \\
\end{array}$ & $\begin{array}{l}3 \\
0 \\
\end{array}$ & & & 1 & 2 & 3 & 4 & 5 & $\begin{array}{l}1 \\
0 \\
\end{array}$ & $\begin{array}{l}1 \\
5 \\
\end{array}$ & $\begin{array}{l}2 \\
0 \\
\end{array}$ & $\begin{array}{l}2 \\
5 \\
\end{array}$ & $\begin{array}{l}3 \\
0 \\
\end{array}$ \\
\hline LA & 1 & & $\mathbf{Y}$ & $\mathbf{Y}$ & $\mathbf{Y}$ & $\mathbf{Y}$ & & & & & & LA & 1 & & $\mathbf{Y}$ & $\mathbf{Y}$ & $\mathbf{Y}$ & $\mathbf{Y}$ & & & & & \\
\hline LA & 2 & & & $\mathbf{Y}$ & $\mathbf{Y}$ & $\mathbf{Y}$ & & & & & & LA & 2 & & & $\mathbf{Y}$ & $\mathbf{Y}$ & $\mathbf{Y}$ & & & & & \\
\hline LA & 3 & & & & $\mathbf{Y}$ & $\mathbf{Y}$ & & & & & & LA & 3 & & & & $\mathbf{Y}$ & $\mathbf{Y}$ & & & & & \\
\hline LA & 4 & & & & & $\mathbf{Y}$ & & & & & & LA & 4 & & & & & $\mathbf{Y}$ & & & & & \\
\hline LA & 5 & & & & & & $\mathbf{Y}$ & $\mathbf{Y}$ & $\mathbf{Y}$ & $\mathbf{Y}$ & $\mathbf{Y}$ & LA & 5 & & & & & & $\mathbf{Y}$ & $\mathbf{Y}$ & $\mathbf{Y}$ & $\mathbf{Y}$ & $\mathbf{Y}$ \\
\hline LA & 10 & & & & & & & $\mathbf{Y}$ & $\mathbf{Y}$ & $\mathbf{Y}$ & $\mathbf{Y}$ & LA & 10 & & & & & & & $\mathbf{Y}$ & $\mathbf{Y}$ & $\mathbf{Y}$ & $Y$ \\
\hline LA & 15 & & & & & & & & $\mathbf{Y}$ & $\mathbf{Y}$ & $\mathbf{Y}$ & LA & 15 & & & & & & & & $\mathbf{Y}$ & $\mathbf{Y}$ & $Y$ \\
\hline LA & 20 & & & & & & & & & $\underline{\mathrm{N}}$ & $\mathbf{Y}$ & LA & 20 & & & & & & & & & $\mathbf{Y}$ & $Y$ \\
\hline LA & 25 & & & & & & & & & & $\underline{\mathrm{N}}$ & LA & 25 & & & & & & & & & & $\mathbf{Y}$ \\
\hline LE & 1 & & $\underline{\mathrm{N}}$ & $\underline{N}$ & $\mathbf{Y}$ & $\mathbf{Y}$ & & & & & & LE & 1 & & $\underline{\mathrm{N}}$ & $\underline{\mathrm{N}}$ & $\mathbf{Y}$ & $\mathbf{Y}$ & & & & & \\
\hline LE & 2 & & & $\underline{N}$ & $\mathrm{Y}$ & $\mathrm{Y}$ & & & & & & LE & 2 & & & $\underline{\mathrm{N}}$ & $\mathrm{Y}$ & $\mathrm{Y}$ & & & & & \\
\hline LE & 3 & & & & $\underline{\mathrm{N}}$ & $\mathbf{Y}$ & & & & & & LE & 3 & & & & $\underline{\mathrm{N}}$ & $\mathbf{Y}$ & & & & & \\
\hline LE & 4 & & & & & $\underline{\mathrm{N}}$ & & & & & & LE & 4 & & & & & $\mathrm{Y}$ & & & & & \\
\hline LE & 5 & & & & & & $\mathbf{Y}$ & $\mathbf{Y}$ & $\mathbf{Y}$ & $\mathbf{Y}$ & $\mathbf{Y}$ & LE & 5 & & & & & & $\mathbf{Y}$ & $\mathbf{Y}$ & $\mathbf{Y}$ & $\mathbf{Y}$ & $\mathbf{Y}$ \\
\hline LE & 10 & & & & & & & $\mathbf{Y}$ & $\mathbf{Y}$ & $\mathbf{Y}$ & $\mathbf{Y}$ & LE & 10 & & & & & & & $\mathbf{Y}$ & $\mathbf{Y}$ & $\mathbf{Y}$ & $Y$ \\
\hline LE & 15 & & & & & & & & $\mathbf{Y}$ & $\mathbf{Y}$ & $\mathbf{Y}$ & LE & 15 & & & & & & & & $\mathbf{Y}$ & $\mathbf{Y}$ & $\mathbf{Y}$ \\
\hline LE & 20 & & & & & & & & & $Y$ & $Y$ & LE & 20 & & & & & & & & & $\mathrm{Y}$ & $Y$ \\
\hline LE & 25 & & & & & & & & & & $\underline{N}$ & LE & 25 & & & & & & & & & & $\underline{N}$ \\
\hline LL & 1 & & $\underline{\mathrm{N}}$ & $\underline{N}$ & $\underline{\mathrm{N}}$ & $\mathrm{Y}$ & & & & & & LL & 1 & & $\underline{\mathrm{N}}$ & $\underline{\mathrm{N}}$ & $\mathrm{Y}$ & $Y$ & & & & & \\
\hline LL & 2 & & & $\underline{N}$ & $\mathrm{Y}$ & $\mathrm{Y}$ & & & & & & LL & 2 & & & $\underline{\mathrm{N}}$ & $Y$ & $Y$ & & & & & \\
\hline LL & 3 & & & & $\underline{N}$ & $\mathbf{Y}$ & & & & & & LL & 3 & & & & $\underline{N}$ & $\mathbf{Y}$ & & & & & \\
\hline
\end{tabular}




\begin{tabular}{|c|c|c|c|c|c|c|c|c|c|c|c|c|c|c|c|c|c|c|c|c|}
\hline LL & 4 & & & & $\underline{N}$ & & & & & $\mathrm{LL}$ & 4 & & & & $\mathrm{Y}$ & & & & & \\
\hline LL & 5 & & & & $\mathbf{Y}$ & $\mathbf{Y}$ & $\mathrm{Y}$ & $\mathbf{Y}$ & $\mathbf{Y}$ & $\mathrm{LL}$ & 5 & & & & & $Y$ & $Y$ & $Y$ & $\mathbf{Y}$ & $Y$ \\
\hline LL & 10 & & & & & $Y$ & $Y$ & $Y$ & $Y$ & $\mathrm{LL}$ & 10 & & & & & & $Y$ & $Y$ & $Y$ & $Y$ \\
\hline LL & 15 & & & & & & $\mathbf{Y}$ & $Y$ & $Y$ & LL & 15 & & & & & & & $Y$ & $Y$ & $Y$ \\
\hline LL & 20 & & & & & & & $Y$ & $Y$ & LL & 20 & & & & & & & & $Y$ & $Y$ \\
\hline LL & 25 & & & & & & & & $\underline{\mathrm{N}}$ & LL & 25 & & & & & & & & & $\underline{\mathrm{N}}$ \\
\hline LS & 1 & $Y$ & $Y$ & $Y$ & $Y$ & & & & & LS & 1 & $Y$ & $\mathrm{Y}$ & $Y$ & $Y$ & & & & & \\
\hline LS & 2 & & $\mathrm{Y}$ & $Y$ & $Y$ & & & & & LS & 2 & & $\mathrm{Y}$ & $Y$ & $Y$ & & & & & \\
\hline LS & 3 & & & $Y$ & $\mathbf{Y}$ & & & & & LS & 3 & & & $\mathbf{Y}$ & $Y$ & & & & & \\
\hline LS & 4 & & & & $\mathbf{Y}$ & & & & & LS & 4 & & & & $Y$ & & & & & \\
\hline LS & 5 & & & & $Y$ & $Y$ & $\mathrm{Y}$ & $Y$ & $Y$ & LS & 5 & & & & & $Y$ & $Y$ & $Y$ & $Y$ & $Y$ \\
\hline LS & 10 & & & & & $\mathbf{Y}$ & $\mathrm{Y}$ & $\mathbf{Y}$ & $\mathbf{Y}$ & LS & 10 & & & & & & $Y$ & $Y$ & $\mathbf{Y}$ & $\mathbf{Y}$ \\
\hline LS & 15 & & & & & & $\mathbf{Y}$ & $Y$ & $\mathbf{Y}$ & LS & 15 & & & & & & & $Y$ & $Y$ & $Y$ \\
\hline LS & 20 & & & & & & & $Y$ & $Y$ & LS & 20 & & & & & & & & $\mathrm{Y}$ & $Y$ \\
\hline LS & 25 & & & & & & & & $\underline{\mathrm{N}}$ & LS & 25 & & & & & & & & & $Y$ \\
\hline $\mathrm{LS}_{\mathrm{b}}$ & 1 & $\underline{N}$ & $\underline{N}$ & $\mathbf{Y}$ & $\mathbf{Y}$ & & & & & $\mathrm{LS}_{\mathrm{b}}$ & 1 & $\underline{N}$ & $\underline{\mathrm{N}}$ & $\underline{N}$ & $\underline{N}$ & & & & & \\
\hline $\mathrm{LS}_{\mathrm{b}}$ & 2 & & $\underline{N}$ & $\underline{N}$ & $\mathrm{Y}$ & & & & & $\mathrm{LS}_{\mathrm{b}}$ & 2 & & $\underline{N}$ & $\underline{N}$ & $\underline{N}$ & & & & & \\
\hline $\mathrm{LS}_{\mathrm{b}}$ & 3 & & & $\underline{\mathrm{N}}$ & $Y$ & & & & & $\mathrm{LS}_{\mathrm{b}}$ & 3 & & & $\underline{N}$ & $\underline{\mathrm{N}}$ & & & & & \\
\hline $\mathrm{LS}_{\mathrm{b}}$ & 4 & & & & $\underline{N}$ & & & & & $\mathrm{LS}_{\mathrm{b}}$ & 4 & & & & $\underline{N}$ & & & & & \\
\hline $\mathrm{LS}_{\mathrm{b}}$ & 5 & & & & $Y$ & $Y$ & $\mathrm{Y}$ & $Y$ & $Y$ & $\mathrm{LS}_{\mathrm{b}}$ & 5 & & & & & $Y$ & $Y$ & $Y$ & $\mathbf{Y}$ & $\mathbf{Y}$ \\
\hline $\mathrm{LS}_{\mathrm{b}}$ & 10 & & & & & $Y$ & $\mathrm{Y}$ & $Y$ & $Y$ & $\mathrm{LS}_{\mathrm{b}}$ & 10 & & & & & & $Y$ & $Y$ & $Y$ & $Y$ \\
\hline $\mathrm{LS}_{\mathrm{b}}$ & 15 & & & & & & $\mathrm{Y}$ & $Y$ & $Y$ & $\mathrm{LS}_{\mathrm{b}}$ & 15 & & & & & & & $Y$ & $Y$ & $Y$ \\
\hline $\mathrm{LS}_{\mathrm{b}}$ & 20 & & & & & & & $\bar{Y}$ & $\mathrm{Y}$ & $\mathrm{LS}_{\mathrm{b}}$ & 20 & & & & & & & & $\mathrm{Y}$ & $\bar{Y}$ \\
\hline $\mathrm{LS}_{\mathrm{b}}$ & 25 & & & & & & & & $\underline{N}$ & $\mathrm{LS}_{\mathrm{b}}$ & 25 & & & & & & & & & $\underline{N}$ \\
\hline $\mathrm{LS}_{\mathrm{u}}$ & 1 & $\underline{N}$ & $\underline{N}$ & $\underline{N}$ & $\underline{N}$ & & & & & $\mathrm{LS}_{\mathrm{u}}$ & 1 & $\underline{N}$ & $\underline{N}$ & $\underline{N}$ & $\underline{N}$ & & & & & \\
\hline $\mathrm{LS}_{\mathrm{u}}$ & 2 & & $\underline{\mathrm{N}}$ & $\underline{\mathrm{N}}$ & $\underline{N}$ & & & & & $\mathrm{LS}_{\mathrm{u}}$ & 2 & . & $\underline{N}$ & $\mathrm{Y}$ & $\mathrm{Y}$ & & & & & \\
\hline $\mathrm{LS}_{\mathrm{u}}$ & 3 & & & $\underline{N}$ & $\underline{N}$ & & & & & $\mathrm{LS}_{\mathrm{u}}$ & 3 & & . & $\underline{N}$ & $Y$ & & & & & \\
\hline $\mathrm{LS}_{\mathrm{u}}$ & 4 & & & & $\underline{\mathrm{N}}$ & & & & & $\mathrm{LS}_{\mathrm{u}}$ & 4 & & & & $\underline{\mathrm{N}}$ & & & & & \\
\hline $\mathrm{LS}_{\mathrm{u}}$ & 5 & & & & $Y$ & $\mathrm{Y}$ & $\mathrm{Y}$ & $\mathrm{Y}$ & $\mathrm{Y}$ & $\mathrm{LS}_{\mathrm{u}}$ & 5 & & & & & $Y$ & $Y$ & $Y$ & $\mathrm{Y}$ & $Y$ \\
\hline $\mathrm{LS}_{\mathrm{u}}$ & 10 & & & & & $Y$ & $\mathrm{Y}$ & $\mathrm{Y}$ & $Y$ & $\mathrm{LS}_{\mathrm{u}}$ & 10 & & & & & & $Y$ & $Y$ & $Y$ & $Y$ \\
\hline $\mathrm{LS}_{\mathrm{u}}$ & 15 & & & & & & $\mathrm{Y}$ & $Y$ & $\mathrm{Y}$ & $\mathrm{LS}_{\mathrm{u}}$ & 15 & & & & & & & $\bar{Y}$ & $Y$ & $\bar{Y}$ \\
\hline $\mathrm{LS}_{\mathrm{u}}$ & 20 & & & & & & & $Y$ & $Y$ & $\mathrm{LS}_{\mathrm{u}}$ & 20 & & & & & & & & $Y$ & $Y$ \\
\hline $\mathrm{LS}_{\mathrm{u}}$ & 25 & & & & & & & & $\underline{N}$ & $\mathrm{LS}_{\mathrm{u}}$ & 25 & & & & & & & & & $\underline{N}$ \\
\hline
\end{tabular}


When considering spirals as primary and barred or unbarred spirals as secondary in Table 5-7, the trend of similarity at fitting ranges below $5^{\circ}$ and dissimilarity above $5^{\circ}$ continues as seen above for elliptical and lenticular primaries. This continues to suggest that the fitting range has a lesser impact on the clustering of $S_{b}$ and $S_{u}$. For spiral primaries, above $5^{\circ}$ dissimilarity is consistently found for all but a very few comparisons. At below $5^{\circ}$ dissimilarity is found consistently for secondary morphologies other than barred or unbarred spirals. Finally for secondaries of lenticular and elliptical at small scales below $5^{\circ}$ a few instances of similarity are found for comparisons of $1^{\circ}$ fitting range to fitting ranges one or two degrees larger.

Table 5-7 2D KS Test Results - comparing two runs having the same primary/secondary filters and varying fitting range filters - limited to spiral primaries.

$\mathrm{Y}=$ dissimilar to the $99 \%$ level. $\mathrm{N}=$ not dissimilar to the $99 \%$ level. FR1 = Fitting Range 1

\begin{tabular}{|c|c|c|c|c|c|c|c|c|c|c|c|c|c|c|c|c|c|c|c|c|c|c|c|}
\hline \multicolumn{12}{|c|}{ North } & \multicolumn{12}{|c|}{ South } \\
\hline \multirow[b]{2}{*}{$\begin{array}{c}\text { Run } \\
1\end{array}$} & \multicolumn{11}{|c|}{ Fitting Range 2 (degree) } & \multicolumn{12}{|c|}{ Fitting Range 2 (degree) } \\
\hline & $\begin{array}{c}\mathrm{FR} \\
1 \\
\end{array}$ & 1 & 2 & 3 & 4 & 5 & $\begin{array}{l}1 \\
0 \\
\end{array}$ & $\begin{array}{l}1 \\
5 \\
\end{array}$ & $\begin{array}{l}2 \\
0 \\
\end{array}$ & $\begin{array}{l}2 \\
5 \\
\end{array}$ & $\begin{array}{l}3 \\
0 \\
\end{array}$ & $\begin{array}{c}\text { Run } \\
1 \\
\end{array}$ & $\begin{array}{c}\text { FR } \\
1 \\
\end{array}$ & 1 & 2 & 3 & 4 & 5 & $\begin{array}{l}1 \\
0 \\
\end{array}$ & $\begin{array}{l}1 \\
5 \\
\end{array}$ & $\begin{array}{l}2 \\
0 \\
\end{array}$ & $\begin{array}{l}2 \\
5 \\
\end{array}$ & $\begin{array}{l}3 \\
0 \\
\end{array}$ \\
\hline SA & 1 & & $\mathbf{Y}$ & $\mathbf{Y}$ & $\mathbf{Y}$ & $\mathbf{Y}$ & & & & & & SA & 1 & & $\mathrm{Y}$ & $\mathrm{Y}$ & $\mathbf{Y}$ & $\mathbf{Y}$ & & & & & \\
\hline SA & 2 & & & $\mathrm{Y}$ & $Y$ & $Y$ & & & & & & SA & 2 & & & $Y$ & $Y$ & Y & & & & & \\
\hline SA & 3 & & & & $\mathrm{Y}$ & $\mathbf{Y}$ & & & & & & SA & 3 & & & & $\mathbf{Y}$ & $\mathbf{Y}$ & & & & & \\
\hline SA & 4 & & & & & $\mathrm{Y}$ & & & & & & SA & 4 & & & & & Y & & & & & \\
\hline SA & 5 & & & & & & $\mathbf{Y}$ & $\mathrm{Y}$ & $\mathrm{Y}$ & $\mathrm{Y}$ & $\mathbf{Y}$ & SA & 5 & & & & & & $\mathbf{Y}$ & $\mathrm{Y}$ & $\mathrm{Y}$ & $\mathbf{Y}$ & $\mathbf{Y}$ \\
\hline SA & 10 & & & & & & & $\mathbf{Y}$ & $\mathbf{Y}$ & $\mathbf{Y}$ & $\mathbf{Y}$ & SA & 10 & & & & & & & $\mathbf{Y}$ & $\mathbf{Y}$ & $\mathrm{Y}$ & $\mathbf{Y}$ \\
\hline SA & 15 & & & & & & & & $Y$ & $Y$ & $\bar{Y}$ & SA & 15 & & & & & & & & $Y$ & $Y$ & $\bar{Y}$ \\
\hline SA & 20 & & & & & & & & & $Y$ & $\mathrm{Y}$ & SA & 20 & & & & & & & & & $Y$ & $Y$ \\
\hline SA & 25 & & & & & & & & & & $\underline{N}$ & SA & 25 & & & & & & & & & & $\mathrm{Y}$ \\
\hline SE & 1 & & $\underline{N}$ & $\mathrm{Y}$ & $\mathbf{Y}$ & $\mathbf{Y}$ & & & & & & SE & 1 & & $\underline{\mathrm{N}}$ & $\underline{\mathrm{N}}$ & $\mathbf{Y}$ & $\mathbf{Y}$ & & & & & \\
\hline SE & 2 & & & $\mathrm{Y}$ & $\mathrm{Y}$ & $\mathbf{Y}$ & & & & & & SE & 2 & & & $\underline{\mathrm{N}}$ & $\mathbf{Y}$ & Y & & & & & \\
\hline SE & 3 & & & & $\mathrm{Y}$ & $\mathrm{Y}$ & & & & & & SE & 3 & & & & $\mathrm{Y}$ & Y & & & & & \\
\hline SE & 4 & & & & & $\mathbf{Y}$ & & & & & & SE & 4 & & & & & $\mathbf{Y}$ & & & & & \\
\hline SE & 5 & & & & & & $\mathrm{Y}$ & $Y$ & $Y$ & $Y$ & $\bar{Y}$ & SE & 5 & & & & & & $\mathrm{Y}$ & $Y$ & $Y$ & $\bar{Y}$ & $\bar{Y}$ \\
\hline SE & 10 & & & & & & & $\mathrm{Y}$ & $\mathrm{Y}$ & $\mathrm{Y}$ & $\mathrm{Y}$ & SE & 10 & & & & & & & $\mathrm{Y}$ & $\mathrm{Y}$ & $\mathrm{Y}$ & $\mathrm{Y}$ \\
\hline SE & 15 & & & & & & & & $Y$ & $Y$ & $Y$ & SE & 15 & & & & & & & & $Y$ & $Y$ & $\bar{Y}$ \\
\hline SE & 20 & & & & & & & & & $Y$ & $\bar{Y}$ & SE & 20 & & & & & & & & & $Y$ & $\bar{Y}$ \\
\hline SE & 25 & & & & & & & & & & $\mathrm{Y}$ & SE & 25 & & & & & & & & & & $Y$ \\
\hline SL & 1 & & $\underline{N}$ & $\underline{N}$ & $Y$ & $Y$ & & & & & & SL & 1 & & $\underline{N}$ & $\underline{N}$ & $Y$ & Y & & & & & \\
\hline$S L$ & 2 & & & $\underline{N}$ & $Y$ & $Y$ & & & & & & $S L$ & 2 & & & $Y$ & $Y$ & $Y$ & & & & & \\
\hline$\overline{S L}$ & 3 & & & & $\mathrm{Y}$ & $\bar{Y}$ & & & & & & $\mathrm{SL}$ & 3 & & & & $Y$ & $Y$ & & & & & \\
\hline SL & 4 & & & & & $\mathrm{Y}$ & & & & & & $\mathrm{SL}$ & 4 & & & & & Y & & & & & \\
\hline
\end{tabular}




\begin{tabular}{|c|c|c|c|c|c|c|c|c|c|c|c|c|c|c|c|c|c|c|c|c|}
\hline SL & 5 & & & & $\mathrm{Y}$ & $\mathrm{Y}$ & $\mathrm{Y}$ & $\mathrm{Y}$ & $Y$ & SL & 5 & & & & & $\mathrm{Y}$ & $\mathrm{Y}$ & $\mathrm{Y}$ & $\mathrm{Y}$ & $Y$ \\
\hline SL & 10 & & & & & $\mathbf{Y}$ & $Y$ & $Y$ & $\mathbf{Y}$ & SL & 10 & & & & & & $\mathbf{Y}$ & $\mathbf{Y}$ & $\mathbf{Y}$ & $\mathbf{Y}$ \\
\hline SL & 15 & & & & & & $\mathbf{Y}$ & $\mathbf{Y}$ & $\mathbf{Y}$ & SL & 15 & & & & & & & $\mathbf{Y}$ & $\mathbf{Y}$ & $Y$ \\
\hline SL & 20 & & & & & & & $Y$ & $Y$ & SL & 20 & & & & & & & & $Y$ & $Y$ \\
\hline SL & 25 & & & & & & & & $Y$ & SL & 25 & & & & & & & & & $Y$ \\
\hline SS & 1 & $\mathbf{Y}$ & $\mathbf{Y}$ & $Y \quad Y$ & $\mathbf{Y}$ & & & & & SS & 1 & $\mathbf{Y}$ & $\mathbf{Y}$ & $\mathbf{Y}$ & $\mathbf{Y}$ & & & & & \\
\hline SS & 2 & & $\mathrm{Y}$ & $Y \quad Y$ & Y & & & & & SS & 2 & & $\mathrm{Y}$ & $\mathrm{Y}$ & $\mathrm{Y}$ & & & & & \\
\hline SS & 3 & & & $Y \quad Y$ & $Y$ & & & & & SS & 3 & & & $\mathbf{Y}$ & $\mathbf{Y}$ & & & & & \\
\hline SS & 4 & & & $Y$ & $Y$ & & & & & SS & 4 & & & & $Y$ & & & & & \\
\hline SS & 5 & & & & $Y$ & $Y$ & $Y$ & $Y$ & $Y$ & SS & 5 & & & & & $Y$ & $Y$ & $Y$ & $Y$ & $Y$ \\
\hline SS & 10 & & & & & $\mathbf{Y}$ & $Y$ & $Y$ & $\mathbf{Y}$ & SS & 10 & & & & & & $\mathbf{Y}$ & $Y$ & $Y$ & $\mathbf{Y}$ \\
\hline SS & 15 & & & & & & $\mathrm{Y}$ & $\mathrm{Y}$ & $Y$ & SS & 15 & & & & & & & $\mathrm{Y}$ & $\mathrm{Y}$ & $Y$ \\
\hline SS & 20 & & & & & & & $Y$ & $Y$ & SS & 20 & & & & & & & & $Y$ & $\mathrm{Y}$ \\
\hline SS & 25 & & & & & & & & $Y$ & SS & 25 & & & & & & & & & $Y$ \\
\hline $\mathrm{SS}_{\mathrm{b}}$ & 1 & $\underline{N}$ & $\underline{N}$ & $\underline{N}$ & $\underline{N}$ & & & & & $\mathrm{SS}_{\mathrm{b}}$ & 1 & $\underline{N}$ & $\underline{\mathrm{N}}$ & $\underline{N}$ & $\underline{N}$ & & & & & \\
\hline $\mathrm{SS}_{\mathrm{b}}$ & 2 & & $\underline{N}$ & $Y \quad Y$ & Y & & & & & $\mathrm{SS}_{\mathrm{b}}$ & 2 & & $\underline{\mathrm{N}}$ & $\underline{\mathrm{N}}$ & $\underline{N}$ & & & & & \\
\hline $\mathrm{SS}_{\mathrm{b}}$ & 3 & & 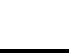 & $\underline{N} \quad Y$ & Y & & & & & $\mathrm{SS}_{\mathrm{b}}$ & 3 & & & $\underline{N}$ & $\underline{N}$ & & & & & \\
\hline $\mathrm{SS}_{\mathrm{b}}$ & 4 & & & $Y$ & $Y$ & & & & & $\mathrm{SS}_{\mathrm{b}}$ & 4 & & & & $\underline{N}$ & & & & & \\
\hline $\mathrm{SS}_{\mathrm{b}}$ & 5 & & & & $\mathrm{Y}$ & $\mathrm{Y}$ & $\mathrm{Y}$ & $\mathrm{Y}$ & $\mathrm{Y}$ & $\mathrm{SS}_{\mathrm{b}}$ & 5 & & & & & $\mathrm{Y}$ & $\mathrm{Y}$ & $\mathrm{Y}$ & $\mathrm{Y}$ & $Y$ \\
\hline $\mathrm{SS}_{\mathrm{b}}$ & 10 & & & & & $\mathrm{Y}$ & $\mathrm{Y}$ & $\mathrm{Y}$ & $\mathrm{Y}$ & $\mathrm{SS}_{\mathrm{b}}$ & 10 & & & & & & $\mathrm{Y}$ & $\mathrm{Y}$ & $\mathrm{Y}$ & $\mathrm{Y}$ \\
\hline $\mathrm{SS}_{\mathrm{b}}$ & 15 & & & & & & $Y$ & $Y$ & $Y$ & $\mathrm{SS}_{\mathrm{b}}$ & 15 & & & & & & & $Y$ & $Y$ & $Y$ \\
\hline $\mathrm{SS}_{\mathrm{b}}$ & 20 & & & & & & & $\mathrm{Y}$ & $\mathrm{Y}$ & $\mathrm{SS}_{\mathrm{b}}$ & 20 & & & & & & & & $\mathrm{Y}$ & $\mathrm{Y}$ \\
\hline $\mathrm{SS}_{\mathrm{b}}$ & 25 & & & & & & & & $Y$ & $\mathrm{SS}_{\mathrm{b}}$ & 25 & & & & & & & & & $Y$ \\
\hline $\mathrm{SS}_{\mathrm{u}}$ & 1 & $\underline{N}$ & $\underline{N}$ & $\underline{N}$ & $Y$ & & & & & $\mathrm{SS}_{\mathrm{u}}$ & 1 & $\underline{N}$ & $\mathrm{Y}$ & $\mathrm{Y}$ & $\mathrm{Y}$ & & & & & \\
\hline $\mathrm{SS}_{\mathrm{u}}$ & 2 & 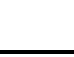 & $\underline{\mathrm{N}}$ & $\begin{array}{l}Y \quad Y \\
\end{array}$ & Y & & & & & $\mathrm{SS}_{\mathrm{u}}$ & 2 & . & $\underline{\mathrm{N}}$ & $\mathrm{Y}$ & $Y$ & & & & & \\
\hline $\mathrm{SS}_{\mathrm{u}}$ & 3 & & & $\underline{N} \quad Y$ & Y & & & & & $\mathrm{SS}_{\mathrm{u}}$ & 3 & & & $\underline{\mathrm{N}}$ & $Y$ & & & & & \\
\hline $\mathrm{SS}_{\mathrm{u}}$ & 4 & & & $Y$ & Y & & & & & $\mathrm{SS}_{\mathrm{u}}$ & 4 & & & & $\underline{N}$ & & & & & \\
\hline $\mathrm{SS}_{\mathrm{u}}$ & 5 & & & & $Y$ & $\mathrm{Y}$ & $\mathrm{Y}$ & $Y$ & $Y$ & $\mathrm{SS}_{\mathrm{u}}$ & 5 & & & & & $Y$ & $\mathrm{Y}$ & $\mathrm{Y}$ & $\mathrm{Y}$ & $\mathrm{Y}$ \\
\hline $\mathrm{SS}_{\mathrm{u}}$ & 10 & & & & & $\mathrm{Y}$ & $\mathrm{Y}$ & $\mathrm{Y}$ & $\mathrm{Y}$ & $\mathrm{SS}_{\mathrm{u}}$ & 10 & & & & & & $\mathrm{Y}$ & $\mathrm{Y}$ & $\mathrm{Y}$ & $\mathrm{Y}$ \\
\hline $\mathrm{SS}_{\mathrm{u}}$ & 15 & & & & & & $\mathrm{Y}$ & $\mathbf{Y}$ & $\mathbf{Y}$ & $\mathrm{SS}_{\mathrm{u}}$ & 15 & & & & & & & $\mathrm{Y}$ & $\mathrm{Y}$ & $Y$ \\
\hline $\mathrm{SS}_{\mathrm{u}}$ & 20 & & & & & & & $Y$ & $\mathrm{Y}$ & $\mathrm{SS}_{\mathrm{u}}$ & 20 & & & & & & & & $\mathrm{Y}$ & $Y$ \\
\hline $\mathrm{SS}_{\mathrm{u}}$ & 25 & & & & & & & & $Y$ & $\mathrm{SS}_{\mathrm{u}}$ & 25 & & & & & & & & & $\underline{N}$ \\
\hline
\end{tabular}


For barred spiral primaries in Table 5-8, the trend for larger scale dissimilarity continues; however, dissimilarity is less common when comparing fitting ranges within one increment $\left(1^{\circ}\right.$ at small scale and $5^{\circ}$ at large scale). It is also noted that below $5^{\circ}$ only secondaries of the spiral morphology show consistent dissimilarity. The trend of similarity below $5^{\circ}$ for secondaries of $S_{b}$ and $S_{u}$ continues as seen for all previous primaries. This continues to suggest that clustering of $S_{b}$ and $S_{u}$ is impacted less by the fitting range. Also interesting, more similarity is noted for early-type secondaries around barred spiral primaries below $5^{\circ}$ than is noted for other primaries.

Table 5-8 2D KS Test Results - comparing two runs having the same primary/secondary filters and varying fitting range filters - limited to barred spiral primaries.

$\mathrm{Y}=$ dissimilar to the $99 \%$ level. $\mathrm{N}=$ not dissimilar to the $99 \%$ level. FR1 = Fitting Range 1

\begin{tabular}{|c|c|c|c|c|c|c|c|c|c|c|c|c|c|c|c|c|c|c|c|c|c|c|c|}
\hline \multicolumn{12}{|c|}{ North } & \multicolumn{12}{|c|}{ South } \\
\hline \multicolumn{12}{|c|}{ Fitting Range 2 (degree) } & \multicolumn{12}{|c|}{ Fitting Range 2 (degree) } \\
\hline $\begin{array}{c}\text { Run } \\
1 \\
\end{array}$ & $\begin{array}{c}\mathrm{FR} \\
1 \\
\end{array}$ & 1 & 2 & 3 & 4 & 5 & $\begin{array}{l}1 \\
0 \\
\end{array}$ & $\begin{array}{l}1 \\
5 \\
\end{array}$ & $\begin{array}{l}2 \\
0 \\
\end{array}$ & $\begin{array}{l}2 \\
5 \\
\end{array}$ & $\begin{array}{l}3 \\
0 \\
\end{array}$ & $\begin{array}{c}\text { Run } \\
1\end{array}$ & $\begin{array}{c}\mathrm{FR} \\
1 \\
\end{array}$ & 1 & 2 & 3 & 4 & 5 & $\begin{array}{l}1 \\
0 \\
\end{array}$ & $\begin{array}{l}1 \\
5 \\
\end{array}$ & $\begin{array}{l}2 \\
0 \\
\end{array}$ & $\begin{array}{l}2 \\
5 \\
\end{array}$ & $\begin{array}{l}3 \\
0 \\
\end{array}$ \\
\hline$S_{b} A$ & 1 & & $\mathbf{Y}$ & $\mathbf{Y}$ & $\mathbf{Y}$ & $\mathbf{Y}$ & & & & & & $S_{b} A$ & 1 & & $\mathbf{Y}$ & $\mathbf{Y}$ & $\mathbf{Y}$ & $\mathbf{Y}$ & & & & & \\
\hline$S_{b} A$ & 2 & & & $\mathbf{Y}$ & $\mathbf{Y}$ & $\mathbf{Y}$ & & & & & & $S_{b} A$ & 2 & & & $\mathbf{Y}$ & $\mathbf{Y}$ & $\mathbf{Y}$ & & & & & \\
\hline$S_{b} A$ & 3 & & & & $\mathbf{Y}$ & $\mathbf{Y}$ & & & & & & $S_{b} A$ & 3 & & & & $\mathbf{Y}$ & $\mathbf{Y}$ & & & & & \\
\hline$S_{b} A$ & 4 & & & & & $\mathbf{Y}$ & & & & & & $S_{b} A$ & 4 & & & & & $\underline{N}$ & & & & & \\
\hline$S_{b} A$ & 5 & & & & & & $\mathbf{Y}$ & $\mathbf{Y}$ & $\mathbf{Y}$ & $\mathbf{Y}$ & $\mathbf{Y}$ & $S_{b} A$ & 5 & & & & & & $\mathbf{Y}$ & $\mathbf{Y}$ & $\mathbf{Y}$ & $\mathbf{Y}$ & $\mathbf{Y}$ \\
\hline$S_{b} A$ & 10 & & & & & & & $\mathbf{Y}$ & $\mathbf{Y}$ & $\mathbf{Y}$ & $\mathbf{Y}$ & $S_{b} A$ & 10 & & & & & & & $\mathbf{Y}$ & $\mathbf{Y}$ & $\mathbf{Y}$ & $Y$ \\
\hline$S_{b} A$ & 15 & & & & & & & & $\underline{N}$ & $\mathbf{Y}$ & $\mathbf{Y}$ & $S_{b} A$ & 15 & & & & & & & & $\underline{\mathrm{N}}$ & $\mathbf{Y}$ & $\mathbf{Y}$ \\
\hline$S_{b} A$ & 20 & & & & & & & & & $\underline{N}$ & $\mathbf{Y}$ & $S_{b} A$ & 20 & & & & & & & & & $\underline{N}$ & $Y$ \\
\hline$S_{b} A$ & 25 & & & & & & & & & & $\underline{N}$ & $S_{b} A$ & 25 & & & & & & & & & & $\underline{N}$ \\
\hline $\mathrm{S}_{\mathrm{b}} \mathrm{E}$ & 1 & & $\underline{N}$ & $\underline{N}$ & $\mathbf{Y}$ & $\mathbf{Y}$ & & & & & & $S_{b} E$ & 1 & & $\underline{N}$ & $\underline{\mathrm{N}}$ & $\underline{N}$ & $\underline{N}$ & & & & & \\
\hline $\mathrm{S}_{\mathrm{b}} \mathrm{E}$ & 2 & & & $\underline{\mathrm{N}}$ & $Y$ & $Y$ & & & & & & $S_{b} E$ & 2 & & & $\underline{N}$ & $\underline{N}$ & $\underline{N}$ & & & & & \\
\hline$S_{b} E$ & 3 & & & & $\underline{\mathrm{N}}$ & $\mathbf{Y}$ & & & & & & $S_{b} E$ & 3 & & & & $\underline{N}$ & $\mathbf{Y}$ & & & & & \\
\hline$S_{b} E$ & 4 & & & & & $\underline{N}$ & & & & & & $S_{b} E$ & 4 & & & & & $\underline{N}$ & & & & & \\
\hline$S_{b} E$ & 5 & & & & & & $\mathbf{Y}$ & $\mathbf{Y}$ & $\mathbf{Y}$ & $\mathbf{Y}$ & $\mathbf{Y}$ & $S_{b} E$ & 5 & & & & & & $\mathbf{Y}$ & $\mathbf{Y}$ & $\mathbf{Y}$ & $\mathbf{Y}$ & $\mathbf{Y}$ \\
\hline $\mathrm{S}_{\mathrm{b}} \mathrm{E}$ & 10 & & & & & & & $\mathbf{Y}$ & $\mathbf{Y}$ & $\mathbf{Y}$ & $\mathbf{Y}$ & $S_{b} E$ & 10 & & & & & & & $\mathbf{Y}$ & $\mathbf{Y}$ & $Y$ & $\mathbf{Y}$ \\
\hline $\mathrm{S}_{\mathrm{b}} \mathrm{E}$ & 15 & & & & & & & & $\mathbf{Y}$ & $\mathbf{Y}$ & $\mathbf{Y}$ & $S_{b} E$ & 15 & & & & & & & & $\underline{N}$ & $Y$ & $\mathbf{Y}$ \\
\hline$S_{b} E$ & 20 & & & & & & & & & $Y$ & $Y$ & $S_{b} E$ & 20 & & & & & & & & & $\underline{N}$ & $Y$ \\
\hline$S_{b} E$ & 25 & & & & & & & & & & $\underline{N}$ & $S_{b} E$ & 25 & & & & & & & & & & $\underline{N}$ \\
\hline$S_{b} L$ & 1 & & $\underline{N}$ & $\underline{\mathrm{N}}$ & $\underline{\mathrm{N}}$ & $\underline{N}$ & & & & & & $S_{b} L$ & 1 & & $\underline{\mathrm{N}}$ & $\underline{N}$ & $\underline{N}$ & $\underline{N}$ & & & & & \\
\hline$S_{b} L$ & 2 & & & $\underline{\mathrm{N}}$ & $Y$ & $Y$ & & & & & & $S_{b} L$ & 2 & & & $\underline{\mathrm{N}}$ & $\underline{N}$ & $Y$ & & & & & \\
\hline$S_{b} L$ & 3 & & & & $\underline{N}$ & $Y$ & & & & & & $S_{b} L$ & 3 & & & & $\underline{N}$ & $Y$ & & & & & \\
\hline$S_{b} L$ & 4 & & & & & $\underline{N}$ & & & & & & $S_{b} L$ & 4 & & & & & $\underline{N}$ & & & & & \\
\hline$S_{b} L$ & 5 & & & & & & $\mathbf{Y}$ & $\mathbf{Y}$ & $\mathbf{Y}$ & $\mathbf{Y}$ & $\mathbf{Y}$ & $S_{b} L$ & 5 & & & & & & $\mathbf{Y}$ & $\mathbf{Y}$ & $\mathbf{Y}$ & $Y$ & $Y$ \\
\hline
\end{tabular}




\begin{tabular}{|c|c|c|c|c|c|c|c|c|c|c|c|c|c|c|c|c|c|c|c|c|}
\hline$S_{b} L$ & 10 & & & & & $\mathbf{Y}$ & $\mathbf{Y}$ & $\mathbf{Y}$ & $\mathbf{Y}$ & $S_{b} L$ & 10 & & & & & & $\mathbf{Y}$ & $\mathbf{Y}$ & $\mathbf{Y}$ & $\mathbf{Y}$ \\
\hline$S_{b} L$ & 15 & & & & & & $\underline{N}$ & $\mathbf{Y}$ & $\mathbf{Y}$ & $S_{b} L$ & 15 & & & & & & & $\underline{\mathrm{N}}$ & $\mathbf{Y}$ & $\mathbf{Y}$ \\
\hline$S_{b} L$ & 20 & & & & & & & $\underline{N}$ & $Y$ & $\mathrm{~S}_{\mathrm{b}} \mathrm{L}$ & 20 & & & & & & & & $\underline{\mathrm{N}}$ & $Y$ \\
\hline$S_{b} L$ & 25 & & & & & & & & $\underline{N}$ & $S_{b} L$ & 25 & & & & & & & & & $\underline{N}$ \\
\hline$S_{b} S$ & 1 & $Y$ & $Y$ & $Y$ & $Y$ & & & & & $\mathrm{~S}_{\mathrm{b}} \mathrm{S}$ & 1 & $Y$ & $Y$ & $Y$ & $Y$ & & & & & \\
\hline$S_{b} S$ & 2 & & $Y$ & Y & $Y$ & & & & & $S_{b} S$ & 2 & & $\underline{N}$ & $Y$ & $Y$ & & & & & \\
\hline$S_{b} S$ & 3 & & & Y & $Y$ & & & & & $S_{b} S$ & 3 & & & $\underline{\mathrm{N}}$ & $Y$ & & & & & \\
\hline$S_{b} S$ & 4 & & & & $Y$ & & & & & $S_{b} S$ & 4 & & & & $\underline{N}$ & & & & & \\
\hline$S_{b} S$ & 5 & & & & $Y$ & $Y$ & $Y$ & $Y$ & $Y$ & $\mathrm{~S}_{\mathrm{b}} \mathrm{S}$ & 5 & & & & & $Y$ & $Y$ & $\mathrm{Y}$ & $Y$ & $\mathrm{Y}$ \\
\hline $\mathrm{S}_{\mathrm{b}} \mathrm{S}$ & 10 & & & & & $Y$ & $Y$ & $Y$ & $Y$ & $\mathrm{~S}_{\mathrm{b}} \mathrm{S}$ & 10 & & & & & & $\mathrm{Y}$ & $Y$ & $Y$ & $Y$ \\
\hline$S_{b} S$ & 15 & & & & & & $\mathbf{Y}$ & $Y$ & $Y$ & $S_{b} S$ & 15 & & & & & & & $\mathbf{Y}$ & $Y$ & $\mathbf{Y}$ \\
\hline$S_{b} S$ & 20 & & & & & & & $Y$ & $Y$ & $\mathrm{~S}_{\mathrm{b}} \mathrm{S}$ & 20 & & & & & & & & $\underline{N}$ & $Y$ \\
\hline$S_{b} S$ & 25 & & & & & & & & $\underline{N}$ & $\mathrm{~S}_{\mathrm{b}} \mathrm{S}$ & 25 & & & & & & & & & $\underline{\mathrm{N}}$ \\
\hline $\mathrm{S}_{\mathrm{b}} \mathrm{S}_{\mathrm{b}}$ & 1 & $Y$ & $\mathrm{Y}$ & $\mathrm{Y}$ & $Y$ & & & & & $\mathrm{~S}_{\mathrm{b}} \mathrm{S}_{\mathrm{b}}$ & 1 & $\underline{N}$ & $\underline{N}$ & $\underline{N}$ & $\underline{N}$ & & & & & \\
\hline $\mathrm{S}_{\mathrm{b}} \mathrm{S}_{\mathrm{b}}$ & 2 & & $\underline{N}$ & $\underline{\mathrm{N}}$ & $\underline{N}$ & & & & & $\mathrm{~S}_{\mathrm{b}} \mathrm{S}_{\mathrm{b}}$ & 2 & & $\underline{N}$ & $\underline{N}$ & $\underline{N}$ & & & & & \\
\hline $\mathrm{S}_{\mathrm{b}} \mathrm{S}_{\mathrm{b}}$ & 3 & & 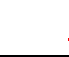 & $\underline{N}$ & $\underline{N}$ & & & & & $\mathrm{~S}_{\mathrm{b}} \mathrm{S}_{\mathrm{b}}$ & 3 & & 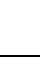 & $\underline{N}$ & $\underline{N}$ & & & & & \\
\hline $\mathrm{S}_{\mathrm{b}} \mathrm{S}_{\mathrm{b}}$ & 4 & & & & $\underline{N}$ & & & & & $\mathrm{~S}_{\mathrm{b}} \mathrm{S}_{\mathrm{b}}$ & 4 & & & & $\underline{N}$ & & & & & \\
\hline $\mathrm{S}_{\mathrm{b}} \mathrm{S}_{\mathrm{b}}$ & 5 & & & & $\mathrm{Y}$ & $Y$ & $Y$ & $Y$ & $Y$ & $\mathrm{~S}_{\mathrm{b}} \mathrm{S}_{\mathrm{b}}$ & 5 & & & & & $Y$ & $Y$ & $Y$ & $Y$ & $Y$ \\
\hline $\mathrm{S}_{\mathrm{b}} \mathrm{S}_{\mathrm{b}}$ & 10 & & & & & $\mathrm{Y}$ & $\mathrm{Y}$ & $\mathrm{Y}$ & $\mathrm{Y}$ & $\mathrm{S}_{\mathrm{b}} \mathrm{S}_{\mathrm{b}}$ & 10 & & & & & & $\mathrm{Y}$ & $\mathrm{Y}$ & $\mathrm{Y}$ & $\mathrm{Y}$ \\
\hline $\mathrm{S}_{\mathrm{b}} \mathrm{S}_{\mathrm{b}}$ & 15 & & & & & & $Y$ & $Y$ & $Y$ & $\mathrm{~S}_{\mathrm{b}} \mathrm{S}_{\mathrm{b}}$ & 15 & & & & & & & $Y$ & $Y$ & $Y$ \\
\hline $\mathrm{S}_{\mathrm{b}} \mathrm{S}_{\mathrm{b}}$ & 20 & & & & & & & $Y$ & $Y$ & $\mathrm{~S}_{\mathrm{b}} \mathrm{S}_{\mathrm{b}}$ & 20 & & & & & & & & $\underline{N}$ & $Y$ \\
\hline $\mathrm{S}_{\mathrm{b}} \mathrm{S}_{\mathrm{b}}$ & 25 & & & & & & & & $\underline{\mathrm{N}}$ & $\mathrm{S}_{\mathrm{b}} \mathrm{S}_{\mathrm{b}}$ & 25 & & & & & & & & & $\underline{\mathrm{N}}$ \\
\hline $\mathrm{S}_{\mathrm{b}} \mathrm{S}_{\mathrm{u}}$ & 1 & $\underline{N}$ & $\underline{\mathrm{N}}$ & $\underline{\mathrm{N}}$ & $\underline{N}$ & & & & & $\mathrm{~S}_{\mathrm{b}} \mathrm{S}_{\mathrm{u}}$ & 1 & $\underline{N}$ & $\underline{N}$ & $\underline{N}$ & $\underline{N}$ & & & & & \\
\hline $\mathrm{S}_{\mathrm{b}} \mathrm{S}_{\mathrm{u}}$ & 2 & 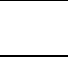 & $\underline{N}$ & $\underline{N}$ & $Y$ & & & & & $\mathrm{~S}_{\mathrm{b}} \mathrm{S}_{\mathrm{u}}$ & 2 & & $\underline{N}$ & $\underline{N}$ & $\underline{N}$ & & & & & \\
\hline $\mathrm{S}_{\mathrm{b}} \mathrm{S}_{\mathrm{u}}$ & 3 & 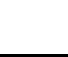 & 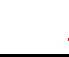 & $\underline{N}$ & $\underline{N}$ & & & & & $\mathrm{~S}_{\mathrm{b}} \mathrm{S}_{\mathrm{u}}$ & 3 & & & $\underline{N}$ & $\underline{N}$ & & & & & \\
\hline $\mathrm{S}_{\mathrm{b}} \mathrm{S}_{\mathrm{u}}$ & 4 & & & & $\underline{N}$ & & & & & $\mathrm{~S}_{\mathrm{b}} \mathrm{S}_{\mathrm{u}}$ & 4 & & & & $\underline{N}$ & & & & & \\
\hline $\mathrm{S}_{\mathrm{b}} \mathrm{S}_{\mathrm{u}}$ & 5 & & & & $Y$ & $Y$ & $Y$ & $Y$ & $Y$ & $\mathrm{~S}_{\mathrm{b}} \mathrm{S}_{\mathrm{u}}$ & 5 & & & & & $\underline{N}$ & $Y$ & $Y$ & $Y$ & $Y$ \\
\hline $\mathrm{S}_{\mathrm{b}} \mathrm{S}_{\mathrm{u}}$ & 10 & & & & & $\mathrm{Y}$ & $\mathrm{Y}$ & $\mathrm{Y}$ & $\mathrm{Y}$ & $\mathrm{S}_{\mathrm{b}} \mathrm{S}_{\mathrm{u}}$ & 10 & & & & & & $\underline{N}$ & $\mathrm{Y}$ & $\mathrm{Y}$ & $\mathrm{Y}$ \\
\hline $\mathrm{S}_{\mathrm{b}} \mathrm{S}_{\mathrm{u}}$ & 15 & & & & & & $Y$ & $Y$ & $Y$ & $\mathrm{~S}_{\mathrm{b}} \mathrm{S}_{\mathrm{u}}$ & 15 & & & & & & & $\underline{N}$ & $Y$ & $Y$ \\
\hline $\mathrm{S}_{\mathrm{b}} \mathrm{S}_{\mathrm{u}}$ & 20 & & & & & & & $\underline{N}$ & $\mathrm{Y}$ & $\mathrm{S}_{\mathrm{b}} \mathrm{S}_{\mathrm{u}}$ & 20 & & & & & & & & $\underline{N}$ & $\underline{N}$ \\
\hline $\mathrm{S}_{\mathrm{b}} \mathrm{S}_{\mathrm{u}}$ & 25 & & & & & & & & $\underline{N}$ & $\mathrm{~S}_{\mathrm{b}} \mathrm{S}_{\mathrm{u}}$ & 25 & & & & & & & & & $\underline{N}$ \\
\hline
\end{tabular}


For primaries of unbarred spiral in Table 5-9, dissimilarity is consistently above $5^{\circ}$ for all secondary morphologies once the difference between fitting ranges compared increases beyond $5^{\circ}$. At small scale only secondaries of spiral exhibit consistent dissimilarity. For other secondaries very little dissimilarity is found at small scale below $5^{\circ}$.

Table 5-9 2D KS Test Results - comparing two runs having the same primary/secondary filters and varying fitting range filters - limited to unbarred spiral primaries.

$\mathrm{Y}=$ dissimilar to the $99 \%$ level. $\mathrm{N}=$ not dissimilar to the $99 \%$ level. FR1 = Fitting Range 1

\begin{tabular}{|c|c|c|c|c|c|c|c|c|c|c|c|c|c|c|c|c|c|c|c|c|c|c|c|}
\hline \multicolumn{12}{|c|}{ North } & \multicolumn{12}{|c|}{ South } \\
\hline \multirow[b]{2}{*}{$\begin{array}{c}\text { Run } \\
1 \\
\end{array}$} & \multirow[b]{2}{*}{$\begin{array}{c}\mathrm{FR} \\
1 \\
\end{array}$} & \multicolumn{10}{|c|}{ Fitting Range 2 (degree) } & \multirow[b]{2}{*}{$\begin{array}{c}\text { Run } \\
1 \\
\end{array}$} & \multirow[b]{2}{*}{$\begin{array}{c}\text { FR } \\
1 \\
\end{array}$} & \multirow[b]{2}{*}{1} & \multirow[b]{2}{*}{2} & \multicolumn{6}{|c|}{ Fitting Range 2 (degree) } & \multirow[b]{2}{*}{$\begin{array}{l}2 \\
5 \\
\end{array}$} & \multirow[b]{2}{*}{$\begin{array}{l}3 \\
0 \\
\end{array}$} \\
\hline & & 1 & 2 & 3 & 4 & 5 & $\begin{array}{l}1 \\
0 \\
\end{array}$ & $\begin{array}{l}1 \\
5 \\
\end{array}$ & $\begin{array}{l}2 \\
0 \\
\end{array}$ & $\begin{array}{l}2 \\
5 \\
\end{array}$ & $\begin{array}{l}3 \\
0 \\
\end{array}$ & & & & & 3 & 4 & 5 & $\begin{array}{l}1 \\
0 \\
\end{array}$ & $\begin{array}{l}1 \\
5 \\
\end{array}$ & $\begin{array}{l}2 \\
0 \\
\end{array}$ & & \\
\hline $\mathrm{S}_{\mathrm{u}} \mathrm{A}$ & 1 & & $\mathrm{Y}$ & $\mathrm{Y}$ & $\mathrm{Y}$ & $Y$ & & & & & & $\mathrm{~S}_{\mathrm{u}} \mathrm{A}$ & 1 & & $\mathrm{Y}$ & $Y$ & $Y$ & $\mathrm{Y}$ & & & & & \\
\hline $\mathrm{S}_{\mathrm{u}} \mathrm{A}$ & 2 & & & $\mathbf{Y}$ & $\mathbf{Y}$ & $\mathbf{Y}$ & & & & & & $\mathrm{S}_{\mathrm{u}} \mathrm{A}$ & 2 & & & $\mathbf{Y}$ & $\mathbf{Y}$ & $\mathbf{Y}$ & & & & & \\
\hline $\mathrm{S}_{\mathrm{u}} \mathrm{A}$ & 3 & & & & $\mathrm{Y}$ & $\mathrm{Y}$ & & & & & & $\mathrm{S}_{\mathrm{u}} \mathrm{A}$ & 3 & & & & $\mathrm{Y}$ & $\mathrm{Y}$ & & & & & \\
\hline $\mathrm{S}_{\mathrm{u}} \mathrm{A}$ & 4 & & & & & $\underline{N}$ & & & & & & $\mathrm{~S}_{\mathrm{u}} \mathrm{A}$ & 4 & & & & & $\underline{N}$ & & & & & \\
\hline $\mathrm{S}_{\mathrm{u}} \mathrm{A}$ & 5 & & & & & & $Y$ & $\mathrm{Y}$ & $\mathrm{Y}$ & $\mathrm{Y}$ & $Y$ & $\mathrm{~S}_{\mathrm{u}} \mathrm{A}$ & 5 & & & & & & $Y$ & $\mathrm{Y}$ & $Y$ & $Y$ & $Y$ \\
\hline $\mathrm{S}_{\mathrm{u}} \mathrm{A}$ & 10 & & & & & & & $\mathrm{Y}$ & $\mathrm{Y}$ & $Y$ & $Y$ & $\mathrm{~S}_{\mathrm{u}} \mathrm{A}$ & 10 & & & & & & & $\mathrm{Y}$ & $\mathrm{Y}$ & $Y$ & $Y$ \\
\hline $\mathrm{S}_{\mathrm{u}} \mathrm{A}$ & 15 & & & & & & & & $\underline{N}$ & $\mathrm{Y}$ & $\mathrm{Y}$ & $\mathrm{S}_{\mathrm{u}} \mathrm{A}$ & 15 & & & & & & & & $\underline{N}$ & $Y$ & $\mathrm{Y}$ \\
\hline $\mathrm{S}_{\mathrm{u}} \mathrm{A}$ & 20 & & & & & & & & & $\underline{N}$ & $Y$ & $\mathrm{~S}_{\mathrm{u}} \mathrm{A}$ & 20 & & & & & & & & & $\underline{N}$ & $\underline{N}$ \\
\hline $\mathrm{S}_{\mathrm{u}} \mathrm{A}$ & 25 & & & & & & & & & & $\underline{N}$ & $\mathrm{~S}_{\mathrm{u}} \mathrm{A}$ & 25 & & & & & & & & & & $\underline{N}$ \\
\hline $\mathrm{S}_{\mathrm{u}} \mathrm{E}$ & 1 & & $\underline{\mathrm{N}}$ & $\underline{\mathrm{N}}$ & $\mathrm{Y}$ & $Y$ & & & & & & $\mathrm{~S}_{\mathrm{u}} \mathrm{E}$ & 1 & & $\underline{\mathrm{N}}$ & $\underline{\mathrm{N}}$ & $\underline{N}$ & $Y$ & & & & & \\
\hline $\mathrm{S}_{\mathrm{u}} \mathrm{E}$ & 2 & & & $\mathrm{Y}$ & $\mathrm{Y}$ & $\mathrm{Y}$ & & & & & & $\mathrm{S}_{\mathrm{u}} \mathrm{E}$ & 2 & & & $\underline{N}$ & $\underline{N}$ & $\mathrm{Y}$ & & & & & \\
\hline $\mathrm{S}_{\mathrm{u}} \mathrm{E}$ & 3 & & & & $\mathrm{Y}$ & $\mathrm{Y}$ & & & & & & $\mathrm{S}_{\mathrm{u}} \mathrm{E}$ & 3 & & & & $\underline{N}$ & $\underline{N}$ & & & & & \\
\hline $\mathrm{S}_{\mathrm{u}} \mathrm{E}$ & 4 & & & & & $\underline{N}$ & & & & & & $\mathrm{~S}_{\mathrm{u}} \mathrm{E}$ & 4 & & & & & $\underline{N}$ & & & & & \\
\hline $\mathrm{S}_{\mathrm{u}} \mathrm{E}$ & 5 & & & & & & $\mathrm{Y}$ & $\mathrm{Y}$ & $\mathrm{Y}$ & $\mathrm{Y}$ & $\mathrm{Y}$ & $\mathrm{S}_{\mathrm{u}} \mathrm{E}$ & 5 & & & & & & $\mathrm{Y}$ & $\mathrm{Y}$ & $\mathrm{Y}$ & $\mathrm{Y}$ & $\mathrm{Y}$ \\
\hline $\mathrm{S}_{\mathrm{u}} \mathrm{E}$ & 10 & & & & & & & $Y$ & $Y$ & $Y$ & $Y$ & $\mathrm{~S}_{\mathrm{u}} \mathrm{E}$ & 10 & & & & & & & $Y$ & $Y$ & $Y$ & $Y$ \\
\hline $\mathrm{S}_{\mathrm{u}} \mathrm{E}$ & 15 & & & & & & & & $Y$ & $Y$ & $Y$ & $\mathrm{~S}_{\mathrm{u}} \mathrm{E}$ & 15 & & & & & & & & $\underline{N}$ & $Y$ & $Y$ \\
\hline $\mathrm{S}_{\mathrm{u}} \mathrm{E}$ & 20 & & & & & & & & & $\mathrm{Y}$ & $\mathrm{Y}$ & $\mathrm{S}_{\mathrm{u}} \mathrm{E}$ & 20 & & & & & & & & & $\underline{N}$ & $Y$ \\
\hline $\mathrm{S}_{\mathrm{u}} \mathrm{E}$ & 25 & & & & & & & & & & $\underline{N}$ & $\mathrm{~S}_{\mathrm{u}} \mathrm{E}$ & 25 & & & & & & & & & & $\underline{N}$ \\
\hline $\mathrm{S}_{\mathrm{u}} \mathrm{L}$ & 1 & & $\underline{N}$ & $\underline{\mathrm{N}}$ & $\underline{\mathrm{N}}$ & $\underline{N}$ & & & & & & $\mathrm{~S}_{\mathrm{u}} \mathrm{L}$ & 1 & & $\underline{\mathrm{N}}$ & $\underline{\mathrm{N}}$ & $\underline{N}$ & $\mathrm{Y}$ & & & & & \\
\hline $\mathrm{S}_{\mathrm{u}} \mathrm{L}$ & 2 & & & $\underline{N}$ & $\underline{N}$ & $Y$ & & & & & & $\mathrm{~S}_{\mathrm{u}} \mathrm{L}$ & 2 & & & $\underline{N}$ & $\underline{N}$ & $Y$ & & & & & \\
\hline $\mathrm{S}_{\mathrm{u}} \mathrm{L}$ & 3 & & & & $\underline{N}$ & $\underline{N}$ & & & & & & $\mathrm{~S}_{\mathrm{u}} \mathrm{L}$ & 3 & & & & $\underline{N}$ & $\mathrm{Y}$ & & & & & \\
\hline $\mathrm{S}_{\mathrm{u}} \mathrm{L}$ & 4 & & & & & $\underline{N}$ & & & & & & $\mathrm{~S}_{\mathrm{u}} \mathrm{L}$ & 4 & & & & & $\underline{N}$ & & & & & \\
\hline $\mathrm{S}_{\mathrm{u}} \mathrm{L}$ & 5 & & & & & & $\mathrm{Y}$ & $\mathrm{Y}$ & $\mathrm{Y}$ & $\mathrm{Y}$ & $\mathrm{Y}$ & $\mathrm{S}_{\mathrm{u}} \mathrm{L}$ & 5 & & & & & & $\mathrm{Y}$ & $\mathrm{Y}$ & $\mathrm{Y}$ & $\mathrm{Y}$ & $\mathrm{Y}$ \\
\hline $\mathrm{S}_{\mathrm{u}} \mathrm{L}$ & 10 & & & & & & & $Y$ & $Y$ & $Y$ & $Y$ & $\mathrm{~S}_{\mathrm{u}} \mathrm{L}$ & 10 & & & & & & & $\underline{N}$ & $Y$ & $Y$ & $Y$ \\
\hline $\mathrm{S}_{\mathrm{u}} \mathrm{L}$ & 15 & & & & & & & & $Y$ & $Y$ & $Y$ & $\mathrm{~S}_{\mathrm{u}} \mathrm{L}$ & 15 & & & & & & & & $\underline{N}$ & $Y$ & $Y$ \\
\hline $\mathrm{S}_{\mathrm{u}} \mathrm{L}$ & 20 & & & & & & & & & $Y$ & $Y$ & $\mathrm{~S}_{\mathrm{u}} \mathrm{L}$ & 20 & & & & & & & & & $\underline{N}$ & $\mathrm{Y}$ \\
\hline$S_{u} L$ & 25 & & & & & & & & & & $\underline{\mathrm{N}}$ & $S_{u} L$ & 25 & & & & & & & & & & $\underline{N}$ \\
\hline
\end{tabular}




\begin{tabular}{|c|c|c|c|c|c|c|c|c|c|c|}
\hline $\mathrm{S}_{\mathrm{u}} \mathrm{S}$ & 1 & $\underline{N}$ & $Y$ & $Y$ & $Y$ & & & & & \\
\hline $\mathrm{S}_{\mathrm{u}} \mathrm{S}$ & 2 & & $\mathbf{Y}$ & $\mathbf{Y}$ & $\mathbf{Y}$ & & & & & \\
\hline $\mathrm{S}_{\mathrm{u}} \mathrm{S}$ & 3 & & & $\mathbf{Y}$ & $\mathrm{Y}$ & & & & & \\
\hline $\mathrm{S}_{\mathrm{u}} \mathrm{S}$ & 4 & & & & $\underline{\mathrm{N}}$ & & & & & \\
\hline $\mathrm{S}_{\mathrm{u}} \mathrm{S}$ & 5 & & & & & $\mathbf{Y}$ & $\mathbf{Y}$ & $\mathbf{Y}$ & $\mathbf{Y}$ & $\mathbf{Y}$ \\
\hline $\mathrm{S}_{\mathrm{u}} \mathrm{S}$ & 10 & & & & & & $\mathbf{Y}$ & $\mathbf{Y}$ & $\mathbf{Y}$ & $\mathbf{Y}$ \\
\hline $\mathrm{S}_{\mathrm{u}} \mathrm{S}$ & 15 & & & & & & & $\underline{\mathrm{N}}$ & $\mathbf{Y}$ & $\mathbf{Y}$ \\
\hline $\mathrm{S}_{\mathrm{u}} \mathrm{S}$ & 20 & & & & & & & & $\underline{N}$ & $\mathbf{Y}$ \\
\hline $\mathrm{S}_{\mathrm{u}} \mathrm{S}$ & 25 & & & & & & & & & $\underline{N}$ \\
\hline$S_{u} S_{b}$ & 1 & $\mathbf{Y}$ & $\mathbf{Y}$ & $\mathbf{Y}$ & $\mathbf{Y}$ & & & & & \\
\hline $\mathrm{S}_{\mathrm{u}} \mathrm{S}_{\mathrm{b}}$ & 2 & & $\underline{N}$ & $\underline{\mathrm{N}}$ & $\underline{\mathrm{N}}$ & & & & & \\
\hline $\mathrm{S}_{\mathrm{u}} \mathrm{S}_{\mathrm{b}}$ & 3 & & & $\underline{\mathrm{N}}$ & $\underline{\mathrm{N}}$ & & & & & \\
\hline $\mathrm{S}_{\mathrm{u}} \mathrm{S}_{\mathrm{b}}$ & 4 & & & & $\underline{\mathrm{N}}$ & & & & & \\
\hline $\mathrm{S}_{\mathrm{u}} \mathrm{S}_{\mathrm{b}}$ & 5 & & & & & $\mathbf{Y}$ & $\mathbf{Y}$ & $\mathbf{Y}$ & $\mathbf{Y}$ & $\mathbf{Y}$ \\
\hline $\mathrm{S}_{\mathrm{u}} \mathrm{S}_{\mathrm{b}}$ & 10 & & & & & & $\mathbf{Y}$ & $\mathbf{Y}$ & $\mathbf{Y}$ & $\mathbf{Y}$ \\
\hline $\mathrm{S}_{\mathrm{u}} \mathrm{S}_{\mathrm{b}}$ & 15 & & & & & & & $Y$ & $Y$ & $Y$ \\
\hline $\mathrm{S}_{\mathrm{u}} \mathrm{S}_{\mathrm{b}}$ & 20 & & & & & & & & $\mathbf{Y}$ & $\mathbf{Y}$ \\
\hline $\mathrm{S}_{\mathrm{u}} \mathrm{S}_{\mathrm{b}}$ & 25 & & & & & & & & & $\underline{\mathrm{N}}$ \\
\hline $\mathrm{S}_{\mathrm{u}} \mathrm{S}_{\mathrm{u}}$ & 1 & $\underline{N}$ & $\underline{\mathrm{N}}$ & $\underline{\mathrm{N}}$ & $\underline{N}$ & & & & & \\
\hline $\mathrm{S}_{\mathrm{u}} \mathrm{S}_{\mathrm{u}}$ & 2 & & $\underline{\mathrm{N}}$ & $\underline{\mathrm{N}}$ & $\mathbf{Y}$ & & & & & \\
\hline $\mathrm{S}_{\mathrm{u}} \mathrm{S}_{\mathrm{u}}$ & 3 & & & $\underline{\mathrm{N}}$ & $\underline{\mathrm{N}}$ & & & & & \\
\hline $\mathrm{S}_{\mathrm{u}} \mathrm{S}_{\mathrm{u}}$ & 4 & & & & $\underline{\mathrm{N}}$ & & & & & \\
\hline $\mathrm{S}_{\mathrm{u}} \mathrm{S}_{\mathrm{u}}$ & 5 & & & & & $\mathbf{Y}$ & $\mathbf{Y}$ & $\mathbf{Y}$ & $\mathbf{Y}$ & $\mathbf{Y}$ \\
\hline $\mathrm{S}_{\mathrm{u}} \mathrm{S}_{\mathrm{u}}$ & 10 & & & & & & $\mathrm{Y}$ & $\mathrm{Y}$ & $\mathrm{Y}$ & $\mathrm{Y}$ \\
\hline $\mathrm{S}_{\mathrm{u}} \mathrm{S}_{\mathrm{u}}$ & 15 & & & & & & & $Y$ & $Y$ & $Y$ \\
\hline $\mathrm{S}_{\mathrm{u}} \mathrm{S}_{\mathrm{u}}$ & 20 & & & & & & & & $\underline{N}$ & $Y$ \\
\hline $\mathrm{S}_{\mathrm{u}} \mathrm{S}_{\mathrm{u}}$ & 25 & & & & & & & & & $\underline{\mathrm{N}}$ \\
\hline
\end{tabular}

\begin{tabular}{|c|c|c|c|c|c|c|c|c|c|c|}
\hline $\mathrm{S}_{\mathrm{u}} \mathrm{S}$ & 1 & $Y$ & $\mathbf{Y}$ & $\mathbf{Y}$ & $\mathbf{Y}$ & & & & & \\
\hline $\mathrm{S}_{\mathrm{u}} \mathrm{S}$ & 2 & & $\underline{N}$ & $\mathbf{Y}$ & $\mathbf{Y}$ & & & & & \\
\hline $\mathrm{S}_{\mathrm{u}} \mathrm{S}$ & 3 & & & $\underline{\mathrm{N}}$ & $\mathbf{Y}$ & & & & & \\
\hline $\mathrm{S}_{\mathrm{u}} \mathrm{S}$ & 4 & & & & $\underline{N}$ & & & & & \\
\hline $\mathrm{S}_{\mathrm{u}} \mathrm{S}$ & 5 & & & & & $\mathbf{Y}$ & $\mathbf{Y}$ & $\mathbf{Y}$ & $\mathbf{Y}$ & $\mathbf{Y}$ \\
\hline $\mathrm{S}_{\mathrm{u}} \mathrm{S}$ & 10 & & & & & & $\mathbf{Y}$ & $\mathbf{Y}$ & $\mathbf{Y}$ & $\mathbf{Y}$ \\
\hline$S_{u} S$ & 15 & & & & & & & $\underline{\mathrm{N}}$ & $\mathbf{Y}$ & $\mathbf{Y}$ \\
\hline $\mathrm{S}_{\mathrm{u}} \mathrm{S}$ & 20 & & & & & & & & $\underline{N}$ & $\mathbf{Y}$ \\
\hline $\mathrm{S}_{\mathrm{u}} \mathrm{S}$ & 25 & & & & & & & & & $\underline{N}$ \\
\hline $\mathrm{S}_{\mathrm{u}} \mathrm{S}_{\mathrm{b}}$ & 1 & $\underline{N}$ & $\underline{N}$ & $\underline{N}$ & $\underline{N}$ & & & & & \\
\hline$S_{u} S_{b}$ & 2 & & $\underline{N}$ & $\underline{\mathrm{N}}$ & $\underline{\mathrm{N}}$ & & & & & \\
\hline $\mathrm{S}_{\mathrm{u}} \mathrm{S}_{\mathrm{b}}$ & 3 & & & $\underline{\mathrm{N}}$ & $\underline{\mathrm{N}}$ & & & & & \\
\hline $\mathrm{S}_{\mathrm{u}} \mathrm{S}_{\mathrm{b}}$ & 4 & & & & $\underline{N}$ & & & & & \\
\hline$S_{u} S_{b}$ & 5 & & & & & $Y$ & $\mathbf{Y}$ & $\mathbf{Y}$ & $\mathbf{Y}$ & $\mathbf{Y}$ \\
\hline $\mathrm{S}_{\mathrm{u}} \mathrm{S}_{\mathrm{b}}$ & 10 & & & & & & $\mathbf{Y}$ & $\mathbf{Y}$ & $\mathbf{Y}$ & $\mathbf{Y}$ \\
\hline $\mathrm{S}_{\mathrm{u}} \mathrm{S}_{\mathrm{b}}$ & 15 & & & & & & & $\underline{\mathrm{N}}$ & $\mathbf{Y}$ & $\mathbf{Y}$ \\
\hline $\mathrm{S}_{\mathrm{u}} \mathrm{S}_{\mathrm{b}}$ & 20 & & & & & & & & $\underline{N}$ & $\underline{N}$ \\
\hline $\mathrm{S}_{\mathrm{u}} \mathrm{S}_{\mathrm{b}}$ & 25 & & & & & & & & & $\underline{N}$ \\
\hline $\mathrm{S}_{\mathrm{u}} \mathrm{S}_{\mathrm{u}}$ & 1 & $\underline{N}$ & $\underline{N}$ & $\underline{N}$ & $\underline{N}$ & & & & & \\
\hline $\mathrm{S}_{\mathrm{u}} \mathrm{S}_{\mathrm{u}}$ & 2 & & $\underline{\mathrm{N}}$ & $\underline{N}$ & $Y$ & & & & & \\
\hline $\mathrm{S}_{\mathrm{u}} \mathrm{S}_{\mathrm{u}}$ & 3 & & & $\underline{\mathrm{N}}$ & $\underline{\mathrm{N}}$ & & & & & \\
\hline $\mathrm{S}_{\mathrm{u}} \mathrm{S}_{\mathrm{u}}$ & 4 & & & & $\underline{N}$ & & & & & \\
\hline $\mathrm{S}_{\mathrm{u}} \mathrm{S}_{\mathrm{u}}$ & 5 & & & & & $\mathrm{Y}$ & $\mathrm{Y}$ & $\mathrm{Y}$ & $Y$ & $Y$ \\
\hline $\mathrm{S}_{\mathrm{u}} \mathrm{S}_{\mathrm{u}}$ & 10 & & & & & & $\underline{N}$ & $\mathrm{Y}$ & $Y$ & $Y$ \\
\hline $\mathrm{S}_{\mathrm{u}} \mathrm{S}_{\mathrm{u}}$ & 15 & & & & & & & $\underline{\mathrm{N}}$ & $\underline{\mathrm{N}}$ & $\underline{N}$ \\
\hline $\mathrm{S}_{\mathrm{u}} \mathrm{S}_{\mathrm{u}}$ & 20 & & & & & & & & $\underline{N}$ & $\underline{N}$ \\
\hline $\mathrm{S}_{\mathrm{u}} \mathrm{S}_{\mathrm{u}}$ & 25 & & & & & & & & & $\underline{N}$ \\
\hline
\end{tabular}

\subsubsection{Conclusions}

The general trend observed is that as the fitting range increases dissimilarity is found. I conclude that there is a relationship between the similarity of environments and the fitting range measured from the primary galaxy such that greater fitting ranges yield dissimilar environments.

Of interest the clustering of $S_{b}$ and $S_{u}$ generally show similarity consistently below $5^{\circ}$ around all primaries, while the clustering of $S$ generally show consistent 
dissimilarity below $5^{\circ}$ around all primaries. Also, the clustering of early-type galaxies around $\mathrm{L}$ primaries shows more similarity below $5^{\circ}$ than the clustering of early-type galaxies around $\mathrm{E}$ primaries below $5^{\circ}$.

\subsubsection{Impact of Secondary Morphology on the Pointwise Dimension}

Data runs where the primary morphology and fitting range are equal with differing secondary morphologies are compared in KS tests.

In all of the following tables $\mathrm{Y}$ indicates that the $p$ value from the $\mathrm{KS}$ test is < 0.01 . This means that there is less than a $1 \%$ chance that the two compared data sets are drawn from the same distribution. That is, the compared data sets are statistically dissimilar to the $99 \%$ level. A value of $\mathrm{N}$ indicates the inverse. That is, the datasets are not statistically dissimilar to the $99 \%$ level. 
When considering ellipticals as primary in Table 5-10, dissimilarity is generally found as the fitting range is increased. The increase in fitting range needed to find dissimilarity is dependent on the secondary morphology. The ESb and ESu comparisons require the larger fitting ranges to find dissimilarity.

The for $E$ primaries below $5^{\circ}$ the clustering of $S_{b}$ and $S_{u}$ spirals appear to have more in common with early-type galaxies than $\mathrm{S}$.

There also is a remarkable disagreement between North and South when comparing EL and EE, with North showing very little dissimilarity at any fitting range.

Table 5-10 2D KS Test Results - comparing two runs having the same primary filter and same fitting range filter with different secondary filters - limited to elliptical primaries.

$\mathrm{Y}=$ dissimilar to the $99 \%$ level. $\mathrm{N}=$ not dissimilar to the $99 \%$ level

\begin{tabular}{|c|c|c|c|c|c|c|c|c|c|c|c|c|c|c|c|c|c|c|c|c|c|c|c|}
\hline \multirow[b]{3}{*}{ Run1 } & \multicolumn{11}{|c|}{ North } & \multicolumn{12}{|c|}{ South } \\
\hline & \multicolumn{11}{|c|}{ Fitting Range (degree) } & \multicolumn{12}{|c|}{ Fitting Range (degree) } \\
\hline & Run2 & 1 & 2 & 3 & 4 & 5 & 10 & 15 & 20 & 25 & 30 & Run1 & Run2 & 1 & 2 & 3 & 4 & 5 & 10 & 15 & 20 & 25 & 30 \\
\hline EA & EE & $\mathbf{Y}$ & $\mathbf{Y}$ & $\mathbf{Y}$ & $\mathbf{Y}$ & $\mathbf{Y}$ & $\mathbf{Y}$ & $\mathbf{Y}$ & $\mathbf{Y}$ & $\mathbf{Y}$ & $\mathbf{Y}$ & EA & EE & $\mathbf{Y}$ & Y & $\mathbf{Y}$ & $\mathbf{Y}$ & $\mathbf{Y}$ & $\mathbf{Y}$ & $\mathbf{Y}$ & $\mathbf{Y}$ & $\mathbf{Y}$ & $\mathbf{Y}$ \\
\hline EA & EL & $\underline{N}$ & $\mathbf{Y}$ & $\mathbf{Y}$ & $\mathbf{Y}$ & $\mathbf{Y}$ & $\mathbf{Y}$ & $\mathbf{Y}$ & $\mathbf{Y}$ & $\mathbf{Y}$ & $\mathbf{Y}$ & EA & EL & $\underline{N}$ & Y & $\mathbf{Y}$ & $\mathbf{Y}$ & $\mathbf{Y}$ & $\mathbf{Y}$ & $\mathbf{Y}$ & $\mathbf{Y}$ & $\mathbf{Y}$ & $\mathbf{Y}$ \\
\hline EA & ES & $\underline{N}$ & $\mathbf{Y}$ & $\mathbf{Y}$ & $\mathbf{Y}$ & $\mathbf{Y}$ & $\mathbf{Y}$ & $\mathbf{Y}$ & $\underline{\mathrm{N}}$ & $\underline{\mathrm{N}}$ & $\underline{N}$ & EA & ES & $\underline{N}$ & $Y$ & $Y$ & $\mathbf{Y}$ & $Y$ & $\mathbf{Y}$ & $Y$ & $\mathbf{Y}$ & $Y$ & $\underline{\mathrm{N}}$ \\
\hline EA & $\mathrm{ES}_{\mathrm{b}}$ & $\underline{\mathrm{N}}$ & $\mathbf{Y}$ & $\mathbf{Y}$ & $\mathbf{Y}$ & $\mathbf{Y}$ & $\mathbf{Y}$ & $\mathbf{Y}$ & $\mathbf{Y}$ & $\mathbf{Y}$ & $\mathbf{Y}$ & EA & $\mathrm{ES}_{\mathrm{b}}$ & $\underline{N}$ & $Y$ & $Y$ & $\mathbf{Y}$ & $Y$ & $\mathbf{Y}$ & $Y$ & $\mathbf{Y}$ & $Y$ & $\mathbf{Y}$ \\
\hline EA & $\mathrm{ES}_{\mathrm{u}}$ & $\underline{\mathrm{N}}$ & $\mathbf{Y}$ & $\mathbf{Y}$ & $\mathbf{Y}$ & $\mathbf{Y}$ & $\mathbf{Y}$ & $\mathbf{Y}$ & $\mathbf{Y}$ & $\mathbf{Y}$ & $\mathbf{Y}$ & EA & $\mathrm{ES}_{\mathrm{u}}$ & $\underline{N}$ & $Y$ & $\mathbf{Y}$ & $\mathbf{Y}$ & $Y$ & $\mathbf{Y}$ & $\mathbf{Y}$ & $\mathbf{Y}$ & $Y$ & $\mathbf{Y}$ \\
\hline EE & EA & $\mathbf{Y}$ & $\mathbf{Y}$ & $\mathbf{Y}$ & $\mathbf{Y}$ & $\mathbf{Y}$ & $\mathbf{Y}$ & $\mathbf{Y}$ & $\mathbf{Y}$ & $Y$ & $\mathbf{Y}$ & EE & EA & $\mathbf{Y}$ & $Y$ & $Y$ & $\mathbf{Y}$ & $Y$ & $\mathbf{Y}$ & $Y$ & $\mathbf{Y}$ & $Y$ & $\mathbf{Y}$ \\
\hline $\mathrm{EE}$ & EL & $\underline{\mathrm{N}}$ & $\mathbf{Y}$ & $\underline{\mathrm{N}}$ & $\underline{N}$ & $\underline{N}$ & $\underline{N}$ & $\underline{N}$ & $\mathbf{Y}$ & $\underline{N}$ & $\mathbf{Y}$ & EE & EL & $\underline{N}$ & $\underline{N}$ & $Y$ & $\mathbf{Y}$ & $Y$ & $\mathbf{Y}$ & $Y$ & $Y$ & $Y$ & $\mathbf{Y}$ \\
\hline $\mathrm{EE}$ & ES & $\underline{\mathrm{N}}$ & $\mathbf{Y}$ & $\mathbf{Y}$ & $\mathbf{Y}$ & $\mathbf{Y}$ & $\mathbf{Y}$ & $\mathbf{Y}$ & $\mathbf{Y}$ & $\mathbf{Y}$ & $\mathbf{Y}$ & EE & ES & $\mathbf{Y}$ & $Y$ & $Y$ & $\mathbf{Y}$ & $Y$ & $\mathbf{Y}$ & $Y$ & $\mathbf{Y}$ & $Y$ & $\mathbf{Y}$ \\
\hline $\mathrm{EE}$ & $\mathrm{ES}_{\mathrm{b}}$ & $\underline{\mathrm{N}}$ & $\underline{\mathrm{N}}$ & $\underline{\mathrm{N}}$ & $Y$ & $Y$ & $\mathbf{Y}$ & $\mathbf{Y}$ & $\mathbf{Y}$ & $\mathbf{Y}$ & $\mathbf{Y}$ & $\mathrm{EE}$ & $\mathrm{ES}_{\mathrm{b}}$ & $\underline{N}$ & $\underline{N}$ & $\underline{N}$ & $\mathbf{Y}$ & $Y$ & $\mathbf{Y}$ & $Y$ & $Y$ & $\mathbf{Y}$ & $Y$ \\
\hline $\mathrm{EE}$ & $\mathrm{ES}_{\mathrm{u}}$ & $\underline{\mathrm{N}}$ & $\underline{\mathrm{N}}$ & $\underline{N}$ & $\mathbf{Y}$ & $Y$ & $\mathbf{Y}$ & $\mathbf{Y}$ & $\mathbf{Y}$ & $\mathbf{Y}$ & $\mathbf{Y}$ & $\mathrm{EE}$ & $\mathrm{ES}_{\mathrm{u}}$ & $\underline{N}$ & $\underline{N}$ & $\underline{N}$ & $\mathbf{Y}$ & $Y$ & $\mathbf{Y}$ & $Y$ & $\mathbf{Y}$ & $Y$ & $\mathbf{Y}$ \\
\hline EL & EA & $\underline{\mathrm{N}}$ & $\mathbf{Y}$ & $\mathbf{Y}$ & $\mathbf{Y}$ & $\mathbf{Y}$ & $\mathbf{Y}$ & $\mathbf{Y}$ & $\mathbf{Y}$ & $\mathbf{Y}$ & $\mathbf{Y}$ & EL & EA & $\underline{\mathrm{N}}$ & $Y$ & $\mathbf{Y}$ & $\mathbf{Y}$ & $Y$ & $\mathbf{Y}$ & $Y$ & $Y$ & $Y$ & $\mathbf{Y}$ \\
\hline EL & EE & $\underline{\mathrm{N}}$ & $\mathbf{Y}$ & $\underline{\mathrm{N}}$ & $\underline{N}$ & $\underline{\mathrm{N}}$ & $\underline{\mathrm{N}}$ & $\underline{N}$ & $\mathbf{Y}$ & $\underline{N}$ & $\mathbf{Y}$ & EL & $\mathrm{EE}$ & $\underline{\mathrm{N}}$ & $\underline{N}$ & $\mathbf{Y}$ & $\mathbf{Y}$ & $Y$ & $\mathbf{Y}$ & $Y$ & $Y$ & $Y$ & $\mathbf{Y}$ \\
\hline EL & ES & $\underline{\mathrm{N}}$ & $\mathbf{Y}$ & $\mathbf{Y}$ & $\mathbf{Y}$ & $\mathbf{Y}$ & $\mathbf{Y}$ & $\mathbf{Y}$ & $\mathbf{Y}$ & $\mathbf{Y}$ & $\mathbf{Y}$ & EL & ES & $\underline{\mathrm{N}}$ & $Y$ & $Y$ & $\mathbf{Y}$ & $Y$ & $\mathbf{Y}$ & $Y$ & $Y$ & $Y$ & $\mathbf{Y}$ \\
\hline EL & $\mathrm{ES}_{\mathrm{b}}$ & $\underline{N}$ & $\underline{\mathrm{N}}$ & $\underline{N}$ & $\mathbf{Y}$ & $\mathbf{Y}$ & $\underline{\mathrm{N}}$ & $\mathbf{Y}$ & $\mathbf{Y}$ & $\mathbf{Y}$ & $\mathbf{Y}$ & EL & $\mathrm{ES}_{\mathrm{b}}$ & $\underline{N}$ & $\underline{N}$ & $\underline{N}$ & $\underline{\mathrm{N}}$ & $Y$ & $\mathbf{Y}$ & $Y$ & $Y$ & $Y$ & $\mathbf{Y}$ \\
\hline EL & $\mathrm{ES}_{\mathrm{u}}$ & $\underline{N}$ & $\underline{N}$ & $\underline{N}$ & $\underline{N}$ & $\underline{N}$ & $\mathbf{Y}$ & $\underline{N}$ & $\underline{N}$ & $\mathbf{Y}$ & $\mathbf{Y}$ & EL & $\mathrm{ES}_{\mathrm{u}}$ & $\underline{N}$ & $\underline{N}$ & $\underline{N}$ & $\underline{N}$ & $\underline{N}$ & $\mathbf{Y}$ & $Y$ & $Y$ & $Y$ & $\mathbf{Y}$ \\
\hline ES & EA & $\underline{\mathrm{N}}$ & $\mathbf{Y}$ & $\mathbf{Y}$ & $\mathbf{Y}$ & $\mathbf{Y}$ & $\mathbf{Y}$ & $\mathbf{Y}$ & $\underline{N}$ & $\underline{N}$ & $\underline{N}$ & ES & EA & $\underline{N}$ & $Y$ & $Y$ & $\mathbf{Y}$ & $Y$ & $\mathbf{Y}$ & $Y$ & $\mathbf{Y}$ & $Y$ & $\underline{\mathrm{N}}$ \\
\hline ES & $\mathrm{EE}$ & $\underline{\mathrm{N}}$ & $\mathbf{Y}$ & $\mathbf{Y}$ & $\mathbf{Y}$ & $\mathbf{Y}$ & $\mathbf{Y}$ & $\mathbf{Y}$ & $\mathbf{Y}$ & $\mathbf{Y}$ & $\mathbf{Y}$ & ES & EE & $\mathbf{Y}$ & $Y$ & $\mathbf{Y}$ & $\mathbf{Y}$ & $Y$ & $\mathbf{Y}$ & $Y$ & $Y$ & $\mathbf{Y}$ & $\mathbf{Y}$ \\
\hline ES & EL & $\underline{\mathrm{N}}$ & $\mathbf{Y}$ & $\mathbf{Y}$ & $\mathbf{Y}$ & $\mathbf{Y}$ & $\mathbf{Y}$ & $\mathbf{Y}$ & $\mathbf{Y}$ & $\mathbf{Y}$ & $\mathbf{Y}$ & ES & EL & $\underline{\mathrm{N}}$ & $Y$ & $Y$ & $\mathbf{Y}$ & $Y$ & $\mathbf{Y}$ & $Y$ & $\mathbf{Y}$ & $\mathbf{Y}$ & $\mathbf{Y}$ \\
\hline ES & $\mathrm{ES}_{\mathrm{b}}$ & $\underline{\mathrm{N}}$ & $\underline{N}$ & $\mathbf{Y}$ & $\mathbf{Y}$ & $\mathbf{Y}$ & $\mathbf{Y}$ & $\mathbf{Y}$ & $\mathbf{Y}$ & $\mathbf{Y}$ & $\mathbf{Y}$ & ES & $\mathrm{ES}_{\mathrm{b}}$ & $\underline{\mathrm{N}}$ & $\underline{N}$ & $Y$ & $\mathbf{Y}$ & $Y$ & $\mathbf{Y}$ & $Y$ & $\mathbf{Y}$ & $\mathbf{Y}$ & $\mathbf{Y}$ \\
\hline ES & $\mathrm{ES}_{\mathrm{u}}$ & $\underline{\mathrm{N}}$ & $\underline{\mathrm{N}}$ & $\mathbf{Y}$ & $\mathbf{Y}$ & $\mathbf{Y}$ & $\mathbf{Y}$ & $\mathbf{Y}$ & $Y$ & $Y$ & $\mathbf{Y}$ & ES & $\mathrm{ES}_{\mathrm{u}}$ & $\underline{\mathrm{N}}$ & $Y$ & $Y$ & $Y$ & $Y$ & $\mathbf{Y}$ & $Y$ & $\mathbf{Y}$ & $\mathbf{Y}$ & $\mathbf{Y}$ \\
\hline $\mathrm{ES}_{\mathrm{b}}$ & EA & $\underline{\mathrm{N}}$ & $\mathbf{Y}$ & $\mathbf{Y}$ & $\mathbf{Y}$ & $\mathbf{Y}$ & $\mathbf{Y}$ & $\mathbf{Y}$ & $\mathbf{Y}$ & $\mathbf{Y}$ & $\mathbf{Y}$ & $\mathrm{ES}_{\mathrm{b}}$ & EA & $\underline{N}$ & $Y$ & $Y$ & $\mathbf{Y}$ & $Y$ & $\mathbf{Y}$ & $Y$ & $\mathbf{Y}$ & $\mathbf{Y}$ & $Y$ \\
\hline
\end{tabular}




\begin{tabular}{|c|c|c|c|c|c|c|c|c|c|c|c|c|c|c|c|c|c|c|c|c|c|c|c|}
\hline $\mathrm{ES}_{\mathrm{b}}$ & $\mathrm{EE}$ & $\underline{\mathrm{N}}$ & $\underline{\mathrm{N}}$ & $\underline{\mathrm{N}}$ & $\mathbf{Y}$ & $\mathbf{Y}$ & $Y$ & $\mathbf{Y}$ & $\mathbf{Y}$ & $\mathbf{Y}$ & $\mathbf{Y}$ & $\mathrm{ES}_{\mathrm{b}}$ & $\mathrm{EE}$ & $\underline{\mathrm{N}}$ & $\underline{N}$ & $\underline{N}$ & $\mathbf{Y}$ & $\mathbf{Y}$ & $\mathbf{Y}$ & $\mathbf{Y}$ & $\mathbf{Y}$ & $\mathbf{Y}$ & $\mathbf{Y}$ \\
\hline $\mathrm{ES}_{\mathrm{b}}$ & EL & $\underline{\mathrm{N}}$ & $\underline{N}$ & $\underline{\mathrm{N}}$ & $\mathbf{Y}$ & $\mathbf{Y}$ & $\underline{N}$ & $\mathbf{Y}$ & $\mathbf{Y}$ & $\mathbf{Y}$ & $\mathbf{Y}$ & $\mathrm{ES}_{\mathrm{b}}$ & EL & $\underline{\mathrm{N}}$ & $\underline{N}$ & $\underline{N}$ & $\underline{N}$ & $\mathbf{Y}$ & $\mathbf{Y}$ & $\mathbf{Y}$ & $Y$ & $\mathbf{Y}$ & $\mathbf{Y}$ \\
\hline $\mathrm{ES}_{\mathrm{b}}$ & ES & $\underline{\mathrm{N}}$ & $\underline{N}$ & $\mathbf{Y}$ & $\mathbf{Y}$ & $\mathbf{Y}$ & $Y$ & $\mathbf{Y}$ & $\mathbf{Y}$ & $\mathbf{Y}$ & $\mathbf{Y}$ & $\mathrm{ES}_{\mathrm{b}}$ & ES & $\underline{\mathrm{N}}$ & $\underline{N}$ & $\mathbf{Y}$ & $\mathbf{Y}$ & $\mathbf{Y}$ & $\mathbf{Y}$ & $\mathbf{Y}$ & $\mathbf{Y}$ & $Y$ & $Y$ \\
\hline $\mathrm{ES}_{\mathrm{b}}$ & $\mathrm{ES}_{\mathrm{u}}$ & $\underline{\mathrm{N}}$ & $\underline{N}$ & $\underline{N}$ & $\underline{N}$ & $\underline{N}$ & $Y$ & $\mathbf{Y}$ & $\mathbf{Y}$ & $Y$ & $\mathbf{Y}$ & $\mathrm{ES}_{\mathrm{b}}$ & $\mathrm{ES}_{\mathrm{u}}$ & $\underline{\mathrm{N}}$ & $\underline{N}$ & $\underline{N}$ & $\underline{N}$ & $\mathbf{Y}$ & $\mathbf{Y}$ & $\mathbf{Y}$ & $Y$ & $\mathbf{Y}$ & $Y$ \\
\hline $\mathrm{ES}_{\mathrm{u}}$ & EA & $\underline{\mathrm{N}}$ & $\mathbf{Y}$ & $Y$ & $\mathbf{Y}$ & $Y$ & $\mathbf{Y}$ & $\mathbf{Y}$ & $Y$ & $Y$ & $\mathbf{Y}$ & $\mathrm{ES}_{\mathrm{u}}$ & EA & $\underline{N}$ & $Y$ & $\mathbf{Y}$ & $\mathbf{Y}$ & $\mathbf{Y}$ & $\mathbf{Y}$ & $\mathbf{Y}$ & $Y$ & $Y$ & $\mathbf{Y}$ \\
\hline $\mathrm{ES}_{\mathrm{u}}$ & $\mathrm{EE}$ & $\underline{N}$ & $\underline{N}$ & $\underline{N}$ & $\mathbf{Y}$ & $\mathbf{Y}$ & $\mathbf{Y}$ & $Y$ & $\mathbf{Y}$ & $\mathbf{Y}$ & $\mathbf{Y}$ & $\mathrm{ES}_{\mathrm{u}}$ & EE & $\underline{\mathrm{N}}$ & $\underline{N}$ & $\underline{\mathrm{N}}$ & $\mathbf{Y}$ & $\mathbf{Y}$ & $\mathbf{Y}$ & $\mathbf{Y}$ & $\mathbf{Y}$ & $Y$ & $Y$ \\
\hline $\mathrm{ES}_{\mathrm{u}}$ & EL & $\underline{\mathrm{N}}$ & $\underline{N}$ & $\underline{\mathrm{N}}$ & $\underline{N}$ & $\underline{N}$ & $\mathbf{Y}$ & $\underline{N}$ & $\underline{\mathrm{N}}$ & $\mathbf{Y}$ & $\mathbf{Y}$ & $\mathrm{ES}_{\mathrm{u}}$ & $E L$ & $\underline{\mathrm{N}}$ & $\underline{N}$ & $\underline{\mathrm{N}}$ & $\underline{N}$ & $\underline{\mathrm{N}}$ & $\mathbf{Y}$ & $\mathbf{Y}$ & $\mathbf{Y}$ & $Y$ & $Y$ \\
\hline $\mathrm{ES}_{\mathrm{u}}$ & ES & $\underline{\mathrm{N}}$ & $\underline{N}$ & $\mathbf{Y}$ & $\mathbf{Y}$ & $\mathbf{Y}$ & $Y$ & $\mathbf{Y}$ & $\mathbf{Y}$ & $\mathbf{Y}$ & $\mathbf{Y}$ & $\mathrm{ES}_{\mathrm{u}}$ & ES & $\underline{\mathrm{N}}$ & $Y$ & $\mathbf{Y}$ & $Y$ & $\mathbf{Y}$ & $\mathbf{Y}$ & $\mathbf{Y}$ & $Y$ & $Y$ & $Y$ \\
\hline $\mathrm{ES}_{\mathrm{u}}$ & $\mathrm{ES}_{\mathrm{b}}$ & $\underline{\mathrm{N}}$ & $\underline{\mathrm{N}}$ & $\underline{N}$ & $\underline{N}$ & $\underline{N}$ & $\mathbf{Y}$ & $Y$ & $\mathbf{Y}$ & $\mathbf{Y}$ & $\mathbf{Y}$ & $\mathrm{ES}_{\mathrm{u}}$ & $\mathrm{ES}_{\mathrm{b}}$ & $\underline{\mathrm{N}}$ & $\underline{N}$ & $\underline{\mathrm{N}}$ & $\underline{\mathrm{N}}$ & $\mathbf{Y}$ & $\mathbf{Y}$ & $\mathbf{Y}$ & $\mathbf{Y}$ & $Y$ & $Y$ \\
\hline
\end{tabular}


For lenticular primaries in Table 5-11, larger fitting ranges generally yield dissimilarity. The LE vs. LL comparisons show little dissimilarity in general. This suggests that clustering of early-type galaxies around $L$ is generally similar for the same fitting range.

It also appears that for $L$ primaries below $5^{\circ} S_{b}$ and $S_{u}$ secondaries cluster more like early-type galaxies than $\mathrm{S}$.

Table 5-11 2D KS Test Results - comparing two runs having the same primary filter and same fitting range filter with different secondary filters - limited to lenticular primaries.

$\mathrm{Y}=$ dissimilar to the $99 \%$ level. $\mathrm{N}=$ not dissimilar to the $99 \%$ level

\begin{tabular}{|c|c|c|c|c|c|c|c|c|c|c|c|c|c|c|c|c|c|c|c|c|c|c|c|}
\hline \multicolumn{12}{|c|}{ North } & \multicolumn{12}{|c|}{ South } \\
\hline \multirow[b]{2}{*}{ Run1 } & \multicolumn{11}{|c|}{ Fitting Range (degree) } & \multicolumn{12}{|c|}{ Fitting Range (degree) } \\
\hline & Run2 & 1 & 2 & 3 & 4 & 5 & 10 & 15 & 20 & 25 & 30 & Run1 & Run2 & 1 & 2 & 3 & 4 & 5 & 10 & 15 & 20 & 25 & 30 \\
\hline LA & LE & $\mathbf{Y}$ & $\mathbf{Y}$ & $\mathbf{Y}$ & $\mathbf{Y}$ & $\mathbf{Y}$ & $\mathbf{Y}$ & $\mathbf{Y}$ & $\mathbf{Y}$ & $\mathbf{Y}$ & $\mathbf{Y}$ & LA & LE & $\underline{N}$ & $\mathbf{Y}$ & $\mathbf{Y}$ & $\mathbf{Y}$ & $\mathbf{Y}$ & $\mathbf{Y}$ & $\mathbf{Y}$ & $\mathbf{Y}$ & $\mathbf{Y}$ & $\mathbf{Y}$ \\
\hline LA & LL & $\underline{N}$ & $\mathbf{Y}$ & $\mathbf{Y}$ & $\mathbf{Y}$ & $\mathbf{Y}$ & $\mathbf{Y}$ & $\mathbf{Y}$ & $\mathbf{Y}$ & 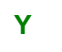 & $\mathbf{Y}$ & LA & LL & $\underline{N}$ & $\mathbf{Y}$ & $\mathbf{Y}$ & $\mathbf{Y}$ & $\mathbf{Y}$ & $\mathbf{Y}$ & $\mathbf{Y}$ & $\mathbf{Y}$ & $\mathbf{Y}$ & $Y$ \\
\hline LA & LS & $\underline{N}$ & $\mathbf{Y}$ & $\mathbf{Y}$ & $\mathbf{Y}$ & $Y$ & $\mathbf{Y}$ & $\mathbf{Y}$ & $\mathbf{Y}$ & $\underline{\mathrm{N}}$ & $\underline{N}$ & LA & LS & $\underline{N}$ & $Y$ & $\mathbf{Y}$ & $Y$ & $\mathbf{Y}$ & $\mathbf{Y}$ & $\mathbf{Y}$ & $\mathbf{Y}$ & $\underline{\mathrm{N}}$ & $\underline{N}$ \\
\hline LA & $\mathrm{LS}_{\mathrm{b}}$ & $\mathbf{Y}$ & $\mathbf{Y}$ & $Y$ & $\mathbf{Y}$ & $Y$ & $\mathbf{Y}$ & $\mathbf{Y}$ & $\mathbf{Y}$ & $Y$ & $\mathbf{Y}$ & LA & $\mathrm{LS}_{\mathrm{b}}$ & $\underline{N}$ & $Y$ & $\mathbf{Y}$ & $Y$ & $\mathbf{Y}$ & $Y$ & $\mathbf{Y}$ & $\mathbf{Y}$ & $\mathbf{Y}$ & $Y$ \\
\hline LA & $\mathrm{LS}_{\mathrm{u}}$ & $\underline{N}$ & $Y$ & $\mathbf{Y}$ & $Y$ & $Y$ & $\mathbf{Y}$ & $\mathbf{Y}$ & $\mathbf{Y}$ & $\mathbf{Y}$ & $\mathbf{Y}$ & LA & $\mathrm{LS}_{\mathrm{u}}$ & $\underline{\mathrm{N}}$ & $Y$ & $\mathbf{Y}$ & $Y$ & $\mathbf{Y}$ & $Y$ & $\mathbf{Y}$ & $\mathbf{Y}$ & $Y$ & $Y$ \\
\hline LE & LA & $\mathbf{Y}$ & $\mathbf{Y}$ & $\mathbf{Y}$ & $\mathbf{Y}$ & $Y$ & $\mathbf{Y}$ & $\mathbf{Y}$ & $\mathbf{Y}$ & $\mathbf{Y}$ & $\mathbf{Y}$ & LE & LA & $\underline{\mathrm{N}}$ & $Y$ & $\mathbf{Y}$ & $Y$ & $\mathbf{Y}$ & $\mathbf{Y}$ & $\mathbf{Y}$ & $\mathbf{Y}$ & $\mathbf{Y}$ & $\mathbf{Y}$ \\
\hline LE & LL & $\underline{N}$ & $\underline{N}$ & $\underline{N}$ & $\underline{N}$ & $\underline{N}$ & $\underline{N}$ & $\underline{N}$ & $\underline{N}$ & $\underline{N}$ & $\underline{N}$ & LE & LL & $\underline{N}$ & $\underline{N}$ & $\underline{N}$ & $\underline{N}$ & $\underline{N}$ & $\underline{\mathrm{N}}$ & $\mathbf{Y}$ & $\mathbf{Y}$ & $Y$ & $Y$ \\
\hline LE & LS & $\underline{\mathrm{N}}$ & $\mathbf{Y}$ & $\mathbf{Y}$ & $\mathbf{Y}$ & $Y$ & $Y$ & $\mathbf{Y}$ & $\mathrm{Y}$ & $\mathbf{Y}$ & $\mathbf{Y}$ & LE & LS & $\underline{N}$ & $Y$ & $\mathbf{Y}$ & $Y$ & $\mathbf{Y}$ & $\mathbf{Y}$ & $\mathbf{Y}$ & $\mathbf{Y}$ & $\mathbf{Y}$ & $Y$ \\
\hline LE & $\mathrm{LS}_{\mathrm{b}}$ & $\underline{N}$ & $\underline{N}$ & $\underline{N}$ & $\mathbf{Y}$ & $\underline{N}$ & $\mathbf{Y}$ & $\mathbf{Y}$ & $\mathbf{Y}$ & $\underline{N}$ & $\underline{N}$ & LE & $\mathrm{LS}_{\mathrm{b}}$ & $\underline{N}$ & $\underline{N}$ & $\underline{\mathrm{N}}$ & $\underline{N}$ & $\underline{N}$ & $\mathbf{Y}$ & $\mathbf{Y}$ & $Y$ & $\mathbf{Y}$ & $\mathbf{Y}$ \\
\hline LE & $\mathrm{LS}_{\mathrm{u}}$ & $\underline{N}$ & $\underline{N}$ & $\underline{N}$ & $\underline{\mathrm{N}}$ & $\underline{N}$ & $\mathbf{Y}$ & $\underline{N}$ & $\mathbf{Y}$ & $Y$ & $\mathbf{Y}$ & LE & $\mathrm{LS}_{\mathrm{u}}$ & $\underline{N}$ & $\underline{N}$ & $\underline{N}$ & $\underline{N}$ & $\underline{N}$ & $\mathbf{Y}$ & $\mathbf{Y}$ & $\mathbf{Y}$ & $Y$ & $Y$ \\
\hline LL & LA & $\underline{N}$ & $Y$ & $\mathbf{Y}$ & $\mathbf{Y}$ & $Y$ & $\mathbf{Y}$ & $\mathbf{Y}$ & $\mathbf{Y}$ & $Y$ & $\mathbf{Y}$ & LL & LA & $\underline{N}$ & $Y$ & $\mathbf{Y}$ & $Y$ & $\mathbf{Y}$ & $\mathbf{Y}$ & $Y$ & $Y$ & $Y$ & $Y$ \\
\hline LL & LE & $\underline{\mathrm{N}}$ & $\underline{\mathrm{N}}$ & $\underline{N}$ & $\underline{\mathrm{N}}$ & $\underline{N}$ & $\underline{\mathrm{N}}$ & $\underline{\mathrm{N}}$ & $\underline{N}$ & $\underline{N}$ & $\underline{N}$ & LL & LE & $\underline{N}$ & $\underline{N}$ & $\underline{\mathrm{N}}$ & $\underline{N}$ & $\underline{N}$ & $\underline{\mathrm{N}}$ & $\mathbf{Y}$ & $\mathbf{Y}$ & $\mathbf{Y}$ & $Y$ \\
\hline LL & LS & $\underline{\mathrm{N}}$ & $\mathbf{Y}$ & $\mathbf{Y}$ & $\mathbf{Y}$ & $\mathbf{Y}$ & $\mathbf{Y}$ & $\mathbf{Y}$ & $\mathbf{Y}$ & $\mathbf{Y}$ & $\mathbf{Y}$ & LL & LS & $\underline{N}$ & $Y$ & $\mathbf{Y}$ & $Y$ & $\mathbf{Y}$ & $\mathbf{Y}$ & $\mathbf{Y}$ & $\mathbf{Y}$ & $\mathbf{Y}$ & $Y$ \\
\hline LL & $\mathrm{LS}_{\mathrm{b}}$ & $\mathbf{Y}$ & $\underline{N}$ & $\underline{N}$ & $\mathbf{Y}$ & $Y$ & $\mathbf{Y}$ & $\underline{N}$ & $\underline{N}$ & $\underline{N}$ & $\mathbf{Y}$ & LL & $\mathrm{LS}_{\mathrm{b}}$ & $\underline{N}$ & $\underline{N}$ & $\underline{N}$ & $Y$ & $\mathbf{Y}$ & $\mathbf{Y}$ & $Y$ & $Y$ & $Y$ & $Y$ \\
\hline LL & $\mathrm{LS}_{\mathrm{u}}$ & $\underline{N}$ & $\underline{\mathrm{N}}$ & $\underline{N}$ & $\mathbf{Y}$ & $Y$ & $\mathbf{Y}$ & $\mathbf{Y}$ & $\mathbf{Y}$ & $Y$ & $\mathbf{Y}$ & LL & $\mathrm{LS}_{\mathrm{u}}$ & $\underline{N}$ & $\underline{N}$ & $\underline{\mathrm{N}}$ & $\underline{N}$ & $Y$ & $Y$ & $Y$ & $Y$ & $Y$ & $Y$ \\
\hline LS & LA & $\underline{N}$ & $\mathbf{Y}$ & $\mathbf{Y}$ & $\mathbf{Y}$ & $\mathbf{Y}$ & $\mathbf{Y}$ & $\mathbf{Y}$ & $\mathbf{Y}$ & $\underline{N}$ & $\underline{N}$ & LS & LA & $\underline{N}$ & $Y$ & $\mathbf{Y}$ & $Y$ & $\mathbf{Y}$ & $\mathbf{Y}$ & $\mathbf{Y}$ & $\mathbf{Y}$ & $\underline{\mathrm{N}}$ & $\underline{N}$ \\
\hline LS & LE & $\underline{N}$ & $\mathbf{Y}$ & $\mathbf{Y}$ & $\mathbf{Y}$ & $\mathbf{Y}$ & $\mathbf{Y}$ & $\mathbf{Y}$ & $\mathbf{Y}$ & $\mathbf{Y}$ & $\mathbf{Y}$ & LS & LE & $\underline{N}$ & $Y$ & $\mathbf{Y}$ & $Y$ & $\mathbf{Y}$ & $\mathbf{Y}$ & $\mathbf{Y}$ & $\mathbf{Y}$ & $\mathbf{Y}$ & $\mathbf{Y}$ \\
\hline LS & LL & $\underline{\mathrm{N}}$ & $\mathbf{Y}$ & $\mathbf{Y}$ & $\mathbf{Y}$ & $Y$ & $\mathbf{Y}$ & $\mathbf{Y}$ & $\mathbf{Y}$ & $\mathbf{Y}$ & $\mathbf{Y}$ & LS & LL & $\underline{N}$ & $Y$ & $\mathbf{Y}$ & $Y$ & $\mathbf{Y}$ & $\mathbf{Y}$ & $\mathbf{Y}$ & $\mathbf{Y}$ & $\mathbf{Y}$ & $\mathbf{Y}$ \\
\hline LS & $\mathrm{LS}_{\mathrm{b}}$ & $\underline{N}$ & $\mathbf{Y}$ & $\mathbf{Y}$ & $\mathbf{Y}$ & $Y$ & $\mathbf{Y}$ & $\mathbf{Y}$ & $Y$ & $Y$ & $\mathbf{Y}$ & LS & $\mathrm{LS}_{\mathrm{b}}$ & $\underline{N}$ & $\underline{N}$ & $\mathbf{Y}$ & $Y$ & $\mathbf{Y}$ & $\mathbf{Y}$ & $Y$ & $Y$ & $Y$ & $Y$ \\
\hline LS & $\mathrm{LS}_{\mathrm{u}}$ & $\underline{N}$ & $\mathbf{Y}$ & $\mathbf{Y}$ & $\mathbf{Y}$ & $\mathbf{Y}$ & $\mathbf{Y}$ & $\mathbf{Y}$ & $Y$ & $Y$ & $\mathbf{Y}$ & LS & $\mathrm{LS}_{\mathrm{u}}$ & $\underline{N}$ & $\mathbf{Y}$ & $\mathbf{Y}$ & $\mathbf{Y}$ & $\mathbf{Y}$ & $\mathbf{Y}$ & $\mathbf{Y}$ & $Y$ & $Y$ & $Y$ \\
\hline $\mathrm{LS}_{\mathrm{b}}$ & LA & $\mathbf{Y}$ & $\mathbf{Y}$ & $\mathbf{Y}$ & $\mathbf{Y}$ & $Y$ & $\mathbf{Y}$ & $\mathbf{Y}$ & $Y$ & $Y$ & $\mathbf{Y}$ & $\mathrm{LS}_{\mathrm{b}}$ & LA & $\underline{N}$ & $\mathbf{Y}$ & $\mathbf{Y}$ & $Y$ & $\mathbf{Y}$ & $Y$ & $Y$ & $Y$ & $Y$ & $Y$ \\
\hline $\mathrm{LS}_{\mathrm{b}}$ & LE & $\underline{\mathrm{N}}$ & $\underline{\mathrm{N}}$ & $\underline{\mathrm{N}}$ & $\mathbf{Y}$ & $\underline{N}$ & $\mathbf{Y}$ & $Y$ & $Y$ & $\underline{N}$ & $\underline{N}$ & $\mathrm{LS}_{\mathrm{b}}$ & LE & $\underline{N}$ & $\underline{N}$ & $\underline{\mathrm{N}}$ & $\underline{N}$ & $\underline{\mathrm{N}}$ & $\mathbf{Y}$ & $\mathbf{Y}$ & $\mathbf{Y}$ & $\mathbf{Y}$ & $Y$ \\
\hline $\mathrm{LS}_{\mathrm{b}}$ & LL & $\mathbf{Y}$ & $\underline{N}$ & $\underline{N}$ & $\mathbf{Y}$ & $Y$ & $\mathbf{Y}$ & $\underline{N}$ & $\underline{N}$ & $\underline{N}$ & $\mathbf{Y}$ & $\mathrm{LS}_{\mathrm{b}}$ & LL & $\underline{N}$ & $\underline{N}$ & $\underline{N}$ & $Y$ & $\mathbf{Y}$ & $\mathbf{Y}$ & $\mathbf{Y}$ & $\mathbf{Y}$ & $\mathbf{Y}$ & $Y$ \\
\hline
\end{tabular}




\begin{tabular}{|c|c|c|c|c|c|c|c|c|c|c|c|c|c|c|c|c|c|c|c|c|c|c|c|}
\hline $\mathrm{LS}_{\mathrm{b}}$ & LS & $\underline{N}$ & $\mathbf{Y}$ & $\mathbf{Y}$ & $\mathbf{Y}$ & $\mathbf{Y}$ & $\mathbf{Y}$ & $\mathbf{Y}$ & $\mathbf{Y}$ & $\mathbf{Y}$ & $\mathbf{Y}$ & $\mathrm{LS}_{\mathrm{b}}$ & LS & $\underline{N}$ & $\underline{\mathrm{N}}$ & $\mathbf{Y}$ & $\mathbf{Y}$ & $\mathbf{Y}$ & $Y$ & $\mathbf{Y}$ & $Y$ & $Y$ & $\mathbf{Y}$ \\
\hline $\mathrm{LS}_{\mathrm{b}}$ & $\mathrm{LS}_{\mathrm{u}}$ & $\underline{\mathrm{N}}$ & $\underline{N}$ & $\underline{N}$ & $\underline{N}$ & $\underline{N}$ & $\underline{N}$ & $\mathbf{Y}$ & $\mathbf{Y}$ & $\mathbf{Y}$ & $\mathbf{Y}$ & $\mathrm{LS}_{\mathrm{b}}$ & $\mathrm{LS}_{\mathrm{u}}$ & $\underline{N}$ & $\underline{\mathrm{N}}$ & $\underline{\mathrm{N}}$ & $\underline{N}$ & $\underline{N}$ & $Y$ & $\mathbf{Y}$ & $Y$ & $Y$ & $\mathrm{Y}$ \\
\hline $\mathrm{LS}_{\mathrm{u}}$ & LA & $\underline{N}$ & $\mathbf{Y}$ & $Y$ & $\mathbf{Y}$ & $Y$ & $\mathbf{Y}$ & $\mathbf{Y}$ & $\mathbf{Y}$ & $Y$ & $\mathbf{Y}$ & $\mathrm{LS}_{\mathrm{u}}$ & LA & $\underline{N}$ & $\mathbf{Y}$ & $Y$ & $Y$ & $\mathbf{Y}$ & $Y$ & $\mathbf{Y}$ & $Y$ & $Y$ & $Y$ \\
\hline $\mathrm{LS}_{\mathrm{u}}$ & LE & $\underline{N}$ & $\underline{N}$ & $\underline{N}$ & $\underline{N}$ & $\underline{N}$ & $\mathbf{Y}$ & $\underline{N}$ & $\mathbf{Y}$ & $\mathbf{Y}$ & $\mathbf{Y}$ & $\mathrm{LS}_{\mathrm{u}}$ & LE & $\underline{N}$ & $\underline{N}$ & $\underline{\mathrm{N}}$ & $\underline{N}$ & $\underline{\mathrm{N}}$ & $Y$ & $Y$ & $Y$ & $Y$ & $Y$ \\
\hline $\mathrm{LS}_{\mathrm{u}}$ & LL & $\underline{N}$ & $\underline{N}$ & $\underline{N}$ & $\mathbf{Y}$ & $\mathbf{Y}$ & $\mathbf{Y}$ & $\mathbf{Y}$ & $\mathbf{Y}$ & $Y$ & $\mathbf{Y}$ & $\mathrm{LS}_{\mathrm{u}}$ & LL & $\underline{N}$ & $\underline{N}$ & $\underline{N}$ & $\underline{N}$ & $\mathbf{Y}$ & $\mathbf{Y}$ & $\mathbf{Y}$ & $Y$ & $Y$ & $Y$ \\
\hline $\mathrm{LS}_{\mathrm{u}}$ & LS & $\underline{N}$ & $Y$ & $Y$ & $Y$ & $Y$ & $\mathbf{Y}$ & $\mathbf{Y}$ & $Y$ & $\mathbf{Y}$ & $\mathbf{Y}$ & $\mathrm{LS}_{\mathrm{u}}$ & LS & $\underline{\mathrm{N}}$ & $Y$ & $\mathbf{Y}$ & $Y$ & $\mathbf{Y}$ & $\mathbf{Y}$ & $\mathbf{Y}$ & $Y$ & $Y$ & $\mathbf{Y}$ \\
\hline $\mathrm{LS}_{\mathrm{u}}$ & $\mathrm{LS}_{\mathrm{b}}$ & $\underline{N}$ & $\underline{N}$ & $\underline{N}$ & $\underline{\mathrm{N}}$ & $\underline{N}$ & $\underline{\mathrm{N}}$ & $\mathbf{Y}$ & $Y$ & $\mathbf{Y}$ & $\mathbf{Y}$ & $\mathrm{LS}_{\mathrm{u}}$ & $\mathrm{LS}_{\mathrm{b}}$ & $\underline{\mathrm{N}}$ & $\underline{N}$ & $\underline{N}$ & $\underline{N}$ & $\underline{N}$ & $\mathbf{Y}$ & $\mathbf{Y}$ & $Y$ & $Y$ & $Y$ \\
\hline
\end{tabular}


For spiral primaries in Table 5-12, dissimilarity is found consistently for SA vs. S with some secondary morphology filter above $1^{\circ}$. SE vs. SL clustering shows a general agreement in similarity between north and south below $5^{\circ}$ however above $5^{\circ}$ the two hemispheres disagree.

The clustering of $S_{b}$ and $S_{u}$ secondaries around $S$ primaries behaves more like early-type galaxies than $\mathrm{S}$. They exhibit similarity to larger fitting ranges than $\mathrm{SS}$ vs. $\mathrm{SS}_{\mathrm{b}}$ and SS vs. SSu.

Finally the $\mathrm{SS}_{\mathrm{b}} \mathrm{vs}$. $\mathrm{SS}_{\mathrm{u}}$ comparisons indicate similarity at sufficiently small fitting ranges. This suggests $\mathrm{SS}_{\mathrm{b}}$ and $\mathrm{SS}_{\mathrm{u}}$ cluster in a similar manner around $\mathrm{S}$ primaries.

Table 5-12 2D KS Test Results - comparing two runs having the same primary filter and same fitting range filter with different secondary filters - limited to spiral primaries.

$\mathrm{Y}=$ dissimilar to the $99 \%$ level. $\mathrm{N}=$ not dissimilar to the $99 \%$ level

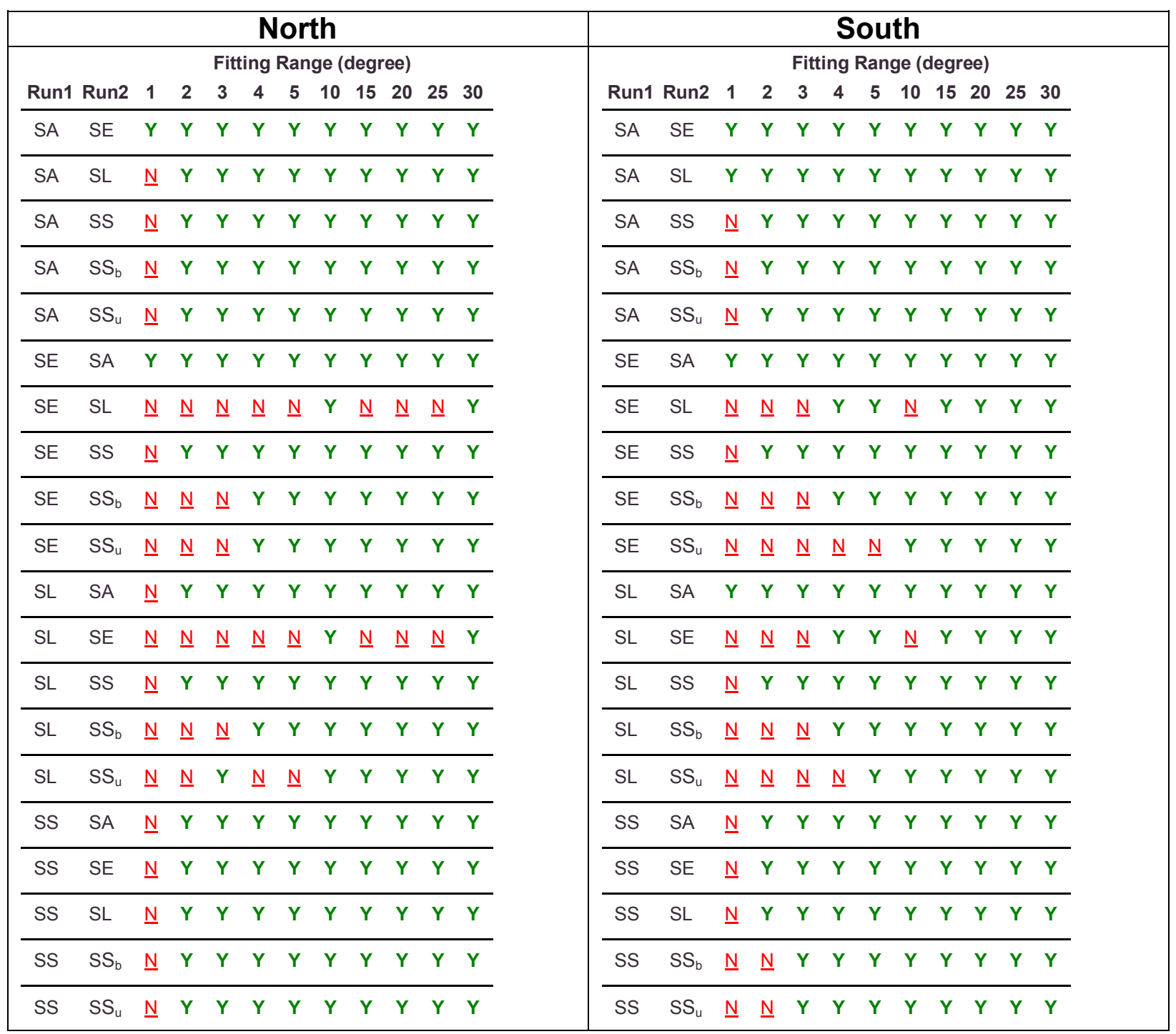




\begin{tabular}{|c|c|c|c|c|c|c|c|c|c|c|c|c|c|c|c|c|c|c|c|c|c|c|c|}
\hline $\mathrm{SS}_{\mathrm{b}}$ & SA & $\underline{N}$ & $\mathbf{Y}$ & $\mathbf{Y}$ & $\mathbf{Y}$ & $\mathbf{Y}$ & $Y$ & $\mathbf{Y}$ & $\mathbf{Y}$ & $\mathbf{Y}$ & $\mathbf{Y}$ & $\mathrm{SS}_{\mathrm{b}}$ & SA & $\underline{\mathrm{N}}$ & $\mathbf{Y}$ & $\mathbf{Y}$ & $\mathbf{Y}$ & $\mathbf{Y}$ & $\mathbf{Y}$ & $\mathbf{Y}$ & $Y$ & $\mathbf{Y}$ & $Y$ \\
\hline $\mathrm{SS}_{\mathrm{b}}$ & SE & $\underline{N}$ & $\underline{\mathrm{N}}$ & $\underline{\mathrm{N}}$ & $\mathbf{Y}$ & $\mathbf{Y}$ & $\mathbf{Y}$ & $\mathbf{Y}$ & $\mathbf{Y}$ & $\mathbf{Y}$ & $\mathbf{Y}$ & $S S_{b}$ & SE & $\underline{\mathrm{N}}$ & $\underline{N}$ & $\underline{N}$ & $\mathbf{Y}$ & $\mathbf{Y}$ & $\mathbf{Y}$ & $Y$ & $Y$ & $Y$ & $Y$ \\
\hline $\mathrm{SS}_{\mathrm{b}}$ & SL & $\underline{\mathrm{N}}$ & $\underline{\mathrm{N}}$ & $\underline{N}$ & $\mathbf{Y}$ & $\mathbf{Y}$ & $\mathbf{Y}$ & $\mathbf{Y}$ & $\mathbf{Y}$ & $\mathbf{Y}$ & $\mathbf{Y}$ & $\mathrm{SS}_{\mathrm{b}}$ & SL & $\underline{\mathrm{N}}$ & $\underline{\mathrm{N}}$ & $\underline{\mathrm{N}}$ & $\mathbf{Y}$ & $\mathbf{Y}$ & $\mathbf{Y}$ & $Y$ & $\mathbf{Y}$ & $\mathbf{Y}$ & $Y$ \\
\hline $\mathrm{SS}_{\mathrm{b}}$ & SS & $\underline{N}$ & $Y$ & $Y$ & $\mathbf{Y}$ & $Y$ & $Y$ & $\mathbf{Y}$ & $\mathbf{Y}$ & $Y$ & $Y$ & $S S_{b}$ & SS & $\underline{\mathrm{N}}$ & $\underline{N}$ & $\mathbf{Y}$ & $Y$ & $\mathbf{Y}$ & $\mathbf{Y}$ & $\mathbf{Y}$ & $\mathbf{Y}$ & $Y$ & $Y$ \\
\hline $\mathrm{SS}_{\mathrm{b}}$ & $\mathrm{SS}_{\mathrm{u}}$ & $\underline{N}$ & $\underline{N}$ & $\underline{N}$ & $\underline{N}$ & $\underline{N}$ & $\underline{\mathrm{N}}$ & $Y$ & $\mathbf{Y}$ & $Y$ & $\mathbf{Y}$ & $S S_{b}$ & $\mathrm{SS}_{\mathrm{u}}$ & $\underline{N}$ & $\underline{N}$ & $\underline{N}$ & $\mathbf{Y}$ & $\mathbf{Y}$ & $\mathbf{Y}$ & $\mathbf{Y}$ & $\mathbf{Y}$ & $\mathbf{Y}$ & $Y$ \\
\hline $\mathrm{SS}_{\mathrm{u}}$ & SA & $\underline{N}$ & $\mathbf{Y}$ & $\mathbf{Y}$ & $\mathbf{Y}$ & $Y$ & $Y$ & $Y$ & $\mathbf{Y}$ & $Y$ & $\mathbf{Y}$ & $\mathrm{SS}_{\mathrm{u}}$ & SA & $\underline{N}$ & $\mathbf{Y}$ & $\mathbf{Y}$ & $\mathbf{Y}$ & $\mathbf{Y}$ & $\mathbf{Y}$ & $\mathbf{Y}$ & $\mathbf{Y}$ & $\mathbf{Y}$ & $Y$ \\
\hline $\mathrm{SS}_{\mathrm{u}}$ & SE & $\underline{N}$ & $\underline{\mathrm{N}}$ & $\underline{\mathrm{N}}$ & $\mathbf{Y}$ & $\mathbf{Y}$ & $\mathbf{Y}$ & $\mathbf{Y}$ & $\mathbf{Y}$ & $\mathbf{Y}$ & $\mathbf{Y}$ & $\mathrm{SS}_{\mathrm{u}}$ & SE & $\underline{\mathrm{N}}$ & $\underline{\mathrm{N}}$ & $\underline{\mathrm{N}}$ & $\underline{N}$ & $\underline{\mathrm{N}}$ & $\mathbf{Y}$ & $\mathbf{Y}$ & $Y$ & $Y$ & $Y$ \\
\hline $\mathrm{SS}_{\mathrm{u}}$ & SL & $\underline{N}$ & $\underline{\mathrm{N}}$ & $\mathbf{Y}$ & $\underline{N}$ & $\underline{N}$ & $\mathbf{Y}$ & $\mathbf{Y}$ & $\mathbf{Y}$ & $\mathbf{Y}$ & $\mathbf{Y}$ & $\mathrm{SS}_{\mathrm{u}}$ & SL & $\underline{\mathrm{N}}$ & $\underline{N}$ & $\underline{N}$ & $\underline{N}$ & $\mathbf{Y}$ & $\mathbf{Y}$ & $\mathbf{Y}$ & $\mathbf{Y}$ & $\mathbf{Y}$ & $Y$ \\
\hline $\mathrm{SS}_{\mathrm{u}}$ & SS & $\underline{N}$ & $\mathbf{Y}$ & $\mathbf{Y}$ & $\mathbf{Y}$ & $\mathbf{Y}$ & $\mathbf{Y}$ & $\mathbf{Y}$ & $\mathbf{Y}$ & $\mathbf{Y}$ & $\mathbf{Y}$ & $\mathrm{SS}_{\mathrm{u}}$ & SS & $\underline{\mathrm{N}}$ & $\underline{N}$ & $\mathbf{Y}$ & $\mathbf{Y}$ & $\mathbf{Y}$ & $\mathbf{Y}$ & $\mathbf{Y}$ & $\mathbf{Y}$ & $\mathbf{Y}$ & $Y$ \\
\hline $\mathrm{SS}_{\mathrm{u}}$ & $\mathrm{SS}_{\mathrm{b}}$ & $\underline{\mathrm{N}}$ & $\underline{\mathrm{N}}$ & $\underline{N}$ & $\underline{N}$ & $\underline{N}$ & $\underline{N}$ & $\mathbf{Y}$ & $\mathbf{Y}$ & $\mathbf{Y}$ & $\mathbf{Y}$ & $\mathrm{SS}_{\mathrm{u}}$ & $\mathrm{SS}_{\mathrm{b}}$ & $\underline{\mathrm{N}}$ & $\underline{\mathrm{N}}$ & $\underline{\mathrm{N}}$ & $\mathbf{Y}$ & $\mathbf{Y}$ & $Y$ & $\mathbf{Y}$ & $Y$ & $\mathbf{Y}$ & $Y$ \\
\hline
\end{tabular}


For barred spiral primaries in Table $5-13, S_{b} L$ vs. $S_{b} E$ shows similarity at all fitting ranges. Thus early-type galaxies cluster around $S_{b}$ primaries similarly at all measured fitting ranges.

Also interesting, $S_{b} S u$ vs. $S_{b} L$ and $S_{b} S_{u}$ vs. $S_{b} E$ show similarity under $5^{\circ}$ in both hemispheres (with North showing similarity at all ranges). This suggests $S_{u}$ clusters similar to early-type galaxies around $\mathrm{S}_{\mathrm{b}}$ primaries.

$\mathrm{S}_{\mathrm{b}} \mathrm{S}_{\mathrm{u}}$ vs. $\mathrm{S}_{\mathrm{b}} \mathrm{S}$ show consistent dissimilarity above $3^{\circ}$. $\mathrm{S}_{b} \mathrm{~S}_{\mathrm{u}}$ vs. $\mathrm{S}_{\mathrm{b}} \mathrm{S}_{\mathrm{b}}$ in the South shows substantial similarity which does not agree with the North's substantial dissimilarity.

Table 5-13 2D KS Test Results - comparing two runs having the same primary filter and same fitting range filter with different secondary filters - limited to barred spiral primaries.

$\mathrm{Y}=$ dissimilar to the $99 \%$ level. $\mathrm{N}=$ not dissimilar to the $99 \%$ level

\begin{tabular}{|c|c|c|c|c|c|c|c|c|c|c|c|c|c|c|c|c|c|c|c|c|c|c|c|}
\hline \multicolumn{12}{|c|}{ North } & \multicolumn{12}{|c|}{ South } \\
\hline \multirow[b]{2}{*}{ Run1 } & \multicolumn{11}{|c|}{ Fitting Range (degree) } & \multicolumn{12}{|c|}{ Fitting Range (degree) } \\
\hline & Run2 & 1 & 2 & 3 & 4 & 5 & 10 & 15 & 20 & 25 & 30 & Run1 & Run2 & 1 & 2 & 3 & 4 & 5 & 10 & 15 & 20 & 25 & 30 \\
\hline $\mathrm{S}_{\mathrm{b}} \mathrm{A}$ & $\mathrm{S}_{\mathrm{b}} \mathrm{E}$ & $\underline{N}$ & $\mathrm{Y}$ & $\mathrm{Y}$ & $\mathrm{Y}$ & $\mathbf{Y}$ & $\mathbf{Y}$ & $\mathbf{Y}$ & $\mathbf{Y}$ & $\mathrm{Y}$ & $\mathbf{Y}$ & $S_{b} A$ & $\mathrm{~S}_{\mathrm{b}} \mathrm{E}$ & $\underline{N}$ & $\mathbf{Y}$ & $\mathbf{Y}$ & $\mathbf{Y}$ & $Y$ & $\mathbf{Y}$ & $Y$ & $\mathbf{Y}$ & $\mathbf{Y}$ & $\mathbf{Y}$ \\
\hline$S_{b} A$ & $\mathrm{~S}_{b} \mathrm{~L}$ & $\underline{N}$ & $\mathrm{Y}$ & $\mathbf{Y}$ & $\mathbf{Y}$ & $\mathbf{Y}$ & $\mathbf{Y}$ & 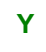 & $\mathbf{Y}$ & $\mathbf{Y}$ & $\mathbf{Y}$ & $S_{b} A$ & $S_{b} L$ & $\underline{N}$ & $Y$ & $\mathbf{Y}$ & $\mathbf{Y}$ & $\mathrm{Y}$ & $\mathrm{Y}$ & $Y$ & $Y$ & $Y$ & $Y$ \\
\hline $\mathrm{S}_{\mathrm{b}} \mathrm{A}$ & $\mathrm{S}_{\mathrm{b}} \mathrm{S}$ & $\underline{N}$ & $\mathbf{Y}$ & $\mathbf{Y}$ & $\mathbf{Y}$ & $\mathbf{Y}$ & $Y$ & $Y$ & $Y$ & $\mathbf{Y}$ & $\underline{N}$ & $S_{b} A$ & $S_{b} S$ & $\underline{N}$ & $\underline{N}$ & $Y$ & $Y$ & $Y$ & $Y$ & $Y$ & $Y$ & $\underline{N}$ & $\underline{N}$ \\
\hline $\mathrm{S}_{\mathrm{b}} \mathrm{A}$ & $\mathrm{S}_{\mathrm{b}} \mathrm{S}_{\mathrm{b}}$ & $Y$ & $Y$ & $\mathrm{Y}$ & $Y$ & $Y$ & $Y$ & $Y$ & $Y$ & $Y$ & $Y$ & $S_{b} A$ & $\mathrm{~S}_{\mathrm{b}} \mathrm{S}_{\mathrm{b}}$ & $\underline{N}$ & $\underline{N}$ & $Y$ & $Y$ & $Y$ & $Y$ & $Y$ & $Y$ & $Y$ & $Y$ \\
\hline$S_{b} A$ & $S_{b} S_{u}$ & $\underline{N}$ & $\mathbf{Y}$ & $\mathbf{Y}$ & $\mathbf{Y}$ & $\mathbf{Y}$ & $\mathbf{Y}$ & $\mathbf{Y}$ & $\mathbf{Y}$ & $\mathbf{Y}$ & $Y$ & $S_{b} A$ & $\mathrm{~S}_{\mathrm{b}} \mathrm{S}_{\mathrm{u}}$ & $\underline{N}$ & $\underline{N}$ & $\mathbf{Y}$ & $\mathbf{Y}$ & $\mathbf{Y}$ & $\mathbf{Y}$ & $Y$ & $Y$ & $Y$ & $Y$ \\
\hline$S_{b} E$ & $S_{b} A$ & $\underline{N}$ & $Y$ & $\mathbf{Y}$ & $Y$ & $Y$ & $\mathbf{Y}$ & $\mathbf{Y}$ & $Y$ & $\mathbf{Y}$ & $Y$ & $S_{b} E$ & $S_{b} A$ & $\underline{N}$ & $Y$ & $Y$ & $\mathbf{Y}$ & $Y$ & $\mathbf{Y}$ & $Y$ & $Y$ & $Y$ & $Y$ \\
\hline$S_{b} E$ & $S_{b} L$ & $\underline{N}$ & $\underline{N}$ & $\underline{N}$ & $\underline{N}$ & $\underline{N}$ & $\underline{N}$ & $\underline{N}$ & $\underline{N}$ & $\underline{N}$ & $\underline{N}$ & $\mathrm{~S}_{\mathrm{b}} \mathrm{E}$ & $S_{b} L$ & $\underline{N}$ & $\underline{N}$ & $\underline{N}$ & $\underline{N}$ & $\underline{N}$ & $\underline{N}$ & $\underline{N}$ & $\underline{N}$ & $\underline{N}$ & $\underline{N}$ \\
\hline$S_{b} E$ & $S_{b} S$ & $\underline{N}$ & $\mathbf{Y}$ & $\mathbf{Y}$ & $Y$ & $Y$ & $\mathbf{Y}$ & $\mathbf{Y}$ & $\mathbf{Y}$ & $Y$ & $Y$ & $S_{b} E$ & $S_{b} S$ & $\underline{N}$ & $\underline{\mathrm{N}}$ & $\underline{N}$ & $\underline{\mathrm{N}}$ & $Y$ & $Y$ & $Y$ & $\mathbf{Y}$ & $Y$ & $Y$ \\
\hline $\mathrm{S}_{\mathrm{b}} \mathrm{E}$ & $\mathrm{S}_{\mathrm{b}} \mathrm{S}_{\mathrm{b}}$ & $\mathbf{Y}$ & $\underline{N}$ & $\underline{N}$ & $\underline{N}$ & $\mathbf{Y}$ & $\mathbf{Y}$ & $\mathbf{Y}$ & $\mathbf{Y}$ & $\mathbf{Y}$ & $\mathbf{Y}$ & $S_{b} E$ & $\mathrm{~S}_{\mathrm{b}} \mathrm{S}_{\mathrm{b}}$ & $\underline{N}$ & $\underline{N}$ & $\underline{N}$ & $\underline{N}$ & $\underline{N}$ & $\mathbf{Y}$ & $\mathbf{Y}$ & $\mathbf{Y}$ & $\mathbf{Y}$ & $\mathbf{Y}$ \\
\hline$S_{b} E$ & $\mathrm{~S}_{\mathrm{b}} \mathrm{S}_{\mathrm{u}}$ & $\underline{N}$ & $\underline{N}$ & $\underline{N}$ & $\underline{N}$ & $\underline{N}$ & $\underline{N}$ & $\underline{N}$ & $\underline{N}$ & $\underline{N}$ & $\underline{N}$ & $\mathrm{~S}_{\mathrm{b}} \mathrm{E}$ & $\mathrm{S}_{\mathrm{b}} \mathrm{S}_{\mathrm{u}}$ & $\underline{N}$ & $\underline{N}$ & $\underline{N}$ & $\underline{N}$ & $\underline{N}$ & $Y$ & $Y$ & $Y$ & $Y$ & $Y$ \\
\hline$S_{b} L$ & $S_{b} A$ & $\underline{N}$ & $Y$ & $\mathbf{Y}$ & $Y$ & $Y$ & $Y$ & $Y$ & $\mathbf{Y}$ & $\mathbf{Y}$ & $Y$ & $S_{b} L$ & $S_{b} A$ & $\underline{N}$ & $Y$ & $Y$ & $Y$ & $Y$ & $Y$ & $Y$ & $Y$ & $Y$ & $Y$ \\
\hline$S_{b} L$ & $\mathrm{~S}_{\mathrm{b}} \mathrm{E}$ & $\underline{N}$ & $\underline{N}$ & $\underline{N}$ & $\underline{N}$ & $\underline{N}$ & $\underline{N}$ & $\underline{N}$ & $\underline{N}$ & $\underline{N}$ & $\underline{N}$ & $S_{b} L$ & $S_{b} E$ & $\underline{N}$ & $\underline{N}$ & $\underline{N}$ & $\underline{N}$ & $\underline{N}$ & $\underline{N}$ & $\underline{N}$ & $\underline{N}$ & $\underline{N}$ & $\underline{N}$ \\
\hline$S_{b} L$ & $S_{b} S$ & $\underline{N}$ & $\underline{N}$ & $Y$ & $Y$ & $Y$ & $Y$ & $Y$ & $Y$ & $Y$ & $Y$ & $S_{b} L$ & $S_{b} S$ & $\underline{N}$ & $Y$ & $\underline{N}$ & $\underline{N}$ & $\underline{N}$ & $Y$ & $Y$ & $Y$ & $Y$ & $Y$ \\
\hline$S_{b} L$ & $S_{b} S_{b}$ & $\mathbf{Y}$ & $\underline{N}$ & $\underline{\mathrm{N}}$ & $\mathbf{Y}$ & $\mathbf{Y}$ & $\mathbf{Y}$ & $\mathbf{Y}$ & $\mathbf{Y}$ & $\mathbf{Y}$ & $Y$ & $S_{b} L$ & $\mathrm{~S}_{\mathrm{b}} \mathrm{S}_{\mathrm{b}}$ & $\underline{N}$ & $\underline{\mathrm{N}}$ & $\underline{N}$ & $\underline{N}$ & $\underline{N}$ & $\mathbf{Y}$ & $Y$ & $Y$ & $\mathbf{Y}$ & $Y$ \\
\hline$S_{b} L$ & $S_{b} S_{u}$ & $\underline{N}$ & $\underline{N}$ & $\underline{\mathrm{N}}$ & $\underline{N}$ & $\underline{N}$ & $\underline{N}$ & $\underline{N}$ & $\underline{N}$ & $\underline{N}$ & $\underline{N}$ & $\mathrm{~S}_{\mathrm{b}} \mathrm{L}$ & $S_{b} S_{u}$ & $\underline{N}$ & $\underline{N}$ & $\underline{N}$ & $\underline{N}$ & $\underline{N}$ & $Y$ & $Y$ & $Y$ & $Y$ & $Y$ \\
\hline$S_{b} S$ & $\mathrm{~S}_{\mathrm{b}} \mathrm{A}$ & $\underline{N}$ & $\mathbf{Y}$ & $\mathbf{Y}$ & $\mathbf{Y}$ & $\mathbf{Y}$ & $\mathbf{Y}$ & $\mathbf{Y}$ & $\mathbf{Y}$ & $\mathbf{Y}$ & $\underline{N}$ & $S_{b} S$ & $\mathrm{~S}_{\mathrm{b}} \mathrm{A}$ & $\underline{N}$ & $\underline{N}$ & $\mathbf{Y}$ & $\mathbf{Y}$ & $\mathbf{Y}$ & $\mathbf{Y}$ & $\mathbf{Y}$ & $\mathbf{Y}$ & $\underline{N}$ & $\underline{N}$ \\
\hline$S_{b} S$ & $\mathrm{~S}_{\mathrm{b}} \mathrm{E}$ & $\underline{N}$ & $Y$ & $Y$ & $Y$ & $Y$ & $Y$ & $Y$ & $Y$ & $Y$ & $Y$ & $S_{b} S$ & $\mathrm{~S}_{\mathrm{b}} \mathrm{E}$ & $\underline{N}$ & $\underline{N}$ & $\underline{N}$ & $\underline{N}$ & $Y$ & $Y$ & $Y$ & $Y$ & $Y$ & $Y$ \\
\hline $\mathrm{S}_{\mathrm{b}} \mathrm{S}$ & $S_{b} L$ & $\underline{N}$ & $\underline{N}$ & $\mathbf{Y}$ & $Y$ & $Y$ & $Y$ & $Y$ & $\mathbf{Y}$ & $\mathbf{Y}$ & $Y$ & $S_{b} S$ & $\mathrm{~S}_{\mathrm{b}} \mathrm{L}$ & $\underline{N}$ & $Y$ & $\underline{N}$ & $\underline{N}$ & $\underline{N}$ & $Y$ & $Y$ & $Y$ & $Y$ & $Y$ \\
\hline $\mathrm{S}_{\mathrm{b}} \mathrm{S}$ & $\mathrm{S}_{\mathrm{b}} \mathrm{S}_{\mathrm{b}}$ & $Y$ & $\underline{N}$ & $Y$ & $Y$ & $Y$ & $Y$ & $Y$ & $Y$ & $Y$ & $Y$ & $S_{b} S$ & $\mathrm{~S}_{\mathrm{b}} \mathrm{S}_{\mathrm{b}}$ & $\underline{N}$ & $\underline{N}$ & $\underline{N}$ & $Y$ & $Y$ & $Y$ & $Y$ & $Y$ & $Y$ & $Y$ \\
\hline
\end{tabular}




\begin{tabular}{|c|c|c|c|c|c|c|c|c|c|c|c|c|c|c|c|c|c|c|c|c|c|c|c|}
\hline$S_{b} S$ & $\mathrm{~S}_{\mathrm{b}} \mathrm{S}_{\mathrm{u}}$ & $\underline{N}$ & $\underline{N}$ & $Y$ & $Y$ & $\mathbf{Y}$ & $\mathbf{Y}$ & $\mathbf{Y}$ & $\mathbf{Y}$ & $\mathbf{Y}$ & $Y$ & $\mathrm{~S}_{\mathrm{b}} \mathrm{S}$ & $\mathrm{S}_{\mathrm{b}} \mathrm{S}_{\mathrm{u}}$ & $\underline{N}$ & $\underline{N}$ & $\underline{N}$ & $Y$ & $Y$ & $\mathbf{Y}$ & $\mathbf{Y}$ & $Y$ & $\mathbf{Y}$ & $Y$ \\
\hline $\mathrm{S}_{\mathrm{b}} \mathrm{S}_{\mathrm{b}}$ & $\mathrm{S}_{\mathrm{b}} \mathrm{A}$ & $\mathbf{Y}$ & $\mathbf{Y}$ & $\mathbf{Y}$ & $\mathbf{Y}$ & $\mathbf{Y}$ & $\mathbf{Y}$ & $\mathbf{Y}$ & $\mathbf{Y}$ & $\mathbf{Y}$ & $\mathbf{Y}$ & $\mathrm{S}_{\mathrm{b}} \mathrm{S}_{\mathrm{b}}$ & $S_{b} A$ & $\underline{N}$ & $\underline{\mathrm{N}}$ & $\mathbf{Y}$ & $\mathbf{Y}$ & $\mathbf{Y}$ & $\mathbf{Y}$ & $\mathbf{Y}$ & $Y$ & $\mathbf{Y}$ & $Y$ \\
\hline$S_{b} S_{b}$ & $\mathrm{~S}_{\mathrm{b}} \mathrm{E}$ & $Y$ & $\underline{N}$ & $\underline{N}$ & $\underline{N}$ & $\mathbf{Y}$ & $\mathbf{Y}$ & $\mathbf{Y}$ & $\mathbf{Y}$ & $\mathbf{Y}$ & $Y$ & $S_{b} S_{b}$ & $S_{b} E$ & $\underline{N}$ & $\underline{N}$ & $\underline{N}$ & $\underline{N}$ & $\underline{N}$ & $\mathbf{Y}$ & $\mathbf{Y}$ & $Y$ & $Y$ & $Y$ \\
\hline$S_{b} S_{b}$ & $S_{b} L$ & $Y$ & $\underline{N}$ & $\underline{N}$ & $\mathbf{Y}$ & $\mathbf{Y}$ & $\mathbf{Y}$ & $\mathbf{Y}$ & $\mathbf{Y}$ & $\mathbf{Y}$ & $Y$ & $\mathrm{~S}_{\mathrm{b}} \mathrm{S}_{\mathrm{b}}$ & $S_{b} L$ & $\underline{N}$ & $\underline{N}$ & $\underline{N}$ & $\underline{N}$ & $\underline{N}$ & $\mathbf{Y}$ & $\mathbf{Y}$ & $Y$ & $Y$ & $Y$ \\
\hline$S_{b} S_{b}$ & $\mathrm{~S}_{\mathrm{b}} \mathrm{S}$ & $\mathbf{Y}$ & $\underline{N}$ & $Y$ & $\mathbf{Y}$ & $Y$ & $\mathbf{Y}$ & $\mathbf{Y}$ & $\mathbf{Y}$ & $\mathbf{Y}$ & $Y$ & $S_{b} S_{b}$ & $S_{b} S$ & $\underline{N}$ & $\underline{N}$ & $\underline{N}$ & $\mathbf{Y}$ & $Y$ & $\mathbf{Y}$ & $Y$ & $Y$ & $Y$ & $Y$ \\
\hline$S_{b} S_{b}$ & $\mathrm{~S}_{\mathrm{b}} \mathrm{S}_{\mathrm{u}}$ & $\underline{N}$ & $\underline{\mathrm{N}}$ & $\mathbf{Y}$ & $\mathbf{Y}$ & $Y$ & $\mathbf{Y}$ & $\mathbf{Y}$ & $\mathbf{Y}$ & $\mathbf{Y}$ & $\mathbf{Y}$ & $S_{b} S_{b}$ & $\mathrm{~S}_{\mathrm{b}} \mathrm{S}_{\mathrm{u}}$ & $\underline{N}$ & $\underline{\mathrm{N}}$ & $\underline{N}$ & $\underline{\mathrm{N}}$ & $\underline{N}$ & $\underline{\mathrm{N}}$ & $\underline{\mathrm{N}}$ & $\underline{N}$ & $\underline{N}$ & $\mathbf{Y}$ \\
\hline $\mathrm{S}_{\mathrm{b}} \mathrm{S}_{\mathrm{u}}$ & $\mathrm{S}_{\mathrm{b}} \mathrm{A}$ & $\underline{N}$ & $\mathbf{Y}$ & $\mathbf{Y}$ & $\mathbf{Y}$ & $\mathbf{Y}$ & $\mathbf{Y}$ & $\mathbf{Y}$ & $\mathbf{Y}$ & $\mathbf{Y}$ & $Y$ & $\mathrm{~S}_{\mathrm{b}} \mathrm{S}_{\mathrm{u}}$ & $S_{b} A$ & $\underline{N}$ & $\underline{\mathrm{N}}$ & $\mathbf{Y}$ & $\mathbf{Y}$ & $\mathbf{Y}$ & $\mathbf{Y}$ & $\mathbf{Y}$ & $\mathbf{Y}$ & $Y$ & $\mathbf{Y}$ \\
\hline $\mathrm{S}_{\mathrm{b}} \mathrm{S}_{\mathrm{u}}$ & $S_{b} E$ & $\underline{N}$ & $\underline{\mathrm{N}}$ & $\underline{N}$ & $\underline{N}$ & $\underline{\mathrm{N}}$ & $\underline{N}$ & $\underline{N}$ & $\underline{N}$ & $\underline{N}$ & $\underline{N}$ & $\mathrm{~S}_{\mathrm{b}} \mathrm{S}_{\mathrm{u}}$ & $\mathrm{S}_{\mathrm{b}} \mathrm{E}$ & $\underline{N}$ & $\underline{\mathrm{N}}$ & $\underline{\mathrm{N}}$ & $\underline{\mathrm{N}}$ & $\underline{\mathrm{N}}$ & $Y$ & $\mathbf{Y}$ & $\mathbf{Y}$ & $Y$ & $\mathbf{Y}$ \\
\hline $\mathrm{S}_{\mathrm{b}} \mathrm{S}_{\mathrm{u}}$ & $\mathrm{S}_{\mathrm{b}} \mathrm{L}$ & $\underline{N}$ & $\underline{N}$ & $\underline{N}$ & $\underline{N}$ & $\underline{N}$ & $\underline{N}$ & $\underline{N}$ & $\underline{N}$ & $\underline{N}$ & $\underline{N}$ & $\mathrm{~S}_{\mathrm{b}} \mathrm{S}_{\mathrm{u}}$ & $S_{b} L$ & $\underline{N}$ & $\underline{N}$ & $\underline{N}$ & $\underline{N}$ & $\underline{N}$ & $Y$ & $Y$ & $\mathbf{Y}$ & $Y$ & $Y$ \\
\hline $\mathrm{S}_{\mathrm{b}} \mathrm{S}_{\mathrm{u}}$ & $S_{b} S$ & $\underline{N}$ & $\underline{\mathrm{N}}$ & $\mathbf{Y}$ & $\mathbf{Y}$ & $\mathbf{Y}$ & $\mathbf{Y}$ & $\mathbf{Y}$ & $\mathbf{Y}$ & $\mathbf{Y}$ & $\mathbf{Y}$ & $\mathrm{S}_{\mathrm{b}} \mathrm{S}_{\mathrm{u}}$ & $S_{b} S$ & $\underline{\mathrm{N}}$ & $\underline{\mathrm{N}}$ & $\underline{\mathrm{N}}$ & $\mathbf{Y}$ & $Y$ & $\mathbf{Y}$ & $\mathbf{Y}$ & $Y$ & $Y$ & $\mathbf{Y}$ \\
\hline$S_{b} S_{u}$ & $\mathrm{~S}_{\mathrm{b}} \mathrm{S}_{\mathrm{b}}$ & $\underline{N}$ & $\underline{N}$ & $Y$ & $Y$ & $Y$ & $\mathbf{Y}$ & $\mathbf{Y}$ & $\mathbf{Y}$ & $Y$ & $Y$ & $\mathrm{~S}_{\mathrm{b}} \mathrm{S}_{\mathrm{u}}$ & $\mathrm{S}_{\mathrm{b}} \mathrm{S}_{\mathrm{b}}$ & $\underline{N}$ & $\underline{N}$ & $\underline{N}$ & $\underline{N}$ & $\underline{N}$ & $\underline{N}$ & $\underline{N}$ & $\underline{N}$ & $\underline{N}$ & $\mathbf{Y}$ \\
\hline
\end{tabular}


When considering unbarred spiral primaries in Table 5-14, early-type galaxies continue to cluster similarity around $S_{u}$, as seen above for other categories of primary. $S_{u}$ and $S_{b}$ clustering also continues to be similar to $L$ and $E$ as seen for other types of primary. Also $S_{b}$ vs. $S_{u}$ clustering around $S_{u}$ is similar to $25^{\circ}$ while $S_{u} S_{b} v s$. $S_{u} S$ and $\mathrm{SuS}_{\mathrm{u}} \mathrm{vs}$. $\mathrm{SuS}$ are dissimilar from $3^{\circ}$ and up.

Table 5-14 2D KS Test Results - comparing two runs having the same primary filter and same fitting range filter with different secondary filters - limited to unbarred spiral primaries.

$\mathrm{Y}=$ dissimilar to the $99 \%$ level. $\mathrm{N}=$ not dissimilar to the $99 \%$ level

\begin{tabular}{|c|c|c|c|c|c|c|c|c|c|c|c|c|c|c|c|c|c|c|c|c|c|c|c|}
\hline \multirow[b]{3}{*}{ Run1 } & \multicolumn{11}{|c|}{ North } & \multicolumn{12}{|c|}{ South } \\
\hline & \multicolumn{11}{|c|}{ Fitting Range (degree) } & \multicolumn{12}{|c|}{ Fitting Range (degree) } \\
\hline & Run2 & 1 & 2 & 3 & 4 & 5 & 10 & 15 & 20 & 25 & 30 & Run1 & Run2 & 1 & 2 & 3 & 4 & 5 & 10 & 15 & 20 & 25 & 30 \\
\hline $\mathrm{S}_{\mathrm{u}} \mathrm{A}$ & $\mathrm{S}_{\mathrm{u}} \mathrm{E}$ & $\underline{N}$ & $\mathbf{Y}$ & $\mathbf{Y}$ & $\mathbf{Y}$ & $\mathbf{Y}$ & $\mathbf{Y}$ & $\mathbf{Y}$ & $\mathbf{Y}$ & $\mathbf{Y}$ & $\mathbf{Y}$ & $\mathrm{S}_{u} \mathrm{~A}$ & $\mathrm{~S}_{\mathrm{u}} \mathrm{E}$ & $\underline{N}$ & $\mathbf{Y}$ & $\mathbf{Y}$ & $\mathbf{Y}$ & $\mathbf{Y}$ & $\mathbf{Y}$ & $\mathbf{Y}$ & $\mathbf{Y}$ & $\mathbf{Y}$ & $\mathbf{Y}$ \\
\hline $\mathrm{S}_{u} \mathrm{~A}$ & $\mathrm{~S}_{\mathrm{u}} \mathrm{L}$ & $\underline{N}$ & $\mathbf{Y}$ & $\mathbf{Y}$ & $\mathbf{Y}$ & $\mathbf{Y}$ & $\mathbf{Y}$ & $\mathbf{Y}$ & $\mathbf{Y}$ & $\mathbf{Y}$ & $\mathbf{Y}$ & $\mathrm{S}_{u} \mathrm{~A}$ & $S_{u} L$ & $\underline{N}$ & $\mathbf{Y}$ & $\mathbf{Y}$ & $\mathbf{Y}$ & $\mathbf{Y}$ & $\mathbf{Y}$ & $\mathbf{Y}$ & $\mathbf{Y}$ & $\mathbf{Y}$ & $\underline{N}$ \\
\hline $\mathrm{S}_{\mathrm{u}} \mathrm{A}$ & $\mathrm{S}_{\mathrm{u}} \mathrm{S}$ & $\underline{N}$ & $\mathbf{Y}$ & $\mathbf{Y}$ & $\mathbf{Y}$ & $\mathbf{Y}$ & $\mathbf{Y}$ & $\mathbf{Y}$ & $\mathbf{Y}$ & $\underline{N}$ & $\underline{N}$ & $\mathrm{~S}_{\mathrm{u}} \mathrm{A}$ & $\mathrm{S}_{\mathrm{u}} \mathrm{S}$ & $\underline{N}$ & $\mathbf{Y}$ & $\mathbf{Y}$ & $\mathbf{Y}$ & $\mathbf{Y}$ & $\mathbf{Y}$ & $Y$ & $\mathbf{Y}$ & $\underline{N}$ & $\underline{N}$ \\
\hline $\mathrm{S}_{\mathrm{u}} \mathrm{A}$ & $S_{u} S_{b}$ & $\mathbf{Y}$ & $\mathbf{Y}$ & $Y$ & $\mathbf{Y}$ & $\mathbf{Y}$ & $\mathbf{Y}$ & $\mathbf{Y}$ & $\mathbf{Y}$ & $\mathbf{Y}$ & $\mathbf{Y}$ & $S_{u} A$ & $\mathrm{~S}_{\mathrm{u}} \mathrm{S}_{\mathrm{b}}$ & $\underline{N}$ & $\mathbf{Y}$ & $\mathbf{Y}$ & $\mathbf{Y}$ & $\mathbf{Y}$ & $Y$ & $Y$ & $Y$ & $\mathbf{Y}$ & $\mathbf{Y}$ \\
\hline $\mathrm{S}_{\mathrm{u}} \mathrm{A}$ & $\mathrm{S}_{\mathrm{u}} \mathrm{S}_{\mathrm{u}}$ & $\underline{N}$ & $\mathbf{Y}$ & $\mathbf{Y}$ & $Y$ & $\mathbf{Y}$ & $\mathbf{Y}$ & $\mathbf{Y}$ & $Y$ & $\mathbf{Y}$ & $Y$ & $S_{u} A$ & $S_{u} S_{u}$ & $\underline{N}$ & $\mathbf{Y}$ & $\mathbf{Y}$ & $\mathbf{Y}$ & $\mathbf{Y}$ & $Y$ & $Y$ & $Y$ & $Y$ & $Y$ \\
\hline$S_{u} E$ & $\mathrm{~S}_{\mathrm{u}} \mathrm{A}$ & $\underline{N}$ & $\mathbf{Y}$ & $\mathbf{Y}$ & $\mathbf{Y}$ & $\mathbf{Y}$ & $\mathbf{Y}$ & $\mathbf{Y}$ & $\mathbf{Y}$ & $\mathbf{Y}$ & $\mathbf{Y}$ & $S_{u} E$ & $S_{u} A$ & $\underline{N}$ & $\mathbf{Y}$ & $\mathbf{Y}$ & $\mathbf{Y}$ & $\mathbf{Y}$ & $Y$ & $\mathbf{Y}$ & $\mathbf{Y}$ & $\mathbf{Y}$ & $\mathbf{Y}$ \\
\hline$S_{u} E$ & $\mathrm{~S}_{\mathrm{u}} \mathrm{L}$ & $\underline{N}$ & $\underline{N}$ & $\underline{N}$ & $\underline{N}$ & $\underline{N}$ & $\underline{N}$ & $\underline{N}$ & $\underline{N}$ & $\underline{N}$ & $\underline{N}$ & $\mathrm{~S}_{\mathrm{u}} \mathrm{E}$ & $\mathrm{S}_{\mathrm{u}} \mathrm{L}$ & $\underline{N}$ & $\underline{N}$ & $\underline{N}$ & $\underline{N}$ & $\underline{N}$ & $\underline{N}$ & $\underline{N}$ & $\underline{N}$ & $\underline{N}$ & $\underline{N}$ \\
\hline$S_{u} E$ & $\mathrm{~S}_{\mathrm{u}} \mathrm{S}$ & $\underline{N}$ & $\mathbf{Y}$ & $\mathbf{Y}$ & $\mathbf{Y}$ & $\mathbf{Y}$ & $\mathbf{Y}$ & $\mathbf{Y}$ & $\mathbf{Y}$ & $\mathbf{Y}$ & $\mathbf{Y}$ & $S_{u} E$ & $\mathrm{~S}_{\mathrm{u}} \mathrm{S}$ & $\underline{N}$ & $\underline{N}$ & $\underline{N}$ & $\mathbf{Y}$ & $\mathbf{Y}$ & $\mathbf{Y}$ & $\underline{N}$ & $\underline{N}$ & $\mathbf{Y}$ & $\mathbf{Y}$ \\
\hline$S_{u} E$ & $S_{u} S_{b}$ & $\underline{N}$ & $\underline{N}$ & $\underline{N}$ & $\underline{N}$ & $\underline{N}$ & $\mathbf{Y}$ & $\mathbf{Y}$ & $\mathbf{Y}$ & $\mathbf{Y}$ & $\underline{N}$ & $\mathrm{~S}_{\mathrm{u}} \mathrm{E}$ & $S_{u} S_{b}$ & $\underline{N}$ & $\underline{N}$ & $\underline{N}$ & $\underline{N}$ & $\underline{N}$ & $\mathbf{Y}$ & $\mathbf{Y}$ & $\mathbf{Y}$ & $\mathbf{Y}$ & $\mathbf{Y}$ \\
\hline$S_{u} E$ & $\mathrm{~S}_{\mathrm{u}} \mathrm{S}_{\mathrm{u}}$ & $\underline{N}$ & $\underline{\mathrm{N}}$ & $\underline{N}$ & $\underline{N}$ & $\underline{N}$ & $\mathbf{Y}$ & $\mathbf{Y}$ & $\mathbf{Y}$ & $\mathbf{Y}$ & $\mathbf{Y}$ & $S_{u} E$ & $\mathrm{~S}_{\mathrm{u}} \mathrm{S}_{\mathrm{u}}$ & $\underline{N}$ & $\underline{N}$ & $\underline{N}$ & $\underline{N}$ & $\underline{N}$ & $\mathbf{Y}$ & $\mathbf{Y}$ & $\mathbf{Y}$ & $\mathbf{Y}$ & $\mathbf{Y}$ \\
\hline $\mathrm{S}_{\mathrm{u}} \mathrm{L}$ & $\mathrm{S}_{\mathrm{u}} \mathrm{A}$ & $\underline{N}$ & $\mathbf{Y}$ & $\mathbf{Y}$ & $\mathrm{Y}$ & $\mathbf{Y}$ & $\mathbf{Y}$ & $\mathbf{Y}$ & $\mathbf{Y}$ & $\mathbf{Y}$ & $\mathbf{Y}$ & $\mathrm{S}_{\mathrm{u}} \mathrm{L}$ & $\mathrm{S}_{\mathrm{u}} \mathrm{A}$ & $\underline{N}$ & $\mathbf{Y}$ & $\mathbf{Y}$ & $\mathbf{Y}$ & $\mathbf{Y}$ & $Y$ & $\mathbf{Y}$ & $\mathbf{Y}$ & $\mathbf{Y}$ & $\underline{N}$ \\
\hline $\mathrm{S}_{\mathrm{u}} \mathrm{L}$ & $\mathrm{S}_{\mathrm{u}} \mathrm{E}$ & $\underline{N}$ & $\underline{N}$ & $\underline{N}$ & $\underline{N}$ & $\underline{N}$ & $\underline{N}$ & $\underline{N}$ & $\underline{N}$ & $\underline{N}$ & $\underline{\mathrm{N}}$ & $S_{u} L$ & $\mathrm{~S}_{\mathrm{u}} \mathrm{E}$ & $\underline{N}$ & $\underline{N}$ & $\underline{N}$ & $\underline{N}$ & $\underline{N}$ & $\underline{N}$ & $\underline{N}$ & $\underline{N}$ & $\underline{N}$ & $\underline{N}$ \\
\hline $\mathrm{S}_{\mathrm{u}} \mathrm{L}$ & $\mathrm{S}_{\mathrm{u}} \mathrm{S}$ & $\underline{N}$ & $\underline{N}$ & $Y$ & $\mathbf{Y}$ & $\mathbf{Y}$ & $\mathbf{Y}$ & $\mathbf{Y}$ & $\mathbf{Y}$ & $\mathbf{Y}$ & $\mathbf{Y}$ & $\mathrm{S}_{\mathrm{u}} \mathrm{L}$ & $\mathrm{S}_{\mathrm{u}} \mathrm{S}$ & $\underline{N}$ & $\underline{N}$ & $\mathbf{Y}$ & $\mathbf{Y}$ & $\mathbf{Y}$ & $\mathbf{Y}$ & $\underline{N}$ & $\underline{N}$ & $\underline{N}$ & $\underline{N}$ \\
\hline $\mathrm{S}_{\mathrm{u}} \mathrm{L}$ & $\mathrm{S}_{\mathrm{u}} \mathrm{S}_{\mathrm{b}}$ & $\underline{N}$ & $\underline{N}$ & $\underline{N}$ & $\mathbf{Y}$ & $\underline{N}$ & $\underline{N}$ & $\underline{N}$ & $\mathbf{Y}$ & $\mathbf{Y}$ & $\mathbf{Y}$ & $\mathrm{S}_{\mathrm{u}} \mathrm{L}$ & $\mathrm{S}_{\mathrm{u}} \mathrm{S}_{\mathrm{b}}$ & $\underline{N}$ & $\underline{N}$ & $\underline{N}$ & $\underline{N}$ & $\underline{N}$ & $\mathbf{Y}$ & $\mathbf{Y}$ & $\mathbf{Y}$ & $\mathbf{Y}$ & $\mathbf{Y}$ \\
\hline $\mathrm{S}_{\mathrm{u}} \mathrm{L}$ & $\mathrm{S}_{\mathrm{u}} \mathrm{S}_{\mathrm{u}}$ & $\underline{N}$ & $\underline{N}$ & $\underline{N}$ & $\underline{N}$ & $\underline{N}$ & $\underline{N}$ & $\underline{N}$ & $\mathbf{Y}$ & $\mathbf{Y}$ & $\mathbf{Y}$ & $\mathrm{S}_{\mathrm{u}} \mathrm{L}$ & $\mathrm{S}_{\mathrm{u}} \mathrm{S}_{\mathrm{u}}$ & $\underline{N}$ & $\underline{N}$ & $\underline{N}$ & $\underline{N}$ & $\underline{N}$ & $\mathbf{Y}$ & $\mathbf{Y}$ & $\mathbf{Y}$ & $\mathbf{Y}$ & $\mathbf{Y}$ \\
\hline $\mathrm{S}_{\mathrm{u}} \mathrm{S}$ & $\mathrm{S}_{\mathrm{u}} \mathrm{A}$ & $\underline{N}$ & $\mathbf{Y}$ & $\mathbf{Y}$ & $\mathbf{Y}$ & $Y$ & $Y$ & $Y$ & $Y$ & $\underline{N}$ & $\underline{N}$ & $\mathrm{~S}_{\mathrm{u}} \mathrm{S}$ & $\mathrm{S}_{\mathrm{u}} \mathrm{A}$ & $\underline{N}$ & $Y$ & $\mathbf{Y}$ & $Y$ & $Y$ & $Y$ & $\mathbf{Y}$ & $\mathbf{Y}$ & $\underline{N}$ & $\underline{N}$ \\
\hline $\mathrm{S}_{\mathrm{u}} \mathrm{S}$ & $S_{u} E$ & $\underline{N}$ & $\mathbf{Y}$ & $\mathbf{Y}$ & $\mathbf{Y}$ & $Y$ & $\mathbf{Y}$ & $\mathbf{Y}$ & $\mathbf{Y}$ & $\mathbf{Y}$ & $Y$ & $\mathrm{~S}_{\mathrm{u}} \mathrm{S}$ & $S_{u} E$ & $\underline{N}$ & $\underline{N}$ & $\underline{N}$ & $\mathbf{Y}$ & $\mathbf{Y}$ & $Y$ & $\underline{N}$ & $\underline{N}$ & $Y$ & $Y$ \\
\hline $\mathrm{S}_{\mathrm{u}} \mathrm{S}$ & $\mathrm{S}_{\mathrm{u}} \mathrm{L}$ & $\underline{N}$ & $\underline{N}$ & $\mathbf{Y}$ & $\mathbf{Y}$ & $\mathbf{Y}$ & $\mathbf{Y}$ & $\mathbf{Y}$ & $\mathbf{Y}$ & $\mathbf{Y}$ & $Y$ & $\mathrm{~S}_{\mathrm{u}} \mathrm{S}$ & $\mathrm{S}_{\mathrm{u}} \mathrm{L}$ & $\underline{N}$ & $\underline{N}$ & $\mathbf{Y}$ & $\mathbf{Y}$ & $\mathbf{Y}$ & $Y$ & $\underline{N}$ & $\underline{N}$ & $\underline{N}$ & $\underline{N}$ \\
\hline $\mathrm{S}_{\mathrm{u}} \mathrm{S}$ & $\mathrm{S}_{\mathrm{u}} \mathrm{S}_{\mathrm{b}}$ & $\underline{N}$ & $\underline{N}$ & $\mathbf{Y}$ & $\mathrm{Y}$ & $\mathbf{Y}$ & $\mathbf{Y}$ & $\mathbf{Y}$ & $\mathbf{Y}$ & $\mathbf{Y}$ & $Y$ & $\mathrm{~S}_{\mathrm{u}} \mathrm{S}$ & $\mathrm{S}_{\mathrm{u}} \mathrm{S}_{\mathrm{b}}$ & $\underline{N}$ & $\underline{N}$ & $\underline{N}$ & $\mathbf{Y}$ & $\mathbf{Y}$ & $\mathbf{Y}$ & $Y$ & $\mathbf{Y}$ & $\mathbf{Y}$ & $\mathbf{Y}$ \\
\hline $\mathrm{S}_{\mathrm{u}} \mathrm{S}$ & $\mathrm{S}_{\mathrm{u}} \mathrm{S}_{\mathrm{u}}$ & $\underline{\mathrm{N}}$ & $\underline{\mathrm{N}}$ & $Y$ & $\mathbf{Y}$ & $\mathbf{Y}$ & $\mathbf{Y}$ & $\mathbf{Y}$ & $\mathbf{Y}$ & $\mathbf{Y}$ & $Y$ & $\mathrm{~S}_{\mathrm{u}} \mathrm{S}$ & $S_{u} S_{u}$ & $\underline{N}$ & $\underline{N}$ & $\underline{N}$ & $\mathbf{Y}$ & $\mathbf{Y}$ & $Y$ & $Y$ & $\mathbf{Y}$ & $Y$ & $Y$ \\
\hline $\mathrm{S}_{\mathrm{u}} \mathrm{S}_{\mathrm{b}}$ & $\mathrm{S}_{\mathrm{u}} \mathrm{A}$ & $\mathbf{Y}$ & $\mathbf{Y}$ & $\mathbf{Y}$ & $\mathbf{Y}$ & $\mathbf{Y}$ & $\mathbf{Y}$ & $\mathbf{Y}$ & $\mathbf{Y}$ & $\mathbf{Y}$ & $Y$ & $S_{u} S_{b}$ & $S_{u} A$ & $\underline{N}$ & $\mathbf{Y}$ & $\mathbf{Y}$ & $\mathbf{Y}$ & $\mathbf{Y}$ & $\mathbf{Y}$ & $\mathbf{Y}$ & $\mathbf{Y}$ & $\mathbf{Y}$ & $\mathbf{Y}$ \\
\hline $\mathrm{S}_{\mathrm{u}} \mathrm{S}_{\mathrm{b}}$ & $\mathrm{S}_{\mathrm{u}} \mathrm{E}$ & $\underline{N}$ & $\underline{N}$ & $\underline{N}$ & $\underline{N}$ & $\underline{N}$ & $\mathbf{Y}$ & $\mathbf{Y}$ & $\mathbf{Y}$ & $\mathbf{Y}$ & $\underline{N}$ & $\mathrm{~S}_{\mathrm{u}} \mathrm{S}_{\mathrm{b}}$ & $\mathrm{S}_{\mathrm{u}} \mathrm{E}$ & $\underline{N}$ & $\underline{N}$ & $\underline{N}$ & $\underline{N}$ & $\underline{N}$ & $\mathbf{Y}$ & $\mathbf{Y}$ & $\mathbf{Y}$ & $\mathbf{Y}$ & $\mathbf{Y}$ \\
\hline$S_{u} S_{b}$ & $S_{u} L$ & $\underline{N}$ & $\underline{\mathrm{N}}$ & $\underline{N}$ & $Y$ & $\underline{\mathrm{N}}$ & $\underline{N}$ & $\underline{N}$ & $\mathbf{Y}$ & $\mathbf{Y}$ & $Y$ & $S_{u} S_{b}$ & $\mathrm{~S}_{\mathrm{u}} \mathrm{L}$ & $\underline{N}$ & $\underline{\mathrm{N}}$ & $\underline{N}$ & $\underline{N}$ & $\underline{N}$ & $Y$ & $\mathbf{Y}$ & $Y$ & $Y$ & $Y$ \\
\hline
\end{tabular}




\begin{tabular}{|c|c|c|c|c|c|c|c|c|c|c|c|c|c|c|c|c|c|c|c|c|c|c|c|}
\hline$S_{u} S_{b}$ & $\mathrm{~S}_{\mathrm{u}} \mathrm{S}$ & $\underline{N}$ & $\underline{N}$ & $\mathbf{Y}$ & $Y$ & $\mathbf{Y}$ & $\mathbf{Y}$ & $\mathbf{Y}$ & $\mathbf{Y}$ & $\mathbf{Y}$ & $\mathbf{Y}$ & $S_{u} S_{b}$ & $\mathrm{~S}_{\mathrm{u}} \mathrm{S}$ & $\underline{\mathrm{N}}$ & $\underline{\mathrm{N}}$ & $\underline{N}$ & $\mathbf{Y}$ & $\mathbf{Y}$ & $\mathbf{Y}$ & $Y$ & $\mathbf{Y}$ & $\mathbf{Y}$ & $\mathbf{Y}$ \\
\hline$S_{u} S_{b}$ & $\mathrm{~S}_{\mathrm{u}} \mathrm{S}_{\mathrm{u}}$ & $\underline{N}$ & $\underline{N}$ & $\underline{N}$ & $\underline{\mathrm{N}}$ & $\underline{\mathrm{N}}$ & $\underline{\mathrm{N}}$ & $\underline{\mathrm{N}}$ & $\underline{\mathrm{N}}$ & $\underline{\mathrm{N}}$ & $\mathbf{Y}$ & $S_{u} S_{b}$ & $S_{u} S_{u}$ & $\underline{\mathrm{N}}$ & $\underline{\mathrm{N}}$ & $\underline{N}$ & $\underline{N}$ & $\underline{\mathrm{N}}$ & $\underline{\mathrm{N}}$ & $\underline{N}$ & $\underline{N}$ & $\underline{\mathrm{N}}$ & $\mathbf{Y}$ \\
\hline $\mathrm{S}_{\mathrm{u}} \mathrm{S}_{\mathrm{u}}$ & $\mathrm{S}_{\mathrm{u}} \mathrm{A}$ & $\underline{N}$ & $Y$ & $Y$ & $Y$ & $\mathbf{Y}$ & $Y$ & $Y$ & $Y$ & $Y$ & $Y$ & $\mathrm{~S}_{\mathrm{u}} \mathrm{S}_{\mathrm{u}}$ & $\mathrm{S}_{\mathrm{u}} \mathrm{A}$ & $\underline{N}$ & $Y$ & $Y$ & $Y$ & $Y$ & $Y$ & $Y$ & $\mathbf{Y}$ & $\mathbf{Y}$ & $Y$ \\
\hline $\mathrm{S}_{\mathrm{u}} \mathrm{S}_{\mathrm{u}}$ & $\mathrm{S}_{\mathrm{u}} \mathrm{E}$ & $\underline{N}$ & $\underline{\mathrm{N}}$ & $\underline{N}$ & $\underline{\mathrm{N}}$ & $\underline{N}$ & $\mathbf{Y}$ & $\mathbf{Y}$ & $\mathbf{Y}$ & $\mathbf{Y}$ & $\mathbf{Y}$ & $\mathrm{S}_{\mathrm{u}} \mathrm{S}_{\mathrm{u}}$ & $\mathrm{S}_{\mathrm{u}} \mathrm{E}$ & $\underline{N}$ & $\underline{N}$ & $\underline{N}$ & $\underline{N}$ & $\underline{N}$ & $\mathbf{Y}$ & $\mathbf{Y}$ & $\mathbf{Y}$ & $\mathbf{Y}$ & $\mathbf{Y}$ \\
\hline $\mathrm{S}_{\mathrm{u}} \mathrm{S}_{\mathrm{u}}$ & $\mathrm{S}_{\mathrm{u}} \mathrm{L}$ & $\underline{N}$ & $\underline{N}$ & $\underline{N}$ & $\underline{N}$ & $\underline{N}$ & $\underline{\mathrm{N}}$ & $\underline{\mathrm{N}}$ & $\mathbf{Y}$ & $\mathbf{Y}$ & $\mathbf{Y}$ & $\mathrm{S}_{\mathrm{u}} \mathrm{S}_{\mathrm{u}}$ & $S_{u} L$ & $\underline{\mathrm{N}}$ & $\underline{\mathrm{N}}$ & $\underline{N}$ & $\underline{\mathrm{N}}$ & $\underline{\mathrm{N}}$ & $Y$ & $Y$ & $\mathbf{Y}$ & $\mathbf{Y}$ & $\mathbf{Y}$ \\
\hline $\mathrm{S}_{\mathrm{u}} \mathrm{S}_{\mathrm{u}}$ & $S_{u} S$ & $\underline{N}$ & $\underline{N}$ & $\mathbf{Y}$ & $\mathbf{Y}$ & $\mathbf{Y}$ & $\mathbf{Y}$ & $\mathbf{Y}$ & $\mathbf{Y}$ & $\mathbf{Y}$ & $\mathbf{Y}$ & $\mathrm{S}_{\mathrm{u}} \mathrm{S}_{\mathrm{u}}$ & $S_{u} S$ & $\underline{\mathrm{N}}$ & $\underline{\mathrm{N}}$ & $\underline{N}$ & $Y$ & $\mathbf{Y}$ & $Y$ & $Y$ & $Y$ & $\mathrm{Y}$ & $\mathbf{Y}$ \\
\hline $\mathrm{S}_{u} \mathrm{~S}_{\mathrm{u}}$ & $S_{u} S_{b}$ & $\underline{N}$ & $\underline{N}$ & $\underline{N}$ & $\underline{N}$ & $\underline{N}$ & $\underline{\mathrm{N}}$ & $\underline{\mathrm{N}}$ & $\underline{N}$ & $\underline{N}$ & $Y$ & $\mathrm{~S}_{\mathrm{u}} \mathrm{S}_{\mathrm{u}}$ & $S_{u} S_{b}$ & $\underline{N}$ & $\underline{N}$ & $\underline{N}$ & $\underline{\mathrm{N}}$ & $\underline{N}$ & $\underline{N}$ & $\underline{N}$ & $\underline{N}$ & $\underline{\mathrm{N}}$ & $\mathbf{Y}$ \\
\hline
\end{tabular}

\subsubsection{Conclusions}

Two significant trends emerge in these tests. First, the clustering of lenticulars and ellipticals around all categories of primary are similar in the vast majority of comparisons. This indicates that lenticulars and ellipticals are possibly related in some way. That is, early-type galaxies cluster similarly.

Second, the clustering of barred and unbarred spirals around all categories of primary require greater fitting ranges to reach dissimilarity than the other categories of secondary. This suggests that barred and unbarred spirals are possibly related as well.

Of interest the clustering of $E$ and $L$ secondaries is similar to $S_{b}$ and $S_{u}$ secondaries at fitting ranges beyond those where similarity is seen for $\mathrm{S}$ secondaries compared to $S_{b}$ and $S_{u}$ secondaries. This suggests 1$) S_{b}$ and $S_{u}$ cluster similarly to early-type galaxies and 2) $S_{b}$ and $S_{u}$ secondaries do not cluster similar to $S$.

\subsubsection{Impact of Primary Morphology on the Pointwise Dimension}

Data runs where the secondary morphology and fitting range are equal and the primary differs are compared using KS tests.

In all of the following tables $\mathrm{Y}$ indicates that the $p$ value from the $\mathrm{KS}$ test is < 0.01 . This means that there is less than a $1 \%$ chance that the two compared data sets are drawn from the same distribution. That is, the compared data sets are statistically dissimilar to the $99 \%$ level. A value of $\mathrm{N}$ indicates the inverse. That is, the datasets are not statistically dissimilar to the $99 \%$ level. 
When considering ellipticals and lenticulars as primary galaxies in Table 5-15, the surrounding environment of all galaxies without morphological filtering is consistently different. In the Southern galactic hemisphere the environment surrounding barred, unbarred, and spirals are not statistically dissimilar from the environment surrounding primary galaxies without regard for morphology. In the Northern galactic hemisphere the environment around primaries of unbarred spirals and spirals are similar to the environment surrounding primary galaxies without regard for morphology. Finally, in the North the environment around barred spirals is statistically dissimilar when compared to the environment around primary galaxies without regard for morphology.

Table 5-15 2D KS Test Results - comparing two runs having the same secondary filter and same fitting range filter with different primary filters - limited to primaries with no particular morphology in Run1. $\mathrm{Y}=$ dissimilar to the $99 \%$ level. $\mathrm{N}=$ not dissimilar to the $99 \%$ level

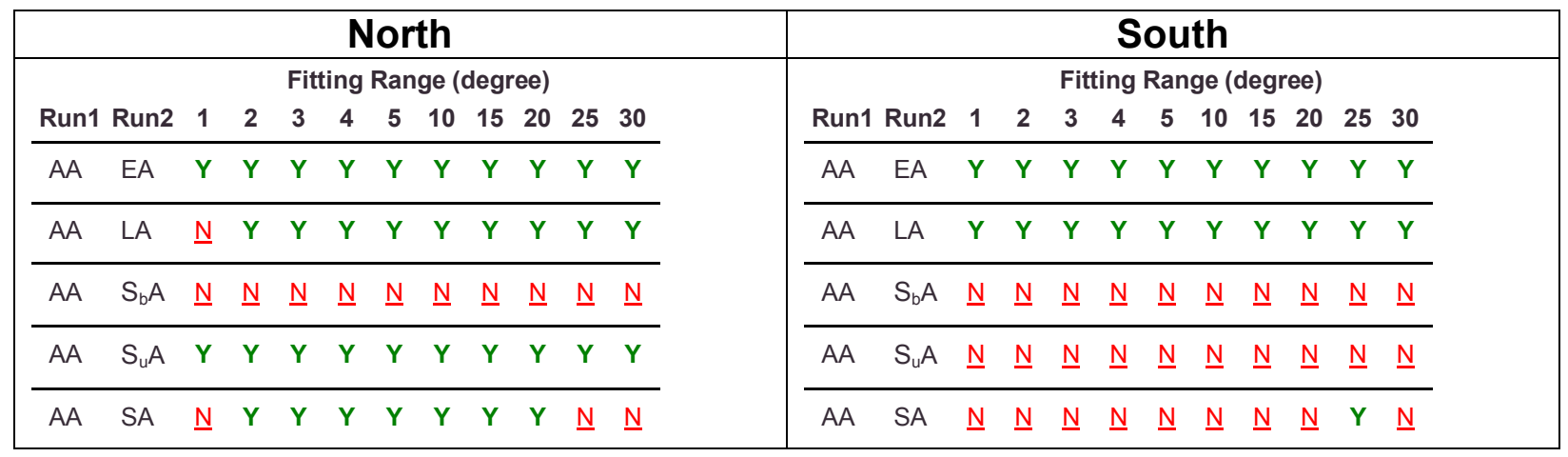


For primaries of elliptical in Table 5-16 vs. other types of primaries few reliable patterns emerge. The salient result is that ES and LS are similar at all fitting ranges observed. There is also a trend for similarity for $E L$ and $L L, E S_{u}$ and $L S_{u}$ and $E A$ and LA (North $5^{\circ}$ and up).

There is a consistent trend for dissimilarity when comparing the environment of elliptical primaries to spirals, barred spirals, and unbarred spirals. This is consistent with the finding of Best [29] that the environments of late-type galaxies and early-type galaxies are statistically dissimilar to the $99 \%$ level.

Table 5-16 2D KS Test Results - comparing two runs having the same secondary filter and same fitting range filter with different primary filters - limited to elliptical primaries in Run1.

$\mathrm{Y}=$ dissimilar to the $99 \%$ level. $\mathrm{N}=$ not dissimilar to the $99 \%$ level

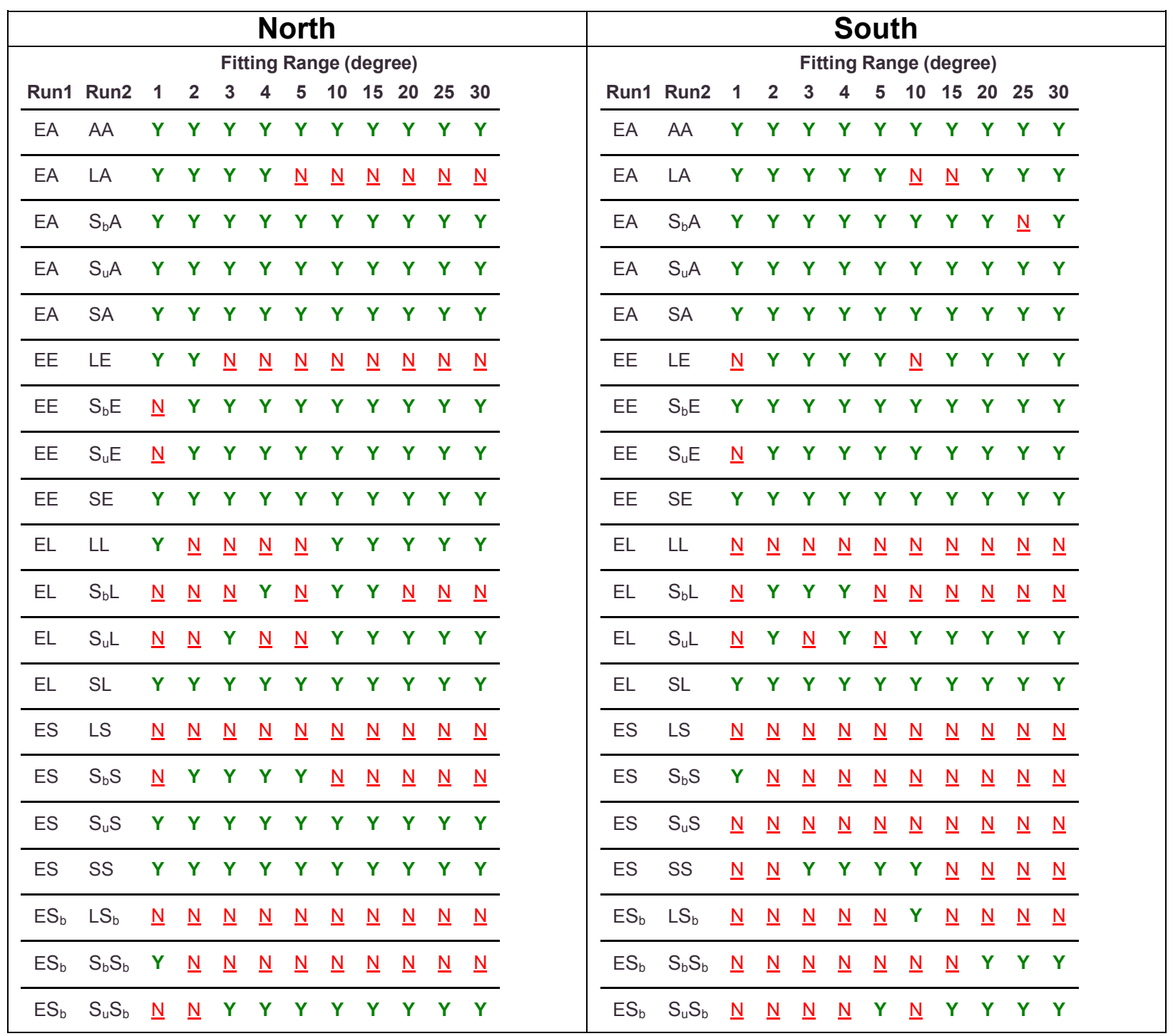




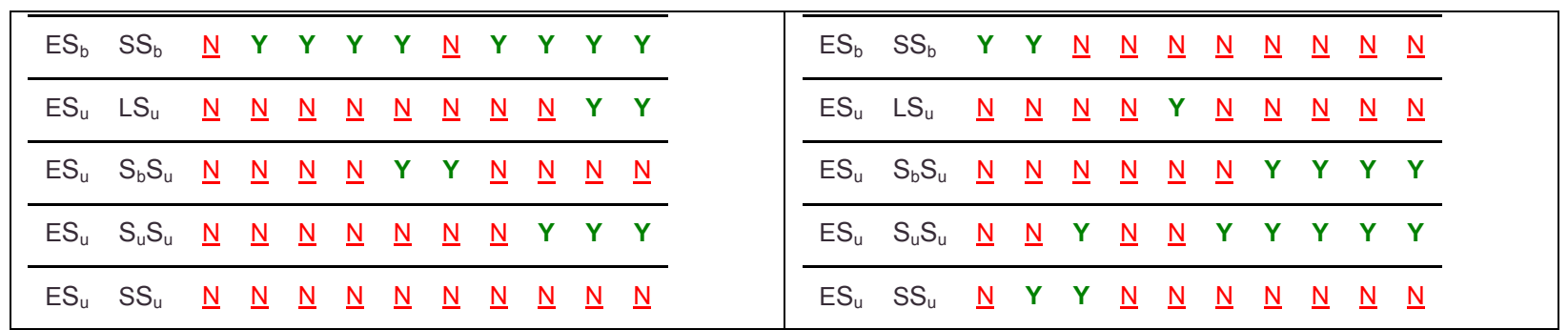


For lenticulars as primary compared to other primaries in Table 5-17, the total surrounding environment generally is dissimilar. Interestingly in a few cases dissimilarity disappears as fitting ranges increase, whereas the trend in the past has been for dissimilarity be observed once the fitting range has become larger. Of interest, the environments of lenticular primaries when compared to the environments of elliptical primaries are similar.

Generally when compared to the environments of spiral primaries the environments of lenticulars are statistically dissimilar. This is consistent with the finding of Best [29] that the environments of late-type galaxies and early-type galaxies are statistically dissimilar to the $99 \%$ level.

Table 5-17 2D KS Test Results - comparing two runs having the same secondary filter and same fitting range filter with different primary filters - limited to lenticular primaries in Run1.

$\mathrm{Y}=$ dissimilar to the $99 \%$ level. $\mathrm{N}=$ not dissimilar to the $99 \%$ level

\begin{tabular}{|c|c|c|c|c|c|c|c|c|c|c|c|c|c|c|c|c|c|c|c|c|c|c|c|}
\hline \multicolumn{12}{|c|}{ North } & \multicolumn{12}{|c|}{ South } \\
\hline \multirow[b]{2}{*}{ Run1 } & \multirow[b]{2}{*}{ Run2 } & \multicolumn{10}{|c|}{ Fitting Range (degree) } & \multicolumn{12}{|c|}{ Fitting Range (degree) } \\
\hline & & 1 & 2 & 3 & 4 & 5 & 10 & 15 & 20 & 25 & 30 & Run1 & Run2 & 1 & 2 & 3 & 4 & 5 & 10 & 15 & 20 & 25 & 30 \\
\hline LA & AA & $\underline{N}$ & $\mathbf{Y}$ & $\mathbf{Y}$ & $\mathbf{Y}$ & $\mathbf{Y}$ & $\mathbf{Y}$ & $\mathbf{Y}$ & $\mathbf{Y}$ & $\mathbf{Y}$ & $\mathbf{Y}$ & LA & AA & $\mathbf{Y}$ & $\mathbf{Y}$ & $\mathbf{Y}$ & $\mathbf{Y}$ & $\mathbf{Y}$ & $\mathbf{Y}$ & $\mathbf{Y}$ & $\mathbf{Y}$ & $\mathbf{Y}$ & $\mathbf{Y}$ \\
\hline LA & EA & $\mathbf{Y}$ & $\mathbf{Y}$ & $\mathbf{Y}$ & $\mathbf{Y}$ & $\underline{N}$ & $\underline{N}$ & $\underline{N}$ & $\underline{N}$ & $\underline{N}$ & $\underline{N}$ & LA & EA & $\mathbf{Y}$ & $\mathbf{Y}$ & $Y$ & $\mathbf{Y}$ & $\mathbf{Y}$ & $\underline{N}$ & $\underline{N}$ & $\mathbf{Y}$ & $\mathbf{Y}$ & $\mathbf{Y}$ \\
\hline LA & $\mathrm{S}_{\mathrm{b}} \mathrm{A}$ & $\underline{N}$ & $\underline{N}$ & $\mathbf{Y}$ & $\mathbf{Y}$ & $\mathbf{Y}$ & $\mathbf{Y}$ & $\mathbf{Y}$ & $\mathbf{Y}$ & $\mathbf{Y}$ & $\mathbf{Y}$ & LA & $\mathrm{S}_{\mathrm{b}} \mathrm{A}$ & $Y$ & $\mathbf{Y}$ & $Y$ & $\mathbf{Y}$ & $Y$ & $\mathbf{Y}$ & $\underline{N}$ & $\underline{N}$ & $\underline{N}$ & $\underline{N}$ \\
\hline LA & $S_{u} A$ & $\mathbf{Y}$ & $Y$ & $\mathbf{Y}$ & $Y$ & $\mathbf{Y}$ & $\mathbf{Y}$ & $\mathbf{Y}$ & $Y$ & $\mathbf{Y}$ & $Y$ & LA & $\mathrm{S}_{\mathrm{u}} \mathrm{A}$ & $\underline{\mathrm{N}}$ & $Y$ & $Y$ & $\mathbf{Y}$ & $Y$ & $\mathbf{Y}$ & $\mathbf{Y}$ & $Y$ & $\mathbf{Y}$ & $\underline{N}$ \\
\hline LA & SA & $Y$ & $Y$ & $\mathbf{Y}$ & $\mathbf{Y}$ & $\mathbf{Y}$ & $Y$ & $\mathbf{Y}$ & $Y$ & $\mathbf{Y}$ & $\mathbf{Y}$ & LA & SA & $Y$ & $Y$ & $Y$ & $\mathbf{Y}$ & $Y$ & $Y$ & $\mathbf{Y}$ & $Y$ & $Y$ & $Y$ \\
\hline LE & EE & $Y$ & $Y$ & $\underline{N}$ & $\underline{N}$ & $\underline{\mathrm{N}}$ & $\underline{N}$ & $\underline{N}$ & $\underline{N}$ & $\underline{\mathrm{N}}$ & $\underline{\mathrm{N}}$ & LE & EE & $\underline{N}$ & $Y$ & $\mathbf{Y}$ & $\mathbf{Y}$ & $Y$ & $\underline{N}$ & $\mathbf{Y}$ & $Y$ & $\mathbf{Y}$ & $Y$ \\
\hline LE & $S_{b} E$ & $\underline{\mathrm{N}}$ & $\underline{N}$ & $\underline{N}$ & $\underline{N}$ & $\mathbf{Y}$ & $Y$ & $\mathbf{Y}$ & $\mathbf{Y}$ & $\mathbf{Y}$ & $Y$ & LE & $\mathrm{S}_{\mathrm{b}} \mathrm{E}$ & $\underline{N}$ & $\mathbf{Y}$ & $Y$ & $\mathbf{Y}$ & $\underline{N}$ & $\underline{N}$ & $\mathbf{Y}$ & $\underline{\mathrm{N}}$ & $\underline{N}$ & $\underline{N}$ \\
\hline LE & $\mathrm{S}_{\mathrm{u}} \mathrm{E}$ & $\underline{N}$ & $\underline{N}$ & $\underline{N}$ & $\mathbf{Y}$ & $\mathbf{Y}$ & $\mathbf{Y}$ & $\mathbf{Y}$ & $Y$ & $\mathbf{Y}$ & $\mathbf{Y}$ & LE & $\mathrm{S}_{\mathrm{u}} \mathrm{E}$ & $\underline{N}$ & $\mathbf{Y}$ & $Y$ & $\mathbf{Y}$ & $Y$ & $\mathbf{Y}$ & $\mathbf{Y}$ & $\mathbf{Y}$ & $Y$ & $Y$ \\
\hline LE & SE & $\underline{N}$ & $\mathbf{Y}$ & $\mathbf{Y}$ & $\mathbf{Y}$ & $\mathbf{Y}$ & $Y$ & $Y$ & $\mathbf{Y}$ & $Y$ & $\mathbf{Y}$ & LE & SE & $\underline{N}$ & $Y$ & $Y$ & $\mathbf{Y}$ & $Y$ & $\mathbf{Y}$ & $\mathbf{Y}$ & $Y$ & $Y$ & $Y$ \\
\hline LL & EL & $\mathbf{Y}$ & $\underline{N}$ & $\underline{N}$ & $\underline{N}$ & $\underline{N}$ & $Y$ & $\mathbf{Y}$ & $\mathbf{Y}$ & $\mathbf{Y}$ & $Y$ & LL & $\mathrm{EL}$ & $\underline{N}$ & $\underline{N}$ & $\underline{N}$ & $\underline{\mathrm{N}}$ & $\underline{N}$ & $\underline{N}$ & $\underline{N}$ & $\underline{N}$ & $\underline{N}$ & $\underline{N}$ \\
\hline LL & $\mathrm{S}_{\mathrm{b}} \mathrm{L}$ & $\underline{N}$ & $\mathbf{Y}$ & $\mathbf{Y}$ & $\mathbf{Y}$ & $\mathbf{Y}$ & $Y$ & $\mathbf{Y}$ & $\mathbf{Y}$ & $\mathbf{Y}$ & $\mathrm{Y}$ & LL & $S_{b} \mathrm{~L}$ & $\underline{N}$ & $\underline{\mathrm{N}}$ & $\underline{N}$ & $\mathbf{Y}$ & $\underline{N}$ & $\mathbf{Y}$ & $\mathbf{Y}$ & $\underline{N}$ & $\underline{N}$ & $\underline{\mathrm{N}}$ \\
\hline LL & $S_{u} L$ & $\underline{N}$ & $\underline{N}$ & $\mathbf{Y}$ & $\mathbf{Y}$ & $\mathbf{Y}$ & $\mathbf{Y}$ & $\mathbf{Y}$ & $\mathbf{Y}$ & $Y$ & $\mathbf{Y}$ & LL & $\mathrm{S}_{\mathrm{u}} \mathrm{L}$ & $\underline{N}$ & $\underline{N}$ & $\underline{N}$ & $Y$ & $\mathbf{Y}$ & $\mathbf{Y}$ & $\mathbf{Y}$ & $\mathbf{Y}$ & $\mathbf{Y}$ & $\mathbf{Y}$ \\
\hline LL & $\mathrm{SL}$ & $\underline{N}$ & $\mathbf{Y}$ & $\mathbf{Y}$ & $\mathbf{Y}$ & $\mathbf{Y}$ & $\mathbf{Y}$ & $\mathbf{Y}$ & $\mathbf{Y}$ & $\mathbf{Y}$ & $\mathbf{Y}$ & LL & $\mathrm{SL}$ & $\underline{N}$ & $\underline{N}$ & $\mathbf{Y}$ & $\mathbf{Y}$ & $\mathbf{Y}$ & $\mathbf{Y}$ & $\mathbf{Y}$ & $Y$ & $\mathbf{Y}$ & $\mathbf{Y}$ \\
\hline LS & ES & $\underline{N}$ & $\underline{N}$ & $\underline{N}$ & $\underline{N}$ & $\underline{N}$ & $\underline{N}$ & $\underline{N}$ & $\underline{N}$ & $\underline{\mathrm{N}}$ & $\underline{\mathrm{N}}$ & LS & ES & $\underline{N}$ & $\underline{N}$ & $\underline{N}$ & $\underline{N}$ & $\underline{N}$ & $\underline{N}$ & $\underline{\mathrm{N}}$ & $\underline{N}$ & $\underline{N}$ & $\underline{N}$ \\
\hline LS & $\mathrm{S}_{\mathrm{b}} \mathrm{S}$ & $\underline{N}$ & $\underline{\mathrm{N}}$ & $\mathbf{Y}$ & $\mathbf{Y}$ & $Y$ & $Y$ & $\underline{N}$ & $\underline{N}$ & $\underline{N}$ & $\underline{\mathrm{N}}$ & LS & $\mathrm{S}_{\mathrm{b}} \mathrm{S}$ & $Y$ & $\underline{\mathrm{N}}$ & $\underline{N}$ & $\underline{N}$ & $\underline{N}$ & $\underline{N}$ & $\underline{\mathrm{N}}$ & $\underline{N}$ & $\underline{N}$ & $\underline{N}$ \\
\hline LS & $\mathrm{S}_{\mathrm{u}} \mathrm{S}$ & $\underline{\mathrm{N}}$ & $\mathbf{Y}$ & $\mathbf{Y}$ & $\mathbf{Y}$ & $\mathbf{Y}$ & $Y$ & $\mathbf{Y}$ & $\mathbf{Y}$ & $\mathbf{Y}$ & $\mathbf{Y}$ & LS & $\mathrm{S}_{\mathrm{u}} \mathrm{S}$ & $\underline{\mathrm{N}}$ & $\underline{N}$ & $\mathbf{Y}$ & $\mathbf{Y}$ & $Y$ & $\mathbf{Y}$ & $\underline{N}$ & $\underline{N}$ & $\underline{N}$ & $\underline{N}$ \\
\hline LS & SS & $\underline{N}$ & $\mathbf{Y}$ & $\mathbf{Y}$ & $Y$ & $Y$ & $Y$ & $Y$ & $Y$ & $Y$ & $Y$ & LS & SS & $\underline{N}$ & $\underline{N}$ & $Y$ & $Y$ & $Y$ & $Y$ & $\mathbf{Y}$ & $Y$ & $\underline{N}$ & $\underline{N}$ \\
\hline $\mathrm{LS}_{\mathrm{b}}$ & $\mathrm{ES}_{\mathrm{b}}$ & $\underline{N}$ & $\underline{N}$ & $\underline{N}$ & $\underline{N}$ & $\underline{N}$ & $\underline{N}$ & $\underline{N}$ & $\underline{N}$ & $\underline{\mathrm{N}}$ & $\underline{\mathrm{N}}$ & $\mathrm{LS}_{\mathrm{b}}$ & $\mathrm{ES}_{\mathrm{b}}$ & $\underline{N}$ & $\underline{N}$ & $\underline{N}$ & $\underline{\mathrm{N}}$ & $\underline{N}$ & $Y$ & $\underline{N}$ & $\underline{N}$ & $\underline{N}$ & $\underline{N}$ \\
\hline $\mathrm{LS}_{\mathrm{b}}$ & $S_{b} S_{b}$ & $Y$ & $\underline{N}$ & $\underline{N}$ & $\underline{N}$ & $\underline{N}$ & $\underline{N}$ & $\underline{N}$ & $\underline{N}$ & $\underline{N}$ & $\underline{N}$ & $\mathrm{LS}_{\mathrm{b}}$ & $S_{b} S_{b}$ & $\underline{N}$ & $\underline{N}$ & $\underline{N}$ & $\mathbf{Y}$ & $Y$ & $\underline{N}$ & $\underline{N}$ & $\underline{N}$ & $Y$ & $\mathbf{Y}$ \\
\hline
\end{tabular}




\begin{tabular}{|c|c|c|c|c|c|c|c|c|c|c|c|c|c|c|c|c|c|c|c|c|c|c|c|}
\hline $\mathrm{LS}_{\mathrm{b}}$ & $\mathrm{S}_{\mathrm{u}} \mathrm{S}_{\mathrm{b}}$ & $\underline{N}$ & $\underline{\mathrm{N}}$ & $\mathbf{Y}$ & $Y$ & $Y$ & $Y$ & $Y$ & $\mathbf{Y}$ & $\mathbf{Y}$ & $\mathbf{Y}$ & $\mathrm{LS}_{\mathrm{b}}$ & $\mathrm{S}_{\mathrm{u}} \mathrm{S}_{\mathrm{b}}$ & $\underline{\mathrm{N}}$ & $\underline{N}$ & $\underline{N}$ & $\underline{N}$ & $\underline{\mathrm{N}}$ & $Y$ & $\mathbf{Y}$ & $\mathbf{Y}$ & $\mathbf{Y}$ & $\mathbf{Y}$ \\
\hline $\mathrm{LS}_{\mathrm{b}}$ & $\mathrm{SS}_{\mathrm{b}}$ & $\underline{\mathrm{N}}$ & $\underline{N}$ & $\underline{N}$ & $Y$ & $Y$ & $Y$ & $Y$ & $\mathbf{Y}$ & $\mathbf{Y}$ & $\mathbf{Y}$ & $\mathrm{LS}_{\mathrm{b}}$ & $\mathrm{SS}_{\mathrm{b}}$ & $\underline{\mathrm{N}}$ & $\underline{N}$ & $\underline{N}$ & $\mathbf{Y}$ & $\mathbf{Y}$ & $\underline{N}$ & $\underline{N}$ & $\underline{N}$ & $\underline{\mathrm{N}}$ & $\mathbf{Y}$ \\
\hline $\mathrm{LS}_{\mathrm{u}}$ & $\mathrm{ES}_{\mathrm{u}}$ & $\underline{N}$ & $\underline{N}$ & $\underline{N}$ & $\underline{N}$ & $\underline{N}$ & $\underline{N}$ & $\underline{N}$ & $\underline{N}$ & $Y$ & $Y$ & $\mathrm{LS}_{\mathrm{u}}$ & $\mathrm{ES}_{\mathrm{u}}$ & $\underline{N}$ & $\underline{N}$ & $\underline{N}$ & $\underline{N}$ & $Y$ & $\underline{N}$ & $\underline{N}$ & $\underline{\mathrm{N}}$ & $\underline{N}$ & $\underline{N}$ \\
\hline $\mathrm{LS}_{\mathrm{u}}$ & $\mathrm{S}_{\mathrm{b}} \mathrm{S}_{\mathrm{u}}$ & $\underline{N}$ & $\underline{N}$ & $\underline{N}$ & $\mathbf{Y}$ & $Y$ & $Y$ & $Y$ & $\underline{N}$ & $Y$ & $\mathbf{Y}$ & $\mathrm{LS}_{\mathrm{u}}$ & $\mathrm{S}_{\mathrm{b}} \mathrm{S}_{\mathrm{u}}$ & $\underline{N}$ & $\mathbf{Y}$ & $\mathbf{Y}$ & $\mathbf{Y}$ & $\underline{\mathrm{N}}$ & $\underline{N}$ & $\mathbf{Y}$ & $\mathbf{Y}$ & $\mathbf{Y}$ & $\mathbf{Y}$ \\
\hline $\mathrm{LS}_{\mathrm{u}}$ & $\mathrm{S}_{\mathrm{u}} \mathrm{S}_{\mathrm{u}}$ & $\underline{\mathrm{N}}$ & $\underline{N}$ & $\underline{N}$ & $Y$ & $\underline{N}$ & $Y$ & $Y$ & $\mathbf{Y}$ & $\mathbf{Y}$ & $\mathbf{Y}$ & $\mathrm{LS}_{\mathrm{u}}$ & $\mathrm{S}_{\mathrm{u}} \mathrm{S}_{\mathrm{u}}$ & $\underline{N}$ & $\underline{N}$ & $\mathbf{Y}$ & $\mathbf{Y}$ & $Y$ & $Y$ & $\mathbf{Y}$ & $Y$ & $\mathbf{Y}$ & $\mathbf{Y}$ \\
\hline $\mathrm{LS}_{\mathrm{u}}$ & $\mathrm{SS}_{\mathrm{u}}$ & $\underline{\mathrm{N}}$ & $\underline{N}$ & $\mathbf{Y}$ & $Y$ & $Y$ & $Y$ & $Y$ & $\underline{N}$ & $\underline{N}$ & $\underline{N}$ & $\mathrm{LS}_{\mathrm{u}}$ & $\mathrm{SS}_{\mathrm{u}}$ & $\underline{\mathrm{N}}$ & $\mathbf{Y}$ & $\mathbf{Y}$ & $Y$ & $Y$ & $\underline{N}$ & $\underline{N}$ & IV & $\underline{\mathrm{N}}$ & $\underline{N}$ \\
\hline
\end{tabular}


In Table 5-18, The environments of spirals compared to the environments of primaries with no morphological filter are dramatically different. In the North dissimilarity is noted for this comparison while dissimilarity is found in the South.

The environment of spirals when compared the environment of ellipticals and lenticulars are consistently dissimilar. This is consistent with the finding of Best [29] that the environments of late-type galaxies and early-type galaxies are statistically dissimilar to the $99 \%$ level.

An important observation is that with few exceptions the environment around spirals when compared to barred spirals and unbarred are similar. This does not suggest environment as a factor in the resultant formation of barred or unbarred variants of spiral galaxies. This is explored further below when considering barred and unbarred spirals as primary against all other primary morphologies.

Table 5-18 2D KS Test Results - comparing two runs having the same secondary filter and same fitting range filter with different primary filters - limited to spiral primaries in Run1.

$\mathrm{Y}=$ dissimilar to the $99 \%$ level. $\mathrm{N}=$ not dissimilar to the $99 \%$ level

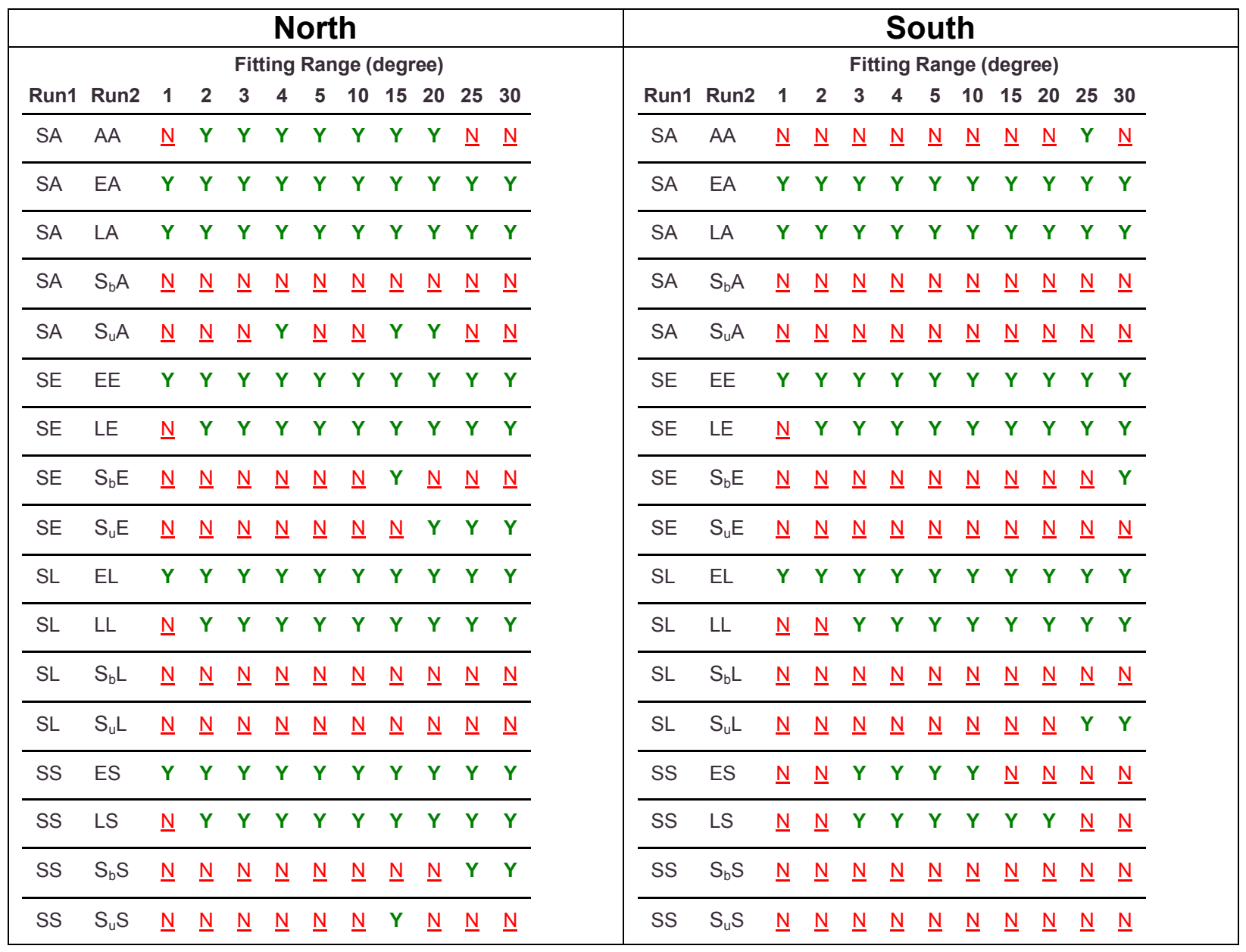




\begin{tabular}{|c|c|c|c|c|c|c|c|c|c|c|c|c|c|c|c|c|c|c|c|c|c|c|c|}
\hline $\mathrm{SS}_{\mathrm{b}}$ & $\mathrm{ES}_{\mathrm{b}}$ & $\underline{\mathrm{N}}$ & $\mathbf{Y}$ & $\mathbf{Y}$ & $\mathbf{Y}$ & $\mathbf{Y}$ & $\underline{\mathrm{N}}$ & $\mathbf{Y}$ & $\mathbf{Y}$ & $\mathbf{Y}$ & $\mathbf{Y}$ & $\mathrm{SS}_{\mathrm{b}}$ & $\mathrm{ES}_{\mathrm{b}}$ & $\mathbf{Y}$ & $\mathbf{Y}$ & $\underline{N}$ & $\underline{\mathrm{N}}$ & $\underline{N}$ & $\underline{N}$ & $\underline{N}$ & $\underline{N}$ & $\underline{N}$ & $\underline{N}$ \\
\hline $\mathrm{SS}_{\mathrm{b}}$ & $\mathrm{LS}_{\mathrm{b}}$ & $\underline{\mathrm{N}}$ & $\underline{\mathrm{N}}$ & $\underline{\mathrm{N}}$ & $Y$ & $\mathbf{Y}$ & $Y$ & $Y$ & $Y$ & $Y$ & $\mathbf{Y}$ & $\mathrm{SS}_{\mathrm{b}}$ & $\mathrm{LS}_{\mathrm{b}}$ & $\underline{N}$ & $\underline{\mathrm{N}}$ & $\underline{N}$ & $\mathbf{Y}$ & $\mathbf{Y}$ & $\underline{N}$ & $\underline{N}$ & $\underline{\mathrm{N}}$ & $\underline{\mathrm{N}}$ & $\mathbf{Y}$ \\
\hline $\mathrm{SS}_{\mathrm{b}}$ & $S_{b} S_{b}$ & $Y$ & $\underline{\mathrm{N}}$ & $\mathbf{Y}$ & $Y$ & $\mathbf{Y}$ & $Y$ & $Y$ & $Y$ & $Y$ & $\mathbf{Y}$ & $\mathrm{SS}_{\mathrm{b}}$ & $\mathrm{S}_{\mathrm{b}} \mathrm{S}_{\mathrm{b}}$ & $\underline{N}$ & $\underline{\mathrm{N}}$ & $\underline{N}$ & $\underline{N}$ & $\underline{N}$ & $\underline{N}$ & $\underline{N}$ & $\underline{N}$ & $\mathbf{Y}$ & $\mathbf{Y}$ \\
\hline $\mathrm{SS}_{\mathrm{b}}$ & $\mathrm{S}_{\mathrm{u}} \mathrm{S}_{\mathrm{b}}$ & $\underline{\mathrm{N}}$ & $\underline{N}$ & $\underline{\mathrm{N}}$ & $\underline{N}$ & $\underline{\mathrm{N}}$ & $\underline{\mathrm{N}}$ & $\underline{\mathrm{N}}$ & $\underline{N}$ & $\underline{N}$ & $\underline{N}$ & $\mathrm{SS}_{\mathrm{b}}$ & $\mathrm{S}_{\mathrm{u}} \mathrm{S}_{\mathrm{b}}$ & $\underline{N}$ & $\underline{\mathrm{N}}$ & $\underline{N}$ & $\underline{N}$ & $\underline{N}$ & $\underline{N}$ & $Y$ & $\mathbf{Y}$ & $\mathbf{Y}$ & $\mathbf{Y}$ \\
\hline $\mathrm{SS}_{\mathrm{u}}$ & $\mathrm{ES}_{\mathrm{u}}$ & $\underline{N}$ & $\underline{N}$ & $\underline{N}$ & $\underline{N}$ & $\underline{\mathrm{N}}$ & $\underline{N}$ & $\underline{\mathrm{N}}$ & $\underline{N}$ & $\underline{N}$ & $\underline{N}$ & $\mathrm{SS}_{\mathrm{u}}$ & $\mathrm{ES}_{\mathrm{u}}$ & $\underline{\mathrm{N}}$ & $Y$ & $Y$ & $\underline{N}$ & $\underline{N}$ & $\underline{N}$ & $\underline{N}$ & $\underline{N}$ & $\underline{N}$ & $\underline{N}$ \\
\hline $\mathrm{SS}_{\mathrm{u}}$ & $\mathrm{LS}_{\mathrm{u}}$ & $\underline{N}$ & $\underline{N}$ & $\mathrm{Y}$ & $\mathbf{Y}$ & $Y$ & $\mathbf{Y}$ & $\mathbf{Y}$ & $\underline{N}$ & $\underline{N}$ & $\underline{N}$ & $\mathrm{SS}_{\mathrm{u}}$ & $\mathrm{LS}_{\mathrm{u}}$ & $\underline{\mathrm{N}}$ & $\mathrm{Y}$ & $\mathrm{Y}$ & $\mathrm{Y}$ & $\mathrm{Y}$ & $\underline{\mathrm{N}}$ & $\underline{N}$ & $\underline{N}$ & $\underline{N}$ & $\underline{N}$ \\
\hline $\mathrm{SS}_{\mathrm{u}}$ & $\mathrm{S}_{\mathrm{b}} \mathrm{S}_{\mathrm{u}}$ & $\underline{N}$ & $\underline{N}$ & $\underline{\mathrm{N}}$ & $\underline{\mathrm{N}}$ & $\underline{N}$ & $\underline{\mathrm{N}}$ & $\underline{N}$ & $\underline{N}$ & $\underline{N}$ & $\underline{N}$ & $\mathrm{SS}_{\mathrm{u}}$ & $S_{b} S_{u}$ & $\underline{N}$ & $\underline{\mathrm{N}}$ & $\underline{\mathrm{N}}$ & $\underline{\mathrm{N}}$ & $\underline{\mathrm{N}}$ & $\underline{N}$ & $\mathbf{Y}$ & $\underline{N}$ & $\mathbf{Y}$ & $\mathbf{Y}$ \\
\hline $\mathrm{SS}_{\mathrm{u}}$ & $\mathrm{S}_{\mathrm{u}} \mathrm{S}_{\mathrm{u}}$ & $\underline{N}$ & $\underline{N}$ & $\underline{N}$ & $\underline{N}$ & $\underline{N}$ & $\mathbf{Y}$ & $\mathbf{Y}$ & $\mathbf{Y}$ & $\mathbf{Y}$ & $\mathbf{Y}$ & $\mathrm{SS}_{\mathrm{u}}$ & $S_{u} S_{u}$ & $\underline{N}$ & $\underline{N}$ & $\underline{N}$ & $\underline{\mathrm{N}}$ & $\underline{N}$ & $Y$ & $Y$ & $Y$ & $Y$ & $\mathbf{Y}$ \\
\hline
\end{tabular}


In Table 5-19, The environments of barred spirals compared to the environments of galaxies with no morphological filtering are similar at all fitting ranges.

Dissimilarity is generally found when comparing the environments of barred spirals to the environments of ellipticals and when comparing the environments of barred spirals to the environments of lenticulars This is consistent with the finding of Best [29] that the environments of late-type galaxies and early-type galaxies are statistically dissimilar to the $99 \%$ level.

Interestingly in the South when comparing the environments of barred spirals to the environments of lenticulars dissimilarity stop at $15^{\circ}$ and above.

No clear patterns emerge that agree in North and South once secondary morphology filters are applied except similarity for $S_{b} S$ vs. SS and $S_{b} L$ vs. $S L$, and $S_{b} L$ vs. and SuL.

The data also clearly suggests that there is a relationship between the environments of barred spirals and spirals because no dissimilarity is found at any of the fitting ranges when comparing those primaries and all secondary morphologies. This suggests that the formation of bars (or not) is not related to the environment.

Table 5-19 2D KS Test Results - comparing two runs having the same secondary filter and same fitting range filter with different primary filters - limited to unbarred spiral primaries in Run1.

$\mathrm{Y}=$ dissimilar to the $99 \%$ level. $\mathrm{N}=$ not dissimilar to the $99 \%$ level

\begin{tabular}{|c|c|c|c|c|c|c|c|c|c|c|c|c|c|c|c|c|c|c|c|c|c|c|c|}
\hline \multicolumn{12}{|c|}{ North } & \multicolumn{12}{|c|}{ South } \\
\hline \multirow[b]{2}{*}{ Run1 } & \multicolumn{11}{|c|}{ Fitting Range (degree) } & \multicolumn{12}{|c|}{ Fitting Range (degree) } \\
\hline & Run2 & 1 & 2 & 3 & 4 & 5 & 10 & 15 & 20 & 25 & 30 & Run1 & Run2 & 1 & 2 & 3 & 4 & 5 & 10 & 15 & 20 & 25 & 30 \\
\hline $\mathrm{S}_{\mathrm{b}} \mathrm{A}$ & $A A$ & $\underline{N}$ & $\underline{N}$ & $\underline{N}$ & $\underline{N}$ & $\underline{N}$ & $\underline{N}$ & $\underline{N}$ & $\underline{N}$ & $\underline{N}$ & $\underline{N}$ & $\mathrm{~S}_{\mathrm{b}} \mathrm{A}$ & AA & $\underline{N}$ & $\underline{N}$ & $\underline{N}$ & $\underline{N}$ & $\underline{N}$ & $\underline{N}$ & $\underline{N}$ & $\underline{N}$ & $\underline{N}$ & $\underline{N}$ \\
\hline $\mathrm{S}_{\mathrm{b}} \mathrm{A}$ & EA & $\mathbf{Y}$ & $\mathbf{Y}$ & $\mathbf{Y}$ & $\mathbf{Y}$ & $\mathbf{Y}$ & $\mathbf{Y}$ & $\mathbf{Y}$ & $\mathbf{Y}$ & $\mathbf{Y}$ & $\mathbf{Y}$ & $\mathrm{S}_{\mathrm{b}} \mathrm{A}$ & EA & $\mathbf{Y}$ & $\mathbf{Y}$ & $\mathbf{Y}$ & $\mathbf{Y}$ & $\mathbf{Y}$ & $\mathbf{Y}$ & $\mathbf{Y}$ & $\mathbf{Y}$ & $\underline{N}$ & $\mathbf{Y}$ \\
\hline$S_{b} A$ & LA & $\underline{N}$ & $\underline{N}$ & $\mathrm{Y}$ & $\mathbf{Y}$ & $Y$ & $\mathrm{Y}$ & $\mathrm{Y}$ & $Y$ & $Y$ & $\mathrm{Y}$ & $\mathrm{S}_{\mathrm{b}} \mathrm{A}$ & LA & $\mathrm{Y}$ & $Y$ & $Y$ & $Y$ & $Y$ & $\mathrm{Y}$ & $\underline{N}$ & $\underline{N}$ & $\underline{N}$ & $\underline{N}$ \\
\hline$S_{b} A$ & $\mathrm{~S}_{\mathrm{u}} \mathrm{A}$ & $\underline{N}$ & $\underline{N}$ & $Y$ & $\mathrm{Y}$ & $Y$ & $Y$ & $Y$ & $Y$ & $Y$ & $Y$ & $S_{b} A$ & $\mathrm{~S}_{\mathrm{u}} \mathrm{A}$ & $\underline{N}$ & $\underline{N}$ & $\underline{N}$ & $\underline{N}$ & $\underline{N}$ & $\underline{N}$ & $\underline{N}$ & $\underline{N}$ & $\underline{N}$ & $\underline{N}$ \\
\hline$S_{b} A$ & SA & $\underline{N}$ & $\underline{N}$ & $\underline{N}$ & $\underline{N}$ & $\underline{N}$ & $\underline{N}$ & $\underline{N}$ & $\underline{N}$ & $\underline{N}$ & $\underline{N}$ & $S_{b} A$ & SA & $\underline{N}$ & $\underline{N}$ & $\underline{N}$ & $\underline{N}$ & $\underline{N}$ & $\underline{N}$ & $\underline{N}$ & $\underline{N}$ & $\underline{N}$ & $\underline{N}$ \\
\hline$S_{b} E$ & EE & $\underline{N}$ & $\mathbf{Y}$ & $\mathbf{Y}$ & $\mathbf{Y}$ & $\mathbf{Y}$ & $\mathbf{Y}$ & $\mathbf{Y}$ & $\mathbf{Y}$ & $\mathbf{Y}$ & $\mathbf{Y}$ & $S_{b} E$ & EE & $\mathbf{Y}$ & $\mathbf{Y}$ & $\mathbf{Y}$ & $\mathbf{Y}$ & $\mathbf{Y}$ & $\mathbf{Y}$ & $\mathbf{Y}$ & $\mathbf{Y}$ & $\mathbf{Y}$ & $\mathbf{Y}$ \\
\hline$S_{b} E$ & LE & $\underline{N}$ & $\underline{N}$ & $\underline{N}$ & $\underline{N}$ & $Y$ & $Y$ & $Y$ & $Y$ & $Y$ & $Y$ & $\mathrm{~S}_{\mathrm{b}} \mathrm{E}$ & LE & $\underline{N}$ & $\mathrm{Y}$ & $Y$ & $Y$ & $\underline{N}$ & $\underline{N}$ & $Y$ & $\underline{N}$ & $\underline{N}$ & $\underline{N}$ \\
\hline$S_{b} E$ & $\mathrm{~S}_{\mathrm{u}} \mathrm{E}$ & $\underline{N}$ & $\underline{N}$ & $\underline{N}$ & $\underline{N}$ & $\underline{N}$ & $\mathbf{Y}$ & $\mathbf{Y}$ & $\mathbf{Y}$ & $\mathbf{Y}$ & $Y$ & $\mathrm{~S}_{\mathrm{b}} \mathrm{E}$ & $S_{u} E$ & $\underline{N}$ & $\underline{N}$ & $\underline{N}$ & $\underline{N}$ & $\underline{\mathrm{N}}$ & $\underline{N}$ & $\underline{\mathrm{N}}$ & $\underline{N}$ & $\underline{N}$ & $\underline{N}$ \\
\hline$S_{b} E$ & SE & $\underline{N}$ & $\underline{N}$ & $\underline{N}$ & $\underline{N}$ & $\underline{N}$ & $\underline{N}$ & $\mathbf{Y}$ & $\underline{N}$ & $\underline{N}$ & $\underline{N}$ & $S_{b} E$ & SE & $\underline{N}$ & $\underline{N}$ & $\underline{N}$ & $\underline{N}$ & $\underline{N}$ & $\underline{N}$ & $\underline{N}$ & $\underline{N}$ & $\underline{N}$ & $\mathbf{Y}$ \\
\hline$S_{b} L$ & $E L$ & $\underline{N}$ & $\underline{N}$ & $\underline{N}$ & $Y$ & $\underline{N}$ & $\mathrm{Y}$ & $Y$ & $\underline{N}$ & $\underline{N}$ & $\underline{N}$ & $\mathrm{~S}_{\mathrm{b}} \mathrm{L}$ & $\mathrm{EL}$ & $\underline{N}$ & $Y$ & $\mathbf{Y}$ & $Y$ & $\underline{N}$ & $\underline{\mathrm{N}}$ & $\underline{N}$ & $\underline{N}$ & $\underline{\mathrm{N}}$ & $\underline{N}$ \\
\hline $\mathrm{S}_{\mathrm{b}} \mathrm{L}$ & LL & $\underline{N}$ & $Y$ & $Y$ & $Y$ & $Y$ & $Y$ & $Y$ & $Y$ & $Y$ & $Y$ & $S_{b} L$ & LL & $\underline{N}$ & $\underline{N}$ & $\underline{N}$ & $Y$ & $\underline{N}$ & $Y$ & $Y$ & $\underline{N}$ & $\underline{N}$ & $\underline{N}$ \\
\hline
\end{tabular}




\begin{tabular}{|c|c|c|c|c|c|c|c|c|c|c|c|c|c|c|c|c|c|c|c|c|c|c|}
\hline$S_{b} L$ & $\mathrm{~S}_{\mathrm{u}} \mathrm{L}$ & $\underline{N}$ & $\underline{N}$ & $\underline{N}$ & $\underline{N}$ & $\underline{N}$ & $\underline{N}$ & $\underline{N}$ & $\underline{N}$ & $\mathbf{Y}$ & $\mathbf{Y}$ & $S_{b} L$ & $\mathrm{~S}_{\mathrm{u}} \mathrm{L}$ & $\underline{N} \quad \underline{N}$ & $\underline{N}$ & $\underline{\mathrm{N}}$ & $\underline{N}$ & $\underline{N}$ & $\underline{N}$ & $\underline{N}$ & $\underline{N}$ & $\underline{N}$ \\
\hline$S_{b} L$ & $\mathrm{SL}$ & $\underline{N}$ & $\underline{N}$ & $\underline{N}$ & $\underline{N}$ & $\underline{N}$ & $\underline{N}$ & $\underline{N}$ & $\underline{N}$ & $\underline{N}$ & $\underline{N}$ & $S_{b} \mathrm{~L}$ & $\mathrm{SL}$ & $\underline{N} \quad \underline{N}$ & $\underline{N}$ & $\underline{N}$ & $\underline{N}$ & $\underline{N}$ & $\underline{N}$ & $\underline{\mathrm{N}}$ & $\underline{N}$ & $\underline{N}$ \\
\hline$S_{b} S$ & ES & $\underline{N}$ & $\mathbf{Y}$ & $Y$ & $\mathbf{Y}$ & $Y$ & $\underline{N}$ & $\underline{N}$ & $\underline{N}$ & $\underline{N}$ & $\underline{N}$ & $S_{b} S$ & ES & $Y \quad \underline{N}$ & $\underline{N}$ & $\underline{N}$ & $\underline{N}$ & $\underline{N}$ & $\underline{N}$ & $\underline{N}$ & $\underline{N}$ & $\underline{N}$ \\
\hline$S_{b} S$ & LS & $\underline{N}$ & $\underline{N}$ & $\mathbf{Y}$ & $\mathbf{Y}$ & $\mathbf{Y}$ & $\mathbf{Y}$ & $\underline{N}$ & $\underline{N}$ & $\underline{N}$ & $\underline{N}$ & $\mathrm{~S}_{\mathrm{b}} \mathrm{S}$ & LS & $Y \quad \underline{N}$ & $\underline{N}$ & $\underline{N}$ & $\underline{N}$ & $\underline{N}$ & $\underline{N}$ & $\underline{N}$ & $\underline{N}$ & $\underline{N}$ \\
\hline$S_{b} S$ & $\mathrm{~S}_{\mathrm{u}} \mathrm{S}$ & $\underline{N}$ & $\underline{N}$ & $\underline{N}$ & $\underline{N}$ & $\underline{N}$ & $\underline{N}$ & $Y$ & $Y$ & $Y$ & $Y$ & $\mathrm{~S}_{\mathrm{b}} \mathrm{S}$ & $\mathrm{S}_{\mathrm{u}} \mathrm{S}$ & $Y \quad \underline{N}$ & $\underline{N}$ & $\underline{N}$ & $\underline{N}$ & $\underline{N}$ & $\underline{N}$ & $\underline{N}$ & $\underline{N}$ & $\underline{N}$ \\
\hline$S_{b} S$ & SS & $\underline{N}$ & $\underline{N}$ & $\underline{N}$ & $\underline{\mathrm{N}}$ & $\underline{N}$ & $\underline{N}$ & $\underline{N}$ & $\underline{N}$ & $Y$ & $\mathbf{Y}$ & $S_{b} S$ & SS & $\underline{N} \quad \underline{N}$ & $\underline{N}$ & $\underline{N}$ & $\underline{N}$ & $\underline{N}$ & $\underline{N}$ & $\underline{N}$ & $\underline{N}$ & $\underline{N}$ \\
\hline$S_{b} S_{b}$ & $\mathrm{ES}_{\mathrm{b}}$ & $Y$ & $\underline{N}$ & $\underline{N}$ & $\underline{\mathrm{N}}$ & $\underline{N}$ & $\underline{N}$ & $\underline{N}$ & $\underline{N}$ & $\underline{N}$ & $\underline{N}$ & $S_{b} S_{b}$ & $\mathrm{ES}_{\mathrm{b}}$ & $\underline{N} \underline{N}$ & $\underline{N}$ & $\underline{N}$ & $\underline{N}$ & $\underline{N}$ & $\underline{N}$ & $\mathbf{Y}$ & $\mathbf{Y}$ & $Y$ \\
\hline$S_{b} S_{b}$ & $\mathrm{LS}_{\mathrm{b}}$ & $\mathbf{Y}$ & $\underline{N}$ & $\underline{N}$ & $\underline{N}$ & $\underline{N}$ & $\underline{N}$ & $\underline{N}$ & $\underline{N}$ & $\underline{N}$ & $\underline{N}$ & $\mathrm{~S}_{\mathrm{b}} \mathrm{S}_{\mathrm{b}}$ & $\mathrm{LS}_{\mathrm{b}}$ & $\underline{N} \underline{N}$ & $\underline{N}$ & $\mathbf{Y}$ & $Y$ & $\underline{N}$ & $\underline{N}$ & $\underline{N}$ & $\mathbf{Y}$ & $\mathbf{Y}$ \\
\hline$S_{b} S_{b}$ & $\mathrm{~S}_{\mathrm{u}} \mathrm{S}_{\mathrm{b}}$ & $Y$ & $\underline{N}$ & $Y$ & $Y$ & $Y$ & $\mathbf{Y}$ & $\mathbf{Y}$ & $Y$ & $Y$ & $Y$ & $\mathrm{~S}_{\mathrm{b}} \mathrm{S}_{\mathrm{b}}$ & $\mathrm{S}_{\mathrm{u}} \mathrm{S}_{\mathrm{b}}$ & $\underline{N} \quad \underline{N}$ & $\underline{N}$ & $\underline{\mathrm{N}}$ & $\underline{N}$ & $\underline{N}$ & $\underline{N}$ & $\underline{N}$ & $\underline{N}$ & $\underline{N}$ \\
\hline $\mathrm{S}_{\mathrm{b}} \mathrm{S}_{\mathrm{b}}$ & $\mathrm{SS}_{\mathrm{b}}$ & $Y$ & $\underline{N}$ & $Y$ & $Y$ & $Y$ & $Y$ & $Y$ & $Y$ & $Y$ & $Y$ & $\mathrm{~S}_{\mathrm{b}} \mathrm{S}_{\mathrm{b}}$ & $\mathrm{SS}_{\mathrm{b}}$ & $\underline{N} \quad \underline{N}$ & $\underline{N}$ & $\underline{N}$ & $\underline{N}$ & $\underline{N}$ & $\underline{N}$ & $\underline{N}$ & $Y$ & $Y$ \\
\hline $\mathrm{S}_{\mathrm{b}} \mathrm{S}_{\mathrm{u}}$ & $\mathrm{ES}_{\mathrm{u}}$ & $\underline{N}$ & $\underline{N}$ & $\underline{N}$ & $\underline{N}$ & $Y$ & $\mathbf{Y}$ & $\underline{N}$ & $\underline{N}$ & $\underline{N}$ & $\underline{N}$ & $\mathrm{~S}_{\mathrm{b}} \mathrm{S}_{\mathrm{u}}$ & $\mathrm{ES}_{\mathrm{u}}$ & $\underline{N} \quad \underline{N}$ & $\underline{N}$ & $\underline{N}$ & $\underline{N}$ & $\underline{N}$ & $Y$ & $\mathbf{Y}$ & $\mathbf{Y}$ & $Y$ \\
\hline $\mathrm{S}_{\mathrm{b}} \mathrm{S}_{\mathrm{u}}$ & $\mathrm{LS}_{\mathrm{u}}$ & $\underline{N}$ & $\underline{N}$ & $\underline{N}$ & $\mathbf{Y}$ & $\mathbf{Y}$ & $Y$ & $\mathbf{Y}$ & $\underline{N}$ & $Y$ & $\mathbf{Y}$ & $\mathrm{S}_{\mathrm{b}} \mathrm{S}_{\mathrm{u}}$ & $\mathrm{LS}_{\mathrm{u}}$ & $\underline{N} \quad Y$ & $\mathbf{Y}$ & $\mathbf{Y}$ & $\underline{N}$ & $\underline{N}$ & $\mathbf{Y}$ & $\mathbf{Y}$ & $\mathbf{Y}$ & $Y$ \\
\hline $\mathrm{S}_{\mathrm{b}} \mathrm{S}_{\mathrm{u}}$ & $\mathrm{S}_{\mathrm{u}} \mathrm{S}_{\mathrm{u}}$ & $\underline{N}$ & $\underline{N}$ & $\underline{N}$ & $\underline{N}$ & $\underline{N}$ & $\underline{N}$ & $\underline{N}$ & $\underline{N}$ & $Y$ & $Y$ & $\mathrm{~S}_{\mathrm{b}} \mathrm{S}_{\mathrm{u}}$ & $\mathrm{S}_{\mathrm{u}} \mathrm{S}_{\mathrm{u}}$ & $\underline{N} \underline{N}$ & $\underline{N}$ & $\underline{N}$ & $\underline{N}$ & $\mathbf{Y}$ & $\mathbf{Y}$ & $\mathbf{Y}$ & $\underline{N}$ & $\underline{N}$ \\
\hline $\mathrm{S}_{\mathrm{b}} \mathrm{S}_{\mathrm{u}}$ & $\mathrm{SS}_{\mathrm{u}}$ & $\underline{N}$ & $\underline{N}$ & $\underline{N}$ & $\underline{N}$ & $\underline{N}$ & $\underline{N}$ & $\underline{N}$ & $\underline{N}$ & $\underline{N}$ & $\underline{N}$ & $\mathrm{~S}_{\mathrm{b}} \mathrm{S}_{\mathrm{u}}$ & $\mathrm{SS}_{\mathrm{u}}$ & $\underline{N} \quad \underline{N}$ & $\underline{N}$ & $\underline{N}$ & $\underline{N}$ & $\underline{N}$ & $Y$ & $\underline{N}$ & $\mathbf{Y}$ & $Y$ \\
\hline
\end{tabular}


In Table 5-20, it is found that the environments of unbarred spirals are almost always dissimilar to the environments of lenticular and elliptical galaxies. This is consistent with the finding of Best [29] that the environments of late-type galaxies and early-type galaxies are statistically dissimilar to the $99 \%$ level.

The environments of unbarred spirals show general dissimilarity when compared to the environments of spirals. The environments of unbarred spirals are generally dissimilar to the environments of barred spirals in the north, but are consistently similar in the south.

Table 5-20 2D KS Test Results - comparing two runs having the same secondary filter and same fitting range filter with different primary filters - limited to unbarred spiral primaries in Run1.

$\mathrm{Y}=$ dissimilar to the $99 \%$ level. $\mathrm{N}=$ not dissimilar to the $99 \%$ level

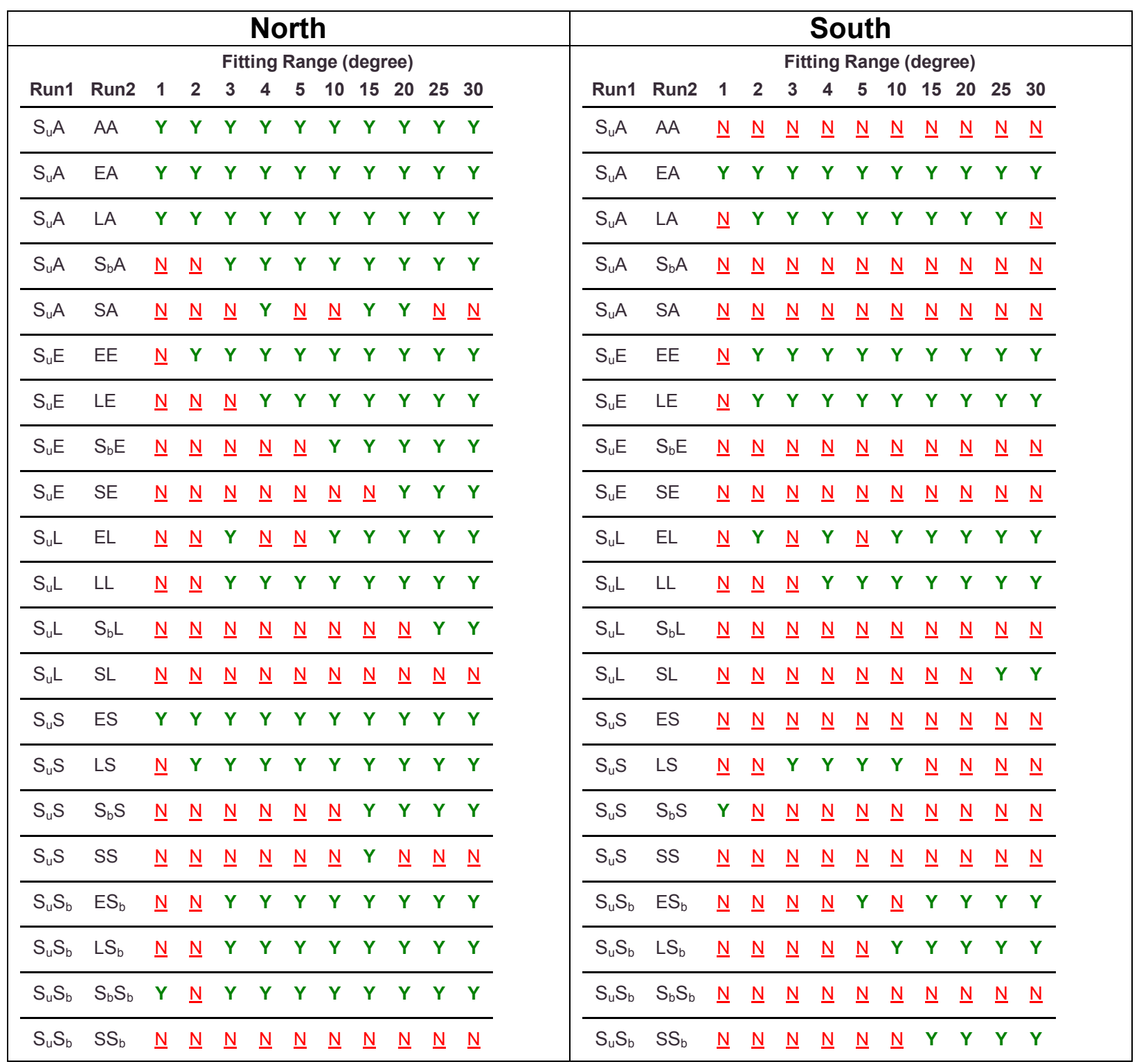




\begin{tabular}{|c|c|c|c|c|c|c|c|c|c|c|c|c|c|c|c|c|c|c|c|c|c|c|}
\hline $\mathrm{S}_{\mathrm{u}} \mathrm{S}_{\mathrm{u}}$ & $\mathrm{ES}_{\mathrm{u}}$ & $\underline{N}$ & $\underline{N}$ & $\underline{N}$ & $\underline{N}$ & $\underline{\mathrm{N}}$ & $\underline{N}$ & $\underline{N}$ & $\mathbf{Y}$ & $\mathbf{Y}$ & $Y$ & $S_{u} S_{u}$ & $\mathrm{ES}_{\mathrm{u}}$ & $\underline{N}$ & $\underline{N}$ & $Y$ & $\underline{N}$ & $\underline{N}$ & $Y$ & $Y$ & & $Y$ \\
\hline $\mathrm{S}_{\mathrm{u}} \mathrm{S}_{\mathrm{u}}$ & $\mathrm{LS}_{\mathrm{u}}$ & $\underline{N}$ & $\underline{N}$ & $\underline{\mathrm{N}}$ & $\mathbf{Y}$ & $\underline{\mathrm{N}}$ & $\mathbf{Y}$ & $\mathbf{Y}$ & $\mathbf{Y}$ & $\mathbf{Y}$ & $\mathbf{Y}$ & $S_{u} S_{u}$ & $\mathrm{LS}_{\mathrm{u}}$ & $\underline{N}$ & $\underline{\mathrm{N}}$ & $\mathbf{Y}$ & $Y$ & $Y$ & $Y$ & $Y$ & & $\mathbf{Y}$ \\
\hline $\mathrm{S}_{\mathrm{u}} \mathrm{S}_{\mathrm{u}}$ & $\mathrm{S}_{\mathrm{b}} \mathrm{S}_{\mathrm{u}}$ & $\underline{\mathrm{N}}$ & $\underline{\mathrm{N}}$ & $\underline{\mathrm{N}}$ & $\underline{\mathrm{N}}$ & $\underline{\mathrm{N}}$ & $\underline{N}$ & $\underline{N}$ & $\underline{\mathrm{N}}$ & $\mathbf{Y}$ & $\mathbf{Y}$ & $\mathrm{S}_{\mathrm{u}} \mathrm{S}_{\mathrm{u}}$ & $\mathrm{S}_{b} \mathrm{~S}_{\mathrm{u}}$ & $\underline{N}$ & $\underline{\mathrm{N}}$ & $\underline{N}$ & $\underline{N}$ & $\underline{N}$ & $Y$ & & 1 & $\underline{N}$ \\
\hline $\mathrm{S}_{\mathrm{u}} \mathrm{S}_{\mathrm{u}}$ & $\mathrm{SS}_{\mathrm{u}}$ & $\underline{N}$ & $\underline{N}$ & $\underline{\mathrm{N}}$ & $\underline{N}$ & $\underline{\mathrm{N}}$ & $\mathbf{Y}$ & $\mathbf{Y}$ & $\mathbf{Y}$ & $\mathbf{Y}$ & $\mathbf{Y}$ & $\mathrm{S}_{\mathrm{u}} \mathrm{S}_{\mathrm{u}}$ & $\mathrm{SS}_{\mathrm{u}}$ & $\underline{\mathrm{N}}$ & $\underline{N}$ & $\underline{N}$ & $\underline{\mathrm{N}}$ & 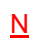 & $Y$ & $Y$ & & $\mathbf{Y}$ \\
\hline
\end{tabular}

\subsubsection{Conclusions}

The important observation from this data is that as primaries ellipticals and lenticulars are generally not surrounded by statistically different environments. This suggests that secular evolution, not the environment, plays a factor in formation of $E$ or L galaxies. 


\subsection{Three Dimensional}

\subsubsection{Averages and Standard Deviations}

Below are the average PD values with error bars for the population standard deviation, $\sigma$. The average is calculated via SQL Server's avg [63] function, and $\sigma$ is calculated using stddevp [64]. The run names describe the parameters of the run as described in section 5.1 of this paper.

For the purposes of this paper I define 3D small scale as fitting ranges from 1 $\mathrm{Mpc}$ to $5 \mathrm{Mpc}$ in $1 \mathrm{Mpc}$ increments. 3D large scale is defined as fitting ranges from 5 $\mathrm{Mpc}$ to $30 \mathrm{Mpc}$ in $5 \mathrm{Mpc}$ increments.

The $\sigma$ values generally decrease as the fitting range increases. This was also the case in 2D. The same smaller average PD value for ellipticals vs. spirals is also seen in $3 \mathrm{D}$ as it was in 2D. Refer to Table 5-21 and Table 5-22 which present the average PD values for SA and EA runs sorted by the average PD in ascending order. No EA run has a higher average PD than an SA run at any of the measured fitting ranges. 
Table 5-21 3D North Elliptical vs. Spiral Avg. PD Sorted by Avg. PD Ascending

\begin{tabular}{|c|c|c|}
\hline \multicolumn{3}{|c|}{ North } \\
\hline Run & Avg. PD & \multicolumn{1}{c|}{$\boldsymbol{\sigma}$ value } \\
\hline EA04 Mpc & 1.548637 & 1.03695 \\
\hline EA03 Mpc & 1.549024 & 1.264794 \\
\hline EA05 Mpc & 1.555154 & 0.763803 \\
\hline EA01 Mpc & 1.606196 & 2.094108 \\
\hline EA02 Mpc & 1.624877 & 1.841042 \\
\hline SA04 Mpc & 1.710717 & 0.769867 \\
\hline SA03 Mpc & 1.717432 & 0.99695 \\
\hline SA05 Mpc & 1.723171 & 0.639963 \\
\hline SA02 Mpc & 1.81942 & 2.511813 \\
\hline SA01 Mpc & 1.863876 & 2.288654 \\
\hline
\end{tabular}

Table 5-22 3D South Elliptical vs. Spiral Avg. PD Sorted by Avg. PD Ascending

\begin{tabular}{|c|c|c|}
\hline \multicolumn{3}{|c|}{ South } \\
\hline Run & Avg. PD & $\boldsymbol{\sigma}$ value \\
\hline EA04 Mpc & 1.519279 & 0.919233 \\
\hline EA05 Mpc & 1.523817 & 0.813827 \\
\hline EA03 Mpc & 1.55674 & 1.237633 \\
\hline EA01 Mpc & 1.581762 & 1.864161 \\
\hline EA02 Mpc & 1.609265 & 1.619966 \\
\hline SA04 Mpc & 1.680809 & 0.840187 \\
\hline SA05 Mpc & 1.685623 & 0.680028 \\
\hline SA03 Mpc & 1.703049 & 1.190826 \\
\hline SA02 Mpc & 1.727282 & 1.346052 \\
\hline SA01 Mpc & 1.931831 & 2.497172 \\
\hline
\end{tabular}

The complete plots for average and $\sigma$ for 3D runs follow. 


\subsubsection{2MRS North Small Scale}

Figure 5-25 2MRS North Small Scale 3D Average PD and $\sigma$ values for All Primaries regardless of morphology

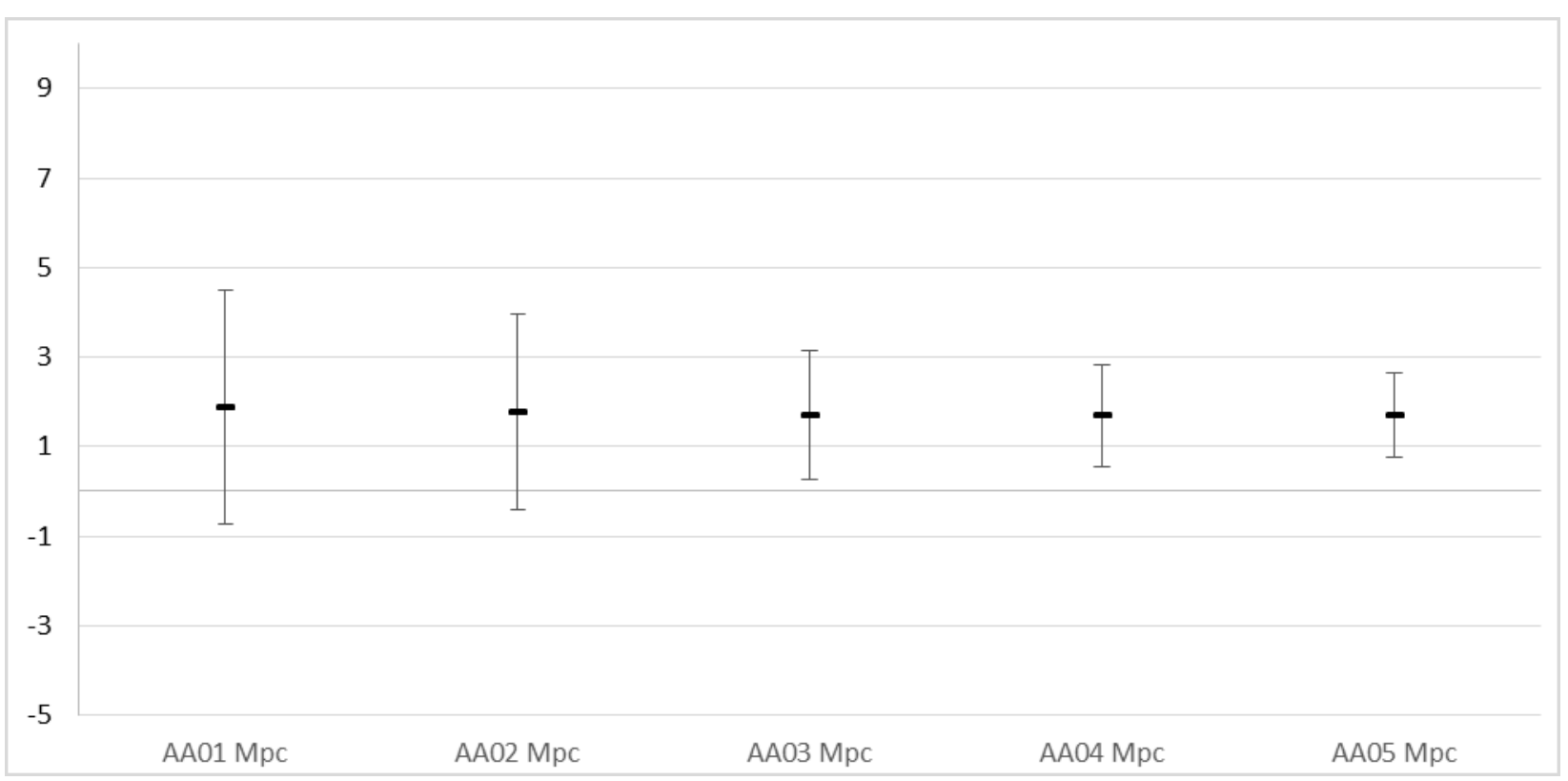

Figure 5-26 2MRS North Small Scale 3D Average PD and $\sigma$ values for Elliptical Primaries

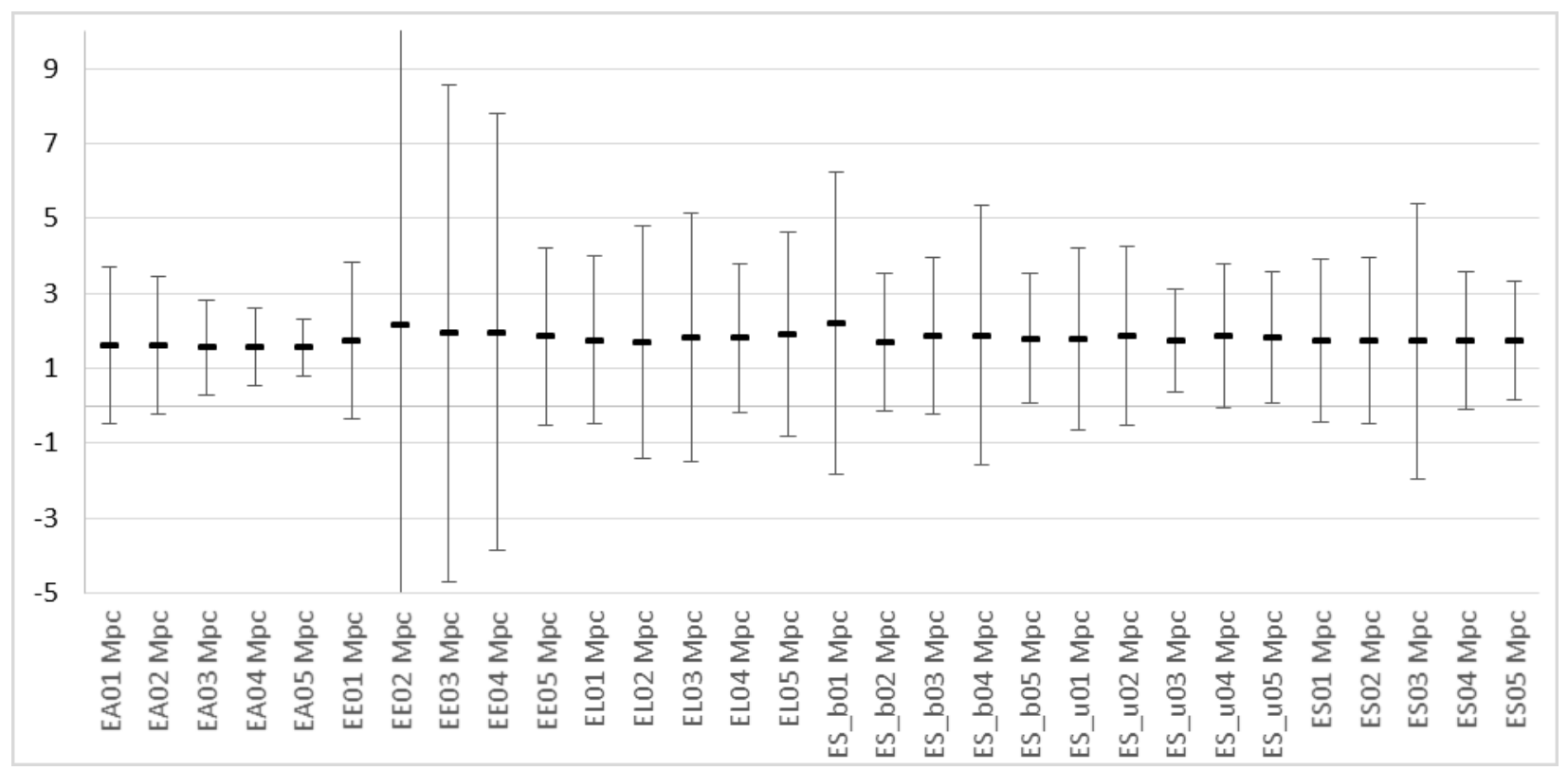


Figure 5-27 2MRS North Small Scale 3D Average PD and $\sigma$ values for Lenticular Primaries

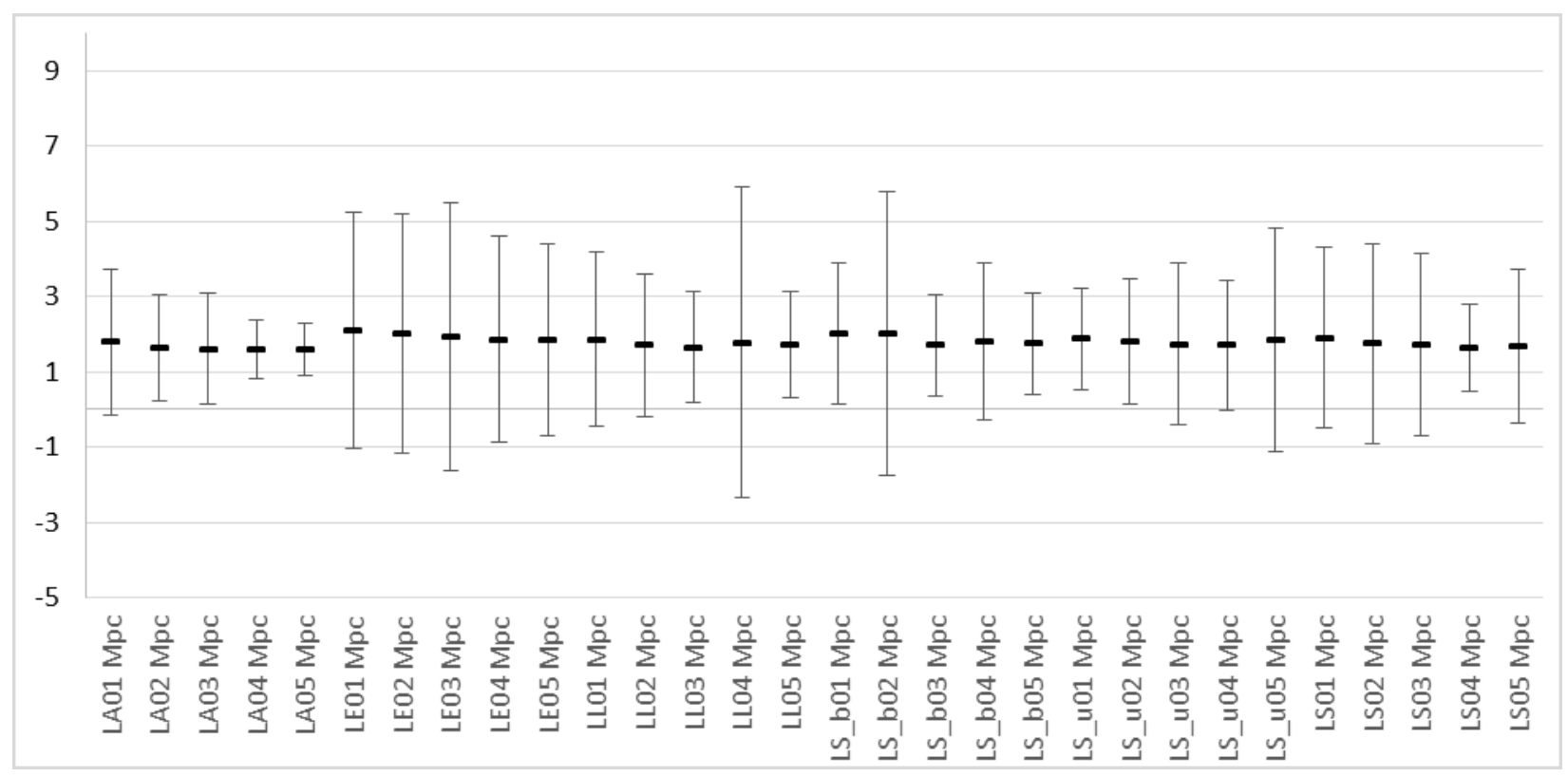

Figure 5-28 2MRS North Small Scale 3D Average PD and $\sigma$ values for Spiral Primaries

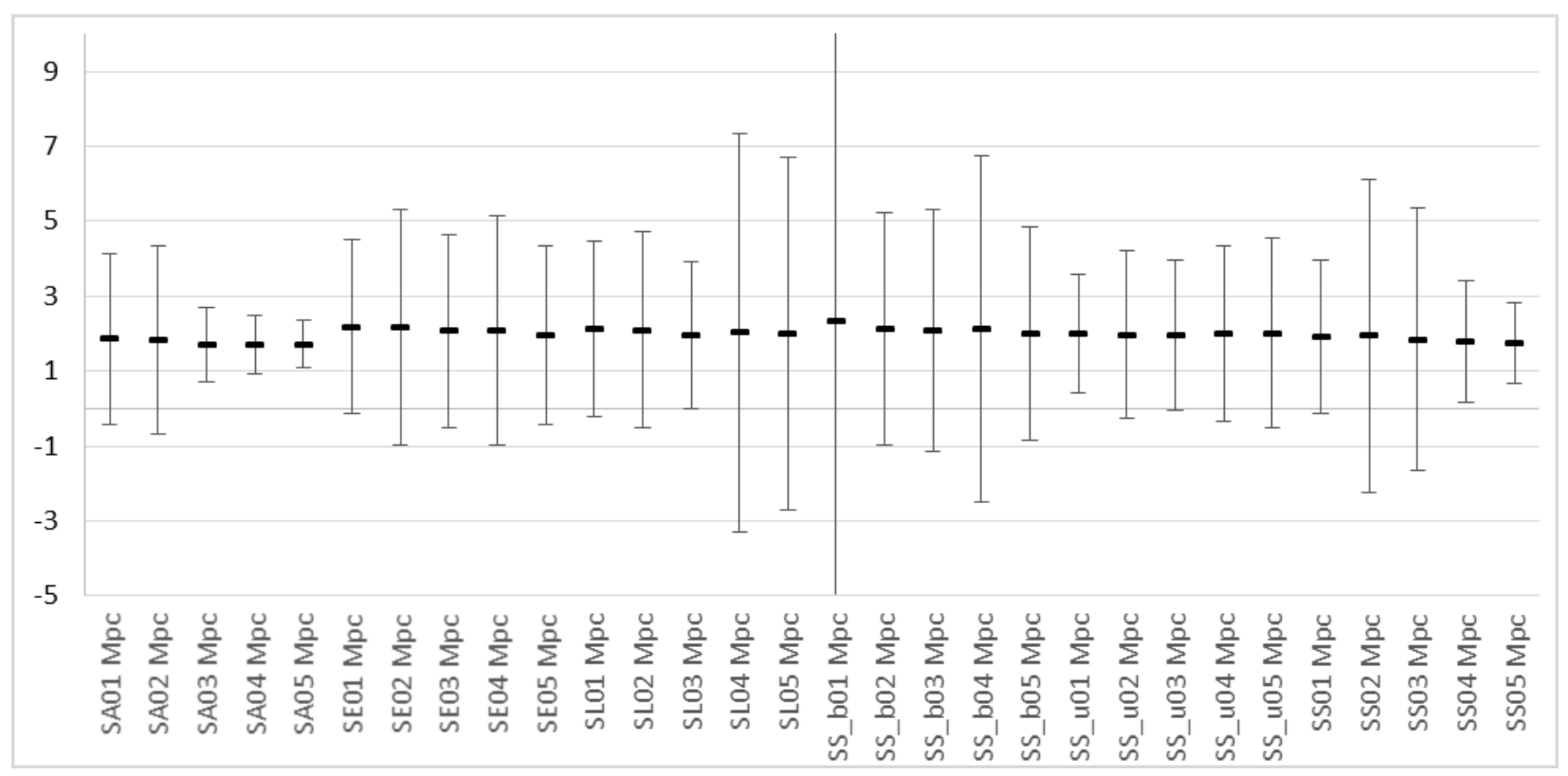


Figure 5-29 2MRS North Small Scale 3D Average PD and $\sigma$ values for Barred Spiral Primaries

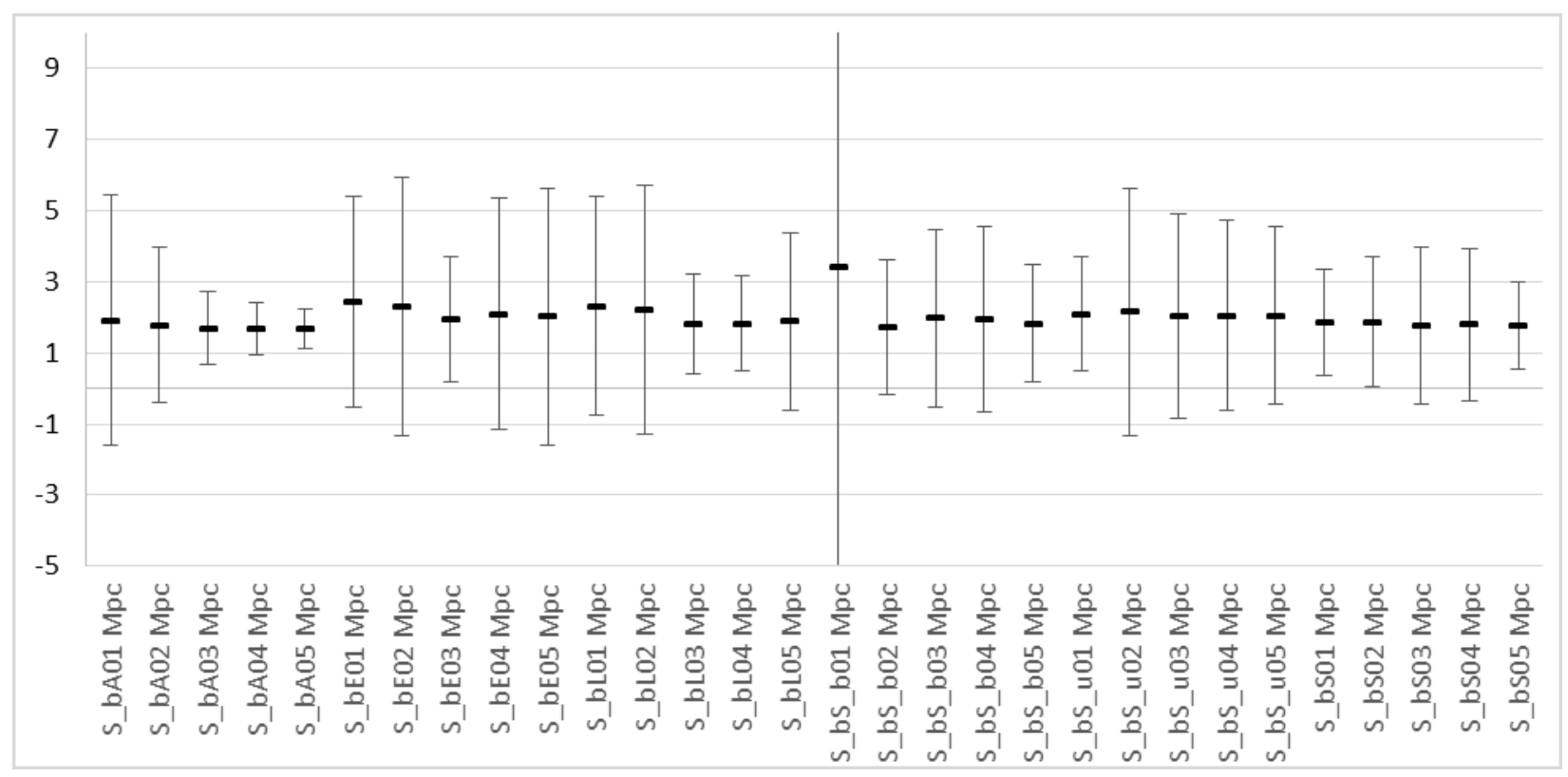

Figure 5-30 2MRS North Small Scale 3D Average PD and $\sigma$ values for Unbarred Spiral Primaries

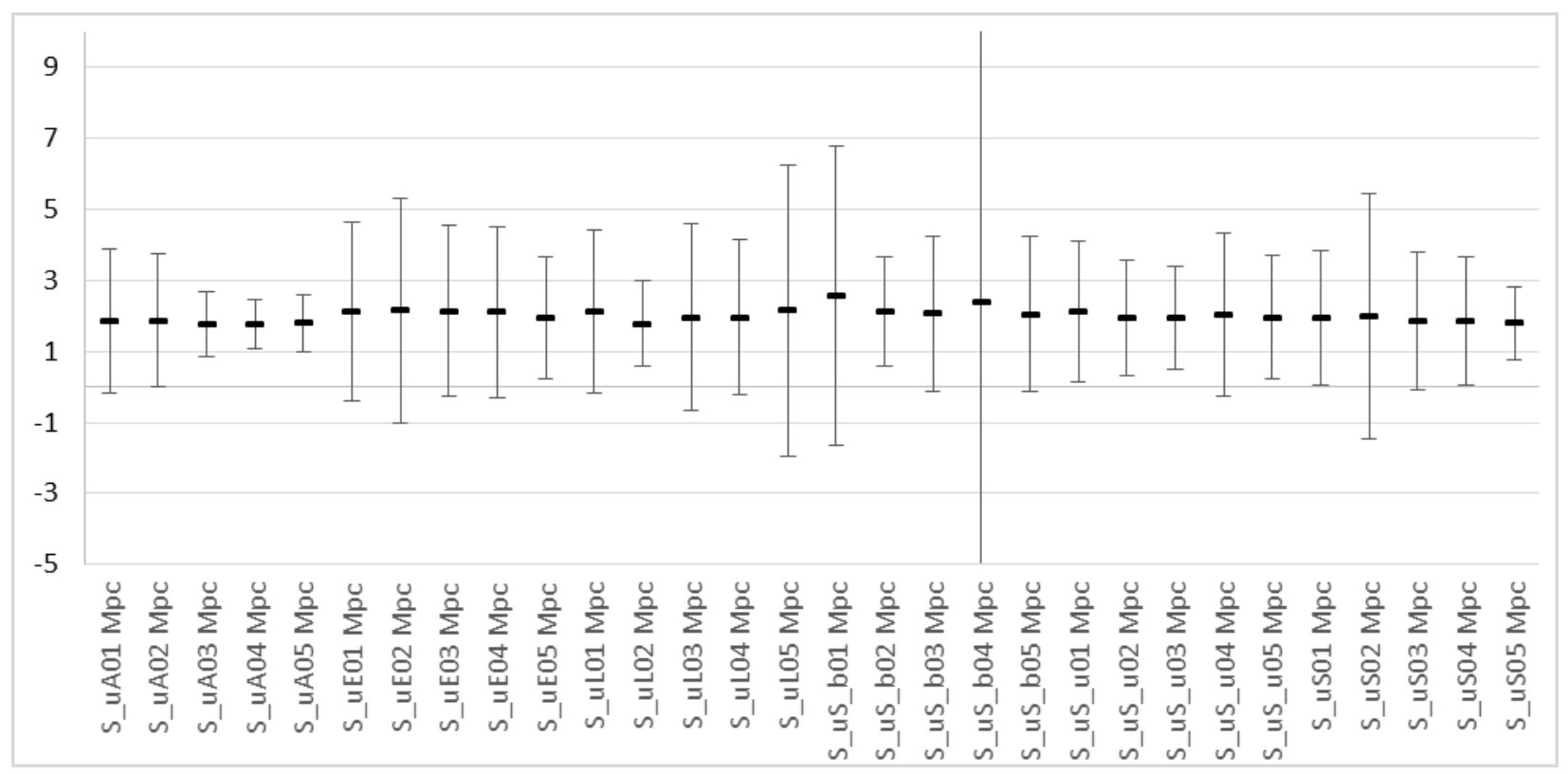




\subsubsection{2MRS South Small Scale}

Figure 5-31 2MRS South Small Scale 3D Average PD and $\sigma$ values for All Primaries regardless of morphology

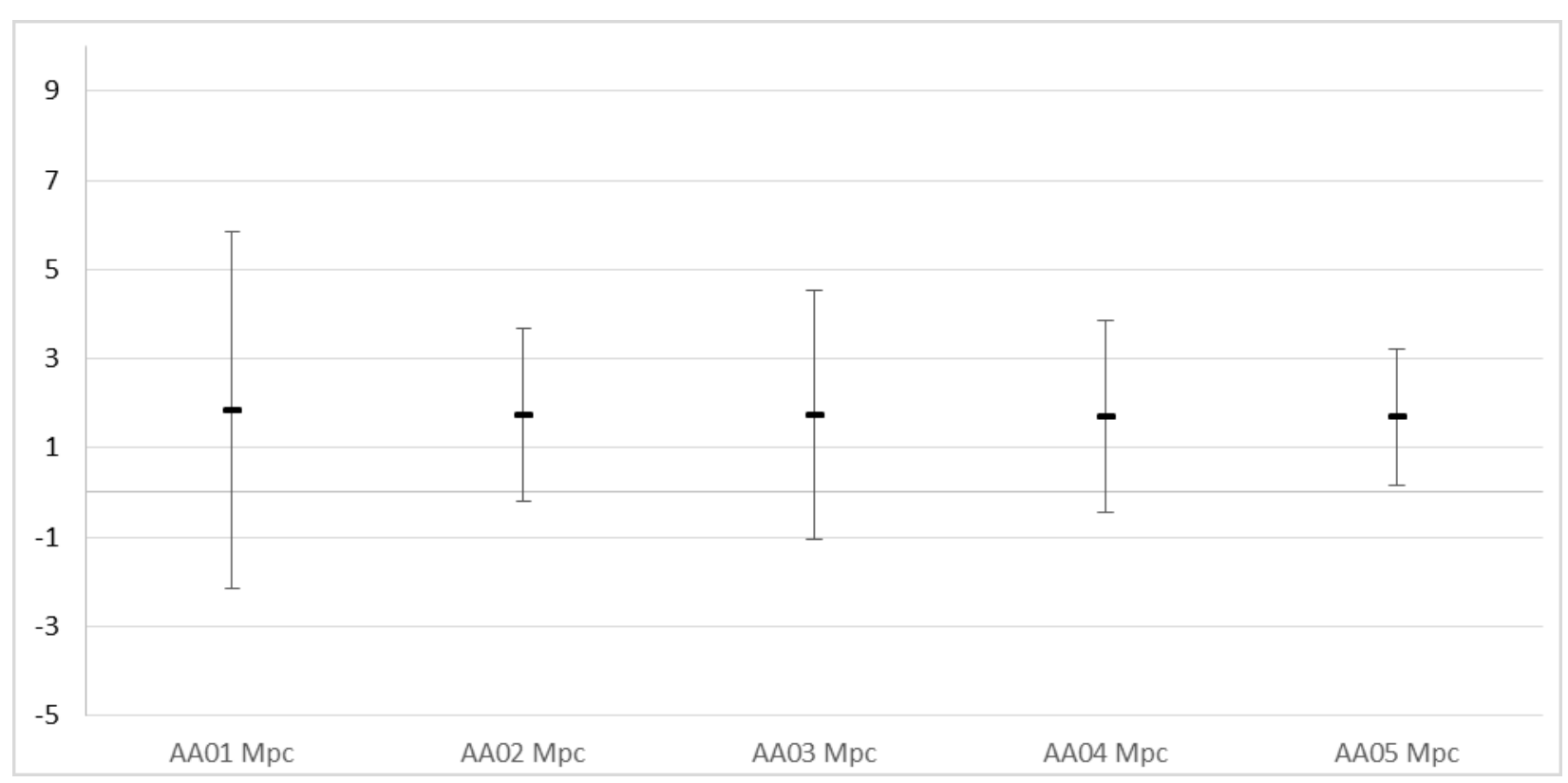

Figure 5-32 2MRS South Small Scale 3D Average PD and $\sigma$ values for Elliptical Primaries

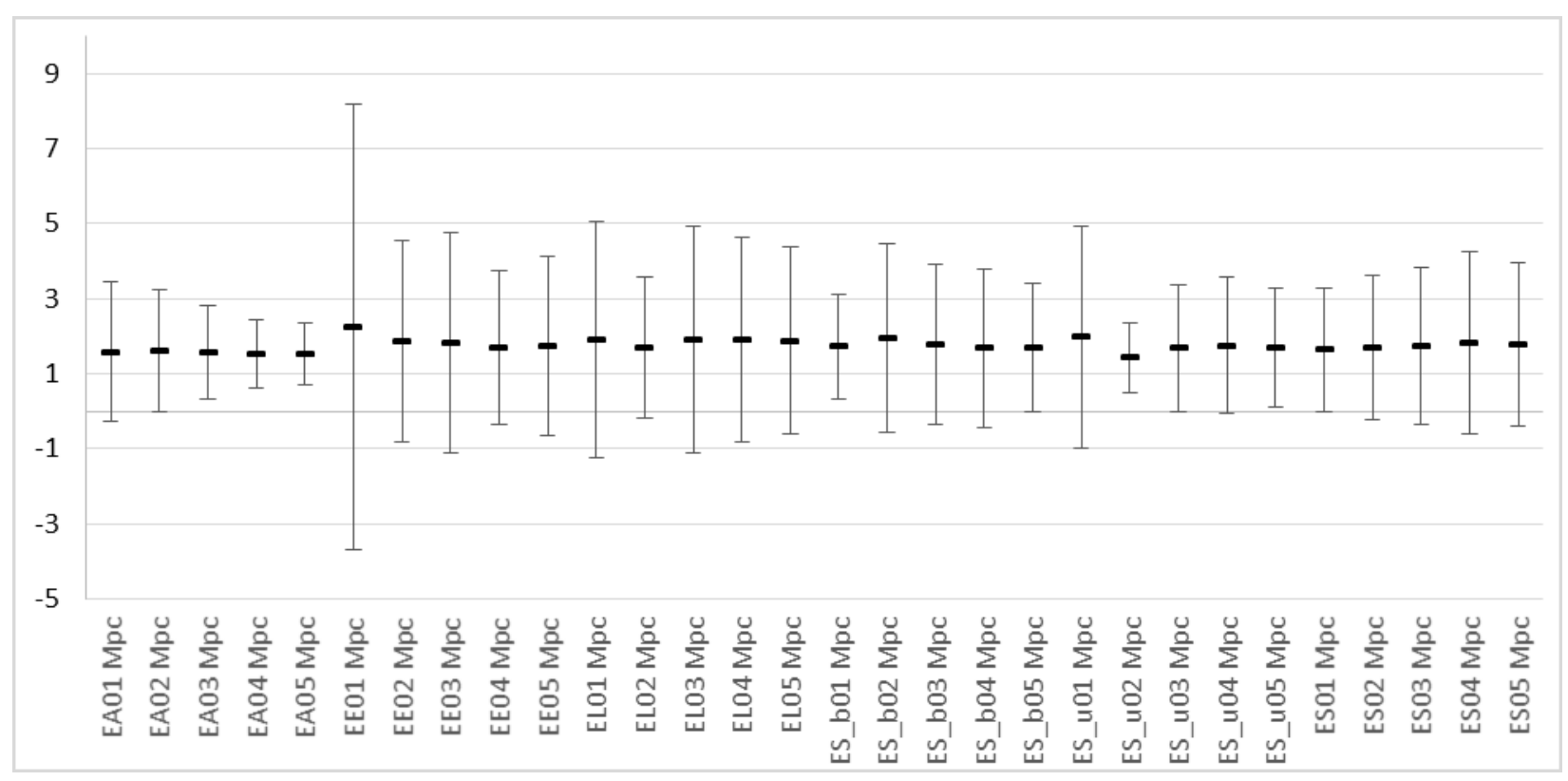


Figure 5-33 2MRS South Small Scale 3D Average PD and $\sigma$ values for Lenticular Primaries

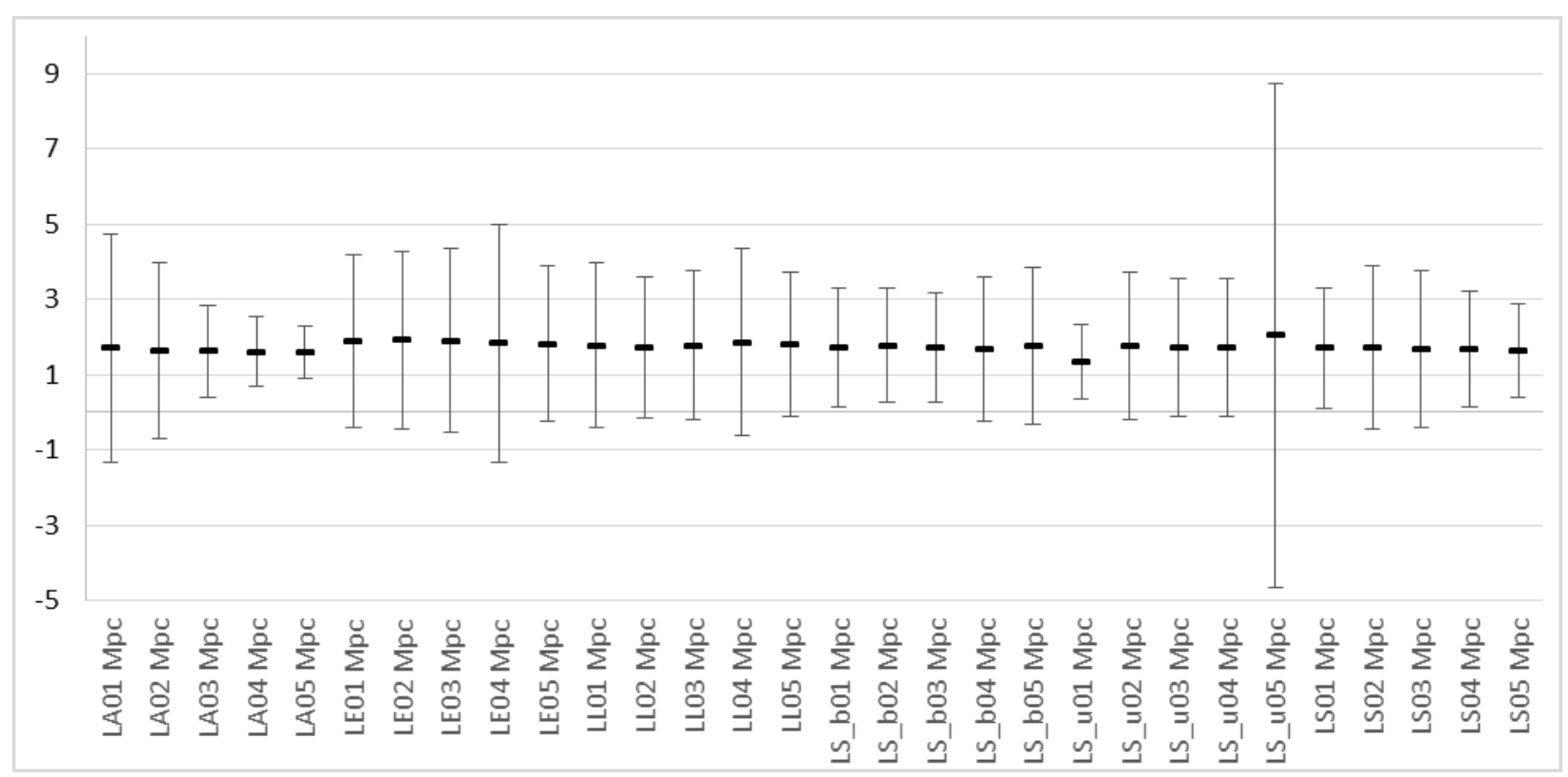

Figure 5-34 2MRS South Small Scale 3D Average PD and $\sigma$ values for Spiral Primaries

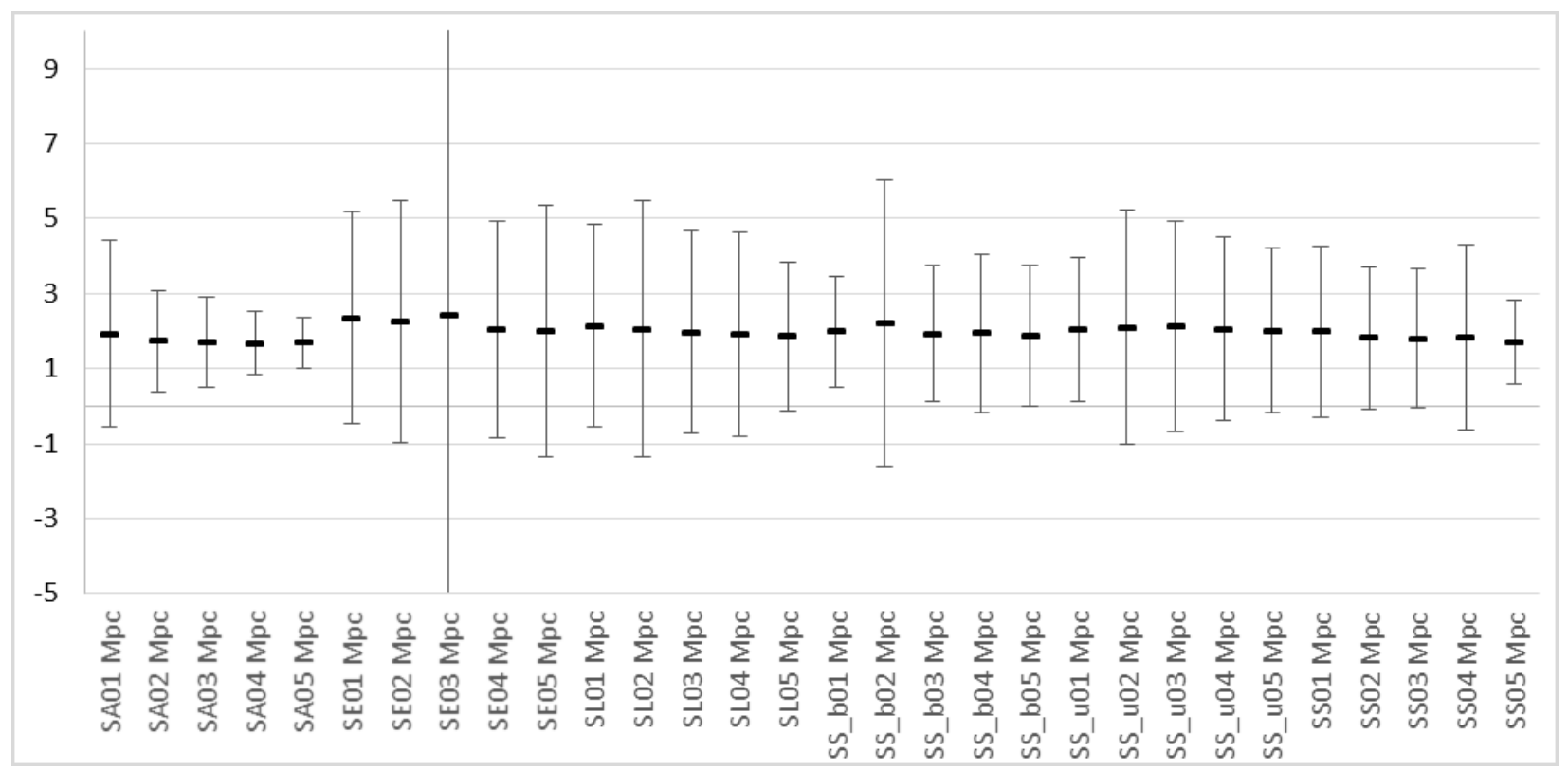


Figure 5-35 2MRS South Small Scale 3D Average PD and $\sigma$ values for Barred Spiral Primaries

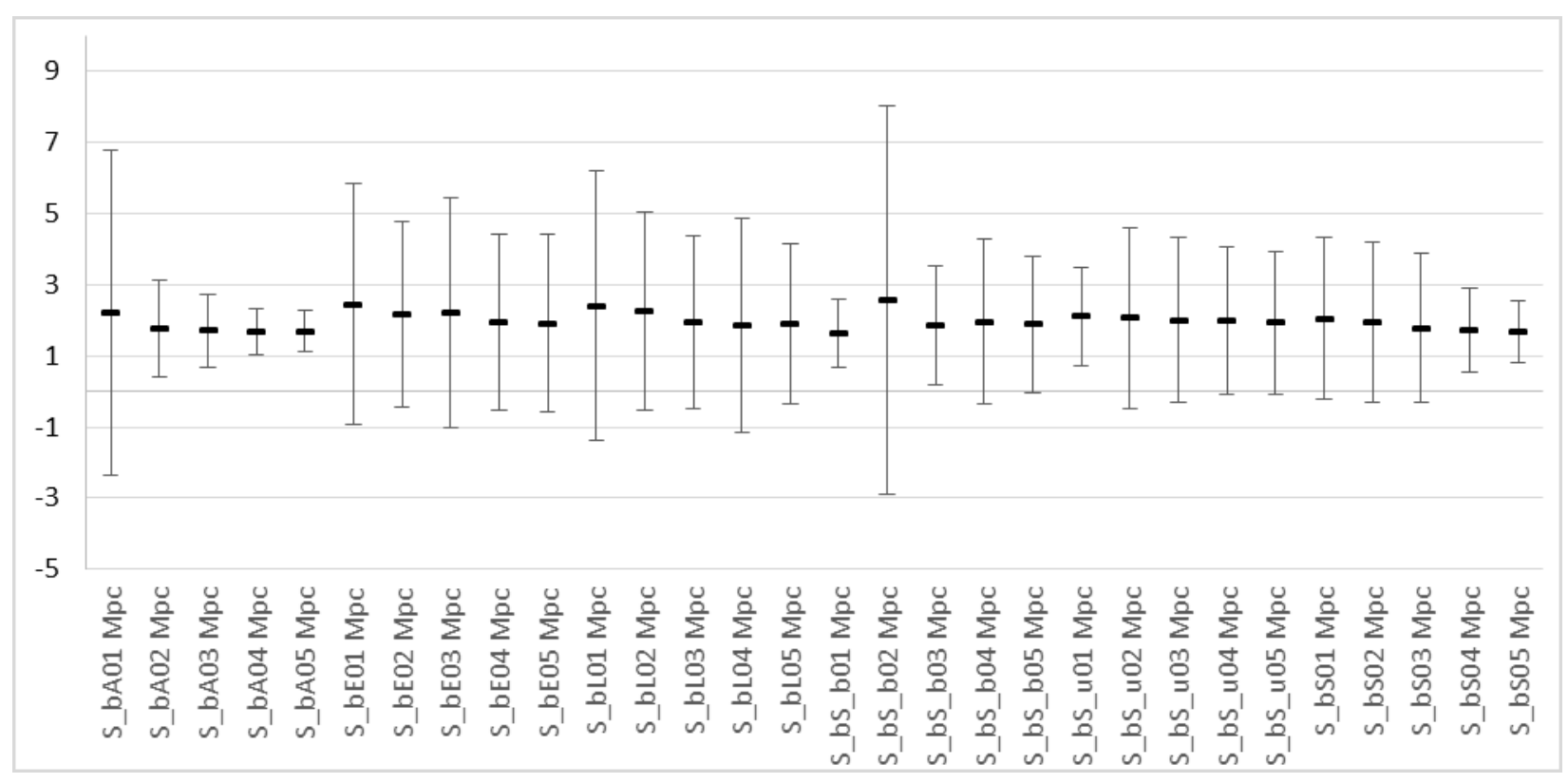

Figure 5-36 2MRS South Small Scale 3D Average PD and $\sigma$ values for Unbarred Spiral Primaries

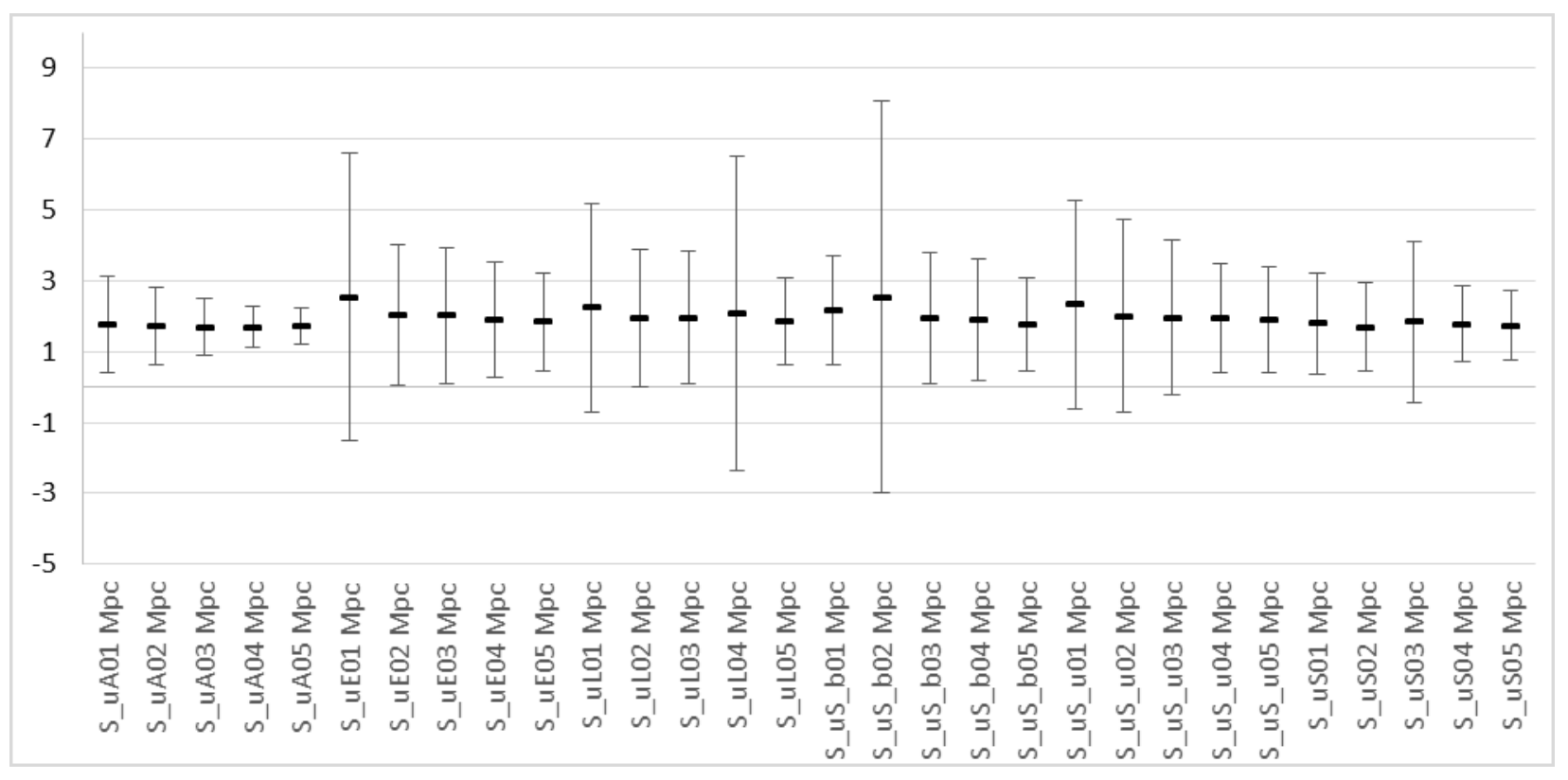




\subsubsection{2MRS North Large Scale}

Figure 5-37 2MRS North Large Scale 3D Average PD and $\sigma$ values for All Primaries regardless of morphology

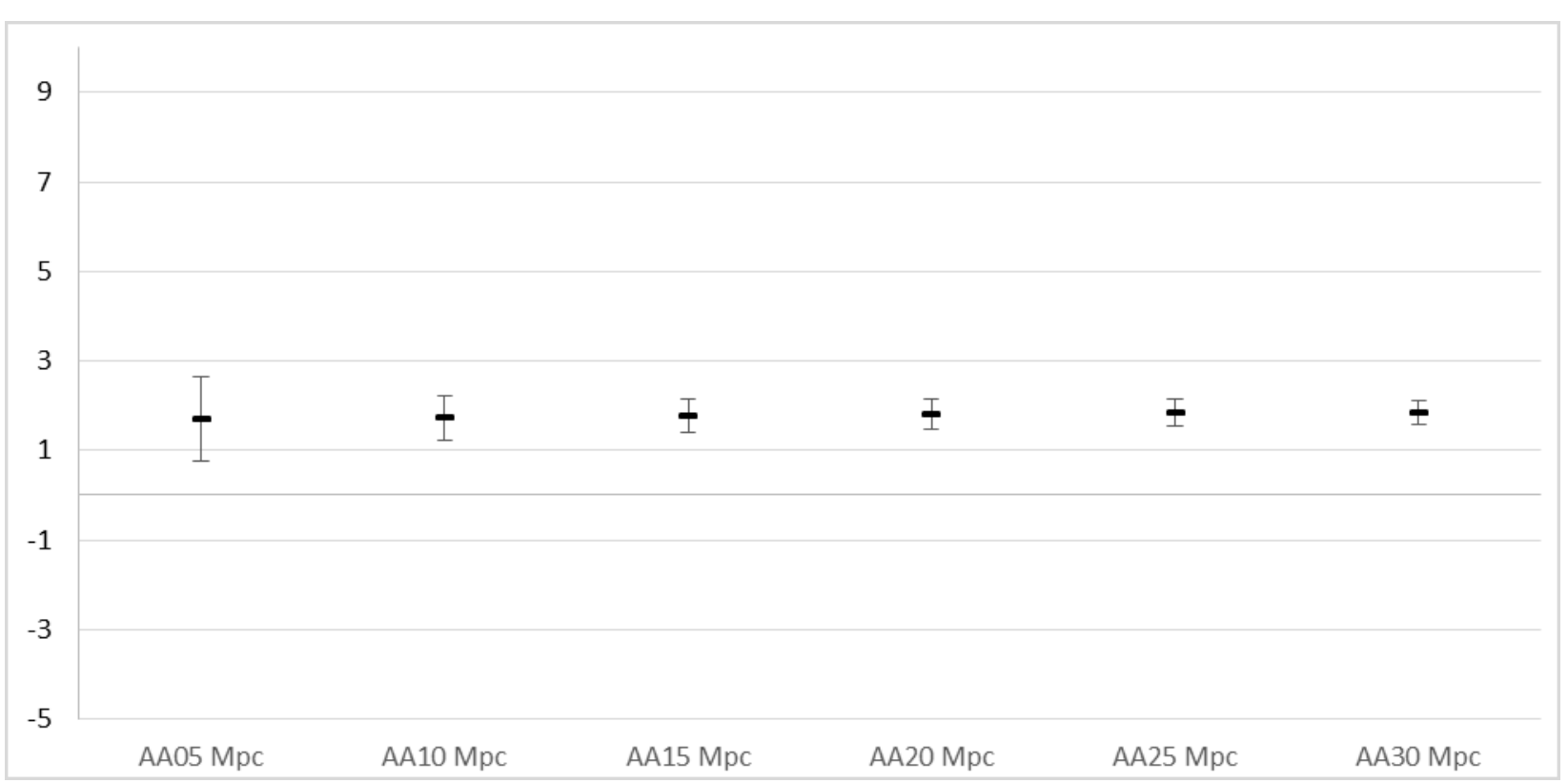

Figure 5-38 2MRS North Large Scale 3D Average PD and $\sigma$ values for Elliptical Primaries

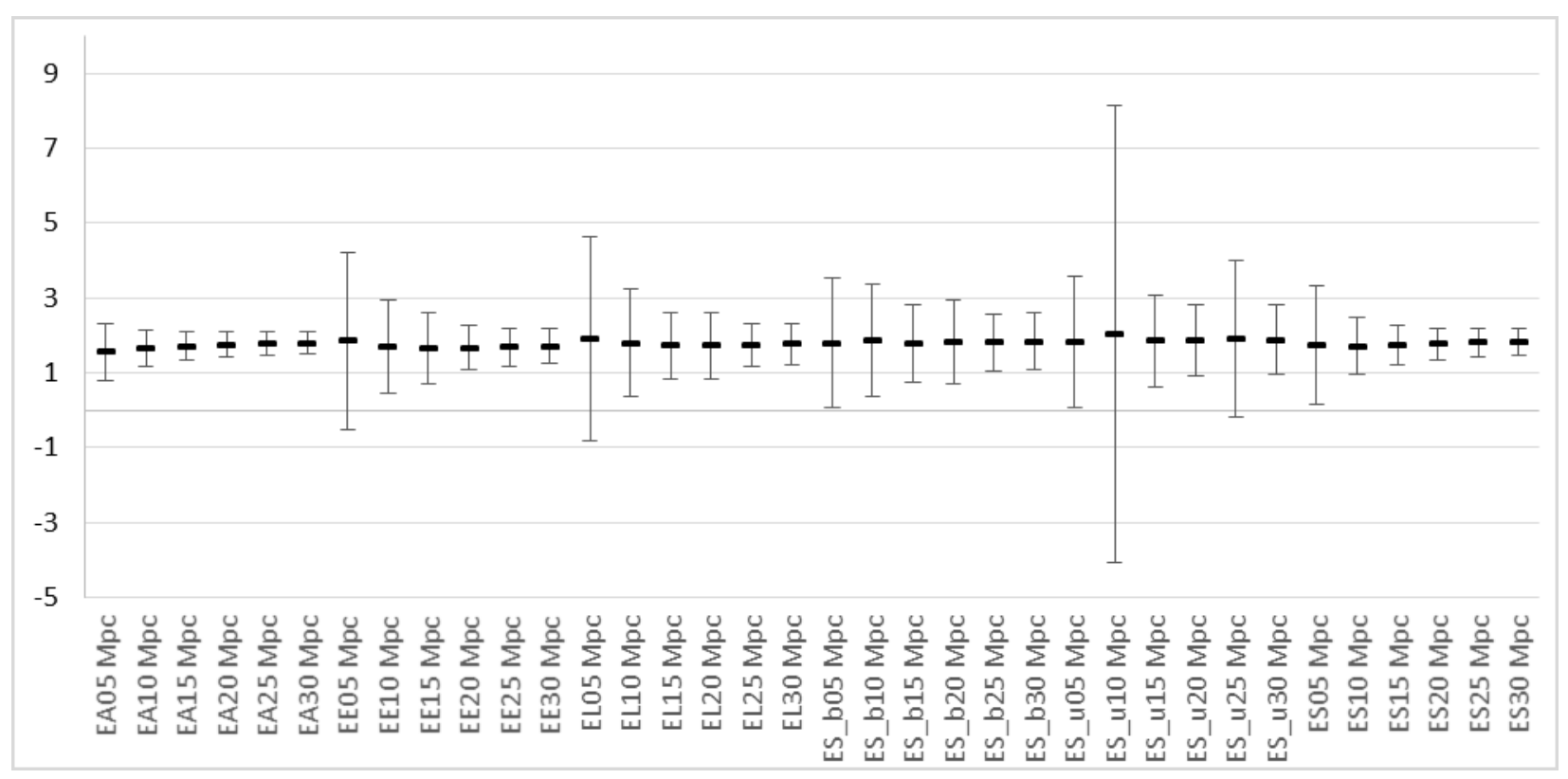


Figure 5-39 2MRS North Large Scale 3D Average PD and $\sigma$ values for Lenticular Primaries

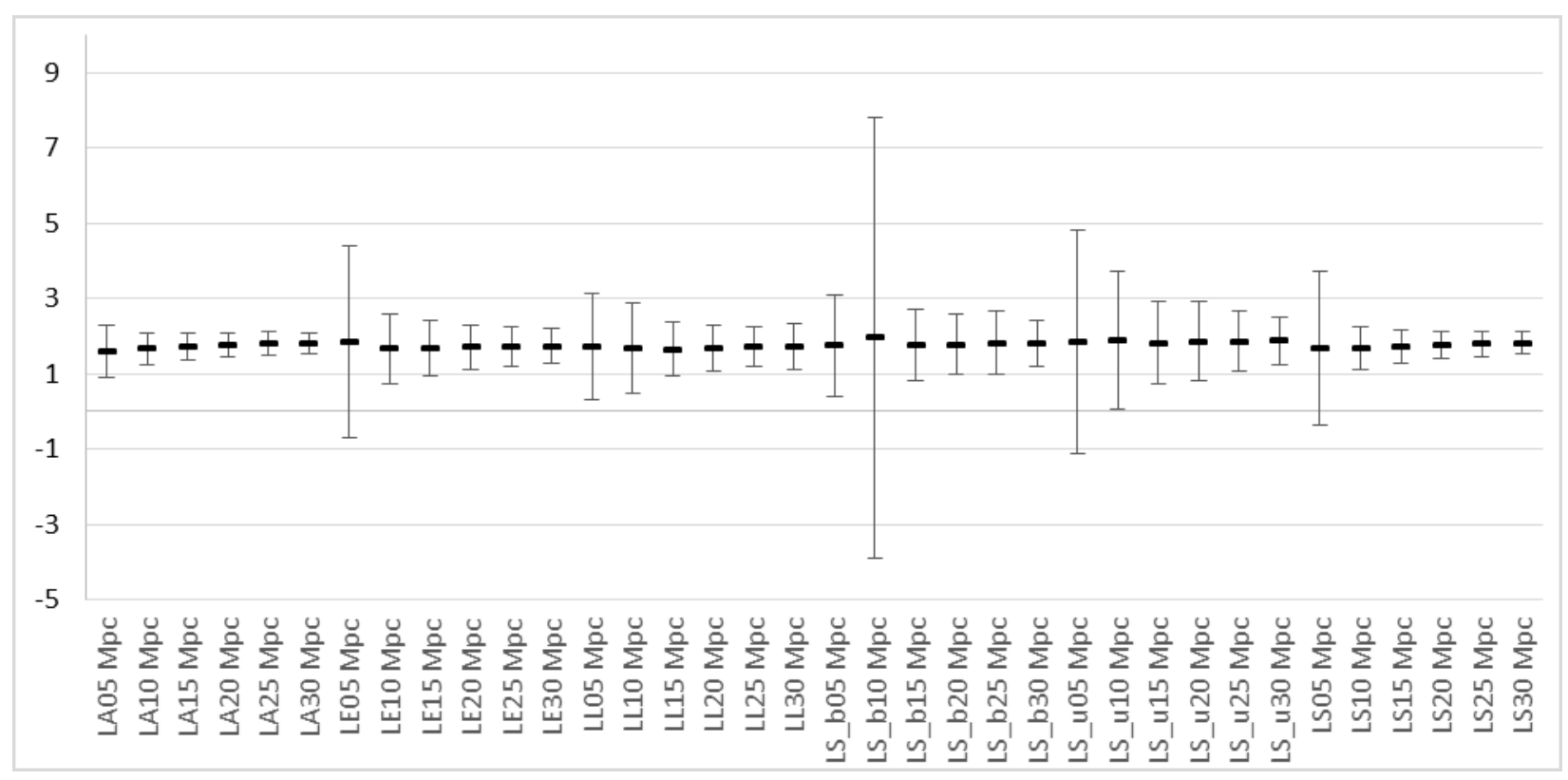

Figure 5-40 2MRS North Large Scale 3D Average PD and $\sigma$ values for Spiral Primaries

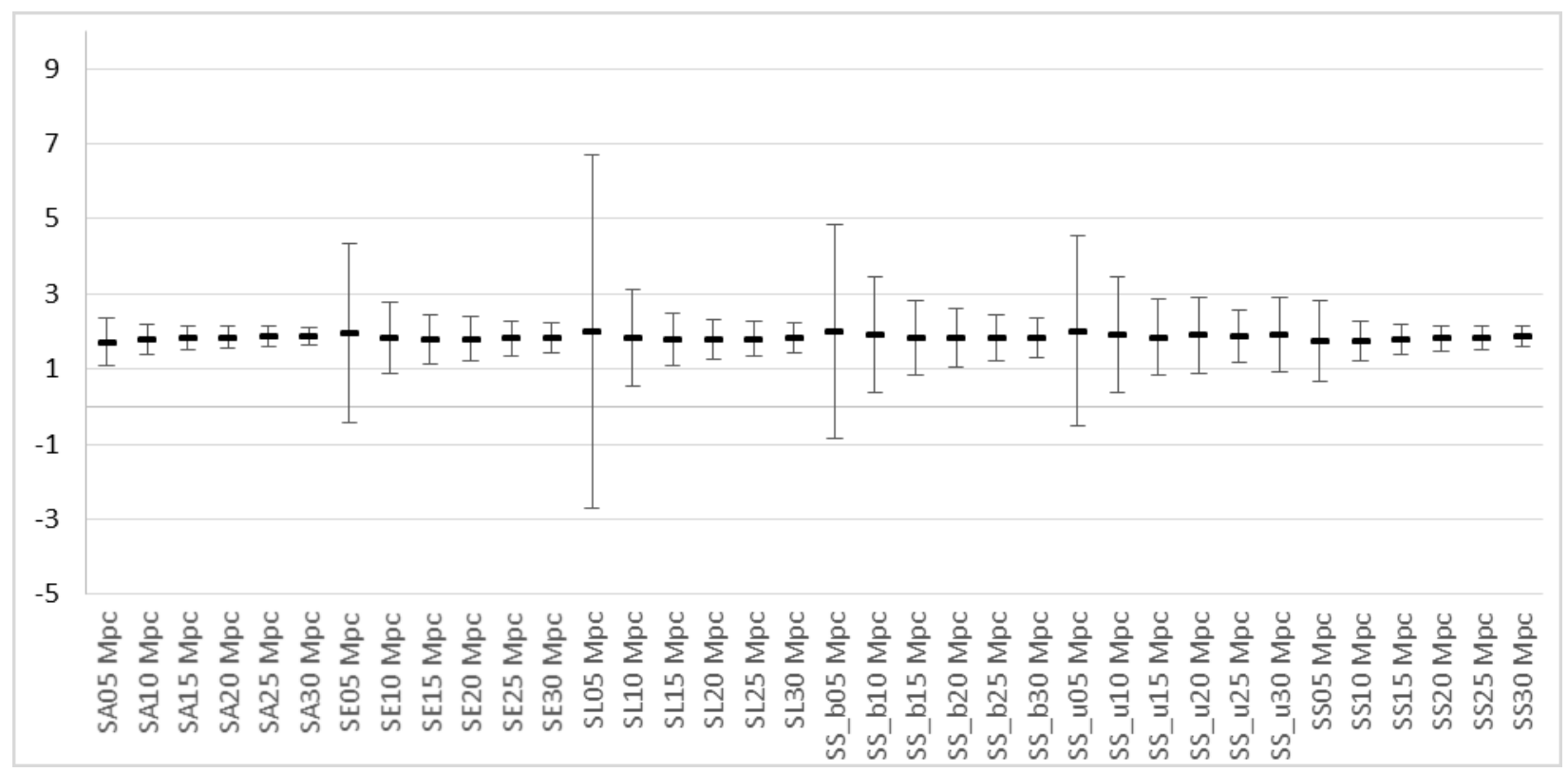


Figure 5-41 2MRS North Large Scale 3D Average PD and $\sigma$ values for Barred Spirals

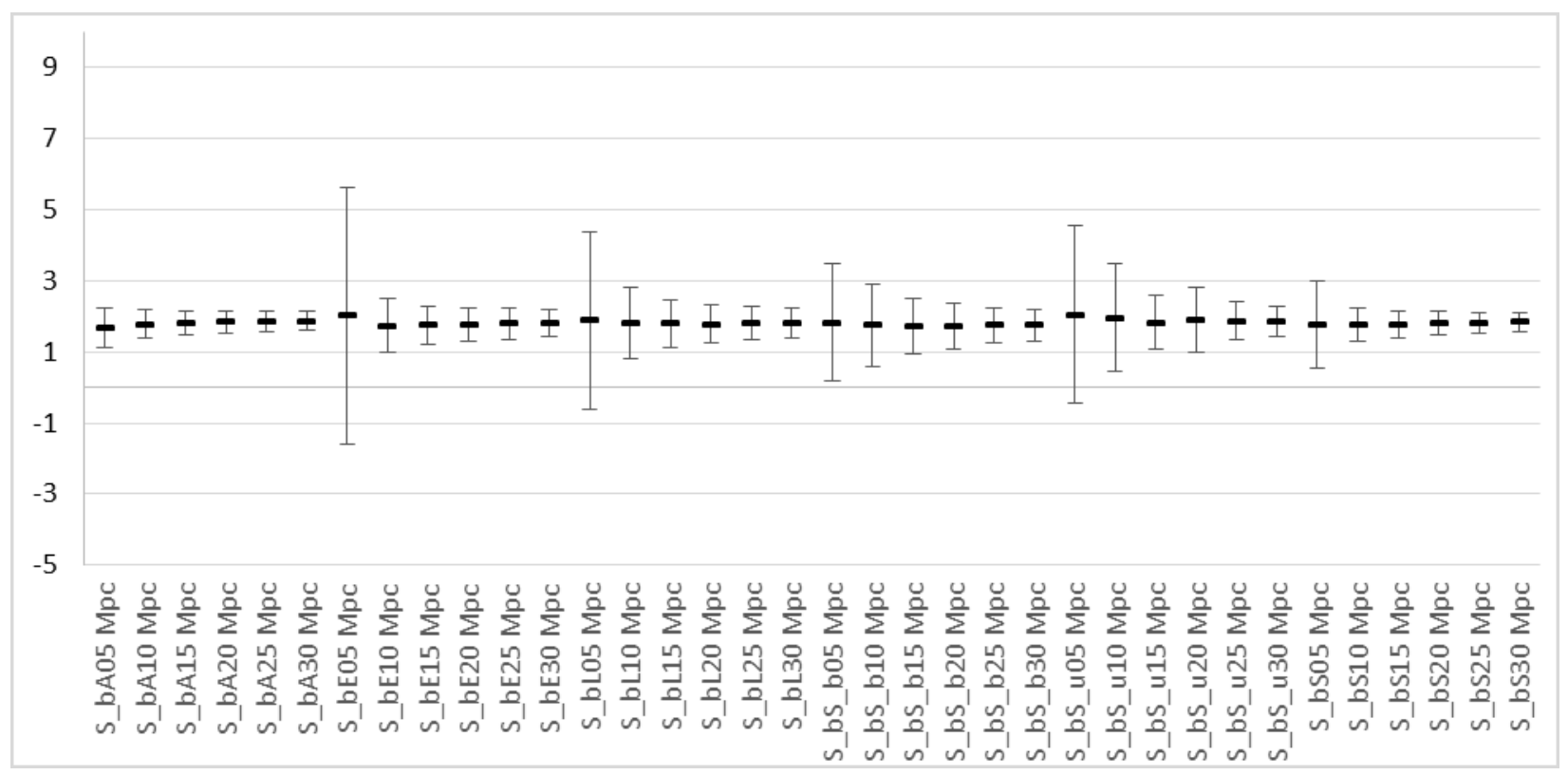

Figure 5-42 2MRS North Large Scale 3D Average PD and $\sigma$ values for Unbarred Spirals

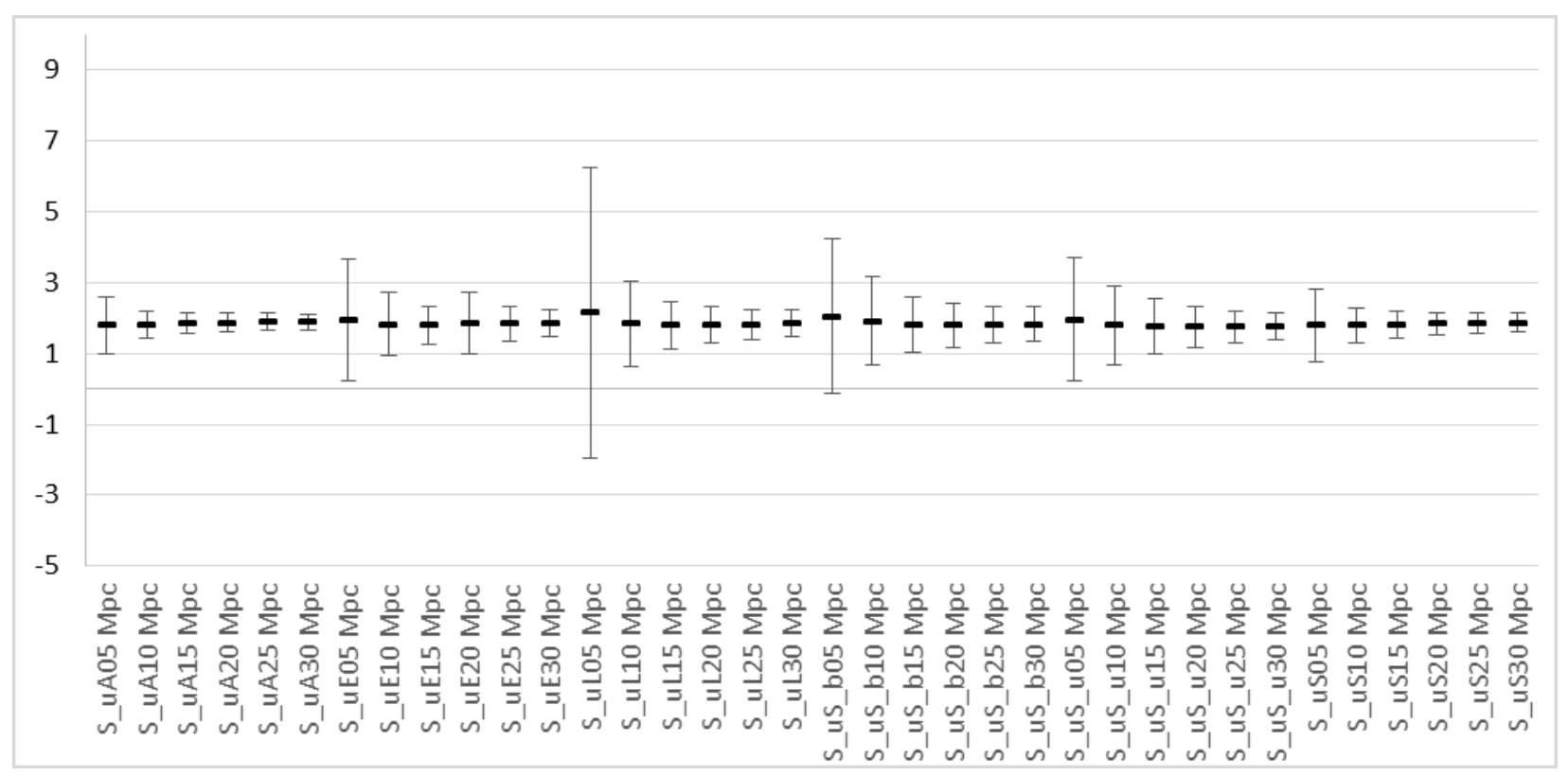




\subsubsection{2MRS South Large Scale}

Figure 5-43 2MRS South Large Scale 3D Average PD and $\sigma$ values for All Primaries regardless of morphology

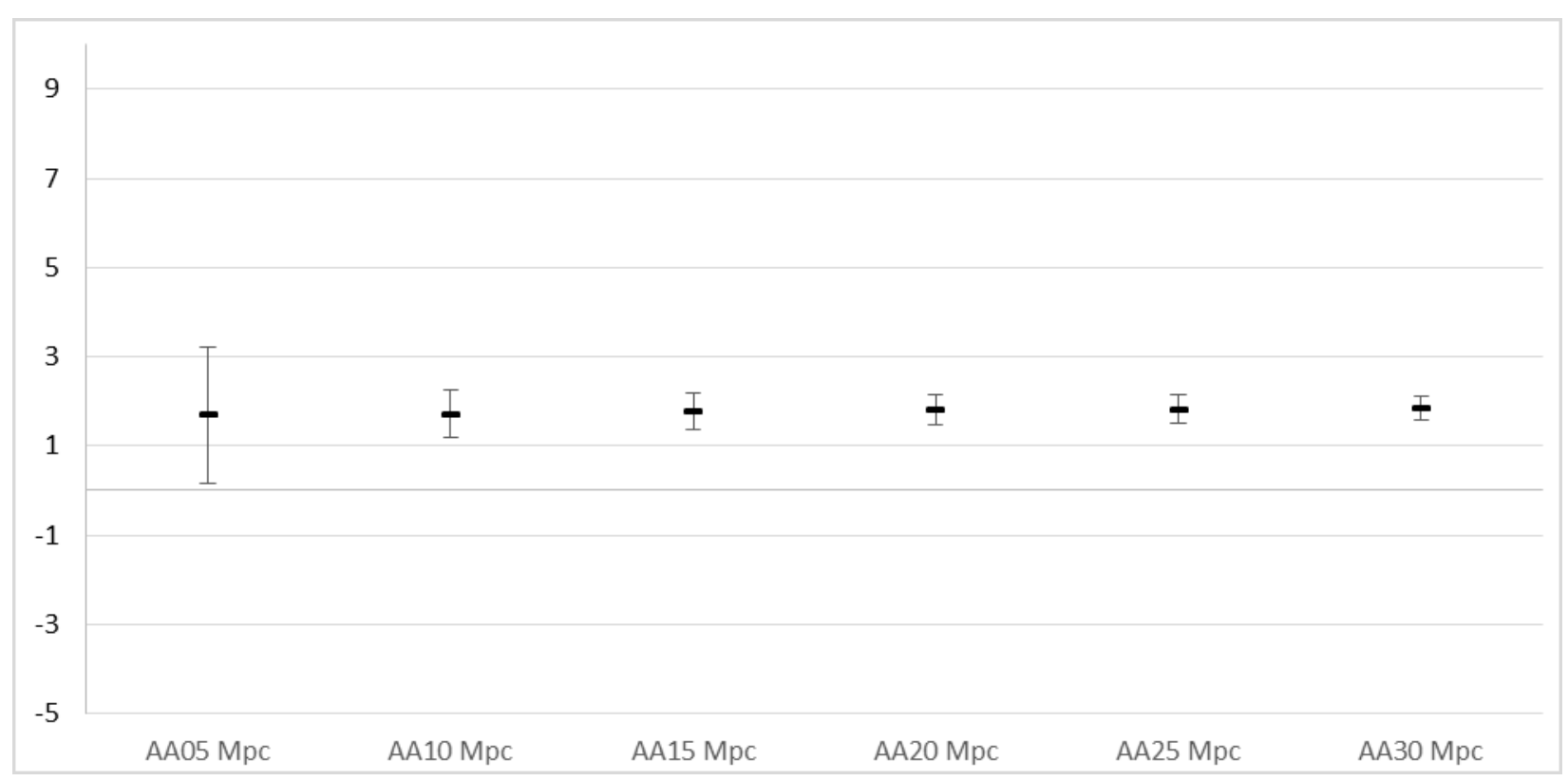

Figure 5-44 2MRS South Large Scale 3D Average PD and $\sigma$ values for Elliptical Primaries

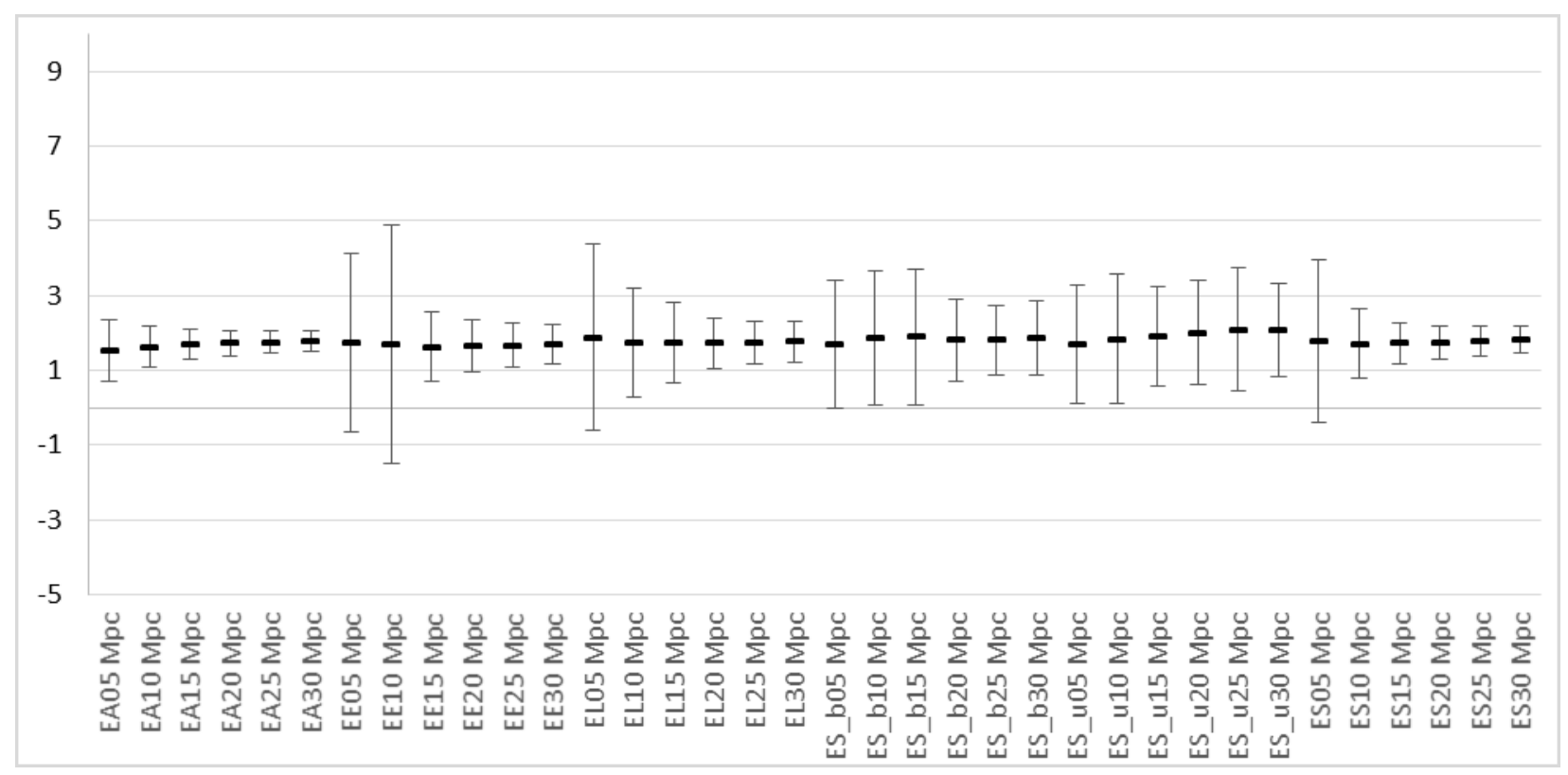


Figure 5-45 2MRS South Large Scale 3D Average PD and $\sigma$ values for Lenticular Primaries

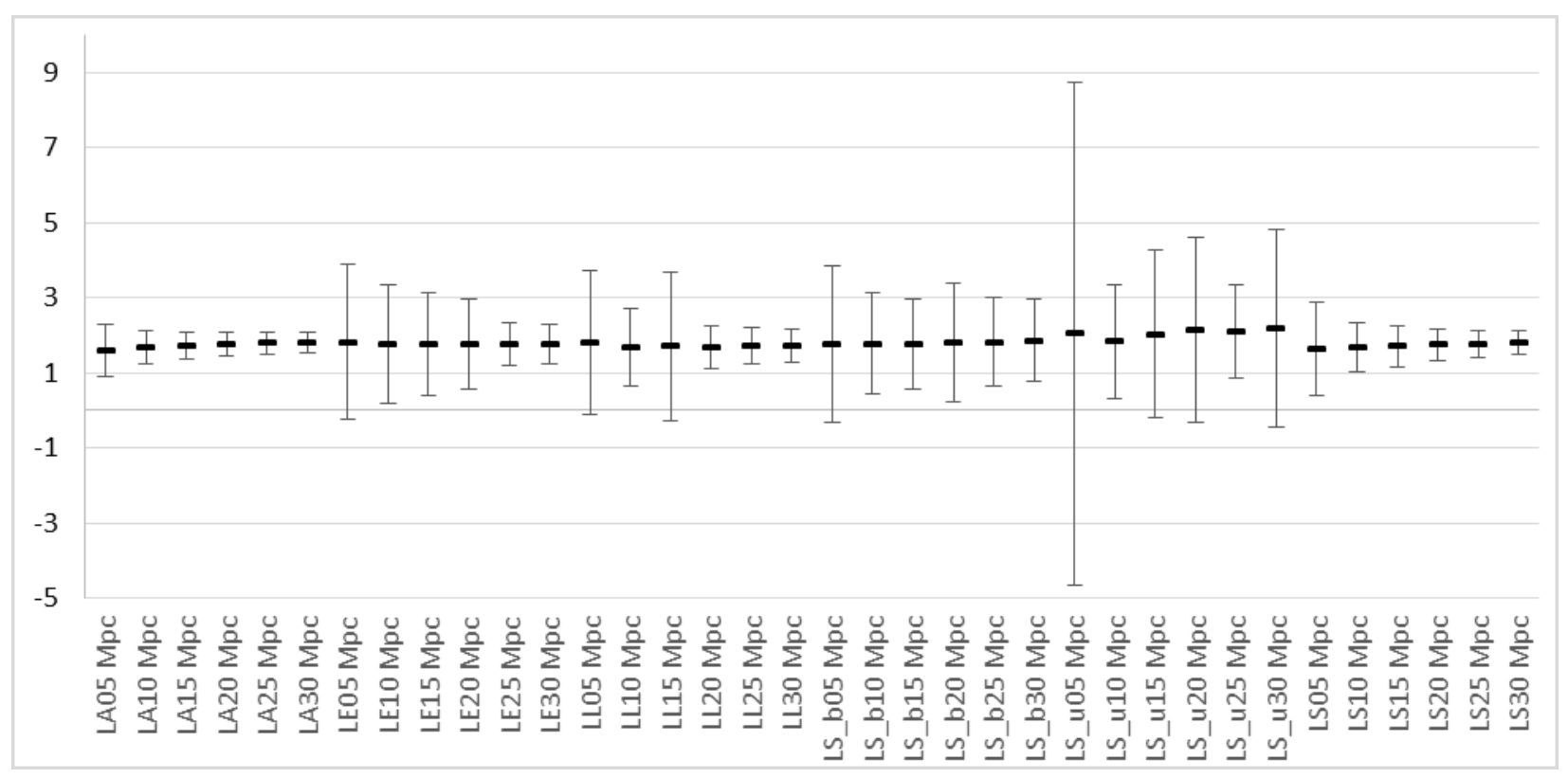

Figure 5-46 2MRS South Large Scale 3D Average PD and $\sigma$ values for Spiral Primaries

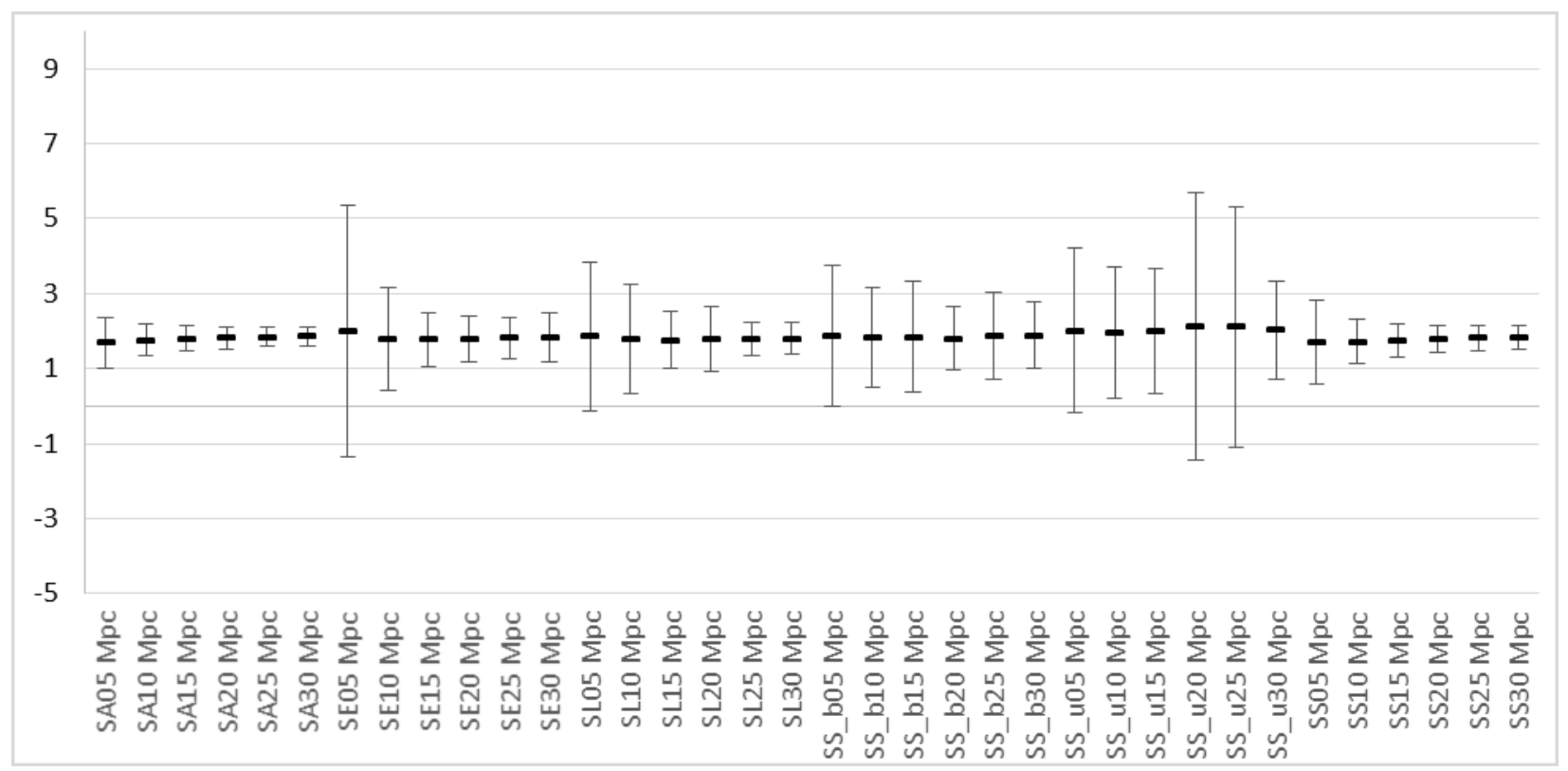


Figure 5-47 2MRS South Large Scale 3D Average PD and $\sigma$ values for Barred Spiral Primaries

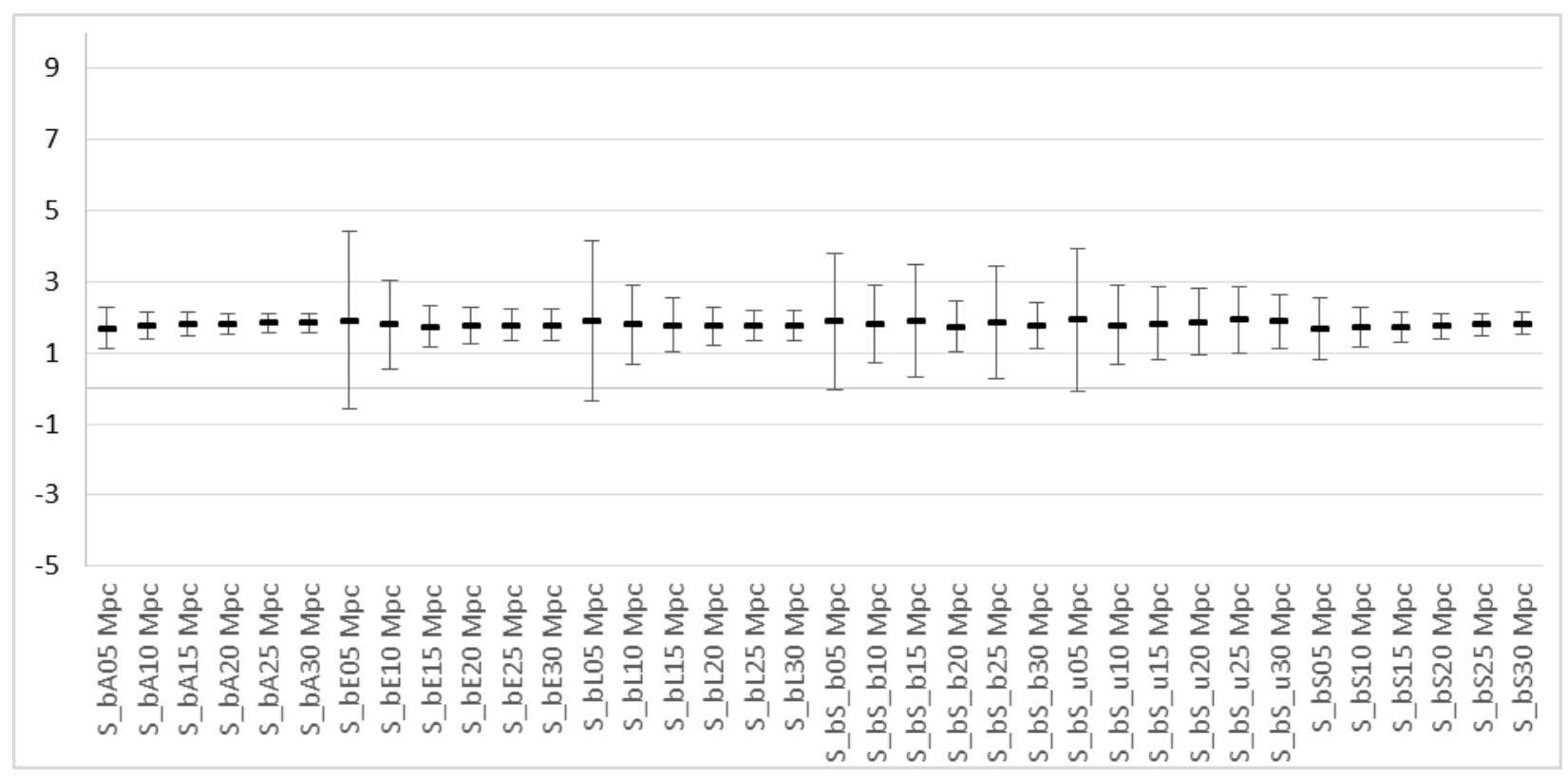

Figure 5-48 2MRS South Large Scale 3D Average PD and $\sigma$ values for Unbarred Spiral Primaries

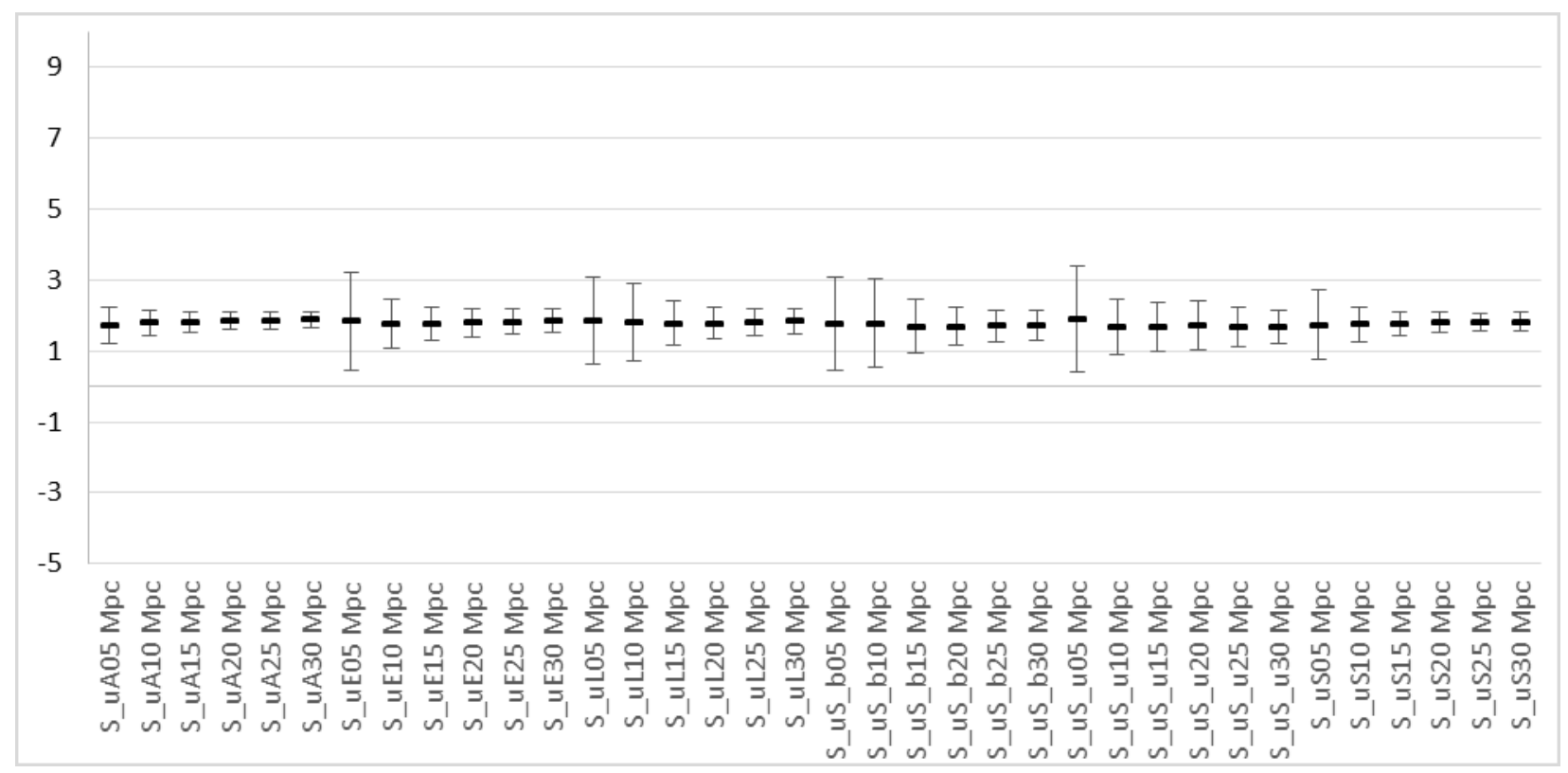




\subsubsection{Impact of Fitting Range on the Pointwise Dimension}

Data runs are created using the same primary and secondary morphological filters at varying fitting ranges. These runs are then analyzed in KS tests.

In all of the following tables $\mathrm{Y}$ indicates that the $p$ value from the $\mathrm{KS}$ test is < 0.01 . This means that there is less than a $1 \%$ chance that the two compared data sets are drawn from the same distribution. That is, the compared data sets are statistically dissimilar to the $99 \%$ level. A value of $\mathrm{N}$ indicates the inverse. That is, the datasets are not statistically dissimilar to the $99 \%$ level. 
When considering all galaxies without morphological filtering in Table 5-23, fitting range clearly is a factor as all comparisons are statistically dissimilar.

Table 5-23 3D KS Test Results - comparing two runs having the same primary/secondary filters and varying fitting range filters - limited to primaries with no specific morphology.

$\mathrm{Y}=$ dissimilar to the $99 \%$ level. $\mathrm{N}=$ not dissimilar to the $99 \%$ level. FR1 = Fitting Range 1

\begin{tabular}{|c|c|c|c|c|c|c|c|c|c|c|c|c|c|c|c|c|c|c|c|c|c|c|c|}
\hline \multirow[b]{3}{*}{$\begin{array}{c}\text { Run } \\
1 \\
\end{array}$} & \multirow[b]{3}{*}{$\begin{array}{c}\mathrm{FR} \\
1 \\
\end{array}$} & \multicolumn{10}{|c|}{ North } & \multicolumn{12}{|c|}{ South } \\
\hline & & \multicolumn{10}{|c|}{ Fitting Range 2 (Mpc) } & \multicolumn{12}{|c|}{ Fitting Range 2 (Mpc) } \\
\hline & & 1 & 2 & 3 & 4 & 5 & $\begin{array}{l}1 \\
0 \\
\end{array}$ & $\begin{array}{l}1 \\
5 \\
\end{array}$ & $\begin{array}{l}2 \\
0 \\
\end{array}$ & $\begin{array}{l}2 \\
5 \\
\end{array}$ & $\begin{array}{l}3 \\
0 \\
\end{array}$ & $\begin{array}{c}\text { Run } \\
1\end{array}$ & $\begin{array}{c}\text { FR } \\
1 \\
\end{array}$ & 1 & 2 & 3 & 4 & 5 & $\begin{array}{l}1 \\
0 \\
\end{array}$ & $\begin{array}{l}1 \\
5 \\
\end{array}$ & $\begin{array}{l}2 \\
0 \\
\end{array}$ & $\begin{array}{l}2 \\
5 \\
\end{array}$ & $\begin{array}{l}3 \\
0 \\
\end{array}$ \\
\hline AA & 1 & & $\mathbf{Y}$ & $\mathbf{Y}$ & $\mathbf{Y}$ & $\mathbf{Y}$ & & & & & & AA & 1 & & $\mathbf{Y}$ & $\mathbf{Y}$ & $\mathbf{Y}$ & $\mathbf{Y}$ & & & & & \\
\hline AA & 2 & & & $Y$ & $\mathbf{Y}$ & $\mathbf{Y}$ & & & & & & AA & 2 & & & $\mathbf{Y}$ & $\mathbf{Y}$ & $\mathbf{Y}$ & & & & & \\
\hline AA & 3 & & & & $\mathbf{Y}$ & $\mathbf{Y}$ & & & & & & AA & 3 & & & & $\mathbf{Y}$ & $\mathbf{Y}$ & & & & & \\
\hline AA & 4 & & & & & $\mathbf{Y}$ & & & & & & AA & 4 & & & & & $\mathbf{Y}$ & & & & & \\
\hline AA & 5 & & & & & & $\mathbf{Y}$ & $\mathbf{Y}$ & $\mathbf{Y}$ & $\mathbf{Y}$ & $\mathbf{Y}$ & AA & 5 & & & & & & $\mathbf{Y}$ & $\mathbf{Y}$ & $\mathbf{Y}$ & $\mathbf{Y}$ & $\mathbf{Y}$ \\
\hline AA & 10 & & & & & & & $\mathbf{Y}$ & $\mathbf{Y}$ & $\mathbf{Y}$ & $\mathbf{Y}$ & AA & 10 & & & & & & & $\mathrm{Y}$ & $\mathbf{Y}$ & $\mathbf{Y}$ & $\mathbf{Y}$ \\
\hline AA & 15 & & & & & & & & $\mathbf{Y}$ & $\mathbf{Y}$ & $\mathbf{Y}$ & $\mathrm{AA}$ & 15 & & & & & & & & $\mathbf{Y}$ & $\mathbf{Y}$ & $\mathbf{Y}$ \\
\hline AA & 20 & & & & & & & & & $\mathbf{Y}$ & $\mathbf{Y}$ & $A A$ & 20 & & & & & & & & & $Y$ & $Y$ \\
\hline AA & 25 & & & & & & & & & & $Y$ & $A A$ & 25 & & & & & & & & & & $\mathbf{Y}$ \\
\hline
\end{tabular}


When ellipticals are considered as the primary galaxies in Table 5-24, statistical dissimilarity is found for the vast majority of comparisons. At small scales below $5 \mathrm{Mpc}$ dissimilarity is very rarely found for the combinations of EE, EL, ES $S_{b}$ and ES

Dissimilarity is found on the upper limit of small scale for the ES combination.

Dissimilarity is found for almost all comparisons for the EA combination, while similarity is found below $5 \mathrm{Mpc}$ for the other types of secondaries. This suggests that the entire environment must be considered as a factor, as opposed to the interaction of an $E$ primary and a single category of secondary.

Table 5-24 3D KS Test Results - comparing two runs having the same primary/secondary filters and varying fitting range filters - limited to elliptical primaries.

$\mathrm{Y}=$ dissimilar to the $99 \%$ level. $\mathrm{N}=$ not dissimilar to the $99 \%$ level. FR1 = Fitting Range 1

\begin{tabular}{|c|c|c|c|c|c|c|c|c|c|c|c|}
\hline \multicolumn{12}{|c|}{ North } \\
\hline \multirow[b]{2}{*}{$\begin{array}{c}\text { Run } \\
1 \\
\end{array}$} & \multirow[b]{2}{*}{$\begin{array}{c}\text { FR } \\
1\end{array}$} & \multirow[b]{2}{*}{1} & \multirow[b]{2}{*}{2} & \multicolumn{6}{|c|}{ Fitting Range 2 (Mpc) } & \multirow[b]{2}{*}{$\begin{array}{l}2 \\
5\end{array}$} & \multirow[b]{2}{*}{$\begin{array}{l}3 \\
0 \\
\end{array}$} \\
\hline & & & & 3 & 4 & 5 & $\begin{array}{l}1 \\
0\end{array}$ & $\begin{array}{l}1 \\
5\end{array}$ & $\begin{array}{l}2 \\
0\end{array}$ & & \\
\hline EA & 1 & & $\underline{N}$ & $\mathbf{Y}$ & $\mathbf{Y}$ & $\mathbf{Y}$ & & & & & \\
\hline EA & 2 & & & $\mathbf{Y}$ & $\mathbf{Y}$ & $\mathbf{Y}$ & & & & & \\
\hline EA & 3 & & & & $\mathbf{Y}$ & $\mathbf{Y}$ & & & & & \\
\hline EA & 4 & & & & & $\mathbf{Y}$ & & & & & \\
\hline EA & 5 & & & & & & $\mathbf{Y}$ & $\mathbf{Y}$ & $\mathbf{Y}$ & $\mathbf{Y}$ & $\mathbf{Y}$ \\
\hline EA & 10 & & & & & & & $\mathbf{Y}$ & $\mathbf{Y}$ & $\mathbf{Y}$ & $\mathbf{Y}$ \\
\hline EA & 15 & & & & & & & & Y & $\mathrm{Y}$ & $\mathrm{Y}$ \\
\hline EA & 20 & & & & & & & & & $\mathbf{Y}$ & $\mathbf{Y}$ \\
\hline EA & 25 & & & & & & & & & & $\mathbf{Y}$ \\
\hline EE & 1 & & $\underline{N}$ & $\underline{\mathrm{N}}$ & $\underline{N}$ & $\mathbf{Y}$ & & & & & \\
\hline EE & 2 & & & $\underline{N}$ & $\underline{N}$ & $\mathbf{Y}$ & & & & & \\
\hline EE & 3 & & & & $\underline{N}$ & $\underline{N}$ & & & & & \\
\hline EE & 4 & & & & & $\underline{N}$ & & & & & \\
\hline EE & 5 & & & & & & $\mathbf{Y}$ & $\mathbf{Y}$ & $\mathbf{Y}$ & $\mathbf{Y}$ & $\mathbf{Y}$ \\
\hline EE & 10 & & & & & & & $\mathbf{Y}$ & $\mathbf{Y}$ & $\mathbf{Y}$ & $\mathbf{Y}$ \\
\hline EE & 15 & & & & & & & & $\mathrm{Y}$ & $\mathrm{Y}$ & $\mathrm{Y}$ \\
\hline EE & 20 & & & & & & & & & $\mathbf{Y}$ & $\mathbf{Y}$ \\
\hline EE & 25 & & & & & & & & & & $\underline{N}$ \\
\hline EL & 1 & & $\underline{N}$ & $\underline{\mathrm{N}}$ & $\underline{\mathrm{N}}$ & $\underline{\mathrm{N}}$ & & & & & \\
\hline EL & 2 & & & $\underline{\mathrm{N}}$ & $\underline{\mathrm{N}}$ & $\mathrm{Y}$ & & & & & \\
\hline EL & 3 & & & & $\underline{\mathrm{N}}$ & $\mathbf{Y}$ & & & & & \\
\hline EL & 4 & & & & & $\underline{\mathrm{N}}$ & & & & & \\
\hline EL & 5 & & & & & & $\mathbf{Y}$ & $\mathbf{Y}$ & $\mathbf{Y}$ & $\mathbf{Y}$ & $\mathbf{Y}$ \\
\hline
\end{tabular}

\begin{tabular}{|c|c|c|c|c|c|c|c|c|c|c|c|}
\hline \multicolumn{12}{|c|}{ South } \\
\hline \multirow[b]{2}{*}{$\begin{array}{c}\text { Run } \\
1\end{array}$} & \multirow[b]{2}{*}{$\begin{array}{c}\mathrm{FR} \\
1\end{array}$} & \multicolumn{10}{|c|}{ Fitting Range 2 (Mpc) } \\
\hline & & 1 & 2 & 3 & 4 & 5 & $\begin{array}{l}1 \\
0\end{array}$ & $\begin{array}{l}1 \\
5\end{array}$ & $\begin{array}{l}2 \\
0\end{array}$ & $\begin{array}{l}2 \\
5\end{array}$ & $\begin{array}{l}3 \\
0\end{array}$ \\
\hline EA & 1 & & $\underline{N}$ & $\mathbf{Y}$ & $\mathbf{Y}$ & $\mathbf{Y}$ & & & & & \\
\hline EA & 2 & & & $\mathbf{Y}$ & $\mathbf{Y}$ & $\mathbf{Y}$ & & & & & \\
\hline EA & 3 & & & & $\mathbf{Y}$ & $\mathbf{Y}$ & & & & & \\
\hline EA & 4 & & & & & $\mathbf{Y}$ & & & & & \\
\hline EA & 5 & & & & & & $\mathbf{Y}$ & $\mathbf{Y}$ & $\mathbf{Y}$ & $\mathbf{Y}$ & $\mathbf{Y}$ \\
\hline EA & 10 & & & & & & & $\mathbf{Y}$ & $\mathbf{Y}$ & $\mathbf{Y}$ & $\mathbf{Y}$ \\
\hline EA & 15 & & & & & & & & $\mathbf{Y}$ & $\mathbf{Y}$ & $\mathbf{Y}$ \\
\hline EA & 20 & & & & & & & & & $\mathbf{Y}$ & $\mathbf{Y}$ \\
\hline EA & 25 & & & & & & & & & & $\mathbf{Y}$ \\
\hline EE & 1 & & $\underline{N}$ & $\underline{\mathrm{N}}$ & $\underline{N}$ & $\underline{N}$ & & & & & \\
\hline EE & 2 & & & $\underline{N}$ & $\underline{N}$ & $\underline{N}$ & & & & & \\
\hline EE & 3 & & & & $\underline{N}$ & $\underline{N}$ & & & & & \\
\hline EE & 4 & & & & & $\underline{N}$ & & & & & \\
\hline EE & 5 & & & & & & $\mathbf{Y}$ & $\mathbf{Y}$ & $\mathbf{Y}$ & $\mathbf{Y}$ & $\mathbf{Y}$ \\
\hline EE & 10 & & & & & & & $\mathbf{Y}$ & $\mathbf{Y}$ & $\mathbf{Y}$ & $\mathbf{Y}$ \\
\hline EE & 15 & & & & & & & & $\mathbf{Y}$ & $\mathbf{Y}$ & $Y$ \\
\hline EE & 20 & & & & & & & & & $\underline{N}$ & $Y$ \\
\hline EE & 25 & & & & & & & & & & $\underline{\mathrm{N}}$ \\
\hline EL & 1 & & $\underline{\mathrm{N}}$ & $\underline{\mathrm{N}}$ & $\underline{\mathrm{N}}$ & $\underline{\mathrm{N}}$ & & & & & \\
\hline$E L$ & 2 & & & $\underline{\mathrm{N}}$ & $\underline{\mathrm{N}}$ & $\mathbf{Y}$ & & & & & \\
\hline EL & 3 & & & & $\underline{\mathrm{N}}$ & $\underline{\mathrm{N}}$ & & & & & \\
\hline EL & 4 & & & & & $\underline{N}$ & & & & & \\
\hline EL & 5 & & & & & & $Y$ & $Y$ & $Y$ & $Y$ & $Y$ \\
\hline
\end{tabular}




\begin{tabular}{|c|c|c|c|c|c|c|c|c|c|c|c|c|c|c|c|c|c|c|c|}
\hline EL & 10 & & & & & $Y$ & $\mathbf{Y}$ & $\mathbf{Y}$ & $\mathbf{Y}$ & EL & 10 & & & & & $Y$ & $\mathbf{Y}$ & $\mathbf{Y}$ & $\mathbf{Y}$ \\
\hline EL & 15 & & & & & & $Y$ & $Y$ & $\mathbf{Y}$ & EL & 15 & & & & & & $\mathbf{Y}$ & $\mathbf{Y}$ & $\mathbf{Y}$ \\
\hline EL & 20 & & & & & & & $Y$ & $Y$ & EL & 20 & & & & & & & $Y$ & $Y$ \\
\hline EL & 25 & & & & & & & & $\mathbf{Y}$ & EL & 25 & & & & & & & & $\mathbf{Y}$ \\
\hline ES & 1 & $\underline{N}$ & $\underline{N} \quad \underline{N}$ & $Y$ & & & & & & ES & 1 & $\underline{N}$ & $\underline{N}$ & $Y$ & $Y$ & & & & \\
\hline ES & 2 & & $\underline{N} \mathrm{Y}$ & $\mathrm{Y}$ & & & & & & ES & 2 & & $\underline{\mathrm{N}}$ & $Y$ & $Y$ & & & & \\
\hline ES & 3 & & $\underline{N}$ & $\mathbf{V} Y$ & & & & & & ES & 3 & & & $\underline{N}$ & $\mathbf{Y}$ & & & & \\
\hline ES & 4 & & & $\mathbf{Y}$ & & & & & & ES & 4 & & & & $\underline{N}$ & & & & \\
\hline ES & 5 & & & & $\mathbf{Y}$ & $Y$ & $\mathbf{Y}$ & $\mathbf{Y}$ & $\mathbf{Y}$ & ES & 5 & & & & $Y$ & $\mathbf{Y}$ & $\mathbf{Y}$ & $\mathbf{Y}$ & $\mathbf{Y}$ \\
\hline ES & 10 & & & & & $Y$ & $Y$ & $Y$ & $Y$ & ES & 10 & & & & & $Y$ & $Y$ & $Y$ & $Y$ \\
\hline ES & 15 & & & & & & $Y$ & $Y$ & $Y$ & ES & 15 & & & & & & $\mathrm{Y}$ & $\mathrm{Y}$ & $Y$ \\
\hline ES & 20 & & & & & & & $Y$ & $Y$ & ES & 20 & & & & & & & $Y$ & $Y$ \\
\hline ES & 25 & & & & & & & & $Y$ & ES & 25 & & & & & & & & $Y$ \\
\hline$E S_{b}$ & 1 & $\underline{N}$ & $\underline{N} \quad \underline{N}$ & $\underline{N}$ & & & & & & $E S_{b}$ & 1 & $\underline{N}$ & $\underline{\mathrm{N}}$ & $\underline{N}$ & $\underline{N}$ & & & & \\
\hline$E S_{b}$ & 2 & 1 & $\underline{N} \quad \underline{N}$ & $\underline{N}$ & & & & & & $\mathrm{ES}_{\mathrm{b}}$ & 2 & 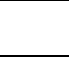 & $\underline{\mathrm{N}}$ & $\underline{N}$ & $\underline{N}$ & & & & \\
\hline$E S_{b}$ & 3 & & $\underline{N}$ & $\underline{N}$ & & & & & & $\mathrm{ES}_{\mathrm{b}}$ & 3 & & 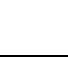 & $\underline{N}$ & $\underline{N}$ & & & & \\
\hline$E S_{b}$ & 4 & & & $\underline{N}$ & & & & & & $E S_{b}$ & 4 & & & & $\underline{\mathrm{N}}$ & & & & \\
\hline $\mathrm{ES}_{\mathrm{b}}$ & 5 & & & & $Y$ & $Y$ & $Y$ & $Y$ & $Y$ & $\mathrm{ES}_{\mathrm{b}}$ & 5 & & & & $Y$ & $Y$ & $\mathrm{Y}$ & $Y$ & $Y$ \\
\hline $\mathrm{ES}_{\mathrm{b}}$ & 10 & & & & & $\mathrm{Y}$ & $\mathrm{Y}$ & $\mathrm{Y}$ & $\mathrm{Y}$ & $\mathrm{ES}_{\mathrm{b}}$ & 10 & & & & & $\mathrm{Y}$ & $\mathrm{Y}$ & $\mathrm{Y}$ & $\mathrm{Y}$ \\
\hline$E S_{b}$ & 15 & & & & & & $Y$ & $Y$ & $Y$ & $E S_{b}$ & 15 & & & & & & $\underline{N}$ & $Y$ & $Y$ \\
\hline$E S_{b}$ & 20 & & & & & & & $Y$ & $Y$ & $\mathrm{ES}_{\mathrm{b}}$ & 20 & & & & & & & $\underline{N}$ & $Y$ \\
\hline$E S_{b}$ & 25 & & & & & & & & $\underline{N}$ & $E S_{b}$ & 25 & & & & & & & & $\underline{N}$ \\
\hline $\mathrm{ES}_{\mathrm{u}}$ & 1 & $\underline{N}$ & $\underline{N} \quad \underline{N}$ & $\underline{N}$ & & & & & & $\mathrm{ES}_{\mathrm{u}}$ & 1 & $\underline{N}$ & $\underline{\mathrm{N}}$ & $\underline{N}$ & $\underline{N}$ & & & & \\
\hline $\mathrm{ES}_{\mathrm{u}}$ & 2 & 1 & $\underline{N} \quad \underline{N}$ & $\underline{N}$ & & & & & & $\mathrm{ES}_{\mathrm{u}}$ & 2 & 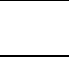 & $\underline{N}$ & $\underline{N}$ & $\underline{N}$ & & & & \\
\hline $\mathrm{ES}_{\mathrm{u}}$ & 3 & 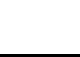 & $\underline{N}$ & $\underline{N}$ & & & & & & $\mathrm{ES}_{\mathrm{u}}$ & 3 & & 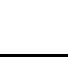 & $\underline{N}$ & $Y$ & & & & \\
\hline $\mathrm{ES}_{\mathrm{u}}$ & 4 & & . & $\underline{N}$ & & & & & & $\mathrm{ES}_{\mathrm{u}}$ & 4 & & & 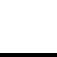 & $\underline{N}$ & & & & \\
\hline $\mathrm{ES}_{\mathrm{u}}$ & 5 & & & & $Y$ & $Y$ & $Y$ & $Y$ & $Y$ & $\mathrm{ES}_{\mathrm{u}}$ & 5 & & & & $Y$ & $Y$ & $Y$ & $\mathrm{Y}$ & $Y$ \\
\hline $\mathrm{ES}_{\mathrm{u}}$ & 10 & & & & & $Y$ & $Y$ & $Y$ & $Y$ & $\mathrm{ES}_{\mathrm{u}}$ & 10 & & & & & $Y$ & $Y$ & $Y$ & $Y$ \\
\hline $\mathrm{ES}_{\mathrm{u}}$ & 15 & & & & & & $Y$ & $Y$ & $Y$ & $\mathrm{ES}_{\mathrm{u}}$ & 15 & & & & & & $\mathrm{Y}$ & $Y$ & $Y$ \\
\hline $\mathrm{ES}_{\mathrm{u}}$ & 20 & & & & & & & $\mathrm{Y}$ & $\mathrm{Y}$ & $\mathrm{ES}_{\mathrm{u}}$ & 20 & & & & & & & $\underline{N}$ & $\mathrm{Y}$ \\
\hline $\mathrm{ES}_{\mathrm{u}}$ & 25 & & & & & & & & $\underline{N}$ & $\mathrm{ES}_{\mathrm{u}}$ & 25 & & & & & & & & $\underline{N}$ \\
\hline
\end{tabular}


When considering lenticulars as primary galaxies in Table $5-25$, dissimilarity is found for most combinations with no secondary morphology filter. No dissimilarity is found below $5 \mathrm{Mpc}$ for the combination of LE, LL, LS , and LS . Some dissimilarity is found for the LS combination below $5 \mathrm{Mpc}$. As with ellipticals, dissimilarity is found for the LA combination where none of the specific secondary morphology combinations show dissimilarity. Again, this suggests the importance of the entire environment.

Table 5-25 3D KS Test Results - comparing two runs having the same primary/secondary filters and varying fitting range filters - limited to lenticular primaries.

$\mathrm{Y}=$ dissimilar to the $99 \%$ level. $\mathrm{N}=$ not dissimilar to the $99 \%$ level. FR1 = Fitting Range 1

\begin{tabular}{|c|c|c|c|c|c|c|c|c|c|c|c|c|c|c|c|c|c|c|c|c|c|c|c|}
\hline \multirow[b]{3}{*}{$\begin{array}{c}\text { Run } \\
1\end{array}$} & \multicolumn{11}{|c|}{ North } & \multicolumn{12}{|c|}{ South } \\
\hline & \multicolumn{11}{|c|}{ Fitting Range 2 (Mpc) } & \multicolumn{12}{|c|}{ Fitting Range 2 (Mpc) } \\
\hline & $\begin{array}{c}\mathrm{FR} \\
1\end{array}$ & 1 & 2 & 3 & 4 & 5 & $\begin{array}{l}1 \\
0\end{array}$ & $\begin{array}{l}1 \\
5\end{array}$ & $\begin{array}{l}2 \\
0\end{array}$ & $\begin{array}{l}2 \\
5\end{array}$ & $\begin{array}{l}3 \\
0\end{array}$ & $\begin{array}{c}\text { Run } \\
1\end{array}$ & $\begin{array}{c}\mathrm{FR} \\
1\end{array}$ & 1 & 2 & 3 & 4 & 5 & $\begin{array}{l}1 \\
0\end{array}$ & $\begin{array}{l}1 \\
5\end{array}$ & $\begin{array}{l}2 \\
0\end{array}$ & $\begin{array}{l}2 \\
5\end{array}$ & $\begin{array}{l}3 \\
0\end{array}$ \\
\hline LA & 1 & & $\underline{N}$ & $\mathbf{Y}$ & $\mathbf{Y}$ & $\mathbf{Y}$ & & & & & & LA & 1 & & $\underline{N}$ & $\mathbf{Y}$ & $\mathbf{Y}$ & $\mathbf{Y}$ & & & & & \\
\hline LA & 2 & & & $\mathbf{Y}$ & $\mathbf{Y}$ & $\mathbf{Y}$ & & & & & & LA & 2 & & & $\mathbf{Y}$ & $\mathbf{Y}$ & $\mathbf{Y}$ & & & & & \\
\hline LA & 3 & & & & $\mathbf{Y}$ & $\mathbf{Y}$ & & & & & & LA & 3 & & & & $\mathbf{Y}$ & $\mathbf{Y}$ & & & & & \\
\hline LA & 4 & & & & & $\mathbf{Y}$ & & & & & & LA & 4 & & & & & $\mathbf{Y}$ & & & & & \\
\hline LA & 5 & & & & & & $Y$ & $Y$ & $\mathrm{Y}$ & $Y$ & $\mathrm{Y}$ & LA & 5 & & & & & & $Y$ & $Y$ & $\mathrm{Y}$ & $\mathrm{Y}$ & $Y$ \\
\hline LA & 10 & & & & & & & $\mathrm{Y}$ & $\mathrm{Y}$ & $\mathrm{Y}$ & $\mathrm{Y}$ & LA & 10 & & & & & & & $\mathrm{Y}$ & $\mathrm{Y}$ & $\mathrm{Y}$ & Y \\
\hline LA & 15 & & & & & & & & $\mathbf{Y}$ & $\mathbf{Y}$ & $\mathbf{Y}$ & LA & 15 & & & & & & & & $\mathbf{Y}$ & $\mathbf{Y}$ & $\mathbf{Y}$ \\
\hline LA & 20 & & & & & & & & & $\underline{N}$ & $\mathbf{Y}$ & LA & 20 & & & & & & & & & $\mathbf{Y}$ & $\mathbf{Y}$ \\
\hline LA & 25 & & & & & & & & & & $\underline{N}$ & LA & 25 & & & & & & & & & & $\underline{N}$ \\
\hline LE & 1 & & $\underline{N}$ & $\underline{N}$ & $\underline{N}$ & $\underline{N}$ & & & & & & LE & 1 & & $\underline{\mathrm{N}}$ & $\underline{N}$ & $\underline{N}$ & $\underline{N}$ & & & & & \\
\hline LE & 2 & & & $\underline{N}$ & $\underline{N}$ & $\underline{N}$ & & & & & & LE & 2 & & & $\underline{\mathrm{N}}$ & $\underline{N}$ & $\underline{N}$ & & & & & \\
\hline LE & 3 & & & & $\underline{N}$ & $\underline{N}$ & & & & & & LE & 3 & & & & $\underline{N}$ & $\underline{N}$ & & & & & \\
\hline LE & 4 & & & & & $\underline{N}$ & & & & & & LE & 4 & & & & & $\underline{N}$ & & & & & \\
\hline LE & 5 & & & & & & $\mathbf{Y}$ & $\mathbf{Y}$ & $\mathbf{Y}$ & $\mathbf{Y}$ & $\mathbf{Y}$ & LE & 5 & & & & & & $\mathbf{Y}$ & $\mathbf{Y}$ & $\mathbf{Y}$ & $\mathbf{Y}$ & $Y$ \\
\hline LE & 10 & & & & & & & $\mathbf{Y}$ & $\mathbf{Y}$ & $\mathbf{Y}$ & $\mathbf{Y}$ & LE & 10 & & & & & & & $\mathbf{Y}$ & $\mathbf{Y}$ & $Y$ & $Y$ \\
\hline LE & 15 & & & & & & & & $\mathbf{Y}$ & $\mathbf{Y}$ & $\mathbf{Y}$ & LE & 15 & & & & & & & & $\mathbf{Y}$ & $\mathbf{Y}$ & $\mathbf{Y}$ \\
\hline LE & 20 & & & & & & & & & $\underline{\mathrm{N}}$ & $\mathbf{Y}$ & LE & 20 & & & & & & & & & $\mathbf{Y}$ & $\mathbf{Y}$ \\
\hline LE & 25 & & & & & & & & & & $\underline{\mathrm{N}}$ & LE & 25 & & & & & & & & & & $\underline{N}$ \\
\hline LL & 1 & & $\underline{\mathrm{N}}$ & $\underline{N}$ & $\underline{N}$ & $\underline{N}$ & & & & & & LL & 1 & & $\underline{N}$ & $\underline{\mathrm{N}}$ & $\underline{N}$ & $\underline{N}$ & & & & & \\
\hline LL & 2 & & & $\underline{N}$ & $\underline{N}$ & $\underline{N}$ & & & & & & LL & 2 & & & $\underline{N}$ & $\underline{N}$ & $\mathrm{Y}$ & & & & & \\
\hline $\mathrm{LL}$ & 3 & & & & $\underline{N}$ & $\underline{N}$ & & & & & & LL & 3 & & & & $\underline{N}$ & $Y$ & & & & & \\
\hline LL & 4 & & & & & $\underline{N}$ & & & & & & LL & 4 & & & & & $\underline{N}$ & & & & & \\
\hline $\mathrm{LL}$ & 5 & & & & & & $Y$ & $Y$ & $Y$ & $Y$ & $Y$ & $\mathrm{LL}$ & 5 & & & & & & $\mathrm{Y}$ & $Y$ & $Y$ & $Y$ & $\bar{Y}$ \\
\hline LL & 10 & & & & & & & $\mathbf{Y}$ & $\mathbf{Y}$ & $\mathbf{Y}$ & $\mathbf{Y}$ & LL & 10 & & & & & & & $\mathbf{Y}$ & $\mathbf{Y}$ & $\mathbf{Y}$ & $\mathbf{Y}$ \\
\hline LL & 15 & & & & & & & & $Y$ & $Y$ & $Y$ & LL & 15 & & & & & & & & $Y$ & $Y$ & $Y$ \\
\hline LL & 20 & & & & & & & & & $\underline{N}$ & $\mathbf{Y}$ & LL & 20 & & & & & & & & & $Y$ & $Y$ \\
\hline
\end{tabular}




\begin{tabular}{|c|c|c|c|c|c|c|c|c|c|c|c|c|c|c|c|c|c|c|c|c|c|}
\hline LL & 25 & & & & & & & & & $\underline{\mathrm{N}}$ & $\mathrm{LL}$ & 25 & & & & & & & & & $\underline{N}$ \\
\hline LS & 1 & $\underline{N}$ & $\underline{N}$ & $\mathrm{Y}$ & $Y$ & & & & & & LS & 1 & $\underline{N}$ & $\underline{N}$ & $\mathrm{Y}$ & $\mathrm{Y}$ & & & & & \\
\hline LS & 2 & & $\underline{N}$ & $Y$ & $\mathrm{Y}$ & & & & & & LS & 2 & & $\underline{N}$ & $Y$ & $Y$ & & & & & \\
\hline LS & 3 & & & $\underline{N}$ & $Y$ & & & & & & LS & 3 & & & $Y$ & $\mathrm{Y}$ & & & & & \\
\hline LS & 4 & & & & $\underline{N}$ & & & & & & LS & 4 & & & & $\underline{\mathrm{N}}$ & & & & & \\
\hline LS & 5 & & & & & $\bar{Y}$ & $\mathrm{Y}$ & $\mathrm{Y}$ & $Y$ & $\mathrm{Y}$ & LS & 5 & & & & & $\mathrm{Y}$ & $Y$ & $Y$ & $\mathrm{Y}$ & $\bar{Y}$ \\
\hline LS & 10 & & & & & & Y & Y & $\mathrm{Y}$ & $Y$ & LS & 10 & & & & & & $Y$ & $\mathrm{Y}$ & $\mathrm{Y}$ & $\mathrm{Y}$ \\
\hline LS & 15 & & & & & & & $\mathrm{Y}$ & $Y$ & $Y$ & LS & 15 & & & & & & & $\mathrm{Y}$ & $Y$ & $\mathrm{Y}$ \\
\hline LS & 20 & & & & & & & & $\mathrm{Y}$ & $\mathrm{Y}$ & LS & 20 & & & & & & & & $\mathrm{Y}$ & $Y$ \\
\hline LS & 25 & & & & & & & & & $Y$ & LS & 25 & & & & & & & & & $Y$ \\
\hline $\mathrm{LS}_{\mathrm{b}}$ & 1 & $\underline{N}$ & $\underline{\mathrm{N}}$ & $\underline{\mathrm{N}}$ & $\underline{\mathrm{N}}$ & & & & & & $\mathrm{LS}_{\mathrm{b}}$ & 1 & $\underline{\mathrm{N}}$ & $\underline{N}$ & $\underline{\mathrm{N}}$ & $\underline{\mathrm{N}}$ & & & & & \\
\hline $\mathrm{LS}_{\mathrm{b}}$ & 2 & & $\underline{N}$ & $\underline{N}$ & $\underline{N}$ & & & & & & $\mathrm{LS}_{\mathrm{b}}$ & 2 & & $\underline{N}$ & $\underline{N}$ & $\underline{N}$ & & & & & \\
\hline $\mathrm{LS}_{\mathrm{b}}$ & 3 & & & $\underline{N}$ & $\underline{N}$ & & & & & & $\mathrm{LS}_{\mathrm{b}}$ & 3 & & & $\underline{N}$ & $\underline{N}$ & & & & & \\
\hline $\mathrm{LS}_{\mathrm{b}}$ & 4 & & & & $\underline{\mathrm{N}}$ & & & & & & $\mathrm{LS}_{\mathrm{b}}$ & 4 & & & & $\underline{N}$ & & & & & \\
\hline $\mathrm{LS}_{\mathrm{b}}$ & 5 & & & & & $Y$ & $Y$ & $\mathrm{Y}$ & $\mathrm{Y}$ & $\mathrm{Y}$ & $\mathrm{LS}_{\mathrm{b}}$ & 5 & & & & & Y & $Y$ & $\mathrm{Y}$ & $Y$ & $Y$ \\
\hline $\mathrm{LS}_{\mathrm{b}}$ & 10 & & & & & & $Y$ & $\mathrm{Y}$ & $Y$ & $Y$ & $\mathrm{LS}_{\mathrm{b}}$ & 10 & & & & & & $Y$ & $\mathrm{Y}$ & $Y$ & $\mathrm{Y}$ \\
\hline $\mathrm{LS}_{\mathrm{b}}$ & 15 & & & & & & & Y & $Y$ & $Y$ & $\mathrm{LS}_{\mathrm{b}}$ & 15 & & & & & & & $\mathrm{Y}$ & $Y$ & $Y$ \\
\hline $\mathrm{LS}_{\mathrm{b}}$ & 20 & & & & & & & & $\underline{\mathrm{N}}$ & $\mathrm{Y}$ & $\mathrm{LS}_{\mathrm{b}}$ & 20 & & & & & & & & $\underline{\mathrm{N}}$ & $Y$ \\
\hline $\mathrm{LS}_{\mathrm{b}}$ & 25 & & & & & & & & & $\underline{N}$ & $\mathrm{LS}_{\mathrm{b}}$ & 25 & & & & & & & & & $\underline{N}$ \\
\hline $\mathrm{LS}_{\mathrm{u}}$ & 1 & $\underline{N}$ & $\underline{\mathrm{N}}$ & $\underline{N}$ & $\underline{N}$ & & & & & & $\mathrm{LS}_{\mathrm{u}}$ & 1 & $\underline{N}$ & $\underline{N}$ & $\underline{N}$ & $\mathrm{Y}$ & & & & & \\
\hline $\mathrm{LS}_{\mathrm{u}}$ & 2 & & $\underline{N}$ & $\underline{N}$ & $\underline{N}$ & & & & & & $\mathrm{LS}_{\mathrm{u}}$ & 2 & & $\underline{N}$ & $\underline{N}$ & $\underline{N}$ & & & & & \\
\hline $\mathrm{LS}_{\mathrm{u}}$ & 3 & & & $\underline{\mathrm{N}}$ & $\underline{\mathrm{N}}$ & & & & & & $\mathrm{LS}_{\mathrm{u}}$ & 3 & & & $\underline{\mathrm{N}}$ & $\underline{N}$ & & & & & \\
\hline $\mathrm{LS}_{\mathrm{u}}$ & 4 & & & & $\underline{N}$ & & & & & & $\mathrm{LS}_{\mathrm{u}}$ & 4 & & & & $\underline{N}$ & & & & & \\
\hline $\mathrm{LS}_{\mathrm{u}}$ & 5 & & & & & $Y$ & $Y$ & Y & $Y$ & $Y$ & $\mathrm{LS}_{\mathrm{u}}$ & 5 & & & & & $\mathrm{Y}$ & $Y$ & $\mathrm{Y}$ & $Y$ & $Y$ \\
\hline $\mathrm{LS}_{\mathrm{u}}$ & 10 & & & & & & $Y$ & $Y$ & $\mathrm{Y}$ & $\mathrm{Y}$ & $\mathrm{LS}_{\mathrm{u}}$ & 10 & & & & & & $Y$ & $\mathrm{Y}$ & $\mathrm{Y}$ & $Y$ \\
\hline $\mathrm{LS}_{\mathrm{u}}$ & 15 & & & & & & & $\mathrm{Y}$ & $\mathrm{Y}$ & $\mathrm{Y}$ & $\mathrm{LS}_{\mathrm{u}}$ & 15 & & & & & & & $\mathrm{Y}$ & $\mathrm{Y}$ & $\mathrm{Y}$ \\
\hline $\mathrm{LS}_{\mathrm{u}}$ & 20 & & & & & & & & $\mathrm{Y}$ & $Y$ & $\mathrm{LS}_{\mathrm{u}}$ & 20 & & & & & & & & $\mathrm{Y}$ & $Y$ \\
\hline $\mathrm{LS}_{\mathrm{u}}$ & 25 & & & & & & & & & $\underline{N}$ & $\mathrm{LS}_{\mathrm{u}}$ & 25 & & & & & & & & & $\underline{N}$ \\
\hline
\end{tabular}


When considering spirals as primary galaxies in Table 5-26, statistical dissimilarity is found at all fitting ranges when no secondary morphology filter is applied. Dissimilarity is found for nearly all comparisons above $5 \mathrm{Mpc}$ having a secondary morphology filter. Dissimilarity is rarely found below $5 \mathrm{Mpc}$ for the SE, SL, SS $\mathrm{b}_{\mathrm{b}}$, and SS combinations. The SS combination consistently shows dissimilarity below $5 \mathrm{Mpc}$. This result indicates that the environment of $E, L, S_{b}$ and $S_{u}$ surrounding $S$ are similar below $5 \mathrm{Mpc}$.

Table 5-26 3D KS Test Results - comparing two runs having the same primary/secondary filters and varying fitting range filters - limited to spiral primaries.

$\mathrm{Y}=$ dissimilar to the $99 \%$ level. $\mathrm{N}=$ not dissimilar to the $99 \%$ level. FR1 = Fitting Range 1

\begin{tabular}{|c|c|c|c|c|c|c|c|c|c|c|c|c|c|c|c|c|c|c|c|c|c|c|c|}
\hline \multicolumn{12}{|c|}{ North } & \multicolumn{12}{|c|}{ South } \\
\hline \multirow[b]{2}{*}{$\begin{array}{c}\text { Run } \\
1\end{array}$} & \multirow[b]{2}{*}{$\begin{array}{c}\text { FR } \\
1\end{array}$} & \multicolumn{10}{|c|}{ Fitting Range 2 (Mpc) } & \multirow[b]{2}{*}{$\begin{array}{c}\text { Run } \\
1\end{array}$} & \multirow[b]{2}{*}{$\begin{array}{c}\text { FR } \\
1\end{array}$} & \multicolumn{10}{|c|}{ Fitting Range 2 (Mpc) } \\
\hline & & 1 & 2 & 3 & 4 & 5 & $\begin{array}{l}1 \\
0\end{array}$ & $\begin{array}{l}1 \\
5\end{array}$ & $\begin{array}{l}2 \\
0\end{array}$ & $\begin{array}{l}2 \\
5\end{array}$ & $\begin{array}{l}3 \\
0\end{array}$ & & & 1 & 2 & 3 & 4 & 5 & $\begin{array}{l}1 \\
0\end{array}$ & $\begin{array}{l}1 \\
5\end{array}$ & $\begin{array}{l}2 \\
0\end{array}$ & $\begin{array}{l}2 \\
5\end{array}$ & $\begin{array}{l}3 \\
0\end{array}$ \\
\hline SA & 1 & & $Y$ & $\mathbf{Y}$ & $\mathbf{Y}$ & $Y$ & & & & & & SA & 1 & & $Y$ & $Y$ & $Y$ & $Y$ & & & & & \\
\hline SA & 2 & & & $\mathrm{Y}$ & $\mathrm{Y}$ & $Y$ & & & & & & SA & 2 & & & $Y$ & $\mathrm{Y}$ & $Y$ & & & & & \\
\hline SA & 3 & & & & $\mathrm{Y}$ & $Y$ & & & & & & SA & 3 & & & & $Y$ & $Y$ & & & & & \\
\hline SA & 4 & & & & & $Y$ & & & & & & SA & 4 & & & & & $Y$ & & & & & \\
\hline SA & 5 & & & & & & $\mathbf{Y}$ & $Y$ & $Y$ & $Y$ & $Y$ & SA & 5 & & & & & & $\mathrm{Y}$ & $Y$ & $Y$ & $Y$ & $Y$ \\
\hline SA & 10 & & & & & & & $\mathrm{Y}$ & $Y$ & $\mathrm{Y}$ & $\bar{Y}$ & SA & 10 & & & & & & & $\mathrm{Y}$ & $\mathrm{Y}$ & $\bar{Y}$ & $\bar{Y}$ \\
\hline SA & 15 & & & & & & & & $\mathrm{Y}$ & $\mathrm{Y}$ & $Y$ & SA & 15 & & & & & & & & $\mathrm{Y}$ & $\mathrm{Y}$ & $\mathrm{Y}$ \\
\hline SA & 20 & & & & & & & & & $\mathrm{Y}$ & $Y$ & SA & 20 & & & & & & & & & $\mathrm{Y}$ & $Y$ \\
\hline SA & 25 & & & & & & & & & & $\underline{N}$ & SA & 25 & & & & & & & & & & $\mathrm{Y}$ \\
\hline SE & 1 & & $\underline{N}$ & $\underline{N}$ & $\underline{N}$ & $\mathrm{Y}$ & & & & & & SE & 1 & & $\underline{N}$ & $\underline{N}$ & $\mathrm{Y}$ & $Y$ & & & & & \\
\hline SE & 2 & & & $\underline{N}$ & $\underline{N}$ & $\mathrm{Y}$ & & & & & & SE & 2 & & & $\underline{N}$ & $\underline{N}$ & $Y$ & & & & & \\
\hline SE & 3 & & & & $\underline{N}$ & $\underline{N}$ & & & & & & SE & 3 & & & & $\underline{N}$ & $Y$ & & & & & \\
\hline SE & 4 & & & & & $\underline{N}$ & & & & & & SE & 4 & & & & & $\underline{N}$ & & & & & \\
\hline SE & 5 & & & & & & $\mathrm{Y}$ & $\mathrm{Y}$ & $Y$ & $\mathrm{Y}$ & $\mathrm{Y}$ & SE & 5 & & & & & & $\mathrm{Y}$ & $\mathrm{Y}$ & $\mathrm{Y}$ & $\mathrm{Y}$ & $\mathrm{Y}$ \\
\hline SE & 10 & & & & & & & $\mathrm{Y}$ & $Y$ & $\mathrm{Y}$ & $Y$ & SE & 10 & & & & & & & $\mathrm{Y}$ & $Y$ & $Y$ & $Y$ \\
\hline SE & 15 & & & & & & & & $Y$ & $Y$ & $Y$ & SE & 15 & & & & & & & & $Y$ & $Y$ & $Y$ \\
\hline SE & 20 & & & & & & & & & $Y$ & $Y$ & SE & 20 & & & & & & & & & $Y$ & $\mathrm{Y}$ \\
\hline SE & 25 & & & & & & & & & & $Y$ & SE & 25 & & & & & & & & & & $\underline{\mathrm{N}}$ \\
\hline SL & 1 & & $\underline{N}$ & $\underline{N}$ & $\underline{N}$ & $\mathbf{Y}$ & & & & & & SL & 1 & & $\underline{\mathrm{N}}$ & $\underline{\mathrm{N}}$ & $\underline{\mathrm{N}}$ & $\underline{N}$ & & & & & \\
\hline SL & 2 & & & $\underline{N}$ & $\underline{N}$ & $\underline{\mathrm{N}}$ & & & & & & SL & 2 & & & $\underline{\mathrm{N}}$ & $\underline{\mathrm{N}}$ & $Y$ & & & & & \\
\hline $\mathrm{SL}$ & 3 & & & & $\underline{N}$ & $\underline{N}$ & & & & & & SL & 3 & & & & $\underline{N}$ & $\underline{N}$ & & & & & \\
\hline $\mathrm{SL}$ & 4 & & & & & $\underline{N}$ & & & & & & $S L$ & 4 & & & & & $\underline{N}$ & & & & & \\
\hline SL & 5 & & & & & & $\mathbf{Y}$ & $Y$ & $Y$ & $Y$ & $Y$ & SL & 5 & & & & & & $Y$ & $Y$ & $Y$ & $Y$ & $\mathbf{Y}$ \\
\hline $\mathrm{SL}$ & 10 & & & & & & & $Y$ & $Y$ & $\mathrm{Y}$ & $\bar{Y}$ & $\mathrm{SL}$ & 10 & & & & & & & $\mathrm{Y}$ & $Y$ & $Y$ & $\mathrm{Y}$ \\
\hline SL & 15 & & & & & & & & $Y$ & $Y$ & $Y$ & SL & 15 & & & & & & & & $\mathbf{Y}$ & $\mathbf{Y}$ & $Y$ \\
\hline
\end{tabular}




\begin{tabular}{|c|c|c|c|c|c|c|c|c|c|c|}
\hline SL & 20 & & & & & & & & $Y$ & $Y$ \\
\hline SL & 25 & & & & & & & & & $\mathbf{Y}$ \\
\hline SS & 1 & $\underline{\mathrm{N}}$ & $\mathbf{Y}$ & $\mathbf{Y}$ & $\mathbf{Y}$ & & & & & \\
\hline SS & 2 & & $\mathbf{Y}$ & $\mathbf{Y}$ & $\mathbf{Y}$ & & & & & \\
\hline SS & 3 & & & $\mathbf{Y}$ & $\mathbf{Y}$ & & & & & \\
\hline SS & 4 & & & & $\mathbf{Y}$ & & & & & \\
\hline SS & 5 & & & & & $\mathbf{Y}$ & $\mathbf{Y}$ & $\mathbf{Y}$ & $\mathbf{Y}$ & $\mathbf{Y}$ \\
\hline SS & 10 & & & & & & $\mathbf{Y}$ & $\mathbf{Y}$ & $\mathbf{Y}$ & $\mathbf{Y}$ \\
\hline SS & 15 & & & & & & & $\mathbf{Y}$ & $\mathbf{Y}$ & $\mathbf{Y}$ \\
\hline SS & 20 & & & & & & & & $\mathbf{Y}$ & $\mathbf{Y}$ \\
\hline SS & 25 & & & & & & & & & $\mathbf{Y}$ \\
\hline $\mathrm{SS}_{\mathrm{b}}$ & 1 & $\underline{N}$ & $\underline{\mathrm{N}}$ & $\underline{\mathrm{N}}$ & $\underline{\mathrm{N}}$ & & & & & \\
\hline $\mathrm{SS}_{\mathrm{b}}$ & 2 & & $\underline{\mathrm{N}}$ & $\underline{N}$ & $\underline{\mathrm{N}}$ & & & & & \\
\hline$S S_{b}$ & 3 & & & $\underline{\mathrm{N}}$ & $\underline{\mathrm{N}}$ & & & & & \\
\hline $\mathrm{SS}_{\mathrm{b}}$ & 4 & & & & $\underline{\mathrm{N}}$ & & & & & \\
\hline $\mathrm{SS}_{\mathrm{b}}$ & 5 & & & & & $\mathbf{Y}$ & $\mathbf{Y}$ & $\mathbf{Y}$ & $Y$ & $\mathbf{Y}$ \\
\hline $\mathrm{SS}_{\mathrm{b}}$ & 10 & & & & & & $\mathbf{Y}$ & $\mathbf{Y}$ & $Y$ & $\mathbf{Y}$ \\
\hline $\mathrm{SS}_{\mathrm{b}}$ & 15 & & & & & & & $Y$ & $Y$ & $\mathrm{Y}$ \\
\hline $\mathrm{SS}_{\mathrm{b}}$ & 20 & & & & & & & & $\mathrm{Y}$ & $\mathrm{Y}$ \\
\hline $\mathrm{SS}_{\mathrm{b}}$ & 25 & & & & & & & & & $Y$ \\
\hline $\mathrm{SS}_{\mathrm{u}}$ & 1 & $\underline{N}$ & $\underline{\mathrm{N}}$ & $\underline{\mathrm{N}}$ & $\underline{\mathrm{N}}$ & & & & & \\
\hline $\mathrm{SS}_{\mathrm{u}}$ & 2 & & $\underline{\mathrm{N}}$ & $\underline{\mathrm{N}}$ & $\underline{\mathrm{N}}$ & & & & & \\
\hline $\mathrm{SS}_{\mathrm{u}}$ & 3 & & & $\underline{N}$ & $\underline{\mathrm{N}}$ & & & & & \\
\hline $\mathrm{SS}_{\mathrm{u}}$ & 4 & & & & $\underline{\mathrm{N}}$ & & & & & \\
\hline $\mathrm{SS}_{\mathrm{u}}$ & 5 & & & & & $Y$ & $Y$ & $Y$ & $Y$ & $Y$ \\
\hline $\mathrm{SS}_{\mathrm{u}}$ & 10 & & & & & & $\mathbf{Y}$ & $\mathbf{Y}$ & $\mathbf{Y}$ & $\mathbf{Y}$ \\
\hline $\mathrm{SS}_{\mathrm{u}}$ & 15 & & & & & & & $\mathbf{Y}$ & $Y$ & $\mathbf{Y}$ \\
\hline $\mathrm{SS}_{\mathrm{u}}$ & 20 & & & & & & & & $Y$ & $Y$ \\
\hline $\mathrm{SS}_{\mathrm{u}}$ & 25 & & & & & & & & & $Y$ \\
\hline
\end{tabular}

\begin{tabular}{|c|c|c|c|c|c|c|c|c|c|c|}
\hline SL & 20 & & & & & & & & $\mathbf{Y}$ & $Y$ \\
\hline SL & 25 & & & & & & & & & $\mathbf{Y}$ \\
\hline SS & 1 & $\underline{\mathrm{N}}$ & $\mathbf{Y}$ & $Y$ & $Y$ & & & & & \\
\hline SS & 2 & & $\mathbf{Y}$ & $\mathbf{Y}$ & $Y$ & & & & & \\
\hline SS & 3 & & & $\mathbf{Y}$ & $\mathbf{Y}$ & & & & & \\
\hline SS & 4 & & & & $\underline{N}$ & & & & & \\
\hline SS & 5 & & & & & $\mathbf{Y}$ & $\mathbf{Y}$ & $\mathbf{Y}$ & $\mathbf{Y}$ & $\mathbf{Y}$ \\
\hline SS & 10 & & & & & & $\mathbf{Y}$ & $\mathbf{Y}$ & $\mathbf{Y}$ & $Y$ \\
\hline SS & 15 & & & & & & & $\mathbf{Y}$ & $\mathbf{Y}$ & $Y$ \\
\hline SS & 20 & & & & & & & & $\mathbf{Y}$ & $Y$ \\
\hline SS & 25 & & & & & & & & & $Y$ \\
\hline $\mathrm{SS}_{\mathrm{b}}$ & 1 & $\underline{N}$ & $\underline{\mathrm{N}}$ & $\underline{N}$ & $\underline{N}$ & & & & & \\
\hline $\mathrm{SS}_{\mathrm{b}}$ & 2 & & $\underline{\mathrm{N}}$ & $\underline{\mathrm{N}}$ & $\underline{\mathrm{N}}$ & & & & & \\
\hline$S S_{b}$ & 3 & & & $\underline{N}$ & $\underline{N}$ & & & & & \\
\hline $\mathrm{SS}_{\mathrm{b}}$ & 4 & & & & $\underline{N}$ & & & & & \\
\hline $\mathrm{SS}_{\mathrm{b}}$ & 5 & & & & & $\mathbf{Y}$ & $\mathbf{Y}$ & $\mathbf{Y}$ & $\mathbf{Y}$ & $\mathbf{Y}$ \\
\hline $\mathrm{SS}_{\mathrm{b}}$ & 10 & & & & & & $Y$ & $\mathrm{Y}$ & $Y$ & $Y$ \\
\hline $\mathrm{SS}_{\mathrm{b}}$ & 15 & & & & & & & $\mathbf{Y}$ & $\mathbf{Y}$ & $Y$ \\
\hline $\mathrm{SS}_{\mathrm{b}}$ & 20 & & & & & & & & $Y$ & $Y$ \\
\hline $\mathrm{SS}_{\mathrm{b}}$ & 25 & & & & & & & & & $\underline{N}$ \\
\hline $\mathrm{SS}_{\mathrm{u}}$ & 1 & $\underline{\mathrm{N}}$ & $\underline{\mathrm{N}}$ & $\underline{N}$ & $\underline{\mathrm{N}}$ & & & & & \\
\hline $\mathrm{SS}_{\mathrm{u}}$ & 2 & . & $\underline{N}$ & $\underline{N}$ & $\underline{N}$ & & & & & \\
\hline $\mathrm{SS}_{\mathrm{u}}$ & 3 & & & $\underline{\mathrm{N}}$ & $\underline{N}$ & & & & & \\
\hline $\mathrm{SS}_{\mathrm{u}}$ & 4 & & & & $\underline{N}$ & & & & & \\
\hline $\mathrm{SS}_{\mathrm{u}}$ & 5 & & & & & $Y$ & $Y$ & $\mathrm{Y}$ & $Y$ & $Y$ \\
\hline $\mathrm{SS}_{\mathrm{u}}$ & 10 & & & & & & $\mathbf{Y}$ & $\mathbf{Y}$ & $\mathbf{Y}$ & $Y$ \\
\hline $\mathrm{SS}_{\mathrm{u}}$ & 15 & & & & & & & $Y$ & $\mathrm{Y}$ & $Y$ \\
\hline $\mathrm{SS}_{\mathrm{u}}$ & 20 & & & & & & & & $Y$ & $Y$ \\
\hline $\mathrm{SS}_{\mathrm{u}}$ & 25 & & & & & & & & & $\underline{N}$ \\
\hline
\end{tabular}


When considering barred spirals as primary galaxies in Table 5-27, statistical dissimilarity is found above $5 \mathrm{Mpc}$ for comparisons with no secondary morphology filter. Under $5 \mathrm{Mpc}$ dissimilarity is also found, however a difference of $1 \mathrm{Mpc}$ or more is required between the two fitting ranges compared. For comparisons having a secondary morphology filter dissimilarity is found above $5 \mathrm{Mpc}$. The environment of barred and unbarred spirals around a barred spiral is consistently similar below $5 \mathrm{Mpc}$.

Table 5-27 3D KS Test Results - comparing two runs having the same primary/secondary filters and varying fitting range filters - limited to barred spiral primaries.

$\mathrm{Y}=$ dissimilar to the $99 \%$ level. $\mathrm{N}=$ not dissimilar to the $99 \%$ level. FR1 = Fitting Range 1

\begin{tabular}{|c|c|c|c|c|c|c|c|c|c|c|c|c|c|c|c|c|c|c|c|c|c|c|c|}
\hline \multicolumn{12}{|c|}{ North } & \multicolumn{12}{|c|}{ South } \\
\hline \multirow[b]{2}{*}{$\begin{array}{c}\text { Run } \\
1 \\
\end{array}$} & \multirow[b]{2}{*}{$\begin{array}{c}\text { FR } \\
1 \\
\end{array}$} & \multirow[b]{2}{*}{1} & \multirow[b]{2}{*}{2} & \multicolumn{6}{|c|}{ Fitting Range 2 (Mpc) } & \multirow[b]{2}{*}{$\begin{array}{l}2 \\
5 \\
\end{array}$} & \multirow[b]{2}{*}{$\begin{array}{l}3 \\
0\end{array}$} & \multirow[b]{2}{*}{$\begin{array}{c}\text { Run } \\
1 \\
\end{array}$} & \multirow[b]{2}{*}{$\begin{array}{c}\text { FR } \\
1 \\
\end{array}$} & \multirow[b]{2}{*}{1} & \multicolumn{7}{|c|}{ Fitting Range 2 (Mpc) } & \multirow[b]{2}{*}{$\begin{array}{r}2 \\
5 \\
\end{array}$} & \multirow[b]{2}{*}{$\begin{array}{l}3 \\
0 \\
\end{array}$} \\
\hline & & & & 3 & 4 & 5 & $\begin{array}{l}1 \\
0 \\
\end{array}$ & $\begin{array}{l}1 \\
5 \\
\end{array}$ & $\begin{array}{l}2 \\
0 \\
\end{array}$ & & & & & & 2 & 3 & 4 & 5 & $\begin{array}{l}1 \\
0 \\
\end{array}$ & $\begin{array}{l}1 \\
5 \\
\end{array}$ & $\begin{array}{l}2 \\
0 \\
\end{array}$ & & \\
\hline $\mathrm{S}_{\mathrm{b}} \mathrm{A}$ & 1 & & $\underline{N}$ & $\mathrm{Y}$ & $\mathrm{Y}$ & $\mathrm{Y}$ & & & & & & $S_{b} A$ & 1 & & $\underline{N}$ & Y & $\mathrm{Y}$ & $Y$ & & & & & \\
\hline$S_{b} A$ & 2 & & & $\underline{\mathrm{N}}$ & $\mathrm{Y}$ & $\mathbf{Y}$ & & & & & & $S_{b} A$ & 2 & & & $\underline{\mathrm{N}}$ & $\mathrm{Y}$ & $\mathbf{Y}$ & & & & & \\
\hline $\mathrm{S}_{\mathrm{b}} \mathrm{A}$ & 3 & & & & $\underline{N}$ & $Y$ & & & & & & $S_{b} A$ & 3 & & & & $\underline{N}$ & $\underline{N}$ & & & & & \\
\hline $\mathrm{S}_{\mathrm{b}} \mathrm{A}$ & 4 & & & & & $\underline{N}$ & & & & & & $S_{b} A$ & 4 & & & & & $\underline{N}$ & & & & & \\
\hline$S_{b} A$ & 5 & & & & & & $\mathbf{Y}$ & $\mathbf{Y}$ & $\mathbf{Y}$ & $\mathrm{Y}$ & $\mathbf{Y}$ & $\mathrm{S}_{\mathrm{b}} \mathrm{A}$ & 5 & & & & & & $\mathrm{Y}$ & $Y$ & $\mathrm{Y}$ & $\mathrm{Y}$ & $\mathbf{Y}$ \\
\hline $\mathrm{S}_{\mathrm{b}} \mathrm{A}$ & 10 & & & & & & & $Y$ & $\mathrm{Y}$ & $Y$ & $Y$ & $S_{b} A$ & 10 & & & & & & & $Y$ & $Y$ & $\mathrm{Y}$ & $\mathrm{Y}$ \\
\hline $\mathrm{S}_{\mathrm{b}} \mathrm{A}$ & 15 & & & & & & & & $\underline{N}$ & $\mathbf{Y}$ & $Y$ & $S_{b} A$ & 15 & & & & & & & & $\underline{N}$ & $\mathrm{Y}$ & $Y$ \\
\hline $\mathrm{S}_{\mathrm{b}} \mathrm{A}$ & 20 & & & & & & & & & $\underline{N}$ & $\underline{N}$ & $S_{b} A$ & 20 & & & & & & & & & $\underline{N}$ & $\underline{N}$ \\
\hline $\mathrm{S}_{\mathrm{b}} \mathrm{A}$ & 25 & & & & & & & & & & $\underline{N}$ & $S_{b} A$ & 25 & & & & & & & & & & $\underline{N}$ \\
\hline $\mathrm{S}_{\mathrm{b}} \mathrm{E}$ & 1 & & $\underline{N}$ & $\underline{N}$ & $Y$ & $Y$ & & & & & & $S_{b} E$ & 1 & & $\underline{N}$ & $\underline{N}$ & $\underline{N}$ & $\underline{N}$ & & & & & \\
\hline $\mathrm{S}_{\mathrm{b}} \mathrm{E}$ & 2 & & & $\underline{N}$ & $\mathrm{Y}$ & $\mathrm{Y}$ & & & & & & $\mathrm{S}_{\mathrm{b}} \mathrm{E}$ & 2 & & & $\underline{N}$ & $\underline{N}$ & $\underline{N}$ & & & & & \\
\hline $\mathrm{S}_{\mathrm{b}} \mathrm{E}$ & 3 & & & & $\underline{N}$ & $\underline{N}$ & & & & & & $S_{b} E$ & 3 & & & & $\underline{N}$ & $\underline{N}$ & & & & & \\
\hline $\mathrm{S}_{\mathrm{b}} \mathrm{E}$ & 4 & & & & & $\underline{N}$ & & & & & & $S_{b} E$ & 4 & & & & & $\underline{N}$ & & & & & \\
\hline$S_{b} E$ & 5 & & & & & & $\mathbf{Y}$ & $\mathbf{Y}$ & $\mathbf{Y}$ & $\mathbf{Y}$ & $\mathbf{Y}$ & $S_{b} E$ & 5 & & & & & & $Y$ & $Y$ & $Y$ & $\mathbf{Y}$ & $\mathbf{Y}$ \\
\hline $\mathrm{S}_{\mathrm{b}} \mathrm{E}$ & 10 & & & & & & & $\mathrm{Y}$ & $\mathrm{Y}$ & $Y$ & $\mathrm{Y}$ & $S_{b} E$ & 10 & & & & & & & $\underline{N}$ & $Y$ & $\mathrm{Y}$ & Y \\
\hline $\mathrm{S}_{\mathrm{b}} \mathrm{E}$ & 15 & & & & & & & & $\underline{N}$ & $Y$ & $\mathrm{Y}$ & $\mathrm{S}_{\mathrm{b}} \mathrm{E}$ & 15 & & & & & & & & $\underline{N}$ & $\mathrm{Y}$ & $\bar{Y}$ \\
\hline $\mathrm{S}_{\mathrm{b}} \mathrm{E}$ & 20 & & & & & & & & & $\underline{N}$ & $\mathbf{Y}$ & $S_{b} E$ & 20 & & & & & & & & & $\underline{N}$ & $\underline{N}$ \\
\hline$S_{b} E$ & 25 & & & & & & & & & & $\underline{N}$ & $S_{b} E$ & 25 & & & & & & & & & & $\underline{N}$ \\
\hline$S_{b} L$ & 1 & & $\underline{N}$ & $\underline{N}$ & $\mathrm{Y}$ & $\underline{N}$ & & & & & & $S_{b} L$ & 1 & & $\underline{N}$ & $\underline{N}$ & $\underline{N}$ & $\underline{N}$ & & & & & \\
\hline $\mathrm{S}_{\mathrm{b}} \mathrm{L}$ & 2 & & & $\underline{N}$ & $\underline{N}$ & $\underline{N}$ & & & & & & $\mathrm{~S}_{\mathrm{b}} \mathrm{L}$ & 2 & & & $\underline{N}$ & $\mathrm{Y}$ & $Y$ & & & & & \\
\hline $\mathrm{S}_{\mathrm{b}} \mathrm{L}$ & 3 & & & & $\underline{N}$ & $\underline{N}$ & & & & & & $S_{b} L$ & 3 & & & & $\underline{N}$ & $\underline{N}$ & & & & & \\
\hline$S_{b} L$ & 4 & & & & & $\underline{N}$ & & & & & & $S_{b} L$ & 4 & & & & & $\underline{N}$ & & & & & \\
\hline$S_{b} L$ & 5 & & & & & & $\mathrm{Y}$ & Y & $\mathrm{Y}$ & $Y$ & $Y$ & $S_{b} L$ & 5 & & & & & & $Y$ & $Y$ & $Y$ & $\mathrm{Y}$ & $\mathrm{Y}$ \\
\hline $\mathrm{S}_{\mathrm{b}} \mathrm{L}$ & 10 & & & & & & & $\mathrm{Y}$ & $\mathrm{Y}$ & $Y$ & $Y$ & $S_{b} L$ & 10 & & & & & & & $\underline{N}$ & $\mathrm{Y}$ & $\mathrm{Y}$ & $\mathrm{Y}$ \\
\hline$S_{b} L$ & 15 & & & & & & & & $\underline{\mathrm{N}}$ & $\mathbf{Y}$ & $\mathbf{Y}$ & $S_{b} L$ & 15 & & & & & & & & $\underline{N}$ & $\mathbf{Y}$ & $\mathbf{Y}$ \\
\hline$S_{b} L$ & 20 & & & & & & & & & $\underline{N}$ & $\underline{N}$ & $S_{b} L$ & 20 & & & & & & & & & $\underline{N}$ & $\underline{N}$ \\
\hline
\end{tabular}




\begin{tabular}{|c|c|c|c|c|c|c|c|c|c|c|c|c|c|c|c|c|c|c|c|c|}
\hline$S_{b} L$ & 25 & & & & & & & & $\underline{N}$ & $S_{b} L$ & 25 & & & & & & & & & $\underline{N}$ \\
\hline$S_{b} S$ & 1 & $\underline{N}$ & $\underline{N}$ & $\mathbf{Y}$ & $\mathbf{Y}$ & & & & & $S_{b} S$ & 1 & $\underline{N}$ & $\underline{N}$ & $\underline{N}$ & $\underline{N}$ & & & & & \\
\hline$S_{b} S$ & 2 & & $\underline{N}$ & $\underline{\mathrm{N}}$ & $\mathbf{Y}$ & & & & & $S_{b} S$ & 2 & & $\underline{N}$ & $\underline{N}$ & $\mathbf{Y}$ & & & & & \\
\hline$S_{b} S$ & 3 & & & $\underline{N}$ & $\mathrm{Y}$ & & & & & $S_{b} S$ & 3 & & & $\underline{N}$ & $\underline{N}$ & & & & & \\
\hline$S_{b} S$ & 4 & & & & $\underline{N}$ & & & & & $\mathrm{~S}_{\mathrm{b}} \mathrm{S}$ & 4 & & & & $\underline{N}$ & & & & & \\
\hline$S_{b} S$ & 5 & & & & $\mathrm{Y}$ & $Y$ & $Y$ & $Y$ & $Y$ & $\mathrm{~S}_{\mathrm{b}} \mathrm{S}$ & 5 & & & & & $Y$ & $Y$ & $Y$ & $Y$ & $Y$ \\
\hline$S_{b} S$ & 10 & & & & & $\mathbf{Y}$ & $\mathbf{Y}$ & $\mathbf{Y}$ & $Y$ & $S_{b} S$ & 10 & & & & & & $\underline{N}$ & $\mathbf{Y}$ & $\mathbf{Y}$ & $Y$ \\
\hline$S_{b} S$ & 15 & & & & & & $\underline{N}$ & $\mathbf{Y}$ & $\mathbf{Y}$ & $S_{b} S$ & 15 & & & & & & & $\underline{N}$ & $\mathbf{Y}$ & $\mathbf{Y}$ \\
\hline$S_{b} S$ & 20 & & & & & & & $\underline{N}$ & $Y$ & $\mathrm{~S}_{\mathrm{b}} \mathrm{S}$ & 20 & & & & & & & & $\underline{N}$ & $Y$ \\
\hline$S_{b} S$ & 25 & & & & & & & & $\underline{N}$ & $S_{b} S$ & 25 & & & & & & & & & $\underline{N}$ \\
\hline$S_{b} S_{b}$ & 1 & $\underline{N}$ & $\underline{\mathrm{N}}$ & $\underline{N}$ & $\underline{\mathrm{N}}$ & & & & & $S_{b} S_{b}$ & 1 & $\underline{N}$ & $\underline{N}$ & $\underline{\mathrm{N}}$ & $\underline{\mathrm{N}}$ & & & & & \\
\hline $\mathrm{S}_{\mathrm{b}} \mathrm{S}_{\mathrm{b}}$ & 2 & & $\underline{N}$ & $\underline{N}$ & $\underline{\mathrm{N}}$ & & & & & $\mathrm{S}_{\mathrm{b}} \mathrm{S}_{\mathrm{b}}$ & 2 & & $\underline{N}$ & $\underline{N}$ & $\underline{N}$ & & & & & \\
\hline$S_{b} S_{b}$ & 3 & & & $\underline{N}$ & $\underline{N}$ & & & & & $\mathrm{~S}_{\mathrm{b}} \mathrm{S}_{\mathrm{b}}$ & 3 & & 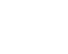 & $\underline{N}$ & $\underline{N}$ & & & & & \\
\hline$S_{b} S_{b}$ & 4 & & & & $\underline{\mathrm{N}}$ & & & & & $S_{b} S_{b}$ & 4 & & & & $\underline{\mathrm{N}}$ & & & & & \\
\hline $\mathrm{S}_{\mathrm{b}} \mathrm{S}_{\mathrm{b}}$ & 5 & & & & $Y$ & $Y$ & $Y$ & $Y$ & $Y$ & $\mathrm{~S}_{\mathrm{b}} \mathrm{S}_{\mathrm{b}}$ & 5 & & & & & $Y$ & $Y$ & $Y$ & $Y$ & $Y$ \\
\hline $\mathrm{S}_{\mathrm{b}} \mathrm{S}_{\mathrm{b}}$ & 10 & & & & & $Y$ & $Y$ & $Y$ & $Y$ & $\mathrm{~S}_{\mathrm{b}} \mathrm{S}_{\mathrm{b}}$ & 10 & & & & & & $\underline{N}$ & $\underline{N}$ & $\mathrm{Y}$ & $Y$ \\
\hline $\mathrm{S}_{\mathrm{b}} \mathrm{S}_{\mathrm{b}}$ & 15 & & & & & & $\underline{N}$ & $Y$ & $Y$ & $\mathrm{~S}_{\mathrm{b}} \mathrm{S}_{\mathrm{b}}$ & 15 & & & & & & & $\underline{N}$ & $\underline{\mathrm{N}}$ & $Y$ \\
\hline $\mathrm{S}_{\mathrm{b}} \mathrm{S}_{\mathrm{b}}$ & 20 & & & & & & & $\underline{N}$ & $Y$ & $\mathrm{~S}_{\mathrm{b}} \mathrm{S}_{\mathrm{b}}$ & 20 & & & & & & & & $\underline{N}$ & $\underline{N}$ \\
\hline$S_{b} S_{b}$ & 25 & & & & & & & & $\underline{N}$ & $S_{b} S_{b}$ & 25 & & & & & & & & & $\underline{N}$ \\
\hline $\mathrm{S}_{\mathrm{b}} \mathrm{S}_{\mathrm{u}}$ & 1 & $\underline{N}$ & $\underline{N}$ & $\underline{N}$ & $\underline{N}$ & & & & & $\mathrm{~S}_{\mathrm{b}} \mathrm{S}_{\mathrm{u}}$ & 1 & $\underline{N}$ & $\underline{N}$ & $\underline{N}$ & $\underline{N}$ & & & & & \\
\hline $\mathrm{S}_{\mathrm{b}} \mathrm{S}_{\mathrm{u}}$ & 2 & & $\underline{N}$ & $\underline{N}$ & $\underline{N}$ & & & & & $\mathrm{~S}_{\mathrm{b}} \mathrm{S}_{\mathrm{u}}$ & 2 & & $\underline{N}$ & $\underline{N}$ & $\underline{N}$ & & & & & \\
\hline $\mathrm{S}_{\mathrm{b}} \mathrm{S}_{\mathrm{u}}$ & 3 & & & $\underline{N}$ & $\underline{N}$ & & & & & $\mathrm{~S}_{\mathrm{b}} \mathrm{S}_{\mathrm{u}}$ & 3 & & 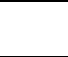 & $\underline{N}$ & $\underline{N}$ & & & & & \\
\hline $\mathrm{S}_{\mathrm{b}} \mathrm{S}_{\mathrm{u}}$ & 4 & & & & $\underline{N}$ & & & & & $\mathrm{~S}_{\mathrm{b}} \mathrm{S}_{\mathrm{u}}$ & 4 & & & & $\underline{N}$ & & & & & \\
\hline $\mathrm{S}_{\mathrm{b}} \mathrm{S}_{\mathrm{u}}$ & 5 & & & & $\underline{N}$ & $Y$ & $Y$ & $Y$ & $Y$ & $\mathrm{~S}_{\mathrm{b}} \mathrm{S}_{\mathrm{u}}$ & 5 & & & & & $\underline{N}$ & $Y$ & $Y$ & $\mathrm{Y}$ & $Y$ \\
\hline $\mathrm{S}_{\mathrm{b}} \mathrm{S}_{\mathrm{u}}$ & 10 & & & & & $\underline{N}$ & $Y$ & $Y$ & $Y$ & $\mathrm{~S}_{\mathrm{b}} \mathrm{S}_{\mathrm{u}}$ & 10 & & & & & & $\underline{N}$ & $Y$ & $Y$ & $Y$ \\
\hline $\mathrm{S}_{\mathrm{b}} \mathrm{S}_{\mathrm{u}}$ & 15 & & & & & & $\mathrm{Y}$ & $\mathrm{Y}$ & $Y$ & $\mathrm{~S}_{\mathrm{b}} \mathrm{S}_{\mathrm{u}}$ & 15 & & & & & & & $\underline{N}$ & $\underline{N}$ & $Y$ \\
\hline $\mathrm{S}_{\mathrm{b}} \mathrm{S}_{\mathrm{u}}$ & 20 & & & & & & & $\underline{N}$ & $Y$ & $\mathrm{~S}_{\mathrm{b}} \mathrm{S}_{\mathrm{u}}$ & 20 & & & & & & & & $\underline{N}$ & $\underline{N}$ \\
\hline $\mathrm{S}_{\mathrm{b}} \mathrm{S}_{\mathrm{u}}$ & 25 & & & & & & & & $\underline{N}$ & $\mathrm{~S}_{\mathrm{b}} \mathrm{S}_{\mathrm{u}}$ & 25 & & & & & & & & & $\underline{N}$ \\
\hline
\end{tabular}


When considering unbarred spirals as primary galaxies in Table 5-28, dissimilarity is found for most comparisons at all fitting ranges. Below $5 \mathrm{Mpc}$ a different of $1 \mathrm{Mpc}$ or more is needed between fitting ranges to find dissimilarity. For comparisons involving a specific morphology filter below $5 \mathrm{Mpc}$ similarity is reliably found.

Table 5-28 3D KS Test Results - comparing two runs having the same primary/secondary filters and varying fitting range filters - limited to unbarred spiral primaries.

$\mathrm{Y}=$ dissimilar to the $99 \%$ level. $\mathrm{N}=$ not dissimilar to the $99 \%$ level. FR1 = Fitting Range 1

\begin{tabular}{|c|c|c|c|c|c|c|c|c|c|c|c|c|c|c|c|c|c|c|c|c|c|c|c|}
\hline \multicolumn{12}{|c|}{ North } & \multicolumn{12}{|c|}{ South } \\
\hline \multirow[b]{2}{*}{$\begin{array}{c}\text { Run } \\
1 \\
\end{array}$} & \multirow[b]{2}{*}{$\begin{array}{c}\text { FR } \\
1 \\
\end{array}$} & \multicolumn{10}{|c|}{ Fitting Range 2 (Mpc) } & \multicolumn{12}{|c|}{ Fitting Range 2 (Mpc) } \\
\hline & & 1 & 2 & 3 & 4 & 5 & $\begin{array}{l}1 \\
0\end{array}$ & $\begin{array}{l}1 \\
5\end{array}$ & $\begin{array}{l}2 \\
0\end{array}$ & $\begin{array}{l}2 \\
5\end{array}$ & $\begin{array}{l}3 \\
0\end{array}$ & $\begin{array}{c}\text { Run } \\
1\end{array}$ & $\begin{array}{c}\mathrm{FR} \\
1\end{array}$ & 1 & 2 & 3 & 4 & 5 & $\begin{array}{l}1 \\
0\end{array}$ & $\begin{array}{l}1 \\
5\end{array}$ & $\begin{array}{l}2 \\
0\end{array}$ & $\begin{array}{l}2 \\
5\end{array}$ & $\begin{array}{l}3 \\
0\end{array}$ \\
\hline$S_{u} A$ & 1 & & $\underline{N}$ & $\underline{N}$ & $\mathbf{Y}$ & $\mathbf{Y}$ & & & & & & $\mathrm{S}_{\mathrm{u}} \mathrm{A}$ & 1 & & $\underline{N}$ & $\mathbf{Y}$ & $\mathbf{Y}$ & $\mathbf{Y}$ & & & & & \\
\hline$S_{u} A$ & 2 & & & $\underline{N}$ & $\mathrm{Y}$ & $\mathrm{Y}$ & & & & & & $\mathrm{S}_{\mathrm{u}} \mathrm{A}$ & 2 & & & $\underline{N}$ & $\mathrm{Y}$ & $\mathrm{Y}$ & & & & & \\
\hline$S_{u} A$ & 3 & & & & $\underline{N}$ & $\mathbf{Y}$ & & & & & & $\mathrm{S}_{\mathrm{u}} \mathrm{A}$ & 3 & & & & $\underline{N}$ & $\mathbf{Y}$ & & & & & \\
\hline$S_{u} A$ & 4 & & & & & $\underline{N}$ & & & & & & $\mathrm{~S}_{\mathrm{u}} \mathrm{A}$ & 4 & & & & & $\underline{N}$ & & & & & \\
\hline $\mathrm{S}_{\mathrm{u}} \mathrm{A}$ & 5 & & & & & & $Y$ & $Y$ & $Y$ & $Y$ & $Y$ & $\mathrm{~S}_{\mathrm{u}} \mathrm{A}$ & 5 & & & & & & $Y$ & $Y$ & $Y$ & $\mathrm{Y}$ & $Y$ \\
\hline $\mathrm{S}_{\mathrm{u}} \mathrm{A}$ & 10 & & & & & & & $\mathrm{Y}$ & $\mathrm{Y}$ & $\mathrm{Y}$ & $\mathrm{Y}$ & $\mathrm{S}_{\mathrm{u}} \mathrm{A}$ & 10 & & & & & & & $\mathrm{Y}$ & $\mathrm{Y}$ & $\mathrm{Y}$ & $\mathrm{Y}$ \\
\hline $\mathrm{S}_{\mathrm{u}} \mathrm{A}$ & 15 & & & & & & & & $\underline{N}$ & $\mathrm{Y}$ & $\mathrm{Y}$ & $\mathrm{S}_{\mathrm{u}} \mathrm{A}$ & 15 & & & & & & & & $\underline{N}$ & $\underline{N}$ & $\mathrm{Y}$ \\
\hline$S_{u} A$ & 20 & & & & & & & & & $\underline{N}$ & $\underline{N}$ & $\mathrm{~S}_{\mathrm{u}} \mathrm{A}$ & 20 & & & & & & & & & $\underline{N}$ & $\underline{N}$ \\
\hline $\mathrm{S}_{u} \mathrm{~A}$ & 25 & & & & & & & & & & $\underline{N}$ & $\mathrm{~S}_{\mathrm{u}} \mathrm{A}$ & 25 & & & & & & & & & & $\underline{N}$ \\
\hline $\mathrm{S}_{\mathrm{u}} \mathrm{E}$ & 1 & & $\underline{N}$ & $\underline{N}$ & $\underline{N}$ & $\underline{N}$ & & & & & & $\mathrm{~S}_{\mathrm{u}} \mathrm{E}$ & 1 & & $\underline{N}$ & $\underline{N}$ & $\underline{N}$ & $\underline{N}$ & & & & & \\
\hline$S_{u} E$ & 2 & & & $\underline{N}$ & $\underline{N}$ & $\underline{N}$ & & & & & & $S_{u} E$ & 2 & & & $\underline{N}$ & $\underline{N}$ & $\underline{N}$ & & & & & \\
\hline $\mathrm{S}_{\mathrm{u}} \mathrm{E}$ & 3 & & & & $\underline{N}$ & $\underline{N}$ & & & & & & $\mathrm{~S}_{\mathrm{u}} \mathrm{E}$ & 3 & & & & $\underline{N}$ & $\underline{N}$ & & & & & \\
\hline $\mathrm{S}_{\mathrm{u}} \mathrm{E}$ & 4 & & & & & $\underline{N}$ & & & & & & $\mathrm{~S}_{\mathrm{u}} \mathrm{E}$ & 4 & & & & & $\underline{N}$ & & & & & \\
\hline $\mathrm{S}_{\mathrm{u}} \mathrm{E}$ & 5 & & & & & & $Y$ & $Y$ & $Y$ & $Y$ & $Y$ & $S_{u} E$ & 5 & & & & & & $Y$ & $Y$ & $Y$ & $Y$ & $Y$ \\
\hline $\mathrm{S}_{\mathrm{u}} \mathrm{E}$ & 10 & & & & & & & $Y$ & $\mathrm{Y}$ & $Y$ & $Y$ & $\mathrm{~S}_{\mathrm{u}} \mathrm{E}$ & 10 & & & & & & & $\mathrm{Y}$ & $\mathrm{Y}$ & $\mathrm{Y}$ & $\mathrm{Y}$ \\
\hline$S_{u} E$ & 15 & & & & & & & & $\underline{N}$ & $Y$ & $Y$ & $S_{u} E$ & 15 & & & & & & & & $\underline{N}$ & $Y$ & $Y$ \\
\hline $\mathrm{S}_{\mathrm{u}} \mathrm{E}$ & 20 & & & & & & & & & $\underline{N}$ & $Y$ & $S_{u} E$ & 20 & & & & & & & & & $\underline{N}$ & $\underline{\mathrm{N}}$ \\
\hline $\mathrm{S}_{\mathrm{u}} \mathrm{E}$ & 25 & & & & & & & & & & $\underline{N}$ & $\mathrm{~S}_{\mathrm{u}} \mathrm{E}$ & 25 & & & & & & & & & & $\underline{N}$ \\
\hline$S_{u} L$ & 1 & & $\underline{N}$ & $\underline{N}$ & $\underline{N}$ & $\underline{N}$ & & & & & & $S_{u} L$ & 1 & & $\underline{\mathrm{N}}$ & $\underline{N}$ & $\underline{N}$ & $\underline{N}$ & & & & & \\
\hline $\mathrm{S}_{\mathrm{u}} \mathrm{L}$ & 2 & & & $\underline{N}$ & $\underline{N}$ & $\underline{N}$ & & & & & & $\mathrm{~S}_{\mathrm{u}} \mathrm{L}$ & 2 & & & $\underline{N}$ & $\underline{N}$ & $\underline{N}$ & & & & & \\
\hline $\mathrm{S}_{\mathrm{u}} \mathrm{L}$ & 3 & & & & $\underline{N}$ & $\underline{N}$ & & & & & & $\mathrm{~S}_{\mathrm{u}} \mathrm{L}$ & 3 & & & & $\underline{N}$ & $\underline{N}$ & & & & & \\
\hline $\mathrm{S}_{\mathrm{u}} \mathrm{L}$ & 4 & & & & & $\underline{N}$ & & & & & & $\mathrm{~S}_{\mathrm{u}} \mathrm{L}$ & 4 & & & & & $\underline{N}$ & & & & & \\
\hline $\mathrm{S}_{\mathrm{u}} \mathrm{L}$ & 5 & & & & & & $Y$ & $Y$ & $Y$ & $\mathrm{Y}$ & $Y$ & $\mathrm{~S}_{\mathrm{u}} \mathrm{L}$ & 5 & & & & & & $\mathrm{Y}$ & $\mathrm{Y}$ & $\mathrm{Y}$ & $\mathrm{Y}$ & $\mathrm{Y}$ \\
\hline$S_{u} L$ & 10 & & & & & & & $Y$ & $Y$ & $Y$ & $Y$ & $S_{u} L$ & 10 & & & & & & & $\underline{N}$ & $\mathrm{Y}$ & $Y$ & $\mathrm{Y}$ \\
\hline $\mathrm{S}_{\mathrm{u}} \mathrm{L}$ & 15 & & & & & & & & $\underline{N}$ & $Y$ & $Y$ & $\mathrm{~S}_{\mathrm{u}} \mathrm{L}$ & 15 & & & & & & & & $\underline{N}$ & $Y$ & $Y$ \\
\hline $\mathrm{S}_{\mathrm{u}} \mathrm{L}$ & 20 & & & & & & & & & $\underline{N}$ & $Y$ & $\mathrm{~S}_{\mathrm{u}} \mathrm{L}$ & 20 & & & & & & & & & $\underline{\mathrm{N}}$ & $\underline{\mathrm{N}}$ \\
\hline $\mathrm{S}_{\mathrm{u}} \mathrm{L}$ & 25 & & & & & & & & & & $\underline{N}$ & $\mathrm{~S}_{\mathrm{u}} \mathrm{L}$ & 25 & & & & & & & & & & $\underline{N}$ \\
\hline $\mathrm{S}_{\mathrm{u}} \mathrm{S}$ & 1 & & $\underline{\mathrm{N}}$ & $\underline{\mathrm{N}}$ & $\underline{N}$ & $\underline{N}$ & & & & & & $\mathrm{~S}_{\mathrm{u}} \mathrm{S}$ & 1 & & $\underline{N}$ & $\underline{N}$ & $\underline{\mathrm{N}}$ & $Y$ & & & & & \\
\hline
\end{tabular}




\begin{tabular}{|c|c|c|c|c|c|c|c|c|c|c|}
\hline $\mathrm{S}_{\mathrm{u}} \mathrm{S}$ & 2 & & $\underline{\mathrm{N}}$ & $\underline{\mathrm{N}}$ & $\mathbf{Y}$ & & & & & \\
\hline $\mathrm{S}_{\mathrm{u}} \mathrm{S}$ & 3 & & & $\underline{\mathrm{N}}$ & $\mathbf{Y}$ & & & & & \\
\hline $\mathrm{S}_{\mathrm{u}} \mathrm{S}$ & 4 & & & & $\underline{\mathrm{N}}$ & & & & & \\
\hline $\mathrm{S}_{\mathrm{u}} \mathrm{S}$ & 5 & & & & & $\mathbf{Y}$ & $\mathbf{Y}$ & $\mathbf{Y}$ & $\mathbf{Y}$ & $\mathbf{Y}$ \\
\hline $\mathrm{S}_{\mathrm{u}} \mathrm{S}$ & 10 & & & & & & $\mathbf{Y}$ & $\mathbf{Y}$ & $Y$ & $\mathbf{Y}$ \\
\hline $\mathrm{S}_{\mathrm{u}} \mathrm{S}$ & 15 & & & & & & & $\mathbf{Y}$ & $Y$ & $\mathbf{Y}$ \\
\hline $\mathrm{S}_{\mathrm{u}} \mathrm{S}$ & 20 & & & & & & & & $\underline{N}$ & $\mathbf{Y}$ \\
\hline $\mathrm{S}_{\mathrm{u}} \mathrm{S}$ & 25 & & & & & & & & & $\underline{N}$ \\
\hline $\mathrm{S}_{\mathrm{u}} \mathrm{S}_{\mathrm{b}}$ & 1 & $\underline{N}$ & $\underline{\mathrm{N}}$ & $\underline{\mathrm{N}}$ & $\underline{N}$ & & & & & \\
\hline$S_{u} S_{b}$ & 2 & & $\underline{N}$ & $\underline{N}$ & $\underline{N}$ & & & & & \\
\hline $\mathrm{S}_{\mathrm{u}} \mathrm{S}_{\mathrm{b}}$ & 3 & & & $\underline{\mathrm{N}}$ & $\underline{N}$ & & & & & \\
\hline $\mathrm{S}_{\mathrm{u}} \mathrm{S}_{\mathrm{b}}$ & 4 & & & & $\underline{N}$ & & & & & \\
\hline $\mathrm{S}_{\mathrm{u}} \mathrm{S}_{\mathrm{b}}$ & 5 & & & & & $\underline{N}$ & $\mathbf{Y}$ & $\mathbf{Y}$ & $\mathbf{Y}$ & $\mathbf{Y}$ \\
\hline $\mathrm{S}_{\mathrm{u}} \mathrm{S}_{\mathrm{b}}$ & 10 & & & & & & $\underline{\mathrm{N}}$ & $\mathbf{Y}$ & $\mathbf{Y}$ & $\mathbf{Y}$ \\
\hline $\mathrm{S}_{\mathrm{u}} \mathrm{S}_{\mathrm{b}}$ & 15 & & & & & & & $\underline{N}$ & $Y$ & $Y$ \\
\hline $\mathrm{S}_{\mathrm{u}} \mathrm{S}_{\mathrm{b}}$ & 20 & & & & & & & & $\underline{N}$ & $\mathbf{Y}$ \\
\hline $\mathrm{S}_{\mathrm{u}} \mathrm{S}_{\mathrm{b}}$ & 25 & & & & & & & & & $\underline{\mathrm{N}}$ \\
\hline $\mathrm{S}_{\mathrm{u}} \mathrm{S}_{\mathrm{u}}$ & 1 & $\underline{N}$ & $\underline{N}$ & $\underline{N}$ & $\underline{N}$ & & & & & \\
\hline $\mathrm{S}_{\mathrm{u}} \mathrm{S}_{\mathrm{u}}$ & 2 & & $\underline{\mathrm{N}}$ & $\underline{\mathrm{N}}$ & $\underline{N}$ & & & & & \\
\hline $\mathrm{S}_{\mathrm{u}} \mathrm{S}_{\mathrm{u}}$ & 3 & & & $\underline{\mathrm{N}}$ & $\underline{N}$ & & & & & \\
\hline $\mathrm{S}_{\mathrm{u}} \mathrm{S}_{\mathrm{u}}$ & 4 & & & & $\underline{N}$ & & & & & \\
\hline $\mathrm{S}_{\mathrm{u}} \mathrm{S}_{\mathrm{u}}$ & 5 & & & & & $\underline{N}$ & $Y$ & $Y$ & $Y$ & $Y$ \\
\hline $\mathrm{S}_{\mathrm{u}} \mathrm{S}_{\mathrm{u}}$ & 10 & & & & & & $\underline{\mathrm{N}}$ & $\mathbf{Y}$ & $\mathbf{Y}$ & $\mathbf{Y}$ \\
\hline $\mathrm{S}_{\mathrm{u}} \mathrm{S}_{\mathrm{u}}$ & 15 & & & & & & & $\underline{N}$ & $Y$ & $\mathbf{Y}$ \\
\hline $\mathrm{S}_{\mathrm{u}} \mathrm{S}_{\mathrm{u}}$ & 20 & & & & & & & & $\underline{N}$ & $Y$ \\
\hline $\mathrm{S}_{\mathrm{u}} \mathrm{S}_{\mathrm{u}}$ & 25 & & & & & & & & & $\underline{N}$ \\
\hline
\end{tabular}

\begin{tabular}{|c|c|c|c|c|c|c|c|c|c|c|}
\hline $\mathrm{S}_{\mathrm{u}} \mathrm{S}$ & 2 & & $\underline{N}$ & $\underline{N}$ & $\mathbf{Y}$ & & & & & \\
\hline $\mathrm{S}_{\mathrm{u}} \mathrm{S}$ & 3 & & & $\underline{N}$ & $\mathbf{Y}$ & & & & & \\
\hline$S_{u} S$ & 4 & & & & $\underline{\mathrm{N}}$ & & & & & \\
\hline$S_{u} S$ & 5 & & & & & $Y$ & $\mathbf{Y}$ & $\mathbf{Y}$ & $Y$ & $\mathbf{Y}$ \\
\hline $\mathrm{S}_{\mathrm{u}} \mathrm{S}$ & 10 & & & & & & $Y$ & $Y$ & $Y$ & $Y$ \\
\hline $\mathrm{S}_{\mathrm{u}} \mathrm{S}$ & 15 & & & & & & & $\underline{\mathrm{N}}$ & $Y$ & $Y$ \\
\hline $\mathrm{S}_{\mathrm{u}} \mathrm{S}$ & 20 & & & & & & & & $\underline{N}$ & $\underline{N}$ \\
\hline $\mathrm{S}_{\mathrm{u}} \mathrm{S}$ & 25 & & & & & & & & & $\underline{N}$ \\
\hline$S_{u} S_{b}$ & 1 & $\underline{N}$ & $\underline{N}$ & $\underline{N}$ & $\underline{N}$ & & & & & \\
\hline $\mathrm{S}_{\mathrm{u}} \mathrm{S}_{\mathrm{b}}$ & 2 & & $\underline{N}$ & $\underline{N}$ & $\underline{N}$ & & & & & \\
\hline $\mathrm{S}_{\mathrm{u}} \mathrm{S}_{\mathrm{b}}$ & 3 & & & $\underline{N}$ & $\underline{N}$ & & & & & \\
\hline$S_{u} S_{b}$ & 4 & & & & $\underline{N}$ & & & & & \\
\hline $\mathrm{S}_{\mathrm{u}} \mathrm{S}_{\mathrm{b}}$ & 5 & & & & & $\mathrm{Y}$ & $\mathrm{Y}$ & $Y$ & $Y$ & $Y$ \\
\hline $\mathrm{S}_{\mathrm{u}} \mathrm{S}_{\mathrm{b}}$ & 10 & & & & & & $\underline{\mathrm{N}}$ & $Y$ & $Y$ & $\mathrm{Y}$ \\
\hline $\mathrm{S}_{\mathrm{u}} \mathrm{S}_{\mathrm{b}}$ & 15 & & & & & & & $\underline{N}$ & $\underline{N}$ & $\mathrm{Y}$ \\
\hline$S_{u} S_{b}$ & 20 & & & & & & & & $\underline{N}$ & $\underline{N}$ \\
\hline $\mathrm{S}_{\mathrm{u}} \mathrm{S}_{\mathrm{b}}$ & 25 & & & & & & & & & $\underline{\mathrm{N}}$ \\
\hline $\mathrm{S}_{\mathrm{u}} \mathrm{S}_{\mathrm{u}}$ & 1 & $\underline{N}$ & $\underline{N}$ & $\underline{N}$ & $\underline{N}$ & & & & & \\
\hline $\mathrm{S}_{\mathrm{u}} \mathrm{S}_{\mathrm{u}}$ & 2 & & $\underline{N}$ & $\underline{N}$ & $\underline{N}$ & & & & & \\
\hline $\mathrm{S}_{\mathrm{u}} \mathrm{S}_{\mathrm{u}}$ & 3 & & & $\underline{N}$ & $\underline{N}$ & & & & & \\
\hline $\mathrm{S}_{\mathrm{u}} \mathrm{S}_{\mathrm{u}}$ & 4 & & & & $\underline{N}$ & & & & & \\
\hline $\mathrm{S}_{\mathrm{u}} \mathrm{S}_{\mathrm{u}}$ & 5 & & & & & $Y$ & $\mathrm{Y}$ & $Y$ & $Y$ & $\mathrm{Y}$ \\
\hline $\mathrm{S}_{\mathrm{u}} \mathrm{S}_{\mathrm{u}}$ & 10 & & & & & & $\underline{N}$ & $\mathrm{Y}$ & $Y$ & $\mathrm{Y}$ \\
\hline$S_{u} S_{u}$ & 15 & & & & & & & $\underline{N}$ & $\underline{N}$ & $Y$ \\
\hline $\mathrm{S}_{\mathrm{u}} \mathrm{S}_{\mathrm{u}}$ & 20 & & & & & & & & $\underline{N}$ & $\underline{N}$ \\
\hline $\mathrm{S}_{u} \mathrm{~S}_{\mathrm{u}}$ & 25 & & & & & & & & & $\underline{N}$ \\
\hline
\end{tabular}

\subsubsection{Conclusions}

As in 2D, the general trend observed is that as the fitting range increases dissimilarity is found. I conclude that there is a relationship between the similarity of environments and the fitting range measured from the primary galaxy such that larger fitting ranges yield dissimilar environments. 


\subsubsection{Impact of Secondary Morphology on the Pointwise Dimension}

Data runs where the primary morphology and fitting range are equal with differing secondary morphologies are compared in KS tests.

In all of the following tables $\mathrm{Y}$ indicates that the $p$ value from the $\mathrm{KS}$ test is < 0.01 . This means that there is less than a $1 \%$ chance that the two compared data sets are drawn from the same distribution. That is, the compared data sets are statistically dissimilar to the $99 \%$ level. A value of $\mathrm{N}$ indicates the inverse. That is, the datasets are not statistically dissimilar to the $99 \%$ level. 
When considering primaries of $E$ in Table 5-29, dissimilarity is consistently found for the higher fitting ranges. The point at which dissimilarity is found varies by the combination of secondary morphology. Comparisons of EE vs. EL consistently require larger fitting ranges for dissimilarity to occur. Also, comparisons involving barred vs. unbarred spirals consistently require larger fitting ranges for dissimilarity to occur. Finally $S_{b}$ and $S_{u}$ as secondaries compared to early-type galaxies as secondaries generally show similarity to larger fitting ranges than $S$ compared to early type.

Table 5-29 3D KS Test Results - comparing two runs having the same primary filter and same fitting range filter with different secondary filters - limited to elliptical primaries.

$\mathrm{Y}=$ dissimilar to the $99 \%$ level. $\mathrm{N}=$ not dissimilar to the $99 \%$ level

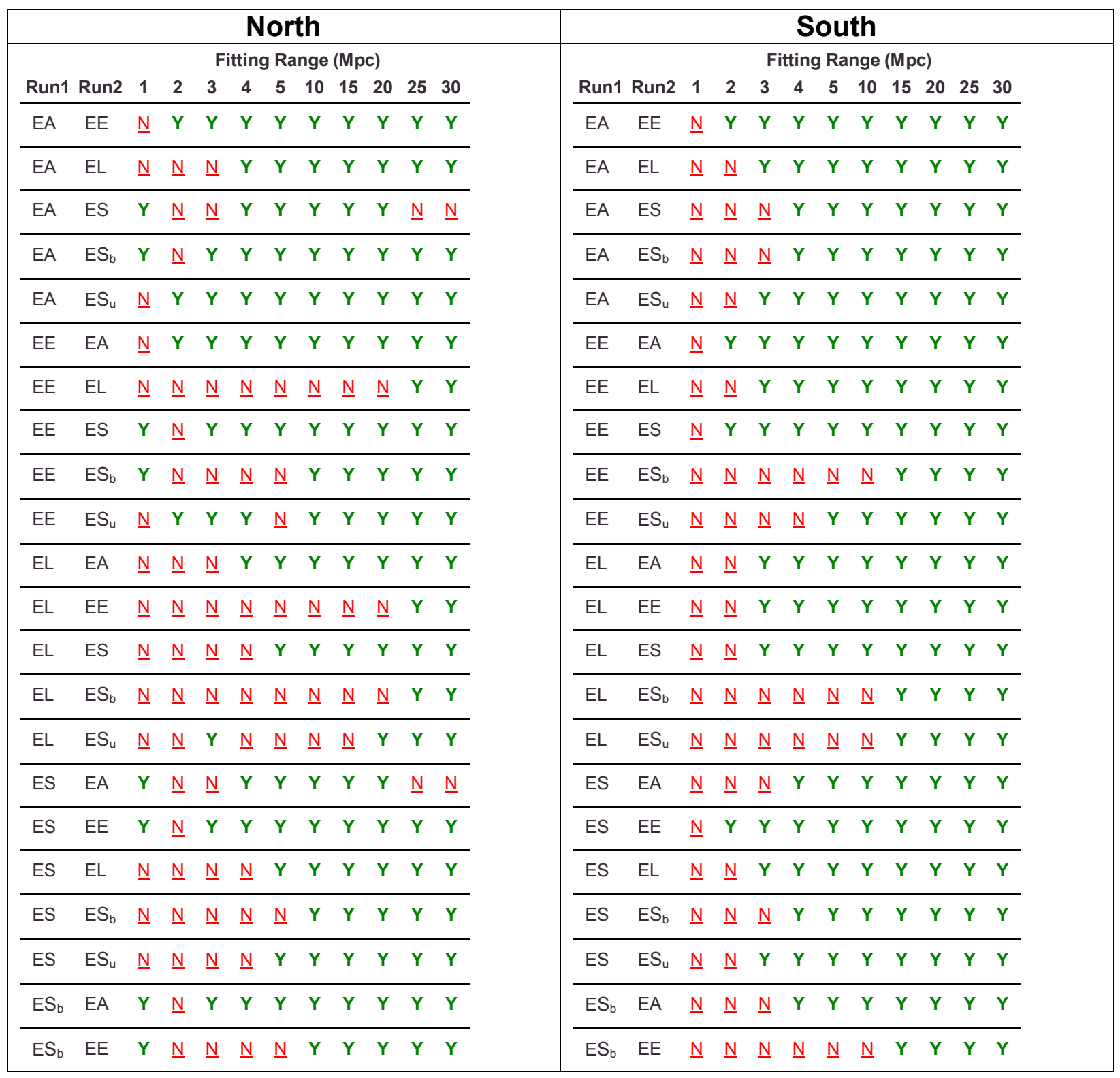




\begin{tabular}{|c|c|c|c|c|c|c|c|c|c|c|c|c|c|c|c|c|c|c|c|c|c|c|c|c|}
\hline $\mathrm{ES}_{\mathrm{b}}$ & EL & $\underline{\mathrm{N}}$ & $\underline{N}$ & $\underline{N}$ & $\underline{N}$ & $\underline{N}$ & $\underline{\mathrm{N}}$ & $\underline{\mathrm{N}}$ & $\underline{N}$ & $\mathbf{Y}$ & $\mathbf{Y}$ & $\mathrm{ES}_{\mathrm{b}}$ & $\mathrm{EL}$ & $\underline{\mathrm{N}}$ & $\underline{N}$ & $\underline{N}$ & $\underline{N}$ & $\underline{N}$ & $\underline{\Lambda}$ & 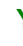 & 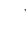 & & Y & \\
\hline $\mathrm{ES}_{\mathrm{b}}$ & ES & $\underline{N}$ & $\underline{\mathrm{N}}$ & $\underline{N}$ & $\underline{\mathrm{N}}$ & $\underline{N}$ & $\mathbf{Y}$ & $\mathbf{Y}$ & $\mathbf{Y}$ & $Y$ & $\mathbf{Y}$ & $\mathrm{ES}_{\mathrm{b}}$ & ES & $\underline{\mathrm{N}}$ & $\underline{N}$ & $\underline{N}$ & $\mathbf{Y}$ & $Y$ & $Y$ & 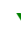 & & & $Y$ & \\
\hline $\mathrm{ES}_{\mathrm{b}}$ & $\mathrm{ES}_{\mathrm{u}}$ & $\underline{\mathrm{N}}$ & $\underline{N}$ & $\underline{N}$ & $\underline{N}$ & $\underline{N}$ & $\mathbf{Y}$ & $\mathbf{Y}$ & $\mathbf{Y}$ & $Y$ & $\mathbf{Y}$ & $\mathrm{ES}_{\mathrm{b}}$ & $\mathrm{ES}_{\mathrm{u}}$ & $\underline{N}$ & $\underline{N}$ & $\underline{N}$ & $\underline{N}$ & $\underline{N}$ & $\Lambda$ & 1 & & & $Y$ & $Y$ \\
\hline $\mathrm{ES}_{\mathrm{u}}$ & EA & $\underline{\mathrm{N}}$ & $\mathbf{Y}$ & $\mathbf{Y}$ & $\mathbf{Y}$ & $\mathbf{Y}$ & $\mathbf{Y}$ & $\mathbf{Y}$ & $\mathbf{Y}$ & $Y$ & $\mathbf{Y}$ & $\mathrm{ES}_{\mathrm{u}}$ & EA & $\underline{\mathrm{N}}$ & $\underline{N}$ & $\mathbf{Y}$ & $\mathbf{Y}$ & $Y$ & $Y$ & 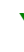 & & & $Y$ & $Y$ \\
\hline $\mathrm{ES}_{\mathrm{u}}$ & $\mathrm{EE}$ & $\underline{\mathrm{N}}$ & $\mathbf{Y}$ & $Y$ & $Y$ & $\underline{N}$ & $\mathbf{Y}$ & $\mathbf{Y}$ & $\mathbf{Y}$ & $Y$ & $\mathbf{Y}$ & $\mathrm{ES}_{\mathrm{u}}$ & $\mathrm{EE}$ & $\underline{N}$ & $\underline{N}$ & $\underline{N}$ & $\underline{N}$ & $Y$ & $Y$ & 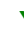 & & & $\mathbf{Y}$ & $Y$ \\
\hline $\mathrm{ES}_{\mathrm{u}}$ & EL & $\underline{\mathrm{N}}$ & $\underline{\mathrm{N}}$ & $\mathrm{Y}$ & $\underline{N}$ & $\underline{N}$ & $\underline{N}$ & $\underline{N}$ & $\mathrm{Y}$ & $\mathbf{Y}$ & $\mathbf{Y}$ & $\mathrm{ES}_{\mathrm{u}}$ & $\mathrm{EL}$ & $\underline{\mathrm{N}}$ & $\underline{N}$ & $\underline{N}$ & $\underline{N}$ & $\underline{N}$ & $\Lambda$ & 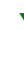 & & & $\mathbf{Y}$ & $Y$ \\
\hline $\mathrm{ES}_{\mathrm{u}}$ & ES & $\underline{\mathrm{N}}$ & $\underline{\mathrm{N}}$ & $\underline{N}$ & $\underline{N}$ & $\mathbf{Y}$ & $\mathbf{Y}$ & $\mathbf{Y}$ & $Y$ & $\mathbf{Y}$ & $\mathbf{Y}$ & $\mathrm{ES}_{\mathrm{u}}$ & ES & $\underline{\mathrm{N}}$ & $\underline{N}$ & $\mathbf{Y}$ & $Y$ & $Y$ & $Y$ & 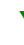 & & & $\mathbf{Y}$ & \\
\hline $\mathrm{ES}_{\mathrm{u}}$ & $\mathrm{ES}_{\mathrm{b}}$ & $\underline{\mathrm{N}}$ & $\underline{\mathrm{N}}$ & $\underline{N}$ & $\underline{\mathrm{N}}$ & $\underline{N}$ & $\mathbf{Y}$ & $\mathbf{Y}$ & $\mathbf{Y}$ & $\mathbf{Y}$ & $\mathbf{Y}$ & $\mathrm{ES}_{\mathrm{u}}$ & $\mathrm{ES}_{\mathrm{b}}$ & $\underline{\mathrm{N}}$ & $\underline{N}$ & $\underline{N}$ & $\underline{N}$ & $\underline{N}$ & & & & & $\mathbf{Y}$ & $Y$ \\
\hline
\end{tabular}


When considering lenticulars as primary galaxies in Table 5-30, dissimilarity is found at the larger fitting ranges. For comparisons involving LE vs. LL similarity is noted for the majority of fitting ranges. $L S_{u}$ vs. LS show similarity under $5 \mathrm{Mpc}$ as does $L S_{u}$ vs. $L E$ and $L S_{u}$ vs. $L L$ (more in North). This suggests $S_{b}$ and $S_{u}$ cluster similarly to $L$ and $E$ secondaries around $L$ primaries. $L S$ vs. $L S_{b}$ and $L S$ vs. $L S_{u}$ show dissimilarity much more frequently which suggests $S_{b}$ and $S_{u}$ do not cluster like $S$ around $L$ primaries.

Table 5-30 3D KS Test Results - comparing two runs having the same primary filter and same fitting range filter with different secondary filters - limited to lenticular primaries.

$Y=$ dissimilar to the $99 \%$ level. $N=$ not dissimilar to the $99 \%$ level

\begin{tabular}{|c|c|c|c|c|c|c|c|c|c|c|c|c|c|c|c|c|c|c|c|c|c|c|c|}
\hline \multicolumn{12}{|c|}{ North } & \multicolumn{12}{|c|}{ South } \\
\hline \multirow[b]{2}{*}{ Run1 } & \multicolumn{11}{|c|}{ Fitting Range (Mpc) } & \multicolumn{12}{|c|}{ Fitting Range (Mpc) } \\
\hline & Run2 & 1 & 2 & 3 & 4 & 5 & 10 & 15 & 20 & 25 & 30 & Run1 & Run2 & 1 & 2 & 3 & 4 & 5 & 10 & 15 & 20 & 25 & 30 \\
\hline LA & LE & $\underline{N}$ & $Y$ & $\mathrm{Y}$ & $\mathrm{Y}$ & $\mathbf{Y}$ & $\mathbf{Y}$ & $Y$ & $\mathbf{Y}$ & $\mathbf{Y}$ & $\mathbf{Y}$ & LA & LE & $\underline{N}$ & $\mathbf{Y}$ & $\mathbf{Y}$ & $\mathbf{Y}$ & $\mathbf{Y}$ & $Y$ & $\mathbf{Y}$ & $\mathbf{Y}$ & $\mathbf{Y}$ & $\mathbf{Y}$ \\
\hline LA & LL & $\underline{N}$ & $\underline{N}$ & $\mathbf{Y}$ & $\mathbf{Y}$ & $\mathbf{Y}$ & $\mathbf{Y}$ & $\mathbf{Y}$ & $\mathbf{Y}$ & $\mathbf{Y}$ & $\mathbf{Y}$ & LA & LL & $\underline{N}$ & $\mathbf{Y}$ & $\mathbf{Y}$ & $\mathbf{Y}$ & $\mathbf{Y}$ & $Y$ & $\mathbf{Y}$ & $\mathbf{Y}$ & $\mathbf{Y}$ & $Y$ \\
\hline LA & LS & $\underline{N}$ & $\underline{N}$ & $\underline{N}$ & $\mathbf{Y}$ & $\mathbf{Y}$ & $\mathbf{Y}$ & $\mathbf{Y}$ & $\mathrm{Y}$ & $\underline{N}$ & $\underline{N}$ & LA & LS & $\underline{N}$ & $\underline{N}$ & $\mathbf{Y}$ & $\mathbf{Y}$ & $\mathbf{Y}$ & $\mathbf{Y}$ & $\mathbf{Y}$ & $\mathbf{Y}$ & $\mathbf{Y}$ & $Y$ \\
\hline LA & $\mathrm{LS}_{\mathrm{b}}$ & $\underline{\mathrm{N}}$ & $\underline{N}$ & $\mathbf{Y}$ & $\mathbf{Y}$ & $Y$ & $\mathbf{Y}$ & $\mathbf{Y}$ & $\mathbf{Y}$ & $\mathbf{Y}$ & $\mathbf{Y}$ & LA & $\mathrm{LS}_{\mathrm{b}}$ & $\underline{N}$ & $\underline{N}$ & $\mathbf{Y}$ & $\mathbf{Y}$ & $Y$ & $Y$ & $\mathbf{Y}$ & $\mathbf{Y}$ & $\mathbf{Y}$ & $\mathbf{Y}$ \\
\hline LA & $\mathrm{LS}_{\mathrm{u}}$ & $\underline{N}$ & $\underline{N}$ & $\underline{N}$ & $Y$ & $Y$ & $\mathbf{Y}$ & $Y$ & $\mathbf{Y}$ & $\mathbf{Y}$ & $\mathbf{Y}$ & LA & $\mathrm{LS}_{\mathrm{u}}$ & $Y$ & $Y$ & $\mathbf{Y}$ & $\mathbf{Y}$ & $Y$ & $Y$ & $Y$ & $\mathbf{Y}$ & $Y$ & $Y$ \\
\hline LE & LA & $\underline{N}$ & $Y$ & $Y$ & $Y$ & $Y$ & $\mathbf{Y}$ & $Y$ & $\mathbf{Y}$ & $\mathbf{Y}$ & $Y$ & LE & LA & $\underline{N}$ & $Y$ & $\mathbf{Y}$ & $Y$ & $\mathbf{Y}$ & $Y$ & $\mathbf{Y}$ & $\mathbf{Y}$ & $Y$ & $Y$ \\
\hline LE & LL & $\underline{\mathrm{N}}$ & $\underline{\mathrm{N}}$ & $\mathbf{Y}$ & $\mathbf{Y}$ & $\underline{\mathrm{N}}$ & $\underline{\mathrm{N}}$ & $\underline{N}$ & $\underline{\mathrm{N}}$ & $\underline{N}$ & $\underline{N}$ & LE & LL & $\underline{\mathrm{N}}$ & $\underline{N}$ & $\underline{\mathrm{N}}$ & $\underline{\mathrm{N}}$ & $\underline{\mathrm{N}}$ & $\underline{N}$ & $\underline{\mathrm{N}}$ & $\mathrm{Y}$ & $\underline{N}$ & $Y$ \\
\hline LE & LS & $\underline{\mathrm{N}}$ & $\underline{N}$ & $\mathbf{Y}$ & $\mathbf{Y}$ & $\mathbf{Y}$ & $\mathbf{Y}$ & $\mathbf{Y}$ & $\mathbf{Y}$ & $\mathbf{Y}$ & $\mathbf{Y}$ & LE & LS & $\underline{N}$ & $Y$ & $\mathbf{Y}$ & $\mathbf{Y}$ & $\mathbf{Y}$ & $Y$ & $\mathbf{Y}$ & $\mathbf{Y}$ & $\mathbf{Y}$ & $\mathbf{Y}$ \\
\hline LE & $\mathrm{LS}_{\mathrm{b}}$ & $\underline{N}$ & $\underline{N}$ & $\underline{N}$ & $\underline{N}$ & $\underline{N}$ & $\underline{\mathrm{N}}$ & $\underline{N}$ & $\underline{\mathrm{N}}$ & $\mathbf{Y}$ & $\mathbf{Y}$ & LE & $\mathrm{LS}_{\mathrm{b}}$ & $\underline{N}$ & $\underline{N}$ & $\underline{\mathrm{N}}$ & $\underline{\mathrm{N}}$ & $\underline{N}$ & $\underline{N}$ & $Y$ & $Y$ & $Y$ & $Y$ \\
\hline LE & $\mathrm{LS}_{\mathrm{u}}$ & $\underline{N}$ & $\underline{N}$ & $\underline{N}$ & $\underline{N}$ & $\underline{N}$ & $\mathbf{Y}$ & $\mathbf{Y}$ & $\mathbf{Y}$ & $\mathbf{Y}$ & $\mathbf{Y}$ & LE & $\mathrm{LS}_{\mathrm{u}}$ & $\underline{\mathrm{N}}$ & $\underline{N}$ & $\underline{N}$ & $\underline{\mathrm{N}}$ & $\underline{\mathrm{N}}$ & $\underline{N}$ & $\mathbf{Y}$ & $\mathbf{Y}$ & $Y$ & $Y$ \\
\hline LL & LA & $\underline{N}$ & $\underline{N}$ & $\mathbf{Y}$ & $Y$ & $\mathbf{Y}$ & $\mathbf{Y}$ & $\mathbf{Y}$ & $\mathbf{Y}$ & $\mathbf{Y}$ & $\mathbf{Y}$ & LL & LA & $\underline{N}$ & $\mathbf{Y}$ & $\mathbf{Y}$ & $\mathbf{Y}$ & $\mathbf{Y}$ & $Y$ & $\mathbf{Y}$ & $\mathbf{Y}$ & $\mathbf{Y}$ & $\mathbf{Y}$ \\
\hline LL & LE & $\underline{\mathrm{N}}$ & $\underline{N}$ & $\mathbf{Y}$ & $\mathbf{Y}$ & $\underline{\mathrm{N}}$ & $\underline{N}$ & $\underline{\mathrm{N}}$ & $\underline{\mathrm{N}}$ & $\underline{N}$ & $\underline{N}$ & LL & LE & $\underline{N}$ & $\underline{N}$ & $\underline{\mathrm{N}}$ & $\underline{\mathrm{N}}$ & $\underline{N}$ & $\underline{N}$ & $\underline{\mathrm{N}}$ & $\mathbf{Y}$ & $\underline{\mathrm{N}}$ & $Y$ \\
\hline LL & LS & $\underline{N}$ & $\underline{N}$ & $\underline{N}$ & $Y$ & $\mathbf{Y}$ & $\mathbf{Y}$ & $Y$ & $\mathbf{Y}$ & $\mathbf{Y}$ & $\mathbf{Y}$ & LL & LS & $\underline{\mathrm{N}}$ & $\underline{N}$ & $\mathbf{Y}$ & $Y$ & $Y$ & $Y$ & $Y$ & $\mathbf{Y}$ & $Y$ & $Y$ \\
\hline LL & $\mathrm{LS}_{\mathrm{b}}$ & $\underline{\mathrm{N}}$ & $\underline{\mathrm{N}}$ & $\underline{N}$ & $\underline{\mathrm{N}}$ & $\underline{N}$ & $\underline{\mathrm{N}}$ & $\mathbf{Y}$ & $\mathbf{Y}$ & $\mathbf{Y}$ & $\underline{\mathrm{N}}$ & LL & $\mathrm{LS}_{\mathrm{b}}$ & $\underline{\mathrm{N}}$ & $\underline{N}$ & $\underline{\mathrm{N}}$ & $\underline{\mathrm{N}}$ & $\underline{\mathrm{N}}$ & $Y$ & $\mathbf{Y}$ & $\mathbf{Y}$ & $Y$ & $Y$ \\
\hline LL & $\mathrm{LS}_{\mathrm{u}}$ & $\underline{N}$ & $\underline{N}$ & $\underline{N}$ & $\underline{N}$ & $\underline{N}$ & $\mathbf{Y}$ & $Y$ & $\mathbf{Y}$ & $Y$ & $\mathbf{Y}$ & LL & $\mathrm{LS}_{\mathrm{u}}$ & $\mathbf{Y}$ & $\underline{N}$ & $\underline{N}$ & $\mathbf{Y}$ & $\mathbf{Y}$ & $\mathbf{Y}$ & $Y$ & $\mathbf{Y}$ & $Y$ & $Y$ \\
\hline LS & LA & $\underline{\mathrm{N}}$ & $\underline{N}$ & $\underline{N}$ & $\mathbf{Y}$ & $Y$ & $\mathbf{Y}$ & $\mathbf{Y}$ & $\mathrm{Y}$ & $\underline{\mathrm{N}}$ & $\underline{N}$ & LS & LA & $\underline{N}$ & $\underline{N}$ & $\mathbf{Y}$ & $\mathbf{Y}$ & $\mathbf{Y}$ & $Y$ & $\mathbf{Y}$ & $\mathbf{Y}$ & $\mathbf{Y}$ & $Y$ \\
\hline LS & LE & $\underline{N}$ & $\underline{N}$ & $\mathbf{Y}$ & $\mathbf{Y}$ & $\mathbf{Y}$ & $\mathbf{Y}$ & $\mathbf{Y}$ & $\mathbf{Y}$ & $\mathbf{Y}$ & $\mathbf{Y}$ & LS & LE & $\underline{N}$ & $Y$ & $\mathbf{Y}$ & $\mathrm{Y}$ & $\mathbf{Y}$ & $\mathbf{Y}$ & $\mathbf{Y}$ & $\mathbf{Y}$ & $\mathbf{Y}$ & $Y$ \\
\hline LS & LL & $\underline{\mathrm{N}}$ & $\underline{N}$ & $\underline{N}$ & $\mathbf{Y}$ & $\mathbf{Y}$ & $\mathbf{Y}$ & $\mathbf{Y}$ & $Y$ & $\mathbf{Y}$ & $\mathbf{Y}$ & LS & LL & $\underline{N}$ & $\underline{N}$ & $\mathbf{Y}$ & $\mathbf{Y}$ & $\mathbf{Y}$ & $Y$ & $\mathbf{Y}$ & $\mathbf{Y}$ & $Y$ & $Y$ \\
\hline LS & $\mathrm{LS}_{\mathrm{b}}$ & $\underline{N}$ & $\underline{N}$ & $\mathbf{Y}$ & $\mathbf{Y}$ & $\mathbf{Y}$ & $\mathbf{Y}$ & $\mathbf{Y}$ & $\mathbf{Y}$ & $\mathbf{Y}$ & $\mathbf{Y}$ & LS & $\mathrm{LS}_{\mathrm{b}}$ & $\underline{\mathrm{N}}$ & $\underline{N}$ & $\underline{N}$ & $\mathbf{Y}$ & $\mathbf{Y}$ & $\mathbf{Y}$ & $Y$ & $\mathbf{Y}$ & $Y$ & $Y$ \\
\hline LS & $\mathrm{LS}_{\mathrm{u}}$ & $\underline{N}$ & $\underline{N}$ & $\underline{N}$ & $\mathbf{Y}$ & $Y$ & $Y$ & $Y$ & $Y$ & $Y$ & $\mathbf{Y}$ & LS & $\mathrm{LS}_{\mathrm{u}}$ & $\mathrm{Y}$ & $\underline{N}$ & $\mathbf{Y}$ & $Y$ & $\mathbf{Y}$ & $\mathbf{Y}$ & $Y$ & $\mathbf{Y}$ & $\mathbf{Y}$ & $\mathbf{Y}$ \\
\hline $\mathrm{LS}_{\mathrm{b}}$ & LA & $\underline{\mathrm{N}}$ & $\underline{N}$ & $\mathbf{Y}$ & $\mathbf{Y}$ & $\mathbf{Y}$ & $\mathbf{Y}$ & $\mathbf{Y}$ & $Y$ & $\mathbf{Y}$ & $\mathbf{Y}$ & $\mathrm{LS}_{\mathrm{b}}$ & LA & $\underline{N}$ & $\underline{N}$ & $\mathbf{Y}$ & $Y$ & $Y$ & $\mathbf{Y}$ & $\mathbf{Y}$ & $\mathbf{Y}$ & $\mathbf{Y}$ & $Y$ \\
\hline $\mathrm{LS}_{\mathrm{b}}$ & LE & $\underline{\mathrm{N}}$ & $\underline{N}$ & $\underline{N}$ & $\underline{N}$ & $\underline{N}$ & $\underline{N}$ & $\underline{\mathrm{N}}$ & $\underline{N}$ & $\mathbf{Y}$ & $\mathbf{Y}$ & $\mathrm{LS}_{\mathrm{b}}$ & LE & $\underline{\mathrm{N}}$ & $\underline{N}$ & $\underline{\mathrm{N}}$ & $\underline{N}$ & $\underline{N}$ & $\underline{\mathrm{N}}$ & $\mathbf{Y}$ & $\mathbf{Y}$ & $\mathbf{Y}$ & $\mathbf{Y}$ \\
\hline $\mathrm{LS}_{\mathrm{b}}$ & LL & $\underline{N}$ & $\underline{N}$ & $\underline{N}$ & $\underline{N}$ & $\underline{N}$ & $\underline{N}$ & $\mathbf{Y}$ & $Y$ & $\mathbf{Y}$ & $\underline{N}$ & $\mathrm{LS}_{\mathrm{b}}$ & LL & $\underline{N}$ & $\underline{N}$ & $\underline{\mathrm{N}}$ & $\underline{N}$ & $\underline{N}$ & $\mathbf{Y}$ & $\mathbf{Y}$ & $\mathbf{Y}$ & $\mathbf{Y}$ & $Y$ \\
\hline
\end{tabular}




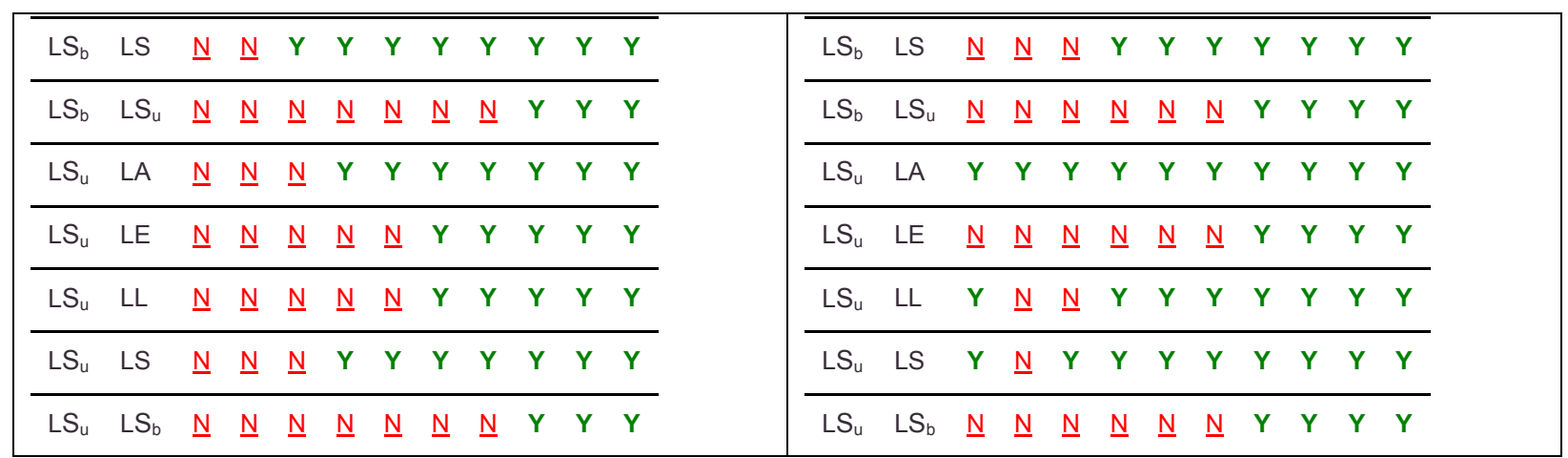


When considering S primaries in Table 5-31, dissimilarity is consistently found for the majority of comparisons, especially at fitting ranges above $5 \mathrm{Mpc}$. For SL vs. SE almost no dissimilarity is found in the Northern hemisphere. Also $S_{b}$ vs. SS require larger fitting ranges to exhibit dissimilarity. This suggests that there is a relationship between lenticular and elliptical galaxies, as well as between barred and unbarred spirals. Finally similarity is found between $S_{b}$ and $S_{u}$ secondaries compared to earlytype secondaries while there is dissimilarity found for $S_{b}$ and $S_{u}$ secondaries compared to $\mathrm{S}$ secondaries.

Table 5-31 3D KS Test Results - comparing two runs having the same primary filter and same fitting range filter with different secondary filters - limited to spiral primaries.

$\mathrm{Y}=$ dissimilar to the $99 \%$ level. $\mathrm{N}=$ not dissimilar to the $99 \%$ level

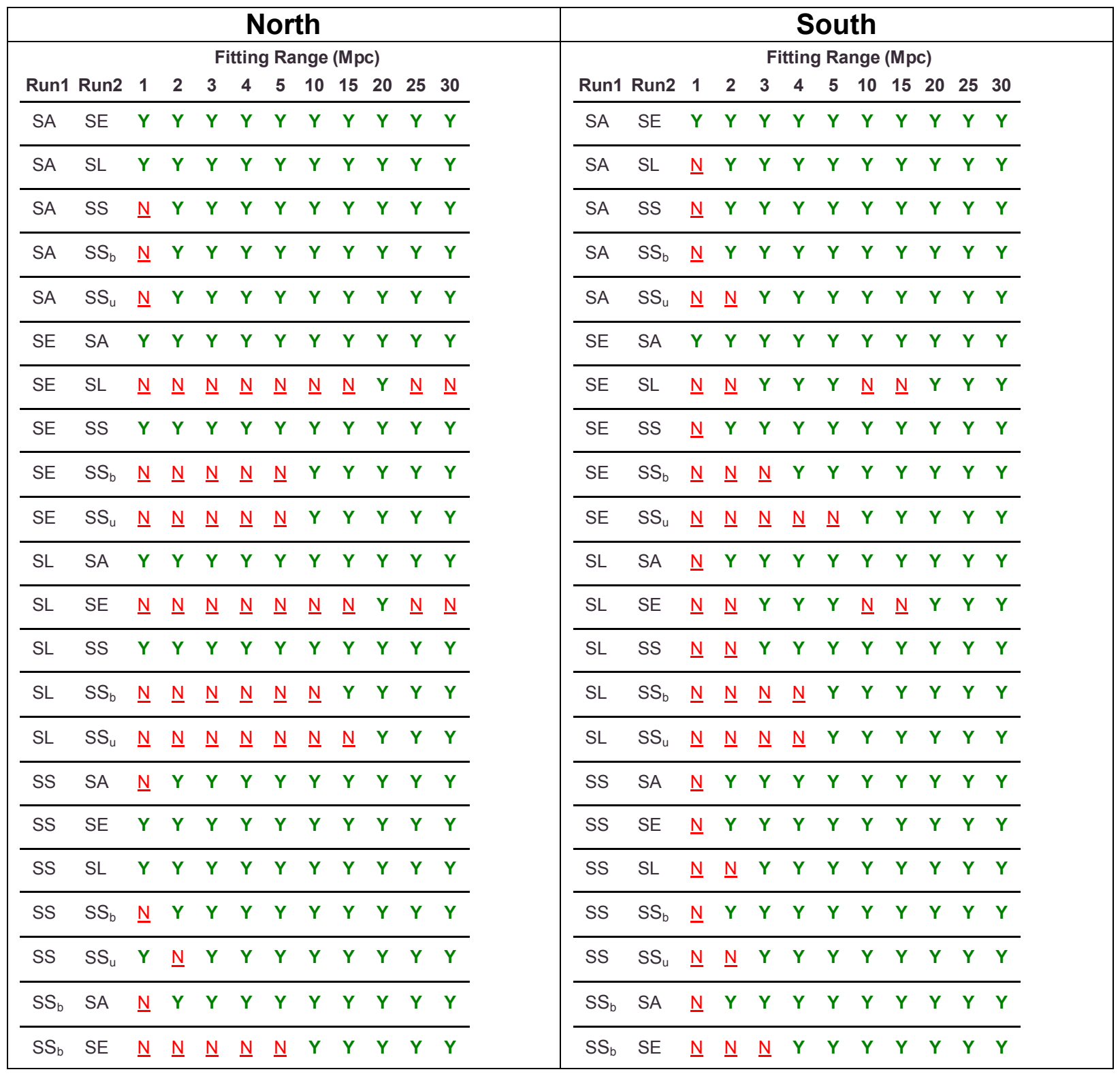




\begin{tabular}{|c|c|c|c|c|c|c|c|c|c|c|c|c|c|c|c|c|c|c|c|c|c|c|c|}
\hline $\mathrm{SS}_{\mathrm{b}}$ & SL & $\underline{\mathrm{N}}$ & $\underline{N}$ & $\underline{\mathrm{N}}$ & $\underline{\mathrm{N}}$ & $\underline{N}$ & $\underline{N}$ & $\mathbf{Y}$ & $\mathbf{Y}$ & $\mathbf{Y}$ & $\mathbf{Y}$ & $\mathrm{SS}_{\mathrm{b}}$ & SL & $\underline{\mathrm{N}}$ & $\underline{N}$ & $\underline{N}$ & $\underline{N}$ & $\mathbf{Y}$ & $\mathbf{Y}$ & $Y$ & $\mathbf{Y}$ & $\mathbf{Y}$ & $Y$ \\
\hline $\mathrm{SS}_{\mathrm{b}}$ & SS & $\underline{\mathrm{N}}$ & $\mathbf{Y}$ & $\mathbf{Y}$ & $\mathbf{Y}$ & $\mathbf{Y}$ & $Y$ & $\mathbf{Y}$ & $\mathbf{Y}$ & $Y$ & $Y$ & $\mathrm{SS}_{\mathrm{b}}$ & SS & $\underline{\mathrm{N}}$ & $\mathbf{Y}$ & $\mathbf{Y}$ & $\mathbf{Y}$ & $\mathbf{Y}$ & $\mathbf{Y}$ & $Y$ & $Y$ & $Y$ & $Y$ \\
\hline $\mathrm{SS}_{\mathrm{b}}$ & $\mathrm{SS}_{\mathrm{u}}$ & $\underline{\mathrm{N}}$ & $\underline{N}$ & $\underline{\mathrm{N}}$ & $\underline{N}$ & $\underline{N}$ & $\underline{\mathrm{N}}$ & $\mathbf{Y}$ & $Y$ & $Y$ & $Y$ & $\mathrm{SS}_{\mathrm{b}}$ & $\mathrm{SS}_{\mathrm{u}}$ & $\underline{N}$ & $\underline{N}$ & $\underline{N}$ & $\underline{N}$ & $\underline{N}$ & $\mathbf{Y}$ & $\mathbf{Y}$ & $\mathbf{Y}$ & $\mathbf{Y}$ & $Y$ \\
\hline $\mathrm{SS}_{\mathrm{u}}$ & SA & $\underline{\mathrm{N}}$ & $Y$ & $Y$ & $\mathbf{Y}$ & $Y$ & $Y$ & $\mathbf{Y}$ & $Y$ & $Y$ & $Y$ & $\mathrm{SS}_{\mathrm{u}}$ & SA & $\underline{\mathrm{N}}$ & $\underline{N}$ & $\mathbf{Y}$ & $Y$ & $\mathbf{Y}$ & $\mathbf{Y}$ & $Y$ & $Y$ & $\mathbf{Y}$ & $Y$ \\
\hline $\mathrm{SS}_{\mathrm{u}}$ & SE & $\underline{\mathrm{N}}$ & $\underline{N}$ & $\underline{N}$ & $\underline{N}$ & $\underline{N}$ & $\mathbf{Y}$ & $\mathbf{Y}$ & $\mathbf{Y}$ & $\mathbf{Y}$ & $\mathbf{Y}$ & $\mathrm{SS}_{\mathrm{u}}$ & SE & $\underline{N}$ & $\underline{N}$ & $\underline{N}$ & $\underline{N}$ & $\underline{N}$ & $\mathbf{Y}$ & $\mathbf{Y}$ & $\mathbf{Y}$ & $\mathbf{Y}$ & $Y$ \\
\hline $\mathrm{SS}_{\mathrm{u}}$ & SL & $\underline{N}$ & $\underline{N}$ & $\underline{N}$ & $\underline{N}$ & $\underline{N}$ & $\underline{N}$ & $\underline{N}$ & $\mathbf{Y}$ & $\mathbf{Y}$ & $\mathbf{Y}$ & $\mathrm{SS}_{\mathrm{u}}$ & SL & $\underline{\mathrm{N}}$ & $\underline{\mathrm{N}}$ & $\underline{\mathrm{N}}$ & $\underline{\mathrm{N}}$ & $\mathrm{Y}$ & $\mathbf{Y}$ & $Y$ & $\mathbf{Y}$ & $\mathbf{Y}$ & $Y$ \\
\hline $\mathrm{SS}_{\mathrm{u}}$ & SS & $\mathbf{Y}$ & $\underline{N}$ & $\mathbf{Y}$ & $\mathbf{Y}$ & $\mathbf{Y}$ & $\mathbf{Y}$ & $\mathbf{Y}$ & $\mathbf{Y}$ & $\mathbf{Y}$ & $Y$ & $\mathrm{SS}_{\mathrm{u}}$ & SS & $\underline{\mathrm{N}}$ & $\underline{\mathrm{N}}$ & $\mathbf{Y}$ & $\mathbf{Y}$ & $\mathbf{Y}$ & $\mathbf{Y}$ & $Y$ & $Y$ & $\mathbf{Y}$ & $Y$ \\
\hline $\mathrm{SS}_{\mathrm{u}}$ & $\mathrm{SS}_{\mathrm{b}}$ & $\underline{\mathrm{N}}$ & $\underline{N}$ & $\underline{\mathrm{N}}$ & $\underline{N}$ & $\underline{N}$ & $\underline{N}$ & $\mathbf{Y}$ & $\mathbf{Y}$ & $\mathbf{Y}$ & $\mathbf{Y}$ & $\mathrm{SS}_{\mathrm{u}}$ & $S S_{b}$ & $\underline{\mathrm{N}}$ & $\underline{N}$ & $\underline{N}$ & $\underline{N}$ & $\underline{N}$ & $\mathbf{Y}$ & $\mathbf{Y}$ & $Y$ & $\mathbf{Y}$ & $Y$ \\
\hline
\end{tabular}


For unbarred spiral primaries in Table 5-32, dissimilarity is consistently found above $5 \mathrm{Mpc}$ for most secondary morphologies, while similarity is found consistently below $5 \mathrm{Mpc}$. Of note, SuE vs. SuL have no dissimilarity at any fitting range. Also for the combination of $S_{b} S_{u} v s$. $S_{b} E$ and $S_{b} S_{u} v s$. $S_{b} L$ no dissimilarity is found for any of the fitting ranges. This suggests that there is a relationship between unbarred spirals and ellipticals, and unbarred spirals and lenticulars surrounding unbarred spirals.

Table 5-32 3D KS Test Results - comparing two runs having the same primary filter and same fitting range filter with different secondary filters - limited to barred spiral primaries.

$\mathrm{Y}=$ dissimilar to the $99 \%$ level. $\mathrm{N}=$ not dissimilar to the $99 \%$ level

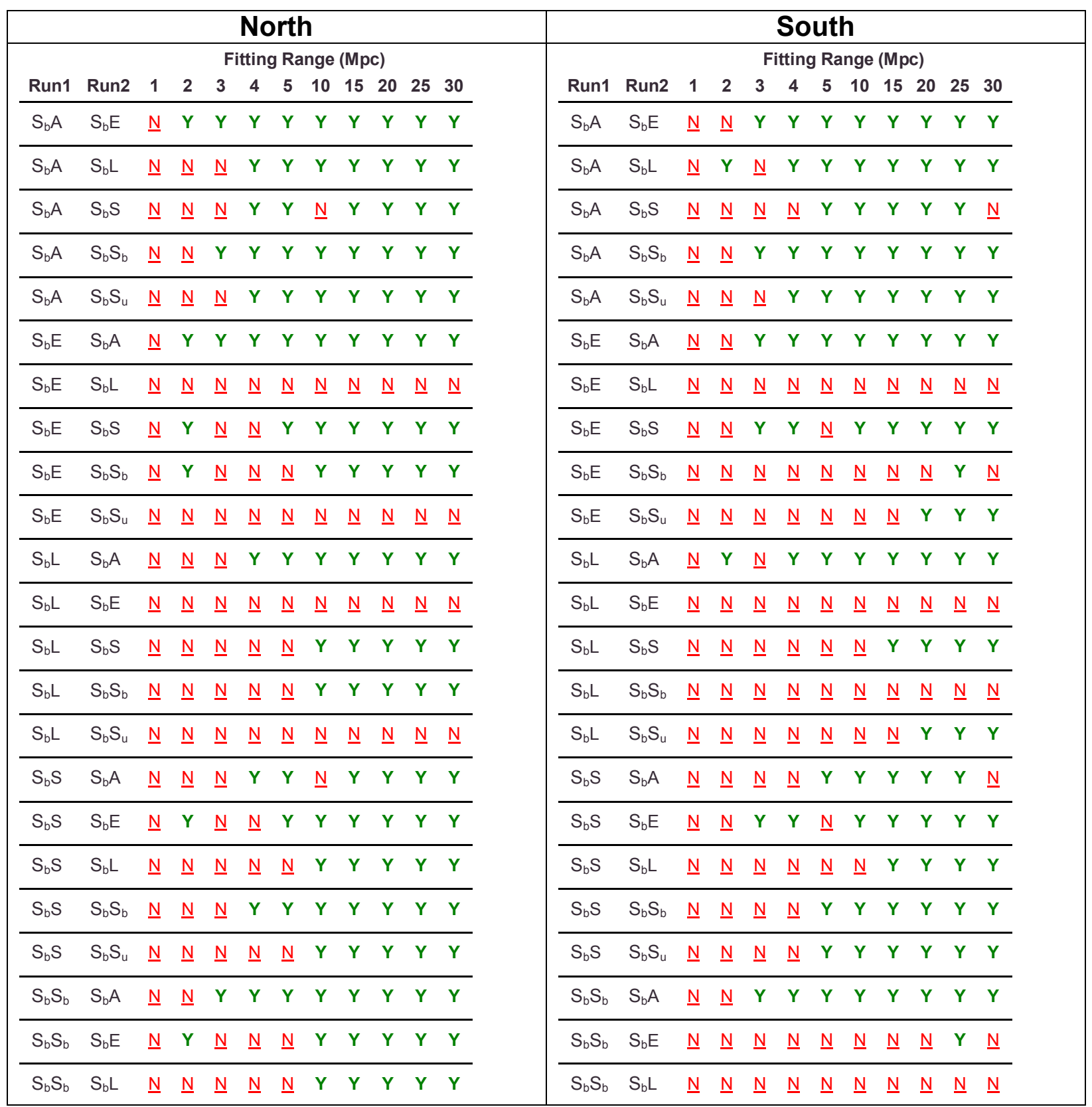




\begin{tabular}{|c|c|c|c|c|c|c|c|c|c|c|c|c|c|c|c|c|c|c|c|c|c|c|c|}
\hline $\mathrm{S}_{\mathrm{b}} \mathrm{S}_{\mathrm{b}}$ & $\mathrm{S}_{\mathrm{b}} \mathrm{S}$ & $\underline{N}$ & $\underline{\mathrm{N}}$ & $\underline{\mathrm{N}}$ & $Y$ & $\mathbf{Y}$ & $\mathbf{Y}$ & $\mathbf{Y}$ & $\mathbf{Y}$ & $\mathbf{Y}$ & $\mathbf{Y}$ & $S_{b} S_{b}$ & $S_{b} S$ & $\underline{N}$ & $\underline{N}$ & $\underline{N}$ & $\underline{\mathrm{N}}$ & $\mathbf{Y}$ & $\mathbf{Y}$ & $\mathbf{Y}$ & $\mathbf{Y}$ & $\mathbf{Y}$ & $\mathbf{Y}$ \\
\hline $\mathrm{S}_{\mathrm{b}} \mathrm{S}_{\mathrm{b}}$ & $\mathrm{S}_{\mathrm{b}} \mathrm{S}_{\mathrm{u}}$ & $\underline{N}$ & $Y$ & $\underline{\mathrm{N}}$ & $\underline{\mathrm{N}}$ & $\mathbf{Y}$ & $\mathbf{Y}$ & $Y$ & $\mathbf{Y}$ & $\mathbf{Y}$ & $\mathbf{Y}$ & $S_{b} S_{b}$ & $\mathrm{~S}_{\mathrm{b}} \mathrm{S}_{\mathrm{u}}$ & $\underline{N}$ & $\underline{N}$ & $\underline{N}$ & $\underline{N}$ & $\underline{N}$ & $\underline{N}$ & $\underline{N}$ & $\underline{N}$ & $\underline{N}$ & $\underline{N}$ \\
\hline $\mathrm{S}_{\mathrm{b}} \mathrm{S}_{\mathrm{u}}$ & $\mathrm{S}_{\mathrm{b}} \mathrm{A}$ & $\underline{N}$ & $\underline{N}$ & $\underline{\mathrm{N}}$ & $\mathbf{Y}$ & $\mathbf{Y}$ & $\mathbf{Y}$ & $Y$ & $\mathbf{Y}$ & $\mathbf{Y}$ & $\mathbf{Y}$ & $\mathrm{S}_{\mathrm{b}} \mathrm{S}_{\mathrm{u}}$ & $\mathrm{S}_{\mathrm{b}} \mathrm{A}$ & $\underline{N}$ & $\underline{N}$ & $\underline{N}$ & $\mathbf{Y}$ & $\mathbf{Y}$ & $Y$ & $\mathbf{Y}$ & $Y$ & $Y$ & $\mathbf{Y}$ \\
\hline $\mathrm{S}_{\mathrm{b}} \mathrm{S}_{\mathrm{u}}$ & $S_{b} E$ & $\underline{N}$ & $\underline{\mathrm{N}}$ & $\underline{\mathrm{N}}$ & $\underline{\mathrm{N}}$ & $\underline{\mathrm{N}}$ & $\underline{\mathrm{N}}$ & $\underline{\mathrm{N}}$ & $\underline{N}$ & $\underline{N}$ & $\underline{N}$ & $\mathrm{~S}_{\mathrm{b}} \mathrm{S}_{\mathrm{u}}$ & $S_{b} E$ & $\underline{N}$ & $\underline{N}$ & $\underline{N}$ & $\underline{N}$ & $\underline{N}$ & $\underline{N}$ & $\underline{\mathrm{N}}$ & $\mathbf{Y}$ & $\mathbf{Y}$ & $\mathbf{Y}$ \\
\hline $\mathrm{S}_{\mathrm{b}} \mathrm{S}_{\mathrm{u}}$ & $\mathrm{S}_{\mathrm{b}} \mathrm{L}$ & $\underline{N}$ & $\underline{N}$ & $\underline{N}$ & $\underline{\mathrm{N}}$ & $\underline{N}$ & $\underline{N}$ & $\underline{N}$ & $\underline{N}$ & $\underline{N}$ & $\underline{N}$ & $\mathrm{~S}_{\mathrm{b}} \mathrm{S}_{\mathrm{u}}$ & $\mathrm{S}_{\mathrm{b}} \mathrm{L}$ & $\underline{N}$ & $\underline{N}$ & $\underline{N}$ & $\underline{N}$ & $\underline{N}$ & $\underline{N}$ & $\underline{\mathrm{N}}$ & $\mathbf{Y}$ & $Y$ & $\mathbf{Y}$ \\
\hline $\mathrm{S}_{\mathrm{b}} \mathrm{S}_{\mathrm{u}}$ & $S_{b} S$ & $\underline{N}$ & $\underline{N}$ & $\underline{N}$ & $\underline{\mathrm{N}}$ & $\underline{N}$ & $\mathrm{Y}$ & $\mathrm{Y}$ & $\mathrm{Y}$ & $\mathrm{Y}$ & $\mathrm{Y}$ & $\mathrm{S}_{\mathrm{b}} \mathrm{S}_{\mathrm{u}}$ & $S_{b} S$ & $\underline{N}$ & $\underline{N}$ & $\underline{N}$ & $\underline{N}$ & $\mathrm{Y}$ & $Y$ & $Y$ & $Y$ & $\mathrm{Y}$ & $\mathbf{Y}$ \\
\hline $\mathrm{S}_{\mathrm{b}} \mathrm{S}_{\mathrm{u}}$ & $S_{b} S_{b}$ & $\underline{N}$ & $\mathbf{Y}$ & $\underline{\mathrm{N}}$ & $\underline{\mathrm{N}}$ & $\mathbf{Y}$ & $\mathbf{Y}$ & $\mathbf{Y}$ & $\mathbf{Y}$ & $\mathbf{Y}$ & $\mathbf{Y}$ & $\mathrm{S}_{\mathrm{b}} \mathrm{S}_{\mathrm{u}}$ & $S_{b} S_{b}$ & $\underline{\mathrm{N}}$ & $\underline{N}$ & $\underline{N}$ & $\underline{N}$ & $\underline{N}$ & $\underline{N}$ & $\underline{N}$ & $\underline{N}$ & $\underline{N}$ & $\underline{\mathrm{N}}$ \\
\hline
\end{tabular}


When considering unbarred spiral primaries in Table 5-33, dissimilarity is consistently found above $5 \mathrm{Mpc}$ for most secondary combinations. As seen above, no dissimilarity is found for secondaries of lenticular vs. elliptical. Also very few comparisons involving secondaries of barred spiral vs. unbarred spiral exhibit dissimilarity.

Table 5-33 3D KS Test Results - comparing two runs having the same primary filter and same fitting range filter with different secondary filters - limited to unbarred spiral primaries.

$\mathrm{Y}=$ dissimilar to the $99 \%$ level. $\mathrm{N}=$ not dissimilar to the $99 \%$ level

\begin{tabular}{|c|c|c|c|c|c|c|c|c|c|c|c|c|c|c|c|c|c|c|c|c|c|c|c|}
\hline \multicolumn{12}{|c|}{ North } & \multicolumn{12}{|c|}{ South } \\
\hline \multirow[b]{2}{*}{ Run1 } & \multicolumn{11}{|c|}{ Fitting Range (Mpc) } & \multicolumn{12}{|c|}{ Fitting Range (Mpc) } \\
\hline & Run2 & 1 & 2 & 3 & 4 & 5 & 10 & 15 & 20 & 253 & 30 & Run1 & Run2 & 1 & 2 & 3 & 4 & 5 & 10 & 15 & 20 & 25 & 30 \\
\hline $\mathrm{S}_{\mathrm{u}} \mathrm{A}$ & $\mathrm{S}_{\mathrm{u}} \mathrm{E}$ & $\underline{N}$ & $\underline{N}$ & $\underline{N}$ & $\mathbf{Y}$ & $\mathbf{Y}$ & $\mathbf{Y}$ & $\mathbf{Y}$ & $\mathbf{Y}$ & $\mathbf{Y}$ & $\mathbf{Y}$ & $\mathrm{S}_{\mathrm{u}} \mathrm{A}$ & $\mathrm{S}_{\mathrm{u}} \mathrm{E}$ & $\underline{N}$ & $\underline{N}$ & $\mathbf{Y}$ & $\mathbf{Y}$ & $\mathbf{Y}$ & $\mathbf{Y}$ & $\mathbf{Y}$ & $\mathbf{Y}$ & $\mathbf{Y}$ & $\mathbf{Y}$ \\
\hline $\mathrm{S}_{\mathrm{u}} \mathrm{A}$ & $\mathrm{S}_{\mathrm{u}} \mathrm{L}$ & $\underline{N}$ & $\underline{N}$ & $\underline{N}$ & $\mathrm{Y}$ & $\mathrm{Y}$ & $\mathrm{Y}$ & $\mathrm{Y}$ & $\mathrm{Y}$ & $\mathrm{Y}$ & Y & $\mathrm{S}_{\mathrm{u}} \mathrm{A}$ & $\mathrm{S}_{\mathrm{u}} \mathrm{L}$ & $\underline{N}$ & $\underline{N}$ & $\mathrm{Y}$ & $\mathrm{Y}$ & $\mathrm{Y}$ & $\mathrm{Y}$ & $\mathrm{Y}$ & $\mathrm{Y}$ & $\mathrm{Y}$ & $\mathrm{Y}$ \\
\hline $\mathrm{S}_{\mathrm{u}} \mathrm{A}$ & $\mathrm{S}_{\mathrm{u}} \mathrm{S}$ & $\underline{N}$ & $\underline{N}$ & $\underline{N}$ & $\mathbf{Y}$ & $\mathbf{Y}$ & $\mathbf{Y}$ & $\mathbf{Y}$ & $\mathbf{Y}$ & $\mathbf{Y}$ & $\mathbf{Y}$ & $\mathrm{S}_{\mathrm{u}} \mathrm{A}$ & $\mathrm{S}_{\mathrm{u}} \mathrm{S}$ & $\underline{N}$ & $\underline{N}$ & $\mathbf{Y}$ & $\mathbf{Y}$ & $\mathbf{Y}$ & $\mathbf{Y}$ & $\mathbf{Y}$ & $\mathbf{Y}$ & $\mathbf{Y}$ & $\mathbf{Y}$ \\
\hline$S_{u} A$ & $S_{u} S_{b}$ & $Y$ & $\underline{N}$ & $\underline{N}$ & $Y$ & $Y$ & $Y$ & $Y$ & $Y$ & $Y$ & $\mathbf{Y}$ & $S_{u} A$ & $S_{u} S_{b}$ & $\underline{N}$ & $\underline{N}$ & $Y$ & $Y$ & $Y$ & $Y$ & $Y$ & $Y$ & $Y$ & $Y$ \\
\hline $\mathrm{S}_{\mathrm{u}} \mathrm{A}$ & $\mathrm{S}_{\mathrm{u}} \mathrm{S}_{\mathrm{u}}$ & $\underline{N}$ & $\underline{N}$ & $\mathbf{Y}$ & $Y$ & $Y$ & $Y$ & $Y$ & $Y$ & $\mathbf{Y}$ & $Y$ & $\mathrm{~S}_{\mathrm{u}} \mathrm{A}$ & $\mathrm{S}_{\mathrm{u}} \mathrm{S}_{\mathrm{u}}$ & $\underline{N}$ & $\underline{N}$ & $Y$ & $Y$ & $Y$ & $Y$ & $Y$ & $Y$ & $\mathbf{Y}$ & $\mathbf{Y}$ \\
\hline$S_{u} E$ & $\mathrm{~S}_{\mathrm{u}} \mathrm{A}$ & $\underline{N}$ & $\underline{N}$ & $\underline{N}$ & $Y$ & $Y$ & $\mathbf{Y}$ & $Y$ & $\mathbf{Y}$ & $Y \quad Y$ & $\mathrm{Y}$ & $S_{u} E$ & $S_{u} A$ & $\underline{N}$ & $\underline{N}$ & $\mathbf{Y}$ & $Y$ & $Y$ & $Y$ & $Y$ & $Y$ & $Y$ & $Y$ \\
\hline $\mathrm{S}_{\mathrm{u}} \mathrm{E}$ & $\mathrm{S}_{\mathrm{u}} \mathrm{L}$ & $\underline{N}$ & $\underline{N}$ & $\underline{N}$ & $\underline{N}$ & $\underline{N}$ & $\underline{N}$ & $\underline{N}$ & $\underline{N}$ & $\underline{N}$ & $\underline{N}$ & $S_{u} E$ & $\mathrm{~S}_{\mathrm{u}} \mathrm{L}$ & $\underline{N}$ & $\underline{N}$ & $\underline{N}$ & $\underline{N}$ & $\underline{N}$ & $\underline{N}$ & $\underline{N}$ & $\underline{N}$ & $\underline{N}$ & $\underline{N}$ \\
\hline $\mathrm{S}_{\mathrm{u}} \mathrm{E}$ & $\mathrm{S}_{\mathrm{u}} \mathrm{S}$ & $\underline{N}$ & $\underline{N}$ & $\underline{N}$ & $Y$ & $Y$ & $\mathbf{Y}$ & $Y$ & $Y$ & $Y$ & $Y$ & $\mathrm{~S}_{\mathrm{u}} \mathrm{E}$ & $\mathrm{S}_{\mathrm{u}} \mathrm{S}$ & $\underline{N}$ & $\underline{N}$ & $\underline{N}$ & $\underline{N}$ & $\underline{N}$ & $\underline{N}$ & $\underline{N}$ & $\underline{N}$ & $\underline{N}$ & $\underline{N}$ \\
\hline$S_{u} E$ & $S_{u} S_{b}$ & $\underline{N}$ & $\underline{N}$ & $\underline{N}$ & $\underline{N}$ & $\underline{N}$ & $\underline{N}$ & $Y$ & $\mathbf{Y}$ & $\mathbf{Y}$ & $\mathbf{Y}$ & $S_{u} E$ & $S_{u} S_{b}$ & $\underline{\mathrm{N}}$ & $\underline{N}$ & $\underline{N}$ & $\underline{N}$ & $\underline{N}$ & $\underline{\mathrm{N}}$ & $\mathbf{Y}$ & $Y$ & $\mathbf{Y}$ & $Y$ \\
\hline$S_{u} E$ & $\mathrm{~S}_{\mathrm{u}} \mathrm{S}_{\mathrm{u}}$ & $\underline{N}$ & $\underline{N}$ & $\underline{N}$ & $\underline{\mathrm{N}}$ & $\underline{N}$ & $Y$ & $Y$ & $\mathbf{Y}$ & $\mathbf{Y}$ & $\mathbf{Y}$ & $S_{u} E$ & $\mathrm{~S}_{\mathrm{u}} \mathrm{S}_{\mathrm{u}}$ & $\underline{N}$ & $\underline{N}$ & $\underline{N}$ & $\underline{N}$ & $\underline{N}$ & $\underline{N}$ & $\mathbf{Y}$ & $\mathbf{Y}$ & $\mathbf{Y}$ & $\mathbf{Y}$ \\
\hline $\mathrm{S}_{\mathrm{u}} \mathrm{L}$ & $\mathrm{S}_{\mathrm{u}} \mathrm{A}$ & $\underline{N}$ & $\underline{N}$ & $\underline{N}$ & $\mathbf{Y}$ & $\mathbf{Y}$ & $\mathbf{Y}$ & $\mathbf{Y}$ & $\mathbf{Y}$ & $\mathbf{Y}$ & $\mathbf{Y}$ & $S_{u} L$ & $S_{u} A$ & $\underline{N}$ & $\underline{N}$ & $\mathbf{Y}$ & $\mathbf{Y}$ & $\mathbf{Y}$ & $\mathbf{Y}$ & $\mathbf{Y}$ & $\mathbf{Y}$ & $\mathbf{Y}$ & $\mathbf{Y}$ \\
\hline$S_{u} L$ & $\mathrm{~S}_{\mathrm{u}} \mathrm{E}$ & $\underline{N}$ & $\underline{N}$ & $\underline{N}$ & $\underline{N}$ & $\underline{N}$ & $\underline{N}$ & $\underline{\mathrm{N}}$ & $\underline{N}$ & $\underline{N}$ & $\underline{N}$ & $\mathrm{~S}_{\mathrm{u}} \mathrm{L}$ & $S_{u} E$ & $\underline{N}$ & $\underline{N}$ & $\underline{N}$ & $\underline{N}$ & $\underline{N}$ & $\underline{N}$ & $\underline{\mathrm{N}}$ & $\underline{\mathrm{N}}$ & $\underline{N}$ & $\underline{N}$ \\
\hline $\mathrm{S}_{\mathrm{u}} \mathrm{L}$ & $\mathrm{S}_{\mathrm{u}} \mathrm{S}$ & $\underline{N}$ & $\underline{N}$ & $\underline{N}$ & $\underline{N}$ & $Y$ & $Y$ & $Y$ & $\mathbf{Y}$ & $\mathbf{Y}$ & $Y$ & $S_{u} L$ & $\mathrm{~S}_{\mathrm{u}} \mathrm{S}$ & $\underline{N}$ & $\underline{N}$ & $\underline{N}$ & $\underline{N}$ & $\underline{N}$ & $Y$ & $\mathbf{Y}$ & $\mathbf{Y}$ & $\underline{N}$ & $\underline{N}$ \\
\hline $\mathrm{S}_{\mathrm{u}} \mathrm{L}$ & $\mathrm{S}_{\mathrm{u}} \mathrm{S}_{\mathrm{b}}$ & $\underline{N}$ & $\underline{N}$ & $\mathbf{Y}$ & $\underline{\mathrm{N}}$ & $\underline{N}$ & $\underline{N}$ & $\underline{N}$ & $\underline{N}$ & $\underline{N}$ & $\underline{N}$ & $S_{u} L$ & $\mathrm{~S}_{\mathrm{u}} \mathrm{S}_{\mathrm{b}}$ & $\underline{N}$ & $\underline{\mathrm{N}}$ & $\underline{N}$ & $\underline{\mathrm{N}}$ & $\mathbf{Y}$ & $\underline{\mathrm{N}}$ & $\mathbf{Y}$ & $\mathbf{Y}$ & $\mathbf{Y}$ & $\mathbf{Y}$ \\
\hline $\mathrm{S}_{\mathrm{u}} \mathrm{L}$ & $\mathrm{S}_{\mathrm{u}} \mathrm{S}_{\mathrm{u}}$ & $\underline{N}$ & $\underline{N}$ & $\underline{N}$ & $\underline{N}$ & $\underline{N}$ & $\underline{N}$ & $\underline{N}$ & $\underline{N}$ & $Y$ & $Y$ & $S_{u} L$ & $\mathrm{~S}_{\mathrm{u}} \mathrm{S}_{\mathrm{u}}$ & $\underline{N}$ & $\underline{N}$ & $\underline{N}$ & $\underline{N}$ & $\underline{N}$ & $\underline{N}$ & $\mathbf{Y}$ & $\mathbf{Y}$ & $\mathbf{Y}$ & $Y$ \\
\hline $\mathrm{S}_{\mathrm{u}} \mathrm{S}$ & $\mathrm{S}_{\mathrm{u}} \mathrm{A}$ & $\underline{N}$ & $\underline{N}$ & $\underline{N}$ & $Y$ & $\mathbf{Y}$ & $\mathbf{Y}$ & $Y$ & $\mathbf{Y}$ & $\mathrm{Y}$ & $\mathbf{Y}$ & $\mathrm{S}_{\mathrm{u}} \mathrm{S}$ & $\mathrm{S}_{\mathrm{u}} \mathrm{A}$ & $\underline{N}$ & $\underline{N}$ & $Y$ & $Y$ & $Y$ & $Y$ & $\mathbf{Y}$ & $\mathbf{Y}$ & $\mathbf{Y}$ & $Y$ \\
\hline$S_{u} S$ & $S_{u} E$ & $\underline{N}$ & $\underline{N}$ & $\underline{N}$ & $Y$ & $\mathbf{Y}$ & $Y$ & $Y$ & $\mathbf{Y}$ & Y Y & $\mathbf{Y}$ & $S_{u} S$ & $S_{u} E$ & $\underline{N}$ & $\underline{N}$ & $\underline{\mathrm{N}}$ & $\underline{N}$ & $\underline{\mathrm{N}}$ & $\underline{N}$ & $\underline{\mathrm{N}}$ & $\underline{\mathrm{N}}$ & $\underline{\mathrm{N}}$ & $\underline{N}$ \\
\hline $\mathrm{S}_{\mathrm{u}} \mathrm{S}$ & $\mathrm{S}_{\mathrm{u}} \mathrm{L}$ & $\underline{N}$ & $\underline{N}$ & $\underline{N}$ & $\underline{\mathrm{N}}$ & $\mathbf{Y}$ & $Y$ & $Y$ & $\mathbf{Y}$ & $Y \quad Y$ & $\mathbf{Y}$ & $\mathrm{S}_{\mathrm{u}} \mathrm{S}$ & $\mathrm{S}_{\mathrm{u}} \mathrm{L}$ & $\underline{N}$ & $\underline{N}$ & $\underline{N}$ & $\underline{N}$ & $\underline{N}$ & $Y$ & $\mathbf{Y}$ & $Y$ & $\underline{\mathrm{N}}$ & $\underline{N}$ \\
\hline$S_{u} S$ & $\mathrm{~S}_{\mathrm{u}} \mathrm{S}_{\mathrm{b}}$ & $\underline{N}$ & $\underline{N}$ & $\underline{N}$ & $\underline{\mathrm{N}}$ & $\mathbf{Y}$ & $\mathbf{Y}$ & $\mathbf{Y}$ & $\mathbf{Y}$ & $Y \quad Y$ & $\mathbf{Y}$ & $\mathrm{S}_{\mathrm{u}} \mathrm{S}$ & $\mathrm{S}_{\mathrm{u}} \mathrm{S}_{\mathrm{b}}$ & $\underline{N}$ & $\underline{\mathrm{N}}$ & $\underline{N}$ & $\mathbf{Y}$ & $\mathbf{Y}$ & $\mathbf{Y}$ & $\mathbf{Y}$ & $\mathbf{Y}$ & $\mathbf{Y}$ & $\mathbf{Y}$ \\
\hline $\mathrm{S}_{\mathrm{u}} \mathrm{S}$ & $\mathrm{S}_{\mathrm{u}} \mathrm{S}_{\mathrm{u}}$ & $\underline{N}$ & $\underline{N}$ & $\underline{N}$ & $\underline{N}$ & $Y$ & $Y$ & $Y$ & $Y$ & $Y \quad Y$ & $Y$ & $\mathrm{~S}_{\mathrm{u}} \mathrm{S}$ & $\mathrm{S}_{\mathrm{u}} \mathrm{S}_{\mathrm{u}}$ & $\underline{N}$ & $\underline{N}$ & $\underline{N}$ & $\underline{N}$ & $\underline{N}$ & $Y$ & $\mathbf{Y}$ & $Y$ & $\mathbf{Y}$ & $Y$ \\
\hline$S_{u} S_{b}$ & $S_{u} A$ & $\mathbf{Y}$ & $\underline{N}$ & $\underline{N}$ & $Y$ & $\mathbf{Y}$ & $\mathbf{Y}$ & $Y$ & $Y$ & $Y \quad Y$ & $\mathrm{Y}$ & $S_{u} S_{b}$ & $S_{u} A$ & $\underline{N}$ & $\underline{N}$ & $\mathbf{Y}$ & $\mathbf{Y}$ & $\mathbf{Y}$ & $Y$ & $\mathbf{Y}$ & $\mathbf{Y}$ & $\mathbf{Y}$ & $Y$ \\
\hline$S_{u} S_{b}$ & $\mathrm{~S}_{\mathrm{u}} \mathrm{E}$ & $\underline{N}$ & $\underline{N}$ & $\underline{N}$ & $\underline{\mathrm{N}}$ & $\underline{N}$ & $\underline{N}$ & $Y$ & $\mathbf{Y}$ & $Y \quad Y$ & $\mathbf{Y}$ & $\mathrm{S}_{\mathrm{u}} \mathrm{S}_{\mathrm{b}}$ & $S_{u} E$ & $\underline{N}$ & $\underline{N}$ & $\underline{N}$ & $\underline{N}$ & $\underline{N}$ & $\underline{\mathrm{N}}$ & $Y$ & $Y$ & $Y$ & $Y$ \\
\hline $\mathrm{S}_{\mathrm{u}} \mathrm{S}_{\mathrm{b}}$ & $\mathrm{S}_{\mathrm{u}} \mathrm{L}$ & $\underline{N}$ & $\underline{N}$ & $\mathbf{Y}$ & $\underline{N}$ & $\underline{N}$ & $\underline{N}$ & $\underline{N}$ & $\underline{N}$ & $\underline{N} \quad$ & $\underline{N}$ & $\mathrm{~S}_{\mathrm{u}} \mathrm{S}_{\mathrm{b}}$ & $\mathrm{S}_{\mathrm{u}} \mathrm{L}$ & $\underline{N}$ & $\underline{N}$ & $\underline{N}$ & $\underline{N}$ & $\mathbf{Y}$ & $\underline{N}$ & $\mathbf{Y}$ & $\mathbf{Y}$ & $\mathbf{Y}$ & $Y$ \\
\hline$S_{u} S_{b}$ & $S_{u} S$ & $\underline{N}$ & $\underline{N}$ & $\underline{N}$ & $\underline{N}$ & $\mathbf{Y}$ & $Y$ & $Y$ & $\mathbf{Y}$ & $Y \quad Y$ & $\mathbf{Y}$ & $S_{u} S_{b}$ & $S_{u} S$ & $\underline{N}$ & $\underline{N}$ & $\underline{N}$ & $\mathbf{Y}$ & $\mathbf{Y}$ & $\mathbf{Y}$ & $\mathbf{Y}$ & $\mathbf{Y}$ & $\mathbf{Y}$ & $Y$ \\
\hline
\end{tabular}




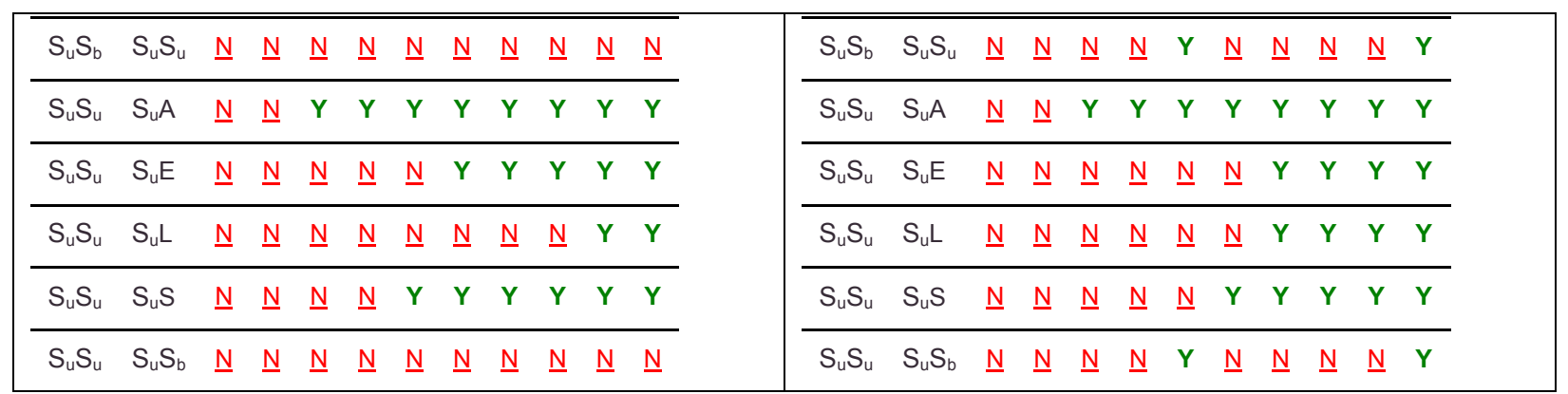

\subsubsection{Conclusions}

As seen in 2D there appears to be a relationship between ellipticals and lenticulars as secondary galaxies. Also a relationship appears to exist between barred and unbarred spirals as secondary galaxies. Finally $S_{b}$ and $S_{u}$ galaxies exhibit clustering similar to early-type galaxies but not similar to $S$ galaxies.

\subsubsection{Impact of Primary Morphology on the Pointwise Dimension}

Data runs where the secondary morphology and fitting range are equal and the primary differs are compared using KS tests.

In all of the following table $Y$ indicates that the $p$ value from the KS test is $<0.01$. This means that there is less than a $1 \%$ chance that the two compared data sets are drawn from the same distribution. That is, the compared data sets are statistically dissimilar to the $99 \%$ level. A value of $\mathrm{N}$ indicates the inverse. That is, the datasets are not statistically dissimilar to the $99 \%$ level. 
In Table 5-34, the environments of all morphological categories compared to the environments of all galaxies are statistically dissimilar in all but a few comparisons.

Table 5-34 3D KS Test Results - comparing two runs having the same secondary filter and same fitting range filter with different primary filters - limited to primaries with no particular morphology in Run1.

$\mathrm{Y}=$ dissimilar to the $99 \%$ level. $\mathrm{N}=$ not dissimilar to the $99 \%$ level

\begin{tabular}{|c|c|c|c|c|c|c|c|c|c|c|c|c|c|c|c|c|c|c|c|c|c|c|c|}
\hline \multicolumn{12}{|c|}{ North } & \multicolumn{12}{|c|}{ South } \\
\hline \multirow[b]{2}{*}{ Run1 } & \multicolumn{11}{|c|}{ Fitting Range (Mpc) } & \multicolumn{12}{|c|}{ Fitting Range (Mpc) } \\
\hline & Run2 & 1 & 2 & 3 & 4 & 5 & 10 & 15 & 20 & 25 & 30 & Run1 & Run2 & 1 & 2 & 3 & 4 & 5 & 10 & 15 & 20 & 25 & 30 \\
\hline AA & EA & $\mathbf{Y}$ & $\mathbf{Y}$ & $\mathbf{Y}$ & $\mathbf{Y}$ & $\mathbf{Y}$ & $\mathbf{Y}$ & $\mathbf{Y}$ & $Y$ & $\mathbf{Y}$ & $\mathbf{Y}$ & AA & EA & $\mathbf{Y}$ & $\mathbf{Y}$ & $\mathbf{Y}$ & $\mathbf{Y}$ & $\mathbf{Y}$ & $\mathbf{Y}$ & $\mathbf{Y}$ & $\mathrm{Y}$ & $\mathbf{Y}$ & $\mathbf{Y}$ \\
\hline AA & LA & $\underline{N}$ & $\mathbf{Y}$ & $\mathbf{Y}$ & $\mathbf{Y}$ & $\mathbf{Y}$ & $Y$ & $\mathbf{Y}$ & $Y$ & $\mathbf{Y}$ & $\mathbf{Y}$ & AA & LA & $\mathbf{Y}$ & $\mathbf{Y}$ & $Y$ & $\mathbf{Y}$ & $\mathbf{Y}$ & $Y$ & $Y$ & $\underline{N}$ & $\mathbf{Y}$ & $\underline{N}$ \\
\hline AA & $\mathrm{S}_{\mathrm{b}} \mathrm{A}$ & $\underline{N}$ & $\mathbf{Y}$ & $\mathbf{Y}$ & $\mathbf{Y}$ & $\mathbf{Y}$ & $\mathbf{Y}$ & $\mathbf{Y}$ & $Y$ & $\mathbf{Y}$ & $\underline{N}$ & AA & $S_{b} A$ & $\underline{N}$ & $\mathbf{Y}$ & $\mathbf{Y}$ & $\mathbf{Y}$ & $\mathbf{Y}$ & $\mathbf{Y}$ & $\mathbf{Y}$ & $\underline{N}$ & $\underline{N}$ & $\underline{\mathrm{N}}$ \\
\hline AA & $S_{u} A$ & $\mathbf{Y}$ & $\mathbf{Y}$ & $\mathbf{Y}$ & $\mathbf{Y}$ & $\mathbf{Y}$ & $Y$ & $\mathbf{Y}$ & $Y$ & $\mathbf{Y}$ & $Y$ & AA & $\mathrm{S}_{\mathrm{u}} \mathrm{A}$ & $\underline{N}$ & $\mathbf{Y}$ & $\mathbf{Y}$ & $Y$ & $\mathbf{Y}$ & $\mathbf{Y}$ & $\mathbf{Y}$ & $Y$ & $Y$ & $\mathbf{Y}$ \\
\hline $\mathrm{AA}$ & SA & $\mathbf{Y}$ & $\mathbf{Y}$ & $\mathbf{Y}$ & $\mathbf{Y}$ & $Y$ & $Y$ & $\mathbf{Y}$ & $Y$ & $\mathbf{Y}$ & $\mathbf{Y}$ & AA & SA & $\mathbf{Y}$ & $\mathbf{Y}$ & $\mathbf{Y}$ & $\mathbf{Y}$ & $\mathbf{Y}$ & $\mathbf{Y}$ & $Y$ & $\mathbf{Y}$ & $\mathbf{Y}$ & $\mathbf{Y}$ \\
\hline
\end{tabular}


The environments of ellipticals are statistically dissimilar when compared to the environments of all galaxies in Table 5-35. When comparing the environments of ellipticals vs. lenticulars very little similarity is found. No similarity is found between the environments of ellipticals and spirals, consistent with Best [29].

Table 5-35 3D KS Test Results - comparing two runs having the same secondary filter and same fitting range filter with different primary filters - limited to elliptical primaries in Run1.

$\mathrm{Y}=$ dissimilar to the $99 \%$ level. $\mathrm{N}=$ not dissimilar to the $99 \%$ level

\begin{tabular}{|c|c|c|c|c|c|c|c|c|c|c|c|c|c|c|c|c|c|c|c|c|c|c|c|}
\hline \multicolumn{12}{|c|}{ North } & \multicolumn{12}{|c|}{ South } \\
\hline \multirow[b]{2}{*}{ Run1 } & \multirow[b]{2}{*}{ Run2 } & \multicolumn{10}{|c|}{ Fitting Range (Mpc) } & \multicolumn{12}{|c|}{ Fitting Range (Mpc) } \\
\hline & & 1 & 2 & 3 & 4 & 5 & 10 & 15 & 20 & 25 & 30 & Run1 & Run2 & 1 & 2 & 3 & 4 & 5 & 10 & 15 & 20 & 25 & 30 \\
\hline $\mathrm{EA}$ & AA & $\mathbf{Y}$ & $\mathbf{Y}$ & $\mathbf{Y}$ & $\mathbf{Y}$ & $\mathbf{Y}$ & $\mathbf{Y}$ & $\mathbf{Y}$ & $\mathbf{Y}$ & $\mathbf{Y}$ & $\mathbf{Y}$ & EA & AA & $\mathbf{Y}$ & $\mathbf{Y}$ & $\mathbf{Y}$ & $\mathbf{Y}$ & $\mathbf{Y}$ & $\mathbf{Y}$ & $\mathbf{Y}$ & $\mathbf{Y}$ & $\mathbf{Y}$ & $\mathbf{Y}$ \\
\hline EA & LA & $\mathrm{Y}$ & $\mathrm{Y}$ & $\mathrm{Y}$ & $\mathrm{Y}$ & $\mathrm{Y}$ & $\mathrm{Y}$ & $\mathrm{Y}$ & $\underline{N}$ & $\underline{N}$ & $\underline{N}$ & EA & LA & $Y$ & $\mathrm{Y}$ & $\mathrm{Y}$ & $\mathrm{Y}$ & $\mathrm{Y}$ & $\mathrm{Y}$ & $\mathrm{Y}$ & $\mathrm{Y}$ & $\mathrm{Y}$ & $Y$ \\
\hline EA & $S_{b} A$ & $\mathbf{Y}$ & $\mathbf{Y}$ & $\mathbf{Y}$ & $\mathbf{Y}$ & $\mathbf{Y}$ & $\mathbf{Y}$ & $\mathbf{Y}$ & $\mathbf{Y}$ & $\mathbf{Y}$ & $\mathbf{Y}$ & EA & $\mathrm{S}_{\mathrm{b}} \mathrm{A}$ & $\mathbf{Y}$ & $\mathbf{Y}$ & $\mathbf{Y}$ & $Y$ & $Y$ & $\mathrm{Y}$ & $\mathbf{Y}$ & $\mathbf{Y}$ & $\mathbf{Y}$ & $Y$ \\
\hline EA & $\mathrm{S}_{\mathrm{u}} \mathrm{A}$ & $\mathbf{Y}$ & $\mathbf{Y}$ & $\mathbf{Y}$ & $\mathbf{Y}$ & $\mathbf{Y}$ & $\mathbf{Y}$ & $\mathbf{Y}$ & $\mathbf{Y}$ & $\mathrm{Y}$ & $\mathbf{Y}$ & EA & $\mathrm{S}_{\mathrm{u}} \mathrm{A}$ & $Y$ & $\mathbf{Y}$ & $\mathbf{Y}$ & $Y$ & $\mathbf{Y}$ & $\mathbf{Y}$ & $\mathbf{Y}$ & $\mathbf{Y}$ & $\mathbf{Y}$ & $Y$ \\
\hline EA & SA & $\mathbf{Y}$ & $\mathbf{Y}$ & $\mathbf{Y}$ & $\mathbf{Y}$ & $\mathbf{Y}$ & $Y$ & $\mathbf{Y}$ & $\mathbf{Y}$ & $\mathbf{Y}$ & $\mathbf{Y}$ & EA & SA & $Y$ & $\mathbf{Y}$ & $\mathbf{Y}$ & $Y$ & $\mathbf{Y}$ & $\mathbf{Y}$ & $\mathbf{Y}$ & $\mathbf{Y}$ & $\mathbf{Y}$ & $Y$ \\
\hline $\mathrm{EE}$ & LE & $\mathbf{Y}$ & $\mathbf{Y}$ & $\mathbf{Y}$ & $\mathbf{Y}$ & $\mathbf{Y}$ & $\underline{N}$ & $\underline{N}$ & $\underline{N}$ & $\underline{N}$ & $\underline{N}$ & EE & LE & $\underline{N}$ & $\mathbf{Y}$ & $\mathbf{Y}$ & $\mathbf{Y}$ & $\mathbf{Y}$ & $\mathbf{Y}$ & $\mathbf{Y}$ & $\mathbf{Y}$ & $\mathbf{Y}$ & $Y$ \\
\hline EE & $\mathrm{S}_{\mathrm{b}} \mathrm{E}$ & $\mathbf{Y}$ & $\mathbf{Y}$ & $\mathbf{Y}$ & $Y$ & $\mathbf{Y}$ & $\mathbf{Y}$ & $Y$ & $\mathbf{Y}$ & $\mathbf{Y}$ & $\mathbf{Y}$ & EE & $S_{b} E$ & $Y$ & $\mathbf{Y}$ & $\mathbf{Y}$ & $Y$ & $Y$ & $\mathbf{Y}$ & $\mathbf{Y}$ & $\mathbf{Y}$ & $\mathbf{Y}$ & $Y$ \\
\hline EE & $\mathrm{S}_{\mathrm{u}} \mathrm{E}$ & $\mathbf{Y}$ & $\mathbf{Y}$ & $\mathbf{Y}$ & $Y$ & $\mathbf{Y}$ & $\mathbf{Y}$ & $\mathbf{Y}$ & $\mathbf{Y}$ & $\mathbf{Y}$ & $\mathbf{Y}$ & EE & $\mathrm{S}_{\mathrm{u}} \mathrm{E}$ & $Y$ & $\mathbf{Y}$ & $\mathbf{Y}$ & $Y$ & $\mathbf{Y}$ & $\mathbf{Y}$ & $\mathbf{Y}$ & $\mathbf{Y}$ & $\mathbf{Y}$ & $Y$ \\
\hline EE & SE & $\mathbf{Y}$ & $\mathbf{Y}$ & $\mathbf{Y}$ & $Y$ & $\mathbf{Y}$ & $\mathbf{Y}$ & $\mathbf{Y}$ & $\mathbf{Y}$ & $\mathbf{Y}$ & $\mathbf{Y}$ & EE & SE & $Y$ & $\mathbf{Y}$ & $\mathbf{Y}$ & $\mathbf{Y}$ & $\mathbf{Y}$ & $\mathbf{Y}$ & $\mathbf{Y}$ & $\mathbf{Y}$ & $\mathbf{Y}$ & $Y$ \\
\hline EL & $\mathrm{LL}$ & $\mathbf{Y}$ & $\underline{\mathrm{N}}$ & $\underline{\mathrm{N}}$ & $\mathbf{Y}$ & $\mathbf{Y}$ & $\underline{N}$ & $\underline{\mathrm{N}}$ & $\underline{N}$ & $\underline{N}$ & $\underline{N}$ & EL & $\mathrm{LL}$ & $\underline{N}$ & $\underline{N}$ & $\underline{N}$ & $\mathbf{Y}$ & $\mathbf{Y}$ & $\underline{N}$ & $\underline{N}$ & $\underline{N}$ & $\underline{N}$ & $\underline{N}$ \\
\hline EL & $S_{b} L$ & $Y$ & $\mathbf{Y}$ & $\mathbf{Y}$ & $\mathbf{Y}$ & $\mathbf{Y}$ & $Y$ & $\mathbf{Y}$ & $\mathbf{Y}$ & $\mathbf{Y}$ & $\mathbf{Y}$ & EL & $S_{b} L$ & $Y$ & $\mathbf{Y}$ & $Y$ & $\mathbf{Y}$ & $\mathbf{Y}$ & $Y$ & $\mathbf{Y}$ & $\mathbf{Y}$ & $\mathbf{Y}$ & $\underline{N}$ \\
\hline EL & $S_{u} L$ & $Y$ & $\mathbf{Y}$ & $\mathbf{Y}$ & $Y$ & $\mathbf{Y}$ & $Y$ & $\mathbf{Y}$ & $Y$ & $Y$ & $\mathbf{Y}$ & $\mathrm{EL}$ & $S_{u} L$ & $\underline{N}$ & $\mathbf{Y}$ & $\mathbf{Y}$ & $Y$ & $\mathbf{Y}$ & $\mathbf{Y}$ & $\mathbf{Y}$ & $Y$ & $\mathbf{Y}$ & $Y$ \\
\hline EL & $\mathrm{SL}$ & $Y$ & $\mathbf{Y}$ & $\mathbf{Y}$ & $Y$ & $\mathbf{Y}$ & $Y$ & $\mathbf{Y}$ & $Y$ & $Y$ & $\mathbf{Y}$ & EL & $\mathrm{SL}$ & $Y$ & $\mathbf{Y}$ & $\mathbf{Y}$ & $Y$ & $\mathbf{Y}$ & $\mathbf{Y}$ & $\mathbf{Y}$ & $Y$ & $\mathbf{Y}$ & $Y$ \\
\hline ES & LS & $Y$ & $\mathbf{Y}$ & $Y$ & $Y$ & $Y$ & $\mathbf{Y}$ & $\underline{N}$ & $\underline{N}$ & $\underline{N}$ & $\underline{N}$ & ES & LS & $\underline{N}$ & $\underline{\mathrm{N}}$ & $\underline{N}$ & $\underline{N}$ & $\underline{\mathrm{N}}$ & $\underline{N}$ & $\underline{N}$ & $\underline{N}$ & $\underline{N}$ & $\underline{N}$ \\
\hline ES & $\mathrm{S}_{\mathrm{b}} \mathrm{S}$ & $\mathbf{Y}$ & $\mathbf{Y}$ & $\mathbf{Y}$ & $Y$ & $Y$ & $\mathbf{Y}$ & $Y$ & $\mathbf{Y}$ & $Y$ & $Y$ & ES & $S_{b} S$ & $Y$ & $\mathbf{Y}$ & $\mathbf{Y}$ & $Y$ & $Y$ & $Y$ & $\mathbf{Y}$ & $\mathbf{Y}$ & $\underline{N}$ & $\underline{N}$ \\
\hline ES & $\mathrm{S}_{\mathrm{u}} \mathrm{S}$ & $Y$ & $\mathbf{Y}$ & $\mathbf{Y}$ & $Y$ & $Y$ & $Y$ & $\mathbf{Y}$ & $\mathbf{Y}$ & $\mathbf{Y}$ & $\mathbf{Y}$ & ES & $S_{u} S$ & $Y$ & $\mathbf{Y}$ & $\mathbf{Y}$ & $Y$ & $\mathbf{Y}$ & $\mathbf{Y}$ & $\mathbf{Y}$ & $\mathbf{Y}$ & $\mathbf{Y}$ & $\mathbf{Y}$ \\
\hline ES & SS & $Y$ & $Y$ & $Y$ & $Y$ & $Y$ & $\mathbf{Y}$ & $Y$ & $\mathbf{Y}$ & $\mathbf{Y}$ & $Y$ & ES & SS & $Y$ & $\mathbf{Y}$ & $\mathbf{Y}$ & $\mathbf{Y}$ & $Y$ & $Y$ & $Y$ & $Y$ & $Y$ & $Y$ \\
\hline $\mathrm{ES}_{\mathrm{b}}$ & $\mathrm{LS}_{\mathrm{b}}$ & $\underline{N}$ & $\underline{N}$ & $\underline{N}$ & $\underline{N}$ & $\underline{N}$ & $\underline{N}$ & $\underline{N}$ & $\underline{N}$ & $\underline{N}$ & $\underline{N}$ & $E S_{b}$ & $\mathrm{LS}_{\mathrm{b}}$ & $\underline{N}$ & $\underline{N}$ & $\underline{N}$ & $\underline{N}$ & $\underline{N}$ & $\underline{N}$ & $\underline{N}$ & $\underline{N}$ & $\underline{N}$ & $\underline{N}$ \\
\hline $\mathrm{ES}_{\mathrm{b}}$ & $\mathrm{S}_{\mathrm{b}} \mathrm{S}_{\mathrm{b}}$ & $\underline{N}$ & $\underline{\mathrm{N}}$ & $\underline{N}$ & $\underline{N}$ & $\underline{N}$ & $Y$ & $\mathbf{Y}$ & $\mathbf{Y}$ & $\underline{\mathrm{N}}$ & $\underline{\mathrm{N}}$ & $\mathrm{ES}_{\mathrm{b}}$ & $S_{b} S_{b}$ & $\underline{N}$ & $\mathbf{Y}$ & $\mathbf{Y}$ & $Y$ & $\mathbf{Y}$ & $Y$ & $\mathbf{Y}$ & $\underline{N}$ & $\underline{N}$ & $\underline{N}$ \\
\hline $\mathrm{ES}_{\mathrm{b}}$ & $S_{u} S_{b}$ & $\underline{N}$ & $Y$ & $Y$ & $Y$ & $Y$ & $Y$ & $\mathbf{Y}$ & $Y$ & $Y$ & $Y$ & $E S_{b}$ & $S_{u} S_{b}$ & $\underline{N}$ & $\underline{N}$ & $\underline{N}$ & $Y$ & $Y$ & $Y$ & $Y$ & $Y$ & $Y$ & $Y$ \\
\hline $\mathrm{ES}_{\mathrm{b}}$ & $\mathrm{SS}_{\mathrm{b}}$ & $\underline{N}$ & $\mathbf{Y}$ & $\mathbf{Y}$ & $\mathbf{Y}$ & $Y$ & $\mathbf{Y}$ & $\mathbf{Y}$ & $\mathbf{Y}$ & $\mathbf{Y}$ & $\mathbf{Y}$ & $\mathrm{ES}_{\mathrm{b}}$ & $\mathrm{SS}_{\mathrm{b}}$ & $\underline{N}$ & $\underline{N}$ & $\mathbf{Y}$ & $Y$ & $Y$ & $Y$ & $Y$ & $Y$ & $\underline{N}$ & $\underline{N}$ \\
\hline $\mathrm{ES}_{\mathrm{u}}$ & $\mathrm{LS}_{\mathrm{u}}$ & $\underline{N}$ & $\underline{N}$ & $\underline{N}$ & $\underline{N}$ & $\underline{N}$ & $\underline{N}$ & $\underline{N}$ & $\underline{N}$ & $\underline{N}$ & $\underline{N}$ & $\mathrm{ES}_{\mathrm{u}}$ & $\mathrm{LS}_{\mathrm{u}}$ & $\underline{N}$ & $\underline{N}$ & $\underline{N}$ & $\underline{N}$ & $\underline{N}$ & $\underline{N}$ & $\underline{N}$ & $\underline{N}$ & $\underline{N}$ & $\underline{N}$ \\
\hline $\mathrm{ES}_{\mathrm{u}}$ & $\mathrm{S}_{\mathrm{b}} \mathrm{S}_{\mathrm{u}}$ & $\underline{N}$ & $Y$ & $Y$ & $Y$ & $\mathrm{Y}$ & $Y$ & $Y$ & $Y$ & $Y$ & $Y$ & $\mathrm{ES}_{\mathrm{u}}$ & $\mathrm{S}_{\mathrm{b}} \mathrm{S}_{\mathrm{u}}$ & $\underline{N}$ & $Y$ & $\mathrm{Y}$ & $Y$ & $\mathrm{Y}$ & $\underline{N}$ & $\underline{N}$ & $\underline{N}$ & $\underline{N}$ & $\underline{N}$ \\
\hline $\mathrm{ES}_{\mathrm{u}}$ & $\mathrm{S}_{\mathrm{u}} \mathrm{S}_{\mathrm{u}}$ & $\underline{N}$ & $Y$ & $Y$ & $Y$ & $\mathbf{Y}$ & $\mathbf{Y}$ & $Y$ & $\mathbf{Y}$ & $\mathbf{Y}$ & $Y$ & $\mathrm{ES}_{\mathrm{u}}$ & $\mathrm{S}_{\mathrm{u}} \mathrm{S}_{\mathrm{u}}$ & $\underline{N}$ & $\underline{N}$ & $\mathbf{Y}$ & $Y$ & $Y$ & $\underline{N}$ & $Y$ & $Y$ & $Y$ & $Y$ \\
\hline $\mathrm{ES}_{\mathrm{u}}$ & $\mathrm{SS}_{\mathrm{u}}$ & $\underline{N}$ & $\mathbf{Y}$ & $\underline{N}$ & $Y$ & $\mathrm{Y}$ & $Y$ & $Y$ & $\mathbf{Y}$ & $\mathbf{Y}$ & $\mathbf{Y}$ & $\mathrm{ES}_{\mathrm{u}}$ & $\mathrm{SS}_{\mathrm{u}}$ & $\underline{N}$ & $\mathbf{Y}$ & $\mathbf{Y}$ & $Y$ & $Y$ & $Y$ & $\underline{N}$ & $\underline{N}$ & $\underline{N}$ & $\underline{N}$ \\
\hline
\end{tabular}


The environments of $L$ primaries in Table 5-36 are consistently dissimilar when compared to the environments of all galaxies. Dissimilarity is consistently found when comparing the environments of $L$ to $S$, which is consistent with Best's finding [29] that the environments of late-type and early-type galaxies are dissimilar.

$S_{b}$ and $S_{u}$ cluster similarly at all fitting ranges around $L$ primaries.

Table 5-36 3D KS Test Results - comparing two runs having the same secondary filter and same fitting range filter with different primary filters - limited to lenticular primaries in Run1.

$\mathrm{Y}=$ dissimilar to the $99 \%$ level. $\mathrm{N}=$ not dissimilar to the $99 \%$ level

\begin{tabular}{|c|c|c|c|c|c|c|c|c|c|c|c|c|c|c|c|c|c|c|c|c|c|c|c|}
\hline \multicolumn{12}{|c|}{ North } & \multicolumn{12}{|c|}{ South } \\
\hline \multirow[b]{2}{*}{ Run1 } & \multicolumn{11}{|c|}{ Fitting Range (Mpc) } & \multicolumn{12}{|c|}{ Fitting Range (Mpc) } \\
\hline & Run2 & 1 & 2 & 3 & 4 & 5 & 10 & 15 & 20 & 25 & 30 & Run1 & Run2 & 1 & 2 & 3 & 4 & 5 & 10 & 15 & 20 & 25 & 30 \\
\hline LA & AA & $\underline{N}$ & $\mathbf{Y}$ & $\mathbf{Y}$ & $\mathbf{Y}$ & $\mathbf{Y}$ & $\mathbf{Y}$ & $\mathbf{Y}$ & $\mathbf{Y}$ & $\mathbf{Y}$ & $\mathbf{Y}$ & LA & $\mathrm{AA}$ & $Y$ & $\mathbf{Y}$ & $\mathbf{Y}$ & $\mathbf{Y}$ & $\mathbf{Y}$ & $\mathbf{Y}$ & $\mathbf{Y}$ & $\underline{N}$ & $\mathbf{Y}$ & $\underline{N}$ \\
\hline LA & EA & $\mathbf{Y}$ & $\mathbf{Y}$ & $\mathbf{Y}$ & $\mathbf{Y}$ & Y & $\mathbf{Y}$ & $\mathbf{Y}$ & $\underline{N}$ & $\underline{\mathrm{N}}$ & $\underline{N}$ & LA & EA & $\mathbf{Y}$ & $\mathbf{Y}$ & $\mathbf{Y}$ & $Y$ & $\mathbf{Y}$ & $\mathbf{Y}$ & $\mathbf{Y}$ & $\mathbf{Y}$ & $\mathbf{Y}$ & $\mathbf{Y}$ \\
\hline LA & $S_{b} A$ & $\underline{N}$ & $\mathbf{Y}$ & $\mathbf{Y}$ & $\mathbf{Y}$ & Y & $\mathbf{Y}$ & $\mathbf{Y}$ & $\mathbf{Y}$ & $\mathbf{Y}$ & $\mathbf{Y}$ & LA & $\mathrm{S}_{\mathrm{b}} \mathrm{A}$ & $Y$ & $\mathbf{Y}$ & $\mathbf{Y}$ & $\mathbf{Y}$ & $\mathbf{Y}$ & $\mathbf{Y}$ & $\mathbf{Y}$ & $\underline{N}$ & $\underline{N}$ & $\underline{N}$ \\
\hline LA & $S_{u} A$ & $\mathbf{Y}$ & $\mathbf{Y}$ & $\mathbf{Y}$ & $\mathbf{Y}$ & $\mathbf{Y}$ & $\mathbf{Y}$ & $\mathbf{Y}$ & $\mathbf{Y}$ & $\mathbf{Y}$ & $\mathbf{Y}$ & LA & $\mathrm{S}_{\mathrm{u}} \mathrm{A}$ & $\underline{N}$ & $\mathbf{Y}$ & $\mathbf{Y}$ & $Y$ & $Y$ & $\mathbf{Y}$ & $\mathbf{Y}$ & $Y$ & $\mathbf{Y}$ & $Y$ \\
\hline LA & SA & $\mathbf{Y}$ & $\mathbf{Y}$ & $\mathbf{Y}$ & $\mathbf{Y}$ & $\mathbf{Y}$ & $\mathbf{Y}$ & $\mathbf{Y}$ & $\mathbf{Y}$ & $\mathbf{Y}$ & $\mathbf{Y}$ & LA & $S A$ & $Y$ & $\mathbf{Y}$ & $\mathbf{Y}$ & $\mathbf{Y}$ & $Y$ & $\mathbf{Y}$ & $\mathbf{Y}$ & $Y$ & $\mathbf{Y}$ & $Y$ \\
\hline LE & $\mathrm{EE}$ & $\mathbf{Y}$ & $\mathbf{Y}$ & $\mathbf{Y}$ & $\mathbf{Y}$ & Y & $\underline{\mathrm{N}}$ & $\underline{N}$ & $\underline{N}$ & $\underline{\mathrm{N}}$ & $\underline{\mathrm{N}}$ & LE & EE & $\underline{N}$ & $\mathbf{Y}$ & $\mathbf{Y}$ & $\mathbf{Y}$ & $Y$ & $\mathbf{Y}$ & $\mathbf{Y}$ & $Y$ & $\mathbf{Y}$ & $Y$ \\
\hline LE & $S_{b} E$ & $\underline{N}$ & $\mathbf{Y}$ & $\mathbf{Y}$ & $\mathbf{Y}$ & $\mathbf{Y}$ & $\mathbf{Y}$ & $\mathbf{Y}$ & $\mathbf{Y}$ & $\mathbf{Y}$ & $\mathbf{Y}$ & LE & $\mathrm{S}_{\mathrm{b}} \mathrm{E}$ & $\underline{N}$ & $\underline{N}$ & $\mathbf{Y}$ & $\mathbf{Y}$ & $Y$ & $\mathbf{Y}$ & $\mathbf{Y}$ & $\mathbf{Y}$ & $\mathbf{Y}$ & $\underline{N}$ \\
\hline LE & $\mathrm{S}_{\mathrm{u}} \mathrm{E}$ & $\underline{N}$ & $\mathbf{Y}$ & $\mathbf{Y}$ & $Y$ & $Y$ & $\mathbf{Y}$ & $Y$ & $\mathbf{Y}$ & $\mathbf{Y}$ & $\mathbf{Y}$ & LE & $\mathrm{S}_{\mathrm{u}} \mathrm{E}$ & $\underline{N}$ & $\underline{N}$ & $\underline{N}$ & $Y$ & $Y$ & $\mathbf{Y}$ & $Y$ & $Y$ & $\mathbf{Y}$ & $Y$ \\
\hline LE & SE & $\underline{N}$ & $\mathbf{Y}$ & $\mathbf{Y}$ & $\mathbf{Y}$ & $\mathbf{Y}$ & $\mathbf{Y}$ & $\mathbf{Y}$ & $\mathbf{Y}$ & $\mathbf{Y}$ & $\mathbf{Y}$ & LE & SE & $Y$ & $\mathbf{Y}$ & $\mathbf{Y}$ & $\mathbf{Y}$ & $Y$ & $\mathbf{Y}$ & $\mathbf{Y}$ & $\mathbf{Y}$ & $Y$ & $Y$ \\
\hline LL & EL & $\mathbf{Y}$ & $\underline{N}$ & $\underline{\mathrm{N}}$ & $\mathbf{Y}$ & $\mathbf{Y}$ & $\underline{N}$ & $\underline{N}$ & $\underline{\mathrm{N}}$ & $\underline{\mathrm{N}}$ & $\underline{\mathrm{N}}$ & LL & EL & $\underline{N}$ & $\underline{\mathrm{N}}$ & $\underline{\mathrm{N}}$ & $\mathbf{Y}$ & $Y$ & $\underline{N}$ & $\underline{N}$ & $\underline{N}$ & $\underline{N}$ & $\underline{N}$ \\
\hline LL & $\mathrm{S}_{\mathrm{b}} \mathrm{L}$ & $\underline{N}$ & $\mathbf{Y}$ & $\mathbf{Y}$ & $Y$ & $\mathbf{Y}$ & $\mathbf{Y}$ & $\mathbf{Y}$ & $\mathbf{Y}$ & $\mathbf{Y}$ & $\mathbf{Y}$ & LL & $\mathrm{S}_{\mathrm{b}} \mathrm{L}$ & $Y$ & $\mathbf{Y}$ & $\mathbf{Y}$ & $\mathbf{Y}$ & $\mathbf{Y}$ & $\mathbf{Y}$ & $\mathbf{Y}$ & $\underline{N}$ & $\underline{N}$ & $\underline{N}$ \\
\hline LL & $\mathrm{S}_{\mathrm{u}} \mathrm{L}$ & $\underline{N}$ & $\mathbf{Y}$ & $\mathbf{Y}$ & $\mathbf{Y}$ & $\mathbf{Y}$ & $\mathbf{Y}$ & $\mathbf{Y}$ & $\mathbf{Y}$ & $\mathbf{Y}$ & $\mathbf{Y}$ & LL & $\mathrm{S}_{\mathrm{u}} \mathrm{L}$ & $\underline{N}$ & $\underline{N}$ & $\mathbf{Y}$ & $\mathbf{Y}$ & $\mathbf{Y}$ & $\mathbf{Y}$ & $\mathbf{Y}$ & $\mathbf{Y}$ & $\mathbf{Y}$ & $Y$ \\
\hline LL & $\mathrm{SL}$ & $\mathbf{Y}$ & $\mathbf{Y}$ & $\mathbf{Y}$ & $\mathbf{Y}$ & $\mathbf{Y}$ & $\mathbf{Y}$ & $\mathbf{Y}$ & $\mathbf{Y}$ & $\mathbf{Y}$ & $\mathbf{Y}$ & LL & $\mathrm{SL}$ & $Y$ & $\mathbf{Y}$ & $\mathbf{Y}$ & $\mathbf{Y}$ & $\mathbf{Y}$ & $\mathbf{Y}$ & $\mathbf{Y}$ & $\mathbf{Y}$ & $\mathbf{Y}$ & $Y$ \\
\hline LS & ES & $\mathbf{Y}$ & $\mathbf{Y}$ & $\mathbf{Y}$ & $\mathbf{Y}$ & $\mathbf{Y}$ & $\mathbf{Y}$ & $\underline{N}$ & $\underline{\mathrm{N}}$ & $\underline{\mathrm{N}}$ & $\underline{\mathrm{N}}$ & LS & ES & $\underline{N}$ & $\underline{N}$ & $\underline{N}$ & $\underline{N}$ & $\underline{N}$ & $\underline{N}$ & $\underline{N}$ & $\underline{N}$ & $\underline{N}$ & $\underline{N}$ \\
\hline LS & $\mathrm{S}_{\mathrm{b}} \mathrm{S}$ & $\underline{\mathrm{N}}$ & $\mathbf{Y}$ & $\mathbf{Y}$ & $\mathbf{Y}$ & $\mathbf{Y}$ & $\mathbf{Y}$ & $\mathbf{Y}$ & $\mathbf{Y}$ & $\mathbf{Y}$ & $\underline{\mathrm{N}}$ & LS & $S_{b} S$ & $\underline{N}$ & $\mathbf{Y}$ & $\mathbf{Y}$ & $\mathbf{Y}$ & $\mathbf{Y}$ & $\mathbf{Y}$ & $\mathbf{Y}$ & $\underline{N}$ & $\underline{\mathrm{N}}$ & $\underline{N}$ \\
\hline LS & $\mathrm{S}_{\mathrm{u}} \mathrm{S}$ & $\underline{N}$ & $\mathbf{Y}$ & $\mathbf{Y}$ & $\mathbf{Y}$ & $\mathbf{Y}$ & $\mathbf{Y}$ & $\mathbf{Y}$ & $\mathbf{Y}$ & $\mathbf{Y}$ & $\mathbf{Y}$ & LS & $\mathrm{S}_{\mathrm{u}} \mathrm{S}$ & $\underline{N}$ & $\underline{\mathrm{N}}$ & $\mathbf{Y}$ & $\mathbf{Y}$ & $\mathbf{Y}$ & $\mathbf{Y}$ & $\mathbf{Y}$ & $\mathbf{Y}$ & $\mathbf{Y}$ & $Y$ \\
\hline LS & SS & $\underline{N}$ & $\mathbf{Y}$ & $\mathbf{Y}$ & $\mathbf{Y}$ & $\mathbf{Y}$ & $\mathbf{Y}$ & $\mathbf{Y}$ & $\mathbf{Y}$ & $\mathbf{Y}$ & $\mathbf{Y}$ & LS & SS & $Y$ & $\mathbf{Y}$ & $\mathbf{Y}$ & $\mathbf{Y}$ & $\mathbf{Y}$ & $\mathbf{Y}$ & $\mathbf{Y}$ & $\mathbf{Y}$ & $\mathbf{Y}$ & $Y$ \\
\hline $\mathrm{LS}_{\mathrm{b}}$ & $\mathrm{ES}_{\mathrm{b}}$ & $\underline{N}$ & $\underline{N}$ & $\underline{N}$ & $\underline{N}$ & $\underline{N}$ & $\underline{N}$ & $\underline{N}$ & $\underline{N}$ & $\underline{N}$ & $\underline{N}$ & $\mathrm{LS}_{\mathrm{b}}$ & $\mathrm{ES}_{\mathrm{b}}$ & $\underline{N}$ & $\underline{N}$ & $\underline{N}$ & $\underline{N}$ & $\underline{N}$ & $\underline{N}$ & $\underline{N}$ & $\underline{N}$ & $\underline{N}$ & $\underline{N}$ \\
\hline $\mathrm{LS}_{\mathrm{b}}$ & $\mathrm{S}_{\mathrm{b}} \mathrm{S}_{\mathrm{b}}$ & $\underline{N}$ & $\underline{N}$ & $\underline{N}$ & $\underline{N}$ & $\underline{N}$ & $\underline{N}$ & $\underline{N}$ & $\underline{N}$ & $\underline{\mathrm{N}}$ & $\underline{N}$ & $\mathrm{LS}_{\mathrm{b}}$ & $\mathrm{S}_{\mathrm{b}} \mathrm{S}_{\mathrm{b}}$ & $\underline{N}$ & $\underline{N}$ & $\underline{N}$ & $\underline{N}$ & $\underline{N}$ & $\mathrm{Y}$ & $\mathbf{Y}$ & $\underline{N}$ & $\underline{N}$ & $\underline{N}$ \\
\hline $\mathrm{LS}_{\mathrm{b}}$ & $\mathrm{S}_{\mathrm{u}} \mathrm{S}_{\mathrm{b}}$ & $\underline{N}$ & $\mathbf{Y}$ & $\mathbf{Y}$ & $\mathbf{Y}$ & $Y$ & $Y$ & $\mathbf{Y}$ & $\mathbf{Y}$ & $\mathbf{Y}$ & $Y$ & $\mathrm{LS}_{\mathrm{b}}$ & $\mathrm{S}_{\mathrm{u}} \mathrm{S}_{\mathrm{b}}$ & $\underline{N}$ & $\underline{N}$ & $\underline{N}$ & $\underline{N}$ & $\underline{N}$ & $\mathbf{Y}$ & $\mathbf{Y}$ & $Y$ & $\mathbf{Y}$ & $Y$ \\
\hline $\mathrm{LS}_{\mathrm{b}}$ & $\mathrm{SS}_{\mathrm{b}}$ & $\underline{\mathrm{N}}$ & $\mathbf{Y}$ & $\mathbf{Y}$ & $\mathbf{Y}$ & $\mathbf{Y}$ & $\mathbf{Y}$ & $\mathbf{Y}$ & $\mathbf{Y}$ & $\mathbf{Y}$ & $\mathbf{Y}$ & $\mathrm{LS}_{\mathrm{b}}$ & $\mathrm{SS}_{\mathrm{b}}$ & $\underline{N}$ & $\underline{N}$ & $\underline{\mathrm{N}}$ & $\mathbf{Y}$ & $\mathbf{Y}$ & $\underline{\mathrm{N}}$ & $\mathbf{Y}$ & $\underline{N}$ & $\mathbf{Y}$ & $\underline{N}$ \\
\hline $\mathrm{LS}_{\mathrm{u}}$ & $\mathrm{ES}_{\mathrm{u}}$ & $\underline{N}$ & $\underline{N}$ & $\underline{N}$ & $\underline{N}$ & $\underline{N}$ & $\underline{N}$ & $\underline{N}$ & $\underline{N}$ & $\underline{N}$ & $\underline{N}$ & $\mathrm{LS}_{\mathrm{u}}$ & $\mathrm{ES}_{\mathrm{u}}$ & $\underline{N}$ & $\underline{N}$ & $\underline{N}$ & $\underline{N}$ & $\underline{N}$ & $\underline{N}$ & $\underline{N}$ & $\underline{N}$ & $\underline{N}$ & $\underline{N}$ \\
\hline
\end{tabular}




\begin{tabular}{|c|c|c|c|c|c|c|c|c|c|c|c|c|c|c|c|c|c|c|c|c|c|c|}
\hline $\mathrm{LS}_{\mathrm{u}}$ & $\mathrm{S}_{\mathrm{b}} \mathrm{S}_{\mathrm{u}}$ & $\underline{N}$ & $\underline{N}$ & $\underline{N}$ & $Y$ & $\mathbf{Y}$ & $Y$ & $\mathbf{Y}$ & $Y$ & $\mathbf{Y} \underline{\mathrm{N}}$ & $\mathrm{LS}_{\mathrm{u}}$ & $\mathrm{S}_{\mathrm{b}} \mathrm{S}_{\mathrm{u}}$ & $\mathbf{Y}$ & $\underline{N}$ & $\underline{N}$ & $\mathbf{Y}$ & $Y$ & $\underline{N}$ & $\underline{N}$ & $\underline{N}$ & $\underline{N}$ & $Y$ \\
\hline $\mathrm{LS}_{\mathrm{u}}$ & $\mathrm{S}_{\mathrm{u}} \mathrm{S}_{\mathrm{u}}$ & $\underline{N}$ & $\underline{N}$ & $\mathbf{Y}$ & $Y$ & $Y$ & $\mathbf{Y}$ & $\mathbf{Y}$ & $\mathbf{Y}$ & $Y \quad Y$ & $\mathrm{LS}_{\mathrm{u}}$ & $S_{u} S_{u}$ & $Y$ & $\underline{N}$ & $Y$ & $Y$ & $Y$ & $Y$ & $Y$ & $Y$ & $Y$ & $Y$ \\
\hline $\mathrm{LS}_{\mathrm{u}}$ & $\mathrm{SS}_{\mathrm{u}}$ & $\underline{N}$ & $\underline{N}$ & $\mathbf{Y}$ & $\mathbf{Y}$ & $\mathbf{Y}$ & $\mathbf{Y}$ & $Y$ & $Y$ & $\mathbf{Y} \underline{\mathrm{N}}$ & $\mathrm{LS}_{\mathrm{u}}$ & $\mathrm{SS}_{\mathrm{u}}$ & $\mathbf{Y}$ & $Y$ & $\mathbf{Y}$ & $\mathbf{Y}$ & $\mathbf{Y}$ & $Y$ & $\underline{N}$ & $\underline{N}$ & $\underline{N}$ & $\underline{N}$ \\
\hline
\end{tabular}


In Table 5-37, when considering $S$ galaxies as primary against other primary morphologies with no secondary filter, dissimilarity is found consistently for $E$ and lenticular L. For comparisons against barred spiral primaries no dissimilarity is found at any fitting range. For unbarred spirals similarity is consistently found below $5 \mathrm{Mpc}$, while generally dissimilarity is found above $5 \mathrm{Mpc}$. $\mathrm{S}$ galaxies have environments different from early-type galaxies.

Table 5-37 3D KS Test Results - comparing two runs having the same secondary filter and same fitting range filter with different primary filters - limited to spiral primaries in Run1.

$\mathrm{Y}=$ dissimilar to the $99 \%$ level. $\mathrm{N}=$ not dissimilar to the $99 \%$ level

\begin{tabular}{|c|c|c|c|c|c|c|c|c|c|c|c|c|c|c|c|c|c|c|c|c|c|c|c|}
\hline \multicolumn{12}{|c|}{ North } & \multicolumn{12}{|c|}{ South } \\
\hline \multirow[b]{2}{*}{ Run1 } & \multirow[b]{2}{*}{ Run2 } & \multicolumn{10}{|c|}{ Fitting Range (Mpc) } & \multicolumn{12}{|c|}{ Fitting Range (Mpc) } \\
\hline & & 1 & 2 & 3 & 4 & 5 & 10 & 15 & 20 & 25 & 30 & Run1 & Run2 & 1 & 2 & 3 & 4 & 5 & 10 & 15 & 20 & 25 & 30 \\
\hline SA & $A A$ & $\mathrm{Y}$ & $Y$ & $\mathrm{Y}$ & $\mathrm{Y}$ & $\mathrm{Y}$ & $\mathrm{Y}$ & $\mathrm{Y}$ & $\mathrm{Y}$ & $Y$ & $\bar{Y}$ & SA & $\mathrm{AA}$ & $Y$ & $\mathrm{Y}$ & $\mathrm{Y}$ & $\mathrm{Y}$ & $\mathrm{Y}$ & $\mathrm{Y}$ & $\mathrm{Y}$ & $\mathrm{Y}$ & $\mathrm{Y}$ & $\mathrm{Y}$ \\
\hline SA & EA & $\mathbf{Y}$ & $\mathbf{Y}$ & $\mathbf{Y}$ & $\mathbf{Y}$ & $\mathbf{Y}$ & $\mathbf{Y}$ & $\mathbf{Y}$ & $\mathbf{Y}$ & $\mathbf{Y}$ & $\mathbf{Y}$ & SA & EA & $\mathbf{Y}$ & $Y$ & $\mathbf{Y}$ & $\mathbf{Y}$ & $\mathbf{Y}$ & $\mathbf{Y}$ & $\mathbf{Y}$ & $Y$ & $\mathrm{Y}$ & $\mathbf{Y}$ \\
\hline SA & LA & $\mathbf{Y}$ & $\mathbf{Y}$ & $\mathbf{Y}$ & $\mathbf{Y}$ & $\mathbf{Y}$ & $\mathbf{Y}$ & $\mathbf{Y}$ & $\mathbf{Y}$ & $\mathrm{Y}$ & $\mathbf{Y}$ & SA & LA & $\mathbf{Y}$ & $\mathbf{Y}$ & $\mathbf{Y}$ & $\mathrm{Y}$ & $\mathbf{Y}$ & $\mathbf{Y}$ & $\mathbf{Y}$ & $\mathrm{Y}$ & $\mathbf{Y}$ & $Y$ \\
\hline $\mathrm{SA}$ & $S_{b} A$ & $\underline{N}$ & $\underline{N}$ & $\underline{N}$ & $\underline{N}$ & $\underline{N}$ & $\underline{N}$ & $\underline{N}$ & $\underline{N}$ & $\underline{N}$ & $\underline{N}$ & SA & $\mathrm{S}_{\mathrm{b}} \mathrm{A}$ & $\underline{N}$ & $\underline{\mathrm{N}}$ & $\underline{N}$ & $\underline{N}$ & $\underline{\mathrm{N}}$ & $\underline{N}$ & $\underline{\mathrm{N}}$ & $\underline{N}$ & $\underline{\mathrm{N}}$ & $\underline{\mathrm{N}}$ \\
\hline $\mathrm{SA}$ & $S_{u} A$ & $\underline{N}$ & $\underline{N}$ & $\underline{N}$ & $\underline{N}$ & $\mathbf{Y}$ & $Y$ & $\underline{N}$ & $Y$ & $Y$ & $\underline{N}$ & SA & $S_{u} A$ & $\underline{N}$ & $\underline{N}$ & $\underline{N}$ & $\underline{N}$ & $\mathbf{Y}$ & $\mathbf{Y}$ & $\mathbf{Y}$ & $Y$ & $\mathbf{Y}$ & $\underline{N}$ \\
\hline SE & $\mathrm{EE}$ & $Y$ & $Y$ & $Y$ & $Y$ & $Y$ & $Y$ & $Y$ & $Y$ & $Y$ & $Y$ & SE & EE & $Y$ & $Y$ & $Y$ & $Y$ & $Y$ & $Y$ & $Y$ & $Y$ & $Y$ & $Y$ \\
\hline SE & LE & $\underline{N}$ & $Y$ & $Y$ & $Y$ & $Y$ & $Y$ & $Y$ & $Y$ & $Y$ & $Y$ & SE & LE & $Y$ & $Y$ & $Y$ & $Y$ & $Y$ & $Y$ & $Y$ & $Y$ & $Y$ & $Y$ \\
\hline SE & $\mathrm{S}_{\mathrm{b}} \mathrm{E}$ & $\underline{N}$ & $\underline{N}$ & $\underline{N}$ & $\underline{N}$ & $\underline{N}$ & $\underline{N}$ & $\underline{N}$ & $\underline{N}$ & $\underline{N}$ & $\underline{\mathrm{N}}$ & SE & $\mathrm{S}_{\mathrm{b}} \mathrm{E}$ & $\underline{N}$ & $\underline{N}$ & $\underline{N}$ & $\underline{N}$ & $\underline{N}$ & $\underline{\mathrm{N}}$ & $\underline{N}$ & $\underline{N}$ & $\underline{\mathrm{N}}$ & $\underline{N}$ \\
\hline SE & $S_{u} E$ & $\underline{N}$ & $\underline{N}$ & $\underline{N}$ & $\underline{N}$ & $\underline{N}$ & $\underline{N}$ & $\underline{N}$ & $\underline{\mathrm{N}}$ & $\underline{N}$ & $\underline{N}$ & SE & $S_{u} E$ & $\underline{N}$ & $\underline{\mathrm{N}}$ & $\underline{\mathrm{N}}$ & $\underline{N}$ & $\underline{N}$ & $\underline{\mathrm{N}}$ & $\mathbf{Y}$ & $Y$ & $\mathbf{Y}$ & $\mathbf{Y}$ \\
\hline $\mathrm{SL}$ & EL & $Y$ & $Y$ & $Y$ & $Y$ & $Y$ & $Y$ & $Y$ & $Y$ & $Y$ & $Y$ & $\mathrm{SL}$ & EL & $Y$ & $Y$ & $Y$ & $Y$ & $Y$ & $Y$ & $Y$ & $\mathrm{Y}$ & $Y$ & $Y$ \\
\hline $\mathrm{SL}$ & LL & $Y$ & $Y$ & $Y$ & $Y$ & $Y$ & $Y$ & $Y$ & $Y$ & $Y$ & $Y$ & SL & LL & $Y$ & $Y$ & $Y$ & $Y$ & $Y$ & $Y$ & $Y$ & $Y$ & $Y$ & $Y$ \\
\hline SL & $S_{b} L$ & $\underline{N}$ & $\underline{N}$ & $\underline{N}$ & $\underline{N}$ & $\underline{N}$ & $\underline{N}$ & $\underline{N}$ & $\underline{N}$ & $\underline{N}$ & $\underline{N}$ & SL & $S_{b} L$ & $\underline{N}$ & $\underline{N}$ & $\underline{N}$ & $\underline{N}$ & $\underline{N}$ & $\underline{N}$ & $\underline{N}$ & $\underline{N}$ & $\underline{\mathrm{N}}$ & $\underline{N}$ \\
\hline SL & $S_{u} L$ & $\underline{N}$ & $\underline{N}$ & $\underline{N}$ & $\underline{N}$ & $\underline{N}$ & $\underline{N}$ & $\underline{N}$ & $\underline{N}$ & $\underline{N}$ & $\underline{\mathrm{N}}$ & SL & $S_{u} L$ & $\underline{N}$ & $\underline{N}$ & $\underline{N}$ & $\underline{N}$ & $\underline{N}$ & $\underline{N}$ & $\underline{N}$ & $\mathbf{Y}$ & $\mathbf{Y}$ & $\mathbf{Y}$ \\
\hline SS & ES & $Y$ & $Y$ & $Y$ & $Y$ & $Y$ & $Y$ & $Y$ & $Y$ & $Y$ & $Y$ & SS & ES & $Y$ & $Y$ & $Y$ & $Y$ & $Y$ & $Y$ & $Y$ & $Y$ & $Y$ & $Y$ \\
\hline SS & LS & $\underline{N}$ & $\mathbf{Y}$ & $Y$ & $\mathbf{Y}$ & $\mathbf{Y}$ & $Y$ & $Y$ & $Y$ & $Y$ & $\mathbf{Y}$ & SS & LS & $Y$ & $\mathbf{Y}$ & $\mathbf{Y}$ & $Y$ & $Y$ & $Y$ & $\mathbf{Y}$ & $Y$ & $\mathbf{Y}$ & $Y$ \\
\hline SS & $S_{b} S$ & $\underline{N}$ & $\underline{N}$ & $\underline{N}$ & $\underline{\mathrm{N}}$ & $\underline{\mathrm{N}}$ & $\underline{N}$ & $\underline{N}$ & $\underline{N}$ & $\underline{\mathrm{N}}$ & $\underline{\mathrm{N}}$ & SS & $S_{b} S$ & $\underline{N}$ & $\underline{N}$ & $\underline{N}$ & $\underline{N}$ & $\underline{N}$ & $\underline{N}$ & $\underline{\mathrm{N}}$ & $\underline{N}$ & $\underline{N}$ & $\underline{N}$ \\
\hline SS & $\mathrm{S}_{\mathrm{u}} \mathrm{S}$ & $\underline{N}$ & $\underline{N}$ & $\underline{\mathrm{N}}$ & $Y$ & $Y$ & $\underline{\mathrm{N}}$ & $\underline{N}$ & $\underline{\mathrm{N}}$ & $\underline{N}$ & $\underline{N}$ & SS & $\mathrm{S}_{\mathrm{u}} \mathrm{S}$ & $\underline{N}$ & $\underline{N}$ & $\underline{N}$ & $\underline{N}$ & $\underline{N}$ & $\underline{N}$ & $\underline{N}$ & $\underline{N}$ & $\underline{N}$ & $\underline{N}$ \\
\hline $\mathrm{SS}_{\mathrm{b}}$ & $\mathrm{ES}_{\mathrm{b}}$ & $\underline{N}$ & $\mathbf{Y}$ & $Y$ & $Y$ & $\mathbf{Y}$ & $Y$ & $Y$ & $Y$ & $Y$ & $\mathbf{Y}$ & $\mathrm{SS}_{\mathrm{b}}$ & $\mathrm{ES}_{\mathrm{b}}$ & $\underline{N}$ & $\underline{N}$ & $\mathbf{Y}$ & $Y$ & $\mathbf{Y}$ & $Y$ & $Y$ & $\mathbf{Y}$ & $\underline{N}$ & $\underline{N}$ \\
\hline $\mathrm{SS}_{\mathrm{b}}$ & $\mathrm{LS}_{\mathrm{b}}$ & $\underline{N}$ & $Y$ & $Y$ & $Y$ & $Y$ & $Y$ & $Y$ & $Y$ & $Y$ & $Y$ & $\mathrm{SS}_{\mathrm{b}}$ & $\mathrm{LS}_{\mathrm{b}}$ & $\underline{N}$ & $\underline{N}$ & $\underline{N}$ & $Y$ & $Y$ & $\underline{N}$ & $Y$ & $\underline{N}$ & $Y$ & $\underline{N}$ \\
\hline$S S_{b}$ & $S_{b} S_{b}$ & $\underline{N}$ & $\underline{N}$ & $\underline{N}$ & $Y$ & $\underline{N}$ & $Y$ & $Y$ & $Y$ & $Y$ & $\mathbf{Y}$ & $\mathrm{SS}_{\mathrm{b}}$ & $S_{b} S_{b}$ & $\underline{N}$ & $\underline{N}$ & $\underline{N}$ & $\underline{N}$ & $\underline{N}$ & $\underline{N}$ & $\underline{N}$ & $\underline{N}$ & $\underline{N}$ & $\underline{N}$ \\
\hline$S S_{b}$ & $S_{u} S_{b}$ & $\underline{N}$ & $\underline{N}$ & $Y$ & $\underline{N}$ & $\underline{N}$ & $\underline{N}$ & $\underline{N}$ & $\underline{N}$ & $\underline{N}$ & $\underline{N}$ & $\mathrm{SS}_{\mathrm{b}}$ & $S_{u} S_{b}$ & $\underline{N}$ & $\underline{N}$ & $\underline{N}$ & $\underline{N}$ & $\underline{N}$ & $\underline{N}$ & $Y$ & $Y$ & $\mathbf{Y}$ & $Y$ \\
\hline $\mathrm{SS}_{\mathrm{u}}$ & $\mathrm{ES}_{\mathrm{u}}$ & $\underline{N}$ & $Y$ & $\underline{N}$ & $Y$ & $Y$ & $Y$ & $Y$ & $Y$ & $\mathrm{Y}$ & $Y$ & $\mathrm{SS}_{\mathrm{u}}$ & $\mathrm{ES}_{\mathrm{u}}$ & $\underline{N}$ & $Y$ & $Y$ & $Y$ & $Y$ & $Y$ & $\underline{N}$ & $\underline{N}$ & $\underline{N}$ & $\underline{N}$ \\
\hline $\mathrm{SS}_{\mathrm{u}}$ & $\mathrm{LS}_{\mathrm{u}}$ & $\underline{N}$ & $\underline{N}$ & $Y$ & $Y$ & $Y$ & $Y$ & $Y$ & $Y$ & $Y$ & $\underline{N}$ & $\mathrm{SS}_{\mathrm{u}}$ & $\mathrm{LS}_{\mathrm{u}}$ & $Y$ & $Y$ & $Y$ & $Y$ & $Y$ & $Y$ & $\underline{N}$ & $\underline{N}$ & $\underline{N}$ & $\underline{N}$ \\
\hline
\end{tabular}




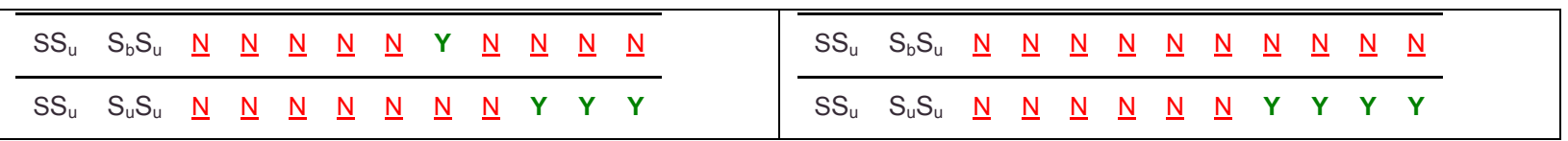


In Table 5-38, when considering $S_{b}$ primaries against other primary morphologies with no secondary morphology filters, dissimilarity is found for $E$ and $L$ primaries. No dissimilarity is found when compared to $S$ primaries. Similarity is consistently found in the South for $S_{u}$ primaries, while in the North dissimilarity is found for most fitting ranges.

Table 5-38 3D KS Test Results - comparing two runs having the same secondary filter and same fitting range filter with different primary filters - limited to unbarred spiral primaries in Run1.

$\mathrm{Y}=$ dissimilar to the $99 \%$ level. $\mathrm{N}=$ not dissimilar to the $99 \%$ level

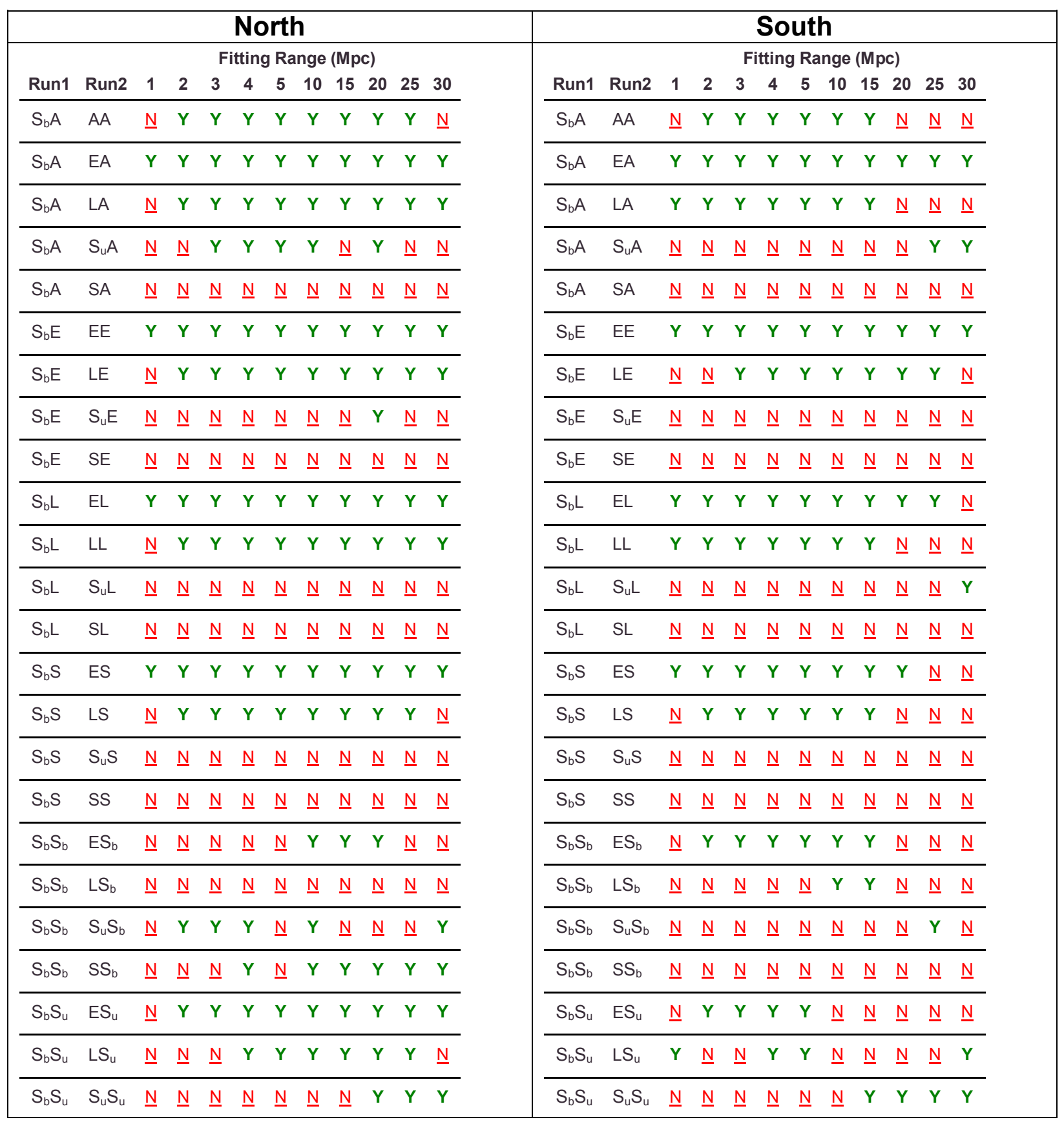




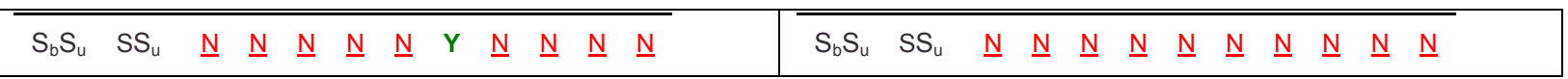


When considering $S_{u}$ primaries vs. other primary morphologies with no secondary morphology filter in Table 5-39, dissimilarity is found for $E$ and $L$ primaries. Similarity is found consistently below $5 \mathrm{Mpc}$ for $\mathrm{S}$ primaries. For $\mathrm{S}_{\mathrm{b}}$ primaries dissimilarity is found for all but two fitting ranges (25 Mpc and $30 \mathrm{Mpc}$ ) in the South. In the North, unbarred spirals do not show a consistent pattern. This suggests that as primary galaxies unbarred and barred spirals share somewhat similar environments.

Table 5-39 3D KS Test Results - comparing two runs having the same secondary filter and same fitting range filter with different primary filters - limited to unbarred spiral primaries in Run1. $Y=$ dissimilar to the $99 \%$ level. $N=$ not dissimilar to the $99 \%$ level

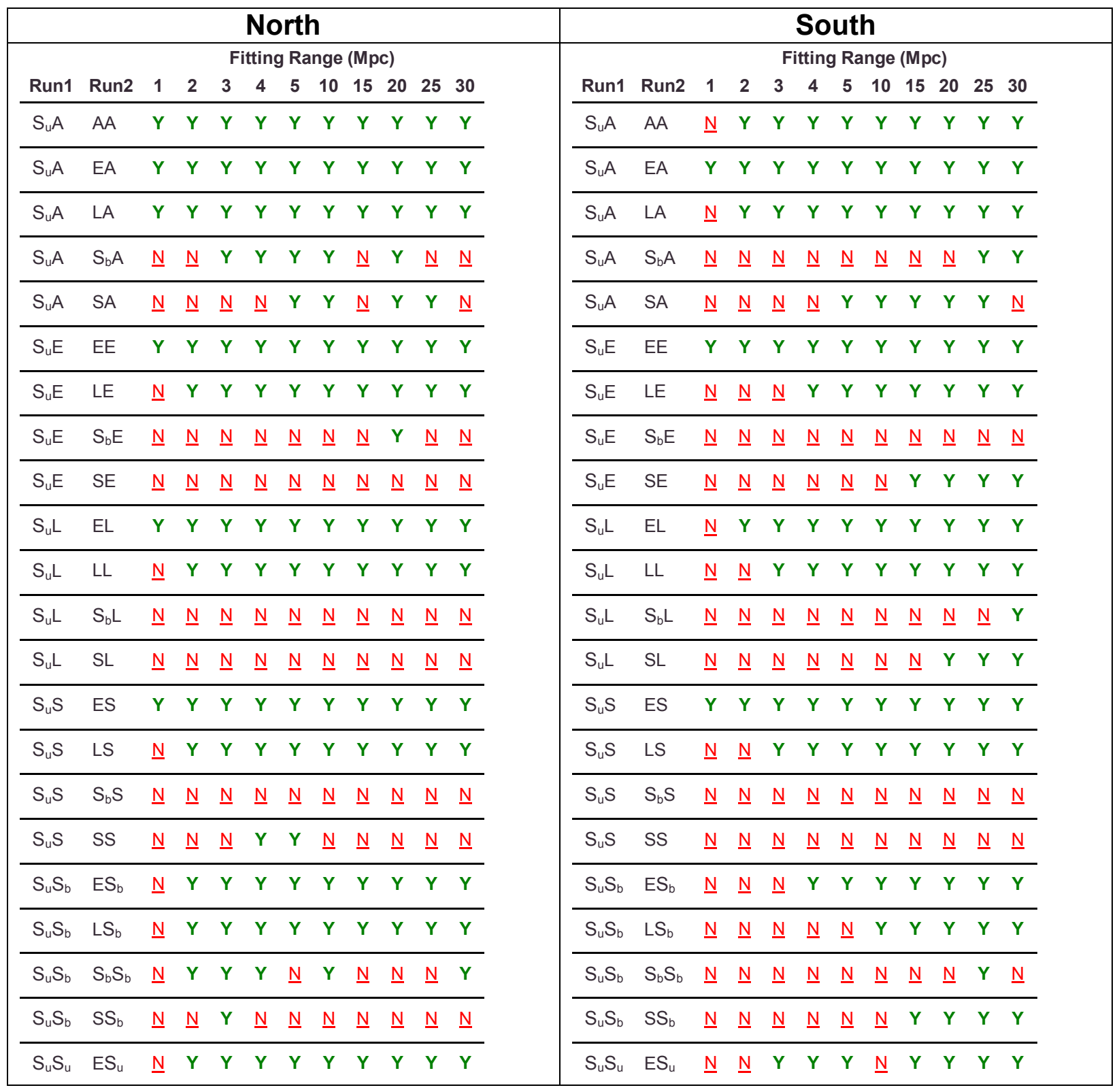




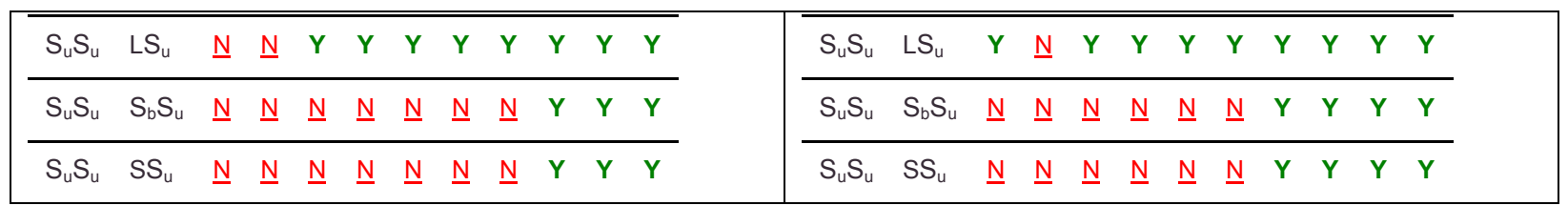

\subsubsection{Conclusions}

As seen above in 2D ellipticals and lenticulars as primary galaxies generally share similar environments. This suggests that secular evolution, not the environment, plays a factor in formation of $E$ or $L$ galaxies.

Also seen here is general similarity between barred and unbarred spirals. Again this suggests some relationship between barred and unbarred spirals. Finally, spirals show consistent similarity in environment for barred spirals, but some dissimilarity is found for unbarred spirals. This suggest that barred spirals are more closely related to spirals than unbarred spirals. 


\subsection{Velocity Bins}

A series of PD runs are created in $3 D$ in two groups. The first group is created with a velocity bin of $3000-6000 \mathrm{~km} / \mathrm{s}$. The second group is created with a velocity bin of $9000-12000 \mathrm{~km} / \mathrm{s}$. Each run has a sibling in the other group using the same primary and secondary morphology as well as the same fitting range. These sibling runs are then compared in KS tests. The count of PD values found given the filters applied is presented as well for both of the bins.

The fitting ranges used mirror those used above. Small scale is defined as 1 to 5 $\mathrm{Mpc}$ in $1 \mathrm{Mpc}$ increments. Large scale is 5 to $30 \mathrm{Mpc}$ in $5 \mathrm{Mpc}$ increments.

In all of the following tables $\mathrm{Y}$ indicates that the $p$ value from the $\mathrm{KS}$ test is < 0.01 . This means that there is less than a $1 \%$ chance that the two compared data sets are drawn from the same distribution. That is, the compared data sets are statistically dissimilar to the $99 \%$ level. A value of $\mathrm{N}$ indicates the inverse. That is, the datasets are not statistically dissimilar to the $99 \%$ level. 
In Table 5-40, for runs without morphological filtering dissimilarity is noted between the velocity bins at fitting ranges of $3 \mathrm{Mpc}$ and larger.

Table 5-40 KS Tests For Velocity Bins of 3000-6000 km/s and $9000-12000 \mathrm{~km} / \mathrm{s}$ limited to all primaries $\mathrm{Y}=$ dissimilar to the $99 \%$ level $\mathrm{N}=$ not dissimilar to the $99 \%$ level

\begin{tabular}{|c|c|c|c|c|c|c|c|c|c|}
\hline \multicolumn{5}{|c|}{ North } & \multicolumn{5}{|c|}{ South } \\
\hline Run & $\begin{array}{l}\text { Fitting } \\
\text { Range } \\
\text { (Mpc) }\end{array}$ & $\begin{array}{c}\text { Num. } \\
\text { PD } \\
\text { Values } \\
\text { in } \\
3000 \\
\text { Bin } \\
\end{array}$ & $\begin{array}{c}\text { Num. } \\
\text { PD } \\
\text { Values } \\
\text { in } \\
9000 \\
\text { Bin } \\
\end{array}$ & Dissimilar & Run & $\begin{array}{l}\text { Fitting } \\
\text { Range } \\
\text { (Mpc) }\end{array}$ & $\begin{array}{c}\text { Num. } \\
\text { PD } \\
\text { Values } \\
\text { in } \\
3000 \\
\text { Bin } \\
\end{array}$ & $\begin{array}{c}\text { Num. } \\
\text { PD } \\
\text { Values } \\
\text { in } \\
9000 \\
\text { Bin } \\
\end{array}$ & Dissimilar \\
\hline$A A$ & 1 & 1672 & 707 & $\underline{N}$ & AA & 1 & 2165 & 635 & $\underline{N}$ \\
\hline $\mathrm{AA}$ & 2 & 2917 & 1630 & $\underline{N}$ & AA & 2 & 3563 & 1589 & $\underline{N}$ \\
\hline $\mathrm{AA}$ & 3 & 3467 & 2551 & $Y$ & $\mathrm{AA}$ & 3 & 4261 & 2422 & $Y$ \\
\hline$A A$ & 4 & 3617 & 3211 & $Y$ & $\mathrm{AA}$ & 4 & 4471 & 3044 & $Y$ \\
\hline AA & 5 & 3645 & 3683 & $Y$ & $\mathrm{AA}$ & 5 & 4493 & 3538 & $Y$ \\
\hline$A A$ & 10 & 3207 & 4309 & $Y$ & AA & 10 & 4033 & 4152 & $Y$ \\
\hline$A A$ & 15 & 2701 & 4266 & $Y$ & $\mathrm{AA}$ & 15 & 3510 & 4095 & $Y$ \\
\hline$A A$ & 20 & 2217 & 4135 & Y & $\mathrm{AA}$ & 20 & 3008 & 4005 & Y \\
\hline$A A$ & 25 & 1798 & 3985 & $\mathrm{Y}$ & AA & 25 & 2491 & 3880 & $Y$ \\
\hline$A A$ & 30 & 1528 & 3824 & $Y$ & $\mathrm{AA}$ & 30 & 2019 & 3746 & $Y$ \\
\hline
\end{tabular}


Of interest when considering elliptical primaries in Table 5-41, in the north similarity is found at $15 \mathrm{Mpc}$ and above when no secondary morphology filter is applied. No dissimilarity is found in the north for EE and only at 25 and $30 \mathrm{Mpc}$ for EL. Interestingly dissimilarity is found for in the north for $E S$ at $10 \mathrm{Mpc}$ and up, for $\mathrm{ES}_{\mathrm{b}}$ at 20 $\mathrm{Mpc}$ and up, and for $\mathrm{ES}_{\mathrm{u}}$ at $15 \mathrm{Mpc}$ and up. This suggests that the clustering of latetype galaxies around $\mathrm{E}$ primaries evolves over time.

Table 5-41 KS Tests For Velocity Bins of $3000-6000 \mathrm{~km} / \mathrm{s}$ and $9000-12000 \mathrm{~km} / \mathrm{s}$ limited to elliptical primaries

$\mathrm{Y}=$ dissimilar to the $99 \%$ level $\mathrm{N}=$ not dissimilar to the $99 \%$ level

\begin{tabular}{|c|c|c|c|c|c|c|c|c|c|}
\hline Run & $\begin{array}{l}\text { Fitting } \\
\text { Range } \\
\text { (Mpc) }\end{array}$ & $\begin{array}{l}\text { Num. } \\
\text { PD } \\
\text { Values } \\
\text { in } \\
3000 \\
\text { Bin } \\
\end{array}$ & $\begin{array}{l}\text { Num. } \\
\text { PD } \\
\text { Values } \\
\text { in } \\
9000 \\
\text { Bin } \\
\end{array}$ & Dissimilar & Run & $\begin{array}{l}\text { Fitting } \\
\text { Range } \\
\text { (Mpc) }\end{array}$ & $\begin{array}{l}\text { Num. } \\
\text { PD } \\
\text { Values } \\
\text { in } \\
3000 \\
\text { Bin } \\
\end{array}$ & $\begin{array}{l}\text { Num. } \\
\text { PD } \\
\text { Values } \\
\text { in } \\
9000 \\
\text { Bin } \\
\end{array}$ & Dissimilar \\
\hline EA & 1 & 231 & 167 & $\underline{N}$ & EA & 1 & 299 & 150 & $\underline{N}$ \\
\hline EA & 2 & 355 & 318 & $\underline{N}$ & $E A$ & 2 & 451 & 281 & $\underline{N}$ \\
\hline EA & 3 & 405 & 484 & $\underline{N}$ & EA & 3 & 508 & 395 & $Y$ \\
\hline EA & 4 & 417 & 586 & $Y$ & $E A$ & 4 & 515 & 454 & $Y$ \\
\hline EA & 5 & 413 & 652 & $Y$ & EA & 5 & 514 & 517 & $Y$ \\
\hline EA & 10 & 358 & 712 & $Y$ & EA & 10 & 436 & 576 & $Y$ \\
\hline EA & 15 & 302 & 701 & $\underline{N}$ & EA & 15 & 363 & 559 & $Y$ \\
\hline EA & 20 & 250 & 681 & $\underline{N}$ & EA & 20 & 312 & 546 & $Y$ \\
\hline EA & 25 & 212 & 654 & $\underline{N}$ & EA & 25 & 250 & 533 & $Y$ \\
\hline EA & 30 & 175 & 633 & $\underline{N}$ & EA & 30 & 195 & 511 & $Y$ \\
\hline EE & 1 & 35 & 22 & $\underline{N}$ & EE & 1 & 70 & 19 & $\underline{N}$ \\
\hline EE & 2 & 93 & 58 & $\underline{N}$ & EE & 2 & 134 & 58 & $\underline{\underline{N}}$ \\
\hline EE & 3 & 164 & 101 & $\underline{N}$ & EE & 3 & 198 & 105 & $\underline{N}$ \\
\hline EE & 4 & 222 & 150 & $\underline{N}$ & EE & 4 & 275 & 150 & $\underline{N}$ \\
\hline EE & 5 & 258 & 210 & $\underline{N}$ & EE & 5 & 328 & 188 & $\underline{N}$ \\
\hline EE & 10 & 329 & 502 & $\underline{N}$ & EE & 10 & 396 & 371 & $\underline{N}$ \\
\hline EE & 15 & 297 & 641 & $\underline{N}$ & EE & 15 & 352 & 479 & $Y$ \\
\hline EE & 20 & 249 & 657 & $\underline{N}$ & EE & 20 & 311 & 509 & $Y$ \\
\hline EE & 25 & 212 & 647 & $\underline{N}$ & EE & 25 & 250 & 519 & $Y$ \\
\hline EE & 30 & 175 & 630 & $\underline{N}$ & EE & 30 & 195 & 504 & $Y$ \\
\hline $\mathrm{EL}$ & 1 & 50 & 7 & $\underline{\underline{N}}$ & $E L$ & 1 & 98 & 5 & $\underline{N}$ \\
\hline EL & 2 & 105 & 27 & $\underline{N}$ & $E L$ & 2 & 192 & 21 & $\underline{N}$ \\
\hline EL & 3 & 168 & 40 & $\underline{N}$ & $E L$ & 3 & 283 & 58 & $\underline{N}$ \\
\hline EL & 4 & 214 & 62 & $\underline{N}$ & $E L$ & 4 & 352 & 85 & $\underline{N}$ \\
\hline EL & 5 & 247 & 107 & $\underline{N}$ & $E L$ & 5 & 393 & 114 & $\underline{N}$ \\
\hline EL & 10 & 326 & 342 & $\underline{N}$ & $E L$ & 10 & 421 & 317 & $\underline{N}$ \\
\hline
\end{tabular}




\begin{tabular}{|c|c|c|c|c|c|c|c|c|c|}
\hline Run & $\begin{array}{l}\text { Fitting } \\
\text { Range } \\
\text { (Mpc) }\end{array}$ & $\begin{array}{l}\text { Num. } \\
\text { PD } \\
\text { Values } \\
\text { in } \\
3000 \\
\text { Bin }\end{array}$ & $\begin{array}{l}\text { Num. } \\
\text { PD } \\
\text { Values } \\
\text { in } \\
9000 \\
\text { Bin }\end{array}$ & Dissimilar & Run & $\begin{array}{l}\text { Fitting } \\
\text { Range } \\
\text { (Mpc) }\end{array}$ & $\begin{array}{l}\text { Num. } \\
\text { PD } \\
\text { Values } \\
\text { in } \\
3000 \\
\text { Bin }\end{array}$ & $\begin{array}{l}\text { Num. } \\
\text { PD } \\
\text { Values } \\
\text { in } \\
9000 \\
\text { Bin }\end{array}$ & Dissimilar \\
\hline EL & 15 & 296 & 517 & $\underline{\mathrm{N}}$ & EL & 15 & 361 & 437 & $Y$ \\
\hline EL & 20 & 249 & 605 & $\underline{\mathrm{N}}$ & EL & 20 & 312 & 497 & $\mathbf{Y}$ \\
\hline EL & 25 & 212 & 635 & $Y$ & EL & 25 & 250 & 514 & $Y$ \\
\hline EL & 30 & 175 & 622 & $Y$ & EL & 30 & 195 & 507 & Y \\
\hline ES & 1 & 126 & 7 & $\underline{N}$ & ES & 1 & 163 & 11 & $\underline{N}$ \\
\hline ES & 2 & 264 & 53 & $\underline{N}$ & ES & 2 & 318 & 53 & $\underline{N}$ \\
\hline ES & 3 & 356 & 131 & $\underline{N}$ & ES & 3 & 439 & 97 & $\underline{N}$ \\
\hline ES & 4 & 390 & 205 & $\underline{\mathrm{N}}$ & ES & 4 & 490 & 152 & $\underline{\mathrm{N}}$ \\
\hline ES & 5 & 395 & 273 & $\underline{N}$ & ES & 5 & 502 & 221 & $Y$ \\
\hline ES & 10 & 357 & 562 & $Y$ & ES & 10 & 434 & 443 & $Y$ \\
\hline ES & 15 & 302 & 675 & $\mathbf{Y}$ & ES & 15 & 363 & 525 & $Y$ \\
\hline ES & 20 & 250 & 676 & $Y$ & ES & 20 & 312 & 541 & $\mathbf{Y}$ \\
\hline ES & 25 & 212 & 653 & $Y$ & ES & 25 & 250 & 533 & $\mathrm{Y}$ \\
\hline ES & 30 & 175 & 633 & $\mathbf{Y}$ & ES & 30 & 195 & 511 & $Y$ \\
\hline$E S_{b}$ & 1 & 11 & 0 & $\mathrm{~N} / \mathrm{A}$ & $E S_{b}$ & 1 & 4 & 0 & $\mathrm{~N} / \mathrm{A}$ \\
\hline $\mathrm{ES}_{\mathrm{b}}$ & 2 & 32 & 7 & $\underline{N}$ & $E S_{b}$ & 2 & 55 & 1 & $\underline{\mathrm{N}}$ \\
\hline $\mathrm{ES}_{\mathrm{b}}$ & 3 & 80 & 17 & $\underline{N}$ & $E S_{b}$ & 3 & 126 & 4 & $\underline{N}$ \\
\hline$E S_{b}$ & 4 & 129 & 41 & $\underline{\mathrm{N}}$ & $E S_{b}$ & 4 & 193 & 11 & $\underline{\mathrm{N}}$ \\
\hline$E S_{b}$ & 5 & 205 & 61 & $\underline{\mathrm{N}}$ & $E S_{b}$ & 5 & 250 & 17 & $\underline{\mathrm{N}}$ \\
\hline $\mathrm{ES}_{\mathrm{b}}$ & 10 & 320 & 166 & $Y$ & $E S_{b}$ & 10 & 343 & 64 & $\underline{\mathrm{N}}$ \\
\hline$E S_{b}$ & 15 & 293 & 246 & $\underline{\mathrm{N}}$ & $E S_{b}$ & 15 & 333 & 148 & $Y$ \\
\hline $\mathrm{ES}_{\mathrm{b}}$ & 20 & 246 & 361 & $Y$ & $E S_{b}$ & 20 & 306 & 209 & $Y$ \\
\hline$E S_{b}$ & 25 & 211 & 450 & $Y$ & $E S_{b}$ & 25 & 249 & 284 & $Y$ \\
\hline $\mathrm{ES}_{\mathrm{b}}$ & 30 & 175 & 537 & $Y$ & $E S_{b}$ & 30 & 195 & 325 & $Y$ \\
\hline $\mathrm{ES}_{\mathrm{u}}$ & 1 & 2 & 0 & $\mathrm{~N} / \mathrm{A}$ & $E S_{u}$ & 1 & 7 & 0 & $\mathrm{~N} / \mathrm{A}$ \\
\hline $\mathrm{ES}_{\mathrm{u}}$ & 2 & 14 & 0 & $\mathrm{~N} / \mathrm{A}$ & $\mathrm{ES}_{\mathrm{u}}$ & 2 & 66 & 0 & $\mathrm{~N} / \mathrm{A}$ \\
\hline $\mathrm{ES}_{\mathrm{u}}$ & 3 & 40 & 1 & $\underline{\mathrm{N}}$ & $\mathrm{ES}_{\mathrm{u}}$ & 3 & 122 & 2 & $Y$ \\
\hline $\mathrm{ES}_{\mathrm{u}}$ & 4 & 93 & 3 & $\underline{N}$ & $\mathrm{ES}_{\mathrm{u}}$ & 4 & 171 & 5 & $Y$ \\
\hline $\mathrm{ES}_{\mathrm{u}}$ & 5 & 146 & 9 & $\underline{\mathrm{N}}$ & $\mathrm{ES}_{\mathrm{u}}$ & 5 & 224 & 6 & $\underline{N}$ \\
\hline $\mathrm{ES}_{\mathrm{u}}$ & 10 & 266 & 89 & $\underline{\mathrm{N}}$ & $\mathrm{ES}_{\mathrm{u}}$ & 10 & 278 & 23 & $\underline{N}$ \\
\hline $\mathrm{ES}_{\mathrm{u}}$ & 15 & 276 & 225 & $Y$ & $E S_{u}$ & 15 & 279 & 46 & $Y$ \\
\hline $\mathrm{ES}_{\mathrm{u}}$ & 20 & 243 & 366 & $\mathbf{Y}$ & $\mathrm{ES}_{\mathrm{u}}$ & 20 & 270 & 98 & $Y$ \\
\hline $\mathrm{ES}_{\mathrm{u}}$ & 25 & 212 & 443 & $Y$ & $E S_{u}$ & 25 & 229 & 140 & $\mathbf{Y}$ \\
\hline $\mathrm{ES}_{\mathrm{u}}$ & 30 & 175 & 486 & $Y$ & $\mathrm{ES}_{\mathrm{u}}$ & 30 & 191 & 169 & Y \\
\hline
\end{tabular}


For lenticular primaries in the north Table 5-42, similarity is found at $15 \mathrm{Mpc}$ and larger. Dissimilarity is not found in the north for LE at any fitting range. Dissimilarity is found in the north for LL at $20 \mathrm{Mpc}$ and above. Finally in the north LS show dissimilarity at $10 \mathrm{Mpc}$ and $u p, \mathrm{LS}_{\mathrm{b}}$ at $30 \mathrm{Mpc}$ and $\mathrm{LS}_{\mathrm{u}}$ at $25 \mathrm{Mpc}$ and up. This suggests that latetype clustering around $L$ primaries is affected by velocity. The introduction of dissimilarity does not seem to be driven by larger fitting ranges (established as a factor previously in this paper), since the LA comparisons in the north are all similar at $15 \mathrm{Mpc}$ and up. It appears that environmental evolution over this difference in time does not introduce dissimilarity for the disk-type environments of lenticular primaries.

Table 5-42 KS Tests For Velocity Bins of $3000-6000$ km/s and $9000-12000$ km/s limited to lenticular primaries

$\mathrm{Y}=$ dissimilar to the $99 \%$ level $\mathrm{N}=$ not dissimilar to the $99 \%$ level

\begin{tabular}{|c|c|c|c|c|}
\hline Run & $\begin{array}{l}\text { Fitting } \\
\text { Range } \\
\text { (Mpc) }\end{array}$ & $\begin{array}{c}\text { Num. } \\
\text { PD } \\
\text { Values } \\
\text { in } \\
3000 \\
\text { Bin }\end{array}$ & $\begin{array}{c}\text { Num. } \\
\text { PD } \\
\text { Values } \\
\text { in } \\
9000 \\
\text { Bin } \\
\end{array}$ & Dissimilar \\
\hline LA & 1 & 358 & 78 & $\underline{\mathrm{N}}$ \\
\hline LA & 2 & 539 & 177 & $\underline{\mathrm{N}}$ \\
\hline LA & 3 & 609 & 267 & $Y$ \\
\hline LA & 4 & 622 & 333 & $Y$ \\
\hline LA & 5 & 631 & 371 & $Y$ \\
\hline LA & 10 & 569 & 420 & $Y$ \\
\hline LA & 15 & 462 & 418 & $\underline{\mathrm{N}}$ \\
\hline LA & 20 & 361 & 412 & $\underline{N}$ \\
\hline LA & 25 & 272 & 397 & $\underline{\underline{N}}$ \\
\hline LA & 30 & 236 & 384 & $\underline{\mathrm{N}}$ \\
\hline LE & 1 & 81 & 15 & $\underline{\mathrm{N}}$ \\
\hline LE & 2 & 168 & 33 & $\underline{\mathrm{N}}$ \\
\hline LE & 3 & 258 & 59 & $\underline{\mathrm{N}}$ \\
\hline LE & 4 & 325 & 83 & $\underline{\mathrm{N}}$ \\
\hline LE & 5 & 386 & 120 & $\underline{\underline{N}}$ \\
\hline LE & 10 & 528 & 302 & $\underline{N}$ \\
\hline LE & 15 & 461 & 376 & $\underline{\mathrm{N}}$ \\
\hline LE & 20 & 361 & 395 & $\underline{N}$ \\
\hline LE & 25 & 272 & 392 & $\underline{\mathrm{N}}$ \\
\hline LE & 30 & 236 & 384 & $\underline{N}$ \\
\hline LL & 1 & 148 & 9 & $\underline{\underline{N}}$ \\
\hline LL & 2 & 246 & 24 & $\underline{\mathrm{N}}$ \\
\hline LL & 3 & 339 & 37 & $\underline{\mathrm{N}}$ \\
\hline
\end{tabular}

\begin{tabular}{|c|c|c|c|c|}
\hline Run & $\begin{array}{l}\text { Fitting } \\
\text { Range } \\
\text { (Mpc) }\end{array}$ & $\begin{array}{c}\text { Num. } \\
\text { PD } \\
\text { Values } \\
\text { in } \\
3000 \\
\text { Bin }\end{array}$ & $\begin{array}{c}\text { Num. } \\
\text { PD } \\
\text { Values } \\
\text { in } \\
9000 \\
\text { Bin }\end{array}$ & Dissimilar \\
\hline LA & 1 & 464 & 88 & $\underline{N}$ \\
\hline LA & 2 & 723 & 202 & $\mathrm{~N}$ \\
\hline LA & 3 & 844 & 302 & Y \\
\hline LA & 4 & 887 & 375 & $Y$ \\
\hline LA & 5 & 887 & 427 & $\mathrm{Y}$ \\
\hline LA & 10 & 791 & 488 & $Y$ \\
\hline LA & 15 & 713 & 483 & $Y$ \\
\hline LA & 20 & 632 & 475 & Y \\
\hline LA & 25 & 542 & 459 & $Y$ \\
\hline LA & 30 & 432 & 447 & $Y$ \\
\hline LE & 1 & 92 & 14 & $\underline{N}$ \\
\hline LE & 2 & 209 & 34 & $\underline{N}$ \\
\hline LE & 3 & 347 & 59 & $\underline{N}$ \\
\hline LE & 4 & 459 & 79 & $\underline{N}$ \\
\hline LE & 5 & 549 & 104 & $\underline{N}$ \\
\hline LE & 10 & 675 & 278 & $\underline{N}$ \\
\hline LE & 15 & 697 & 364 & $Y$ \\
\hline LE & 20 & 632 & 416 & $Y$ \\
\hline LE & 25 & 542 & 437 & $Y$ \\
\hline LE & 30 & 432 & 445 & $Y$ \\
\hline LL & 1 & 134 & 8 & $\underline{N}$ \\
\hline LL & 2 & 290 & 15 & $\underline{N}$ \\
\hline LL & 3 & 485 & 38 & $\underline{N}$ \\
\hline
\end{tabular}




\begin{tabular}{|c|c|c|c|c|c|c|c|c|c|}
\hline Run & $\begin{array}{l}\text { Fitting } \\
\text { Range } \\
\text { (Mpc) }\end{array}$ & $\begin{array}{c}\text { Num. } \\
\text { PD } \\
\text { Values } \\
\text { in } \\
3000 \\
\text { Bin }\end{array}$ & $\begin{array}{l}\text { Num. } \\
\text { PD } \\
\text { Values } \\
\text { in } \\
9000 \\
\text { Bin }\end{array}$ & Dissimilar & Run & $\begin{array}{l}\text { Fitting } \\
\text { Range } \\
\text { (Mpc) }\end{array}$ & $\begin{array}{c}\text { Num. } \\
\text { PD } \\
\text { Values } \\
\text { in } \\
\mathbf{3 0 0 0} \\
\text { Bin }\end{array}$ & $\begin{array}{c}\text { Num. } \\
\text { PD } \\
\text { Values } \\
\text { in } \\
9000 \\
\text { Bin }\end{array}$ & Dissimilar \\
\hline LL & 4 & 428 & 53 & $\underline{N}$ & LL & 4 & 630 & 70 & $\underline{N}$ \\
\hline LL & 5 & 495 & 81 & $\underline{N}$ & LL & 5 & 734 & 99 & $\underline{N}$ \\
\hline LL & 10 & 548 & 231 & $Y$ & LL & 10 & 774 & 266 & $Y$ \\
\hline $\mathrm{LL}$ & 15 & 458 & 325 & $\underline{N}$ & LL & 15 & 712 & 404 & $Y$ \\
\hline LL & 20 & 361 & 375 & $Y$ & LL & 20 & 632 & 445 & $Y$ \\
\hline LL & 25 & 272 & 390 & $Y$ & LL & 25 & 542 & 446 & $\mathbf{Y}$ \\
\hline LL & 30 & 236 & 381 & $Y$ & LL & 30 & 432 & 447 & $\mathrm{Y}$ \\
\hline LS & 1 & 222 & 11 & $\underline{N}$ & LS & 1 & 250 & 9 & $\underline{N}$ \\
\hline LS & 2 & 414 & 45 & $\underline{N}$ & LS & 2 & 525 & 33 & $\underline{N}$ \\
\hline LS & 3 & 544 & 85 & $\underline{\mathrm{N}}$ & LS & 3 & 714 & 82 & $\underline{N}$ \\
\hline LS & 4 & 593 & 136 & $\underline{N}$ & LS & 4 & 803 & 126 & $\underline{N}$ \\
\hline LS & 5 & 611 & 190 & $\underline{N}$ & LS & 5 & 853 & 183 & $\underline{N}$ \\
\hline LS & 10 & 569 & 346 & $\mathrm{Y}$ & LS & 10 & 788 & 389 & $Y$ \\
\hline LS & 15 & 462 & 400 & $Y$ & LS & 15 & 713 & 460 & $Y$ \\
\hline LS & 20 & 361 & 409 & $\mathbf{Y}$ & LS & 20 & 632 & 474 & $\mathbf{Y}$ \\
\hline LS & 25 & 272 & 397 & $Y$ & LS & 25 & 542 & 459 & $Y$ \\
\hline LS & 30 & 236 & 384 & $Y$ & LS & 30 & 432 & 447 & $Y$ \\
\hline $\mathrm{LS}_{\mathrm{b}}$ & 1 & 23 & 0 & $\mathrm{~N} / \mathrm{A}$ & $\mathrm{LS}_{\mathrm{b}}$ & 1 & 9 & 0 & $\mathrm{~N} / \mathrm{A}$ \\
\hline $\mathrm{LS}_{\mathrm{b}}$ & 2 & 65 & 7 & $Y$ & $\mathrm{LS}_{\mathrm{b}}$ & 2 & 86 & 1 & $\underline{\mathrm{N}}$ \\
\hline $\mathrm{LS}_{\mathrm{b}}$ & 3 & 115 & 16 & $Y$ & $\mathrm{LS}_{\mathrm{b}}$ & 3 & 171 & 4 & $\underline{\mathrm{N}}$ \\
\hline $\mathrm{LS}_{\mathrm{b}}$ & 4 & 208 & 31 & $\underline{\mathrm{N}}$ & $\mathrm{LS}_{\mathrm{b}}$ & 4 & 264 & 5 & $\underline{\mathrm{N}}$ \\
\hline $\mathrm{LS}_{\mathrm{b}}$ & 5 & 307 & 36 & $\underline{\mathrm{N}}$ & $\mathrm{LS}_{\mathrm{b}}$ & 5 & 335 & 8 & $\underline{\mathrm{N}}$ \\
\hline $\mathrm{LS}_{\mathrm{b}}$ & 10 & 502 & 116 & $\underline{\mathrm{N}}$ & $\mathrm{LS}_{\mathrm{b}}$ & 10 & 549 & 37 & $\underline{N}$ \\
\hline $\mathrm{LS}_{\mathrm{b}}$ & 15 & 448 & 177 & $\underline{\mathrm{N}}$ & $\mathrm{LS}_{\mathrm{b}}$ & 15 & 608 & 90 & $Y$ \\
\hline $\mathrm{LS}_{\mathrm{b}}$ & 20 & 357 & 226 & $\underline{\mathrm{N}}$ & $\mathrm{LS}_{\mathrm{b}}$ & 20 & 589 & 148 & $Y$ \\
\hline $\mathrm{LS}_{\mathrm{b}}$ & 25 & 271 & 280 & $\underline{\mathrm{N}}$ & $\mathrm{LS}_{\mathrm{b}}$ & 25 & 523 & 187 & $Y$ \\
\hline $\mathrm{LS}_{\mathrm{b}}$ & 30 & 235 & 323 & $\mathrm{Y}$ & $\mathrm{LS}_{\mathrm{b}}$ & 30 & 428 & 220 & $Y$ \\
\hline $\mathrm{LS}_{\mathrm{u}}$ & 1 & 1 & 0 & $\mathrm{~N} / \mathrm{A}$ & $\mathrm{LS}_{\mathrm{u}}$ & 1 & 3 & 0 & $\mathrm{~N} / \mathrm{A}$ \\
\hline $\mathrm{LS}_{\mathrm{u}}$ & 2 & 29 & 0 & $\mathrm{~N} / \mathrm{A}$ & $\mathrm{LS}_{\mathrm{u}}$ & 2 & 85 & 0 & $\mathrm{~N} / \mathrm{A}$ \\
\hline $\mathrm{LS}_{\mathrm{u}}$ & 3 & 81 & 0 & $\mathrm{~N} / \mathrm{A}$ & $\mathrm{LS}_{\mathrm{u}}$ & 3 & 162 & 0 & $\mathrm{~N} / \mathrm{A}$ \\
\hline $\mathrm{LS}_{\mathrm{u}}$ & 4 & 152 & 0 & $\mathrm{~N} / \mathrm{A}$ & $\mathrm{LS}_{\mathrm{u}}$ & 4 & 235 & 1 & $Y$ \\
\hline $\mathrm{LS}_{\mathrm{u}}$ & 5 & 203 & 2 & $\underline{\mathrm{N}}$ & $\mathrm{LS}_{\mathrm{u}}$ & 5 & 288 & 2 & $\underline{\mathrm{N}}$ \\
\hline $\mathrm{LS}_{\mathrm{u}}$ & 10 & 401 & 58 & $\underline{\mathrm{N}}$ & $\mathrm{LS}_{\mathrm{u}}$ & 10 & 385 & 9 & $\underline{\mathrm{N}}$ \\
\hline $\mathrm{LS}_{\mathrm{u}}$ & 15 & 402 & 130 & $\underline{\mathrm{N}}$ & $\mathrm{LS}_{\mathrm{u}}$ & 15 & 465 & 32 & $\underline{\mathrm{N}}$ \\
\hline $\mathrm{LS}_{\mathrm{u}}$ & 20 & 344 & 212 & $\underline{\mathrm{N}}$ & $\mathrm{LS}_{\mathrm{u}}$ & 20 & 499 & 69 & $\underline{\mathrm{N}}$ \\
\hline $\mathrm{LS}_{\mathrm{u}}$ & 25 & 271 & 244 & $Y$ & $\mathrm{LS}_{\mathrm{u}}$ & 25 & 495 & 85 & $Y$ \\
\hline $\mathrm{LS}_{\mathrm{u}}$ & 30 & 236 & 274 & $Y$ & $\mathrm{LS}_{\mathrm{u}}$ & 30 & 426 & 102 & $Y$ \\
\hline
\end{tabular}


For spiral primaries in Table 5-43, dissimilarity is found in both north and south for secondaries with no morphological filter at $5 \mathrm{Mpc}$ and up. Of interest very little dissimilarity is found for $E$ secondaries in the north while dissimilarity is found for $L$ and late-type galaxies. This trend is not observed in the south. It appears that environmental evolution over this difference in time does not introduce dissimilarity for $\mathrm{E}$ environments of spiral primaries.

Table 5-43 KS Tests For Velocity Bins of 3000-6000 km/s and $9000-12000 \mathrm{~km} / \mathrm{s}$ limited to spiral primaries

$\mathrm{Y}=$ dissimilar to the $99 \%$ level $\mathrm{N}=$ not dissimilar to the $99 \%$ level

\begin{tabular}{|c|c|c|c|c|c|c|c|c|c|}
\hline Run & $\begin{array}{l}\text { Fitting } \\
\text { Range } \\
\text { (Mpc) }\end{array}$ & $\begin{array}{l}\text { Num. } \\
\text { PD } \\
\text { Values } \\
\text { in } \\
3000 \\
\text { Bin } \\
\end{array}$ & $\begin{array}{l}\text { Num. } \\
\text { PD } \\
\text { Values } \\
\text { in } \\
9000 \\
\text { Bin } \\
\end{array}$ & Dissimilar & Run & $\begin{array}{l}\text { Fitting } \\
\text { Range } \\
\text { (Mpc) }\end{array}$ & $\begin{array}{l}\text { Num. } \\
\text { PD } \\
\text { Values } \\
\text { in } \\
3000 \\
\text { Bin } \\
\end{array}$ & $\begin{array}{l}\text { Num. } \\
\text { PD } \\
\text { Values } \\
\text { in } \\
9000 \\
\text { Bin } \\
\end{array}$ & Dissimilar \\
\hline SA & 1 & 723 & 146 & $\underline{N}$ & SA & 1 & 864 & 121 & $\underline{N}$ \\
\hline SA & 2 & 1397 & 372 & $\underline{N}$ & SA & 2 & 1562 & 335 & $\underline{N}$ \\
\hline SA & 3 & 1716 & 573 & $\underline{N}$ & SA & 3 & 1923 & 556 & $Y$ \\
\hline SA & 4 & 1820 & 732 & $\underline{N}$ & SA & 4 & 2025 & 699 & $Y$ \\
\hline SA & 5 & 1831 & 841 & $Y$ & SA & 5 & 2037 & 821 & $Y$ \\
\hline SA & 10 & 1612 & 1009 & $Y$ & SA & 10 & 1876 & 985 & $Y$ \\
\hline SA & 15 & 1376 & 1001 & $Y$ & SA & 15 & 1639 & 983 & $Y$ \\
\hline SA & 20 & 1141 & 977 & $Y$ & SA & 20 & 1382 & 960 & $Y$ \\
\hline SA & 25 & 923 & 952 & $Y$ & SA & 25 & 1145 & 927 & $Y$ \\
\hline SA & 30 & 790 & 930 & $Y$ & SA & 30 & 946 & 903 & $Y$ \\
\hline SE & 1 & 95 & 20 & $\underline{N}$ & SE & 1 & 123 & 11 & $\underline{N}$ \\
\hline SE & 2 & 324 & 57 & $\underline{N}$ & SE & 2 & 332 & 50 & $\underline{\underline{N}}$ \\
\hline SE & 3 & 566 & 108 & $\underline{N}$ & SE & 3 & 632 & 89 & $\underline{N}$ \\
\hline SE & 4 & 813 & 189 & $\underline{N}$ & SE & 4 & 944 & 132 & $\underline{N}$ \\
\hline SE & 5 & 986 & 274 & $\underline{N}$ & SE & 5 & 1119 & 185 & $\underline{N}$ \\
\hline SE & 10 & 1458 & 703 & $\underline{N}$ & SE & 10 & 1606 & 486 & $Y$ \\
\hline SE & 15 & 1356 & 885 & $\underline{N}$ & SE & 15 & 1603 & 696 & $Y$ \\
\hline SE & 20 & 1140 & 938 & $\underline{N}$ & SE & 20 & 1381 & 802 & $\mathrm{Y}$ \\
\hline SE & 25 & 923 & 943 & $\underline{N}$ & SE & 25 & 1145 & 874 & $Y$ \\
\hline SE & 30 & 790 & 928 & $\underline{N}$ & SE & 30 & 946 & 892 & $Y$ \\
\hline SL & 1 & 167 & 7 & $\underline{\underline{N}}$ & SL & 1 & 165 & 5 & $\underline{N}$ \\
\hline SL & 2 & 413 & 24 & $\underline{N}$ & $S L$ & 2 & 558 & 20 & $\underline{N}$ \\
\hline SL & 3 & 668 & 57 & $\underline{N}$ & $S L$ & 3 & 950 & 60 & $\underline{N}$ \\
\hline SL & 4 & 933 & 95 & $\underline{N}$ & SL & 4 & 1255 & 95 & $\underline{N}$ \\
\hline SL & 5 & 1125 & 158 & $\underline{N}$ & $S L$ & 5 & 1466 & 158 & $\underline{N}$ \\
\hline SL & 10 & 1474 & 494 & $Y$ & SL & 10 & 1782 & 509 & $Y$ \\
\hline
\end{tabular}




\begin{tabular}{|c|c|c|c|c|c|c|c|c|c|}
\hline Run & $\begin{array}{l}\text { Fitting } \\
\text { Range } \\
\text { (Mpc) }\end{array}$ & $\begin{array}{c}\text { Num. } \\
\text { PD } \\
\text { Values } \\
\text { in } \\
3000 \\
\text { Bin }\end{array}$ & $\begin{array}{c}\text { Num. } \\
\text { PD } \\
\text { Values } \\
\text { in } \\
9000 \\
\text { Bin }\end{array}$ & Dissimilar & Run & $\begin{array}{l}\text { Fitting } \\
\text { Range } \\
\text { (Mpc) }\end{array}$ & $\begin{array}{c}\text { Num. } \\
\text { PD } \\
\text { Values } \\
\text { in } \\
\mathbf{3 0 0 0} \\
\text { Bin }\end{array}$ & $\begin{array}{c}\text { Num. } \\
\text { PD } \\
\text { Values } \\
\text { in } \\
9000 \\
\text { Bin }\end{array}$ & Dissimilar \\
\hline SL & 15 & 1351 & 729 & Y & SL & 15 & 1630 & 765 & Y \\
\hline SL & 20 & 1137 & 876 & $Y$ & SL & 20 & 1382 & 862 & Y \\
\hline SL & 25 & 923 & 923 & $Y$ & SL & 25 & 1145 & 887 & $Y$ \\
\hline $\mathrm{SL}$ & 30 & 790 & 921 & $Y$ & SL & 30 & 946 & 893 & $Y$ \\
\hline SS & 1 & 359 & 15 & $\underline{N}$ & SS & 1 & 386 & 5 & $\underline{N}$ \\
\hline SS & 2 & 981 & 77 & $\underline{N}$ & SS & 2 & 1071 & 46 & $\underline{N}$ \\
\hline SS & 3 & 1420 & 170 & $\underline{N}$ & SS & 3 & 1576 & 140 & $\underline{N}$ \\
\hline SS & 4 & 1656 & 273 & $\underline{N}$ & SS & 4 & 1837 & 230 & $\underline{N}$ \\
\hline SS & 5 & 1759 & 384 & $Y$ & SS & 5 & 1944 & 324 & $\mathbf{Y}$ \\
\hline SS & 10 & 1610 & 795 & $Y$ & SS & 10 & 1873 & 761 & $Y$ \\
\hline SS & 15 & 1376 & 947 & $Y$ & SS & 15 & 1638 & 932 & $Y$ \\
\hline SS & 20 & 1141 & 963 & $Y$ & SS & 20 & 1382 & 952 & $Y$ \\
\hline SS & 25 & 923 & 950 & $Y$ & SS & 25 & 1145 & 927 & $Y$ \\
\hline SS & 30 & 790 & 929 & $Y$ & SS & 30 & 946 & 903 & $Y$ \\
\hline$S_{b}$ & 1 & 21 & 2 & $\underline{N}$ & $S S_{b}$ & 1 & 7 & 0 & $\mathrm{~N} / \mathrm{A}$ \\
\hline$S S_{b}$ & 2 & 111 & 10 & $\underline{N}$ & $S S_{b}$ & 2 & 143 & 1 & $\underline{\mathrm{N}}$ \\
\hline$S S_{b}$ & 3 & 293 & 23 & $\underline{\mathrm{N}}$ & $S S_{b}$ & 3 & 313 & 6 & $\underline{N}$ \\
\hline$S S_{b}$ & 4 & 519 & 47 & $\underline{\mathrm{N}}$ & $S S_{b}$ & 4 & 521 & 10 & $\underline{\mathrm{N}}$ \\
\hline$S S_{b}$ & 5 & 782 & 76 & $\underline{\mathrm{N}}$ & $S S_{b}$ & 5 & 733 & 15 & $\underline{\mathrm{N}}$ \\
\hline $\mathrm{SS}_{\mathrm{b}}$ & 10 & 1425 & 249 & $\underline{\mathrm{N}}$ & $S S_{b}$ & 10 & 1310 & 71 & $\underline{\mathrm{N}}$ \\
\hline$S S_{b}$ & 15 & 1336 & 371 & $\underline{\mathrm{N}}$ & $S S_{b}$ & 15 & 1394 & 154 & $Y$ \\
\hline $\mathrm{SS}_{\mathrm{b}}$ & 20 & 1129 & 487 & $Y$ & $S S_{b}$ & 20 & 1301 & 266 & $Y$ \\
\hline$S S_{b}$ & 25 & 920 & 626 & $Y$ & $S S_{b}$ & 25 & 1117 & 363 & $Y$ \\
\hline $\mathrm{SS}_{\mathrm{b}}$ & 30 & 790 & 746 & $Y$ & $S S_{b}$ & 30 & 940 & 445 & $Y$ \\
\hline $\mathrm{SS}_{\mathrm{u}}$ & 1 & 5 & 0 & $\mathrm{~N} / \mathrm{A}$ & $\mathrm{SS}_{\mathrm{u}}$ & 1 & 11 & 0 & $\mathrm{~N} / \mathrm{A}$ \\
\hline $\mathrm{SS}_{\mathrm{u}}$ & 2 & 63 & 0 & $\mathrm{~N} / \mathrm{A}$ & $\mathrm{SS}_{\mathrm{u}}$ & 2 & 111 & 0 & N/A \\
\hline $\mathrm{SS}_{\mathrm{u}}$ & 3 & 188 & 2 & $\underline{\mathrm{N}}$ & $\mathrm{SS}_{\mathrm{u}}$ & 3 & 283 & 1 & $\underline{\mathrm{N}}$ \\
\hline $\mathrm{SS}_{\mathrm{u}}$ & 4 & 390 & 6 & $\underline{N}$ & $\mathrm{SS}_{\mathrm{u}}$ & 4 & 467 & 1 & $\underline{N}$ \\
\hline $\mathrm{SS}_{\mathrm{u}}$ & 5 & 584 & 12 & $\underline{\mathrm{N}}$ & $\mathrm{SS}_{\mathrm{u}}$ & 5 & 636 & 6 & $\underline{\mathrm{N}}$ \\
\hline $\mathrm{SS}_{\mathrm{u}}$ & 10 & 1173 & 143 & $\underline{\mathrm{N}}$ & $\mathrm{SS}_{\mathrm{u}}$ & 10 & 984 & 36 & $Y$ \\
\hline $\mathrm{SS}_{\mathrm{u}}$ & 15 & 1227 & 300 & $\underline{N}$ & $S_{\mathrm{u}}$ & 15 & 1126 & 72 & $Y$ \\
\hline $\mathrm{SS}_{\mathrm{u}}$ & 20 & 1102 & 463 & $Y$ & $\mathrm{SS}_{\mathrm{u}}$ & 20 & 1137 & 151 & $Y$ \\
\hline $\mathrm{SS}_{\mathrm{u}}$ & 25 & 922 & 581 & $Y$ & $\mathrm{SS}_{\mathrm{u}}$ & 25 & 1057 & 201 & $Y$ \\
\hline $\mathrm{SS}_{\mathrm{u}}$ & 30 & 790 & 659 & $Y$ & $\mathrm{SS}_{\mathrm{u}}$ & 30 & 941 & 242 & $Y$ \\
\hline
\end{tabular}


For barred spiral primaries in Table 5-44, very little dissimilarity is noted in either north or south. Since dissimilarity was noted for spiral primaries this is interesting and suggests that the environments of barred spirals do not evolve over this difference in time whereas the environments of spirals do evolve over this difference in time.

Table 5-44 KS Tests For Velocity Bins of $3000-6000 \mathrm{~km} / \mathrm{s}$ and $9000-12000 \mathrm{~km} / \mathrm{s}$ limited to barred spiral primaries

$\mathrm{Y}=$ dissimilar to the $99 \%$ level $\mathrm{N}=$ not dissimilar to the $99 \%$ level

\begin{tabular}{|c|c|c|c|c|c|c|c|c|c|}
\hline Run & $\begin{array}{l}\text { Fitting } \\
\text { Range } \\
\text { (Mpc) }\end{array}$ & $\begin{array}{l}\text { Num. } \\
\text { PD } \\
\text { Values } \\
\text { in } \\
3000 \\
\text { Bin } \\
\end{array}$ & $\begin{array}{c}\text { Num. } \\
\text { PD } \\
\text { Values } \\
\text { in } \\
9000 \\
\text { Bin } \\
\end{array}$ & Dissimilar & Run & $\begin{array}{l}\text { Fitting } \\
\text { Range } \\
\text { (Mpc) }\end{array}$ & $\begin{array}{c}\text { Num. } \\
\text { PD } \\
\text { Values } \\
\text { in } \\
3000 \\
\text { Bin } \\
\end{array}$ & $\begin{array}{c}\text { Num. } \\
\text { PD } \\
\text { Values } \\
\text { in } \\
9000 \\
\text { Bin } \\
\end{array}$ & Dissimilar \\
\hline$S_{b} A$ & 1 & 116 & 34 & $\underline{N}$ & $\mathrm{~S}_{\mathrm{b}} \mathrm{A}$ & 1 & 128 & 12 & $\underline{N}$ \\
\hline$S_{b} A$ & 2 & 220 & 73 & $\underline{N}$ & $\mathrm{~S}_{\mathrm{b}} \mathrm{A}$ & 2 & 204 & 32 & $\underline{N}$ \\
\hline $\mathrm{S}_{\mathrm{b}} \mathrm{A}$ & 3 & 278 & 113 & $\underline{N}$ & $S_{b} A$ & 3 & 248 & 50 & $\underline{\underline{N}}$ \\
\hline $\mathrm{S}_{\mathrm{b}} \mathrm{A}$ & 4 & 302 & 132 & $\underline{N}$ & $\mathrm{~S}_{b} \mathrm{~A}$ & 4 & 256 & 67 & $\underline{N}$ \\
\hline $\mathrm{S}_{\mathrm{b}} \mathrm{A}$ & 5 & 303 & 142 & $\underline{N}$ & $\mathrm{~S}_{\mathrm{b}} \mathrm{A}$ & 5 & 259 & 80 & $\underline{N}$ \\
\hline $\mathrm{S}_{\mathrm{b}} \mathrm{A}$ & 10 & 273 & 170 & $\underline{N}$ & $S_{b} A$ & 10 & 237 & 91 & $Y$ \\
\hline $\mathrm{S}_{\mathrm{b}} \mathrm{A}$ & 15 & 240 & 171 & $\underline{N}$ & $S_{b} A$ & 15 & 203 & 92 & $Y$ \\
\hline $\mathrm{S}_{\mathrm{b}} \mathrm{A}$ & 20 & 201 & 169 & $\underline{N}$ & $\mathrm{~S}_{\mathrm{b}} \mathrm{A}$ & 20 & 159 & 89 & $Y$ \\
\hline $\mathrm{S}_{\mathrm{b}} \mathrm{A}$ & 25 & 165 & 167 & $\underline{N}$ & $\mathrm{~S}_{b} \mathrm{~A}$ & 25 & 124 & 85 & $Y$ \\
\hline $\mathrm{S}_{\mathrm{b}} \mathrm{A}$ & 30 & 146 & 165 & $\underline{N}$ & $S_{b} A$ & 30 & 100 & 84 & $\underline{N}$ \\
\hline$S_{b} E$ & 1 & 12 & 6 & $\underline{N}$ & $S_{b} E$ & 1 & 15 & 1 & $\underline{N}$ \\
\hline $\mathrm{S}_{\mathrm{b}} \mathrm{E}$ & 2 & 51 & 18 & $\underline{N}$ & $\mathrm{~S}_{\mathrm{b}} \mathrm{E}$ & 2 & 53 & 10 & $\underline{N}$ \\
\hline$S_{b} E$ & 3 & 88 & 24 & $\underline{N}$ & $S_{b} E$ & 3 & 100 & 16 & $\underline{N}$ \\
\hline$S_{b} E$ & 4 & 124 & 43 & $\underline{N}$ & $S_{b} E$ & 4 & 136 & 18 & $\underline{N}$ \\
\hline $\mathrm{S}_{\mathrm{b}} \mathrm{E}$ & 5 & 157 & 57 & $\underline{N}$ & $\mathrm{~S}_{\mathrm{b}} \mathrm{E}$ & 5 & 160 & 24 & $\underline{N}$ \\
\hline $\mathrm{S}_{\mathrm{b}} \mathrm{E}$ & 10 & 245 & 126 & $\underline{N}$ & $\mathrm{~S}_{\mathrm{b}} \mathrm{E}$ & 10 & 221 & 54 & $\underline{N}$ \\
\hline $\mathrm{S}_{\mathrm{b}} \mathrm{E}$ & 15 & 237 & 149 & $\underline{N}$ & $S_{b} E$ & 15 & 200 & 74 & $Y$ \\
\hline $\mathrm{S}_{\mathrm{b}} \mathrm{E}$ & 20 & 200 & 164 & $\underline{N}$ & $S_{b} E$ & 20 & 159 & 86 & $\underline{N}$ \\
\hline$S_{b} E$ & 25 & 165 & 167 & $\underline{N}$ & $S_{b} E$ & 25 & 124 & 85 & $Y$ \\
\hline $\mathrm{S}_{\mathrm{b}} \mathrm{E}$ & 30 & 146 & 165 & $\underline{N}$ & $\mathrm{~S}_{\mathrm{b}} \mathrm{E}$ & 30 & 100 & 84 & $Y$ \\
\hline$S_{b} L$ & 1 & 21 & 2 & $\underline{N}$ & $S_{b} L$ & 1 & 25 & 0 & $\mathrm{~N} / \mathrm{A}$ \\
\hline$S_{b} L$ & 2 & 48 & 7 & $\underline{\mathrm{N}}$ & $S_{b} L$ & 2 & 79 & 0 & N/A \\
\hline $\mathrm{S}_{b} \mathrm{~L}$ & 3 & 84 & 17 & $\underline{N}$ & $\mathrm{~S}_{\mathrm{b}} \mathrm{L}$ & 3 & 130 & 6 & $\underline{N}$ \\
\hline$S_{b} L$ & 4 & 126 & 21 & $\underline{N}$ & $S_{b} L$ & 4 & 162 & 8 & $\underline{N}$ \\
\hline$S_{b} L$ & 5 & 167 & 35 & $\underline{N}$ & $S_{b} L$ & 5 & 193 & 15 & $\underline{N}$ \\
\hline$S_{b} L$ & 10 & 245 & 94 & $\underline{N}$ & $S_{b} L$ & 10 & 227 & 50 & $\underline{N}$ \\
\hline$S_{b} L$ & 15 & 238 & 130 & $\underline{N}$ & $S_{b} L$ & 15 & 201 & 72 & $\underline{N}$ \\
\hline $\mathrm{S}_{\mathrm{b}} \mathrm{L}$ & 20 & 201 & 152 & $\underline{N}$ & $S_{b} L$ & 20 & 159 & 81 & $Y$ \\
\hline$S_{b} L$ & 25 & 165 & 162 & $\underline{\mathrm{N}}$ & $S_{b} L$ & 25 & 124 & 84 & $Y$ \\
\hline
\end{tabular}




\begin{tabular}{|c|c|c|c|c|}
\hline Run & $\begin{array}{l}\text { Fitting } \\
\text { Range } \\
\text { (Mpc) }\end{array}$ & $\begin{array}{l}\text { Num. } \\
\text { PD } \\
\text { Values } \\
\text { in } \\
3000 \\
\text { Bin } \\
\end{array}$ & $\begin{array}{c}\text { Num. } \\
\text { PD } \\
\text { Values } \\
\text { in } \\
9000 \\
\text { Bin } \\
\end{array}$ & Dissimilar \\
\hline$S_{b} L$ & 30 & 146 & 164 & $\underline{N}$ \\
\hline$S_{b} S$ & 1 & 49 & 6 & $\underline{N}$ \\
\hline$S_{b} S$ & 2 & 149 & 23 & $\underline{N}$ \\
\hline$S_{b} S$ & 3 & 231 & 34 & $\underline{N}$ \\
\hline$S_{b} S$ & 4 & 277 & 55 & $\underline{N}$ \\
\hline$S_{b} S$ & 5 & 291 & 85 & $\underline{N}$ \\
\hline$S_{b} S$ & 10 & 273 & 138 & $Y$ \\
\hline$S_{b} S$ & 15 & 240 & 161 & $\underline{N}$ \\
\hline$S_{b} S$ & 20 & 201 & 168 & $Y$ \\
\hline$S_{b} S$ & 25 & 165 & 167 & $\underline{N}$ \\
\hline$S_{b} S$ & 30 & 146 & 165 & $\underline{N}$ \\
\hline$S_{b} S_{b}$ & 1 & 4 & 0 & N/A \\
\hline$S_{b} S_{b}$ & 2 & 25 & 4 & $\underline{N}$ \\
\hline$S_{b} S_{b}$ & 3 & 75 & 5 & $\underline{N}$ \\
\hline$S_{b} S_{b}$ & 4 & 115 & 10 & $\underline{N}$ \\
\hline$S_{b} S_{b}$ & 5 & 150 & 22 & $\underline{N}$ \\
\hline$S_{b} S_{b}$ & 10 & 242 & 66 & $\underline{N}$ \\
\hline$S_{b} S_{b}$ & 15 & 236 & 95 & $Y$ \\
\hline$S_{b} S_{b}$ & 20 & 201 & 112 & $\underline{\underline{N}}$ \\
\hline$S_{b} S_{b}$ & 25 & 165 & 127 & $Y$ \\
\hline$S_{b} S_{b}$ & 30 & 146 & 147 & $Y$ \\
\hline $\mathrm{S}_{\mathrm{b}} \mathrm{S}_{\mathrm{u}}$ & 1 & 1 & 0 & $\mathrm{~N} / \mathrm{A}$ \\
\hline $\mathrm{S}_{\mathrm{b}} \mathrm{S}_{\mathrm{u}}$ & 2 & 14 & 0 & N/A \\
\hline $\mathrm{S}_{\mathrm{b}} \mathrm{S}_{\mathrm{u}}$ & 3 & 37 & 0 & N/A \\
\hline$S_{b} S_{u}$ & 4 & 76 & 0 & $\mathrm{~N} / \mathrm{A}$ \\
\hline $\mathrm{S}_{\mathrm{b}} \mathrm{S}_{\mathrm{u}}$ & 5 & 116 & 0 & $N / A$ \\
\hline $\mathrm{S}_{\mathrm{b}} \mathrm{S}_{\mathrm{u}}$ & 10 & 222 & 32 & $\underline{N}$ \\
\hline $\mathrm{S}_{\mathrm{b}} \mathrm{S}_{\mathrm{u}}$ & 15 & 222 & 75 & $Y$ \\
\hline $\mathrm{S}_{\mathrm{b}} \mathrm{S}_{\mathrm{u}}$ & 20 & 197 & 108 & $\underline{N}$ \\
\hline $\mathrm{S}_{\mathrm{b}} \mathrm{S}_{\mathrm{u}}$ & 25 & 165 & 126 & $Y$ \\
\hline$S_{b} S_{u}$ & 30 & 146 & 137 & $\underline{N}$ \\
\hline
\end{tabular}

\begin{tabular}{|c|c|c|c|c|}
\hline Run & $\begin{array}{l}\text { Fitting } \\
\text { Range } \\
\text { (Mpc) }\end{array}$ & $\begin{array}{l}\text { Num. } \\
\text { PD } \\
\text { Values } \\
\text { in } \\
3000 \\
\text { Bin }\end{array}$ & $\begin{array}{c}\text { Num. } \\
\text { PD } \\
\text { Values } \\
\text { in } \\
9000 \\
\text { Bin }\end{array}$ & Dissimilar \\
\hline$S_{b} L$ & 30 & 100 & 84 & Y \\
\hline$S_{b} S$ & 1 & 67 & 2 & $\underline{N}$ \\
\hline$S_{b} S$ & 2 & 155 & 6 & $\underline{N}$ \\
\hline$S_{b} S$ & 3 & 213 & 13 & $\underline{N}$ \\
\hline$S_{b} S$ & 4 & 234 & 23 & $\underline{N}$ \\
\hline$S_{b} S$ & 5 & 248 & 28 & $\underline{N}$ \\
\hline$S_{b} S$ & 10 & 237 & 67 & $Y$ \\
\hline$S_{b} S$ & 15 & 203 & 88 & Y \\
\hline$S_{b} S$ & 20 & 159 & 89 & $Y$ \\
\hline$S_{b} S$ & 25 & 124 & 85 & $Y$ \\
\hline$S_{b} S$ & 30 & 100 & 84 & $Y$ \\
\hline$S_{b} S_{b}$ & 1 & 0 & 0 & N/A \\
\hline$S_{b} S_{b}$ & 2 & 19 & 0 & $\mathrm{~N} / \mathrm{A}$ \\
\hline$S_{b} S_{b}$ & 3 & 49 & 1 & $\underline{N}$ \\
\hline$S_{b} S_{b}$ & 4 & 78 & 1 & $\underline{N}$ \\
\hline $\mathrm{S}_{b} \mathrm{~S}_{\mathrm{b}}$ & 5 & 120 & 4 & $\underline{N}$ \\
\hline$S_{b} S_{b}$ & 10 & 193 & 13 & $\underline{N}$ \\
\hline$S_{b} S_{b}$ & 15 & 190 & 22 & $\underline{N}$ \\
\hline $\mathrm{S}_{b} \mathrm{~S}_{\mathrm{b}}$ & 20 & 156 & 36 & $\mathrm{Y}$ \\
\hline$S_{b} S_{b}$ & 25 & 123 & 49 & $Y$ \\
\hline$S_{b} S_{b}$ & 30 & 100 & 61 & $Y$ \\
\hline $\mathrm{S}_{\mathrm{b}} \mathrm{S}_{\mathrm{u}}$ & 1 & 2 & 0 & N/A \\
\hline $\mathrm{S}_{\mathrm{b}} \mathrm{S}_{\mathrm{u}}$ & 2 & 19 & 0 & N/A \\
\hline $\mathrm{S}_{b} \mathrm{~S}_{\mathrm{u}}$ & 3 & 50 & 1 & $Y$ \\
\hline$S_{b} S_{u}$ & 4 & 79 & 1 & $\underline{N}$ \\
\hline $\mathrm{S}_{\mathrm{b}} \mathrm{S}_{\mathrm{u}}$ & 5 & 112 & 3 & $\underline{N}$ \\
\hline $\mathrm{S}_{b} \mathrm{~S}_{\mathrm{u}}$ & 10 & 165 & 9 & $\underline{N}$ \\
\hline $\mathrm{S}_{\mathrm{b}} \mathrm{S}_{\mathrm{u}}$ & 15 & 174 & 16 & $\underline{N}$ \\
\hline $\mathrm{S}_{b} \mathrm{~S}_{\mathrm{u}}$ & 20 & 147 & 31 & $Y$ \\
\hline $\mathrm{S}_{b} \mathrm{~S}_{\mathrm{u}}$ & 25 & 119 & 34 & $Y$ \\
\hline $\mathrm{S}_{b} \mathrm{~S}_{\mathrm{u}}$ & 30 & 100 & 50 & $Y$ \\
\hline
\end{tabular}


For unbarred spiral primaries in Table 5-45, little dissimilarity is noted in north or south. Since dissimilarity is found for spiral galaxies this is interesting and suggests that the environments of unbarred spirals do not evolve over this difference in time while the environments of spirals evolve over this difference in time.

Table 5-45 KS Tests For Velocity Bins of $3000-6000$ km/s and $9000-12000$ km/s limited to unbarred spiral primaries

$\mathrm{Y}=$ dissimilar to the $99 \%$ level $\mathrm{N}=$ not dissimilar to the $99 \%$ level

\begin{tabular}{|c|c|c|c|c|c|c|c|c|c|}
\hline Run & $\begin{array}{l}\text { Fitting } \\
\text { Range } \\
\text { (Mpc) }\end{array}$ & $\begin{array}{l}\text { Num. } \\
\text { PD } \\
\text { Values } \\
\text { in } \\
3000 \\
\text { Bin } \\
\end{array}$ & $\begin{array}{c}\text { Num. } \\
\text { PD } \\
\text { Values } \\
\text { in } \\
9000 \\
\text { Bin } \\
\end{array}$ & Dissimilar & Run & $\begin{array}{l}\text { Fitting } \\
\text { Range } \\
\text { (Mpc) }\end{array}$ & $\begin{array}{c}\text { Num. } \\
\text { PD } \\
\text { Values } \\
\text { in } \\
3000 \\
\text { Bin } \\
\end{array}$ & $\begin{array}{c}\text { Num. } \\
\text { PD } \\
\text { Values } \\
\text { in } \\
9000 \\
\text { Bin } \\
\end{array}$ & Dissimilar \\
\hline$S_{u} A$ & 1 & 88 & 10 & $\underline{N}$ & $\mathrm{~S}_{\mathrm{u}} \mathrm{A}$ & 1 & 105 & 11 & $\underline{N}$ \\
\hline$S_{u} A$ & 2 & 182 & 35 & $\underline{N}$ & $\mathrm{~S}_{\mathrm{uA}}$ & 2 & 203 & 19 & $\underline{N}$ \\
\hline$S_{u} A$ & 3 & 249 & 73 & $\underline{N}$ & $\mathrm{SuA}_{\mathrm{u}}$ & 3 & 238 & 31 & $\underline{\underline{N}}$ \\
\hline$S_{u} A$ & 4 & 280 & 96 & $\underline{N}$ & $\mathrm{SuA}_{\mathrm{u}}$ & 4 & 247 & 44 & $\underline{N}$ \\
\hline$S_{u} A$ & 5 & 287 & 112 & $\underline{N}$ & $\mathrm{SuA}_{u}$ & 5 & 246 & 49 & $\underline{N}$ \\
\hline$S_{u} A$ & 10 & 263 & 137 & $\underline{N}$ & $\mathrm{SuA}_{\mathrm{u}}$ & 10 & 222 & 62 & $\underline{N}$ \\
\hline$S_{u} A$ & 15 & 232 & 137 & $\underline{N}$ & $S_{u} A$ & 15 & 184 & 60 & $\underline{N}$ \\
\hline$S_{u} A$ & 20 & 203 & 136 & $\underline{N}$ & $\mathrm{SuA}_{\mathrm{u}}$ & 20 & 164 & 59 & $\underline{N}$ \\
\hline$S_{u} A$ & 25 & 167 & 135 & $\underline{N}$ & $\mathrm{SuA}_{\mathrm{u}}$ & 25 & 131 & 56 & $\underline{N}$ \\
\hline$S_{u} A$ & 30 & 153 & 133 & $\underline{N}$ & $\mathrm{SuA}_{\mathrm{u}}$ & 30 & 110 & 55 & $\underline{N}$ \\
\hline$S_{u} E$ & 1 & 11 & 0 & N/A & $S_{u} E$ & 1 & 17 & 0 & N/A \\
\hline$S_{u} E$ & 2 & 34 & 2 & $\underline{N}$ & $\mathrm{~S}_{\mathrm{u}} \mathrm{E}$ & 2 & 53 & 2 & $\underline{N}$ \\
\hline$S_{u} E$ & 3 & 67 & 10 & $\underline{N}$ & $\mathrm{SuE}_{\mathrm{u}}$ & 3 & 99 & 5 & $\underline{N}$ \\
\hline$S_{u} E$ & 4 & 104 & 20 & $\underline{N}$ & $\mathrm{SuE}_{\mathrm{u}}$ & 4 & 134 & 6 & $\underline{N}$ \\
\hline$S_{u} E$ & 5 & 129 & 35 & $\underline{N}$ & $\mathrm{~S}_{\mathrm{u}} \mathrm{E}$ & 5 & 156 & 12 & $\underline{N}$ \\
\hline$S_{u} E$ & 10 & 235 & 96 & $\underline{N}$ & $\mathrm{~S}_{\mathrm{u}} \mathrm{E}$ & 10 & 207 & 32 & $\underline{N}$ \\
\hline$S_{u} E$ & 15 & 228 & 119 & $\underline{N}$ & $\mathrm{~S}_{\mathrm{u}} \mathrm{E}$ & 15 & 183 & 41 & $\underline{N}$ \\
\hline$S_{u} E$ & 20 & 203 & 135 & $\underline{N}$ & $\mathrm{SuE}_{\mathrm{u}}$ & 20 & 164 & 51 & $\underline{N}$ \\
\hline$S_{u} E$ & 25 & 167 & 135 & $\underline{N}$ & $S_{u} E$ & 25 & 131 & 54 & $\underline{N}$ \\
\hline$S_{u} E$ & 30 & 153 & 133 & $\underline{N}$ & $\mathrm{Su}_{\mathrm{u}}$ & 30 & 110 & 54 & $\underline{N}$ \\
\hline$S_{u L}$ & 1 & 18 & 1 & $\underline{N}$ & $\mathrm{Su}_{\mathrm{u}}$ & 1 & 20 & 0 & $\mathrm{~N} / \mathrm{A}$ \\
\hline$S_{u L}$ & 2 & 42 & 3 & $\underline{\mathrm{N}}$ & SuL & 2 & 75 & 0 & $\mathrm{~N} / \mathrm{A}$ \\
\hline$S_{u L}$ & 3 & 60 & 5 & $\underline{N}$ & $\mathrm{SuL}_{\mathrm{u}}$ & 3 & 117 & 3 & $\underline{N}$ \\
\hline SuL & 4 & 106 & 8 & $\underline{N}$ & SuL & 4 & 147 & 5 & $\underline{N}$ \\
\hline$S_{u L}$ & 5 & 140 & 13 & $\underline{N}$ & $\mathrm{SuL}_{\mathrm{u}}$ & 5 & 175 & 8 & $\underline{N}$ \\
\hline$S_{u L}$ & 10 & 234 & 63 & $\underline{N}$ & $\mathrm{SuL}_{\mathrm{u}}$ & 10 & 211 & 21 & $\underline{N}$ \\
\hline SuL & 15 & 227 & 103 & $\underline{N}$ & SuL & 15 & 184 & 41 & $\underline{N}$ \\
\hline$S_{u L}$ & 20 & 203 & 124 & $\underline{N}$ & SuL & 20 & 164 & 50 & $\underline{N}$ \\
\hline$S_{u L}$ & 25 & 167 & 132 & $\underline{\mathrm{N}}$ & $\mathrm{Su}_{u}$ & 25 & 131 & 51 & $\underline{\mathrm{N}}$ \\
\hline
\end{tabular}




\begin{tabular}{|c|c|c|c|c|}
\hline Run & $\begin{array}{l}\text { Fitting } \\
\text { Range } \\
\text { (Mpc) }\end{array}$ & $\begin{array}{l}\text { Num. } \\
\text { PD } \\
\text { Values } \\
\text { in } \\
3000 \\
\text { Bin } \\
\end{array}$ & $\begin{array}{c}\text { Num. } \\
\text { PD } \\
\text { Values } \\
\text { in } \\
9000 \\
\text { Bin } \\
\end{array}$ & Dissimilar \\
\hline$S_{u} L$ & 30 & 153 & 131 & $\underline{N}$ \\
\hline $\mathrm{SuS}$ & 1 & 42 & 1 & $\underline{N}$ \\
\hline SuS & 2 & 114 & 6 & $\underline{N}$ \\
\hline SuS & 3 & 193 & 21 & $\underline{N}$ \\
\hline SuS & 4 & 250 & 37 & $\underline{N}$ \\
\hline $\mathrm{SuS}$ & 5 & 272 & 53 & $\mathrm{~N}$ \\
\hline SuS & 10 & 262 & 112 & $\underline{N}$ \\
\hline SuS & 15 & 232 & 128 & $\mathbf{Y}$ \\
\hline SuS & 20 & 203 & 135 & $Y$ \\
\hline SuS & 25 & 167 & 135 & $\underline{N}$ \\
\hline $\mathrm{SuS}_{\mathrm{u}}$ & 30 & 153 & 133 & $\underline{N}$ \\
\hline$S_{u} S_{b}$ & 1 & 2 & 0 & N/A \\
\hline$S_{u} S_{b}$ & 2 & 11 & 0 & N/A \\
\hline$S_{u} S_{b}$ & 3 & 31 & 1 & $\underline{N}$ \\
\hline$S_{u} S_{b}$ & 4 & 64 & 4 & $\underline{N}$ \\
\hline$S_{u} S_{b}$ & 5 & 112 & 9 & $\underline{N}$ \\
\hline$S_{u} S_{b}$ & 10 & 236 & 38 & $\underline{N}$ \\
\hline$S_{u} S_{b}$ & 15 & 229 & 55 & $\underline{N}$ \\
\hline$S_{u} S_{b}$ & 20 & 203 & 79 & $\underline{\underline{N}}$ \\
\hline$S_{u} S_{b}$ & 25 & 167 & 104 & $\underline{N}$ \\
\hline$S_{u} S_{b}$ & 30 & 153 & 114 & $\underline{N}$ \\
\hline $\mathrm{S}_{\mathrm{u}} \mathrm{S}_{\mathrm{u}}$ & 1 & 2 & 0 & $\mathrm{~N} / \mathrm{A}$ \\
\hline $\mathrm{S}_{\mathrm{u}} \mathrm{S}_{\mathrm{u}}$ & 2 & 12 & 0 & N/A \\
\hline $\mathrm{S}_{\mathrm{u}} \mathrm{S}_{\mathrm{u}}$ & 3 & 34 & 0 & N/A \\
\hline $\mathrm{S}_{\mathrm{u}} \mathrm{S}_{\mathrm{u}}$ & 4 & 80 & 1 & $\underline{\mathrm{N}}$ \\
\hline $\mathrm{S}_{\mathrm{u}} \mathrm{S}_{\mathrm{u}}$ & 5 & 119 & 4 & $\underline{N}$ \\
\hline $\mathrm{SuS}_{\mathrm{u}}$ & 10 & 234 & 38 & $\underline{N}$ \\
\hline $\mathrm{SuS}_{\mathrm{u}}$ & 15 & 227 & 58 & $\underline{N}$ \\
\hline $\mathrm{S}_{\mathrm{u}} \mathrm{S}_{\mathrm{u}}$ & 20 & 203 & 82 & $Y$ \\
\hline $\mathrm{SuS}_{\mathrm{u}}$ & 25 & 167 & 112 & $Y$ \\
\hline $\mathrm{S}_{\mathrm{u}} \mathrm{S}_{\mathrm{u}}$ & 30 & 153 & 126 & $Y$ \\
\hline
\end{tabular}

\begin{tabular}{|c|c|c|c|c|}
\hline Run & $\begin{array}{l}\text { Fitting } \\
\text { Range } \\
\text { (Mpc) }\end{array}$ & $\begin{array}{l}\text { Num. } \\
\text { PD } \\
\text { Values } \\
\text { in } \\
3000 \\
\text { Bin }\end{array}$ & $\begin{array}{l}\text { Num. } \\
\text { PD } \\
\text { Values } \\
\text { in } \\
9000 \\
\text { Bin }\end{array}$ & Dissimilar \\
\hline$S_{u} L$ & 30 & 110 & 54 & $\underline{N}$ \\
\hline SuS & 1 & 43 & 0 & $\mathrm{~N} / \mathrm{A}$ \\
\hline SuS & 2 & 133 & 2 & $\underline{N}$ \\
\hline SuS & 3 & 202 & 6 & $\underline{N}$ \\
\hline SuS & 4 & 228 & 11 & $\underline{N}$ \\
\hline SuS & 5 & 237 & 16 & $\underline{N}$ \\
\hline SuS & 10 & 222 & 41 & $\underline{N}$ \\
\hline SuS & 15 & 184 & 57 & $Y$ \\
\hline SuS & 20 & 164 & 59 & $Y$ \\
\hline SuS & 25 & 131 & 56 & $Y$ \\
\hline SuS & 30 & 110 & 55 & $Y$ \\
\hline$S_{u} S_{b}$ & 1 & 3 & 0 & $\mathrm{~N} / \mathrm{A}$ \\
\hline $\mathrm{SuS}_{\mathrm{b}}$ & 2 & 26 & 1 & $\underline{N}$ \\
\hline$S_{u} S_{b}$ & 3 & 52 & 1 & $\underline{N}$ \\
\hline $\mathrm{SuS}_{\mathrm{b}}$ & 4 & 94 & 2 & $\underline{N}$ \\
\hline$S_{u} S_{b}$ & 5 & 129 & 2 & $\underline{N}$ \\
\hline $\mathrm{SuS}_{\mathrm{b}}$ & 10 & 197 & 11 & $\underline{N}$ \\
\hline$S_{u} S_{b}$ & 15 & 176 & 18 & $Y$ \\
\hline$S_{u} S_{b}$ & 20 & 164 & 32 & $Y$ \\
\hline$S_{u} S_{b}$ & 25 & 131 & 42 & $\mathbf{Y}$ \\
\hline$S_{u} S_{b}$ & 30 & 110 & 50 & $Y$ \\
\hline $\mathrm{SuS}_{\mathrm{u}}$ & 1 & 1 & 0 & $\mathrm{~N} / \mathrm{A}$ \\
\hline $\mathrm{SuS}_{\mathrm{u}}$ & 2 & 19 & 0 & $\mathrm{~N} / \mathrm{A}$ \\
\hline $\mathrm{SuS}_{\mathrm{u}}$ & 3 & 67 & 0 & $\mathrm{~N} / \mathrm{A}$ \\
\hline $\mathrm{S}_{\mathrm{u}} \mathrm{S}_{\mathrm{u}}$ & 4 & 102 & 0 & $\mathrm{~N} / \mathrm{A}$ \\
\hline $\mathrm{SuS}_{\mathrm{u}}$ & 5 & 142 & 2 & $\underline{N}$ \\
\hline $\mathrm{SuS}_{\mathrm{u}}$ & 10 & 193 & 8 & $\underline{\mathrm{N}}$ \\
\hline $\mathrm{SuS}_{\mathrm{u}}$ & 15 & 168 & 17 & $\underline{N}$ \\
\hline $\mathrm{SuS}_{\mathrm{u}}$ & 20 & 160 & 25 & $\underline{N}$ \\
\hline $\mathrm{SuS}_{\mathrm{u}}$ & 25 & 128 & 36 & $\underline{N}$ \\
\hline $\mathrm{S}_{\mathrm{u}} \mathrm{S}_{\mathrm{u}}$ & 30 & 110 & 46 & $\mathrm{~N}$ \\
\hline
\end{tabular}




\subsubsection{Conclusions}

The lack of dissimilarity for the environments of $S_{b}$ and $S_{u}$ combined with the finding of dissimilarity for the environments of $S$ is astounding. Remember, barred and unbarred spirals are still spiral galaxies; we just do not have clear data on the presence or lack of a bar structure for many of the S galaxies in 2MRS [19]. It does not make sense from the data that the environments of $S_{b}$ and $S_{u}$ are not impacted by this difference in time while the environments of $S$ are. This suggests further study and classification of barred or unbarred within the spiral category is critical.

Also interesting is the trend for disk-type galaxies to cluster differently around $\mathrm{L}$ and $\mathrm{S}$ primaries across the two bins. Perhaps the initial impetus for velocity during the era of galaxy formation imparted some rotation that lead to a different distribution of disk-type galaxies at various velocities.

Both of these findings demand further research on the impact of velocity on galactic environments. 


\section{Conclusions and Future Work}

\subsection{Scientific Results}

The scientific research conducted and discussed in this thesis is the first I am aware of that analyzes the environments of barred and unbarred spirals. The scientific findings of this thesis suggest new avenues of research and potential constraints on new theories of galactic evolution. Findings include:

- The environments of early-type and late-type galaxies are dissimilar. This aligns with research conducted by Dr. Best in 1999 [29].

- The environments of elliptical and lenticular galaxies are similar. This suggests the environment is not a factor in the formation of elliptical or lenticular galaxies.

- The environments of spirals, barred spirals, and unbarred spirals are generally similar. This suggests that secular evolution is responsible for the formation (or not) of bars in spiral galaxies.

- The clustering of disk-type (lenticular and spiral) galaxies around disk-type galaxies appears to evolve over time while the clustering of elliptical galaxies around all types of galaxies does not.

\subsection{Ease of Use}

The creation of Galactic provides a software tool that is easier to use than previous software. Users of Galactic do not need any formal experience in software development to add new data sets or to use the software. Galactic streamlines a process that, in the past, required four separate software tools as well as programming skills to maintain the software and analyze new data sets.

\subsection{Performance}

Galactic provides a substantial performance improvement over the previous software. Galactic is capable of processing data runs in parallel, whereas the previous software could only process serially. Performance improvements are also found through 
set based operations, made possible by the use of SQL Server. Improvements also are realized by eliminating the need to iterate the entire dataset. The previous software took hours or days to process a data run, while Galactic processes data runs in minutes or hours.

\subsection{Importance of Domain Knowledge and Understanding the User Experience}

We know from the literature that an engineer with expert level domain knowledge will better understand the needs of a system and make fewer mistakes in design [37]. This is confirmed by my experience during the creation of Galactic. Understanding the science was a critical early step in the design phase of the project.

I also find that understanding a user's interaction with the system provides the engineer with insight into design deficiencies. Because I used Galactic to conduct scientific research I was able to experience and acknowledge design flaws. I then was able to correct them. Microsoft refers to this as "dogfooding", because the engineer is eating his or her own dog food [65].

\subsection{Benefits of Cross-Field Collaboration}

The collaboration between myself, a software engineer, and Dr. Best, a scientist, has resulted in the creation of a software package that greatly eases the analyses of galactic environments. The formal training and experience in software development I have is directly related to the success of this project. I find that collaboration of this nature will advance computational science research. This aligns with work conducted very recently at the University of Washington [66] where the database group created software to assist the astrophysics group. Scientists should actively seek the assistance of software engineers who are experts in their field to assist in development of research software.

\subsection{Future Work}

Of interest for future work is the evolution of Galactic as it is used by Dr. Best and his students for research into other potential factors in the evolution of galaxies.

Further performance increases will be sought. One potential avenue for performance gains is distributed computing. Remote clients could communicate with the 
system over the existing presenter interface to check out a data run and download the data needed. Collecting the entire North or South dataset in JSON format over the presenter interface takes about 2 seconds. De-serializing from JSON to C\# objects would be acceptably fast. These client can process and asynchronously push the PD values for each galaxy via the JSON web service.

This is an approach worth serious consideration because this would allow a lab of workstations, such as Dr. Best has, to be pooled for more processing power.

\subsection{Source Code}

The source code to Galactic is available at the Galactic project page on Codeplex at http://galactic.codeplex.com. 


\section{References}

[1] Intel, "Moore's Law...," [Online]. Available:

http://www.intel.com/content/www/us/en/history/museum-gordon-moorelaw.html. [Accessed 0206 2014].

[2] Wikimedia Commons, "Transistor Count and Moore's Law," [Online]. Available: http://commons.wikimedia.org/wiki/File:Transistor_Count_and_Moore\%27s_La w_-_2011.svg. [Accessed 0206 2014].

[3] NASA, "Hubble Story," [Online]. Available: http://www.nasa.gov/mission_pages/hubble/story/index.html\#.U4y21nJdWgs. [Accessed 0206 2014].

[4] NASA, "Hubble Servicing Missions," [Online]. Available: http://www.nasa.gov/mission_pages/hubble/servicing/index.html\#.U4y3iXJdWg s. [Accessed 0206 2014].

[5] NASA, "Chandra X-Ray Observatory," [Online]. Available: http://chandra.harvard.edu/about/axaf_mission.html. [Accessed 0206 2014].

[6] E. Hubble, "Extra-Galactic Nebulae," The Astrophysical Journal, no. 64, pp. 321-369, 1926.

[7] NASA, "Hubblesite.org - Hubble Tuning Fork Diagram," [Online]. Available: http://hubblesite.org/newscenter/archive/releases/1999/34/image/o/format/large _web/. [Accessed 0206 2014].

[8] NASA, "Dark Energy and Dark Matter," [Online]. Available: http://science.nasa.gov/astrophysics/focus-areas/what-is-dark-energy/. [Accessed 0206 2014].

[9] R. Tully and J. Fisher, Astronomy and Astrophysics, vol. 54, p. 661, 1977.

[10] S. Faber and R. Jackson, "Velocity Dispersions and Mass-To-Light Ratios for Elliptical Galaxies," The Astrophysical Journal, vol. 204, p. 668, 1976. 
[11] NASA/STSI, "hubblesite.org," [Online]. Available: http://hubblesite.org/newscenter/archive/releases/2008/07/image/a/. [Accessed 0907 2014].

[12] NASA, [Online]. Available: http://www.nasa.gov/content/goddard/hubble-eyesa-mysterious-old-spiral/\#.U73OKPIdWtI. [Accessed 0907 2014].

[13] NASA, [Online]. Available:

http://www.nasa.gov/multimedia/imagegallery/image_feature_2132.html. [Accessed 0907 2014].

[14] NASA, "hubblesite.org," [Online]. Available: http://hubblesite.org/newscenter/archive/releases/2005/01/image/a/. [Accessed 0907 2014].

[15] NASA, [Online]. Available:

http://hubblesite.org/newscenter/archive/releases/1999/25/image/a/. [Accessed 0907 2014].

[16] Wikipedia, "Visible Light Spectrum," [Online]. Available: http://en.wikipedia.org/wiki/Visible_spectrum\#mediaviewer/File:Linear_visible_s pectrum.svg. [Accessed 1007 2014].

[17] E. Hubble, "A relation between distance and radial velocity among extragalactic nebulae," Proceedings of the National Academy of Sciences, vol. 15, p. 168, 1929.

[18] E. Hubble and M. Humason, "The Velocity-Distance Relation Among ExtraGalactic Nebulae," The Astrophysical Journal, vol. 74, p. 43, 1931.

[19] J. Huchra, L. Macri, L. Masters and et. al, "The 2MASS Redshift Survey Description and Data Release," The Astrophysical Journal Supplement Series, vol. 199, no. 2, 2012.

[20] R. Buta, Four lectures to be published in Secular Evolution of Galaxies, Cambridge University Press, in press, 2013.

[21] A. Oemler, "The Systematic Properties of Clusters of Galaxies. I. Photometry of 15 Clusters," The Astrophysical Journal, vol. 194, pp. 1-19, 1974. 
[22] M. Davis and M. Geller, "Galaxy Correlatins as a Function of Morphological Type," The Astrophysical Journal, vol. 208, pp. 13-19, 1976.

[23] A. Dressler, "Galaxy morphology in rich clusters - Implications for the formation and evolution of galaxies," The Astrophysical Journal, vol. 236, p. 351, 1980.

[24] Y. Peng and e. al, "MASS AND ENVIRONMENT AS DRIVERS OF GALAXY EVOLUTION IN SDSS AND zCOSMOS AND THE ORIGIN OF THE SCHECHTER FUNCTION," The Astrophysical Journal, vol. 721, p. 193, 2010.

[25] J. Lotz and e. al., "THE MAJOR AND MINOR GALAXY MERGER RATES AT z<1.5," The Astrophysical Journal, vol. 742, p. 103, 2011.

[26] J. Hibbard and J. van Gorken, "H I, H II, and R-band Observations of a Galactic Merger Sequence," The Astronomical Journal, vol. 111, no. 2, p. 655, 1996.

[27] T. Naab, P. Johansson and J. Ostriker, "Minor mergers and the size evolution of elliptical galaxies," The Astrophysical Journal Letters, vol. 699, no. 2, 2009.

[28] J. Best, "An Examination of the Large-Scale Clustering of the Las Campanas Redshift Survey," The Astrophysical Journal, no. 541, p. 519, 2000.

[29] J. Best, "Analyzing the Dressler Catalog using the Pointwise Dimension," The Astrophysical Journal, no. 520, p. 507, 1999.

[30] J. Best, J. Charlton and G. Mayer-Kress, "Analysis of Galaxy Morphology and Evolution using the Pointwise Dimension," The Astrophysical Journal, no. 496, p. 55, 1996.

[31] M. D. Moore, "Galactic," [Online]. Available: http://galactic.codeplex.com.

[32] S. Schectman and e. al., "The Las Campanas Redshift Survey," The Astrophysical Journal, no. 470, p. 172, 1996.

[33] S. Shandarin and C. Yess, "Detection of network structure in the Las Campanas Redshift Survey," The Astrophysical Journal, no. 505, p. 12, 1998.

[34] K. Wu, O. Lahav and M. Rees, "The large-scale smoothness of the universe," Nature, vol. 397, p. 225, 1999. 
[35] A. Dressler, "A catalog of morphological types in 55 rich clusters of galaxies," The Astrophysical Journal Supplement Series, no. 42, pp. 565-609, 1980.

[36] B. Mandelbrot, The Fractal Geometry of Nature, New York: Freeman, 1983.

[37] A. von Mayrhauser and A. Vans, "Program Comprehension During Software Maintenance and Evolution," Computer, vol. 28, no. 8, pp. 44-55, 1995.

[38] "Model View Presenter," [Online]. Available:

http://en.wikipedia.org/wiki/Model\%E2\%80\%93view\%E2\%80\%93presenter\#me diaviewer/File:Model_View_Presenter.png. [Accessed 0807 2014].

[39] S. Haefliger, G. von Krogh and S. Spaeth, "Code Reuse in Open Source Software," MANAGEMENT SCIENC, vol. 54, no. 1, pp. 180-193, 2008.

[40] The jQuery Foundation, "jQuery," [Online]. Available: http://jquery.com.

[41] The jQuery Foundation, "jQuery UI," [Online]. Available: http:/jqueryui.com.

[42] D. Gandy, "Font Awesome," [Online]. Available: http://fontawesome.github.io.

[43] IOLA and O. Laursen, "Flot," [Online]. Available: http://www.flotcharts.org.

[44] J. Newton-King, "Json.NET," [Online]. Available: http://json.codeplex.com.

[45] C. R. de Souza, "Accord.Net Framework," [Online]. Available: http://accordframework.net.

[46] S. Rutzky, "SQL Sharp," [Online]. Available: http://www.sqlsharp.com/.

[47] Newegg, "Newegg," [Online]. Available: http://www.newegg.com.

[48] Microsoft, "Codeplex," [Online]. Available: http://www.codeplex.com.

[49] Oracle, VirtualBox, "VirtualBox," [Online]. Available: http://www.virtualbox.org.

[50] 2dFGRS Team, "VizieR Astronomical Server," [Online]. Available: vizier.cfa.harvard.edu. [Accessed 0306 2013]. 
[51] B. Love and J. Best, "Analysis of Luminosity and Redshift Dependence of the Large-Scale Distribtion of Quasars within the Sloan Digital Sky Survey," Proceedings of the West Virginia Academy of Science, 2014, in press.

[52] A. Bianchi, D. Caivano, V. Marengo and G. Visaggio, "Iterative Reengineering of Legacy Systems," IEEE TRANSACTIONS ON SOFTWARE ENGINEERING, vol. 29, no. 3, p. 225, 2003.

[53] D. L. Parnas, "Software Aging," Proceedings of the 16th international conference on Software engineering, pp. 279-287, 1994.

[54] J. D. Mooney, "Bringing Portability to the Software Process," 05 1997. [Online]. Available: http://www.csee.wvu.edu/ jdm/classes/cs533/notes/refs/trsproc.html. [Accessed 1007 2014].

[55] Superblock, LLC, "Pushover," [Online]. Available: https://pushover.net.

[56] G. Wilson and et al, "Best practices for scientific computing," PLoS biology, vol. 12, no. 1, 2014.

[57] Microsoft, "Technet," [Online]. Available: http://technet.microsoft.com/enus/library/bb964737.aspx. [Accessed 2105 2014].

[58] Microsoft, "Technet," [Online]. Available: http://technet.microsoft.com/enus/library/bb933952.aspx. [Accessed 2105 2014].

[59] Microsoft, "Technet," [Online]. Available: http://technet.microsoft.com/enus/library/bb964707.aspx. [Accessed 2105 2014].

[60] Microsoft, "Technet," [Online]. Available: http://msdn.microsoft.com/enus/library/ms179859.aspx. [Accessed 2105 2014].

[61] Microsoft, "Using PIVOT and UNPIVOT," [Online]. Available: http://technet.microsoft.com/en-us/library/ms177410(v=sql.105).aspx.

[62] $\mathrm{NIH}$, "Color blindness," [Online]. Available: http://www.ncbi.nlm.nih.gov/pubmedhealth/PMH0001997/. [Accessed 1007 2014]. 
[63] Microsoft, "MSDN - SQL AVG," [Online]. Available: http://msdn.microsoft.com/en-us/library/ms177677.aspx. [Accessed 1206 2014].

[64] Microsoft, "MSDN - SQL STDEVP," [Online]. Available: http://msdn.microsoft.com/en-us/library/ms176080.aspx. [Accessed 1206 2014].

[65] Microsoft, "Technet - About Early Technology Adoption (Dogfooding)," [Online]. Available: http://technet.microsoft.com/en-us/library/cc627315.aspx.

[66] S. Loebman, "Big-Data Management Use-Case: A Cloud Service for Creating and Analyzing Galactic Merger Trees," 2014. [Online]. Available: http://homes.cs.washington.edu/ magda/papers/loebman-danac14.pdf. 